



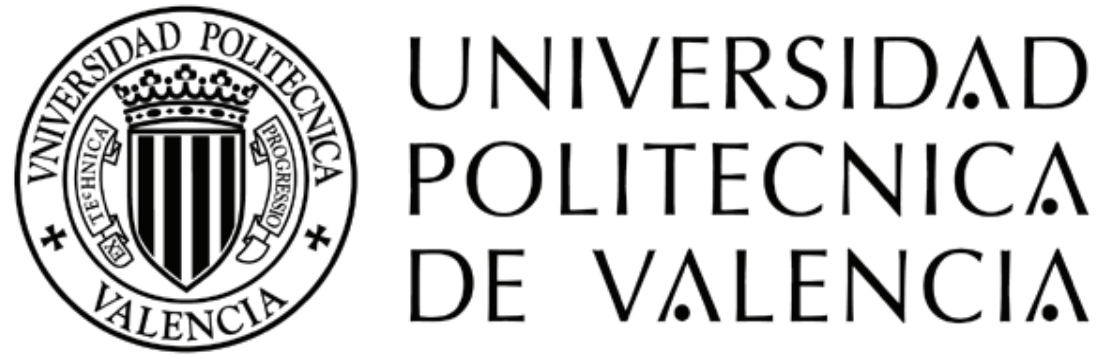

\section{DEPARTAMENTO DE INGENIERÍA ELÉCTRICA}

\section{TESIS DOCTORAL}

SIGNIFICADO Y MEDIDA DE LOS FENÓMENOS DE DESFASE

EN LOS SISTEMAS TRIFÁSICOS DESEQUILIBRADOS, LINEALES.

APLICACIÓN A LA MEDIDA EN SISTEMAS CON CONDUCTOR NEUTRO.

Doctorando: Manuel Ángel Graña López

Dirigida por: Dr. Vicente León Martínez

Dr. Joaquín Montañana Romeu 



\section{Agradecimientos.}

En la vida casi nada surge de manera espontánea, y la mayor parte de las cosas se logran con un mayor o menor esfuerzo, por mi parte he de reconocer y agradecer públicamente que para que pudiera llevarse a cabo esta tesis doctoral, además de mi esfuerzo he contado con el apoyo de otros muchos que en su medida han estado ahí para que ahora yo pueda escribir estas líneas.

No me cabe duda alguna que mi mayor agradecimiento, respeto y amistad es para mis dos directores de tesis los profesores D. Vicente León y D. Joaquín Montañana. A Vicente por lo mucho que me ha enseñado y la facilidad con la que me ha transmitido sus conocimientos y sobre todo la paciencia y generosidad que ha tenido para ello, además de sus palabras de motivación y ánimo siempre que me hicieron falta. Por otra parte, Joaquín me ha mostrado que un ingeniero además de pensar ha de saber llevar sus pensamientos a la práctica, y que no todo sale siempre a la primera, lo que no debe ser razón para abandonar, sino que debe servirnos de acicate, también ha demostrado su paciencia conmigo a la vez que me inculcaba nuevas inquietudes.

También quisiera recordar a las personas, que siempre me han mostrado su incondicional apoyo y que forman parte de los "otros muchos", entre los que están Ana, Jesús, Antonio y Carlos. 

PRóLOGO 



\section{Prólogo.}

Dentro del mundo de la electrotecnia, la identificación y cuantificación de los fenómenos presentes en toda transferencia de energía eléctrica es clave tanto por aspectos técnicos, como económicos. Desde principios del siglo $X X$, se vio que era necesario además de contabilizar la energía activa consumida, también la energía reactiva, siendo ambas magnitudes fundamentales para llevar a cabo la facturación de la energía consumida.

La energía reactiva es una ineficiencia de los sistemas eléctricos, puesto que no se transforma en otro tipo de energía aprovechable y, por tanto, no puede ser utilizada. Además, la energía reactiva disminuye el valor del factor de potencia y reduce la capacidad de los sistemas eléctricos para transferir la energía útil o activa, siendo una de las causas de los cortes en el suministro. Estos efectos de la energía reactiva se traducen en la práctica en un sobredimensionado de las instalaciones, transformadores y generadores eléctricos, que deben ser diseñados para mayores valores de corrientes y potencias, redundando todo ello en un encarecimiento de los sistemas eléctricos, que repercute finalmente sobre los usuarios, en la facturación. Asimismo, el suministro de energía reactiva está reglamentado. El Reglamento Electrotécnico para Baja Tensión prohíbe que las instalaciones eléctricas puedan llegar a ser capacitivas, es decir, que sus dispositivos de compensación suministren energía reactiva a la red eléctrica. También está reglamentado el suministro de energía reactiva en parques eólicos. Los países productores de este tipo de energía establecen, en los llamados códigos de red, las condiciones para el suministro de energía reactiva bajo determinados regímenes de funcionamiento, como en presencia de huecos de tensión. Todo lo anterior justifica la necesidad de conocer y medir correctamente el fenómeno de la energía reactiva.

La presente Tesis Doctoral está dedicada al estudio de la energía reactiva en sistemas trifásicos sinusoidales, desequilibrados, con hilo neutro, aunque las 
conclusiones pueden ser aplicadas también a sistemas no sinusoidales, como se explicará más adelante.

En primer lugar, se ha analizado críticamente el concepto y formulación de la potencia reactiva establecido por las principales teorías de la potencia eléctrica desarrolladas desde finales del siglo XIX. Estas teorías atribuyen el fenómeno de la reactiva a dos causas: 1) la presencia de reactancias en los sistemas eléctricos y 2) el funcionamiento de los convertidores electrónicos controlados existentes en los sistemas eléctricos, para la regulación de motores, la inversión de la corriente continua en generadores de energía solar fotovoltaica, entre otras aplicaciones. Sin embargo, ninguna de estas teorías ha propuesto la existencia de fenómenos reactivos causados únicamente por los desequilibrios. Únicamente Jeon [104] insinuó en 2006 la posible existencia de una potencia reactiva debida a los desequilibrios, aunque sin llegar a formularla y más recientemente [32], cuando la presente tesis ya había sido finalizada, se ha tenido conocimiento de una potencia reactiva debida a los desequilibrios propuesta por L.S. Czarnecki, cuando el autor de esta tesis ya había formulado y modelizado este tipo de potencia reactiva con bastante anterioridad $[113,114]$.

La potencia reactiva definida por L.S. Czarnecki para los sistemas trifásicos, desequilibrados, lineales, tiene dos componentes una debida a las reactancias y otra debida a los desequilibrios. Ambas se obtienen a partir de la potencia reactiva tradicional y sus expresiones sólo son válidas para sistemas a tres hilos (sin neutro), que son los menos usuales, y no pueden ser aplicadas a sistemas no sinusoidales (distorsionados). Además, las corrientes reactivas que definen y transfieren a estas potencias no verifican la ley de Conservación de la Carga Eléctrica (Primera Ley de Kirchhoff) y, por tanto, no son magnitudes físicas, sino sólo formulaciones matemáticas; todo ello debido a que para la obtención de estas corrientes no se utiliza la carga real sino un modelo equivalente en cuanto a las potencias activa y reactiva, ni tampoco se trabaja con las tensiones reales sino con un sistema de tensiones simples (fase-neutro) que tiene las mismas componentes directa e inversa que el sistema real, pero sin componente homopolar. 
La explicación de los fenómenos reactivos que se hace en la presente Tesis Doctoral sigue las leyes y teoremas conocidos de la Teoría de Circuitos. En este sentido todas las magnitudes que se establecen tienen significado físico, verifican las leyes de Kirchhoff y el Principio de Superposición Lineal. Los fenómenos reactivos se manifiestan siempre que haya desfase entre las ondas de tensión y de corriente, de aquí que deberían ser llamados más correctamente como fenómenos de desfase y no reactivos, como tradicionalmente se les conoce, porque pueden presentarse incluso sin la presencia de reactancias en el sistema. En la Tesis se les continuará denominando fenómenos reactivos o de desfase, indistintamente.

Las expresiones de las potencias reactivas se obtienen en esta Tesis Doctoral por aplicación de la Teoría Unificadora de la Potencia Eléctrica [123]. El empleo de esta teoría está avalado por la IEEE Standard 1459-2000 y la expresión de la potencia reactiva que se deduce de ella es aplicable a todo tipo de sistemas eléctricos: equilibrados y desequilibrados, sinusoidales y no sinusoidales, a tres y a cuatro hilos; por tanto, es una formulación general, en contraposición a la planteada por L.S, Czarnecki, que está muy limitada en cuanto a resultados y a su aplicación, como se señaló en párrafos anteriores.

La potencia reactiva que se formula para los sistemas trifásicos, en general, se manifiesta siempre que haya desfases entre las componentes fundamentales de secuencia directa de las tensiones y corrientes. Tiene dos componentes: la tradicionalmente conocida debida a las reactancias $y / 0$ convertidores electrónicos, y la producida exclusivamente por los desequilibrios. Se demuestra en la tesis que esta última potencia reactiva depende de los desequilibrios de excitaciones y cargas, conjuntamente; no aparece cuando las tensiones o las cargas son equilibradas, o cuando exista una cierta simetría en el circuito.

Además, la potencia reactiva debida a los desequilibrios puede manifestarse incluso con cargas resistivas, ya que depende de la existencia de desfases entre tensiones y corrientes. Esta es una diferencia respecto de la potencia reactiva 
debida a los desequilibrios de Czarnecki*, que sólo existe cuando hay cargas reactivas.

Las dos componentes de la potencia reactiva pueden tener los mismos signos o contrarios y, en este último caso, se compensan.

En el primer capítulo de la Tesis se presentan y analizan críticamente las principales teorías de la potencia eléctrica partiendo de Steinmetz, quién a finales del siglo XIX fue el primero en definir y formular la potencia reactiva, aunque sólo en sistemas monofásicos lineales. También se han estudiado las teorías de la potencia eléctrica desarrolladas por Budeanu y por Fryze durante las décadas de los 20 y 30 del pasado siglo XX, dado que a partir de ellas se han desarrollado las modernas teorías de la potencia eléctrica aplicadas a los sistemas trifásicos. Finalmente, se han analizado las principales teorías de los autores contemporáneos, algunas de las cuales están incluidas en la IEEE Standard 14592000, siendo este estudio aplicado al fenómeno de la reactiva en los sistemas trifásicos.

El segundo capítulo de la Tesis está dedicado a la formulación de la potencia reactiva en los sistemas trifásicos. Constituye este capítulo la aportación fundamental de la Tesis desde el punto de vista teórico. Las expresiones de la potencia reactiva se obtienen para sistemas a tres y a cuatro hilos, siendo estos últimos el objetivo perseguido en la Tesis. Se comparan finalmente las expresiones de la potencia reactiva con las que resultan de las teorías Clásicas (Budeanu).

En el tercer capítulo, los fenómenos reactivos son representados gráficamente mediante asociaciones trifásicas de elementos de circuito. Estas asociaciones son útiles para visualizar por separado los efectos de los desequilibrios y de las

\footnotetext{
* L.S. Czarnecki. "Physical Interpretation of the Reactive Power in Terms of the CPC Power Theory". Electrical Power Quality and Utilisation Journal. Vol XIII, nº 1, 2007, pp. 89-95.
} 
reactancias sobre el fenómeno de la reactiva. Asimismo, también permiten obtener las expresiones de la potencia reactiva, como suma de las potencias reactivas correspondientes a cada uno de sus elementos.

La parte experimental de la Tesis se encuentra desarrollada en el cuarto y quinto capítulos. En el cuarto capítulo se describe el instrumento de medida que se ha utilizado para la medida de la potencia reactiva, basada en las formulaciones desarrolladas en el segundo capítulo. Este dispositivo de medida es original y no existe en el mercado. En el quinto capítulo se ha comprobado prácticamente la bondad de la formulación propuesta para la potencia reactiva, tanto utilizando un software de simulación apropiado, como utilizando el instrumento de medida sobre instalaciones industriales y urbanas. Con la simulación, se han obtenido resultados muy interesantes en cuanto a la evolución de la potencia reactiva debida a los desequilibrios en función del grado de desequilibrio y de los argumentos de las tensiones. Estos resultados pueden ser muy útiles para la generación o la compensación de este tipo de potencia reactiva.

Finalmente, los capítulos VI y VII se han dedicado a "Conclusiones y realizaciones futuras" y "Bibliografía", respectivamente. Se ha utilizado un total de 186 referencias bibliográficas relacionadas con el tema de la Tesis y con los fenómenos de los sistemas eléctricos, en general. Unas referencias están más próximas a la principal aportación de la Tesis, aunque también se han utilizado otras más alejadas, como es el caso de las teorías p-q-r, muy utilizadas actualmente para ala realización de circuitos de control de filtros activos, pero que no son útiles, a mi juicio, para explicar y formular los fenómenos reactivos, dado que no separan adecuadamente los fenómenos correspondientes a las ineficiencias (reactiva, desequilibrios y distorsión).

En resumen, la nueva formulación de la potencia reactiva desarrollada en esta Tesis Doctoral, constituye, a mi juicio, una importante herramienta para mejor comprender el funcionamiento de los sistemas eléctricos, así como para proponer soluciones para el cumplimiento de las normativas vigentes y para el diseño de dispositivos de mejora de la eficiencia. 

ÍNDICE 

ÍNDICE.

\section{CAPÍTULO I. ANTECEDENTES.}

I.1. Introducción

I.2. Teoría de Steinmetz

I.2.1. Sistema monofásico lineal

$\begin{array}{lr}\text { I.2.2. Sistema trifásico lineal } & 8\end{array}$

I.3. Teoría de Budeanu 13

I.4. Teoría de Fryze 18

I.5. Teoría de la Potencia Instantánea de Akagi 22

I.6. Teoría de Czarnecki 29

I.7. Teoría Unificadora de la Potencia Eléctrica 34

I.8. IEEE Std. 1459/2000 39

I.8.1. Teoría de Emanuel $\quad 42$

$\begin{array}{ll}\text { I.9. Conclusiones } & 48\end{array}$

\section{CAPÍtUlO II. FENÓMENOS DE DESFASE EN SISTEMAS TRIFÁSICOS DESEQUILIBRADOS LINEALES.}

II.1. Introducción

II.2. Fenómenos de desfase en sistemas desequilibrados lineales en

Triángulo. Tensiones Equilibradas

II.2.1. Teoría Clásica

II.2.2. Teoría Unificadora

II.3. Fenómenos de desfase en sistemas desequilibrados lineales en Triángulo. Tensiones Desequilibradas.

II.3.1. Teoría Clásica

II.3.2. Teoría Unificadora

II.4. Fenómenos de desfase en sistemas desequilibrados lineales con neutro. Tensiones Equilibradas. 
II.5. Fenómenos de desfase en sistemas desequilibrados lineales con neutro. Tensiones Desequilibradas. 73

II.5.1. Teoría Clásica

II.5.2. Teoría Unificadora

II.6. Conclusiones 86

\section{CAPÍTULO III. REPRESENTACIÓN DEL FENÓMENO DEL DESFASE.}

$\begin{array}{ll}\text { III.1. Introducción. } & 91\end{array}$

III.2. Representación del desfase en sistemas en triángulo. 93

III.2.1. Tensiones equilibradas. 93

III.2.2. Tensiones desequilibradas. 95

III.2.3. Caso particular de receptor puramente resistivo. 98

III.3. Representación del desfase en sistemas en estrella con neutro. 102

$\begin{array}{ll}\text { III.3.1. Tensiones equilibradas. } & 102\end{array}$

III.3.2. Tensiones desequilibradas. 104

III.3.3. Caso particular de receptor puramente resistivo. 108

III.4. Conclusiones. 112

\section{CAPÍTULO IV. DESCRIPCIÓN DEL DISPOSITIVO DE MEDIDA.}

$\begin{array}{ll}\text { IV.1. Introducción. } & 117\end{array}$

IV.2. Constitución del sistema de medida. Hardware y software. 118

$\begin{array}{ll}\text { IV.2.1. Hardware } & 118\end{array}$

IV.2.2. Software. Procedimiento de medida de los módulos de programa. 119

IV.3. Descripción de las funciones del dispositivo 124

IV.3.1. Pantalla principal 124

$\begin{array}{ll}\text { IV.4. Imágenes del dispositivo de medida } & 127\end{array}$

$\begin{array}{ll}\text { IV.5. Esquemas de montaje } & 128\end{array}$

IV.5.1. Medida directa de hasta 25 A 129

$\begin{array}{ll}\text { IV.5.2. Medida indirecta hasta } 10.000 \mathrm{~A} & 130\end{array}$ 


\section{COMPROBACIÓN EXPERIMENTAL.}

$\begin{array}{lr}\text { V.1. Introducción. } & 135\end{array}$

$\begin{array}{ll}\text { V.2. Proceso experimental. } & 136\end{array}$

V.3. Medidas experimentales obtenidas con la simulación. 136

V.3.1. Carga monofásica entre fase y neutro. 137

V.3.2. Cargas conectadas entre dos fases y el neutro. 222

V.3.3. Cargas monofásicas entre fase y neutro. Vario módulos de tensión. 246

V.4. Medidas experimentales obtenidas en centros de transformación. 254

V.4.1. C.T. Industrial no 1.

V.4.2. C.T. Industrial no 2 . 256

V.4.3. C.T. Industrial no 3.

V.4.4. C.T. Industrial no 4.

V.4.5. C.T. Higinio Noja. $\quad 259$

V.4.6. C.T. Polígono de Viviendas. 260

V.4.7. CT Línea Residencial no 56 de la Calle Paz 261

V.4.8. CT Línea Residencial no 57 de la Calle Paz 262

V.5. Fuente trifásica programable 263

V.6. Medidas experimentales obtenidas en el laboratorio 266

$\begin{array}{ll}\text { V.7. Conclusiones. } & 274\end{array}$

CAPÍTULO VI. CONCLUSIONES Y FUTURAS REALIZACIONES.

$\begin{array}{lr}\text { VI.1. Conclusiones. } & 279\end{array}$

$\begin{array}{ll}\text { VI.2. Futuras realizaciones. } & 281\end{array}$

\section{CAPÍTULO VII. BIBLIOGRAFÍA}

$\begin{array}{ll}\text { VII. Bibliografía. } & 285\end{array}$ 

CAPÍTULO I ANTECEDENTES. 



\section{I.- Antecedentes.}

\section{I.1.- Introducción.}

Desde el origen de la distribución de la energía eléctrica mediante el uso de la corriente alterna, se puso de manifiesto a través de trabajos como los de Shallenberg y Santley ${ }^{1}$ en el 1888, y de manera independiente, la presencia de fenómenos reactivos. Por lo tanto desde finales del siglo XIX y hasta nuestros días, el fenómeno de la reactiva ha sido un tema que interesa en gran medida a los ingenieros eléctricos, ya que la medida de la energía que se transfiere en un sistema posee unas connotaciones comerciales, y es necesario identificar y cuantificar los fenómenos energéticos que se ponen en juego.

En 1897 C.P. Steinmetz explica de manera analítica el funcionamiento de los sistemas monofásicos lineales, estableciendo el fenómeno de la reactiva que se ponía de manifiesto mediante flujos de potencia reactiva instantánea sinusoidales, siendo la amplitud de estos flujos lo que definió como la potencia reactiva Q. Esta teoría aún esta vigente en nuestros días y queda recogida en la IEEE Standard $1459 / 2000$ [102].

Igualmente, se halla recogido implícitamente en [102] que el fenómeno de la reactiva en los sistemas trifásicos lineales, equilibrados y desequilibrados en cargas, se manifiesta mediante tres flujos de potencia reactiva instantánea, equilibrados y sinusoidales, producidos por las tensiones y corrientes de la misma secuencia de fases que las excitaciones. Las discrepancias en la Comunidad Científica se presentan al explicar el fenómeno de la reactiva cuando las tensiones de excitación son desequilibradas.

\footnotetext{
${ }^{1}$ W.Stanley. "Phenomena of retardation in the Induction Coil". A.I.E.E., Vol. V, № 4, Juanary 1888, pp.97-115.

O.B. Shallenberger. "The Disribution of Electricity by Alternative Current". Electrical World, March 3, 1888, pp.114-115.
} 
Antecedentes.

A juicio del autor de esta Tesis, el fenómeno de la reactiva en los sistema trifásicos se manifiesta siempre de la misma manera: mediante tres flujos sinusoidales y equilibrados, debidos a las tensiones y corrientes de secuencia directa y frecuencia fundamental, independientemente de que el sistema de generación sea equilibrado o desequilibrado o de que el sistema sea o no lineal.

También Emanuel asume implícitamente, esta tesis al definir la potencia de desequilibrio y la potencia aparente no fundamental separándolas de las potencias activa y reactiva de secuencia directa y frecuencia fundamental, tal y como establece en [102].

Esta descripción de los fenómenos reactivos permite conocerlos mejor e, incluso, encontrar nuevas causas que los producen. Así, los primeros fenómenos reactivos conocidos fueron los producidos por las reactancias, de aquí el nombre que se les asignó. Más reciente es la presencia de fenómenos reactivos en los sistemas con convertidores electrónicos controlados, aún no existiendo reactancias en ellos.

En esta Tesis se describen y formulan los fenómenos reactivos causados por los desequilibrios en los sistemas lineales, cuya existencia ya se había adelantado en [113] y [114], y que recientemente han sido apuntados por Jeon [104].

A lo largo de este capítulo se expondrán las teorías sobre el fenómeno reactivo en los sistemas eléctricos de: Steinmetz, Budeanu, Fryze, Akagi, Czarnecki, Emanuel, León, y la norma IEEE Standard 1459/2000. 


\section{2.- Teoría de Steinmetz.}

\section{I.2.1.- Sistema Monofásico Lineal.}

En 1897, C. P. Steinmetz ${ }^{2}$ establece su "Teoría del fenómeno de la corriente alterna", en el que explicaba porqué en corriente alterna no toda la potencia eléctrica se transformaba en energía útil. Tomando como valores instantáneos de la tensión e intensidad de un generador monofásico los siguientes:

$$
\begin{aligned}
& v(t)=V \cdot \sqrt{2} \cdot \operatorname{sen} \omega t \\
& i(t)=I \cdot \sqrt{2} \cdot \operatorname{sen}(\omega t-\varphi)
\end{aligned}
$$
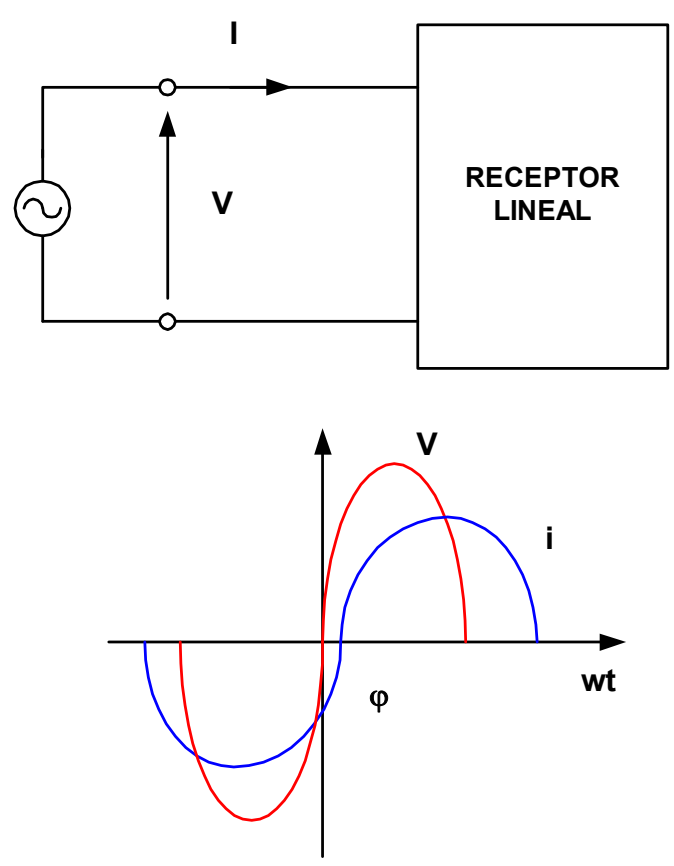

Figura I.1. Teoría Steinmetz. Monofásico lineal.

La corriente que consume el receptor, como se muestra en la figura I.1 es sinusoidal al igual que la tensión, y de la misma frecuencia, pero desfasada un ángulo $\varphi$. Podemos descomponer esta corriente en dos términos, según el fenómeno que representen:

\footnotetext{
${ }^{2}$ C. P. Steinmetz, 'Theory and Calculation of Alternating Current Phenomena', McGraw-Hill, New York,
} 1897. 


$$
\begin{aligned}
& i(t)=i_{a}(t)+i_{r}(t) \\
& i_{a}(t)=I \sqrt{2} \cdot \cos \varphi \cdot \operatorname{sen} \omega t \\
& i_{r}(t)=I \sqrt{2} \cdot \operatorname{sen} \varphi \cdot \operatorname{sen}(\omega t-\pi / 2)
\end{aligned}
$$

Tenemos por lo tanto, una corriente $i_{a}(t)$ que es la corriente activa, y que es la responsable del fenómeno de la transferencia de energía neta; y la $i_{r}(t)$ que representa la corriente reactiva y es la responsable del fenómeno del desfase.

Los fenómenos energéticos, los hallamos reflejados en las componentes de la potencia instantánea que viene dada por la siguiente expresión:

$$
\begin{aligned}
& p(t)=v(t) \cdot i(t) \\
& p(t)=V \cdot I \cdot \cos \varphi \cdot(1-\cos 2 \omega t)-V \cdot I \cdot \operatorname{sen} \varphi \cdot \operatorname{sen} 2 \omega t
\end{aligned}
$$

en la que podemos observar dos términos, el primer término denominado potencia activa instantánea $p a(t)$, que caracteriza la transferencia neta de energía:

$$
p a(t)=p(t)=V \cdot I \cdot \cos \varphi \cdot(1-\cos 2 \omega t)
$$

y cuyo valor medio es distinto de cero e igual a la Potencia Activa:

$$
P=\frac{1}{T} \int_{0}^{T} p(t) \cdot d t=V \cdot I \cdot \cos \varphi
$$

y el segundo término es la denominada potencia reactiva instantánea $\operatorname{pr}(t)$.

$$
\operatorname{pr}(t)=-V \cdot I \cdot \operatorname{sen} \varphi \cdot \operatorname{sen} 2 \omega t
$$

en donde observamos que la potencia reactiva instantánea es senoidal y de frecuencia doble que la tensión y la corriente, siendo además su valor medio nulo. 
La energía reactiva esta expresada pues por la componente reactiva de la potencia instantánea y se manifiesta mediante un flujo senoidal de potencia, que es cuantificado por lo que denominamos Potencia Reactiva .

$$
Q=V \cdot I \cdot \operatorname{sen} \varphi
$$

Steinmetz define la potencia reactiva como la amplitud de la potencia reactiva instantánea en base a las siguientes razones: a) por ser nulo el valor medio de la potencia reactiva instantánea; b) por la semejanza existente con la expresión de la potencia activa; c) y fundamentalmente por la relación que existe entre su valor y el fenómeno que cuantifica (en este caso, las oscilaciones de potencia entre el generador y el receptor debidas al desfase), cuyo origen se halla en la carga y descarga de la energía que en forma de campos electromagnéticos se almacena en las bobinas y condensadores.

Por convenio se considera que un condensador cede energía reactiva a la red, que la consume y por lo tanto se le da un valor negativo. Realmente sabemos que lo que se produce es un intercambio de energía entre el condensador y la red de valor medio nulo, este intercambio de energía entre la red y el condensador se corresponde con el almacenamiento y cesión de la energía electrostática en el dieléctrico del condensador. Para el caso de una bobina se toma que esta absorbe energía reactiva de la red, dándole a esta energía un valor positivo, y el intercambio de energía se corresponde al almacenamiento y liberación de energía electromagnética en el circuito magnético formado por el arrollamiento de la bobina.

Por lo tanto podemos considerar correcta y perfectamente válida la Teoría de Steinmetz, para los sistemas monofásicos y lineales, ya que identifica y cuantifica con precisión cada uno de los fenómenos energéticos presentes en el sistema. 


\section{I.2.2.- Sistema Trifásico Lineal.}

La traslación directa de la Teoría de Steinmetz a los sistemas trifásicos lineales, llevada a cabo a principios del siglo XX, llevo a los mismos resultados que se habían obtenido para los sistemas monofásicos lineales, estableciéndose los fenómenos energéticos en los sistemas trifásicos como una generalización de los sistemas monofásicos lineales. Atendiendo a esta teoría tan sólo se consideran dos fenómenos que son el de transferencia de energía, y el de los fenómenos reactivos. Para ello se parte del desarrollo de la potencia instantánea del sistema, considerando inicialmente que tengamos un sistema equilibrado y se analizara posteriormente un sistema desequilibrado.

\section{I.2.2.1.- Sistemas Equilibrados.}

Partimos de un sistema trifásico, equilibrado en tensiones como el de la figura I.2:

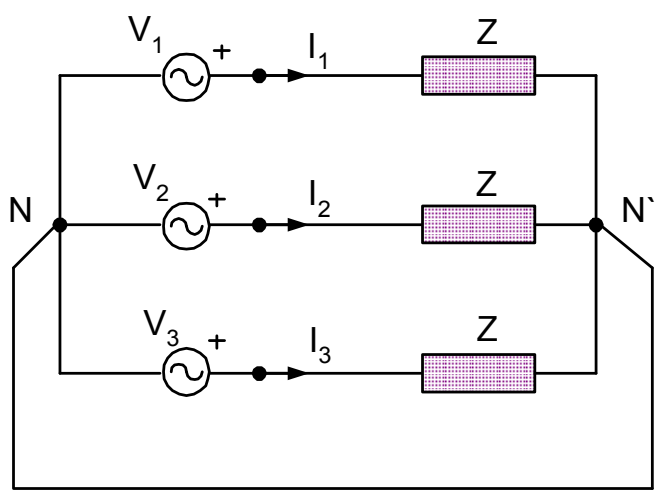

Figura I.2. Teoría Steinmetz. Trifásico equilibrado lineal.

Siendo el sistema de tensiones el que se muestra:

$$
\begin{aligned}
& v_{1}(t)=V \cdot \sqrt{2} \cdot \operatorname{sen} \omega t \\
& v_{2}(t)=V \cdot \sqrt{2} \cdot \operatorname{sen}\left(\omega t-120^{\circ}\right) \\
& v_{3}(t)=V \cdot \sqrt{2} \cdot \operatorname{sen}\left(\omega t-240^{\circ}\right)
\end{aligned}
$$

por el que circulan las siguientes corrientes: 


$$
\begin{aligned}
& i_{1}(t)=I \cdot \sqrt{2} \cdot \operatorname{sen}(\omega t-\varphi) \\
& i_{2}(t)=I \cdot \sqrt{2} \cdot \operatorname{sen}\left(\omega t-\varphi-120^{\circ}\right) \\
& i_{3}(t)=I \cdot \sqrt{2} \cdot \operatorname{sen}\left(\omega t-\varphi-240^{\circ}\right)
\end{aligned}
$$

aplicando el Principio de Conservación de la Energía, sabemos que la potencia instantánea total será la suma de las potencias instantáneas de cada una de sus fases:

$$
p(t)=\sum_{z=1}^{3} v_{z}(t) \cdot i_{z}(t)=v_{1}(t) \cdot i_{1}(t)+v_{2}(t) \cdot i_{2}(t)+v_{3}(t) \cdot i_{3}(t)
$$

en el desarrollo de la expresión anterior, vamos a encontrar las dos componentes de la potencia instantánea:

1. La potencia activa instantánea.

$$
\begin{aligned}
& p_{a}(t)=V \cdot I \cdot \cos \varphi \cdot(1-\cos 2 \omega t)+V \cdot I \cdot \cos \varphi \cdot\left(1-\cos 2\left(\omega t-120^{\circ}\right)\right)+ \\
& +V \cdot I \cdot \cos \varphi \cdot\left(1-\cos 2\left(\omega t-240^{\circ}\right)\right)
\end{aligned}
$$

2. La potencia reactiva instantánea.

$$
\begin{aligned}
& p_{r}(t)=-V \cdot I \cdot \operatorname{sen} \varphi \cdot \operatorname{sen} 2 \omega t-V \cdot I \cdot \operatorname{sen} \varphi \cdot \operatorname{sen} 2\left(\omega t-120^{\circ}\right)- \\
& -V \cdot I \cdot \operatorname{sen} \varphi \cdot \operatorname{sen} 2\left(\omega t-240^{\circ}\right)
\end{aligned}
$$

El fenómeno de la transferencia de energía neta, se halla cuantificada por la potencia activa:

$$
P=\frac{1}{T} \int_{0}^{T} p(t) \cdot d t=3 \cdot V \cdot I \cdot \cos \varphi
$$

El fenómeno reactivo, se deduce de la expresión desarrollada de la potencia reactiva instantánea, en donde su valor medio es cero, y es obtenido como suma de las 
amplitudes de las oscilaciones de potencia reactiva de cada fase, como se había considerado en los sistemas monofásicos.

La potencia reactiva se expresa entonces como:

$$
Q=3 \cdot V \cdot I \cdot \operatorname{sen} \varphi
$$

\section{I.2.2.2.- Sistemas Desequilibrados.}

Sea el siguiente sistema desequilibrado en tensiones y en cargas, mostrado en la figura I.3:

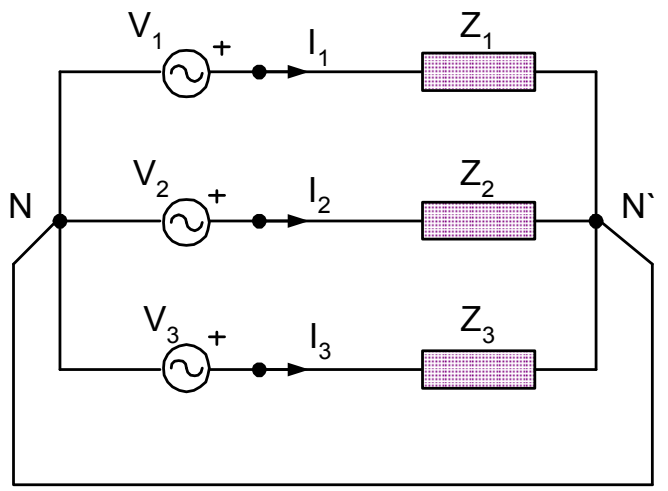

Figura I.3. Teoría Steinmetz. Trifásico desequilibrado lineal.

En donde el sistema de tensiones viene dado por:

$$
\begin{aligned}
& v_{1}(t)=V \cdot \sqrt{2} \cdot \operatorname{sen}\left(\omega t+\alpha_{1}\right) \\
& v_{2}(t)=V \cdot \sqrt{2} \cdot \operatorname{sen}\left(\omega t+\alpha_{2}\right) \\
& v_{3}(t)=V \cdot \sqrt{2} \cdot \operatorname{sen}\left(\omega t+\alpha_{3}\right)
\end{aligned}
$$

siendo las intensidades desequilibradas resultantes en el sistema las siguientes:

$$
\begin{aligned}
& i_{1}(t)=I \cdot \sqrt{2} \cdot \operatorname{sen}\left(\omega t+\alpha_{1}-\varphi_{1}\right) \\
& i_{2}(t)=I \cdot \sqrt{2} \cdot \operatorname{sen}\left(\omega t+\alpha_{2}-\varphi_{2}\right) \\
& i_{3}(t)=I \cdot \sqrt{2} \cdot \operatorname{sen}\left(\omega t+\alpha_{3}-\varphi_{3}\right)
\end{aligned}
$$


La generalización de la Teoría de Steinmetz a los sistemas desequilibrados lineales, se llevo a cabo partiendo de la potencia instantánea del sistema.

$$
p(t)=\sum_{z=1}^{3} v_{z}(t) \cdot i_{z}(t)=v_{1}(t) \cdot i_{1}(t)+v_{2}(t) \cdot i_{2}(t)+v_{3}(t) \cdot i_{3}(t)
$$

En donde se consideran tan sólo los dos fenómenos energéticos, que se contemplaban en los sistemas equilibrados, y que se hallan caracterizados por la potencia activa instantánea $\mathrm{p}_{\mathrm{a}}(\mathrm{t})$ y la potencia reactiva instantánea $\mathrm{p}_{\mathrm{r}}(\mathrm{t})$, no teniendo en cuenta las energías procedentes de los desequilibrios:

$$
\begin{aligned}
& p_{a}(t)=V_{1} \cdot I_{1} \cdot \cos \varphi_{1} \cdot\left(1-\cos \left(2 \omega t+\alpha_{1}\right)\right)+V_{2} \cdot I_{2} \cdot \cos \varphi_{2} \cdot\left(1-\cos 2\left(\omega t+\alpha_{2}\right)\right)+ \\
& +V_{3} \cdot I_{3} \cdot \cos \varphi_{3} \cdot\left(1-\cos 2\left(\omega t+\alpha_{3}\right)\right) \\
& p_{r}(t)=-V_{1} \cdot I_{1} \cdot \operatorname{sen} \varphi_{1} \cdot \operatorname{sen}\left(2 \omega t+\alpha_{1}\right)-V_{2} \cdot I_{2} \cdot \operatorname{sen} \varphi_{2} \cdot \operatorname{sen} 2\left(\omega t+\alpha_{2}\right)- \\
& -V_{3} \cdot I_{3} \cdot \operatorname{sen} \varphi_{3} \cdot \operatorname{sen} 2\left(\omega t+\alpha_{3}\right)
\end{aligned}
$$

los cuales son:

- La transferencia de energía neta, que se halla definida por tres flujos unidireccionales de diferente valor en cada fase y cuantificada por la Potencia Activa:

$$
P=V_{1} \cdot I_{1} \cdot \cos \varphi_{1}+V_{2} \cdot I_{2} \cdot \cos \varphi_{2}+V_{3} \cdot I_{3} \cdot \cos \varphi_{3}
$$

- Los fenómenos reactivos, que se hallan definidos por tres flujos sinusoidales de distinta amplitud en cada fase, y cuantificados por la Potencia Reactiva:

$$
Q=V_{1} \cdot I_{1} \cdot \operatorname{sen} \varphi_{1}+V_{2} \cdot I_{2} \cdot \operatorname{sen} \varphi_{2}+V_{3} \cdot I_{3} \cdot \operatorname{sen} \varphi_{3}
$$


En donde al igual que en los sistemas monofásicos, estos fenómenos energéticos se ponen de manifiesto en las componentes de la corrientes que circulan por el sistema, las corrientes activas (transferencia de energía neta) y las reactivas (fenómenos reactivos).

Corrientes activas:

$$
\begin{aligned}
& i_{a 1}(t)=I_{1} \sqrt{2} \cdot \cos \left(\alpha_{1}-\varphi_{1}\right) \cdot \operatorname{sen} \omega t \\
& i_{a 2}(t)=I_{2} \sqrt{2} \cdot \cos \left(\alpha_{2}-\varphi_{2}\right) \cdot \operatorname{sen} \omega t \\
& i_{a 3}(t)=I_{3} \sqrt{2} \cdot \cos \left(\alpha_{3}-\varphi_{3}\right) \cdot \operatorname{sen} \omega t
\end{aligned}
$$

Corrientes reactivas:

$$
\begin{aligned}
& i_{r 1}(t)=I_{1} \sqrt{2} \cdot \operatorname{sen}\left(\alpha_{1}-\varphi_{1}\right) \cdot \cos \omega t \\
& i_{r 2}(t)=I_{2} \sqrt{2} \cdot \operatorname{sen}\left(\alpha_{2}-\varphi_{2}\right) \cdot \cos \omega t \\
& i_{r 3}(t)=I_{3} \sqrt{2} \cdot \operatorname{sen}\left(\alpha_{3}-\varphi_{3}\right) \cdot \cos \omega t
\end{aligned}
$$

Observamos que en los sistemas lineales el fenómeno de la transferencia de energía neta, se lleva a cabo por medio de flujos de potencia unidireccionales, mientrás que el fenómeno de la energía reactiva se produce mediante flujos de potencia sinusoidales.

Para el autor de esta Tesis, en este caso de sistema trifásico desequilibrado y lineal, la formulación que se plantea de la potencia reactiva es erronea, ya que basa en la suma de las amplitudes de tres flujos sinuoidales que alcanzan sus valores máximos en distintos instantes, lo cual no es correcto, opinión esta compartida por León, Czarnecki y Emanuel en sus publicaciones. Formulación usada tradicionalmente por la Teoría de Circuitos y que sale reflejada en la IEEE Std. 1459/2000, en función de las componetes simétricas del sistema, pero se ha de resaltar que en la expresión de la potencia reactiva total del sistema como suma de las debidas a la secuencia directa, inversa y homopolar, la inversa y la homopolar son el efecto del desequilibrio sobre la potencia reactiva, ya que si no existieran desequilibrios de tensiones, esos terminos serian nulos. 
Vamos ahora a abordar dos enfoques fundamentales que surgen a finales de los años veinte y principios de los treinta sobre las definiciones de la potencia eléctrica en corriente alterna, uno de ellos realizado en el dominio frecuencial, el debido a Budeanu, y el llevado a cabo en el dominio temporal y elaborado por Fryze.

\section{I.3. Teoría de Budeanu.}

Se desarrolla esta teoría ${ }^{3}$ para sistemas monofásicos no lineales en el dominio de la frecuencia, como vemos en la figura I.4, usando para ello las series de Fourier, partiendo de la formulación de la potencia instantánea del sistema.

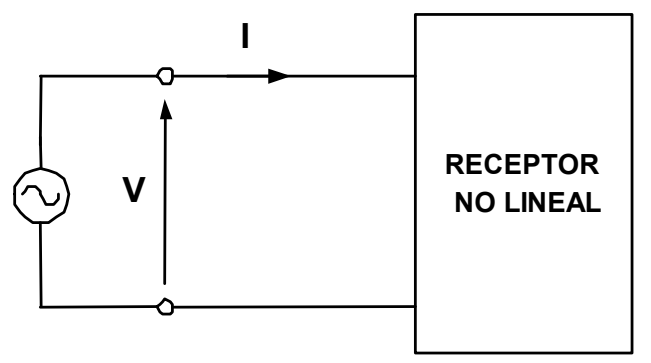

Figura I.4. Teoría Budeanu. Monofásico no lineal. Dominio frecuencia.

En este sistema tenemos las siguientes tensionesy corrientes:

$$
\begin{aligned}
& v(t)=V_{0}+\sum_{n=1}^{\infty} V_{n} \cdot \sqrt{2} \cdot \operatorname{sen}\left(n \omega t-\alpha_{n}\right) \\
& i(t)=I_{0}+\sum_{m=1}^{\infty} I_{m} \cdot \sqrt{2} \cdot \operatorname{sen}\left(m \omega t-\beta_{m}\right)
\end{aligned}
$$

en donde el valor eficaz de dichos valores viene dado por:

$$
V=\sqrt{V_{0}^{2}+\sum_{n=1}^{\infty} V_{n}^{2}} ; \quad I=\sqrt{I_{0}^{2}+\sum_{m=1}^{\infty} I_{m}^{2}}
$$

\footnotetext{
${ }^{3}$ C.I. Budeanu, "Puissances reactives et fictives", Instytut Romain de l'Energie. Bucharest, Romania, 1927.
} 
El valor de la potencia instantánea en este caso está compuesta por tres términos.

$$
p(t)=v(t) \cdot i(t)=p_{a}(t)+p_{r}(t)+p_{d}(t)
$$

el primer término que representa la potencia activa instantánea y viene dada por:

$$
p_{a}(t)=V_{0} \cdot I_{0}+\sum_{n=1}^{\infty} V_{n} \cdot I_{n} \cdot \cos \varphi_{n} \cdot\left[1-\cos 2\left(n \omega t-\alpha_{n}\right)\right]
$$

el segundo nos da la potencia reactiva instantánea:

$$
p_{r}(t)=-\left(V_{0} \cdot I_{0}+\sum_{n=1}^{\infty} V_{n} \cdot I_{n} \cdot \operatorname{sen} \varphi_{n} \cdot \operatorname{sen} 2\left(n \omega t-\alpha_{n}\right)\right)
$$

siendo el tercer término el que nos informa sobre la potencia de distorsión instantánea, estando formada por los productos de armónicos de tensión y corriente de diferentes frecuencias

La potencia activa determinada como el valor medio de la potencia instantánea, se expresa como:

$$
P_{B}=\sum_{n=m} V_{n} \cdot I_{n} \cdot \cos \varphi_{n}
$$

y la potencia reactiva toma el valor:

$$
Q_{B}=\sum_{n=m} V_{n} \cdot I_{n} \cdot \operatorname{sen} \varphi_{n}
$$

La potencia aparente la determina como el producto de los valores eficaces de tensión y corriente:

$$
S=V \cdot I=\sqrt{\sum_{n}^{\infty} V_{n}^{2} \cdot \sum_{m}^{\infty} I_{m}^{2}}
$$


Budeanu descompone la potencia aparente, como dos componentes ortogonales, la potencia activa $\mathrm{P}$ y la potencia ficticia $\mathrm{F}$.

$$
S=\sqrt{P^{2}+F^{2}}
$$

A esta potencia ficticia F, Budeanu la descompone a su vez en la potencia reactiva (Q) y de distorsión (D), también ortogonales entre ellas, quedando entonces:

$$
S=\sqrt{P^{2}+Q^{2}+D^{2}}
$$

que pasamos a representar en la figura. I.5.

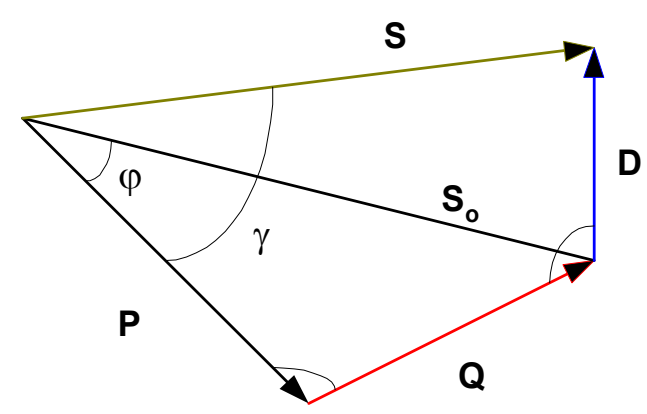

Figura I.5. Triángulo potencias Budeanu.

Las definiciones de Budeanu, han sido muy difundidas en diversos libros y publicaciones técnicas, aunque ha sufrido también numerosas objeciones, de las cuales pasamos a comentar algunas de ellas.

- Fryze, le objeta que para poder determinar la potencia reactiva se ha de tener la descomposición de tensiones y corrientes usando las series de Fourier, y este modelo no puede usarse cuando las ondas no son periódicas.

- Shepherd y Zakikhani, objetan que en la formulación de la Teoría de Budeanu, la potencia reactiva de Budeanu $\mathrm{Q}_{\mathrm{B}}$, es una cantidad que no tiene sentido físico. 
- Czarnecki en [53], manifiesta que la $Q_{B}$ y la $D_{B}$, no poseen los atributos que permitan mostrar los fenómenos de la potencia electrica en los circuitos y que sus valores no valen para diseñar circuitos de compensación. $L a Q_{B}$ no es conservativa, ya que es la suma de las amplitudes $Q_{n}$ de los diversos armónicos, sin tener en cuenta que estas amplitudes tienen diferentes ángulos de fase y por lo tanto no es válida esa suma. $L a D_{B}$ introducida por Budeanu, no es capaz de cuantificar la distorsión de la onda de tensión con respecto a la corriente, ya que al aplicar una onda distorsionada de tensión a una carga resistiva, la forma de onda de la intensidad será idéntica y la potencia de distorsión de Budeanu, sería nula, lo cual no es correcto.

- Czarnecki en [37], plantea que en la expresión de la potencia instantánea de un sistema con tensiones e intensidades no senoidales, la potencia reactiva se considera como la amplitud de la oscilación de energía que evoluciona con (sen2wt); esto se ha generalizado por los ingenieros eléctricos a los sistemas no senoidales de manera que a las oscilaciones de energía se las interpreta como potencia reactiva, esto lleva a que las oscilaciones de energía de los diversos armónicos de un sistema no senoidal, sean considerados como terminos constituyentes de la potencia reactiva total del sistema, cometiendose así el grave error de sumar oscilaciones que poseen diferente frecuencia.

- Pilipski en [83], critica la definición de la potencia reactiva contenida en el IEE Estándar Diccionario, al ser la expuesta por Budeanu, ya que la potencia reactiva de cada armónico puede tener diferente signo y habrá potencias de armónicos del mismo signo, pero habrá otras de diverso signo y por lo tanto no se pueden sumar todas ellas para dar la potencia reactiva total del sistema. Además la $\mathrm{Q}_{\mathrm{B}}$ no tiene sentido físico y no es capaz de cuantificar de forma correcta la reactiva del sistema en aras de lograr el equipo óptimo de compensación de reactiva del sistema.

- León en [123], indica que la $P_{B}$ no informa sobre la eficiencia del sistema y la calidad del suministro, además de no cuantificar de forma adecuada sobre la energía útil. 
El autor de esta Tesis, coincide con la afirmación de Czarnecki, de que la potencia reactiva total del sistema sea la suma de las potencias reactivas de cada armónico, es erronea ya que esta suma no tiene sentido al tener cada armonico angulos de fase diferentes, y al igual que sucedía en los sistemas trifásicos desequilibrados lineales, los valores máximos de las amplitudes no tienen porque darse en el mismo instante, esto es debido a que Budeanu asigna al fenómeno de la potencia reactiva todos los flujos de potencia sinusoidales, aún cuando tengan diferente frecuencia; lo mismo le sucede con el fenómeno de la transferencia de energía neta, en la que considera todos los flujos unidireccionales aunque sean de diferente frecuencia.

El autor coincide también con León, en que el valor de la potencia reactiva de Budeanu no vale para cuantificar de manera correcta el fenómeno de la reactiva, ya que lo obtiene mediante la suma de las amplitudes de diferentes frecuencias, lo que no es matemáticamente correcto. 


\section{I.4. Teoría de Fryze.}

Tal vez la limitación de la teoría de Budeanu de tan sólo poder ser aplicada a sistemas con señales periódicas y por lo tanto desarrollables en series de Fourier, llevo a que S. Fryze en al año 1932, plantee el modelo de funcionamiento de los sistemas monofásicos no lineales en el dominio del tiempo.

Por lo tanto la formulación de la teoría de la potencia eléctrica propuesta por Fryze, se desarrolla como acabamos de mencionar en el dominio del tiempo, el cual como comenta Czarnecki [36] es el espacio natural para la representación de los fenómenos eléctricos y su medida, mientras que el dominio frecuencial es una herramienta matemática usada para representar fenómenos físicos.

La Teoría de Fryze, se basa en que para un sistema monofásico no lineal, alimentado por excitaciones cualesquiera $v(t)$, con un valor eficaz $v$, se puede proceder a una descomposición de la corriente absorbida por el receptor en dos: una corriente activa $i_{a}, y$ una corriente ficticia $i_{f}$, de manera que ambas sean ortogonales, lo que se muestra en la figura I.6.

$$
i(t)=i_{a}(t)+i_{f}(t)
$$

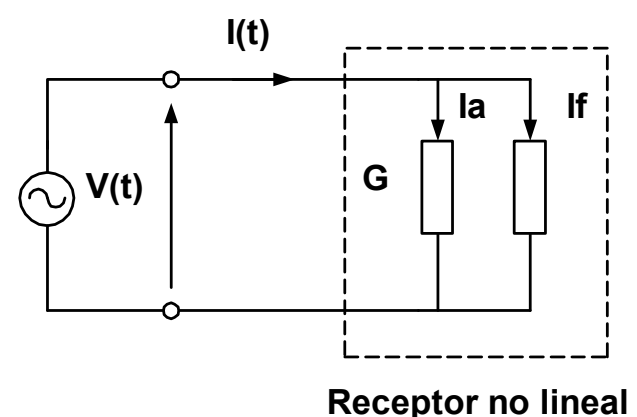

Figura I.6. Teoría Fryze. Monofásico no lineal. Dominio tiempo.

en donde la corriente activa (que se halla en fase y tiene la misma forma de onda que la tensión) sería la corriente consumida por la resistencia equivalente del 
receptor original, que consuma la potencia activa $\mathrm{P}$, a la misma tensión de la fuente de alimentación.

$$
i_{a}(t)=\left(P / V^{2}\right) \cdot v(t)=G_{e} \cdot v(t)
$$

en donde $G_{e}$, representa la conductancia equivalente del receptor.

La diferencia entre las formas de onda de la tensión de excitación y de la corriente que absorbe el circuito, lleva a la existencia de una corriente que entrega la fuente y que no es la corriente activa, a la que Fryze denomina corriente ficticia y tiene por valor:

$$
i_{f}(t)=i(t)-i_{a}(t)
$$

cumpliéndose la relación de ortogonalidad:

$$
\frac{1}{T} \int_{0}^{T} i_{a} \cdot i_{f} \cdot d t=\frac{1}{T} \int_{0}^{T} i_{a} \cdot\left(i-i_{a}\right) \cdot d t=0
$$

pudiéndose sacar la siguiente relación de valores eficaces:

$$
I^{2}=I_{a}^{2}+I_{f}^{2}
$$

Las potencias que nos permiten cuantificar estos fenómenos, que son distintos, ya que cada uno vendrá dado por una corriente y que ambas son ortogonales entre sí, se obtienen mediante el producto de los valores eficaces de la tensión por cada una de las corrientes.

La Potencia Activa se define como:

$$
P=V \cdot I_{a}
$$

y la Potencia Ficticia será:

$$
Q_{F}=V \cdot I_{f}
$$


siendo por lo tanto la Potencia Aparente:

$$
S=V \cdot I=V \cdot \sqrt{I_{a}^{2}+I_{f}^{2}}=\sqrt{P^{2}+Q_{F}^{2}}
$$

de la Teoría de la potencia de Fryze, podemos sacar las siguientes conclusiones:

- La posibilidad de describir propiedades y fenómenos de los circuitos eléctricos en el dominio del tiempo, es más fácil y natural que usar el dominio frecuencial, lo que facilita la implementación de dispositivos de medida mucho más simples.

- Fryze considera como corriente activa a toda aquella que vaya en fase con la tensión aplicada.

- La no concordancia de las formas de onda de la tensión y la corriente implica la presencia de una corriente ficticia (en la que se incluyen desfases, distorsiones, etc) y desfasada $90^{\circ}$ con respecto de esta.

- La $I_{a}$, representa la menor corriente necesaria para que la carga consuma su potencia activa.

Existiendo además una serie de objeciones que pasamos a mencionar:

- Czarnecki en [37], comenta severas limitaciones de la Teoría de Fryze, con respecto a la interpretación de los fenómenos eléctricos presentes en el sistema, así como respecto a su implementación práctica. Da una visión muy superficial de la interpretación del fenómeno de la potencia eléctrica, ya que aunque es buena la idea de la componente de la corriente activa, su interpretación como "componente útil" de la corriente que entrega la fuente no es del todo convincente, ya que "potencia activa no es sinónimo de potencia útil" (punto este en el que coincide con León en [123]), ya que la potencia activa con armónicos no es útil para las máquinas rotativas. Con respecto a la corriente ficticia, la única interpretación que hace de ella es que es una corriente inútil, pero no distingue los fenómenos que en la carga provocan la presencia de esa corriente, y por lo tanto la Teoría de Fryze no 
relaciona esta corriente ficticia $\mathrm{i}_{\mathrm{F}}$, con las propiedades y parámetros que tenga la carga.

- León en [123], plantea lo siguiente: las corrientes en el modelo de teoría propuesto por Fryze no cumplen la $1^{\text {a }}$ Ley de Kirchoff, y además en un sistema eléctrico en el cual la forma de onda de la tensión e intensidad sean iguales, implica la máxima eficiencia, aunque las ondas sean distorsionadas, lo cual no es correcto.

El autor de esta Tesis, concuerda con la opinión de León, en que la teoría de Fryze, no cumple la $1^{a}$ Ley de Kirchoff, y que define mal la corriente activa, lo que lleva a que la que Fryze denomina corriente ficticia, y que es ortogonal a la activa (para nosotros es el equivalente de la corriente reactiva), este por lo tanto también mal definida y la potencia ficticia (reactiva) de Fryze también sea errónea en su concepción y formulación.

A pesar de estas objeciones, la Teoría de Fryze esta recomendada por el C.E.I., y la potencia ficticia $\mathrm{Q}_{\mathrm{f}}$, se encuentra implementada en gran cantidad de aparatos de medida de la potencia reactiva en sistemas no lineales. 
Antecedentes.

\section{I.5. Teoría de la Potencia Instantánea de Akagi.}

En 1984, Akagi y otros coautores plantean una nueva teoría [6], en la que las tensiones y corrientes de un sistema trifásico a tres hilos se expresaba vectorialmente usando la transformación de Park, lo cual se extendió a sistemas trifásicos a cuatro hilos [2].

Akagi, propone nuevos conceptos de la potencia activa instantánea y de la potencia reactiva instantánea y tiene la ventaja de que puede ser usada en régimen transitorio y con tensiones e intensidades senoidales y no senoidales.

El objetivo de Akagi es la obtención de las intensidades activa instantánea y la intensidad reactiva instantánea, de forma que se puedan explicar flujos de potencia entre el sistema de generación y el sistema receptor, ya que el objetivo inicial de la Teoría de la Potencia Reactiva Instantánea es el diseño de compensadores estáticos de potencia que no necesiten de elementos que almacene energía para poder llevar a cabo la compensación.

En el caso de un sistema a tres hilos, se produce la conversión de los sistemas vectoriales de tensiones e intensidades del sistema a un sistema de ejes ortogonales $\alpha, \beta$, usando para ello la transformación de Park.

Sea por lo tanto el sistema trifásico definido por las fases RST, tenemos lo siguiente:

Para las tensiones,

$$
\bar{V}_{\alpha \beta}=\left[\begin{array}{l}
V_{\alpha} \\
V_{\beta}
\end{array}\right]=\frac{\sqrt{2}}{3} \cdot\left[\begin{array}{ccc}
1 & -1 / 2 & -1 / 2 \\
0 & \sqrt{3} / 2 & -\sqrt{3} / 2
\end{array}\right] \cdot\left[\begin{array}{l}
V_{R} \\
V_{S} \\
V_{T}
\end{array}\right]=[P] \cdot\left[\begin{array}{l}
V_{R} \\
V_{S} \\
V_{T}
\end{array}\right]
$$

en donde la matriz $[\mathrm{P}]$, es la matriz de transformación de Park. 
Para las corrientes,

$$
\bar{I}_{\alpha \beta}=\left[\begin{array}{c}
i_{\alpha} \\
i_{\beta}
\end{array}\right]=\frac{\sqrt{2}}{3} \cdot\left[\begin{array}{ccc}
1 & -1 / 2 & -1 / 2 \\
0 & \sqrt{3} / 2 & -\sqrt{3} / 2
\end{array}\right] \cdot\left[\begin{array}{c}
i_{R} \\
i_{S} \\
i_{T}
\end{array}\right]=[P] \cdot\left[\begin{array}{c}
i_{R} \\
i_{S} \\
i_{T}
\end{array}\right]
$$

Usando estas variables transformadas Akagi, define las siguientes potencias:

$$
\left[\begin{array}{l}
p_{\alpha \beta} \\
q_{\alpha \beta}
\end{array}\right]=\left[\begin{array}{cc}
v_{\alpha} & v_{\beta} \\
-v_{\beta} & v_{\alpha}
\end{array}\right] \cdot\left[\begin{array}{c}
i_{\alpha} \\
i_{\beta}
\end{array}\right]
$$

La $p_{\alpha \beta}$, que se definió por Akagi como la Potencia real instantánea, y se obtiene como producto de tensiones y corrientes de la misma fase.

$$
p_{\alpha \beta}=v_{\alpha} i_{\alpha}+v_{\beta} i_{\beta}=v_{R} i_{R}+v_{s} i_{s}+v_{T} i_{T}
$$

que podemos expresar como:

$$
p(t)=p_{\alpha \beta}=\bar{P}+\tilde{p}
$$

en la expresión anterior, el primer término $\bar{P}$ es constante y representa la potencia activa del sistema, mientras que el segundo término $\tilde{p}$ surge en los sistemas trifásicos cuando existen desequilibrios y/o presencia de elementos no lineales.

La $q_{\alpha \beta}$, fue la verdadera innovación de la formulación de la Teoría de Akagi, que denomino como Potencia imaginaria instantánea. Desde el punto de vista físico, Akagi establece que la reactiva no se puede medir en al plano real, sino sobre un eje ortogonal, definido por el vector potencia imaginaria instantánea, y que surge como producto de tensiones y corrientes de fases distintas del sistema transformado. Es por ello que Akagi, propone como unidad para esta potencia imaginaria el IVA (Imaginary Volt-Ampere).

$$
q_{\alpha \beta}=v_{\alpha} i_{\beta}-v_{\beta} i_{\alpha}
$$


La expresión de esta potencia en el sistema original RST será:

$$
q=-\frac{1}{\sqrt{3}}\left[\left(v_{R}-v_{S}\right) i_{T}+\left(v_{S}-v_{T}\right) i_{R}+\left(v_{T}-v_{R}\right) i_{S}\right]
$$

que es la expresión usada para medir la potencia reactiva de los sistema trifásicos lineales.

Esta potencia imaginaria instantánea formulada por Akagi, al igual que sucedía con la potencia real instantánea se puede descomponer en dos términos:

$$
q(t)=q_{\alpha \beta}=\bar{Q}+\tilde{q}
$$

en la anterior expresión, el primer término es constante y representa la potencia reactiva del sistema y el segundo término surge en los sistemas desequilibrados y no lineales como suma de términos senoidales.

Watanabe en [178], expone la anterior expresión y aclara que la potencia instantánea imaginaria $q_{\alpha \beta}$ no contribuye al transporte de la energía, aunque las corrientes reactivas presentes en cada fase provocan un mal aprovechamiento de los conductores usados para el transporte de la energía. Así como define la potencia armónica $\mathrm{H}$ :

$$
H=\sqrt{P^{2}(\approx)+Q^{2}(\approx)}
$$

en donde $P(\approx)$ y $Q(\approx)$ son respectivamente los valores eficaces de $\tilde{p}$ y $\tilde{q}$.

En sistemas que sean senoidales en generación, nos encontramos que la potencia armónica de Watanabe, coincide con la potencia de distorsión de Budeanu y además se verifica la siguiente expresión de la potencia aparente: 


$$
S=\sqrt{P^{2}+Q^{2}+H^{2}}
$$

Si en el nuevo sistema de referencia formulado por Akagi, se realiza una descomposición de las corrientes en el presentes, tenemos una componente activa $\left(i_{\alpha p}, i_{p p}\right)$ y otra reactiva $\left(i_{\alpha q}, i_{\beta q}\right)$, operando en las ecuaciones de la potencia real instantánea y reactiva imaginaria de Akagi, podemos expresar las corrientes como:

$$
\begin{aligned}
& i_{\alpha}=\frac{v_{\alpha}}{v_{\alpha}^{2}+v_{\beta}^{2}} \cdot p-\frac{v_{\beta}}{v_{\alpha}^{2}+v_{\beta}^{2}} \cdot q=i_{\alpha p}+i_{\alpha q} \\
& i_{\beta}=\frac{v_{\beta}}{v_{\alpha}^{2}+v_{\beta}^{2}} \cdot p+\frac{v_{\alpha}}{v_{\alpha}^{2}+v_{\beta}^{2}} \cdot q=i_{\beta p}+i_{\beta q}
\end{aligned}
$$

La teoría de la potencia instantánea es válida para sistemas trifásicos a cuatro hilos, sin más que proceder a introducir vectores espaciales de tensión y corriente de secuencia homopolar $\left(\mathrm{v}_{0}, \mathrm{i}_{0}\right)$ que definen un nuevo eje de secuencia homopolar. Lo que conlleva a una nueva definición de la potencia real instantánea en este sistema a cuatro hilos, cuya formulación es la siguiente:

$$
p(t)=p_{\alpha \beta}=v_{\alpha} i_{\alpha}+v_{\beta} i_{\beta}+v_{0} i_{0}=v_{R} i_{R}+v_{S} i_{S}+v_{T} i_{T}
$$

en la cual podemos observar que se compone de la potencia real instantánea del sistema a tres hilos, más el término debido a la potencia instantánea de secuencia cero $p_{0}(t)=v_{0} i_{0}$ y que al igual que antes la podemos descomponer en dos términos:

$$
p_{0}(t)=\bar{P}_{0}+\tilde{p}_{0}
$$

en donde el primer término es constante y el segundo es oscilante.

No sucede esto mismo en cambio para la potencia imaginaria instantánea, ya que Akagi considera que esta no depende de la componente homopolar y por lo tanto coincide con la vista en los sistemas a tres hilos. 
Antecedentes.

La Teoría de la potencia instantánea de Akagi, ha sido tomada como base por numerosos autores, pero en todos ellos transciende la idea de que más que una teoría de la potencia eléctrica es una brillante formulación matemática usada para el diseño de sistemas de compensación, ya que no entra a analizar todos los fenómenos presentes en un sistema eléctrico. Su gran novedad como ya se ha comentado es el establecer el concepto de potencia imaginaria instantánea.

En 1992, Willems [181], generaliza la teoría de la potencia instantánea a sistemas de $m$-fases, y plantea los vectores de corrientes activas $I_{p}, y$ el de corrientes no activas $I_{q}$, obtenido este como diferencia del vector de corrientes original $I$, menos el de corrientes activas, descomposición que ya realizaba también Fryze, sin embargo discrepa con la teoría original de Akagi, en que para él la componente de secuencia homopolar afecta tanto a la potencia real instantánea como a la imaginaria.

En 1996, Nabae [155] plantea la teoría pq modificada, en la que si se cumple el principio de conservación de la potencia. También en 1996, Zheng y Lai [186] generalizan la teoría de la potencia reactiva instantánea en los sistema trifásicos. Akagi ya había introducido que el vector de potencia imaginaria de un sistema trifásico era siempre perpendicular al plano $\alpha \beta$, Zheng propone como vector de potencia imaginaria el producto vectorial siguiente:

$$
\bar{q}=\bar{V} \times \bar{I}
$$

y para un sistema de referencia RST se tendría:

$$
\bar{q}=\left[\begin{array}{l}
q_{R} \\
q_{S} \\
q_{T}
\end{array}\right]=\left[\begin{array}{rrr}
0 & -v_{T} & v_{S} \\
v_{T} & 0 & -v_{R} \\
-v_{S} & v_{R} & 0
\end{array}\right] \cdot\left[\begin{array}{c}
i_{R} \\
i_{S} \\
i_{T}
\end{array}\right]=[V] \cdot\left[\begin{array}{c}
i_{R} \\
i_{S} \\
i_{T}
\end{array}\right]
$$

en la expresión anterior, la matriz [V] tiene por valor cero, al igual que le sucedía a teoría pq modificada propuesta por Nabae, tenemos pues tres potencias imaginarias 
que no son totalmente independientes, y en las que su relación y su significado físico es difícil de explicar.

Zheng y Lai, enunciaron y justificaron varios teoremas que recopilaban los postulados y modificaciones de la teoría de la potencia instantánea en los que se pone de manifiesto su concordancia con la teoría de Fryze, ya que en uno de ellos se justifica que un sistema posee la máxima eficiencia si la suma de corrientes no activas es nula, con total independencia del tipo de onda que posean la tensión y la corriente.

Watanabe en [177], realiza una serie de puntualizaciones:

- La potencia de secuencia homopolar $p_{0}$, coincide con la potencia instantánea de un sistema monofásico lineal ya que se halla compuesta de un término de valor medio y otro oscilante, pero sin embargo, en la definición de la potencia reactiva instantánea, esta potencia de secuencia homopolar no es considerada.

- Admite que la potencia reactiva del sistema pueda ser producida por las componentes de secuencia directa e inversa.

León en [123], objeta a esta teoría:

- Que no refleja de forma adecuada los fenómenos que suceden en el sistema eléctrico.

- Al seguir la Teoría de Fryze, comete el mismo error de cuantificar la eficiencia máxima del sistema a aquel en el que las formas de onda de la tensión y la corriente coinciden.

Czarnecki en [33] y [34], plantea también una serie de críticas a esta teoría:

- Que sólo sea capaz de describir los fenómenos de un sistema eléctrico en las condiciones de equilibrio y linealidad, ya que en los sistemas lineales pero desequilibrados falla en la identificación de las propiedades del sistema. 
- Le desconcierta que en circuitos lineales sin elementos reactivos, y alimentados por un generador equilibrado y senoidal no sea nula la potencia reactiva instantánea.

- También le preocupa, que en sistemas formados por elementos lineales y alimentados por generadores equilibrados y senoidales, surjan corrientes no lineales, o lo que es lo mismo que aparezcan armónicos, en sistema lineales.

El autor de esta Tesis, no esta de acuerdo con que a la hora de determinar las potencias activas y reactivas de un sistema eléctrico, como establece Akagi se siga incluyendo la secuencia inversa a la contribución de estas potencias aunque se excluya de manera correcta la secuencia homopolar, además comete el error de que se sumen las componentes directa e inversa de la potencia sin tener en cuenta su signo. 


\section{I.6. Teoría de Czarnecki.}

Desde que en 1988, L.S. Czarnecki en [52] expuso su Teoría de la Potencia Eléctrica, hasta nuestros días, podemos asegurar que ha sido uno de los autores más prolíficos en este campo, como se puede observar en las referencias bibliográficas [32-53].

Una de las influencias que queda patente en su teoría, es la recibida por Fryze, ya que desde sus primeros trabajos Czarnecki, sigue la filosofía que queda plasmada en [52], en la que sugiere la siguiente idea:

"La descomposición de la corriente suministrada por el generador, en sus componentes ortogonales, nos permite relacionar con claridad a estas, con los fenómenos energéticos a ellas asociadas y que se producen en el circuito analizado"

En [50], Czarnecki, sigue con el desarrollo de su teoría, volviendo a hacer énfasis en que su teoría propone indicar cuales de las componentes de la corriente suministrada por el generador son "inútiles", además de indicar cual es el fenómeno asociado responsable de ella, por lo que concluye que es deseable el medir estas corrientes de manera que luego se pueda proceder a su compensación, ya que lo único que provocan estas "corrientes inútiles", es un incremento del valor RMS de la corriente que tiene que suministrar el generador.

Czarnecki, desarrolla su teoría realizando un amplio estudio de diversas topologías: para el caso de que el generador (equilibrado, lineal ó no) sea monofásico o trifásico y que el receptor sea monofásico ó trifásico (equilibrado y/o desequilibrado), y con tensiones senoidales ó no.

Para el caso general, de sistemas trifásicos desequilibrados en cargas y no lineales, nos encontramos con cuatro posibles causas que provocarían la presencia de corrientes que deben de ser compensadas (ya que no producen ningún aprovechamiento y conllevan un peor rendimiento) a saber: 
1. Flujos oscilantes de energía entre el generador y la carga, que provocan la presencia de una corriente reactiva $i_{r}$.

2. Variaciones de la conductancia del receptor dependientes de la frecuencia, lo que conlleva la presencia de una corriente de dispersión $i_{s}$.

3. Asimetrías de la carga, lo que provoca la existencia de una corriente de desequilibrio $i_{\iota \cdot}$.

4. Presencia de cargas no lineales, lo que implica la aparición de corrientes armónicas $i_{g}$.

Además de estas corrientes "inútiles", tenemos la corriente activa $i_{a}$ que sería la responsable de la transmisión de la potencia activa.

El generador entrega por lo tanto una corriente $i$, que puede ser descompuesta en sus componentes ortogonales entre sí, que nos permiten identificar los fenómenos energéticos que se producen en la carga.

$$
\bar{i}=\bar{i}_{a}+\bar{i}_{s}+\bar{i}_{r}+\bar{i}_{u}+\bar{i}_{g}
$$

Nosotros nos vamos a centrar en el caso de Sistemas trifásicos desequilibrados y lineales, lo que podemos modelizar en la siguiente figura I.7.

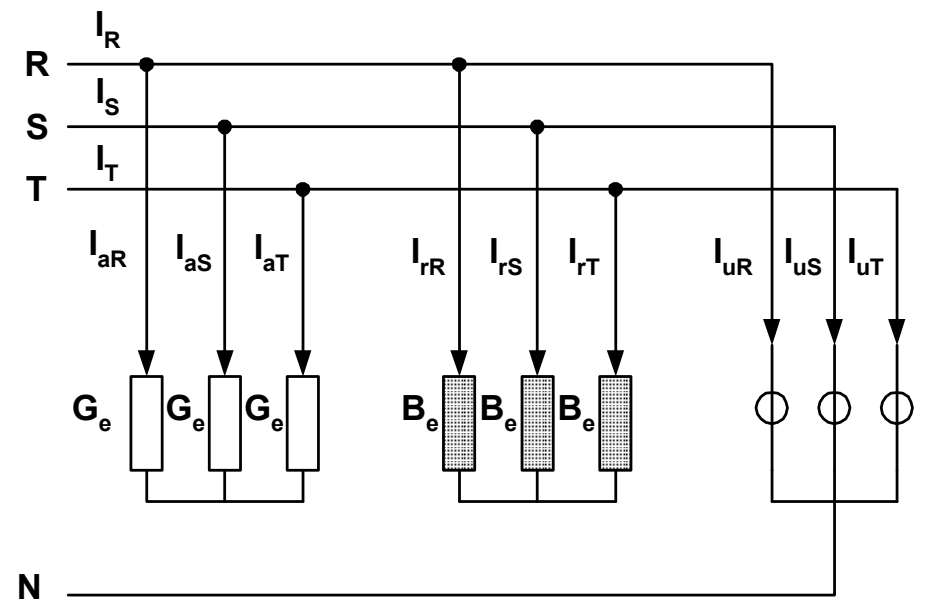

Figura I.7. Teoría Czarnecki. Trifásico desequilibrado lineal. 
Las corrientes y tensiones del sistema tienen tres componentes, correspondientes a cada una de las fases:

$$
\begin{aligned}
& \bar{v}=\left(\begin{array}{lll}
v_{R} & v_{S} & v_{T}
\end{array}\right) \\
& \bar{i}=\left(\begin{array}{lll}
i_{R} & i_{S} & i_{T}
\end{array}\right)
\end{aligned}
$$

y se definen las siguientes componentes ortogonales entre sí de la corriente:

1. Corriente Activa: $\quad \bar{i}_{a}=G_{e} \cdot \bar{V}$

2. Corriente Reactiva: $\quad \bar{i}_{r}=B_{e} \cdot \frac{d}{d w t} \bar{v}$

3. Corriente de Desequilibrio: $\quad \bar{i}_{u}=\bar{i}_{-}-\bar{i}_{a}-\bar{i}_{r}$

que estarían asociadas con las siguientes potencias, que reflejarían el correspondiente fenómeno energético en la carga:

1. Potencia Activa:

$$
\begin{aligned}
& P=V \cdot I_{a} \\
& Q=V \cdot I_{r}
\end{aligned}
$$

2. Potencia Reactiva:

3. Potencia de Desequilibrio: $D_{u}=V \cdot I_{u}$

Lo que nos daría la ecuación de potencias siguiente:

$$
S^{2}=P^{2}+Q^{2}+D_{u}^{2}
$$

en esta ecuación queda de manifiesto que la potencia aparente en la carga es función de tres fenómenos independientes entre sí, y que caracterizan cada una de 
Antecedentes.

las corrientes en las que podemos descomponer la corriente entregada por el generador.

Como ya se ha comentado la teoría de la potencia de Czarnecki, es una de las aportaciones más destacables en este campo, debido su concepción, desarrollo y rigurosidad. El relacionar cada una de las corrientes con el fenómeno energético asociado en el receptor, le permite el poder diseñar redes de compensación independientes para cada uno de ellos.

De entre sus numerosas publicaciones, en [41] y [43], pone de manifiesto su rechazo a que oscilaciones de energía, en el término de la potencia instantánea, debidas a asimetrías de carga puedan dar lugar a un incremento de la potencia aparente, lo que si acepta A. Emanuel [76] y [77].

En [34], critica la Teoría p-q, al poner de relieve que en el caso de tener un sistema desequilibrado con una carga resistiva, no sea nulo el término de la potencia reactiva, que lo atribuye a la inconsistencia de llamar "corriente reactiva" a la descomposición que se realiza de corrientes, debiéndole llamar deactiva como había sugerido Willems.

En [35], reconoce que en algunos sistemas en particular existe potencia reactiva, sin la presencia de oscilaciones de energía, así como que no sean necesarios elementos que almacenen energía en el circuito (bobinas y/o condensadores), pero que no se deben de generalizar. Pone de manifiesto estos casos, pero le cuesta llamar potencia reactiva a un fenómeno energético presente en el circuito, pero sin la presencia de los elementos "reactivos" de los que coge su nombre, pero no niega el desfase que se produce entre la tensión y la intensidad en estos circuitos, sino la aceptación general de que este desfase tan sólo es provocado por los elementos reactivos.

León en [123], realiza una serie de matizaciones a su teoría, entre las que pasamos a remarcar algunas de ellas: 
- Su teoría al estar influenciada por la de Fryze, le lleva a que no se cumpla la $1^{\text {a }}$ Ley de Kirchoff, para la descomposición en componentes de corrientes que realiza, entre los terminales de un mismo elemento del circuito. Esto provoca que esta descomposición de corrientes efectuada, no nos conduzca a reflejar de manera exacta los fenómenos energéticos presentes en el circuito.

- Al definir la corriente activa y de dispersión en función de la conductancia equivalente, $G_{e}=P / V^{2}$, se viola el principio de superposición lineal, al ser una función cuadrática.

El autor de esta Tesis, coincide con las afirmaciones realizadas por León, además de matizar que:

- Los inversos de la reactancia y de la resistencia no siempre coinciden con la susceptancia y conductancia respectivamente, lo que conlleva a que la potencia reactiva $Q$, y la potencia de dispersión $D_{s}$, no sean conservativas.

- Al igual que le sucede a las teorías inspiradas en Fryze, la corriente activa es aquella que tiene la misma forma de onda que la tensión, lo que lleva a que no se pueda alcanzar la máxima eficiencia. 
Antecedentes.

\section{I.7. Teoría Unificadora de la Potencia Eléctrica.}

La Teoría Unificadora de la Potencia Eléctrica, es establecida por V. León en 1997 [123], basándose en que los fenómenos inherentes a toda transferencia de energía en un sistema eléctrico, los hallamos reflejados en la expresión de la potencia instantánea, además dichos fenómenos se hallan caracterizados por componentes de las tensiones y corrientes de cada fase, que verifican las leyes y teoremas de la Teoría de Circuitos.

En cualquier sistema eléctrico genérico, en régimen permanente, nos podemos encontrar con los siguientes fenómenos energéticos presentes en el mismo:

- Transferencia de energía neta o flujo unidireccional de potencia.

- Un flujo bidireccional de potencia, debido a los desfases.

- La distorsión armónica, debida a la falta de linealidad.

- La asimetría en los sistemas polifásicos.

Los tres últimos fenómenos energéticos, son en sí ineficiencias del sistema, ya que dan lugar a la presencia de flujos energéticos en el sistema que no son aprovechables.

Cada uno de los fenómenos energéticos anteriores, se caracteriza por un par de componentes de tensión e intensidad, de manera que las tensiones y corrientes de cada fase ( $\mathrm{z}$ ) se descomponen en primer lugar en una de frecuencia fundamental $\left(v_{1 z}, i_{1 z}\right)$ y una de distorsión, formada por armónicos de frecuencia diferente a la fundamental ( $\Sigma \mathrm{v}_{\mathrm{nz}}, \Sigma \mathrm{i}_{\mathrm{mz}}$ ), que caracteriza el fenómeno de la distorsión armónica; a su vez las componentes fundamentales pueden descomponerse en otras tres de secuencia directa, inversa y homopolar $\left(\mathrm{v}_{\mathrm{d} 1 \mathrm{z}}, \mathrm{i}_{\mathrm{d} 1 \mathrm{z}}, \mathrm{v}_{\mathrm{i} 1 \mathrm{z}}, \mathrm{i}_{\mathrm{i} 1 \mathrm{z}}, \mathrm{v}_{\mathrm{h} 1 \mathrm{z}}, \mathrm{i}_{\mathrm{h} 1 \mathrm{z}}\right)$, siendo las componentes inversa y homopolar las que caracterizan el fenómeno de la asimetría; finalmente, las componentes de secuencia directa se descomponen en una componente efectiva $\left(v_{a d 1 z}, i_{a d 1 z}\right)$, que caracteriza el fenómeno de la transferencia de energía útil, y otra componente de desfase $\left(\mathrm{v}_{\mathrm{rd} 1 \mathrm{z}}, \mathrm{i}_{\mathrm{rd} 1 \mathrm{z}}\right)$, que caracteriza los fenómenos de desfase (reactivos), que seguidamente pasamos a ver: 
- Transferencia de energía neta, que representa la energía que es realmente aprovechable y por lo tanto se puede transformar en otro tipo de energía (térmica, mecánica, etc.), se caracteriza por las componentes efectivas de tensión e intensidad.

- Flujo bidireccional de potencia, debido a los desfases, y se caracteriza por la existencia de componentes reactivas de tensión e intensidad.

- La distorsión, se halla caracterizada por la existencia de componentes de frecuencia distinta de la fundamental en las tensiones e intensidades.

- El fenómeno de la asimetría, se halla caracterizado por la presencia de componentes de secuencia directa, inversa y homopolar en las tensiones e intensidades.

Como acabamos de mencionar, todos los fenómenos energéticos presentes en un sistema eléctrico, se hallan contemplados en la expresión de la potencia instantánea, que clásicamente se ha formulado para los sistemas trifásicos como:

$$
p(t)=\sum_{z=1}^{3} v_{z} \cdot i_{z}
$$

La ecuación (I.54), no clarifica de forma adecuada, los diversos fenómenos energéticos presentes en el sistema y por lo tanto no permite una cuantificación de los mismos, para este fin es más conveniente realizar una descomposición de las tensiones e intensidades en sus posibles componentes, tal y como se puede observar a continuación:

$$
\begin{aligned}
& p(t)=\sum_{z=1}^{3} v_{z} \cdot i_{z}=\sum_{z=1}^{3}\left(v_{a d 1 z} i_{a d 1 z}+v_{r d 1 z} i_{r d 1 z}+\sum_{\substack{j \neq k \\
j, k=a, r}} v_{j d 1 z} i_{k d 1 z}+\sum_{\substack{u=i, h \\
j, k=a, r}} v_{j u 1 z} i_{k u 1 z}+\right. \\
& \left.+\sum_{\substack{x \neq y \\
x, y=d, i, h \\
j, k=a, r}} v_{j x 1 z} i_{k x 1 z}+\sum_{\substack{p=2 \\
x, y=d, i, h \\
j, k=a, r}} v_{j x p z} i_{k y p z}+\sum_{\substack{n \neq m \\
n, m=1 \\
x, y=d, i, h \\
j, k=a, r}} v_{j x n z} i_{k y m z}\right)
\end{aligned}
$$


Realizada esta descomposición, pasamos a identificar los términos que forman la ecuación (I.56), el primer término nos da la potencia efectiva instantánea $\mathbf{p}_{\mathbf{e}}(\mathbf{t})$, debida a las componentes activa de secuencia directa y frecuencia fundamental, el segundo y tercer término representa la potencia reactiva instantánea $\mathbf{p}_{\mathbf{r}}(\mathbf{t})$, debida a las componentes reactivas de tensión e intensidad, el cuarto y quinto término son producidos por las componentes de secuencia inversa y homopolar y definen la potencia instantánea de asimetría $\mathbf{p}_{\mathbf{A}}(\mathbf{t})$, siendo el sexto y séptimo términos los que son debidos a la falta de linealidad y representan la potencia instantánea de distorsión $\mathbf{p}_{\mathrm{D}}(\mathbf{t})$.

Lo que podemos poner de manera que quedan perfectamente diferenciados todos los fenómenos energéticos, que se hallan presentes en la transferencia de energía en el sistema eléctrico:

$$
p(t)=p_{e}(t)+p_{r}(t)+p_{A}(t)+p_{D}(t)
$$

Usando la analogía existente entre las potencias instantáneas y aparente [114], que se fundamenta en:

1. La potencia aparente y la instantánea poseen las mismas componentes, y dichas componentes poseen el mismo número de términos.

2. Los términos de la potencia instantánea son productos de valores instantáneos y los de la potencia aparente son el producto de valores eficaces.

3. Los términos que componen la potencia instantánea definen los flujos de energía que caracterizan a cada fenómeno y los términos de la potencia aparente cuantifican dichos fenómenos.

Ponemos la ecuación (I.56) como sigue: 


$$
\begin{aligned}
& S^{2}=9 \cdot V_{a d 1}^{2} \cdot I_{a d 1}^{2}+9 \cdot V_{r d 1}^{2} \cdot I_{r d 1}^{2}+9 \cdot \sum_{\substack{j \neq k \\
j, k=a, r}} V_{j d 1}^{2} \cdot I_{k d 1}^{2}+9 \cdot \sum_{\substack{u=i, h \\
j, k=a, r}} V_{j u 1}^{2} \cdot I_{k u 1}^{2}+ \\
& \text { +9. } \sum_{\substack{x \neq y \\
x, y=d, i, h \\
j, k=a, r}} V_{j \times 1}^{2} \cdot I_{k y 1}^{2}+9 \cdot \sum_{\begin{array}{l}
p=2 \\
x, y=d, i, h \\
j, k=a, r
\end{array}} V_{j \times p}^{2} \cdot I_{k y p}^{2}+9 \cdot \sum_{\begin{array}{l}
n \neq m \\
n, m=1 \\
x, y=d, i, h \\
j, k=a, r
\end{array}} V_{j \times n}^{2} \cdot I_{k y m}^{2}
\end{aligned}
$$

De donde la potencia aparente del sistema la podemos expresar como:

$$
S=3 \cdot \sqrt{\sum_{\substack{n=1 \\ z=d, i, h \\ j=a, r}} V_{j x n z}^{2} \cdot \sum_{\substack{m=1 \\ y=d, i, h \\ k=a, r}} I_{k y m z}^{2}}=\sqrt{P_{E}^{2}+Q^{2}+A^{2}+D^{2}}
$$

En donde cada una de estas componentes, ortogonales entre sí, ya cuantifica cada uno de los fenómenos energéticos, que exponemos:

- Potencia efectiva: está dada por el producto de tensiones e intensidades activas fundamentales y de secuencia directa, cuantifica la energía que es realmente aprovechable:

$$
P_{E}=3 \cdot V_{a d 1} \cdot I_{a d 1}=3 \cdot V_{d 1} \cdot I_{d 1} \cdot \cos \varphi_{d 1}
$$

- Potencia reactiva: se halla compuesta por dos términos, uno de ellos representa la potencia activa residual provocada por los elementos reactivos del sistema, y el otro es la potencia reactiva debida al desfase:

$$
Q=3 \cdot \sqrt{V_{r d 1}^{2} \cdot I_{r d 1}^{2}+\sum_{\substack{j \neq k \\ j=a, r}} V_{j d 1}^{2} \cdot I_{k d 1}^{2}}=3 \cdot V_{a d 1} \cdot I_{d 1} \cdot \operatorname{sen} \varphi_{d}
$$

- Potencia de asimetría: se compone de dos términos, uno de ellos representa la potencias activas y reactivas residuales, debidas a las asimetrías y el otro término representa el fenómeno en sí de la asimetría: 


$$
A=3 \cdot \sqrt{\sum_{\substack{u=i, h \\ j, k=a, r}} V_{j u 1}^{2} \cdot I_{k u 1}^{2}+\sum_{\substack{x \neq y \\ x, y=d, i, h \\ j=a, r}} V_{j x 1}^{2} \cdot I_{k y 1}^{2}}
$$

- Potencia de distorsión: es la magnitud que cuantifica los efectos debidos a las ineficiencias por distorsión:

$$
D=3 \cdot \sqrt{\sum_{\substack{p=2 \\
x, y=d, i, h \\
j, k=a, r}} V_{j x p}^{2} \cdot I_{k y p}^{2}+\sum_{\begin{array}{l}
n \neq m \\
n, m=1 \\
x, y=d, i, h \\
j=a, r
\end{array}} V_{j \times n}^{2} \cdot I_{k y m}^{2}}
$$

Hemos visto que la potencia instantánea y la potencia aparente poseen las mismas componentes, y se han definido las potencias efectiva, reactiva, asimetría y distorsión, que cuantifican los fenómenos energéticos del sistema.

Para la eficiencia del sistema, se define el factor de eficiencia $(\varepsilon)$, que nos cuantifica la energía que es realmente aprovechable por el sistema.

$$
\varepsilon=\frac{P_{E}}{S}=\frac{P_{E}}{\sqrt{P_{E}^{2}+Q^{2}+A^{2}+D^{2}}}
$$




\section{I.8. IEEE Std. 1459/2000.}

La IEEE Std. 1459/2000 [102], es una norma que resume las diversas Teorías existentes usadas para la medida de cantidades relacionadas con la potencia eléctrica, bajo las diversas condiciones que pueden darse en un sistema y que podemos resumir en: linealidad, no linealidad, equilibrio y desequilibrio del mismo. Además de incluir la Teoría de Emanuel, que trataremos en un apartado específico.

En ella se ponen de manifiesto las expresiones matemáticas que fueron usadas en el pasado, así como las nuevas expresiones y explica que características poseen las nuevas definiciones.

Las definiciones clásicas de potencia activa, reactiva y aparente, frecuentemente usadas se basan en el conocimiento desarrollado en los años 40 del siglo XX, y que fueron y algunas aún son válidas sobre todo para propósitos comerciales, siempre y cuando las formas de onda de la tensión sean senoidales.

Pero en los últimos 50 años, se han producido una serie de cambios en los sistemas provocados entre otras cosas por:

1. Profusa utilización de equipos electrónicos, tales como variadores de velocidad, rectificadores, balastos electrónicos, hornos de arco de inducción, ordenadores personales, etc., lo que ha generado la presencia de flujos de energía no activa que distorsionan las tensiones y corrientes, siendo los causantes de la presencia de armónicos en los sistemas.

2. Surgimiento de nuevas definiciones de la potencia eléctrica, que conllevan sus correspondientes discusiones, aprobaciones y rechazos, como podemos ver en [83].

3. La instrumentación usada hoy en día en la mayoría de aplicaciones es analógica y diseñada para el trabajo con ondas senoidales, a la frecuencia fundamental $(50 / 60 \mathrm{~Hz})$, lo que provoca que con ondas distorsionadas se produzcan errores no aceptables, lo que ha derivado en el uso de sistemas de adquisición y medida, que conectados con un ordenador en el que se halle 
implementado el procedimiento de medida más adecuado, nos facilite la cuantificación de los parámetros eléctricos de nuestro interés.

En esta norma, surgen nuevas definiciones de la potencia eléctrica, que ya se plantean en las siguientes situaciones más acordes con nuestros días, tales como:

1. Presencia en los sistemas de tensiones y corrientes no senoidales.

2. Existencia de sistemas desequilibrados en la generación y/o en carga.

Las nuevas definiciones, son desarrolladas como base de las cantidades que deben de ser medidas y sobre todo siempre bajo el punto de vista de la transacción comercial que lleva aparejada el consumo de esa energía eléctrica.

La norma comienza con el planteamiento de una serie de definiciones, a la frecuencia fundamental tales como la potencia activa, reactiva y aparente, como cantidades básicas para poder llevar a cabo el flujo de energía en los sistemas eléctricos.

Desde un punto de vista global podemos decir que en la norma se realiza un planteamiento para los sistemas monofásicos lineales y no lineales, pasando luego a los sistemas trifásicos equilibrados, desequilibrados y luego los no lineales; estando el interés de este estudio centrado en los sistemas desequilibrados y lineales, no se abordaran los otros planteamientos posibles.

En los sistemas monofásicos lineales, la norma plantea las definiciones de las potencias activa y reactiva, que se basan en la formulación que fue ya presentada para estos sistemas por C. P. Steinmetz.

En los sistemas trifásicos senoidales lineales, se realiza un doble planteamiento, que el sistema este equilibrado ó que sea desequilibrado.

En los equilibrados, se muestran las definiciones que coinciden con las ya vistas en el apartado I.2.2.1. , para cuantificar la potencia activa y reactiva, y que seguidamente pasamos a recordar: 
Potencia Activa:

Potencia Reactiva:

$$
P=3 \cdot V_{\ln } \cdot I \cdot \cos \vartheta=\sqrt{3} \cdot V_{/ /} \cdot I \cdot \cos \vartheta
$$

En los desequilibrados, aparecen las mismas expresiones que hemos visto para expresar la potencia activa y reactiva, tal y como aparecía en el apartado I.2.2.2, en las que a mayores se plantea una descomposición de estas potencias activas y reactivas totales en función de sus respectivas componentes de secuencia directa, inversa y homopolar.

$$
\text { Potencia Activa: } \quad P=P_{a}+P_{b}+P_{c}=P_{d}+P_{i}+P_{h}
$$

En donde $\mathrm{P}_{\mathrm{a}, \mathrm{b}, \mathrm{c}}$ representan las potencias activas de cada fase, $\mathrm{y} \mathrm{P}_{\mathrm{d}, \mathrm{r}, \mathrm{h}}$ son las potencias activas de secuencia directa $\left(P_{d}\right)$, secuencia inversa $\left(P_{i}\right)$, y homopolar $\left(P_{h}\right)$.

$$
\text { Potencia Reactiva: } \quad Q=Q_{a}+Q_{b}+Q_{c}=Q_{d}+Q_{i}+Q_{h}
$$

En donde $\mathrm{Q}_{\mathrm{a}, \mathrm{b}, \mathrm{c}}$ representan las potencias reactivas de cada fase, y $\mathrm{Q}_{\mathrm{dri,h}}$ son las potencias reactivas de secuencia directa $\left(Q_{d}\right)$, secuencia inversa $\left(Q_{i}\right)$, y homopolar $\left(\mathrm{Q}_{\mathrm{h}}\right)$.

Observamos por lo tanto que en las expresiones que presenta la Std. 1459/2000, se basan tanto en sistemas monofásicos lineales, como en los sistemas trifásicos lineales equilibrados y desequilibrados en la Teoría de Steinmetz de sistemas monofásicos y su generalización posterior a los sistemas trifásicos, basándose por lo tanto en que los únicos fenómenos energéticos presentes en un sistema eléctrico se hallan inmersos en la expresión de la potencia instantánea.

Las teorías clásicas que se basan en la Teoría de Steinmetz generalizada que se tratan en esta norma, tan sólo contemplan dos fenómenos, que son la transferencia 
Antecedentes.

de energía neta (potencia activa) y la existencia de fenómenos reactivos (potencia reactiva), no considerando por tanto la presencia de flujos de energía procedentes de los desequilibrios del sistema (ya que no se tienen en cuenta la interacción de tensiones y corrientes de diferente secuencia), y que serían procedentes de las asimetrías que presente el sistema eléctrico.

Es de destacar que en las expresiones que se plantean en la Std. 1459/2000, para los sistemas desequilibrados el valor de la potencia activa total del sistema se realiza como suma de tres flujos unidireccionales de distinto valor y cuyos valores máximos no tienen porque alcanzarse en el mismo instante, sucediendo algo similar para el caso de la potencia reactiva total, que se formula como suma de tres flujos sinusoidales desequilibrados y de forma general de distintas amplitudes y cuyos valores máximos pueden ser alcanzados en distintos momentos, y por lo tanto para el autor de esta Tesis, la norma tan sólo plantea algunas formulaciones novedosas para el caso de sistemas no lineales, pero no sucede así con los sistemas lineales, ya que el planteamiento que se presenta es el de las teorías clásicas basadas como ya se ha comentado en la Teoría de Steinmetz generalizada y reproduciendo sus conceptos erróneos ya comentados con anterioridad.

\section{I.8.1. Teoría de Emanuel.}

A. Emanuel ha sido un gran propulsor de las Teorías de la Energía Eléctrica, desde los años 70 del siglo XX. En [78], pone de manifiesto su intento de relacionar el sentido físico de las componentes de la potencia instantánea, con los valores que toman, con el fin de lograr modelos de la potencia aparente que las tengan en consideración. Realiza su exposición partiendo de sistemas sinusoidales y luego procede a abordar los no senoidales. Se basa en la Teoría de Fryze, de la potencia aparente:

$$
S^{2}=P^{2}+Q_{F}^{2}
$$


en donde la $Q_{F}$, engloba a diversos tipos de potencia reactiva, procediendo a realizar su identificación por sus amplitudes en el término de la potencia instantánea. En este artículo, plantea que debe de procederse a una separación de la $Q_{F}$ en una $Q_{1}$, que sería la potencia reactiva del sistema a la frecuencia fundamental, y otra $Q_{H}$ en la que se considere la potencia reactiva debida a los armónicos. También manifiesta que la potencia reactiva de frecuencia fundamental $Q_{1}$, no puede ser sumada algebraicamente con la potencia reactiva debida a los armónicos $Q_{H}$ ya que sus amplitudes máximas no se alcanzan simultáneamente. Dentro de sus conclusiones deja claro que el término de la potencia reactiva de frecuencia fundamental : $Q_{1}=V_{1} I_{1} \operatorname{sen} \vartheta_{1}$, es el término dominante dentro de toda la potencia reactiva, y por lo tanto debe de tratarse de manera independiente, ya que es la que hace incrementar de manera considerable los valores "rms" de las corrientes en las líneas.

En [77], analiza la potencia aparente, en los sistema trifásicos a tres hilos haciendo uso de las componentes simétricas, y el producto entre la tensión y corriente equivalentes, que se había usado en el pasado $^{4}$ y que resurge de nuevo, basadas en:

$$
\begin{aligned}
& V_{e}=\sqrt{\left(V_{a}^{2}+V_{b}^{2}+V_{c}^{2}\right) / 3} \\
& I_{e}=\sqrt{\left(I_{a}^{2}+I_{b}^{2}+I_{c}^{2}\right) / 3}
\end{aligned}
$$

La potencia aparente queda definida como:

$$
S=3 \cdot V_{e} \cdot I_{e}
$$

Emanuel, haciendo uso de las componentes simétricas, define la potencia aparente como:

$$
S=3 \cdot \sqrt{V_{d}^{2}+V_{i}^{2}+V_{h}^{2}} \cdot \sqrt{I_{d}^{2}+I_{i}^{2}+I_{h}^{2}}
$$

\footnotetext{
${ }^{4}$ Lurie, L.S. : Kajushchyasya Mojnost Trehfaznoi Systemi. Elektrichestvo 1 (1951) pp.47-52.
} 
En base a esto último, hace hincapié en que cada componente de la potencia aparente, así definida, posee un claro sentido físico, y que es necesario separar las potencias activas de secuencia directa, de las de secuencia inversa y homopolar.

A la hora de las descomposiciones que realiza, de la potencia aparente, en potencia activa y reactiva, vuelve a recalcar la necesidad de separar la potencia activa de secuencia directa y frecuencia fundamental, de la provocada por la presencia de armónicos, al igual que le sucede a la potencia reactiva, que debe de descomponerse en potencia reactiva de secuencia directa y frecuencia fundamental, de la procedente de los armónicos, debido a su importancia en los sistemas en relación con esta última.

En [73], expone la idea de que los sistemas trifásicos desequilibrados, y no lineales, a cuatro hilos se pueden explicar en base a un circuito virtual equilibrado que posea las mismas pérdidas de potencia que el sistema trifásico real, que consuma una potencia aparente efectiva:

$$
S_{e}=3 \cdot V_{e} I_{e}
$$

que se obtiene usando los valores efectivos de tensión $\left(V_{e}\right)$ y corriente $\left(I_{e}\right)$ presentes en el circuito equivalente.

En donde $I_{e}$, se define de la ecuación de igualdad siguiente, de pérdidas de potencia del circuito real y equivalente en las líneas:

$$
3 r I_{e}^{2}=r \cdot\left(I_{a}^{2}+I_{b}^{2}+I_{c}^{2}\right)+r_{n} I_{n}^{2}
$$

en la anterior expresión $r$, representa la resistencia de cada fase de las líneas, y $r_{n_{r}}$ la del neutro del sistema, así como $I_{a, b, c}$, son las corrientes de línea del sistema, y la $I_{n}$ es la corriente del neutro.

$$
I_{e}=\sqrt{\left(I_{a}^{2}+I_{b}^{2}+I_{c}^{2}+\rho I_{n}^{2}\right) / 3} ; \rho=r_{n} / r
$$

Manea, F. :The powers of unsymmetrical and unbalenced systems. Stud. Res. in Energet. 10 (1960), n04, pp.771-777. 
Procediendo de manera similar, se obtiene el valor de la tensión equivalente, que toma por valor:

$$
V_{e}=\sqrt{\left(V_{a n}^{2}+V_{b n}^{2}+V_{c n}^{2}+V_{a b}^{2}+V_{b c}^{2}+V_{c a}^{2}\right) / 4}
$$

En la misma idea, pero con mayor profundidad, establece Emanuel en la IEEE Std.1459/2000 [102], lo siguiente que ya había esbozado en anteriores publicaciones, se parte de una potencia aparente efectiva, $S_{e}=3 \cdot V_{e} I_{e}$, que se obtiene con los valores efectivos de tensión y corriente, cuyos valores ya se han expuesto con anterioridad, pero ahora procede a realizar una descomposición en: componente fundamental y no fundamental, quedando:

$$
S_{e}=\sqrt{S_{e 1}^{2}+S_{e N}^{2}}
$$

Teniendo por tanto la potencia aparente fundamental $S_{e 1 \text {, }}$ tres componentes ortogonales entre sí, la potencia activa de secuencia directa fundamental $P_{d 1}$, la potencia reactiva de secuencia directa fundamental $Q_{d 1}$ y la potencia de desequilibrio de secuencia directa fundamental $S_{u 1}$.

$$
S_{e 1}=3 \cdot V_{e 1} I_{e 1}=\sqrt{P_{d 1}^{2}+Q_{d 1}^{2}+S_{u 1}^{2}}
$$

en donde:

$$
\begin{aligned}
& P_{d 1}=3 \cdot V_{d 1} I_{d 1} \cos \vartheta_{d 1} \\
& Q_{d 1}=3 \cdot V_{d 1} I_{d 1} \operatorname{sen} \vartheta_{d 1} \\
& S_{u 1}=\sqrt{S_{e 1}^{2}-S_{d 1}^{2}}=\sqrt{S_{e 1}^{2}-P_{d 1}^{2}-Q_{d 1}^{2}}
\end{aligned}
$$

Y el otro término es el de la potencia aparente efectiva no fundamental $S_{e N}$

$$
S_{e N}=\sqrt{S_{e}^{2}-S_{e 1}^{2}}=\sqrt{D_{e 1}^{2}+D_{e V}^{2}+S_{e H}^{2}}
$$


compuesto por tres términos también ortogonales entre sí, que son la potencia de distorsión en corrientes $D_{e 1}$, la potencia de distorsión en tensiones $D_{e v}$ y la potencia aparente armónica $S_{e H}$, y cuyos valores son los siguientes:

$$
\begin{aligned}
& D_{e 1}=3 \cdot V_{e 1} I_{e H} \\
& D_{e V}=3 \cdot V_{e H} I_{e 1} \\
& S_{e H}=3 \cdot V_{e H} I_{e H}
\end{aligned}
$$

Es de destacar que Emanuel, acierte en:

- La separación de las potencias activa y reactiva de secuencia directa y frecuencia fundamental, ya que de manera implícita reconoce el significado de los fenómenos de energía útil y reactiva. Así como la ubicación de las potencias activas y reactivas de otras frecuencias y secuencias de fase no directa en los fenómenos de la distorsión y el desequilibrio, ya que estas potencias son fruto de la perturbación de los desequilibrios y distorsiones en los flujos de potencia de energía útil y reactiva, tal y como ya estableció V. León mediante la Teoría Unificadora de la Potencia Eléctrica en 1997 [123].

No obstante, existen una serie de puntualizaciones que seguidamente mencionamos:

- La definición de la potencia aparente efectiva, no es adecuada para poder cuantificar los fenómenos del sistema. Aunque para su obtención parte del hecho físico de las pérdidas de potencia en las líneas del sistema, se basa en un circuito equivalente virtual, en el cual no están presentes los fenómenos energéticos del receptor real.

- En los sistemas a tres hilos, no considera los fenómenos debidos a la componente homopolar de tensión y corriente de las fases del receptor, pero en los sistemas a cuatro hilos magnifica de manera abusiva, el efecto de hilo neutro sobre la distorsión y el desequilibrio. 
- La potencia aparente efectiva, no determina la potencia aparente del receptor real, lo que lleva a que las potencias de desequilibrio y no fundamental definidas por Emanuel, no nos permitan cuantificar los fenómenos del desequilibrio y distorsión en el receptor.

- A la hora de determinar el factor de potencia, lo hace en base a la relación entre la potencia activa (en la que incluye las potencias activas de frecuencia fundamental y de secuencia inversa, homopolar y la de distorsión) y la potencia aparente efectiva, con lo cual en la potencia activa incluye términos que no contribuyen a la eficiencia.

- No se pueden diseñar dispositivos de mejora de la eficiencia, ya que no define las componentes de tensión y corriente que caracterizan los fenómenos del desequilibrio y la distorsión. 


\section{I.9. Conclusiones.}

A lo largo de este capítulo se han expuesto diversas teorías de gran importancia para el desarrollo y conocimiento de la potencia eléctrica, y que han surgido a lo largo del tiempo desde finales del siglo XIX hasta nuestros días.

La idea del autor de esta Tesis es dejar patente, que desde las primeras publicaciones en las cuales se trata el tema de la Potencia Reactiva [108],[125], pasando por autores tales como Steinmetz, Budeanu, Fryze, Akagi, Czarnecki, Emanuel, etc, no existe un acuerdo total al respecto de la definición de lo que se debe de considerar como Potencia Reactiva, a no ser que se trate de sistemas monofásicos y lineales tal y como menciona Emanuel en [77], ya que este caso todos entendemos que la potencia reactiva $Q$, es la amplitud de las oscilaciones de la potencia instantánea.

En 2006, Jeon [104], manifiesta que aún siendo nula la potencia reactiva instantánea, esto no es garantía de que la potencia reactiva sea nula cuando nos hallamos en sistemas con desequilibrios de tensiones, conclusión que comparte y que ya había puesto de manifiesto el autor de esta Tesis, tal y como figura en [114], presentado en la 9th International Conference, E.P.Q.U. en 2007, y en [113] presentado en el T.I.E.M.A. en 2005.

En el siguiente capítulo se establecerá el concepto de potencia reactiva (o de desfase), y se expresarán por separado los fenómenos reactivos (o de desfase) debidos a la presencia de reactancias en el sistema, y los provocados por los desequilibrios, siendo estos últimos desconocidos en la literatura técnica, y para lo que se ha usado la Teoría Unificadora de la Potencia Eléctrica [123]. 


\section{CAPÍTULO II}

FENÓMENOS DE DESFASE EN SISTEMAS TRIFÁSICOS DESEQUILIBRADOS LINEALES. 



\section{II.- Fenómenos de desfase en sistemas desequilibrados lineales.}

\section{II.1.- Introducción.}

En este capítulo, se tratarán los fenómenos de desfase en los sistemas trifásicos desequilibrados y lineales.

A la hora de proceder al análisis de los fenómenos de desfase, hemos de tener en cuenta que el fenómeno del desfase está constituido por la energía eléctrica que no se transforma, siendo ello provocado como sabemos por diversos factores, tales como la presencia de reactancias en el sistema, presencia de dispositivos electrónicos como los convertidores, y la existencia de desequilibrios, son por lo tanto muy diversas las causas que pueden llevar a la aparición del fenómeno del desfase, pero todos ellos poseen un punto de conexión y que es que provocan el desfase entre tensiones y corrientes.

Hasta ahora los fenómenos de desfase se habían estudiado como un todo sin realizar una separación de cuales eran los causantes del mismo, en esta Tesis se analizan los fenómenos de desfase provocados por los desequilibrios, expresándolos por separado de los provocados por otras causas, hecho este que no se había planteado y llevado a cabo en la literatura técnica hasta el momento.

Estos fenómenos de desfase son en sí energías no aprovechadas que no se transforman por el sistema, y constituyen por lo tanto una ineficiencia del mismo, es por ello que para poder identificarlos se ha partido de la potencia instantánea, ya que esta magnitud está directamente ligada con la energía y existe consenso dentro del seno de la Comunidad Científica sobre esta cuestión, siendo la potencia instantánea, como la velocidad con la que se transfiere la energía en un circuito.

$$
p(t)=\frac{\partial W}{\partial t}
$$


Para poder obtener la expresión del fenómeno del desfase (en sistemas en triángulo y en estrella con neutro), el procedimiento que se ha llevado a cabo ha sido el mismo, se parte inicialmente de un sistema trifásico equilibrado y lineal, y se plantea primeramente la expresión de la potencia instantánea del sistema según establece la Teoría Clásica, ya que en ella se halla inmersa la potencia reactiva instantánea, posteriormente se formula la potencia instantánea usando la Teoría Unificadora, y la potencia reactiva instantánea, que al igual que expone Emanuel en la IEEE Standard $1459 / 200$ se establece para la frecuencia fundamental del sistema y secuencia directa, posteriormente se trabaja con el sistema trifásico desequilibrado y lineal y se obtiene la nueva expresión de la potencia reactiva instantánea de secuencia directa y frecuencia fundamental para este caso. Una vez obtenidas estas dos expresiones de la potencia reactiva instantánea la del sistema equilibrado y la del desequilibrado, se observa por comparación la existencia de términos en el caso desequilibrado que no aparecían cuando el sistema estaba equilibrado, siendo estos términos provocados por el desequilibrio y los que nos permiten expresar el fenómeno del desfase debido al desequilibrio del sistema.

Al igual que realizo Steinmetz, la potencia reactiva instantánea o de desfase que cuantifica el fenómeno se obtiene como la amplitud de los flujos de potencia reactiva instantánea, y que podemos definir como aquella energía que no se transforma y que se manifiesta cuando hay desfases entre las ondas de tensión y de intensidad de cada fase, provocados por las reactancias, los convertidores controlados o los desequilibrios. 


\section{II.2.- Fenómenos de desfase en sistemas desequilibrados lineales en triángulo. Tensiones equilibradas.}

\section{II.2.1.- Teoría Clásica.}

Sea un generador trifásico equilibrado de secuencia directa, que alimenta a un receptor trifásico lineal desequilibrado conectado en triángulo, formado por las impedancias $Z_{12}, Z_{23}, Z_{31}$ (consideradas inductivas), y que para mayor facilidad a la hora de poder identificar de forma clara los fenómenos de desfase, se ha procedido a descomponer como se muestra en la figura II.1, en las resistencias y reactancias equivalentes en paralelo $R_{i j}, X_{i j},(i, j=1,2,3$ e $i \neq j)$, y siendo el valor de las tensiones aplicadas el siguiente:

$$
\begin{aligned}
& V_{12}(t)=V_{12 d}(t)=V_{d} \cdot \sqrt{2} \cdot \operatorname{sen}\left(w t+\alpha_{d}\right) \\
& V_{23}(t)=V_{23 d}(t)=V_{d} \cdot \sqrt{2} \cdot \operatorname{sen}\left(w t+\alpha_{d}-120^{\circ}\right) \\
& V_{31}(t)=V_{31 d}(t)=V_{d} \cdot \sqrt{2} \cdot \operatorname{sen}\left(w t+\alpha_{d}-240^{\circ}\right)
\end{aligned}
$$

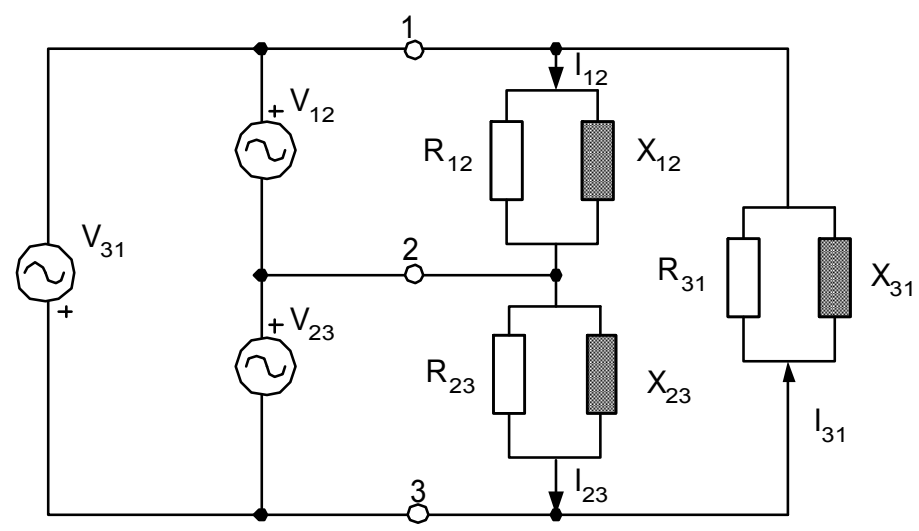

Figura II.1. Sistema en triángulo. Tensiones equilibradas.

Al ser un sistema equilibrado en tensiones de secuencia directa, estas tensiones tan sólo tendrán por lo tanto componente directa.

La aplicación de este sistema de tensiones sobre el receptor, da lugar a la circulación de corrientes en las fases del mismo, en cuyas expresiones se han usado las conductancias $\left(G_{z}\right)$ y susceptancias $\left(B_{z}\right)$ equivalentes y cuyos valores expresados en forma fasorial son: 


$$
\begin{aligned}
& \bar{I}_{12}=\bar{V}_{12 d} \cdot\left(G_{12}+\bar{B}_{12}\right) \\
& \bar{I}_{23}=\bar{V}_{23 d} \cdot\left(G_{23}+\bar{B}_{23}\right)=a^{2} \cdot \bar{V}_{12 d} \cdot\left(G_{23}+\bar{B}_{23}\right) \\
& \bar{I}_{31}=\bar{V}_{31 d} \cdot\left(G_{31}+\bar{B}_{31}\right)=a \cdot \bar{V}_{12 d} \cdot\left(G_{31}+\bar{B}_{31}\right)
\end{aligned}
$$

Como es un sistema desequilibrado en corrientes, podemos aplicar el Teorema de Stokvis y pasamos a determinar las componentes simétricas de las corrientes de fase del circuito anterior, y procedemos a su descomposición en función de los fenómenos resistivos y reactivos (asociados con las susceptancias) del receptor, que para el que se halla conectado entre 1 y 2 vale:

$$
\begin{aligned}
\bar{I}_{12 d} & =\frac{1}{3} \cdot \bar{V}_{12 d} \cdot\left(G_{12}+G_{23}+G_{31}\right)+\frac{1}{3} \cdot \bar{V}_{12 d} \cdot\left(\bar{B}_{12}+\bar{B}_{23}+\bar{B}_{31}\right)= \\
& =\bar{I}_{12 d a}+\bar{I}_{12 d r} \\
\bar{I}_{12 i} & =\frac{1}{3} \cdot \bar{V}_{12 d} \cdot\left(G_{12}+a \cdot G_{23}+a^{2} \cdot G_{31}\right)+\frac{1}{3} \cdot \bar{V}_{12 d} \cdot\left(\bar{B}_{12}+a \cdot \bar{B}_{23}+a^{2} \cdot \bar{B}_{31}\right)= \\
& =\bar{I}_{12 i R}+\bar{I}_{12 i x} \\
\bar{I}_{12 h} & =\frac{1}{3} \cdot \bar{V}_{12 d} \cdot\left(G_{12}+a^{2} \cdot G_{23}+a \cdot G_{31}\right)+\frac{1}{3} \cdot \bar{V}_{12 d} \cdot\left(\bar{B}_{12}+a^{2} \cdot \bar{B}_{23}+a \cdot \bar{B}_{31}\right)= \\
& =\bar{I}_{12 h R}+\bar{I}_{12 h x}
\end{aligned}
$$

Los valores de las corrientes de secuencia directa para las otras fases valen:

$$
\begin{aligned}
& \bar{I}_{23 d}=a^{2} \cdot \bar{I}_{12 d} \\
& \bar{I}_{31 d}=a \cdot \bar{I}_{12 d}
\end{aligned}
$$


Los fenómenos energéticos que se hallan presentes en el sistema, los podemos deducir de las componentes de la potencia instantánea, que pasamos a expresar en función de las componentes simétricas (directa, inversa y homopolar, con los subíndices $d, i, h)$ de las tensiones y corrientes de cada fase como se ve a continuación tal y como establece la Teoría Clásica, en donde las corrientes de secuencia directa se han descompuesto en su componente activa y reactiva (subíndices a y $r$ respectivamente):

$$
\begin{aligned}
p(t)= & v_{12 d} \cdot\left(i_{12 d a}+i_{12 d r}+i_{12 i}+i_{12 h}\right)+ \\
& +v_{23 d} \cdot\left(i_{23 d a}+i_{23 d r}+i_{23 i}+i_{23 h}\right)+ \\
& +v_{31 d} \cdot\left(i_{31 d a}+i_{31 d r}+i_{31 i}+i_{31 h}\right)
\end{aligned}
$$

El fenómeno reactivo, para la Teoría Clásica se halla definido por la potencia reactiva instantánea $Q_{r}(t)$, y que se halla inmersa en la expresión anteriormente expuesta de la potencia instantánea, y que vendrá determinada por el producto de las tensiones y corrientes reactivas de cada fase e idéntica secuencia, tal y como se muestra:

$$
\begin{aligned}
& Q_{r}(t)=v_{12 d} \cdot i_{12 d r}+v_{23 d} \cdot i_{23 d r}+v_{31 d} \cdot i_{31 d r}= \\
& =Q_{r 12 d}(t)+Q_{r 23 d}(t)+Q_{r 31 d}(t)
\end{aligned}
$$

Vamos a proceder a determinar el primero de los términos de la ecuación (II.6), para lo que necesitamos los términos de la tensión y la corriente directa reactiva.

La ecuación (II.3) la podemos expresar como:

$$
\bar{I}_{12 d}=\bar{V}_{12 d} \cdot\left(G_{e}+\bar{B}_{e}\right)
$$

en donde $G_{e}$ y $B_{e}$ son las conductancias y susceptancias directas del receptor, y que vienen dadas por: 


$$
G_{e}=\frac{1}{3} \cdot\left(G_{12}+G_{23}+G_{31}\right) ; \quad \bar{B}_{e}=\frac{1}{3} \cdot\left(\bar{B}_{12}+\bar{B}_{23}+\bar{B}_{31}\right)
$$

La componente directa reactiva de la corriente en su expresión temporal será:

$$
i_{12 d r}(t)=V_{d} \cdot \sqrt{2} \cdot B_{e} \cdot \operatorname{sen}\left(w t+\alpha_{d}-90^{\circ}\right)
$$

y la tensión directa la tenemos en la ecuación (II.1) siendo su valor:

$$
V_{12 d}(t)=V_{d} \cdot \sqrt{2} \cdot \operatorname{sen}\left(w t+\alpha_{d}\right)
$$

Por lo tanto la potencia reactiva instantánea directa para el receptor conectado entre 1 y $2, Q_{r 12 d}(t)$ vale:

$$
\begin{aligned}
& Q_{r 12 d}(t)=V_{12 d}(t) \cdot i_{12 d r}(t)= \\
& =V_{d} \cdot \sqrt{2} \cdot \operatorname{sen}\left(w t+\alpha_{d}\right) \cdot V_{d} \cdot \sqrt{2} \cdot B_{e} \cdot \operatorname{sen}\left(w t+\alpha_{d}-90^{\circ}\right)= \\
& =-V_{d}^{2} \cdot(\sqrt{2})^{2} \cdot B_{e} \cdot \operatorname{sen}\left(w t+\alpha_{d}\right) \cdot \cos \left(w t+\alpha_{d}\right)=-B_{e} \cdot V_{d}^{2} \cdot \operatorname{sen} 2\left(w t+\alpha_{d}\right)
\end{aligned}
$$

Siguiendo el mismo procedimiento para los términos $\mathrm{Q}_{\mathrm{r} 23 \mathrm{~d}}(\mathrm{t})$ y $\mathrm{Q}_{\mathrm{r} 31 \mathrm{~d}}(\mathrm{t})$ de la ecuación (II.6), obtenemos la potencia reactiva instantánea del sistema como:

$$
\begin{aligned}
& Q_{r}(t)=-B_{e} \cdot V_{d}^{2} \cdot \operatorname{sen} 2\left(w t+\alpha_{d}\right) \\
& -B_{e} \cdot V_{d}^{2} \cdot \operatorname{sen} 2\left(w t+\alpha_{d}-120^{\circ}\right) \\
& -B_{e} \cdot V_{d}^{2} \cdot \operatorname{sen} 2\left(w t+\alpha_{d}-240^{\circ}\right)
\end{aligned}
$$

Observamos, después de analizada la expresión de la potencia reactiva instantánea, dada por la ecuación (II.12), que los fenómenos reactivos (o de desfase) que tengamos en el sistema son debidos exclusivamente a la presencia de las reactancias (ó susceptancias) del sistema. 


\section{II.2.2.- Teoría Unificadora.}

Vamos ahora a determinar el fenómeno reactivo, usando la Teoría Unificadora de la Potencia Eléctrica, partimos también de la expresión de la potencia instantánea del sistema, que en este caso de tensiones de alimentación equilibradas no difiere a la hora de analizar el fenómeno reactivo de lo visto en la Teoría Clásica, ya que tan sólo se considera que las corrientes de secuencia directa poseen componentes activa y reactiva, quedándonos entonces la siguiente expresión para la potencia instantánea:

$$
\begin{aligned}
p(t)= & v_{12 d} \cdot\left(i_{12 d a}+i_{12 d r}+i_{12 i}+i_{12 h}\right)+ \\
& +v_{23 d} \cdot\left(i_{23 d a}+i_{23 d r}+i_{23 i}+i_{23 h}\right)+ \\
& +v_{31 d} \cdot\left(i_{31 d a}+i_{31 d r}+i_{31 i}+i_{31 h}\right)
\end{aligned}
$$

La potencia reactiva instantánea, según la Teoría Unificadora, en este caso de que las tensiones de alimentación estén equilibradas, coincide con lo planteado por la Teoría Clásica obteniéndose las expresiones mostradas en las ecuaciones (II.6, 11 y 12), observando que el fenómeno reactivo, se pone de manifiesto en los sistemas eléctricos con tensiones de alimentación equilibradas por medio de tres flujos de potencia sinusoidales y equilibrados constituyendo la potencia reactiva instantánea total del sistema y que seguidamente pasamos a recordar:

$$
\begin{aligned}
& Q_{r}(t)=-B_{e} \cdot V_{d}^{2} \cdot \operatorname{sen} 2\left(w t+\alpha_{d}\right) \\
& -B_{e} \cdot V_{d}^{2} \cdot \operatorname{sen} 2\left(w t+\alpha_{d}-120^{\circ}\right) \\
& -B_{e} \cdot V_{d}^{2} \cdot \operatorname{sen} 2\left(w t+\alpha_{d}-240^{\circ}\right)
\end{aligned}
$$

La magnitud que nos permite cuantificar este fenómeno, es la potencia reactiva $\mathrm{Q}_{r}$, y cuyo valor es igual a la suma de las amplitudes de la ecuación (II.14).

$$
Q_{r}=3 \cdot B_{e} \cdot V_{d}^{2}
$$




\section{II.3.- Fenómenos de desfase en sistemas desequilibrados lineales en} triángulo. Tensiones desequilibradas.

\section{II.3.1.- Teoría Clásica.}

Ahora procederemos al análisis del sistema mostrado en la figura II.2, en el cual un receptor trifásico desequilibrado es alimentado por un generador trifásico también desequilibrado, y por lo tanto realizamos la descomposición en sus componentes simétricas, de las tensiones de sus fases:

$$
\begin{aligned}
& V_{12}(t)=V_{d} \cdot \sqrt{2} \cdot \operatorname{sen}\left(w t+\alpha_{d}\right)+V_{i} \cdot \sqrt{2} \cdot \operatorname{sen}\left(w t+\alpha_{i}\right) \\
& V_{23}(t)=V_{d} \cdot \sqrt{2} \cdot \operatorname{sen}\left(w t+\alpha_{d}-120^{\circ}\right)+V_{i} \cdot \sqrt{2} \cdot \operatorname{sen}\left(w t+\alpha_{i}+120^{\circ}\right) \\
& V_{31}(t)=V_{d} \cdot \sqrt{2} \cdot \operatorname{sen}\left(w t+\alpha_{d}-240^{\circ}\right)+V_{i} \cdot \sqrt{2} \cdot \operatorname{sen}\left(w t+\alpha_{i}+240^{\circ}\right)
\end{aligned}
$$

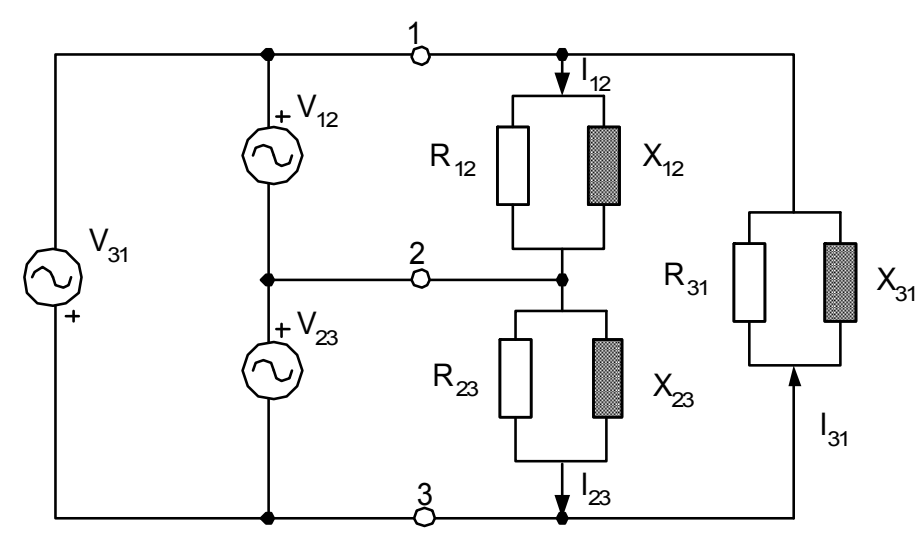

Figura II.2. Sistema en triángulo. Tensiones desequilibradas.

Expresando la ecuación (II.16) en su forma fasorial tenemos:

$$
\begin{aligned}
& \bar{V}_{12}=\bar{V}_{12 d}+\bar{V}_{12 i} \\
& \bar{V}_{23}=\bar{V}_{23 d}+\bar{V}_{23 i}=a^{2} \cdot \bar{V}_{12 d}+a \cdot \bar{V}_{12 i} \\
& \bar{V}_{31}=\bar{V}_{31 d}+\bar{V}_{31 i}=a \cdot \bar{V}_{12 d}+a^{2} \cdot \bar{V}_{12 i}
\end{aligned}
$$

Aplicando la Ley de Ohm al circuito de la figura II.2, obtenemos las corrientes de las fases del receptor, en su forma fasorial: 


$$
\begin{aligned}
& \bar{I}_{12}=\bar{V}_{12} \cdot\left(G_{12}+\bar{B}_{12}\right)=\left(\bar{V}_{12 d}+\bar{V}_{12 i}\right) \cdot\left(G_{12}+\bar{B}_{12}\right) \\
& \bar{I}_{23}=\bar{V}_{23} \cdot\left(G_{23}+\bar{B}_{23}\right)=\left(a^{2} \cdot \bar{V}_{12 d}+a \cdot \bar{V}_{12 i}\right) \cdot\left(G_{23}+\bar{B}_{23}\right) \\
& \bar{I}_{31}=\bar{V}_{31} \cdot\left(G_{31}+\bar{B}_{31}\right)=\left(a \cdot \bar{V}_{12 d}+a^{2} \cdot \bar{V}_{12 i}\right) \cdot\left(G_{31}+\bar{B}_{31}\right)
\end{aligned}
$$

A continuación aplicamos el Teorema de Stokvis al sistema de ecuaciones dado por (II.18), y determinamos las componentes simétricas de las corrientes de fase del receptor conectado entre 1 y 2 :

$$
\begin{aligned}
& \bar{I}_{12 d}=\frac{1}{3} \bar{V}_{12 d} \cdot\left(G_{12}+G_{23}+G_{31}\right)+\frac{1}{3} \bar{V}_{12 d} \cdot\left(\bar{B}_{12}+\bar{B}_{23}+\bar{B}_{31}\right)+ \\
& +\frac{1}{3} \bar{V}_{12 i} \cdot\left(G_{12}+a^{2} \cdot G_{23}+a \cdot G_{31}\right)+\frac{1}{3} \bar{V}_{12 i} \cdot\left(\bar{B}_{12}+a^{2} \cdot \bar{B}_{23}+a \cdot \bar{B}_{31}\right) \\
& \bar{I}_{12 i}=\frac{1}{3} \bar{V}_{12 d} \cdot\left(G_{12}+a \cdot G_{23}+a^{2} \cdot G_{31}\right)+\frac{1}{3} \bar{V}_{12 d} \cdot\left(\bar{B}_{12}+a \cdot \bar{B}_{23}+a^{2} \cdot \bar{B}_{31}\right)+ \\
& +\frac{1}{3} \bar{V}_{12 i} \cdot\left(G_{12}+G_{23}+G_{31}\right)+\frac{1}{3} \bar{V}_{12 i} \cdot\left(\bar{B}_{12}+\bar{B}_{23}+\bar{B}_{31}\right) \\
& \bar{I}_{12 h}=\frac{1}{3} \bar{V}_{12 d} \cdot\left(G_{12}+a^{2} \cdot G_{23}+a \cdot G_{31}\right)+\frac{1}{3} \bar{V}_{12 d} \cdot\left(\bar{B}_{12}+a^{2} \cdot \bar{B}_{23}+a \cdot \bar{B}_{31}\right)+ \\
& +\frac{1}{3} \bar{V}_{12 i} \cdot\left(G_{12}+a \cdot G_{23}+a^{2} \cdot G_{31}\right)+\frac{1}{3} \bar{V}_{12 i} \cdot\left(\bar{B}_{12}+a \cdot \bar{B}_{23}+a^{2} \cdot \bar{B}_{31}\right)
\end{aligned}
$$

Y que para las otras fases, las componentes de secuencia directa toman los valores siguientes:

$$
\begin{aligned}
& \bar{I}_{23 d}=a^{2} \cdot \bar{I}_{12 d} \\
& \bar{I}_{23 i}=a \cdot \bar{I}_{12 i} \\
& \bar{I}_{31 d}=a \cdot \bar{I}_{12 d} \\
& \bar{I}_{31 i}=a^{2} \cdot \bar{I}_{12 i}
\end{aligned}
$$

Del análisis de la ecuación (II.19) podemos relacionar diversos elementos del circuito, que pasaremos a denominar como sigue: 
1. Consideramos como susceptancia directa e inversa a:

$$
\bar{B}_{e}=\frac{1}{3} \cdot\left(\bar{B}_{12}+\bar{B}_{23}+\bar{B}_{31}\right)
$$

2. Admitancia básica de desequilibrio para la secuencia directa a:

$$
\overline{Y^{\prime}}=Y_{-\alpha^{\prime}}^{\prime}=\frac{1}{3} \cdot\left[G_{12}+\bar{B}_{12}+a^{2} \cdot\left(G_{23}+\bar{B}_{23}\right)+a \cdot\left(G_{31}+\bar{B}_{31}\right)\right]
$$

3. Admitancia básica de desequilibrio para la secuencia inversa a:

$$
\overline{Y^{\prime \prime}}=Y_{-\alpha^{\prime \prime}}^{\prime \prime}=\frac{1}{3} \cdot\left[G_{12}+\bar{B}_{12}+a \cdot\left(G_{23}+\bar{B}_{23}\right)+a^{2} \cdot\left(G_{31}+\bar{B}_{31}\right)\right]
$$

Que podemos expresar también como:

$$
\begin{aligned}
& \bar{B}_{e}=\frac{1}{\bar{X}_{e}}=\frac{1}{3} \cdot\left(\frac{1}{\bar{X}_{12}}+\frac{1}{\bar{X}_{23}}+\frac{1}{\bar{X}_{31}}\right) \\
& \bar{Y}^{\prime}=Y_{-\alpha^{\prime}}^{\prime}=\frac{1}{3}\left(\bar{Y}_{12}+a^{2} \cdot \bar{Y}_{23}+a \cdot \bar{Y}_{31}\right) \\
& \bar{Y}^{\prime \prime}=Y_{-\alpha^{\prime \prime}}^{\prime \prime}=\frac{1}{3}\left(\bar{Y}_{12}+a \cdot \bar{Y}_{23}+a^{2} \cdot \bar{Y}_{31}\right)
\end{aligned}
$$

Al igual que habíamos realizado en los sistemas equilibrados en tensiones, partimos de la expresión de la potencia instantánea, en donde la corrientes de cada secuencia se han descompuesto en su parte activa y reactiva, tal y como establece la Teoría Clásica, y ahora al ser un sistema desequilibrado en tensiones, se ha procedido a descomponer en sus secuencias directa e inversa la tensión de cada fase. 


$$
\begin{aligned}
p(t) & =\left(v_{12 d}+v_{12 i}\right) \cdot\left(i_{12 d a}+i_{12 d r}+i_{12 i a}+i_{12 i r}+i_{12 h a}+i_{12 h r}\right)+ \\
& +\left(v_{23 d}+v_{23 i}\right) \cdot\left(i_{23 d a}+i_{23 d r}+i_{23 i a}+i_{23 i r}+i_{23 h a}+i_{23 h r}\right)+ \\
& +\left(v_{31 d}+v_{31 i}\right) \cdot\left(i_{31 d a}+i_{31 d r}+i_{31 i a}+i_{31 i r}+i_{31 h a}+i_{31 h r}\right)
\end{aligned}
$$

Los fenómenos reactivos se deducen de la componente reactiva de la potencia instantánea $\mathrm{Q}_{\mathrm{r}}(\mathrm{t})$, y como establece la Teoría Clásica será la suma de las potencias reactivas debidas a las secuencias directa $Q_{r d}(t)$ e inversa $Q_{r i}(t)$, al ser un sistema desequilibrado en tensiones.

Siendo la potencia reactiva de secuencia directa la provocada por los productos de la tensiones directas por las componentes directas reactivas de las corrientes en cada fase, y la potencia reactiva de secuencia inversa el producto de las tensiones inversas de cada fase por la correspondiente corriente inversa reactiva, tal y como se muestra a continuación:

$$
\begin{aligned}
& Q_{r}(t)=Q_{r d}(t)+Q_{r i}(t)= \\
& =\left(Q_{r 12 d}(t)+Q_{r 23 d}(t)+Q_{r 31 d}(t)\right)+\left(Q_{r 12 i}(t)+Q_{r 23 i}(t)+Q_{r 31 i}(t)\right)= \\
& =\left(v_{12 d} \cdot i_{12 d r}+v_{23 d} \cdot i_{23 d r}+v_{31 d} \cdot i_{31 d r}\right)+ \\
& +\left(v_{12 i} \cdot i_{12 i r}+v_{23 i} \cdot i_{23 i r}+v_{31 i} \cdot i_{31 i r}\right)
\end{aligned}
$$

Vamos a desarrollar los términos $Q_{r 12 d}$ y $Q_{r 12 i}$ de la ecuación (II.26), que nos darían la potencia reactiva instantánea de la primera fase.

De la ecuación (II.19), conjuntamente con las (II.21 a 24), obtenemos:

$$
\bar{I}_{12 d}=\bar{V}_{12 d} \cdot\left(G_{e}+\bar{B}_{e}\right)+\bar{V}_{12 i} \cdot \bar{Y}^{\prime}
$$

Para analizar el fenómeno reactivo que nos interesa, hemos de considerar la componente reactiva de la corriente mostrada en la ecuación (II.27), además al 
ser un sistema desequilibrado en tensiones se define el grado de desequilibrio $\left(\delta_{\mathrm{u}}\right)$ de las tensiones de las fases como:

$$
\bar{\delta}_{u}=\frac{\bar{V}_{12 i}}{\bar{V}_{12 d}}=\frac{V_{i} \angle \alpha_{i}}{V_{d} \angle \alpha_{d}}=\delta_{u} \angle \alpha_{i}-\alpha_{d}
$$

Usando el grado de desequilibrio del sistema $\left(\delta_{\mathrm{u}}\right)$, la ecuación (II.27) nos queda:

$$
\bar{I}_{12 d}=\bar{V}_{12 d} \cdot\left(G_{e}+\bar{B}_{e}+\bar{\delta}_{u} \cdot \bar{Y}^{\prime}\right)
$$

De acuerdo con la ecuación (II.29), vemos que es posible la existencia de desfases entre tensiones y corrientes de secuencia directa, no provocados tan sólo por la presencia de elementos reactivos en el sistema, sino también por los desequilibrios. Esta ecuación (II.29) expresada en forma fasorial, pasaremos a desarrollarla de manera que obtengamos su parte real y su parte imaginaria, siendo su parte imaginaria la que nos proporciona información sobre la componente reactiva de esa corriente.

$$
\begin{aligned}
& \bar{\delta}_{u} \cdot \bar{Y}^{\prime}=\delta_{u} \angle\left(\alpha_{i}-\alpha_{d}\right) \cdot Y^{\prime} \angle-\alpha^{\prime}=\delta_{u} \cdot Y^{\prime} \angle\left(\alpha_{i}-\alpha_{d}-\alpha^{\prime}\right)= \\
& =\delta_{u} \cdot Y^{\prime} \cdot \cos \left(\alpha_{i}-\alpha_{d}-\alpha^{\prime}\right)+j \cdot \delta_{u} \cdot Y^{\prime} \cdot \operatorname{sen}\left(\alpha_{i}-\alpha_{d}-\alpha^{\prime}\right)= \\
& =\delta_{u} \cdot Y^{\prime} \cdot \cos \left(\alpha_{i}-\alpha_{d}-\alpha^{\prime}\right)-j \cdot \delta_{u} \cdot Y^{\prime} \cdot \operatorname{sen}\left(\alpha_{d}-\alpha_{i}+\alpha^{\prime}\right)= \\
& =\delta_{u} \cdot Y^{\prime} \cdot \cos \left(\alpha_{i}-\alpha_{d}-\alpha^{\prime}\right)-j \cdot \delta_{u} \cdot Y^{\prime} \cdot \operatorname{sen}\left(\varphi_{i}^{\prime}\right) \\
& \varphi_{i}^{\prime}=\left(\alpha_{d}-\alpha_{i}+\alpha^{\prime}\right)
\end{aligned}
$$

Quedándonos entonces:

$$
\bar{I}_{12 d}=V_{d} \angle \alpha_{d}\left[\left(G_{e}+\delta_{u} \cdot Y^{\prime} \cdot \cos \left(\alpha_{i}-\alpha_{d}-\alpha^{\prime}\right)\right)-j\left(B_{e}+\delta_{u} \cdot Y^{\prime} \cdot \operatorname{sen}\left(\varphi_{i}^{\prime}\right)\right)\right]
$$

Y siendo por lo tanto la componente reactiva de la corriente mostrada en la ecuación (II.31), en su forma temporal la siguiente: 


$$
i_{12 d r}(t)=V_{d} \cdot \sqrt{2} \cdot\left[B_{e}+\delta_{u} \cdot Y^{\prime} \cdot \operatorname{sen} \varphi_{i}^{\prime}\right] \cdot \operatorname{sen}\left(w t+\alpha_{d}-90^{\circ}\right)
$$

Podemos entonces determinar la potencia reactiva directa $\mathrm{Q}_{\mathrm{r} 12 \mathrm{~d}}(\mathrm{t})$ :

$$
\begin{aligned}
& Q_{r 12 d}(t)=V_{12 d}(t) \cdot i_{12 d r}(t)= \\
& =\left(V_{d} \cdot \sqrt{2} \cdot \operatorname{sen}\left(w t+\alpha_{d}\right)\right) \cdot\left(V_{d} \cdot \sqrt{2} \cdot\left[B_{e}+\delta_{u} \cdot Y^{\prime} \cdot \operatorname{sen} \varphi_{i}^{\prime}\right] \cdot \operatorname{sen}\left(w t+\alpha_{d}-90^{\circ}\right)\right)= \\
& =\left(V_{d} \cdot \sqrt{2} \cdot \operatorname{sen}\left(w t+\alpha_{d}\right)\right) \cdot\left(-V_{d} \cdot \sqrt{2} \cdot\left[B_{e}+\delta_{u} \cdot Y^{\prime} \cdot \operatorname{sen} \varphi_{i}^{\prime}\right] \cdot \cos \left(w t+\alpha_{d}\right)\right)= \\
& =\left(-2 V_{d}{ }^{2}\right) \cdot\left[B_{e}+\delta_{u} \cdot Y^{\prime} \cdot \operatorname{sen} \varphi_{i}^{\prime}\right] \cdot \operatorname{sen}\left(w t+\alpha_{d}\right) \cdot \cos \left(w t+\alpha_{d}\right)= \\
& =-V_{d}{ }^{2} \cdot\left[B_{e}+\left(V_{i} / V_{d}\right) \cdot Y^{\prime} \cdot \operatorname{sen} \varphi_{i}^{\prime}\right] \cdot \operatorname{sen} 2\left(w t+\alpha_{d}\right)= \\
& =-\left(B_{e} \cdot V_{d}^{2}+V_{d} \cdot V_{i} \cdot Y^{\prime} \cdot \operatorname{sen} \varphi_{i}^{\prime}\right) \cdot \operatorname{sen} 2\left(w t+\alpha_{d}\right)
\end{aligned}
$$

Para determinar la $\mathrm{Q}_{\mathrm{r} 12 \mathrm{i}}(\mathrm{t})$, partimos de la corriente inversa dada en la ecuación (II. 19), y determinamos su parte reactiva, siguiendo el proceso usado anteriormente para la determinación de la componente reactiva de la corriente de secuencia directa, después de obtenida procederemos a realizar el producto por la componente inversa de la tensión teniendo ya la potencia reactiva instantánea de secuencia inversa para la fase 12.

La expresión de la corriente es:

$$
\bar{I}_{12 i}=\bar{V}_{12 i} \cdot\left(G_{e}+\bar{B}_{e}\right)+\bar{V}_{12 d} \cdot \overline{Y^{\prime \prime}}
$$

que usando el grado de desequilibrio del sistema nos queda:

$$
\bar{I}_{12 i}=\bar{V}_{12 i} \cdot\left(G_{e}+\bar{B}_{e}+\overline{Y^{\prime \prime}} / \bar{\delta}_{u}\right)
$$


en la anterior ecuación nos interesa la componente reactiva, realizamos la descomposición en parte real e imaginaria, esta última nos dará la parte reactiva de esa corriente.

$$
\begin{aligned}
& \bar{Y}^{\prime \prime} / \bar{\delta}_{u}=Y^{\prime \prime} \angle-\alpha^{\prime \prime} / \delta_{u} \angle\left(\alpha_{i}-\alpha_{d}\right)=\bar{Y}^{\prime \prime} / \bar{\delta}_{u} \angle\left(\alpha_{d}-\alpha_{i}-\alpha^{\prime \prime}\right)= \\
& =\bar{Y}^{\prime \prime} / \bar{\delta}_{u} \cdot \cos \left(\alpha_{d}-\alpha_{i}-\alpha^{\prime \prime}\right)+j \cdot \bar{Y}^{\prime \prime} / \bar{\delta}_{u} \cdot \operatorname{sen}\left(\alpha_{d}-\alpha_{i}-\alpha^{\prime \prime}\right)= \\
& =\bar{Y}^{\prime \prime} / \bar{\delta}_{u} \cdot \cos \left(\alpha_{d}-\alpha_{i}-\alpha^{\prime \prime}\right)-j \cdot \bar{Y}^{\prime \prime} / \bar{\delta}_{u} \cdot \operatorname{sen}\left(\alpha_{i}-\alpha_{d}+\alpha^{\prime \prime}\right)= \\
& =\bar{Y}^{\prime \prime} / \bar{\delta}_{u} \cdot \cos \left(\alpha_{d}-\alpha_{i}-\alpha^{\prime \prime}\right)-j \cdot \bar{Y}^{\prime \prime} / \bar{\delta}_{u} \cdot \operatorname{sen}\left(\varphi_{d}^{\prime \prime}\right) \\
& \varphi_{d}^{\prime \prime}=\left(\alpha_{i}-\alpha_{d}+\alpha^{\prime \prime}\right)
\end{aligned}
$$

Nos queda por lo tanto:

$$
\left.\bar{I}_{12 i}=V_{i} \angle \alpha_{i} \mid\left(G_{e}+\overline{Y^{\prime \prime}} / \delta_{u} \cdot \cos \left(\alpha_{d}-\alpha_{i}-\alpha^{\prime \prime}\right)\right)-j\left(B_{e}+\overline{Y^{\prime \prime}} / \delta_{u} \cdot \operatorname{sen}\left(\varphi_{d}^{\prime \prime}\right)\right)\right]
$$

Siendo la componente reactiva en su expresión temporal la siguiente:

$$
i_{12 \text { ir }}(t)=V_{i} \cdot \sqrt{2} \cdot\left[B_{e}+Y^{\prime \prime} / \delta_{u} \cdot \operatorname{sen} \varphi_{d}^{\prime \prime \prime}\right] \cdot \operatorname{sen}\left(w t+\alpha_{i}-90^{\circ}\right)
$$

Determinamos ahora la $\mathrm{Q}_{\mathrm{r} 12 \mathrm{i}}(\mathrm{t})$ : 


$$
\begin{aligned}
& Q_{r 12 i}(t)=V_{12 i}(t) \cdot i_{12 i r}(t)= \\
& =\left(V_{i} \cdot \sqrt{2} \cdot \operatorname{sen}\left(w t+\alpha_{i}\right)\right) \cdot\left(V_{i} \cdot \sqrt{2} \cdot\left[B_{e}+\left(Y^{\prime \prime} / \delta_{u}\right) \cdot \operatorname{sen} \varphi_{d}^{\prime \prime}\right] \cdot \operatorname{sen}\left(w t+\alpha_{i}-90^{\circ}\right)\right)= \\
& =\left(V_{i} \cdot \sqrt{2} \cdot \operatorname{sen}\left(w t+\alpha_{i}\right)\right) \cdot\left(-V_{d} \cdot \sqrt{2} \cdot\left[B_{e}+\left(Y^{\prime \prime} / \delta_{u}\right) \cdot \operatorname{sen} \varphi_{d}^{\prime \prime}\right] \cdot \cos \left(w t+\alpha_{i}\right)\right)= \\
& =\left(-2 V_{i}^{2}\right) \cdot\left[B_{e}+\left(Y^{\prime \prime} / \delta_{u}\right) \cdot \operatorname{sen} \varphi_{d}^{\prime \prime}\right] \cdot \operatorname{sen}\left(w t+\alpha_{i}\right) \cdot \cos \left(w t+\alpha_{i}\right)= \\
& =-V_{i}^{2} \cdot\left[B_{e}+\left(V_{d} \cdot Y^{\prime \prime} / V_{i}\right) \cdot \operatorname{sen} \varphi_{d}^{\prime \prime}\right] \cdot \operatorname{sen} 2\left(w t+\alpha_{i}\right)= \\
& =-\left(B_{e} \cdot V_{i}^{2}+V_{i} \cdot V_{d} \cdot Y^{\prime \prime} \cdot \operatorname{sen} \varphi_{d}^{\prime \prime}\right) \cdot \operatorname{sen} 2\left(w t+\alpha_{i}\right)
\end{aligned}
$$

Procediendo de la misma manera en el desarrollo de los términos que componen la ecuación (II.26), obtenemos finalmente la potencia reactiva instantánea total del sistema $\mathrm{Q}_{\mathrm{r}}(\mathrm{t})$, cuya expresión es:

$$
\begin{aligned}
& Q_{r}(t)=Q_{r d}(t)+Q_{r i}(t)= \\
& =-\left(B_{e} \cdot V_{d}^{2}+Y^{\prime} \cdot V_{d} \cdot V_{i} \cdot \operatorname{sen} \varphi_{i}^{\prime}\right) \cdot \operatorname{sen} 2\left(w t+\alpha_{d}\right)- \\
& -\left(B_{e} \cdot V_{i}^{2}+Y^{\prime \prime} \cdot V_{d} \cdot V_{i} \cdot \operatorname{sen} \varphi_{d}^{\prime \prime}\right) \cdot \operatorname{sen} 2\left(w t+\alpha_{i}\right)- \\
& -\left(B_{e} \cdot V_{d}^{2}+Y^{\prime} \cdot V_{d} \cdot V_{i} \cdot \operatorname{sen} \varphi_{i}^{\prime}\right) \cdot \operatorname{sen} 2\left(w t+\alpha_{d}-120^{\circ}\right)- \\
& -\left(B_{e} \cdot V_{i}^{2}+Y^{\prime \prime} \cdot V_{d} \cdot V_{i} \cdot \operatorname{sen} \varphi_{d}^{\prime \prime}\right) \cdot \operatorname{sen} 2\left(w t+\alpha_{i}+120^{\circ}\right)- \\
& -\left(B_{e} \cdot V_{d}^{2}+Y^{\prime} \cdot V_{d} \cdot V_{i} \cdot \operatorname{sen} \varphi_{i}^{\prime}\right) \cdot \operatorname{sen} 2\left(w t+\alpha_{d}-240^{\circ}\right)- \\
& -\left(B_{e} \cdot V_{i}^{2}+Y^{\prime \prime} \cdot V_{d} \cdot V_{i} \cdot \operatorname{sen} \varphi_{d}^{\prime \prime}\right) \cdot \operatorname{sen} 2\left(w t+\alpha_{i}+240^{\circ}\right)
\end{aligned}
$$




\section{II.3.2.- Teoría Unificadora.}

Si consideramos la Teoría Unificadora para determinar la potencia reactiva instantánea, hemos de recordar que tan sólo las tensiones de secuencia directa y las corrientes de secuencia directa reactiva nos la determinan, tal y como implícitamente apunta Emanuel en la IEEE Standard 1459/2000.

La potencia reactiva instantánea $\mathrm{Q}_{\mathrm{r}}(\mathrm{t})$ en los sistemas desequilibrados viene dada por:

$$
\begin{aligned}
& Q_{r}(t)=Q_{r d}(t)=\left(q_{r 12 d}+q_{r 23 d}+q_{r 31 d}\right)= \\
& =v_{12 d} \cdot i_{12 d r}+v_{23 d} \cdot i_{23 d r}+v_{31 d} \cdot i_{31 d r}= \\
& =-\left(B_{e} \cdot V_{d}^{2}+Y^{\prime} \cdot V_{d} \cdot V_{i} \cdot \operatorname{sen} \varphi_{i}^{\prime}\right) \cdot \operatorname{sen} 2\left(w t+\alpha_{d}\right)- \\
& -\left(B_{e} \cdot V_{d}^{2}+Y^{\prime} \cdot V_{d} \cdot V_{i} \cdot \operatorname{sen} \varphi_{i}^{\prime}\right) \cdot \operatorname{sen} 2\left(w t+\alpha_{d}-120^{\circ}\right)- \\
& -\left(B_{e} \cdot V_{d}^{2}+Y^{\prime} \cdot V_{d} \cdot V_{i} \cdot \operatorname{sen} \varphi_{i}^{\prime}\right) \cdot \operatorname{sen} 2\left(w t+\alpha_{d}-240^{\circ}\right)
\end{aligned}
$$

Procediendo a comparar la expresión obtenida en el caso de tensiones equilibradas dada por la ecuación (II.14), y la obtenida para el caso de tensiones desequilibradas mostrada en la ecuación (II.41), vemos que existe un flujo de potencia reactiva instantánea, en cada una de las fases, debida a las reactancias, cuya expresión es idéntica a la de la ecuación (II.14) y que denominamos por $\mathrm{Q}_{\mathrm{rr}}(\mathrm{t})$,

$$
\begin{aligned}
& Q_{r r}(t)=-B_{e} \cdot V_{d}^{2} \cdot \operatorname{sen} 2\left(w t+\alpha_{d}\right) \\
& -B_{e} \cdot V_{d}^{2} \cdot \operatorname{sen} 2\left(w t+\alpha_{d}-120^{\circ}\right) \\
& -B_{e} \cdot V_{d}^{2} \cdot \operatorname{sen} 2\left(w t+\alpha_{d}-240^{\circ}\right)
\end{aligned}
$$


y se añade a este flujo debido a las reactancias, otros flujos sinusoidales y también equilibrados de potencia reactiva instantánea, que mostramos a continuación y cuyas amplitudes dependen de los desequilibrios de tensiones y cargas del sistema, y que denominamos por $\mathrm{Q}_{\mathrm{ru}}(\mathrm{t})$ :

$$
\begin{aligned}
& Q_{r u}(t)=-\left(Y^{\prime} \cdot V_{d} \cdot V_{i} \cdot \operatorname{sen} \varphi_{i}^{\prime}\right) \cdot \operatorname{sen} 2\left(w t+\alpha_{d}\right)- \\
& -\left(Y^{\prime} \cdot V_{d} \cdot V_{i} \cdot \operatorname{sen} \varphi_{i}^{\prime}\right) \cdot \operatorname{sen} 2\left(w t+\alpha_{d}-120^{\circ}\right)- \\
& -\left(Y^{\prime} \cdot V_{d} \cdot V_{i} \cdot \operatorname{sen} \varphi_{i}^{\prime}\right) \cdot \operatorname{sen} 2\left(w t+\alpha_{d}-240^{\circ}\right)
\end{aligned}
$$

Estos flujos de potencia reactiva instantánea, debidos al desequilibrio del sistema se suman a los flujos de potencia reactiva del sistema provocados por las reactancias presentes en el sistema, siendo por lo tanto la potencia reactiva instantánea del sistema compuesta por la suma de los efectos propios de las reactancias y los debidos al desequilibrio del sistema.

$$
Q_{r}(t)=Q_{r r}(t)+Q_{r u}(t)
$$




\section{II.4.- Fenómenos de desfase en sistemas desequilibrados lineales con neutro. Tensiones equilibradas.}

\section{II.4.1.- Teoría Clásica.}

Sea un generador trifásico equilibrado, de secuencia directa, que alimenta a un receptor trifásico lineal desequilibrado conectado en estrella con neutro, formado por las impedancias $Z_{1}, Z_{2}, Z_{3}$ (consideradas inductivas), y que para mayor facilidad a la hora de poder identificar de forma clara los fenómenos de desfase, se ha procedido a descomponer como se muestra en la figura II.3, en las resistencias y reactancias equivalentes en paralelo $R_{i}, X_{i},(i=1,2,3)$, y siendo el valor de las tensiones aplicadas el siguiente:

$$
\begin{aligned}
& V_{1}(t)=V_{1 d}(t)=V_{d} \cdot \sqrt{2} \cdot \operatorname{sen}\left(w t+\alpha_{d}\right) \\
& V_{2}(t)=V_{2 d}(t)=V_{d} \cdot \sqrt{2} \cdot \operatorname{sen}\left(w t+\alpha_{d}-120^{\circ}\right) \\
& V_{3}(t)=V_{3 d}(t)=V_{d} \cdot \sqrt{2} \cdot \operatorname{sen}\left(w t+\alpha_{d}-240^{\circ}\right)
\end{aligned}
$$

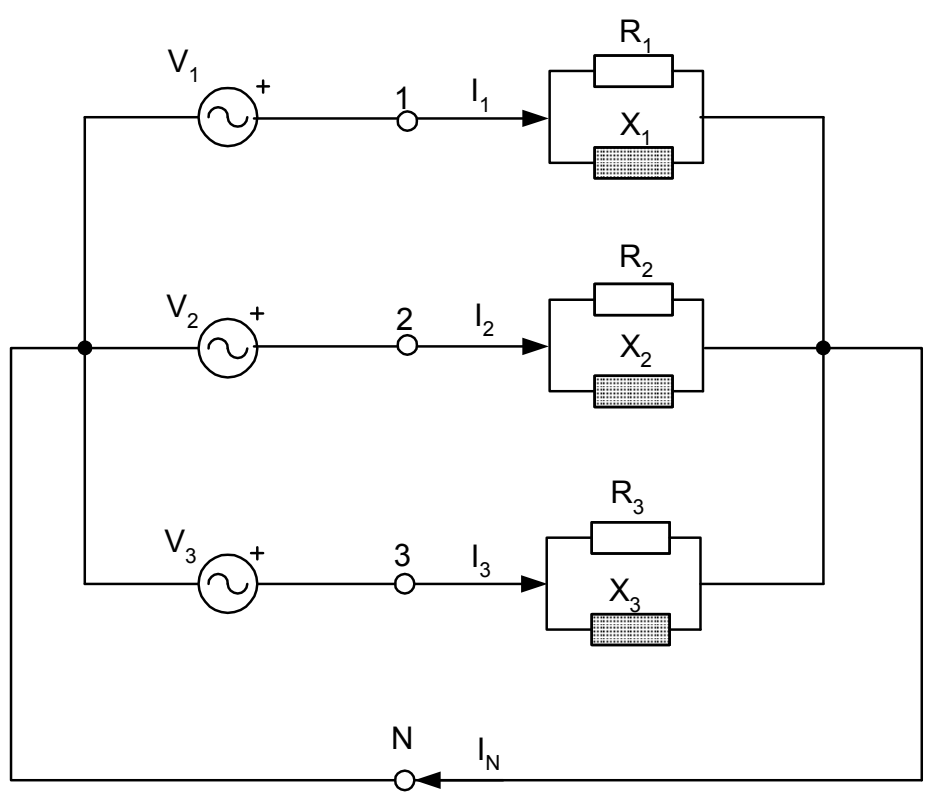

Figura II.3. Sistema con neutro. Tensiones equilibradas.

Lo que da lugar a la circulación de corrientes en las fases del receptor en notación fasorial, cuyo valor es entonces: 


$$
\begin{aligned}
& \bar{I}_{1}=\overline{V_{1 d}} \cdot\left(G_{1}+\bar{B}_{1}\right) \\
& \bar{I}_{2}=\bar{V}_{2 d} \cdot\left(G_{2}+\bar{B}_{2}\right)=a^{2} \cdot \bar{V}_{1 d} \cdot\left(G_{2}+\bar{B}_{2}\right) \\
& \bar{I}_{3}=\bar{V}_{3 d} \cdot\left(G_{3}+\bar{B}_{3}\right)=a \cdot \bar{V}_{1 d} \cdot\left(G_{3}+\bar{B}_{3}\right)
\end{aligned}
$$

Expresando las componentes simétricas de estas corrientes, en función de la contribución a los fenómenos resistivos y reactivos del receptor para la primera fase, tenemos:

$$
\begin{aligned}
\bar{I}_{1 d} & =\frac{1}{3} \cdot \bar{V}_{1 d} \cdot\left(G_{1}+G_{2}+G_{3}\right)+\frac{1}{3} \cdot \bar{V}_{1 d} \cdot\left(\bar{B}_{1}+\bar{B}_{2}+\bar{B}_{3}\right)= \\
& =\bar{I}_{1 d a}+\bar{I}_{1 d r} \\
\bar{I}_{1 i} & =\frac{1}{3} \cdot \bar{V}_{1 d} \cdot\left(G_{1}+a \cdot G_{2}+a^{2} \cdot G_{3}\right)+\frac{1}{3} \cdot \bar{V}_{1 d} \cdot\left(\bar{B}_{1}+a \cdot \bar{B}_{2}+a^{2} \cdot \bar{B}_{3}\right)= \\
& =\bar{I}_{1 i R}+\bar{I}_{1 i x} \\
\bar{I}_{1 h} & =\frac{1}{3} \cdot \bar{V}_{1 d} \cdot\left(G_{1}+a^{2} \cdot G_{2}+a \cdot G_{3}\right)+\frac{1}{3} \cdot \bar{V}_{1 d} \cdot\left(\bar{B}_{1}+a^{2} \cdot \bar{B}_{2}+a \cdot \bar{B}_{3}\right)= \\
& =\bar{I}_{1 h R}+\bar{I}_{1 h x}
\end{aligned}
$$

Que para las otras fases, las componentes de secuencia directa del sistema nos quedan:

$$
\begin{aligned}
& \bar{I}_{2 d}=a^{2} \cdot \bar{I}_{1 d} \\
& \bar{I}_{2 i}=a \cdot \bar{I}_{1 i} \\
& \bar{I}_{2 h}=\bar{I}_{1 h} \\
& \bar{I}_{3 d}=a \cdot \bar{I}_{1 d} \\
& \bar{I}_{3 i}=a^{2} \cdot \bar{I}_{1 i} \\
& \bar{I}_{3 h}=\bar{I}_{1 h}
\end{aligned}
$$


Al igual que en los sistemas a tres hilos, partimos de la potencia instantánea expresada en función de las componentes simétricas de las tensiones y corrientes del sistema, ya que en sus componentes se hallan inmersos todos los fenómenos energéticos del sistema:

$$
\begin{aligned}
p(t)= & v_{1 d} \cdot\left(i_{1 d a}+i_{1 d r}+i_{1 i a}+i_{1 i r}+i_{1 h a}+i_{1 h r}\right)+ \\
& +v_{2 d} \cdot\left(i_{2 d a}+i_{2 d r}+i_{2 i a}+i_{2 i r}+i_{2 h a}+i_{2 h r}\right)+ \\
& +v_{3 d} \cdot\left(i_{3 d a}+i_{3 d r}+i_{3 i a}+i_{3 i r}+i_{3 h a}+i_{3 h r}\right)
\end{aligned}
$$

Y por lo tanto, como ya hemos planteado, los fenómenos reactivos (o de desfase), se encuentran inmersos en la expresión de la potencia reactiva instantánea $\mathrm{Q}_{\mathrm{r}}(\mathrm{t})$ que según la Teoría Clásica queda formulada como sigue:

$$
\begin{aligned}
& Q_{r}(t)=Q_{r d}(t)=v_{1 d} \cdot i_{1 d r}+v_{2 d} \cdot i_{2 d r}+v_{3 d} \cdot i_{3 d r}= \\
& =Q_{r 1 d}(t)+Q_{r 2 d}(t)+Q_{r 3 d}(t)
\end{aligned}
$$

Observamos por lo tanto que la potencia reactiva instantánea del sistema, se halla compuesta por la suma de las potencias reactivas instantáneas de cada fase, a continuación se realiza el desarrollo para el primero de los términos, que nos da la potencia reactiva instantánea de la primera fase, determinando para ello sus componentes de tensión e intensidad.

Expresamos la ecuación (II.47) como:

$$
\bar{I}_{1 d}=\bar{V}_{1 d} \cdot\left(G_{e}+\bar{B}_{e}\right)
$$

en donde $G_{e}$ y $B_{e}$, son la conductancia y susceptancia equivalente:

$$
G_{e}=\frac{1}{3} \cdot\left(G_{1}+G_{2}+G_{3}\right) ; \quad \bar{B}_{e}=\frac{1}{3} \cdot\left(\bar{B}_{1}+\bar{B}_{2}+\bar{B}_{3}\right)
$$


La expresión temporal de la componente directa reactiva de la corriente es:

$$
i_{1 d r}(t)=V_{d} \cdot \sqrt{2} \cdot B_{e} \cdot \operatorname{sen}\left(w t+\alpha_{d}-90^{\circ}\right)
$$

y la tensión directa la tenemos de la ecuación (II.44) siendo su valor:

$$
V_{1 d}(t)=V_{d} \cdot \sqrt{2} \cdot \operatorname{sen}\left(w t+\alpha_{d}\right)
$$

Por lo tanto la potencia reactiva instantánea directa para el receptor de la fase 1 , $\mathrm{Q}_{\mathrm{rld}}(\mathrm{t})$ vale:

$$
\begin{aligned}
& Q_{r 1 d}(t)=V_{1 d}(t) \cdot i_{1 d r}(t)= \\
& =V_{d} \cdot \sqrt{2} \cdot \operatorname{sen}\left(w t+\alpha_{d}\right) \cdot V_{d} \cdot \sqrt{2} \cdot B_{e} \cdot \operatorname{sen}\left(w t+\alpha_{d}-90^{\circ}\right)= \\
& =-V_{d}^{2} \cdot(\sqrt{2})^{2} \cdot B_{e} \cdot \operatorname{sen}\left(w t+\alpha_{d}\right) \cdot \cos \left(w t+\alpha_{d}\right)=-B_{e} \cdot V_{d}^{2} \cdot \operatorname{sen} 2\left(w t+\alpha_{d}\right)
\end{aligned}
$$

Operando de idéntica manera obtenemos las potencias reactivas instantáneas de las otras dos fases, la $\mathrm{Q}_{\mathrm{r} 2 \mathrm{~d}}(\mathrm{t})$ y la $\mathrm{Q}_{\mathrm{r} 3 \mathrm{~d}}(\mathrm{t})$, siendo la potencia reactiva instantánea del sistema entonces:

$$
\begin{aligned}
& Q_{r}(t)=-B_{e} \cdot V_{d}^{2} \cdot \operatorname{sen} 2\left(w t+\alpha_{d}\right) \\
& -B_{e} \cdot V_{d}^{2} \cdot \operatorname{sen} 2\left(w t+\alpha_{d}-120^{\circ}\right) \\
& -B_{e} \cdot V_{d}^{2} \cdot \operatorname{sen} 2\left(w t+\alpha_{d}-240^{\circ}\right)
\end{aligned}
$$

Como ocurría en el caso de sistemas sin hilo neutro, y después de analizar la expresión de la potencia reactiva instantánea dada por la ecuación (II.56), observamos que, en los sistemas equilibrados en tensiones, el fenómeno del desfase es provocado exclusivamente por la presencia de reactancias en el sistema. 


\section{II.4.2.- Teoría Unificadora.}

Procedemos ahora a determinar la potencia reactiva instantánea por medio de la Teoría Unificadora, para lo que partimos de la expresión de la potencia instantánea del sistema, en la que se ha procedido a descomponer tan sólo las corrientes directas de cada fase en sus partes activas y reactivas tal y como postula, siendo su expresión la siguiente:

$$
\begin{aligned}
p(t) & =v_{1 d} \cdot\left(i_{1 d a}+i_{1 d r}+i_{1 i}+i_{1 h}\right)+ \\
& +v_{2 d} \cdot\left(i_{2 d a}+i_{2 d r}+i_{2 i}+i_{2 h}\right)+ \\
& +v_{3 d} \cdot\left(i_{3 d a}+i_{3 d r}+i_{3 i}+i_{3 h}\right)
\end{aligned}
$$

Al igual que sucedía en los sistemas a tres hilos, cuando el sistema de alimentación esta equilibrado, la potencia reactiva instantánea usando la Teoría Unificadora coincide con la planteada usando la Teoría Clásica, dada por la ecuación (II.56), manifestándose por medio de tres flujos de potencia sinusoidales equilibrados, siendo estos provocados única y exclusivamente por la presencia de elementos reactivos en el sistema, tal y como podemos observar en la siguiente ecuación:

$$
\begin{aligned}
& Q_{r}(t)=-B_{e} \cdot V_{d}^{2} \cdot \operatorname{sen} 2\left(w t+\alpha_{d}\right) \\
& -B_{e} \cdot V_{d}^{2} \cdot \operatorname{sen} 2\left(w t+\alpha_{d}-120^{\circ}\right) \\
& -B_{e} \cdot V_{d}^{2} \cdot \operatorname{sen} 2\left(w t+\alpha_{d}-240^{\circ}\right)
\end{aligned}
$$




\section{II.5.- Fenómenos de desfase en sistemas desequilibrados lineales con neutro. Tensiones desequilibradas.}

\section{II.5.1.- Teoría Clásica.}

Sea ahora un generador desequilibrado, como el mostrado en la figura II.4, y por lo tanto aplicando componentes simétricas, pasamos a expresar sus tensiones, observando que posee componentes de secuencia directa, inversa y homopolar:

$$
\begin{aligned}
V_{1}(t)= & V_{d} \cdot \sqrt{2} \cdot \operatorname{sen}\left(w t+\alpha_{d}\right)+V_{i} \cdot \sqrt{2} \cdot \operatorname{sen}\left(w t+\alpha_{i}\right)+ \\
& +V_{h} \cdot \sqrt{2} \cdot \operatorname{sen}\left(w t+\alpha_{h}\right) \\
V_{2}(t) & =V_{d} \cdot \sqrt{2} \cdot \operatorname{sen}\left(w t+\alpha_{d}-120^{\circ}\right)+V_{i} \cdot \sqrt{2} \cdot \operatorname{sen}\left(w t+\alpha_{i}+120^{\circ}\right)+ \\
& +V_{h} \cdot \sqrt{2} \cdot \operatorname{sen}\left(w t+\alpha_{h}\right) \\
V_{3}(t) & =V_{d} \cdot \sqrt{2} \cdot \operatorname{sen}\left(w t+\alpha_{d}+240^{\circ}\right)+V_{i} \cdot \sqrt{2} \cdot \operatorname{sen}\left(w t+\alpha_{i}-240^{\circ}\right)+ \\
& +V_{h} \cdot \sqrt{2} \cdot \operatorname{sen}\left(w t+\alpha_{h}\right)
\end{aligned}
$$

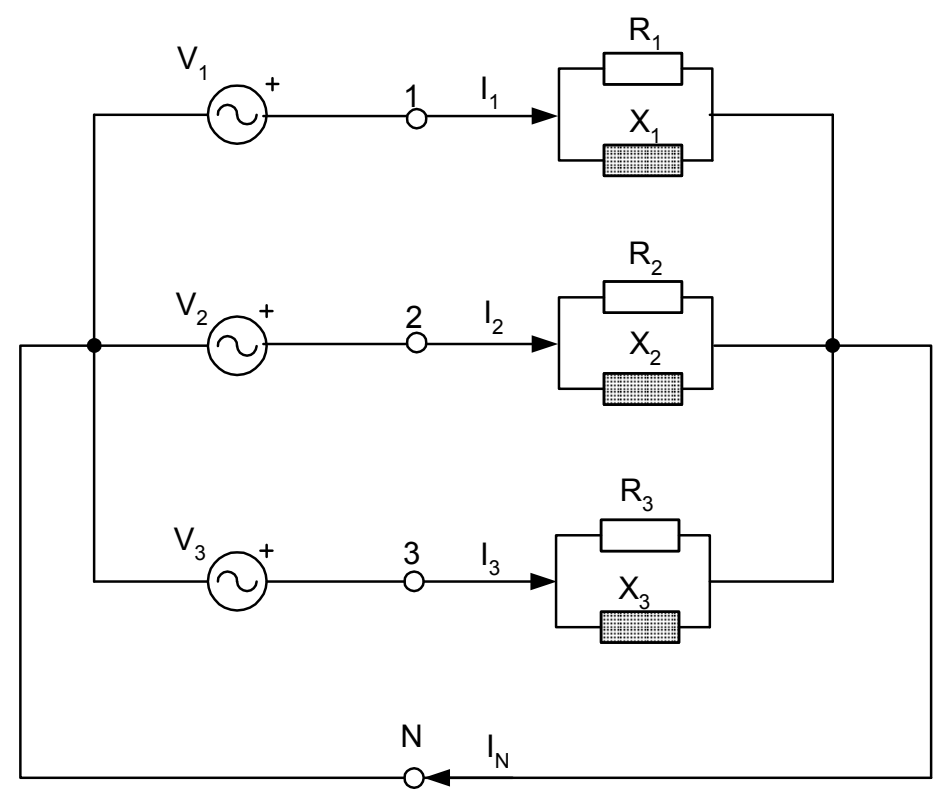

Figura II.4. Sistema con neutro. Tensiones desequilibradas.

Expresando estas tensiones en su forma fasorial tenemos: 


$$
\begin{aligned}
& \overline{V_{1}}=\bar{V}_{1 d}+\bar{V}_{1 i}+\bar{V}_{1 h}=V_{d \angle \alpha_{d}}+V_{i \angle \alpha_{i}}+V_{h \angle \alpha_{h}} \\
& \overline{V_{2}}=\bar{V}_{2 d}+\bar{V}_{2 i}+\bar{V}_{2 h}=a^{2} \cdot \bar{V}_{1 d}+a \cdot \bar{V}_{1 i}+\bar{V}_{1 h} \\
& \overline{V_{3}}=\bar{V}_{3 d}+\bar{V}_{3 i}+\bar{V}_{3 h}=a \cdot \bar{V}_{1 d}+a^{2} \cdot \bar{V}_{1 i}+\bar{V}_{1 h}
\end{aligned}
$$

Siguiendo el mismo proceso que los casos anteriores, pasamos a determinar las corrientes que circulan por las fases del sistema:

$$
\begin{aligned}
& \bar{I}_{1}=\overline{V_{1}} \cdot\left(G_{1}+\bar{B}_{1}\right)=\left(\overline{V_{1 d}}+\overline{V_{1 i}}+\overline{V_{1 h}}\right) \cdot\left(G_{1}+\bar{B}_{1}\right) \\
& \bar{I}_{2}=\overline{V_{2}} \cdot\left(G_{2}+\bar{B}_{2}\right)=\left(a^{2} \cdot \bar{V}_{1 d}+a \cdot \bar{V}_{1 i}+\bar{V}_{1 h}\right) \cdot\left(G_{2}+\bar{B}_{2}\right) \\
& \bar{I}_{3}=\overline{V_{3}} \cdot\left(G_{3}+\bar{B}_{3}\right)=\left(a \cdot \bar{V}_{1 d}+a^{2} \cdot \bar{V}_{1 i}+\bar{V}_{1 h}\right) \cdot\left(G_{3}+\bar{B}_{3}\right)
\end{aligned}
$$

Descomponiendo estas corrientes en sus componentes simétricas, para la primera fase obtenemos:

$$
\begin{aligned}
& \bar{I}_{1 d}=\frac{1}{3} \bar{V}_{1 d} \cdot\left(G_{1}+G_{2}+G_{3}\right)+\frac{1}{3} \bar{V}_{1 d} \cdot\left(\bar{B}_{1}+\bar{B}_{2}+\bar{B}_{3}\right)+ \\
& +\frac{1}{3} \bar{V}_{1 i} \cdot\left(G_{1}+a^{2} \cdot G_{2}+a \cdot G_{3}\right)+\frac{1}{3} \bar{V}_{1 i} \cdot\left(\bar{B}_{1}+a^{2} \cdot \bar{B}_{2}+a \cdot \bar{B}_{3}\right)+ \\
& +\frac{1}{3} \bar{V}_{1 h} \cdot\left(G_{1}+a \cdot G_{2}+a^{2} \cdot G_{3}\right)+\frac{1}{3} \bar{V}_{1 h} \cdot\left(\bar{B}_{1}+a \cdot \bar{B}_{2}+a^{2} \cdot \bar{B}_{3}\right) \\
& \bar{I}_{1 i}=\frac{1}{3} \bar{V}_{1 d} \cdot\left(G_{1}+a \cdot G_{2}+a^{2} \cdot G_{3}\right)+\frac{1}{3} \bar{V}_{1 d} \cdot\left(\bar{B}_{1}+a \cdot \bar{B}_{2}+a^{2} \cdot \bar{B}_{3}\right)+ \\
& +\frac{1}{3} \bar{V}_{1 i} \cdot\left(G_{1}+G_{2}+G_{3}\right)+\frac{1}{3} \bar{V}_{1 i} \cdot\left(\bar{B}_{1}+\bar{B}_{2}+\bar{B}_{3}\right)+ \\
& +\frac{1}{3} \bar{V}_{1 h} \cdot\left(G_{1}+a^{2} \cdot G_{2}+a \cdot G_{3}\right)+\frac{1}{3} \bar{V}_{1 h} \cdot\left(\bar{B}_{1}+a^{2} \cdot \bar{B}_{2}+a \cdot \bar{B}_{3}\right) \\
& \bar{I}_{1 h}=\frac{1}{3} \bar{V}_{1 d} \cdot\left(G_{1}+a^{2} \cdot G_{2}+a \cdot G_{3}\right)+\frac{1}{3} \bar{V}_{1 d} \cdot\left(\bar{B}_{1}+a^{2} \cdot \bar{B}_{2}+a \cdot \bar{B}_{3}\right)+ \\
& +\frac{1}{3} \bar{V}_{1 i} \cdot\left(G_{1}+a \cdot G_{2}+a^{2} \cdot G_{3}\right)+\frac{1}{3} \bar{V}_{1 i} \cdot\left(\bar{B}_{1}+a \cdot \bar{B}_{2}+a^{2} \cdot \bar{B}_{3}\right)+ \\
& +\frac{1}{3} \bar{V}_{1 h} \cdot\left(G_{1}+G_{2}+G_{3}\right)+\frac{1}{3} \bar{V}_{1 h} \cdot\left(\bar{B}_{1}+\bar{B}_{2}+\bar{B}_{3}\right)
\end{aligned}
$$


Que para las otras fases del sistema, las componentes directas de las corrientes son:

$$
\begin{aligned}
& \bar{I}_{2 d}=a^{2} \cdot \bar{I}_{1 d} \\
& \bar{I}_{2 i}=a \cdot \bar{I}_{1 i} \\
& \bar{I}_{2 h}=\bar{I}_{1 h} \\
& \bar{I}_{3 d}=a \cdot \bar{I}_{1 d} \\
& \bar{I}_{3 i}=a^{2} \cdot \bar{I}_{1 i} \\
& \bar{I}_{3 h}=\bar{I}_{1 h}
\end{aligned}
$$

Al igual que sucedía en sistemas a tres hilos, y del análisis de la ecuación (II.62) podemos agrupar a diversos elementos del circuito, que pasaremos a denominar como sigue:

1. Consideramos como susceptancia directa, inversa y homopolar:

$$
\bar{B}_{e}=\frac{1}{3} \cdot\left(\bar{B}_{1}+\bar{B}_{2}+\bar{B}_{3}\right)
$$

2. Siendo las admitancias básicas de desequilibrio para las secuencias directa, inversa y homopolar las siguientes:

$$
\begin{aligned}
& \overline{Y^{\prime}}=Y_{-\alpha^{\prime}}^{\prime}=\frac{1}{3} \cdot\left[G_{1}+\bar{B}_{1}+a^{2} \cdot\left(G_{2}+\bar{B}_{2}\right)+a \cdot\left(G_{3}+\bar{B}_{3}\right)\right]=\bar{Y}_{d i}=\bar{Y}_{i h}=\bar{Y}_{h d} \\
& \overline{Y^{\prime \prime}}=Y_{-\alpha^{\prime \prime}}^{\prime \prime}=\frac{1}{3} \cdot\left[G_{1}+\bar{B}_{1}+a \cdot\left(G_{2}+\bar{B}_{2}\right)+a^{2} \cdot\left(G_{3}+\bar{B}_{3}\right)\right]=\bar{Y}_{d h}=\bar{Y}_{i d}=\bar{Y}_{h i}
\end{aligned}
$$

Que podemos formular también como sigue: 


$$
\begin{aligned}
& \bar{B}_{e}=\frac{1}{\bar{X}_{e}}=\frac{1}{3} \cdot\left(\frac{1}{\bar{X}_{1}}+\frac{1}{\bar{X}_{2}}+\frac{1}{\bar{X}_{3}}\right) \\
& \bar{Y}^{\prime}=Y_{-\alpha^{\prime}}^{\prime}=\frac{1}{3}\left(\bar{Y}_{1}+a^{2} \cdot \bar{Y}_{2}+a \cdot \bar{Y}_{3}\right) \\
& \bar{Y}^{\prime \prime}=Y_{-\alpha^{\prime \prime}}^{\prime \prime}=\frac{1}{3}\left(\bar{Y}_{1}+a \cdot \bar{Y}_{2}+a^{2} \cdot \bar{Y}_{3}\right)
\end{aligned}
$$

La potencia instantánea del sistema viene dada por la siguiente expresión:

$$
\begin{aligned}
p(t)= & \left(v_{1 d}+v_{1 i}+v_{1 h}\right) \cdot\left(i_{1 d a}+i_{1 d r}+i_{1 i a}+i_{1 i r}+i_{1 h a}+i_{1 h r}\right)+ \\
& +\left(v_{2 d}+v_{2 i}+v_{2 h}\right) \cdot\left(i_{2 d a}+i_{2 d r}+i_{2 i a}+i_{2 i r}+i_{2 h a}+i_{2 h r}\right)+ \\
& +\left(v_{3 d}+v_{3 i}+v_{3 h}\right) \cdot\left(i_{3 d a}+i_{3 d r}+i_{3 i a}+i_{3 i r}+i_{3 h a}+i_{3 h r}\right)
\end{aligned}
$$

Para proceder al análisis del fenómeno reactivo, nos centramos en la expresión de la potencia reactiva instantánea según la Teoría Clásica.

$$
\begin{aligned}
& Q_{r}(t)=v_{1 d} \cdot i_{1 d r}+v_{1 i} \cdot i_{1 i r}+v_{1 h} \cdot i_{1 h r}+ \\
& +v_{2 d} \cdot i_{2 d r}+v_{2 i} \cdot i_{2 i r}+v_{2 h} \cdot i_{2 h r}+ \\
& +v_{3 d} \cdot i_{3 d r}+v_{3 i} \cdot i_{3 i r}+v_{3 h} \cdot i_{3 h r}= \\
& =\left(v_{1 d} \cdot i_{1 d r}+v_{2 d} \cdot i_{2 d r}+v_{3 d} \cdot i_{3 d r}\right)+ \\
& +\left(v_{1 i} \cdot i_{1 i r}+v_{2 i} \cdot i_{2 i r}+v_{3 i} \cdot i_{3 i r}\right)+ \\
& +\left(v_{1 h} \cdot i_{1 h r}+v_{2 h} \cdot i_{2 h r}+v_{3 h} \cdot i_{3 h r}\right)= \\
& =Q_{r d}(t)+Q_{r i}(t)+Q_{r h}(t)
\end{aligned}
$$

En la anterior expresión observamos que la potencia reactiva instantánea se halla formada por los productos de las tensiones e intensidades reactivas de la misma secuencia, lo que nos da la potencia reactiva de secuencia directa $Q_{r d}(t)$, la 
potencia reactiva de secuencia inversa $Q_{r i}(t)$ y la potencia reactiva de secuencia homopolar $\mathrm{Q}_{\mathrm{rh}}(\mathrm{t})$.

$$
\begin{aligned}
& Q_{r}(t)=Q_{r d}(t)+Q_{r i}(t)+Q_{r h}(t)= \\
& =\left(Q_{r 1 d}(t)+Q_{r 2 d}(t)+Q_{r 3 d}(t)\right)+\left(Q_{r 1 i}(t)+Q_{r 2 i}(t)+Q_{r 3 i}(t)\right)+ \\
& +\left(Q_{r 1 h}(t)+Q_{r 2 h}(t)+Q_{r 3 h}(t)\right)
\end{aligned}
$$

Seguidamente pasamos a desarrollar, los primeros términos para cada una de las secuencias, la $\mathrm{Q}_{\mathrm{r} 1 \mathrm{~d}}, \mathrm{Q}_{\mathrm{r} 1 \mathrm{i}}$ y la $\mathrm{Q}_{\mathrm{r} 1 \mathrm{~h}}$ usando para ello las expresiones temporales de las tensiones y las intensidades reactivas.

Comenzamos con la determinación de la $\mathrm{Q}_{\mathrm{r} 1 \mathrm{~d}}(\mathrm{t})$, la ecuación (II.62), haciendo uso de las ecuaciones (II.63 y 64), la expresamos como sigue:

$$
\bar{I}_{1 d}=\bar{V}_{1 d} \cdot\left(G_{e}+\bar{B}_{e}\right)+\bar{V}_{1 i} \cdot \bar{Y}^{\prime}+\bar{V}_{1 h} \cdot \bar{Y}^{\prime \prime}
$$

Que considerando el grado de desequilibrio del sistema $\left(\delta_{\mathrm{u}}\right)$, y el grado de asimetría $\left(\delta_{\mathrm{A}}\right)$ del mismo como:

$$
\begin{aligned}
& \bar{\delta}_{u}=\frac{\bar{V}_{1 i}}{\bar{V}_{1 d}}=\frac{V_{i} \angle \alpha_{i}}{V_{d} \angle \alpha_{d}}=\delta_{u} \angle \alpha_{i}-\alpha_{d} \\
& \bar{\delta}_{A}=\frac{\bar{V}_{1 h}}{\bar{V}_{1 d}}=\frac{V_{h} \angle \alpha_{h}}{V_{d} \angle \alpha_{d}}=\delta_{u} \angle \alpha_{h}-\alpha_{d}
\end{aligned}
$$

La ecuación (II.69) nos queda:

$$
\bar{I}_{1 d}=\bar{V}_{1 d} \cdot\left(G_{e}+\bar{B}_{e}+\bar{\delta}_{u} \cdot \bar{Y}^{\prime}+\bar{\delta}_{A} \cdot \bar{Y}^{\prime \prime}\right)
$$

Pasamos a desarrollar la ecuación (II.72), de manera que obtengamos la información de la parte reactiva de la misma que es la que nos interesa para 
determinar el fenómeno reactivo, el término $\left(\delta_{\mathrm{u}} Y^{`}\right)$ ya se había calculado en la ecuación (II.30), por lo que nos centramos en el término $\left(\delta_{A} Y^{\prime \prime}\right)$.

$$
\begin{aligned}
& \bar{\delta}_{u} \cdot \bar{Y}^{\prime}=\delta_{u} \cdot Y^{\prime} \cdot \cos \left(\alpha_{i}-\alpha_{d}-\alpha^{\prime}\right)-j \cdot \delta_{u} \cdot Y^{\prime} \cdot \operatorname{sen}\left(\varphi_{i}^{\prime}\right) \\
& \varphi_{i}^{\prime}=\left(\alpha_{d}-\alpha_{i}+\alpha^{\prime}\right) \\
& \bar{\delta}_{A} \cdot \bar{Y}^{\prime \prime}=\delta_{A} \angle\left(\alpha_{h}-\alpha_{d}\right) \cdot Y^{\prime \prime} \angle-\alpha^{\prime \prime}=\delta_{A} \cdot Y^{\prime \prime} \angle\left(\alpha_{h}-\alpha_{d}-\alpha^{\prime \prime}\right)= \\
& =\delta_{A} \cdot Y^{\prime \prime} \cdot \cos \left(\alpha_{h}-\alpha_{d}-\alpha^{\prime \prime}\right)+j \cdot \delta_{A} \cdot Y^{\prime \prime} \cdot \operatorname{sen}\left(\alpha_{h}-\alpha_{d}-\alpha^{\prime \prime}\right)= \\
& =\delta_{A} \cdot Y^{\prime \prime} \cdot \cos \left(\alpha_{h}-\alpha_{d}-\alpha^{\prime \prime}\right)-j \cdot \delta_{A} \cdot Y^{\prime \prime} \cdot \operatorname{sen}\left(\alpha_{d}-\alpha_{h}+\alpha^{\prime \prime}\right)= \\
& =\delta_{A} \cdot Y^{\prime \prime} \cdot \cos \left(\alpha_{h}-\alpha_{d}-\alpha^{\prime \prime}\right)-j \cdot \delta_{A} \cdot Y^{\prime \prime} \cdot \operatorname{sen}\left(\varphi_{h}^{\prime \prime}\right) \\
& \varphi_{h}^{\prime \prime}=\left(\alpha_{d}-\alpha_{h}+\alpha^{\prime \prime}\right)
\end{aligned}
$$

Quedándonos entonces:

$$
\begin{aligned}
& \bar{I}_{1 d}=V_{d \angle \alpha_{d}}\left[\left(G_{e}+\delta_{u} Y^{\prime} \cos \left(\alpha_{i}-\alpha_{d}-\alpha^{\prime}\right)+\delta_{A} Y^{\prime \prime} \cos \left(\alpha_{h}-\alpha_{d}-\alpha^{\prime \prime}\right)\right)-\right. \\
& \left.-j\left(B_{e}+\delta_{u} Y^{\prime} \operatorname{sen}\left(\varphi_{i}^{\prime}\right)+\delta_{A} Y^{\prime \prime} \operatorname{sen}\left(\varphi_{h}^{\prime \prime}\right)\right)\right]
\end{aligned}
$$

La componente reactiva de la corriente mostrada en la ecuación (II.73), en su forma temporal será:

$$
i_{1 d r}(t)=V_{d} \cdot \sqrt{2} \cdot\left[B_{e}+\delta_{u} Y^{\prime} \operatorname{sen} \varphi_{i}^{\prime}+\delta_{A} Y^{\prime \prime} \operatorname{sen} \varphi_{h}^{\prime \prime}\right] \cdot \operatorname{sen}\left(w t+\alpha_{d}-90^{\circ}\right)
$$

Podemos entonces determinar la potencia reactiva directa $\mathrm{Q}_{\mathrm{rld}}(\mathrm{t})$ : 


$$
\begin{aligned}
& Q_{r 1 d}(t)=V_{1 d}(t) \cdot i_{1 d r}(t)= \\
& =\left(V_{d} \sqrt{2} \operatorname{sen}\left(w t+\alpha_{d}\right)\right) \cdot\left(V_{d} \sqrt{2} \cdot\left[B_{e}+\delta_{u} Y^{\prime} \operatorname{sen} \varphi_{i}^{\prime}+\delta_{A} Y^{\prime \prime} \operatorname{sen} \varphi_{h}^{\prime \prime}\right] \cdot \operatorname{sen}\left(w t+\alpha_{d}-90^{\circ}\right)\right)= \\
& =\left(V_{d} \sqrt{2} \operatorname{sen}\left(w t+\alpha_{d}\right)\right) \cdot\left(-V_{d} \sqrt{2} \cdot\left[B_{e}+\delta_{u} Y^{\prime} \operatorname{sen} \varphi_{i}^{\prime}+\delta_{A} Y^{\prime \prime} \operatorname{sen} \varphi_{h}^{\prime \prime}\right] \cdot \cos \left(w t+\alpha_{d}\right)\right)= \\
& =\left(-2 V_{d}^{2}\right) \cdot\left[B_{e}+\delta_{u} Y^{\prime} \operatorname{sen} \varphi_{i}^{\prime}+\delta_{A} Y^{\prime \prime} \operatorname{sen} \varphi_{i}^{\prime \prime}\right] \cdot \operatorname{sen}\left(w t+\alpha_{d}\right) \cdot \cos \left(w t+\alpha_{d}\right)= \\
& =-V_{d}{ }^{2} \cdot\left[B_{e}+\left(V_{i} / V_{d}\right) \cdot Y^{\prime} \operatorname{sen} \varphi_{i}^{\prime}+\left(V_{h} / V_{d}\right) \cdot Y^{\prime \prime} \operatorname{sen} \varphi_{h}^{\prime \prime}\right] \cdot \operatorname{sen} 2\left(w t+\alpha_{d}\right)= \\
& =-\left(B_{e} \cdot V_{d}^{2}+V_{d} \cdot V_{i} \cdot Y^{\prime} \cdot \operatorname{sen} \varphi_{i}^{\prime}+V_{d} \cdot V_{h} \cdot Y^{\prime \prime} \cdot \operatorname{sen} \varphi_{h}^{\prime \prime}\right) \cdot \operatorname{sen} 2\left(w t+\alpha_{d}\right)
\end{aligned}
$$

Ahora se procederá al cálculo de la $\mathrm{Q}_{\mathrm{rli}}(\mathrm{t})$, partiendo de la ecuación (II.62), podemos expresar la componente inversa de la corriente $\mathrm{I}_{1 \mathrm{i}}$ como sigue:

$$
\bar{I}_{1 i}=\bar{V}_{1 i} \cdot\left(G_{e}+\bar{B}_{e}\right)+\bar{V}_{1 d} \cdot \bar{Y}^{\prime \prime}+\bar{V}_{1 h} \cdot \bar{Y}^{\prime}
$$

Usando las definiciones del grado de desequilibrio del sistema $\left(\delta_{\mathrm{u}}\right)$ y grado de asimetría $\left(\delta_{\mathrm{A}}\right)$, vistos en la ecuación (II.71), la ecuación (II.77) nos queda:

$$
\bar{I}_{1 i}=\bar{V}_{1 i} \cdot\left(G_{e}+\bar{B}_{e}+\overline{Y^{\prime \prime}} / \bar{\delta}_{u}+\left(\bar{\delta}_{A} / \bar{\delta}_{u}\right) \cdot \bar{Y}^{\prime}\right)
$$

En la ecuación anterior, nos interesa la parte reactiva de la misma, por lo que vamos descomponer en su parte activa y reactiva, el término $\left(Y^{\prime \prime} / \delta_{u}\right)$ se había determinado por la ecuación (II.36), y seguidamente hallamos el término $\left(\delta_{\mathrm{A}} \mathrm{Y}^{\prime} / \delta_{\mathrm{u}}\right)$ : 


$$
\begin{aligned}
& \left(\bar{\delta}_{A} \overline{Y^{\prime}}\right) / \bar{\delta}_{u}=\left(\delta_{A} Y^{\prime}\right) / \delta_{u} \angle\left(\alpha_{h}-\alpha_{i}-\alpha^{\prime}\right)= \\
& =\left(\delta_{A} Y^{\prime}\right) / \delta_{u} \cdot \cos \left(\alpha_{h}-\alpha_{i}-\alpha^{\prime}\right)+\left(\delta_{A} Y^{\prime}\right) / \delta_{u} \cdot \operatorname{sen}\left(\alpha_{h}-\alpha_{i}-\alpha^{\prime}\right)= \\
& =\left(\delta_{A} Y^{\prime}\right) / \delta_{u} \cdot \cos \left(\alpha_{h}-\alpha_{i}-\alpha^{\prime}\right)-j \cdot\left(\delta_{A} Y^{\prime}\right) / \delta_{u} \cdot \operatorname{sen}\left(\alpha_{i}-\alpha_{h}+\alpha^{\prime}\right)= \\
& =\left(\delta_{A} Y^{\prime}\right) / \delta_{u} \cdot \cos \left(\alpha_{h}-\alpha_{i}-\alpha^{\prime}\right)-j \cdot\left(\delta_{A} Y^{\prime}\right) / \delta_{u} \cdot \operatorname{sen}\left(\varphi_{h}^{\prime}\right) \\
& \varphi_{h}^{\prime}=\left(\alpha_{i}-\alpha_{h}+\alpha^{\prime}\right)
\end{aligned}
$$

Siendo entonces la componente inversa de la corriente la siguiente:

$$
\begin{aligned}
& \bar{I}_{1 i}=V_{i \angle \alpha i}\left[\left(G_{e}+Y^{\prime \prime} / \delta_{u} \cos \left(\alpha_{d}-\alpha_{i}-\alpha^{\prime \prime}\right)+\left(\delta_{A} Y^{\prime} / \delta_{u}\right) \cos \left(\alpha_{h}-\alpha_{i}-\alpha^{\prime}\right)\right)-\right. \\
& \left.-j\left(B_{e}+Y^{\prime \prime} / \delta_{u} \operatorname{sen}\left(\varphi_{d}^{\prime \prime}\right)+\left(\delta_{A} Y^{\prime} / \delta_{u}\right) \operatorname{sen}\left(\varphi_{h}^{\prime}\right)\right)\right]
\end{aligned}
$$

Y la expresión temporal de la parte reactiva será:

$$
i_{1 i r}(t)=V_{i} \cdot \sqrt{2} \cdot\left[B_{e}+Y^{\prime \prime} / \delta_{u} \operatorname{sen} \varphi_{d}^{\prime \prime}+\left(\delta_{A} Y^{\prime} / \delta_{u}\right) \operatorname{sen} \varphi_{h}^{\prime}\right] \cdot \operatorname{sen}\left(w t+\alpha_{i}-90^{\circ}\right)
$$

Siendo entonces la potencia reactiva inversa $\mathrm{Q}_{\mathrm{r1i}}(\mathrm{t})$ :

$$
\begin{aligned}
& Q_{r 1 i}(t)=V_{1 i}(t) \cdot i_{1 i r}(t)= \\
& =\left(V_{i} \sqrt{2} \operatorname{sen}\left(w t+\alpha_{i}\right)\right) \cdot\left(V_{i} \sqrt{2} \cdot\left[B_{e}+Y^{\prime \prime} / \delta_{u} \operatorname{sen} \varphi_{d}^{\prime \prime}+\delta_{A} Y^{\prime} / \delta_{u} \operatorname{sen} \varphi_{h}^{\prime}\right] \cdot \operatorname{sen}\left(w t+\alpha_{i}-90^{\circ}\right)\right)= \\
& =\left(V_{i} \sqrt{2} \operatorname{sen}\left(w t+\alpha_{i}\right)\right) \cdot\left(-V_{i} \sqrt{2} \cdot\left[B_{e}+Y^{\prime \prime} / \delta_{u} \operatorname{sen} \varphi_{d}^{\prime \prime}+\delta_{A} Y^{\prime} / \delta_{u} \operatorname{sen} \varphi_{h}^{\prime}\right] \cdot \cos \left(w t+\alpha_{i}\right)\right)= \\
& =\left(-2 V_{i}^{2}\right) \cdot\left[B_{e}+Y^{\prime \prime} / \delta_{u} \operatorname{sen} \varphi_{d}^{\prime \prime}+\delta_{A} Y^{\prime} / \delta_{u} \operatorname{sen} \varphi_{h}^{\prime}\right] \cdot \operatorname{sen}\left(w t+\alpha_{i}\right) \cdot \cos \left(w t+\alpha_{i}\right)= \\
& =-V_{i}^{2} \cdot\left[B_{e}+\left(V_{d} / V_{i}\right) \cdot Y^{\prime \prime \prime} \operatorname{sen} \varphi_{d}^{\prime \prime}+\left(V_{h} / V_{i}\right) \cdot Y^{\prime} \operatorname{sen} \varphi_{h}^{\prime}\right] \cdot \operatorname{sen} 2\left(w t+\alpha_{i}\right)= \\
& =-\left(B_{e} \cdot V_{i}^{2}+V_{i} \cdot V_{d} \cdot Y^{\prime \prime} \cdot \operatorname{sen} \varphi_{d}^{\prime \prime}+V_{i} \cdot V_{h} \cdot Y^{\prime} \cdot \operatorname{sen} \varphi_{h}^{\prime}\right) \cdot \operatorname{sen} 2\left(w t+\alpha_{i}\right)
\end{aligned}
$$


Pasamos a determinar la $\mathrm{Q}_{\mathrm{r} 1 \mathrm{~h}}(\mathrm{t})$, partiendo de la ecuación (II.62), la componente homopolar de la corriente $I_{1 h}$ es:

$$
\bar{I}_{1 h}=\bar{V}_{1 h} \cdot\left(G_{e}+\bar{B}_{e}\right)+\bar{V}_{1 d} \cdot \bar{Y}^{\prime}+\bar{V}_{1 i} \cdot \bar{Y}^{\prime \prime}
$$

Usando las definiciones del grado de desequilibrio del sistema $\left(\delta_{\mathrm{u}}\right)$ y grado de asimetría $\left(\delta_{\mathrm{A}}\right)$, vistos en la ecuación (II.71), la ecuación (II.83) nos queda:

$$
\bar{I}_{1 h}=\bar{V}_{1 h} \cdot\left(G_{e}+\bar{B}_{e}+\overline{Y^{\prime}} / \bar{\delta}_{A}+\left(\bar{\delta}_{u} / \bar{\delta}_{A}\right) \cdot \bar{Y}^{\prime \prime}\right)
$$

Procediendo como en anteriores ocasiones, y como nos interesa la parte reactiva de la ecuación (II.84), vamos a descomponerla en su parte activa y reactiva, para lo que hallamos los términos $\left(\mathrm{Y}^{\prime} / \delta_{\mathrm{A}}\right)$ y el término $\left(\delta_{\mathrm{u}} \mathrm{Y}^{\prime \prime} / \delta_{\mathrm{A}}\right)$ :

$$
\begin{aligned}
& \bar{Y}^{\prime} / \bar{\delta}_{A}=Y^{\prime} \angle-\alpha^{\prime} / \delta_{A} \angle\left(\alpha_{h}-\alpha_{d}\right)=Y^{\prime} / \delta_{A} \angle\left(\alpha_{d}-\alpha_{h}-\alpha^{\prime}\right)= \\
& =Y^{\prime} / \delta_{A} \cdot \cos \left(\alpha_{d}-\alpha_{h}-\alpha^{\prime}\right)+j \cdot Y^{\prime} / \delta_{A} \cdot \operatorname{sen}\left(\alpha_{d}-\alpha_{h}-\alpha^{\prime}\right)= \\
& =Y^{\prime} / \delta_{A} \cdot \cos \left(\alpha_{d}-\alpha_{h}-\alpha^{\prime}\right)-j \cdot Y^{\prime} / \delta_{A} \cdot \operatorname{sen}\left(\alpha_{h}-\alpha_{d}+\alpha^{\prime}\right)= \\
& =Y^{\prime} / \delta_{A} \cdot \cos \left(\alpha_{d}-\alpha_{h}-\alpha^{\prime}\right)-j \cdot Y^{\prime} / \delta_{A} \cdot \operatorname{sen}\left(\varphi_{d}^{\prime}\right) \\
& \varphi_{d}^{\prime}=\left(\alpha_{h}-\alpha_{d}+\alpha^{\prime}\right)
\end{aligned}
$$




$$
\begin{aligned}
& \left(\bar{\delta}_{u} \bar{Y}^{\prime \prime}\right) / \bar{\delta}_{A}=\left(\delta_{u} Y^{\prime \prime}\right) / \delta_{A} \angle\left(\alpha_{i}-\alpha_{h}-\alpha^{\prime \prime}\right)= \\
& =\left(\delta_{u} Y^{\prime \prime}\right) / \delta_{A} \cdot \cos \left(\alpha_{i}-\alpha_{h}-\alpha^{\prime \prime}\right)+\left(\delta_{u} Y^{\prime \prime}\right) / \delta_{A} \cdot \operatorname{sen}\left(\alpha_{i}-\alpha_{h}-\alpha^{\prime \prime}\right)= \\
& =\left(\delta_{u} Y^{\prime \prime}\right) / \delta_{A} \cdot \cos \left(\alpha_{i}-\alpha_{h}-\alpha^{\prime \prime}\right)-j \cdot\left(\delta_{u} Y^{\prime \prime}\right) / \delta_{A} \cdot \operatorname{sen}\left(\alpha_{h}-\alpha_{i}+\alpha^{\prime \prime}\right)= \\
& =\left(\delta_{u} Y^{\prime \prime}\right) / \delta_{A} \cdot \cos \left(\alpha_{h}-\alpha_{i}-\alpha^{\prime}\right)-j \cdot\left(\delta_{u} Y^{\prime \prime}\right) / \delta_{A} \cdot \operatorname{sen}\left(\varphi_{i}^{\prime \prime}\right) \\
& \varphi_{i}^{\prime \prime}=\left(\alpha_{h}-\alpha_{i}+\alpha^{\prime \prime}\right)
\end{aligned}
$$

La componente homopolar de la corriente se puede poner como:

$$
\begin{aligned}
& \bar{I}_{1 h}=V_{h \angle \alpha h}\left[\left(G_{e}+Y^{\prime} / \delta_{A} \cos \left(\alpha_{d}-\alpha_{h}-\alpha^{\prime}\right)+\left(\delta_{u} Y^{\prime \prime} / \delta_{A}\right) \cos \left(\alpha_{i}-\alpha_{h}-\alpha^{\prime \prime}\right)\right)-\right. \\
& \left.-j\left(B_{e}+Y^{\prime} / \delta_{A} \operatorname{sen}\left(\varphi_{d}^{\prime}\right)+\left(\delta_{u} Y^{\prime \prime} / \delta_{A}\right) \operatorname{sen}\left(\varphi_{i}^{\prime \prime}\right)\right)\right]
\end{aligned}
$$

Siendo la expresión temporal de la parte reactiva:

$$
i_{1 h r}(t)=V_{h} \cdot \sqrt{2} \cdot\left[B_{e}+Y^{\prime} / \delta_{A} \operatorname{sen} \varphi_{d}^{\prime}+\left(\delta_{u} Y^{\prime \prime} / \delta_{A}\right) \operatorname{sen} \varphi_{i}^{\prime \prime}\right] \cdot \operatorname{sen}\left(w t+\alpha_{h}-90^{\circ}\right)
$$

La potencia reactiva homopolar $\mathrm{Q}_{\mathrm{r} 1 \mathrm{~h}}(\mathrm{t})$ vale:

$$
\begin{aligned}
& Q_{r 1 h}(t)=V_{1 h}(t) \cdot i_{1 h r}(t)= \\
& =\left(V_{h} \sqrt{2} \operatorname{sen}\left(w t+\alpha_{h}\right)\right) \cdot\left(V_{h} \sqrt{2} \cdot\left[B_{e}+Y^{\prime} / \delta_{A} \operatorname{sen} \varphi_{d}^{\prime}+\delta_{u} Y^{\prime \prime} / \delta_{A} \operatorname{sen} \varphi_{i}^{\prime \prime}\right] \cdot \operatorname{sen}\left(w t+\alpha_{h}-90^{\circ}\right)\right)= \\
& =\left(V_{h} \sqrt{2} \operatorname{sen}\left(w t+\alpha_{h}\right)\right) \cdot\left(-V_{h} \sqrt{2} \cdot\left[B_{e}+Y^{\prime} / \delta_{A} \operatorname{sen} \varphi_{d}^{\prime}+\delta_{u} Y^{\prime \prime} / \delta_{A} \operatorname{sen} \varphi_{i}^{\prime \prime}\right] \cdot \cos \left(w t+\alpha_{h}\right)\right)= \\
& =\left(-2 V_{h}^{2}\right) \cdot\left[B_{e}+Y^{\prime} / \delta_{A} \operatorname{sen} \varphi_{d}^{\prime}+\delta_{u} Y^{\prime \prime} / \delta_{A} \operatorname{sen} \varphi_{i}^{\prime \prime}\right] \cdot \operatorname{sen}\left(w t+\alpha_{h}\right) \cdot \cos \left(w t+\alpha_{h}\right)= \\
& =-V_{h}^{2} \cdot\left[B_{e}+\left(V_{d} / V_{h}\right) \cdot Y^{\prime} \operatorname{sen} \varphi_{d}^{\prime}+\left(V_{i} / V_{h}\right) \cdot Y^{\prime \prime} \operatorname{sen} \varphi_{i}^{\prime \prime}\right] \cdot \operatorname{sen} 2\left(w t+\alpha_{h}\right)= \\
& =-\left(B_{e} \cdot V_{h}^{2}+V_{h} \cdot V_{d} \cdot Y^{\prime} \cdot \operatorname{sen} \varphi_{d}^{\prime}+V_{h} \cdot V_{i} \cdot Y^{\prime \prime} \cdot \operatorname{sen} \varphi_{i}^{\prime \prime}\right) \cdot \operatorname{sen} 2\left(w t+\alpha_{h}\right)
\end{aligned}
$$


Operando de idéntica manera para los otros términos que componen la ecuación (II.68), obtenemos la potencia reactiva instantánea total del sistema $\mathrm{Q}_{\mathrm{r}}(\mathrm{t})$ :

$$
\begin{aligned}
& Q_{r}(t)=Q_{r d}(t)+Q_{r i}(t)+Q_{r h}(t)= \\
& =-\left(B_{e} \cdot V_{d}^{2}+Y^{\prime} \cdot V_{d} \cdot V_{i} \cdot \operatorname{sen} \varphi_{i}^{\prime}+Y^{\prime \prime} \cdot V_{d} \cdot V_{h} \cdot \operatorname{sen} \varphi_{h}^{\prime \prime}\right) \cdot \operatorname{sen} 2\left(w t+\alpha_{d}\right)- \\
& -\left(B_{e} \cdot V_{i}^{2}+Y^{\prime \prime} \cdot V_{d} \cdot V_{i} \cdot \operatorname{sen} \varphi_{d}^{\prime \prime}+Y^{\prime} \cdot V_{i} \cdot V_{h} \cdot \operatorname{sen} \varphi_{h}^{\prime}\right) \cdot \operatorname{sen} 2\left(w t+\alpha_{i}\right)- \\
& -\left(B_{e} \cdot V_{h}^{2}+Y^{\prime} \cdot V_{d} \cdot V_{h} \cdot \operatorname{sen} \varphi_{d}^{\prime}+Y^{\prime \prime} \cdot V_{i} \cdot V_{h} \cdot \operatorname{sen} \varphi_{i}^{\prime \prime}\right) \cdot \operatorname{sen} 2\left(w t+\alpha_{h}\right)- \\
& -\left(B_{e} \cdot V_{d}^{2}+Y^{\prime} \cdot V_{d} \cdot V_{i} \cdot \operatorname{sen} \varphi_{i}^{\prime}+Y^{\prime \prime} \cdot V_{d} \cdot V_{h} \cdot \operatorname{sen} \varphi_{h}^{\prime \prime}\right) \cdot \operatorname{sen} 2\left(w t+\alpha_{d}-120^{\circ}\right)- \\
& -\left(B_{e} \cdot V_{i}^{2}+Y^{\prime \prime} \cdot V_{d} \cdot V_{i} \cdot \operatorname{sen} \varphi_{d}^{\prime \prime}+Y^{\prime} \cdot V_{i} \cdot V_{h} \cdot \operatorname{sen} \varphi_{h}^{\prime}\right) \cdot \operatorname{sen} 2\left(w t+\alpha_{i}+120^{\circ}\right)- \\
& -\left(B_{e} \cdot V_{h}^{2}+Y^{\prime} \cdot V_{d} \cdot V_{h} \cdot \operatorname{sen} \varphi_{d}^{\prime}+Y^{\prime \prime} \cdot V_{i} \cdot V_{h} \cdot \operatorname{sen} \varphi_{i}^{\prime \prime}\right) \cdot \operatorname{sen} 2\left(w t+\alpha_{h}\right)- \\
& -\left(B_{e} \cdot V_{i}^{2}+Y^{\prime \prime} \cdot V_{d} \cdot V_{d} \cdot V_{i} \cdot \operatorname{sen} \varphi_{i}^{\prime}+Y^{\prime \prime} \cdot V_{d} \cdot V_{h} \cdot \operatorname{sen} \varphi_{d}^{\prime \prime}+Y^{\prime \prime} \cdot V_{i} \cdot V_{h} \cdot \operatorname{sen} \varphi_{h}^{\prime}\right) \cdot \operatorname{sen} 2\left(w t+\alpha_{d}-240^{\circ}\right)- \\
& -\left(B_{e} \cdot V_{h}^{2}+Y^{\prime} \cdot V_{d} \cdot V_{h} \cdot \operatorname{sen} \varphi_{d}^{\prime}+Y^{\prime \prime} \cdot V_{i} \cdot V_{h} \cdot \operatorname{sen} \varphi_{i}^{\prime \prime}\right) \cdot \operatorname{sen} 2\left(w t+\alpha_{h}\right)
\end{aligned}
$$

De la observación de la ecuación (II.90), se ve que la potencia reactiva instantánea del sistema según la Teoría Clásica esta formada por los términos de las potencias reactivas debidas a las secuencias directa, inversa y homopolar, como queda reflejado en la IEEE Standard 1459/2000. 


\section{II.5.2.- Teoría Unificadora.}

Siguiendo el procedimiento usado en anteriores ocasiones determinamos ahora la potencia reactiva instantánea basándonos en la Teoría Unificadora, usando para ello tan sólo las componentes directas de tensión y las intensidades directas reactivas de cada fase, como también establece Emanuel en la citada IEEE Standard 1459/2000, siendo entonces la potencia reactiva instantánea $Q_{r}(t)$ la siguiente:

$$
\begin{aligned}
& Q_{r}(t)=Q_{r d}=\left(Q_{r 1 d}+Q_{q 2 d}+Q_{r 3 d}\right)= \\
& =\left(v_{1 d} \cdot i_{1 d r}+v_{2 d} \cdot i_{2 d r}+v_{3 d} \cdot i_{3 d r}\right)= \\
& -\left(B_{e} \cdot V_{d}^{2}+Y^{\prime} \cdot V_{d} \cdot V_{i} \cdot \operatorname{sen} \varphi_{i}^{\prime}+Y^{\prime \prime} \cdot V_{d} \cdot V_{h} \cdot \operatorname{sen} \varphi_{h}^{\prime \prime}\right) \cdot \operatorname{sen} 2\left(w t+\alpha_{d}\right)- \\
& -\left(B_{e} \cdot V_{d}^{2}+Y^{\prime} \cdot V_{d} \cdot V_{i} \cdot \operatorname{sen} \varphi_{i}^{\prime}+Y^{\prime \prime} \cdot V_{d} \cdot V_{h} \cdot \operatorname{sen} \varphi_{h}^{\prime \prime}\right) \cdot \operatorname{sen} 2\left(w t+\alpha_{d}-120^{\circ}\right)- \\
& -\left(B_{e} \cdot V_{d}^{2}+Y^{\prime} \cdot V_{d} \cdot V_{i} \cdot \operatorname{sen} \varphi_{i}^{\prime}+Y^{\prime \prime} \cdot V_{d} \cdot V_{h} \cdot \operatorname{sen} \varphi_{h}^{\prime \prime}\right) \cdot \operatorname{sen} 2\left(w t+\alpha_{d}-240^{\circ}\right)
\end{aligned}
$$

Comparando esta ecuación (II.91) con la ecuación (II.58) de los sistemas equilibrados en tensiones, observamos que existen en el caso de tensiones desequilibradas flujos de potencia reactiva instantánea que no aparecen en el caso de tensiones equilibradas, pero si permanece un flujo de potencia reactiva instantánea en ambos casos, este flujo es el que se corresponde con la presencia de elementos reactivos en el sistema, y que denominamos como $\mathrm{Q}_{\mathrm{rr}}(\mathrm{t})$, y cuya expresión es:

$$
\begin{aligned}
& Q_{r r}(t)=-B_{e} \cdot V_{d}^{2} \cdot \operatorname{sen} 2\left(w t+\alpha_{d}\right) \\
& -B_{e} \cdot V_{d}^{2} \cdot \operatorname{sen} 2\left(w t+\alpha_{d}-120^{\circ}\right) \\
& -B_{e} \cdot V_{d}^{2} \cdot \operatorname{sen} 2\left(w t+\alpha_{d}-240^{\circ}\right)
\end{aligned}
$$


y otro término que sólo surge en el caso de los sistemas con tensiones desequilibradas también formado por flujos sinusoidales y equilibrados, cuyas amplitudes dependen del desequilibrio en tensiones y cargas que posea el sistema, y que denominamos por $\mathrm{Q}_{\mathrm{ru}}(\mathrm{t})$ :

$$
\begin{aligned}
& Q_{r u}(t)=-\left(Y^{\prime} \cdot V_{d} \cdot V_{i} \cdot \operatorname{sen} \varphi_{i}^{\prime}+Y^{\prime \prime} \cdot V_{d} \cdot V_{h} \cdot \operatorname{sen} \varphi_{h}^{\prime \prime}\right) \cdot \operatorname{sen} 2\left(w t+\alpha_{d}\right)- \\
& -\left(Y^{\prime} \cdot V_{d} \cdot V_{i} \cdot \operatorname{sen} \varphi_{i}^{\prime}+Y^{\prime \prime} \cdot V_{d} \cdot V_{h} \cdot \operatorname{sen} \varphi_{h}^{\prime \prime}\right) \cdot \operatorname{sen} 2\left(w t+\alpha_{d}-120^{\circ}\right)- \\
& -\left(Y^{\prime} \cdot V_{d} \cdot V_{i} \cdot \operatorname{sen} \varphi_{i}^{\prime}+Y^{\prime \prime} \cdot V_{d} \cdot V_{h} \cdot \operatorname{sen} \varphi_{h}^{\prime \prime}\right) \cdot \operatorname{sen} 2\left(w t+\alpha_{d}-240^{\circ}\right)
\end{aligned}
$$

Estos dos flujos de potencia reactiva instantánea, el debido al propio efecto de la presencia de elementos reactivos en el sistema, y el provocado por los desequilibrios del sistema, son los que contribuyen para dar la potencia reactiva instantánea total del sistema $\mathrm{Q}_{\mathrm{r}}(\mathrm{t})$, quedando como mostramos:

$$
Q_{r}(t)=Q_{r r}(t)+Q_{r u}(t)
$$




\section{II.6.- Conclusiones.}

A lo largo del capítulo hemos obtenido las expresiones que ponen de manifiesto la presencia de Fenómenos de Desfase en sistemas trifásicos desequilibrados y lineales, así como una serie de conclusiones que pasamos a remarcar:

- Existencia de dos flujos de potencia reactiva instantánea:

- Los debidos a la presencia de reactancias en el sistema.

- Los provocados por los desequilibrios, que no son contemplados por las Teorías Clásicas.

- Los flujos de potencia reactiva presentes en las ecuaciones (II.42, 43, 92 y 93), tanto los debidos a las presencia de elementos reactivos en el sistema, como los provocados por los desequilibrios, tienen un origen común, que es el desfase entre las tensiones y las corrientes de secuencia directa de cada fase.

- Este origen común, lleva a denominar como potencia de desfase $Q_{d}$, a la magnitud que cuantifica los efectos de estos fenómenos, siendo su valor igual a la suma de las amplitudes de las potencia reactivas instantáneas de secuencia directa de cada una de las fases del sistema, cuyos valores para los sistemas a tres y cuatro hilos se muestran en las tablas II.1 y II.2.

La Potencia de Desfase (o reactiva), definida por las Teorías Clásicas para los sistemas desequilibrados lineales, como ya hemos visto en el Capítulo I (Antecedentes), tiene en cuenta las potencias de desfase debidas a la secuencia inversa y homopolar, concepto erróneo a nuestro entender, ya que tan sólo la secuencia directa sería la que debería de considerarse, ya que los valores máximos de las otras secuencias no coinciden y además no van en fase, tal y como establece la Teoría Unificadora de la Potencia Eléctrica y Emmanuel en la IEEE Std. 1459-2000, para la cual la Potencia de Desfase (o reactiva) viene determinada por el producto de la tensión directa y la intensidad directa reactiva, cuya expresión pasamos a recordar: 


$$
Q=3 \cdot V_{d} \cdot I_{d r}=3 \cdot V_{d} \cdot I_{d} \cdot \operatorname{sen} \varphi_{d}
$$

A lo largo de este capítulo, se han realizado los desarrollos pertinentes para los sistemas trifásicos desequilibrados lineales en triángulo y con hilo neutro, identificando los diferentes términos que contribuyen al fenómeno del Desfase, y que a continuación pasamos a mostrar en las Tablas II.1 y II.2:

Sistemas en triángulo.

\begin{tabular}{||l|l||}
\hline $\begin{array}{l}\text { Potencia de desfase debida a la presencia } \\
\text { de elementos reactivos }\end{array}$ & $Q_{d r r}=3 \cdot B_{e} \cdot V_{d}^{2}$ \\
\hline $\begin{array}{l}\text { Potencia de desfase debida a desequilibrios } \\
\text { de tensiones y cargas }\end{array}$ & $Q_{d r u}=3 \cdot Y^{\prime} \cdot V_{d} \cdot V_{i} \cdot \operatorname{sen} \varphi_{i}^{\prime}$ \\
\hline
\end{tabular}

Tabla II.1. Términos potencia desfase en sistemas en triángulo.

\section{Sistemas con hilo neutro.}

\begin{tabular}{||l|l||}
\hline $\begin{array}{l}\text { Potencia de desfase debida } \\
\text { a la presencia de elementos } \\
\text { reactivos }\end{array}$ & $Q_{d r r}=3 \cdot B_{e} \cdot V_{d}^{2}$ \\
\hline $\begin{array}{l}\text { Potencia de desfase debida } \\
\text { a desequilibrios de tensiones } \\
\text { y cargas }\end{array}$ & $Q_{d r u}=3 \cdot\left(Y^{\prime} \cdot V_{d} \cdot V_{i} \cdot \operatorname{sen} \varphi_{i}^{\prime}+Y^{\prime \prime} \cdot V_{d} \cdot V_{h} \cdot \operatorname{sen} \varphi_{h}^{\prime \prime}\right)$ \\
\hline
\end{tabular}

Tabla II.2. Términos potencia desfase en sistemas con hilo neutro.

- Además como veremos en capítulos posteriores, los flujos de potencia reactiva de desfase, provocados por los desequilibrios, pueden aumentar 0 disminuir los efectos producidos por las reactancias del sistema.

- En sistemas puramente resistivos, se observan flujos de potencia reactiva de desfase, que son atribuidos de forma exclusiva a los desequilibrios de cargas y excitaciones existentes, ya que no existen elementos reactivos. 



\section{CAPÍTULO III}

REPRESENTACIÓN DEL FENÓMENO

DEL DESFASE. 



\section{III.- Representación del Fenómeno del Desfase.}

\section{III.1.- Introducción.}

En el capitulo I, se ha expuesto la Teoría Unificadora de la Potencia Eléctrica, y en el capítulo II ha quedado de manifiesto la presencia de algunos fenómenos energéticos que pueden manifestarse durante la transferencia de energía en los sistemas eléctricos y que no han sido previstos por la mayoría de las principales teorías de la potencia eléctrica. Uno de estos fenómenos antes aludidos es el fenómeno de desfase o reactivo causado por los desequilibrios en los sistemas trifásicos, fenómeno cuyo estudio, como es conocido, es el objeto principal de esta tesis doctoral.

La Teoría de Circuitos nos muestra que los fenómenos energéticos en los circuitos eléctricos pueden ser representados mediante elementos de circuito o asociaciones de dichos elementos. Así es conocido que una resistencia representa el fenómeno de la energía eléctrica que se transforma, ya sea en calor o, en general, en cualquier otro tipo de energía. Una bobina representa los fenómenos de tipo electromagnético y un condensador los fenómenos de tipo electrostático.

La teoría Unificadora de la Potencia Eléctrica permite obtener representaciones simbólicas de los distintos fenómenos que se manifiestan en los sistemas eléctricos, que son conocidas como circuitos equivalentes [100]. Los circuitos equivalentes de los receptores trifásicos son conexiones trifásicas de impedancias, que absorben las mismas corrientes y potencias que el receptor real y permiten caracterizar y mostrar gráficamente cada fenómeno, así como determinar sus efectos.

De las diferentes conexiones o redes trifásicas que se pueden establecer en los circuitos equivalentes, en esta tesis doctoral nos interesará, sobre cualquier otra, la red de desfase, es decir, aquella que caracteriza los fenómenos de desfase o reactivos. La red de desfase es aquella que absorbe las corrientes reactivas de 
secuencia directa y frecuencia fundamental, tal como establece la teoría Unificadora de la Potencia Eléctrica y como se deduciría de aplicar, para este cometido, la teoría de Emmanuel recogida en la IEEE Standard 1459-2000. Frente a los circuitos equivalentes que se deducen de otras teorías [40], los que aquí se utilizan, derivados de la teoría Unificadora, pueden ser aplicados a sistemas a cuatro hilos y se caracterizan por representar fielmente el fenómeno físico, ya que dan lugar al mismo valor de la potencia instantánea que el circuito real y, por tanto, no son representaciones formales, meramente matemáticas.

Para determinar los distintos fenómenos de desfase a partir de las redes de desfase correspondientes a circuitos trifásicos sinusoidales y no sinusoidales, en este capítulo se ha procedido de la siguiente manera:

Primero. Partiendo de un receptor desequilibrado y lineal, primero en triángulo y después en estrella, alimentado por un sistema de excitaciones equilibrado y de secuencia directa, se calculan las corrientes reactivas de secuencia directa y frecuencia fundamental de cada una de las fases. Con estas corrientes y aplicando la ley de Ohm, se obtienen las expresiones de las impedancias de la red de desfase con tensiones equilibradas, como relación entre las tensiones de frecuencia fundamental y de secuencia directa de cada fase y las corrientes reactivas de frecuencia fundamental y secuencia directa de cada fase.

Segundo. Siguiendo el mismo proceso anterior, se obtienen las impedancias de las redes de desfase correspondientes a receptores desequilibrados, primero en triángulo y después en estrella, alimentados con excitaciones desequilibradas.

Tercero. Se comparan los resultados obtenidos de los dos casos anteriores para distinguir el origen de los distintos fenómenos de desfase o reactivos. 


\section{III.2- Representación del desfase en sistemas en triángulo.}

\section{2.1.- Tensiones Equilibradas.}

Tal y como se ha expuesto en la introducción de este capítulo, para la representación del fenómeno del desfase y como ya se había planteado en el capítulo II, y haciendo uso de la Teoría Unificadora, nos es necesario conocer las intensidades reactivas de secuencia directa y frecuencia fundamental, que son las que caracterizan al fenómeno del desfase en el sistema.

Partiendo de un sistema desequilibrado lineal en triángulo, y excitado por un sistema de tensiones equilibradas, como se ve en la figura III.1, y como el usado en el apartado II.2.1, del capítulo II, habíamos obtenido, según la ecuación (II.3) y las ecuaciones (II.7 y 9), que la corriente directa reactiva para el receptor conectado entre las fases 1 y 2 vale:

$$
i_{12 d r}(t)=V_{d} \cdot \sqrt{2} \cdot B_{e} \cdot \operatorname{sen}\left(w t+\alpha_{d}-90^{\circ}\right)
$$

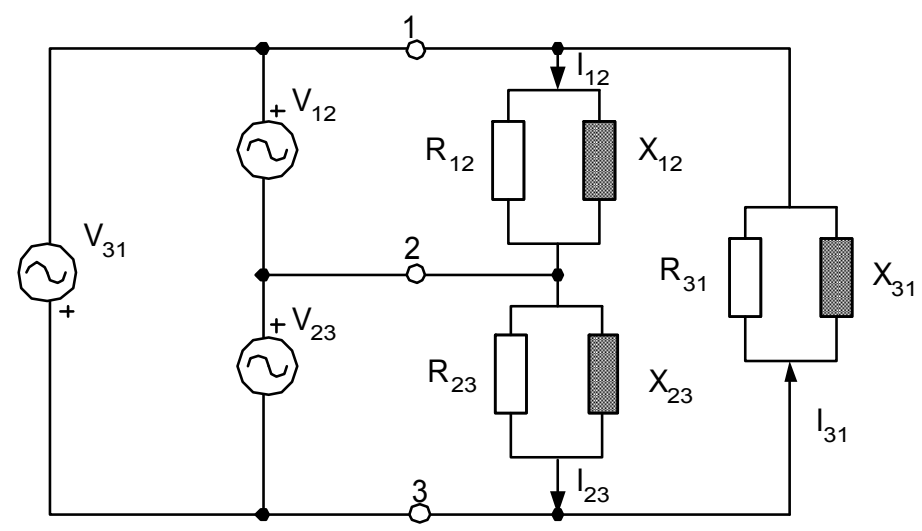

Figura III.1. Sistema en triángulo.

Tensiones equilibradas.

Que en su representación fasorial es: 


$$
\begin{aligned}
& \bar{I}_{12 d r}=\bar{V}_{12 d} \cdot \bar{B}_{e} \\
& \bar{B}_{e}=1 / 3 \cdot\left(\bar{B}_{12}+\bar{B}_{23}+\bar{B}_{31}\right)=1 / \bar{X}_{e}
\end{aligned}
$$

Al ser un sistema equilibrado de excitaciones, tan sólo tenemos componente directa de tensión $\mathrm{V}_{12 \mathrm{~d}}$, por lo tanto las impedancias que caracteriza el fenómeno del desfase, serán:

$$
\begin{aligned}
& \bar{Z}_{12 d d}=\frac{\bar{V}_{12 d}}{\bar{I}_{12 d r}}=\frac{\bar{V}_{12 d}}{\bar{V}_{12 d} \cdot \bar{B}_{e}}=\frac{1}{\bar{B}_{e}}=\bar{X}_{e} \\
& \bar{Z}_{23 d d}=\frac{\bar{V}_{23 d}}{\bar{I}_{23 d r}}=\frac{a^{2} \cdot \bar{V}_{12 d}}{a^{2} \cdot \bar{V}_{12 d} \cdot \bar{B}_{d}}=\frac{1}{\bar{B}_{e}}=\bar{X}_{e} \\
& \bar{Z}_{31 d d}=\frac{\bar{V}_{31 d}}{\bar{I}_{31 d r}}=\frac{a \cdot \bar{V}_{12 d}}{a \cdot \bar{V}_{12 d} \cdot \bar{B}_{d}}=\frac{1}{\bar{B}_{e}}=\bar{X}_{e}
\end{aligned}
$$

Las ecuaciones (III.3) muestran que en los sistemas con tensiones equilibradas el fenómeno del desfase es debido única y exclusivamente a la presencia de elementos reactivos (bobinas y condensadores) en el sistema. Este hecho se aprecia gráficamente en la red de desfase que, para estos sistemas, es tal como muestra la figura III.2.

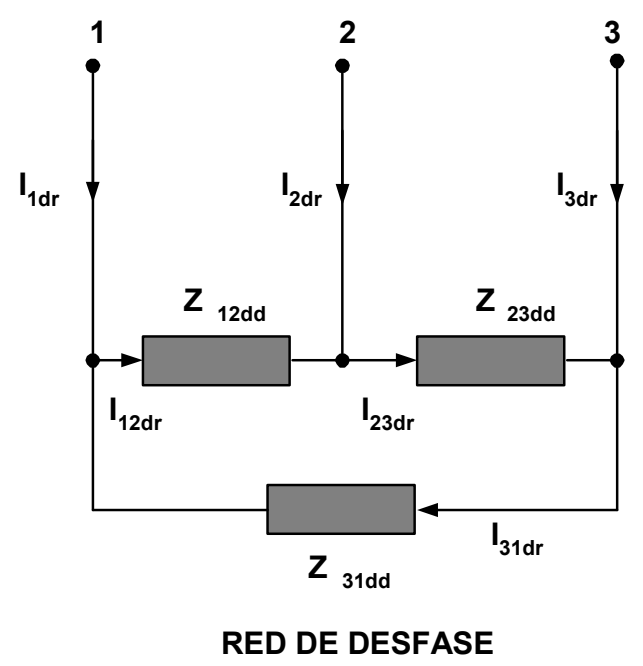

Figura III.2. Red de desfase. Sistema en triángulo y tensiones equilibradas. 


\section{2.2.- Tensiones Desequilibradas.}

El circuito del que partimos es el mostrado en la figura III.1, pero en este caso se halla alimentado por un sistema de excitación desequilibrado, cuyas tensiones de fase, aplicando el Teorema de Stokvis, tienen una componente de secuencia directa, $\mathrm{V}_{12 \mathrm{~d}}$, $\mathrm{y}$ otra de secuencia inversa, $\mathrm{V}_{12 \mathrm{i}}$.

Siguiendo el mismo procedimiento que en el apartado anterior, para caracterizar el fenómeno del desfase tenemos que conocer la intensidad reactiva de secuencia directa. Tal como se vio en el capítulo II, para el circuito que estamos analizando esta corriente se deduce de la ecuación (II.31):

$$
\bar{I}_{12 d r}=V_{d} \angle \alpha_{d}\left[-j\left(B_{e}+\delta_{u} \cdot Y^{\prime} \cdot \operatorname{sen}\left(\varphi_{i}^{\prime}\right)\right)\right]
$$

Seguidamente pasamos a recordar lo que significaba cada uno de los términos de la ecuación (III.4), la $V_{d}$ representa la componente directa de la tensión, la $B_{e}$ era la susceptancia directa del receptor, $\delta_{u}$ nos indica el grado de desequilibrio de tensiones del sistema, la $Y^{`}$ es la admitancia básica de desequilibrio y el ángulo $\varphi_{i}$ 'es función de los ángulos de la componente directa e inversa de tensión y del propio ángulo de la carga.

Las impedancias que caracterizan el desfase son, aplicando la ley de Ohm:

$$
\begin{aligned}
& \bar{Z}_{12 d}=\frac{\bar{V}_{12}}{\bar{I}_{12 d r}}=\frac{\bar{V}_{12 d}+\bar{V}_{12 i}}{\bar{I}_{12 d r}}=\frac{\bar{V}_{12 d}}{\bar{I}_{12 d r}}+\frac{\bar{V}_{12 i}}{\bar{I}_{12 d r}}= \\
& =\frac{V_{12 d}}{-j \cdot V_{12 d} \cdot\left(B_{e}+\delta_{u} \cdot Y^{\prime} \cdot \operatorname{sen} \varphi_{i}^{\prime}\right)}+\frac{V_{12 i}}{-j \cdot V_{12 d} \cdot\left(B_{e}+\delta_{u} \cdot Y^{\prime} \cdot \operatorname{sen} \varphi_{i}^{\prime}\right)}= \\
& =\frac{j}{\left(B_{e}+\delta_{u} \cdot Y^{\prime} \cdot \operatorname{sen} \varphi_{i}^{\prime}\right)}+\frac{j \cdot \delta_{u}}{\left(B_{e}+\delta_{u} \cdot Y^{\prime} \cdot \operatorname{sen} \varphi_{i}^{\prime}\right)}=\bar{Z}_{12 d d}+\bar{Z}_{12 d i}
\end{aligned}
$$


En la ecuación (III.5), observamos que la $Z_{12 d}$, se halla formada por dos términos, el debido a la tensión directa $\mathrm{V}_{12 \mathrm{~d}} \mathrm{y}$ la corriente directa reactiva que hemos denominado $Z_{12 d d}$, y que caracteriza el fenómeno del desfase, y el otro término, $\mathrm{Z}_{12 \mathrm{di}}$ que surge como relación entre la componente inversa de la tensión $\mathrm{V}_{12 \mathrm{i}}$ y la corriente directa reactiva, que aparece debido al desequilibrio de tensiones.

El término $Z_{12 d d}$, vamos a proceder a descomponerlo de manera que podamos diferenciar el fenómeno del desfase debido a la presencia de elementos reactivos en el sistema, y el provocado por el desequilibrio de tensiones

$$
\frac{1}{\bar{Z}_{12 d d}}=\frac{1}{\frac{j}{\left(B_{e}+\delta_{u} \cdot Y^{\prime} \cdot \operatorname{sen} \varphi_{i}^{\prime}\right)}}=-j \cdot B_{e}-j \cdot\left(\delta_{u} \cdot Y^{\prime} \cdot \operatorname{sen} \varphi_{i}^{\prime}\right)
$$

y por lo tanto:

$$
\begin{aligned}
& \frac{1}{\bar{Z}_{12 d d}}=\frac{1}{\bar{Z}_{12 d}^{r}}+\frac{1}{\bar{Z}_{12 d}^{u}} \\
& \bar{Z}_{12 d}^{r}=\frac{1}{B_{e}} ; \quad \bar{Z}_{12 d}^{u}=\frac{1}{\left(\delta_{u} \cdot Y^{\prime} \cdot \operatorname{sen} \varphi_{i}^{\prime}\right)}
\end{aligned}
$$

En la ecuación (III.7) denominamos por $Z_{12 d}^{r}$ al término que es fruto única y exclusivamente de los elementos reactivos presentes en el sistema, y el otro término $Z_{12 d}^{u}$ que sólo surge si hay desequilibrios en el sistema de excitación.

Tenemos entonces: 


$$
\begin{aligned}
& \bar{Z}_{12 d}=\bar{Z}_{12 d d}+\bar{Z}_{12 d i} \\
& \bar{Z}_{23 d}=\bar{Z}_{12 d d}+a^{2} \cdot \bar{Z}_{12 d i}=\bar{Z}_{23 d d}+\bar{Z}_{23 d i} \\
& \bar{Z}_{31 d}=\bar{Z}_{12 d d}+a \cdot \bar{Z}_{12 d i}=\bar{Z}_{31 d d}+\bar{Z}_{31 d i}
\end{aligned}
$$

Las impedancias que caracterizan el fenómeno del desfase valen:

$$
\begin{aligned}
& \bar{Z}_{12 d}^{r}=\bar{Z}_{23 d}^{r}=\bar{Z}_{31 d}^{r}=\left[\frac{1}{B_{e}}\right] \\
& \bar{Z}_{12 d}^{u}=\bar{Z}_{23 d}^{u}=\bar{Z}_{31 d}^{u}=\left[\frac{1}{\delta_{u} \cdot Y^{\prime} \cdot \operatorname{sen} \varphi_{i}^{\prime}}\right]
\end{aligned}
$$

Estas reactancias las pasamos a representar en la siguiente red de desfase, figura III.3, para el caso de un sistema desequilibrado en tensiones.

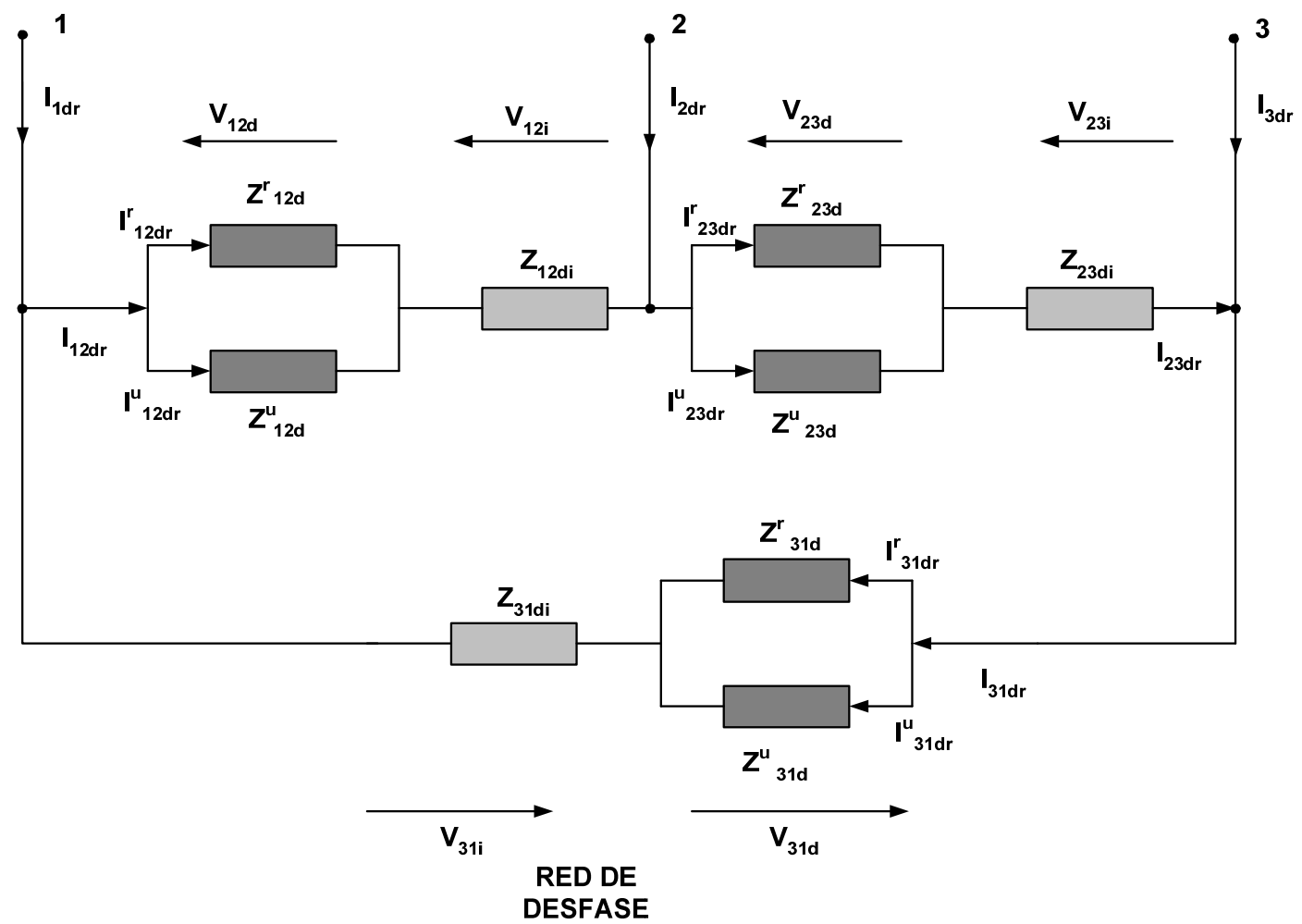

Figura III.3. Red de desfase. Sistema en triángulo y tensiones desequilibradas. 
En el circuito de la figura III.3, se ha procedido a descomponer la $\bar{Z}_{12 d d}$, en dos, para lo que se ha separado la intensidad de secuencia directa reactiva $\bar{I}_{12 d r}$ en dos intensidades en función de las tensiones que las producen: $\bar{I}_{12 d r}=\bar{I}_{12 d}^{r}+\bar{I}_{12 d}^{u}$, la debida a la tensión de secuencia directa, representada por

$\bar{I}_{12 d}^{r}$ y la provocada por la tensión de secuencia inversa, representada por $\bar{I}_{12 d}^{u}$. Esta última constituye, pues, el efecto del desequilibrio sobre la corriente reactiva de secuencia directa.

\section{2.3.- Caso particular de receptor puramente resistivo.}

\section{Sistema de excitación equilibrado y receptor desequilibrado.}

Vamos a analizar el caso de que tengamos un receptor lineal, desequilibrado y puramente resistivo, alimentado por un sistema de tensiones equilibrado y de secuencia directa, como ya pusimos de manifiesto a lo largo de este capítulo con las expresiones obtenidas, en este caso al ser el sistema de generación equilibrado y aún siendo desequilibrado el receptor, no se pone de manifiesto el fenómeno del desfase a no ser que el receptor sea reactivo, sea por lo tanto el siguiente circuito mostrado en la figura III.4.

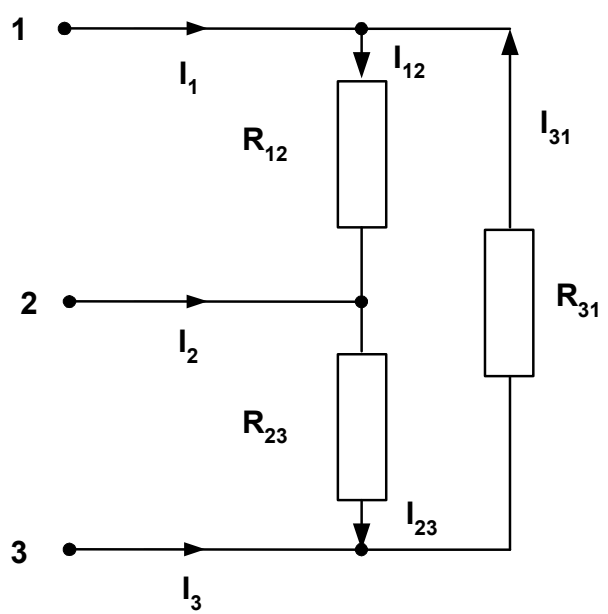

Figura III.4. Receptor resistivo desequilibrado y tensiones equilibradas. 
Haciendo uso de la ecuación (III.3), tenemos lo siguiente:

$$
\bar{Z}_{12 d d}=\bar{Z}_{23 d d}=\bar{Z}_{31 d d}=\frac{3}{\left(\bar{B}_{12}+\bar{B}_{23}+\bar{B}_{31}\right)}=\infty
$$

lo que evidencia que este caso no se produce el fenómeno del desfase.

\section{Sistema de excitación y receptor desequilibrado.}

Veamos ahora el caso de un sistema formado por un receptor desequilibrado lineal y puramente resistivo, pero ahora alimentado por un sistema de generación desequilibrado, por lo tanto el grado de desequilibrio es: $\bar{\delta}_{u}=\bar{V}_{12 i} /{\overline{V_{12 d}}} \neq 0$. Un circuito de este tipo se muestra en la figura III.5.

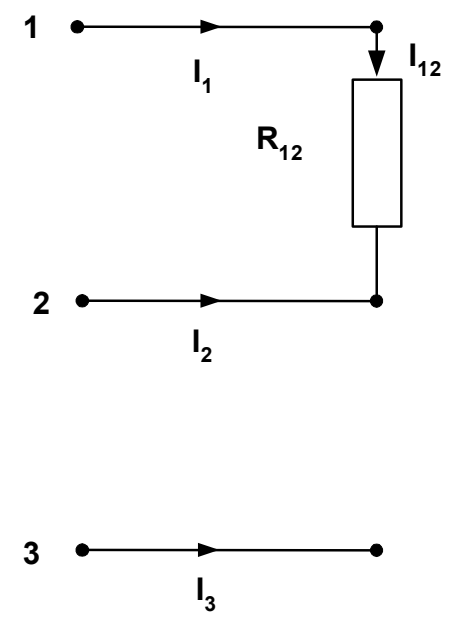

Figura III.5. Receptor resistivo desequilibrado y tensiones desequilibradas.

Es un sistema desequilibrado en tensiones y tal y como hemos visto, la red de desfase se halla formada por varios términos como se vio en la ecuación (III.7), en donde el término $\bar{Z}_{12 d}^{r}$ al igual que en el caso de tensiones equilibradas, su valor es infinito, lo que representa un circuito abierto, ya que no existen elementos reactivos en el sistema, pero ahora el término $\bar{Z}_{12 d}^{u}$ ya no es nulo, como pasamos a ver: 


$$
\begin{aligned}
& \bar{\delta}_{u}=\frac{\bar{V}_{12 i}}{\bar{V}_{12 d}} \neq 0, \overline{Y^{\prime}}=\frac{1}{3} \cdot G_{12} \neq 0 \\
& \operatorname{sen} \varphi_{i}^{\prime}=\operatorname{sen}\left(\alpha_{d}-\alpha_{i}\right) \neq 0 \quad \text { y } \quad \alpha^{\prime}=0 \\
& \bar{Z}_{12 d}^{u}=\frac{1}{\bar{\delta}_{u} \cdot \bar{Y}^{\prime} \cdot \operatorname{sen} \varphi_{i}^{\prime}} \neq \infty
\end{aligned}
$$

Lo que pone de manifiesto la presencia de desfase, provocado por un elemento puramente resistivo.

La red que representa por lo tanto el fenómeno de desfase será la que se muestra en la figura III. 6

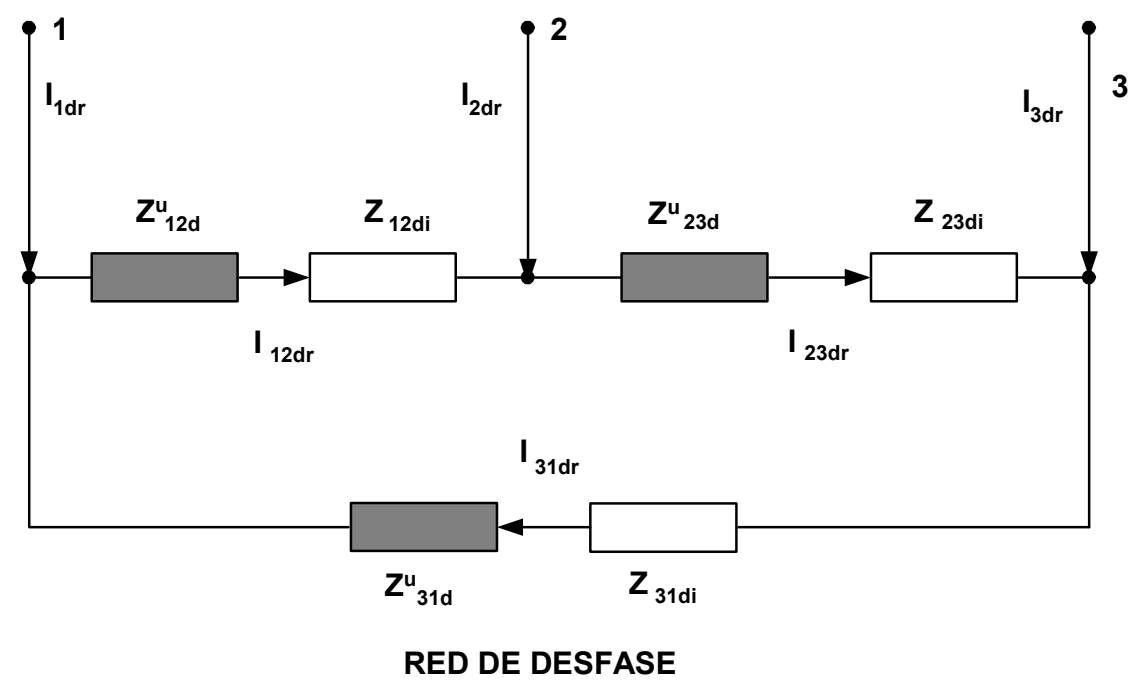

Figura III.6. Red de desfase del sistema desequilibrado.

Los valores que conforman esa red de desfase son los siguientes:

$$
\begin{aligned}
& \bar{Z}_{12 d}^{u}=\bar{Z}_{23 d}^{u}=\bar{Z}_{31 d}^{u}=\left[\frac{1}{\bar{\delta}_{u} \cdot \overline{Y^{\prime}} \cdot \operatorname{sen} \varphi_{i}^{\prime}}\right] \neq \infty \\
& \bar{Z}_{12 d i}=\bar{Z}_{23 d i}=\bar{Z}_{31 d i} \neq \infty
\end{aligned}
$$




\section{Sistema de excitación desequilibrado y receptor equilibrado.}

Se trata ahora el caso de un receptor equilibrado lineal y resistivo, alimentado por un sistema de excitación desequilibrado, tal como el mostrado en la figura III.7.

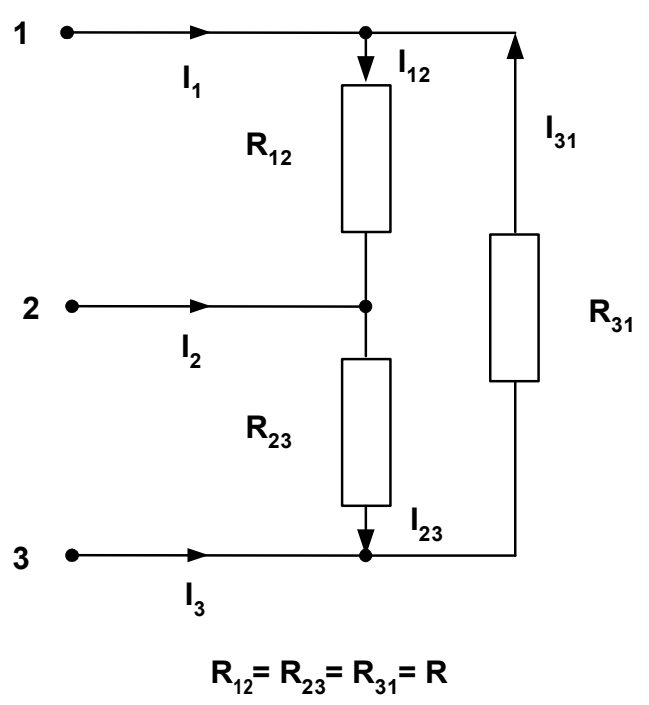

Figura III.7. Receptor resistivo equilibrado y tensiones desequilibradas.

Como sucedía en el caso anteriormente planteado, y haciendo uso de la ecuación (III.7), vemos que el término $\bar{Z}_{12 d}^{r}$ es infinito, siendo por lo tanto un circuito abierto, al no existir elementos reactivos, y se observa que el término $\bar{Z}_{12 d}^{u}$ es también un circuito abierto, debido ello a ser un receptor equilibrado aún siendo alimentado por un sistema desequilibrado de tensiones, no habiendo consumo alguno de reactiva por este sistema.

$$
\begin{aligned}
& \bar{\delta}_{u}=\frac{\overline{V_{12 i}}}{\bar{V}_{12 d}} \neq 0, G_{12}=G_{23}=G_{31}=G \\
& \overline{Y^{\prime}}=\frac{1}{3} \cdot\left(G_{12}+a^{2} \cdot G_{23}+a \cdot G_{31}\right)=\frac{1}{3} \cdot\left[G \cdot\left(1+a+a^{2}\right)\right]=0 \\
& \operatorname{sen} \varphi_{i}^{\prime}=\operatorname{sen}\left(\alpha_{d}-\alpha_{i}\right) \neq 0 \quad y \quad \alpha^{\prime}=0 \\
& \bar{Z}_{12 d}^{u}=\frac{1}{\bar{\delta} \cdot Y^{\prime} \cdot \operatorname{sen} \varphi_{i}^{\prime}}=\infty
\end{aligned}
$$




\section{3.- Representación del desfase en sistemas en estrella con neutro.}

\section{III.3.1.- Tensiones Equilibradas.}

Sea el circuito de la figura III.8. constituido por un receptor lineal y desequilibrado, alimentado por un sistema de excitación equilibrado de secuencia directa, en el Capítulo II, para este circuito habíamos obtenido la intensidad directa reactiva, que extraída de la ecuación (II.53) vale:

$$
i_{1 d r}(t)=V_{d} \cdot \sqrt{2} \cdot B_{e} \cdot \operatorname{sen}\left(w t+\alpha_{d}-90^{\circ}\right)
$$

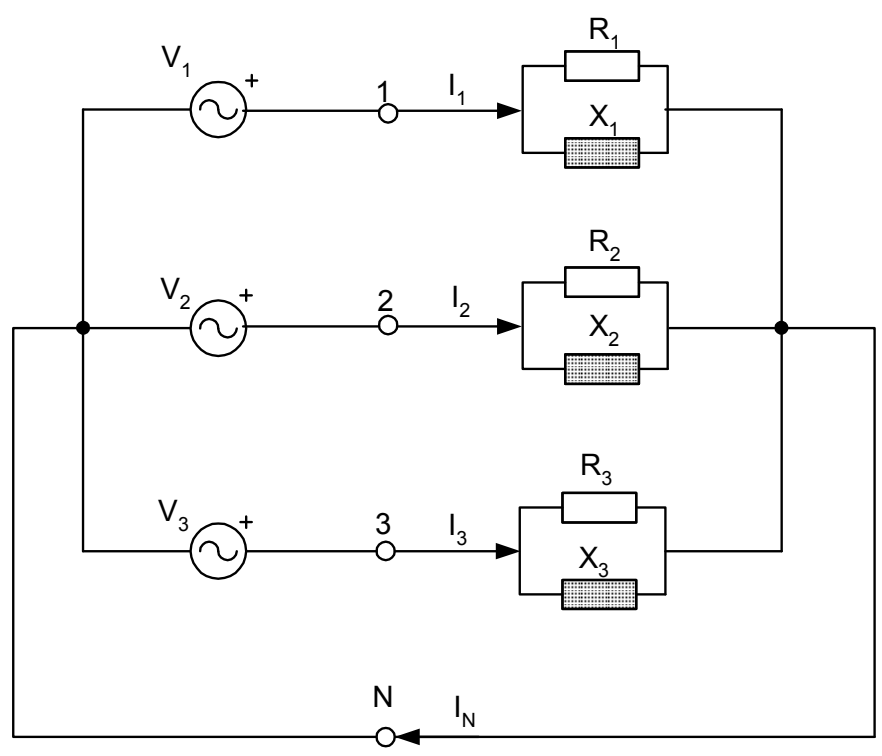

Figura III.8. Sistema con neutro. Tensiones equilibradas.

Ecuación que podemos poner en su forma fasorial como:

$$
\begin{aligned}
& \bar{I}_{1 d r}=\bar{V}_{1 d} \cdot \bar{B}_{e} \\
& \bar{B}_{e}=1 / 3 \cdot\left(\bar{B}_{1}+\bar{B}_{2}+\bar{B}_{3}\right)=1 / \bar{X}_{e}
\end{aligned}
$$


Como el sistema de alimentación es equilibrado, tan sólo existe componente directa de tensión $\mathrm{V}_{1 \mathrm{~d}}$, y por lo tanto las impedancias que representan el desfase, serán:

$$
\begin{aligned}
& \bar{Z}_{1 d d}=\frac{\bar{V}_{1 d}}{\bar{I}_{1 d r}}=\frac{\bar{V}_{1 d}}{\bar{V}_{1 d} \cdot \bar{B}_{e}}=\frac{1}{\bar{B}_{e}}=\bar{X}_{e} \\
& \bar{Z}_{2 d d}=\frac{\bar{V}_{2 d}}{\bar{I}_{2 d r}}=\frac{a^{2} \cdot \bar{V}_{1 d}}{a^{2} \cdot \bar{V}_{1 d} \cdot \bar{B}_{d}}=\frac{1}{\bar{B}_{e}}=\bar{X}_{e} \\
& \bar{Z}_{3 d d}=\frac{\bar{V}_{3 d}}{\bar{I}_{3 d r}}=\frac{a \cdot \bar{V}_{1 d}}{a \cdot \bar{V}_{1 d} \cdot \bar{B}_{d}}=\frac{1}{\bar{B}_{e}}=\bar{X}_{e}
\end{aligned}
$$

Al igual que sucedía en los sistemas en triángulo, el fenómeno del desfase se debe tan sólo a la presencia de elementos reactivos en el sistema, siendo la red de desfase para este caso la mostrada en la figura III.9

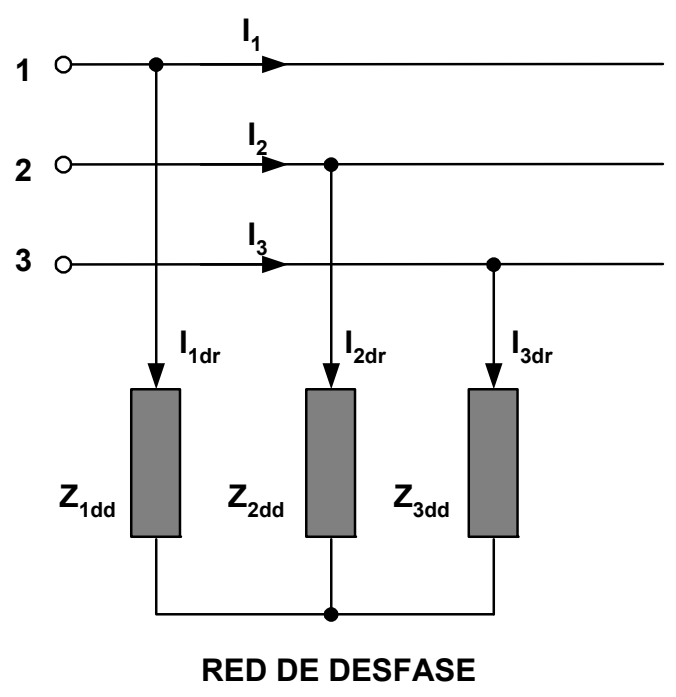

Figura III.9. Red de desfase.

Sistema con neutro y tensiones equilibradas. 


\section{III.3.2.- Tensiones Desequilibradas.}

Partiendo del mismo sistema mostrado en la figura III.8, ahora tenemos un sistema que se halla desequilibrado en tensiones, en el que generalmente habrá una componente directa de tensión $\mathrm{V}_{1 \mathrm{~d}}$, una componente inversa $\mathrm{V}_{1 \mathrm{i}}$, $\mathrm{y}$ una componente homopolar $V_{1 \mathrm{~h}}$, para la primera fase, $\mathrm{y}$ asimismo ocurre para las otras dos fases.

Nuevamente utilizamos la corriente directa reactiva para conocer el fenómeno del desfase. Tal como se estableció en el capítulo II, en estos circuitos la corriente de secuencia directa se expresaba según la ecuación (II.74), de donde se obtiene la componente reactiva como:

$$
\bar{I}_{1 d r}=V_{d \angle \alpha_{d}}\left[-j\left(B_{e}+\delta_{u} Y^{\prime} \operatorname{sen}\left(\varphi_{i}^{\prime}\right)+\delta_{A} Y^{\prime \prime} \operatorname{sen}\left(\varphi_{h}^{\prime \prime}\right)\right)\right]
$$

en la anterior expresión $\mathrm{V}_{\mathrm{d}}$, es la componente directa de la tensión, la $\mathrm{B}_{\mathrm{e}}$ es la susceptancia directa, siendo las $Y^{\prime}, Y^{\prime \prime}$ las admitancias básicas de desequilibrio, $\delta_{\mathrm{u}}$ representa el grado de desequilibrio del sistema, así como $\delta_{\mathrm{A}}$ nos indica el grado de asimetría del mismo, el ángulo $\varphi_{i}^{`}$ es función del ángulo de la componente directa de tensión, del de la componente inversa y de la propia carga, y el ángulo $\varphi_{\mathrm{h}}$ depende del ángulo de la componente directa y homopolar de tensión, así como el de la propia carga.

La impedancia que representa el desfase para la fase 1, vendrá dada por: 


$$
\begin{aligned}
& \bar{Z}_{1 d}=\frac{\bar{V}_{1}}{\bar{I}_{1 d r}}=\frac{\bar{V}_{1 d}+\bar{V}_{1 i}+\bar{V}_{1 h}}{\bar{I}_{1 d r}}=\frac{V_{1 d}}{-j \cdot V_{1 d} \cdot\left(B_{e}+\delta_{u} \cdot Y^{\prime} \cdot \operatorname{sen} \varphi_{i}^{\prime}+\delta_{A} \cdot Y^{\prime \prime} \cdot \operatorname{sen} \varphi_{h}^{\prime \prime}\right)}+ \\
& +\frac{V_{1 i}}{I_{1 d r}}+\frac{V_{1 h}}{I_{1 d r}}=\frac{j}{\left(B_{e}+\delta_{u} \cdot Y^{\prime} \cdot \operatorname{sen} \varphi_{i}^{\prime}+\delta_{A} \cdot Y^{\prime \prime} \cdot \operatorname{sen} \varphi_{h}^{\prime \prime}\right)}+\frac{\bar{V}_{1 i}}{\bar{I}_{1 d r}}+\frac{\bar{V}_{1 h}}{\bar{I}_{1 d r}}= \\
& =\bar{Z}_{1 d d}+\bar{Z}_{1 d i}+\bar{Z}_{1 d h}
\end{aligned}
$$

Al igual que sucedía en los sistemas a tres hilos, se observa que la impedancia $Z_{1 d}$, se halla formada por tres términos, el debido a la componente directa de tensión $\mathrm{V}_{1 \mathrm{~d}} \mathrm{y}$ la corriente reactiva directa, $\mathrm{y}$ denominado por $\mathrm{Z}_{1 \mathrm{dd}, \mathrm{Y}} \mathrm{Y}$ que representa el fenómeno del desfase, y los otros dos términos que surgen de la componente inversa de tensión $\mathrm{V}_{1 \mathrm{i}}$ y la corriente directa reactiva y el debido a la componente homopolar de tensión y la corriente directa reactiva, que denominamos respectivamente por $Z_{1 \mathrm{di}}$ y $Z_{1 \mathrm{dh}}$, $y$ que deben ser atribuidos al fenómeno del desequilibrio.

El término $Z_{1 d d}$, lo vamos a descomponer, para poder diferenciar el efecto del desfase provocado por la presencia de elementos reactivos en el sistema, y los debidos al desequilibrio de tensiones del sistema.

$$
\begin{aligned}
& \frac{1}{\bar{Z}_{1 d d}}=\frac{1}{\frac{j}{\left(B_{e}+\delta_{u} \cdot Y^{\prime} \cdot \operatorname{sen} \varphi_{i}^{\prime}+\delta_{A} \cdot Y^{\prime \prime} \cdot \operatorname{sen} \varphi_{h}^{\prime \prime}\right)}}= \\
& =-j \cdot B_{e}-j \cdot\left(\delta_{u} \cdot Y^{\prime} \cdot \operatorname{sen} \varphi_{i}^{\prime}\right)-j \cdot\left(\delta_{A} \cdot Y^{\prime \prime} \cdot \operatorname{sen} \varphi_{h}^{\prime \prime}\right)
\end{aligned}
$$

y por lo tanto: 


$$
\begin{aligned}
& \frac{1}{\bar{Z}_{1 d d}}=\frac{1}{\bar{Z}_{1 d}^{r}}+\frac{1}{\bar{Z}_{1 d}^{u 1}}+\frac{1}{Z_{1 d}^{u 2}} \\
& \bar{Z}_{1 d}^{r}=\frac{1}{B_{e}} ; \quad \bar{Z}_{1 d}^{u 1}=\frac{1}{\left(\delta_{u} \cdot Y^{\prime} \cdot \operatorname{sen} \varphi_{i}^{\prime}\right)} ; \quad \bar{Z}_{1 d}^{u 2}=\frac{1}{\left(\delta_{A} \cdot Y^{\prime \prime} \cdot \operatorname{sen} \varphi_{h}^{\prime \prime}\right)}
\end{aligned}
$$

En la ecuación (III.20) denominamos por $Z_{1 d}^{r}$ al término que es fruto única y exclusivamente de los elementos reactivos presentes en el sistema, y los otros términos $Z^{\mathrm{u} 1}{ }_{1 \mathrm{~d}} \mathrm{y}^{\mathrm{u} 2}{ }_{1 \mathrm{~d}}$ que sólo surgen si hay desequilibrios en las tensiones del sistema. (presencia de componentes inversa y homopolar de tensión).

Tenemos entonces:

$$
\begin{aligned}
& \bar{Z}_{1 d}=\bar{Z}_{1 d d}+\bar{Z}_{1 d i}+\bar{Z}_{1 d h} \\
& \bar{Z}_{2 d}=\bar{Z}_{1 d d}+a^{2} \cdot \bar{Z}_{1 d i}+a \cdot \bar{Z}_{1 d h}=\bar{Z}_{2 d d}+\bar{Z}_{2 d i}+\bar{Z}_{2 d h} \\
& \bar{Z}_{3 d}=\bar{Z}_{1 d d}+a \cdot \bar{Z}_{1 d i}+a^{2} \cdot \bar{Z}_{1 d h}=\bar{Z}_{3 d d}+\bar{Z}_{3 d i}+\bar{Z}_{3 d h}
\end{aligned}
$$

Las impedancias que caracterizan el fenómeno del desfase valen:

$$
\begin{aligned}
& \bar{Z}_{1 d}^{r}=\bar{Z}_{2 d}^{r}=\bar{Z}_{3 d}^{r}=\left[\frac{1}{B_{e}}\right] \\
& \bar{Z}_{1 d}^{u 1}=\bar{Z}_{2 d}^{u 1}=\bar{Z}_{3 d}^{u 1}=\left[\frac{1}{\delta_{u} \cdot Y^{\prime} \cdot \operatorname{sen} \varphi_{i}^{\prime}}\right] \\
& \bar{Z}_{1 d}^{u 2}=\bar{Z}_{2 d}^{u 2}=\bar{Z}_{3 d}^{u 2}=\left[\frac{1}{\delta_{A} \cdot Y^{\prime \prime} \cdot \operatorname{sen} \varphi_{h}^{\prime \prime}}\right]
\end{aligned}
$$

En la siguiente figura III.10, mostramos la red de desfase para este sistema desequilibrado en tensiones. 


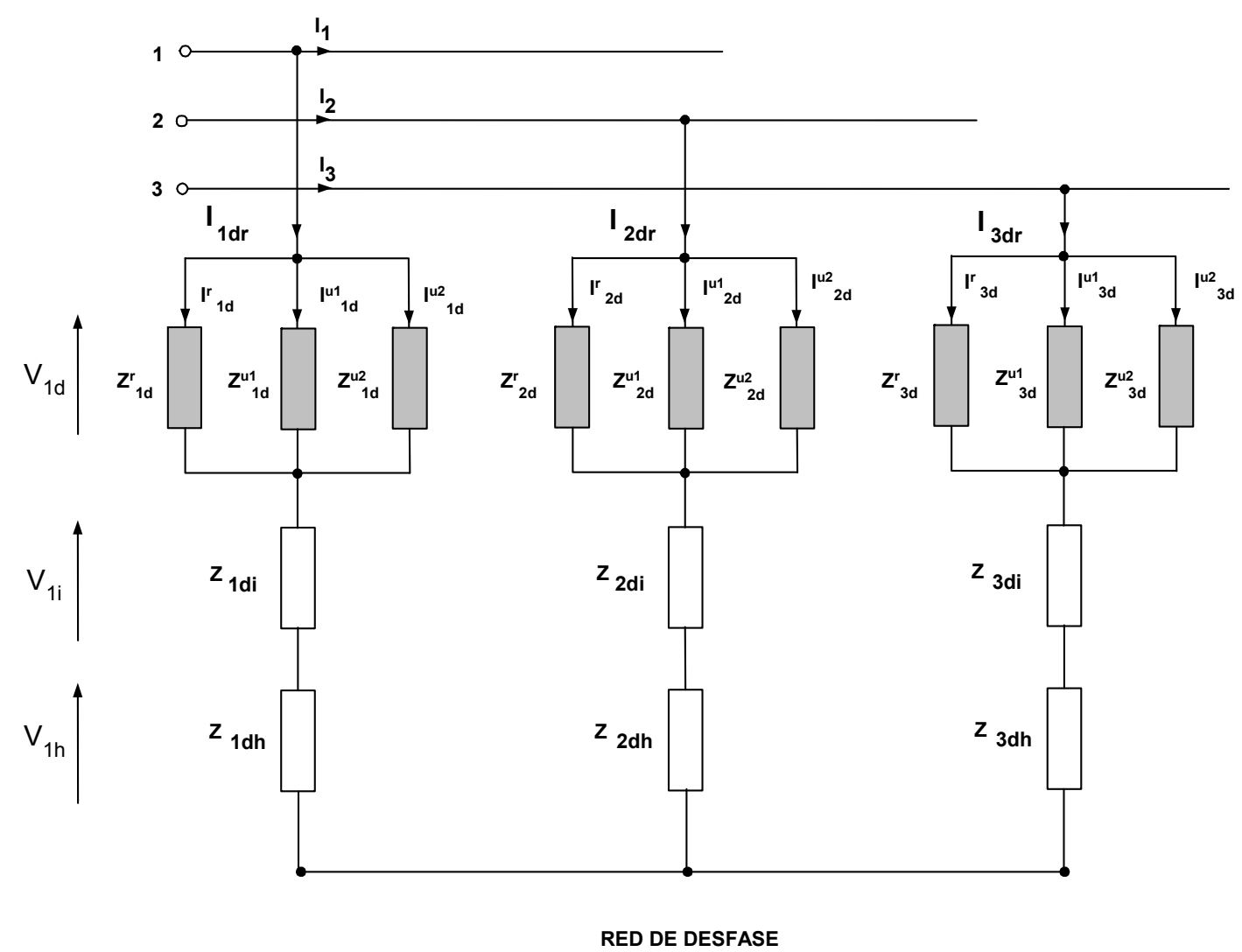

Figura III.10. Red de desfase. Sistema con neutro y tensiones desequilibradas.

En la red de la figura III.10, se ha descompuesto la impedancia $Z_{1 d d}$, en tres, separando para ello la intensidad directa reactiva $\mathrm{I}_{1 \mathrm{dr}}$, en función de las tensiones que las producen: $\bar{I}_{1 d r}=\bar{I}_{1 d}^{r}+\bar{I}_{1 d}^{u 1}+\bar{I}_{1 d}^{u 2}$, la debida a la tensión de secuencia directa, representada por $\bar{I}_{1 d}^{r}$ y la provocada por las tensiones de secuencia inversa, y homopolar representadas respectivamente por $\bar{I}_{1 d}^{u 1}$ y la $\bar{I}_{1 d}^{u 2}$. 


\section{3.3.- Caso particular de receptor puramente resistivo.}

\section{Sistema de excitación equilibrado y receptor desequilibrado.}

Al igual que se realizo para el caso de sistemas en triángulo, se va a analizar el siguiente circuito mostrado en la figura III.11, formado por un receptor desequilibrado lineal y alimentado por un sistema de excitación equilibrado. Como ya hemos visto, en este caso no se pone de manifiesto el fenómeno del desfase, al no existir elementos reactivos que conformen el receptor, y al ser un sistema equilibrado en tensiones.

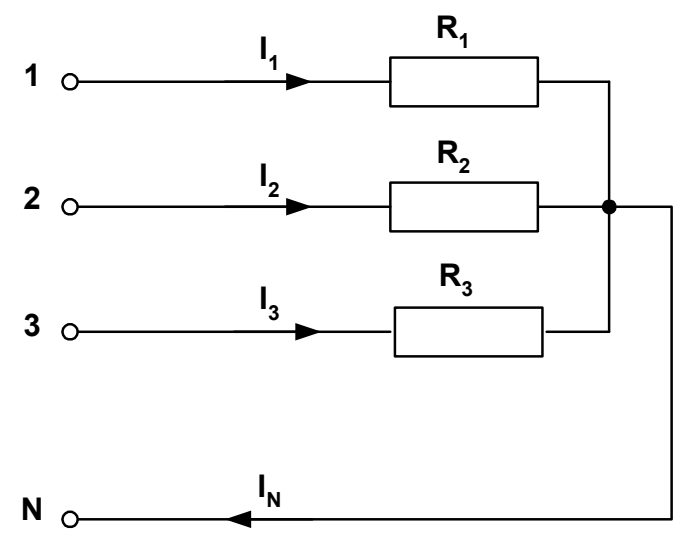

Figura III.11. Receptor resistivo desequilibrado y tensiones equilibradas.

Aplicando la ecuación (III.16), anteriormente desarrollada, tenemos:

$$
\bar{Z}_{1 d d}=\bar{Z}_{2 d d}=\bar{Z}_{3 d d}=\frac{3}{\left(\bar{B}_{1}+\bar{B}_{2}+\bar{B}_{3}\right)}=\infty
$$

por lo tanto en este circuito y en las condiciones de trabajo comentadas, no hay elementos que produzcan el fenómeno del desfase. 


\section{Sistema de excitación y receptor desequilibrado.}

Sea ahora el caso de un sistema formado por un receptor desequilibrado lineal y resistivo, pero alimentado por un sistema de tensiones desequilibrado, al ser un sistema en estrella con neutro, tenemos las siguientes componentes de tensión: secuencia directa, inversa y homopolar, y por lo tanto, los grados de desequilibrio y de asimetría de las tensiones no son nulos:

$$
\bar{\delta}_{u}=\bar{V}_{1 i} / \bar{V}_{1 d} \neq 0, \bar{\delta}_{A}=\bar{V}_{1 h} / \bar{V}_{1 d} \neq 0
$$

Se muestra un circuito de este tipo en la figura III.12.

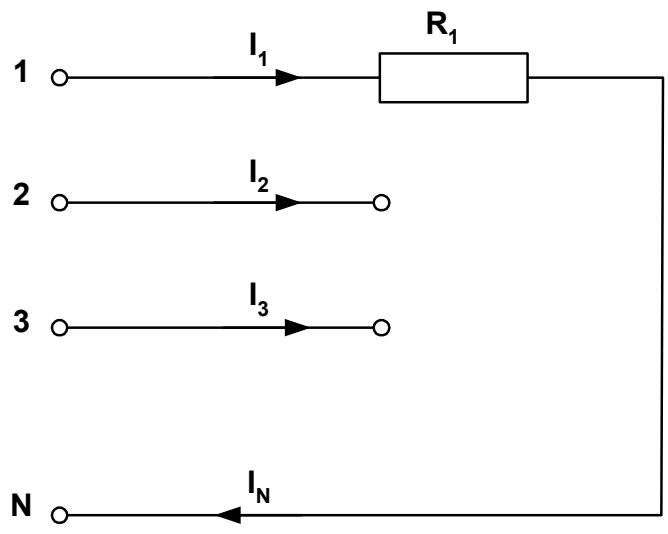

Figura III.12. Receptor desequilibrado resistivo bajo tensiones desequilibradas.

Para este caso, la red que caracteriza el desfase se halla formada por varios términos como se mostró en la ecuación (III.20) el término $\bar{Z}_{1 d}^{r}$ al no existir elementos reactivos, toma el valor de infinito, lo que representa un circuito abierto, no sucediendo así con los otros términos el $\bar{Z}_{1 d}^{u 1}$ y el $\bar{Z}_{1 d}^{u 2}$ que surgen de la existencia de desequilibrios de tensiones en el sistema, tal y como vemos a continuación: 


$$
\begin{aligned}
& \bar{\delta}_{u}=\frac{\bar{V}_{1 i}}{\bar{V}_{1 d}} \neq 0, \bar{\delta}_{A}=\frac{\bar{V}_{1 h}}{\bar{V}_{1 d}} \neq 0, \overline{Y^{\prime \prime}}=\overline{Y^{\prime}}=\frac{1}{3} \cdot G_{1} \neq 0 \\
& \operatorname{sen} \varphi_{i}^{\prime}=\operatorname{sen}\left(\alpha_{d}-\alpha_{i}\right) \neq 0 \quad y \quad \alpha^{\prime}=0 \\
& \operatorname{sen} \varphi_{h}^{\prime \prime}=\operatorname{sen}\left(\alpha_{d}-\alpha_{h}\right) \neq 0 \quad y \quad \alpha^{\prime \prime}=0 \\
& \bar{Z}_{1 d}^{u 1}=\frac{1}{\bar{\delta}_{u} \cdot \overline{Y^{\prime}} \cdot \operatorname{sen} \varphi_{i}^{\prime}} \neq \infty \\
& \bar{Z}_{1 d}^{u 2}=\frac{1}{\bar{\delta}_{A} \cdot \overline{Y^{\prime \prime}} \cdot \operatorname{sen} \varphi_{h}^{\prime \prime}} \neq \infty
\end{aligned}
$$

De la anterior expresión, observamos que se produce el fenómeno del desfase, aún estando con un sistema que se halla formado por tan sólo elementos resistivos.

La red que representa el desfase es la mostrada en la figura III.13.

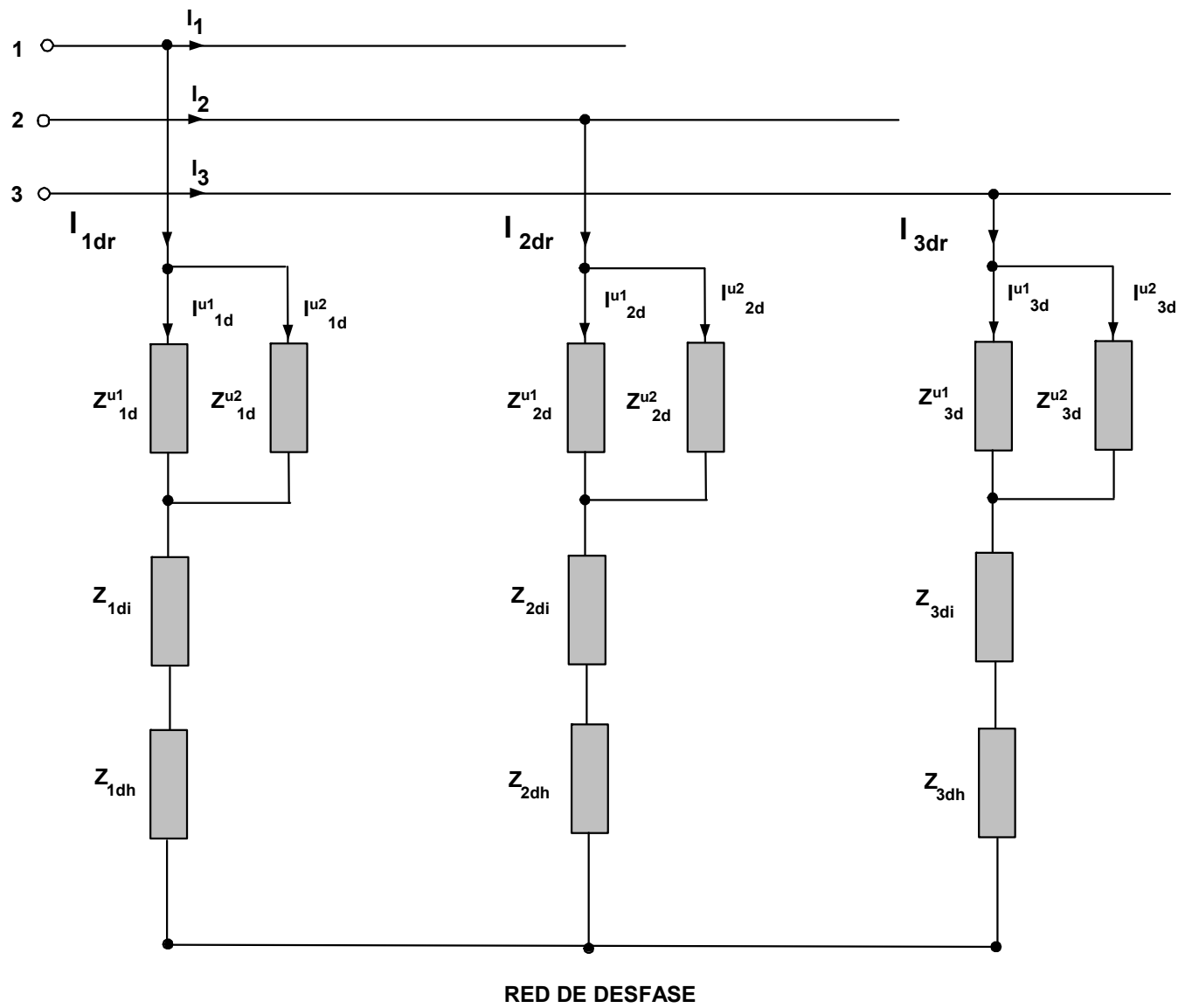

Figura III.13. Red desfase del sistema desequilibrado. 


\section{Sistema de excitación desequilibrado y receptor equilibrado.}

Veamos ahora el caso de un receptor equilibrado, lineal y resistivo, pero alimentado por un sistema de tensiones desequilibrado, como se muestra en la figura III.14.

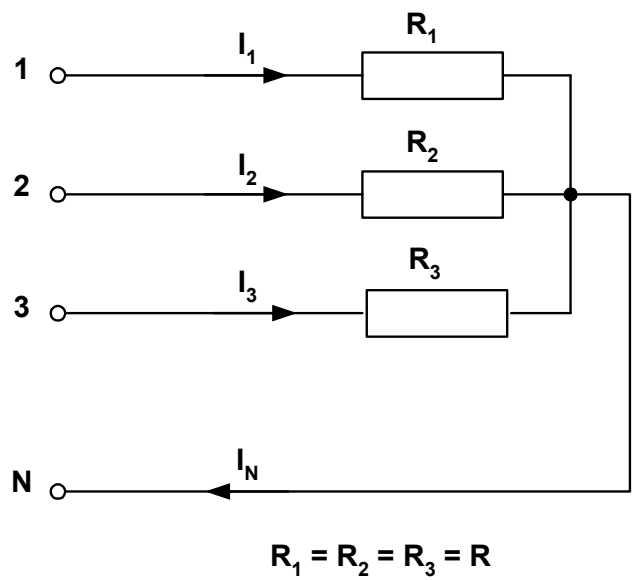

Figura III.14. Receptor resistivo equilibrado y tensiones desequilibradas.

De la ecuación (III.20) de la impedancia que representa el fenómeno del desfase, todos los términos, que la integran el $\bar{Z}_{1 d}^{r}$ y los $\bar{Z}_{1 d}^{u 1}$ y $\bar{Z}_{1 d}^{u 2}$ toman ahora el valor de infinito lo que hace que la red de desfase se comporte como si fuera un circuito abierto, no produciéndose ningún consumo de reactiva en este circuito.

$$
\begin{aligned}
& \bar{\delta}_{u}=\frac{\bar{V}_{1 i}}{\bar{V}_{1 d}} \neq 0, \quad \bar{\delta}_{A}=\frac{\bar{V}_{1 h}}{\bar{V}_{1 d}} \neq 0, \quad G_{1}=G_{2}=G_{3}=G \\
& \overline{Y^{\prime}}=\frac{1}{3} \cdot\left(G_{1}+a^{2} \cdot G_{2}+a \cdot G_{3}\right)=\frac{1}{3} \cdot\left[G \cdot\left(1+a+a^{2}\right)\right]=0 \\
& \overline{Y^{\prime \prime}}=\frac{1}{3} \cdot\left(G_{1}+a \cdot G_{2}+a^{2} \cdot G_{3}\right)=\frac{1}{3} \cdot\left[G \cdot\left(1+a+a^{2}\right)\right]=0 \\
& \operatorname{sen} \varphi_{i}^{\prime} \neq 0 \quad y \quad \operatorname{sen} \varphi_{h}^{\prime \prime} \neq 0, \quad \alpha^{\prime}=0, \alpha^{\prime \prime}=0 \\
& \bar{Z}_{1 d}^{u 1}=\frac{1}{\bar{\delta}_{u} \cdot \overline{Y^{\prime}} \cdot \operatorname{sen} \varphi_{i}^{\prime}}=\infty \\
& \bar{Z}_{1 d}^{u 2}=\frac{1}{\bar{\delta}_{A} \cdot \overline{Y^{\prime \prime}} \cdot \operatorname{sen} \varphi_{h}^{\prime \prime}}=\infty
\end{aligned}
$$




\section{4.- Conclusiones.}

A lo largo de este capítulo, y basándose en la Teoría Unificadora de la Potencia Eléctrica, se ha tratado y analizado la representación del fenómeno del desfase, en sistemas en triángulo y en estrella con neutro, partiendo en ambos casos de sistemas de alimentación equilibrados, para posteriormente trabajar con un sistema de alimentación desequilibrado.

Del análisis se ha comprobado que el fenómeno del desfase puede ser representado mediante conexiones de impedancias, denominadas redes de desfase, que absorben las corrientes reactivas de secuencia directa y frecuencia fundamental, y que dan lugar a las mismas potencias reactivas o de desfase instantáneas que el sistema real. Estas impedancias son reactancias positivas y negativas (no tienen parte resistiva, lógicamente), dependiendo del carácter inductivo o capacitivo del fenómeno. Asimismo, del análisis de las redes de desfase se ha constatado:

1) La existencia del fenómeno del desfase o reactivo causado por los desequilibrios, exclusivamente, que había sido sugerida (pero no analizada) recientemente por Jeon [104], aunque con posterioridad a nuestra publicación [114].

2) El fenómeno de desfase debido a los desequilibrios puede tener el mismo o diferente carácter (inductivo o capacitivo) que el fenómeno de desfase causado por las reactancias, incrementando o compensando en cada caso el efecto de estas últimas.

3) El fenómeno del desfase puede manifestarse incluso en circuitos resistivos, siempre que haya desfase entre las tensiones y corrientes de secuencia directa y frecuencia fundamental, de aquí que en esta tesis se hable de fenómenos de desfase como forma más apropiada de denominar a estos fenómenos que la tradicionalmente utilizada, que sólo alude al fenómeno causado por las reactancias. 
4) El fenómeno de desfase debido a los desequilibrios ocurre cuando hay desequilibrios de tensiones y cargas al mismo tiempo y siempre que no haya ningún tipo de simetría en el sistema. 



\section{CAPÍTULO IV}

DESCRIPCIÓN DEL DISPOSITIVO DE

MEDIDA. 



\section{IV.- Descripción del Dispositivo de Medida.}

\section{IV.1.- Introducción.}

En este capítulo, se muestran las características del dispositivo de medida desarrollado para realizar la comprobación experimental del fenómeno del desfase, puesto de manifiesto en el Capítulo II.

Tomando como base las expresiones de la potencia formuladas por la Teoría Unificadora de la Potencia Eléctrica, se han implementado estas usando para ello el programa LabVIEW, en un dispositivo de medida portátil [141].

El dispositivo de medida, consta esencialmente de dos partes: el hardware, dentro del que se encuentran los sistemas de medida y adquisición de las señales eléctricas de tensión e intensidad del sistema a analizar, y el software, que es el programa implementado para la medida de la potencia eléctrica.

El proceso de la medida se puede resumir básicamente en: adquisición de señales de tensión y corriente mediante unos sensores de efecto Hall, acondicionamiento de estas señales para su entrada en una tarjeta de adquisición de datos, y a partir de este momento, mediante el software LabVIEW, estas muestras de tensión y corriente se procesan usando la transformada rápida de Fourier (FFT), obteniéndose así una matriz de datos (módulos y fase) para cada frecuencia múltiplo de la fundamental, de esta matriz, se determinan las componentes simétricas de las tensiones e intensidades, que una vez elegida la topología del sistema y usando estas matrices obtenemos la cuantificación de los fenómenos energéticos formulados por la Teoría Unificadora. 
IV.2.- Constitución del sistema de medida. Hardware y software.

El sistema de medida SIMPELEC Desfase, puede esquematizarse como vemos en la figura IV.1

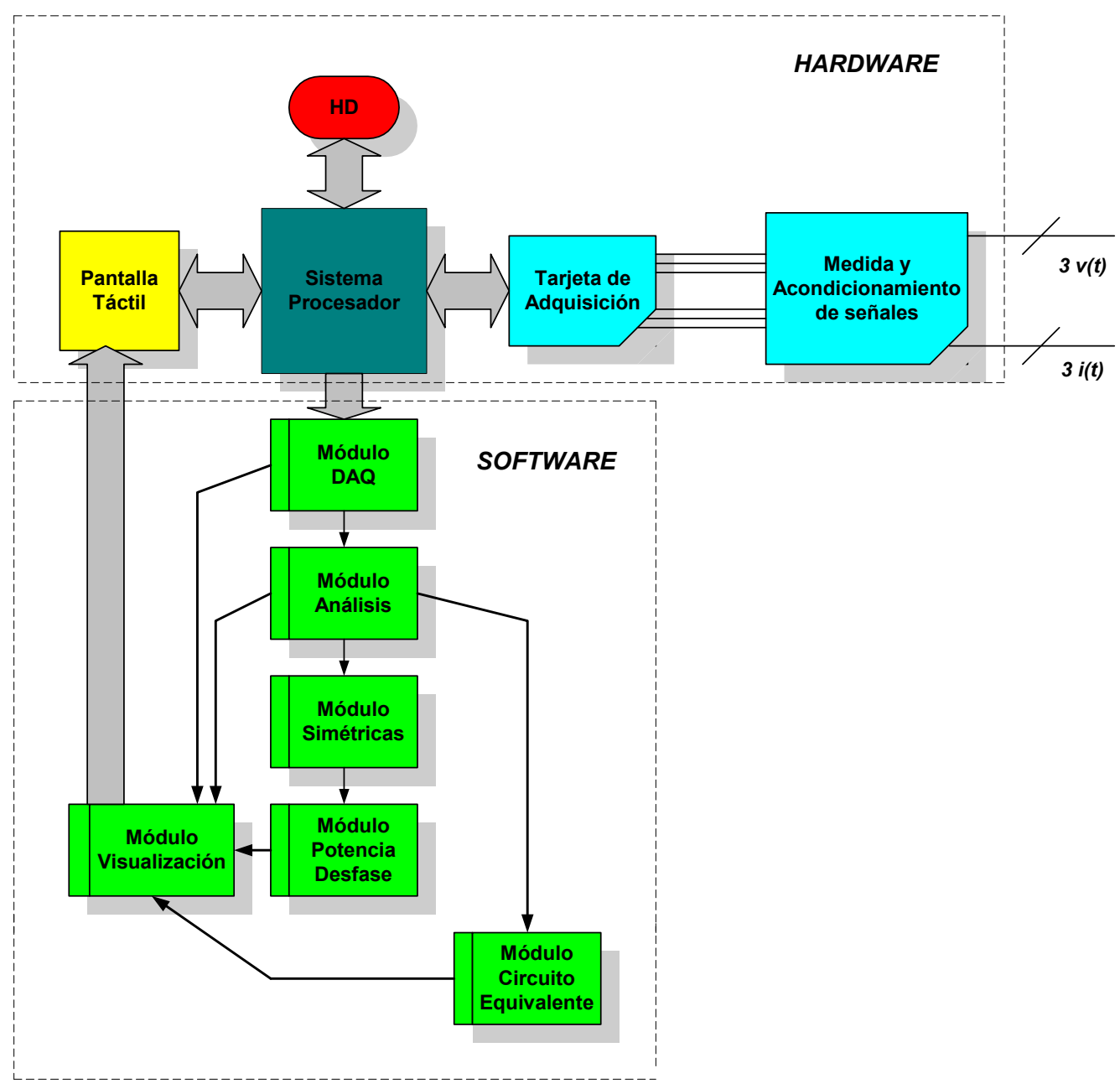

Figura IV.1. Esquema del equipo de medida.

En el esquema podemos ver los sistemas que componen el hardware y el software, y que seguidamente pasamos a comentar.

\section{IV.2.1- Hardware.}

- Características de las entradas.

Tensión: 4 canales hasta 660 V; nivel de aislamiento de 1,5 kV; precisión del $1 \%$. 
Corriente: 3 canales; medida directa hasta 25 A, y medida indirecta usando sondas desde $10 \mathrm{~A}$ hasta $10.000 \mathrm{~A}$; precisión del $1 \%$.

- Sensores de medida de tensión y corriente. Se han usado sensores de efecto Hall, que son los encargados de medir los valores de tensión y corriente instantáneos. Los valores máximos son de $5 \mathrm{~A}$ para los de intensidad y $660 \mathrm{~V}$ para los de tensión. Siendo la salida de los secundarios de estos sensores en una señal de corriente, proporcional a la de entrada con un valor máximo de $25 \mathrm{~mA}$.

- Acondicionamiento de la señal. Que nos permiten convertir las señales de corriente en señales de tensión en el rango entre $\pm 5 \mathrm{~V}$, que entran en las entradas analógicas de la tarjeta de adquisición.

- Tarjeta de adquisición. Se ha usado una tarjeta NI 6220 de National Instruments, compatible con LabVIEW, con 16 entradas analógicas simples, para montaje en bus PCI, con una resolución de 16 bits, una frecuencia de muestreo de $250 \mathrm{kS} / \mathrm{s}$, y una entrada máxima de tensión de $\pm 10 \mathrm{~V}$ y que es la encargada de convertir las señales analógicas de tensión y corriente, en una serie de muestras discretas, que procesa el programa de medida de la potencia eléctrica.

- Sistema procesador. Compuesto por una placa base PCM-9581 de la casa Advantech, equipada con un procesador Pentium M $1.4 \mathrm{GHz}$, con un máximo de 2 GB de RAM, y un slot PCI de $32 \mathrm{bit} / 33 \mathrm{MHz}$ en donde se inserta la tarjeta de adquisición. El sistema posee un disco duro HD de 100 Gb.

- Pantalla táctil LCD. Se ha usado una pantalla táctil de 8,4 pulgadas de diagonal, en la cual se visualiza la información de las diferentes magnitudes eléctricas así como las formas de onda de las mismas.

\section{IV.2.2- Software. Procedimiento de medida de los módulos de programa.}

El programa SIMPELEC Desfase, tiene implementado las expresiones que surgen de la Teoría Unificadora, usando para ello diferentes módulos de programa que 
nos permiten poder visualizar en la pantalla, las diferentes variables eléctricas muestreadas del sistema y procesadas por la tarjeta de adquisición.

La estructura del programa SIMPELEC Desfase, se halla formada por módulos de programa que hacen las funciones básicas de:

- Adquirir muestras de tensión e intensidad.

- Procesar digitalmente las señales adquiridas.

- Obtención de componentes simétricas.

- Obtener el circuito equivalente del circuito.

- Medida de la potencia de desfase.

A continuación se muestra en la figura IV.2, el procedimiento de medida usado.

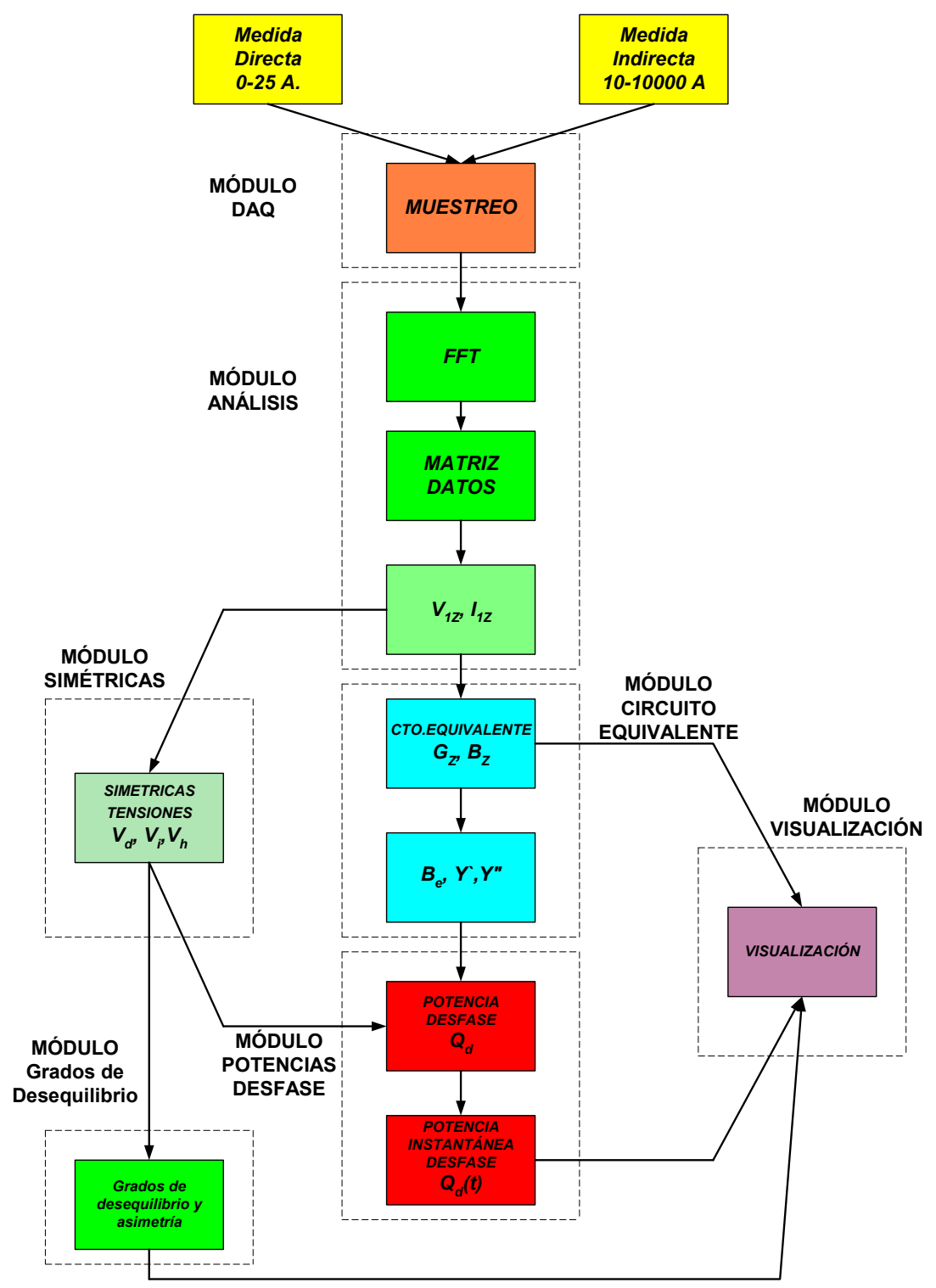

Figura IV.2. Procedimiento de medida. 
Módulo DAQ. Es el encargado de adquirir las muestras de señales de tensión e intensidad a partir del sistema de medida (hardware) a una frecuencia de muestreo determinada en 40,96 kHz/canal, con una resolución en frecuencia de $10 \mathrm{~Hz}$. Guardándose estas muestras en un vector de longitud el número de muestras total programadas.

Módulo análisis. Este módulo se halla formado por varios submódulos, el FFT, que realiza el procesado digital del vector de muestras de tensión e intensidad, que consiste en la aplicación de la transformada rápida de Fourier al vector de muestras obtenido en el módulo DAQ. A partir de la FFT se obtiene una Matriz de Datos formada por los valores eficaces (módulos) y fases para cada frecuencia múltiplo de $\Delta \mathrm{f}=10 \mathrm{~Hz}$ y mediante la detección posterior de los múltiplos de la frecuencia fundamental, se crea una matriz de módulos y fases para esas frecuencias, y de esta Matriz de Datos extraemos el valor eficaz a la frecuencia fundamental $(50 \mathrm{~Hz})$. Usando el Teorema de Parseval, que nos dice que la potencia media de una señal periódica es igual a la suma de los cuadrados de los módulos de los términos de Fourier, obtenemos las siguientes expresiones para el valor eficaz de cada armónico de orden k, para la tensión y la corriente:

$$
V_{n}=\sqrt{2} \cdot \frac{|V(n)|}{N} ; \quad I_{n}=\sqrt{2} \cdot \frac{|I(n)|}{N}
$$

Al ser la FFT una variable compleja, la fase inicial de cada armónico viene dada por:

$$
\alpha_{k}=\operatorname{arctg} \frac{\operatorname{Im}[X(k)]}{\operatorname{Re}[X(k)]}
$$

Y para la frecuencia fundamental tenemos:

$$
\begin{aligned}
& V_{1}=\sqrt{2} \cdot \frac{|V(1)|}{N} ; \quad I_{1}=\sqrt{2} \cdot \frac{|I(1)|}{N} \\
& \alpha_{1}=\operatorname{arctg} \frac{\operatorname{Im}[V(1)]}{\operatorname{Re}[V(1)]} ; \quad \overline{V_{1}}=V_{1} \angle \alpha_{1}
\end{aligned}
$$


Módulo de componentes simétricas. Este módulo es el encargado de obtener los vectores de los fasores de las componentes simétricas (directa, inversa y homopolar), en valor eficaz y fase, de las tensiones y de las corrientes para cada frecuencia múltiplo de la fundamental, partiendo de los valores obtenidos en el módulo anterior. Usando para ello el Teorema de Stokvis.

Módulo de grados de desequilibrio. En este módulo usando la información proporcionada por el módulo de componentes simétricas, se determinan los grados de desequilibrio $\bar{\delta}_{U}$ y de asimetría $\bar{\delta}_{A}$ de las tensiones, mediante las siguientes ecuaciones:

$$
\begin{gathered}
\bar{\delta}_{u}=\frac{\overline{V_{i}}}{\overline{V_{d}}} ; \\
\bar{\delta}_{A}=\frac{\overline{V_{h}}}{\overline{V_{d}}}
\end{gathered}
$$

Módulo de circuito equivalente. En este módulo se obtienen las conductancias y susceptancias por fase del circuito estudiado $\left(G_{z}, \bar{B}_{z} ; z=1,2,3\right)$, mediante la simple aplicación de la Ley de Ohm, una vez obtenidas las tensiones y corrientes: $\left(\bar{V}_{1}, \bar{V}_{2}, \bar{V}_{3} ; \bar{I}_{1}, \bar{I}_{2}, \bar{I}_{3}\right)$, del módulo de análisis. Posteriormente usamos las expresiones mostradas en el capítulo II para determinar la susceptancia equivalente $\left(\bar{B}_{e}\right)$ y las admitancias básicas de desequilibrio $\left(\overline{Y^{\prime}}, \overline{Y^{\prime \prime}}\right)$, y que seguidamente pasamos a recordar:

$$
\begin{aligned}
& \bar{B}_{e}=\frac{1}{3} \cdot\left(\bar{B}_{1}+\bar{B}_{2}+\bar{B}_{3}\right) \\
& \overline{Y^{\prime}}=\frac{1}{3} \cdot\left(\bar{Y}_{1}+a^{2} \bar{Y}_{2}+a \bar{Y}_{3}\right) ; \overline{Y^{\prime \prime}}=\frac{1}{3} \cdot\left(\bar{Y}_{1}+a \bar{Y}_{2}+a^{2} \bar{Y}_{3}\right)
\end{aligned}
$$


Módulo de potencia de desfase. Este módulo se halla compuesto por dos, el submodulo de potencia de desfase, en el cual se determinan las potencias de desfase en base a la Teoría Unificadora, discerniendo la que es debida a la presencia de reactancias en el sistema:

$$
Q_{r r}=3 \cdot B_{e} \cdot V_{d}^{2}
$$

y la debida a los desequilibrios

$$
Q_{r u}=3 \cdot\left(Y^{\prime} \cdot V_{d} \cdot V_{i} \cdot \operatorname{sen} \varphi_{i}^{\prime}+Y^{\prime \prime} \cdot V_{d} \cdot V_{h} \cdot \operatorname{sen} \varphi_{h}^{\prime \prime}\right)
$$

y en el otro submodulo se determinan los valores instantáneos de estas potencias, teniendo en cuenta que se hallan formadas por tres flujos sinusoidales y equilibrados, tal y como se había desarrollado en el capítulo II.

Módulo visualización. Gracias a este módulo podemos visualizar la información procedente del módulo del circuito equivalente, de manera que se observe los circuitos equivalentes de cada fase, así como del módulo de potencias de desfase podemos ver las formas de onda, de las potencias instantáneas. 


\section{IV.3.- Descripción de las funciones del dispositivo.}

A continuación se van a describir cada una de las funciones del dispositivo de medida SIMPELEC Desfase. El instrumento de medida, puede trabajar como un analizador de la calidad de la red eléctrica, trabajando bajo un entorno Windows, y comunicación con el usuario a través de una pantalla táctil, pero además puede trabajar como un Simulador, para lo que posee la capacidad de leer ficheros de datos procedentes del análisis de alguna instalación, y la opción de entrada de datos que le introduce el usuario y que le permite analizar casos con valores concretos.

\section{IV.3.1- Pantalla Principal.}

En la pantalla principal del dispositivo de medida, tenemos los botones que nos dan acceso a diversas subpantallas en cada una de las cuales se realizan diversas funciones y que se muestra en la figura IV.3.

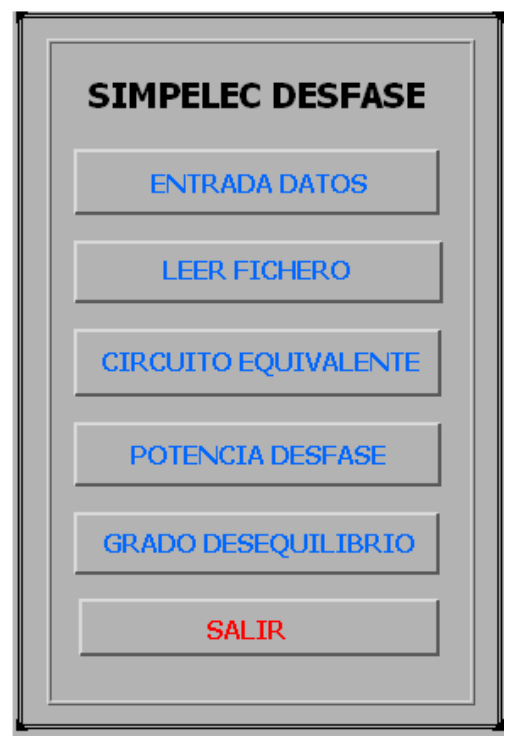

Figura IV.3. Pantalla principal. 


\section{IV.3.1.1- Pantalla Entrada de Datos.}

Gracias a esta pantalla, podemos introducir los datos de tensiones y corrientes (módulos y argumentos), en cada una de las fases, pudiendo así realizar simulaciones que nos permiten ver el comportamiento de una red bajo cualquier condición que se desee, esta pantalla la observamos en la figura IV.4.

\begin{tabular}{|c|c|c|c|c|c|c|c|c|}
\hline \multicolumn{9}{|c|}{ 氕Entrada de DATOS } \\
\hline \multicolumn{3}{|c|}{ Tensión Fase R } & \multicolumn{3}{|c|}{ Tensón Fase 5} & \multicolumn{3}{|c|}{ Tensión Fase T } \\
\hline (5) 0 & 0 & 0 & 7) 0 & 0 & 0 & (5) 0 & 0 & 0 \\
\hline (5) 0 & 0 & 0 & 5) 0 & 0 & 0 & 75) 0 & 0 & 0 \\
\hline \multicolumn{3}{|c|}{ Corriente Fase R } & \multicolumn{3}{|c|}{ Corriente Fase S } & \multicolumn{3}{|c|}{ Corriente Fase T } \\
\hline 5) 0 & 0 & 0 & (3) 0 & 0 & 0 & 7) 0 & 0 & 0 \\
\hline (5) 0 & 0 & 0 & 3) 0 & 0 & 0 & 5) 0 & 0 & 0 \\
\hline
\end{tabular}

Figura IV.4. Pantalla de entrada de datos.

\section{IV.3.1.2- Pantalla Leer Fichero.}

La opción de leer fichero, abre un cuadro de diálogo en donde se nos pide el fichero que se debe leer, tal y como podemos observar en la figura IV.5, el fichero comienza por Simpelec seguido de la fecha año-mes-día y de la hora en formato HH-MM-SS (por ejemplo Simpelec 2006-11-28-14-03-02), con una extensión .lv.

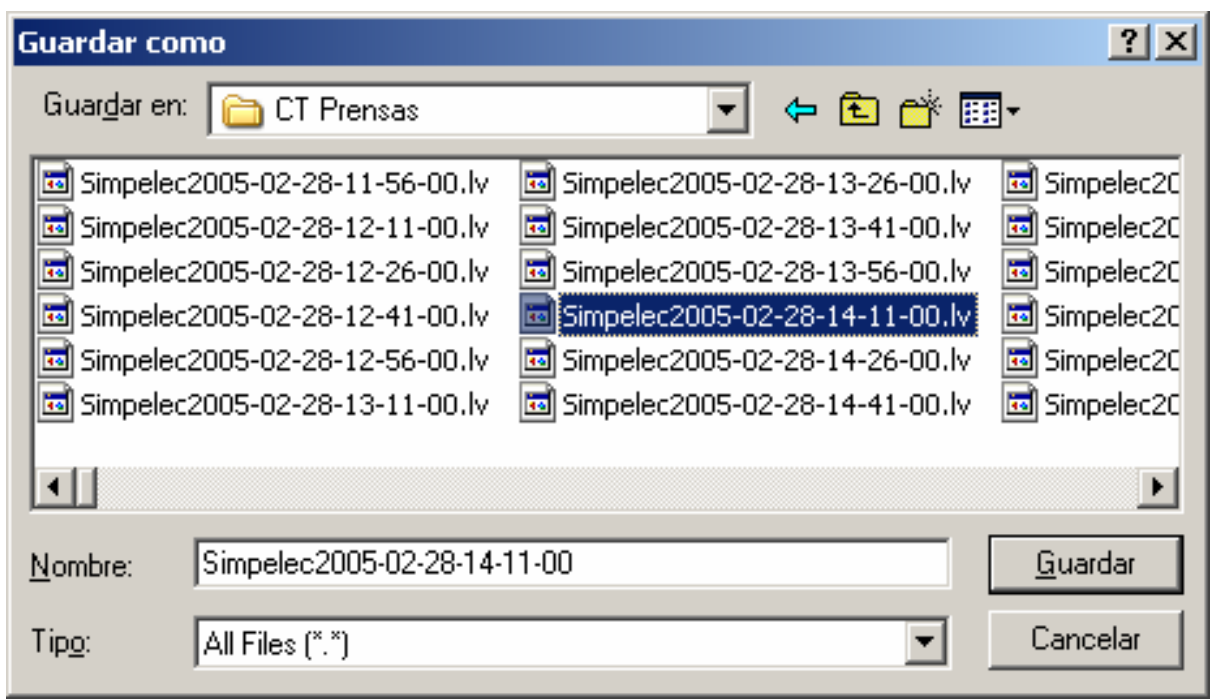

Figura IV.5. Pantalla de lectura de fichero. 


\section{IV.3.1.3- Pantalla Circuito Equivalente.}

En esta pantalla se nos presentan las conductancias y susceptancias equivalente para cada fase del sistema, como podemos observar en la figura IV.6.

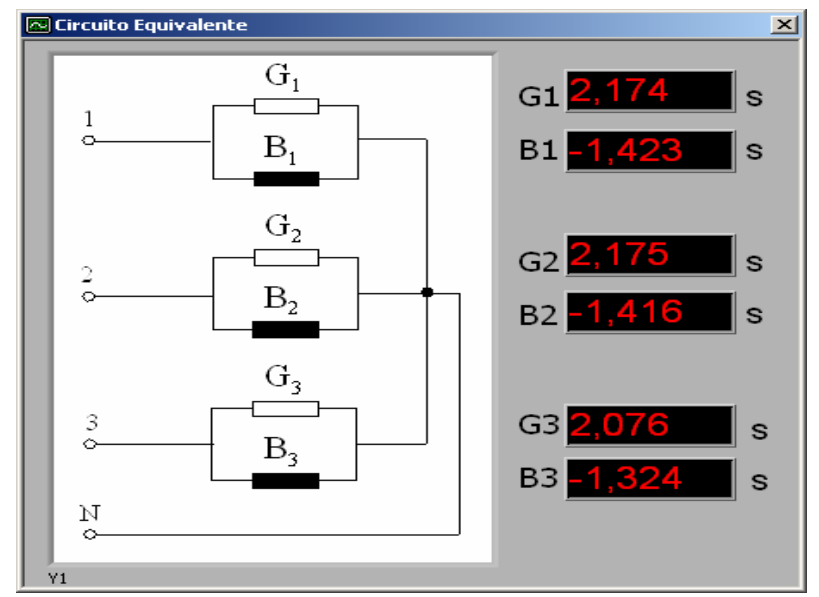

Figura IV.6. Pantalla del circuito equivalente.

\section{IV.3.1.4- Pantalla Potencia de Desfase.}

En esta pantalla, que vemos en la figura IV.7, visualizamos (con indicación de si es capacitiva mediante el flag "CAP") la potencia de desfase debida a la presencia de reactancias en el sistema $\mathrm{Q}_{\mathrm{rr}}(\mathrm{t})$, la procedente de los desequilibrios del mismo $\mathrm{Q}_{\mathrm{ru}}(\mathrm{t})$, y la potencia de desfase total $\mathrm{Q}_{\mathrm{r}}(\mathrm{t})$.

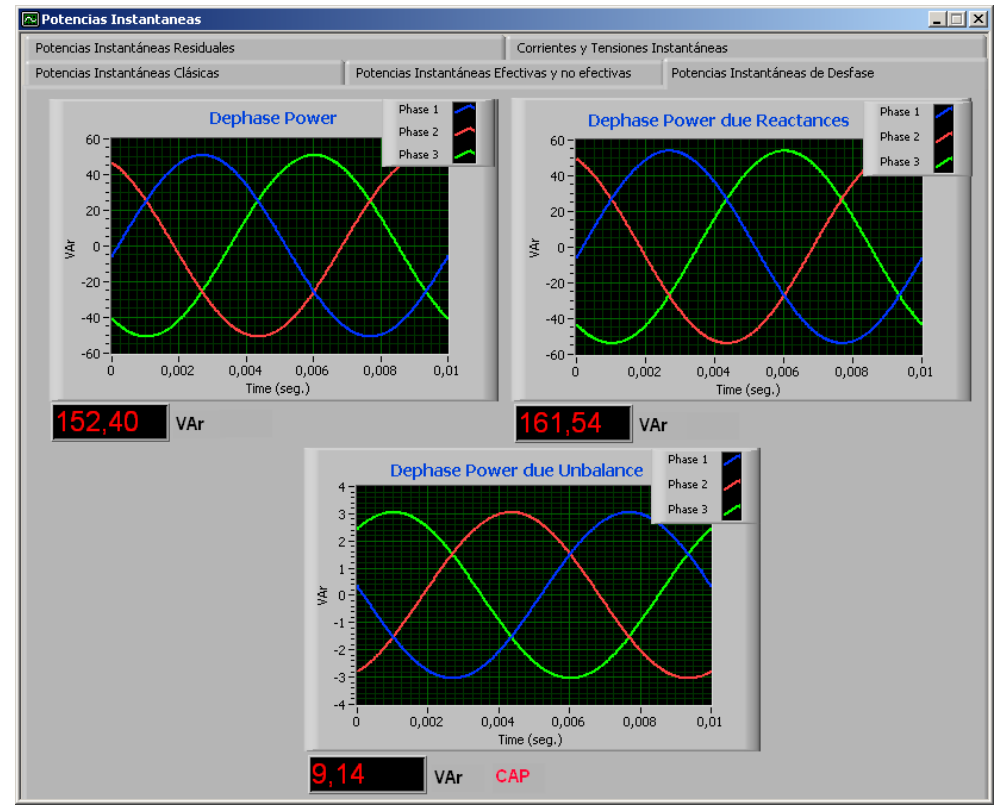

Figura IV.7. Pantalla de potencias de desfase. 


\section{IV.3.1.5- Pantalla Grados de Desequilibrio.}

A través de esta pantalla que se muestra en la figura IV. 8, podemos apreciar el grado de desequilibrio y de asimetría que posee el sistema que estemos estudiando.

\begin{tabular}{|c|c|c|c|}
\hline \multicolumn{4}{|c|}{ GRADOS DE DESEQUILIBRIO Y ASIMETRIAA } \\
\hline \multicolumn{2}{|c|}{ TENSIONES } & \multicolumn{2}{|c|}{ CORRIENTES } \\
\hline $\begin{array}{l}\text { Grado de } \\
\text { Desequilibrio }\end{array}$ & 5,09 & $\begin{array}{l}\text { Grado de } \\
\text { Desequilibrio }\end{array}$ & 11,03 \\
\hline Grado de Asimetría & 2,84 & Grado de Asimetría & 34,70 \\
\hline
\end{tabular}

Figura IV.8. Pantalla de grados de desequilibrio.

\section{IV.4.- Imágenes del Dispositivo de Medida.}

Se muestran a continuación una serie de imágenes del dispositivo de medida en diferentes vistas, que permiten al lector hacerse una idea del mismo.

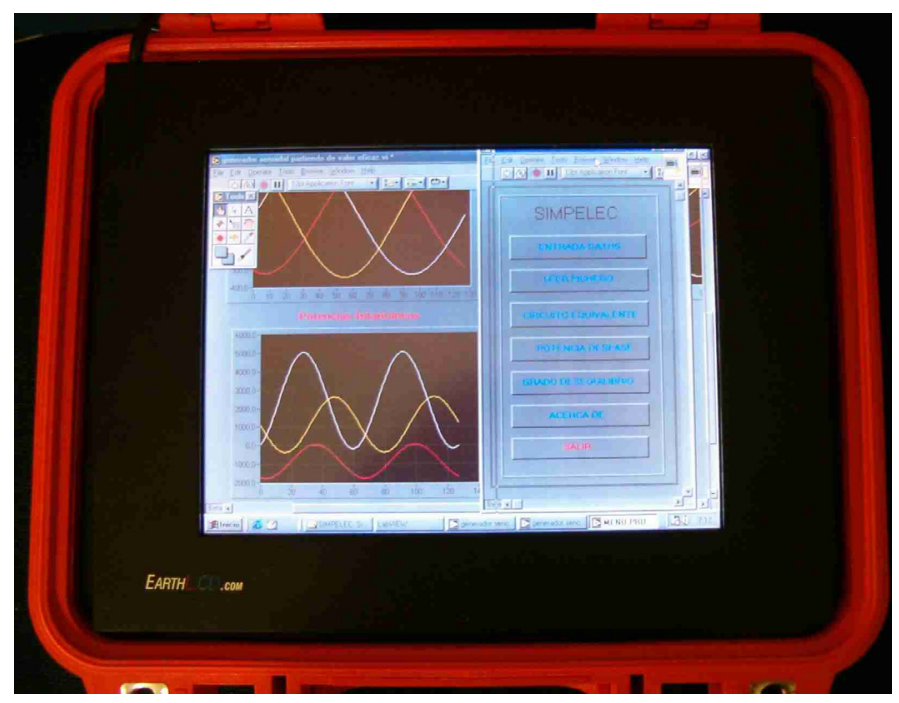

Figura IV.9. Vista Frontal del dispositivo de medida. 


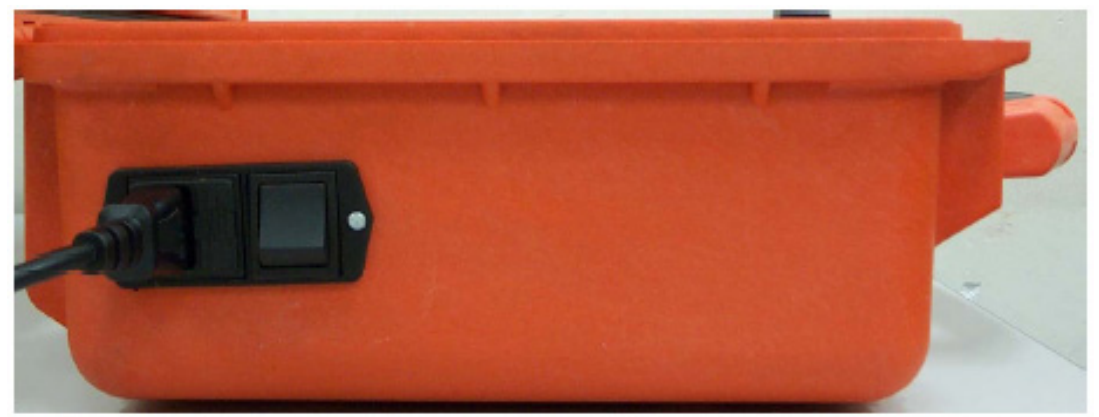

Figura IV.10. Vista lateral izquierda del dispositivo de medida.

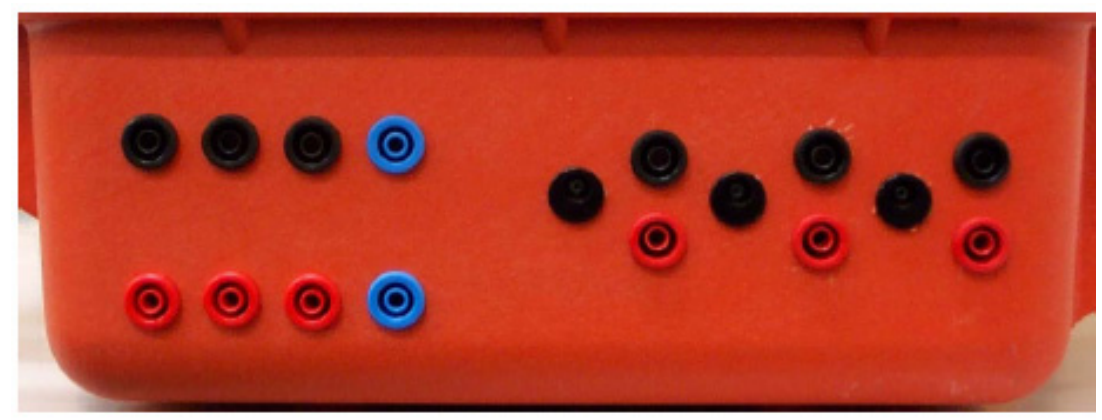

Figura IV.11. Vista lateral derecha del dispositivo de medida.

\section{IV.5.- Esquemas de Montaje.}

A la hora de realizar las medidas con el dispositivo, podemos usar dos diferentes esquemas de montaje en función de la corriente que se vaya a medir:

- Medida directa de hasta $25 \mathrm{~A}$.

- Medida indirecta a través de sonda amperimétrica para medidas de corriente desde 10 a $10.000 \mathrm{~A}$.

En la figura IV. 12 podemos observar el sinóptico incorporado en el propio dispositivo de medida, de manera que se le facilite al usuario del mismo su conexionado. 


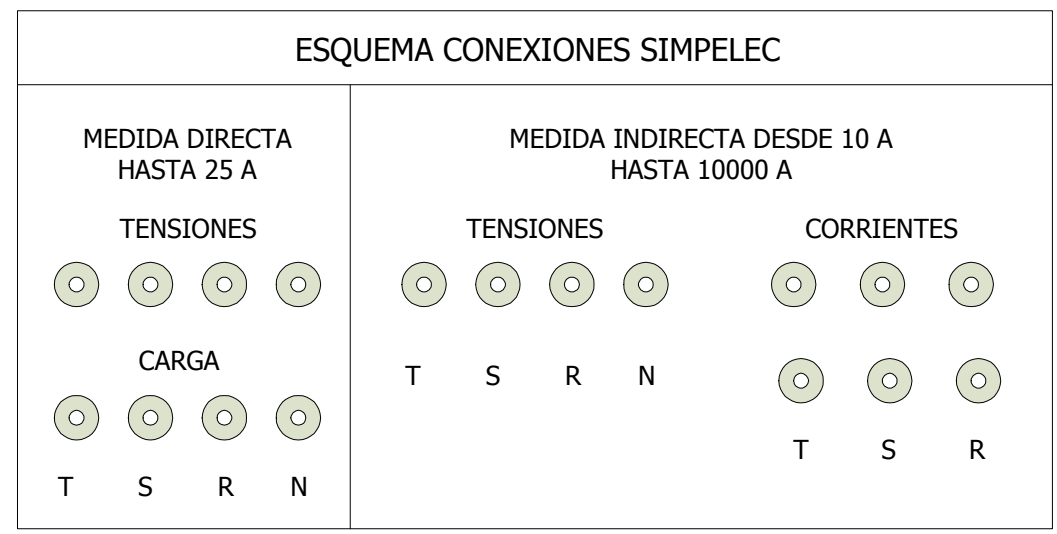

Figura IV.12. Esquemas de Conexión.

El uso de la medida directa es posible cuando se tiene acceso a los receptores de lo cuales se quiere efectuar la medida, siendo necesario desconectar la instalación mientras que con la medida indirecta podemos efectuar la medida sin tener que desconectar la instalación. La medida de las tensiones se efectúa mediante los cables suministrados de pinza y es común para los dos montajes como se observa en la figura IV.12.

\section{IV.5.1.- Medida directa de hasta 25 A.}

Es usado de forma exclusiva si es posible el acceder a desconectar a los receptores de la instalación. Se usa tal y como se puede observar en la figura IV.13 centrándonos en los bornes de la parte izquierda:

- Para las fases se han usado los bornes superiores, de color negro y azul para el neutro, de manera que el receptor queda conectado a las tensiones de alimentación.

- En los bornes inferiores, se ha usado el color rojo para las corrientes de cada fase y el azul para el neutro, conectándose a la carga a través de ellos. 
Se ha de tener en cuenta que al ser un esquema de montaje con un máximo admisible de corriente de $25 \mathrm{~A}$, se debe de conocer previamente a su conexión el consumo máximo de esos receptores a conectar, de manera que no se dañe el dispositivo.

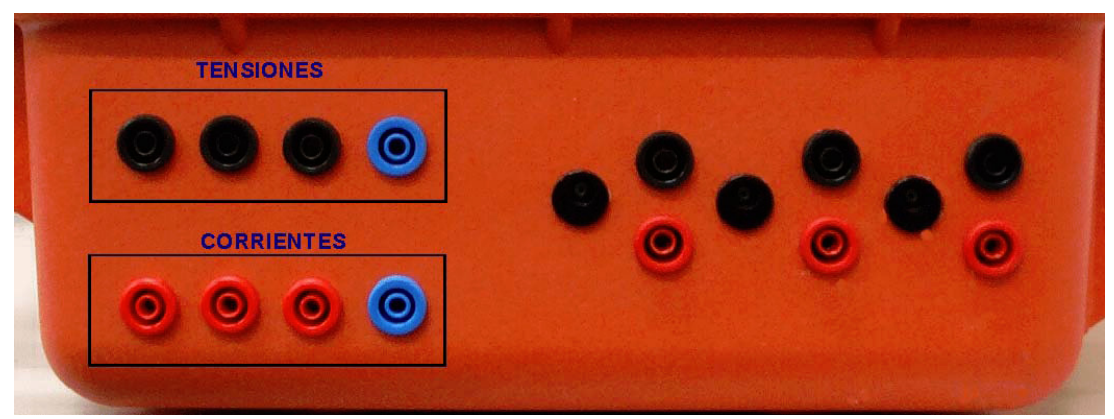

Figura IV.13. Conexionado de bornes para medida directa de hasta $25 \mathrm{~A}$.

\section{IV.5.2.- Medida indirecta hasta 10.000 A.}

Cuando no existe la posibilidad de poder desconectar a los receptores de la instalación, o la medida prevista supera los 25 A., se procederá a usar este esquema de medida y cuyo conexionado se muestra en la figura IV.14.

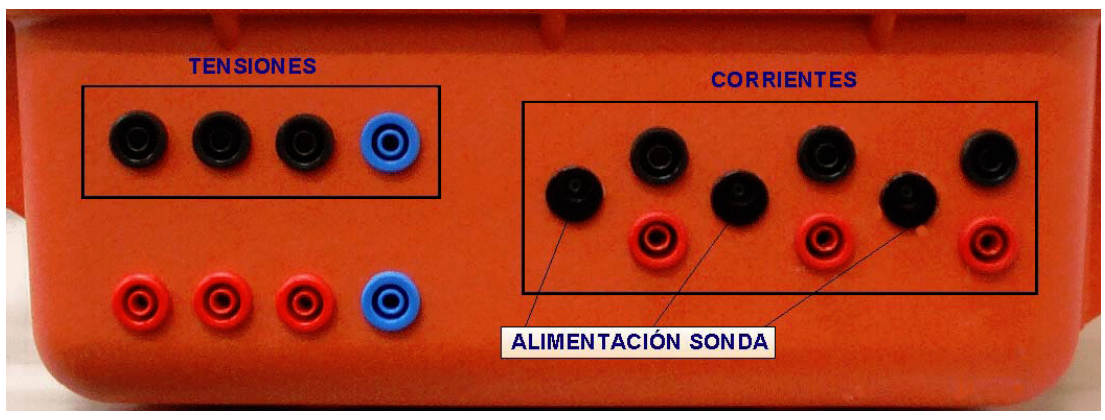

Figura IV.14. Conexionado de bornes para medida indirecta.

- Como se indico en el apartado de medida directa, los bornes de tensión son comunes para ambos esquemas de conexionada, y son por lo tanto los que se observan en la parte superior izquierda de la 
figura IV.14, siendo de color negro para las fases y azul para el neutro, quedando pues conectados los bornes a las tensiones de alimentación.

- Los bornes de la parte derecha de la figura IV.14 son usados para efectuar la conexión de las sondas amperimétricas, siendo el borne negro el común (o masa) y el rojo el de entrada de la señal, teniendo la precaución de hacer coincidir los colores de los bornes de la sonda con los del dispositivo de medida.

- El conector central tipo jack, de color negro y que se indica en la figura IV.14, permite la alimentación autónoma de las sondas del tipo AMPLEX (anillo Rogowski). 



\section{CAPÍTULO $v$}

\section{COMPROBACIÓN EXPERIMENTAL.}





\section{V.- Comprobación Experimental}

\section{V.1.- Introducción.}

En el Capítulo II, se han mostrado las expresiones que ponían de manifiesto el fenómeno del desfase, de manera que por primera vez dentro de la comunidad científica se da a conocer que la presencia del desfase puede ser provocado por los desequilibrios existentes en el sistema, además de los ya conocidos debidos a la presencia de elementos reactivos (bobinas y condensadores). A lo largo de este capítulo se realizará la comprobación experimental que las verifica.

En el proceso de la comprobación experimental, hemos llevado a cabo más de 30.000 medidas "in situ" efectuadas mediante el instrumento de medida SIMPELEC Desfase, y efectuadas en Centros de Transformación de instalaciones industriales y en Centros de Transformación residenciales, así como las efectuadas en las instalaciones que el GINTEC posee en el Instituto de Ingeniería Energética y las medidas virtuales de simulación llevadas a cabo mediante el programa SIMPELEC Simulador. 


\section{V.2.- Proceso Experimental.}

Como acabamos de comentar en el apartado anterior, vamos a realizar las medidas que nos permiten comprobar la presencia del fenómeno del desfase puesto de manifiesto en el Capítulo II.

A la hora de realizar estas medidas, hemos de indicar que se han realizado varios tipos de medidas:

- Medidas resultado de la simulación efectuada con el programa SIMPELEC Simulador.

- Medidas de campo efectuadas en centros industriales, polígonos residenciales y en el laboratorio, realizadas con el instrumento de medida SIMPELEC Desfase.

La serie de medidas efectuadas por medio de la simulación, se han realizado de manera que recoja la mayor cantidad de casos posibles, para ello se han utilizado receptores resistivos, inductivos y capacitivos, efectuando conexiones de estos en las diversas fases y el neutro del sistema, de manera que el sistema se halle cargado en una sola fase, en dos fases y en las tres fases. Para poder mostrar el fenómeno del desfase, como ya habíamos visto ha de existir desequilibrios de cargas y de tensiones, para lo que se han efectuado los pertinentes desequilibrios, como veremos en un apartado siguiente.

\section{V.3.- Medidas Experimentales Obtenidas con la Simulación. (Programa SIMPELEC Simulador).}

Los casos desarrollados a la hora de efectuar las medidas, se resumen a continuación: 
1. Una carga entre una fase y el neutro. Se conectara de manera sucesiva en cada fase del sistema una carga resistiva, inductiva y capacitiva, provocándose los pertinentes grados de desequilibrio en el sistema.

2. Cargas conectadas entre dos fases y el neutro, quedando una fase abierta. Se procede a conectar cargas de diverso carácter en dos de las fases del sistema, y alternando en estas conexiones los caracteres de las cargas, a la vez que provocamos desequilibrios en el sistema.

\section{V.3.1.- Carga monofásica entre fase y neutro.}

El proceso llevado a cabo se ha basado en partir de un sistema en el cual dejamos constantes los módulos de las tensiones, y se realizan variaciones de los argumentos de las fases en las que no existe carga, de manera que conseguimos ángulos crecientes y decrecientes, al estar tan sola una fase cargada, vamos produciendo secuencialmente variaciones en los argumentos de las otras fases, mediante la suma de ángulos positivos y negativos a los mismos.

a) Carga Resistiva: Se analiza el comportamiento de este elemento frente a diversos desequilibrios que se producen, estando esta carga de manera sucesiva en cada fase.

Carga resistiva entre la fase 1 y el neutro, con un consumo de $1 \angle 0^{\circ} \mathrm{A}, \mathrm{y}$ producimos el desequilibrio sumando ángulos positivos al argumento de la fase 2.

Introducimos gracias a la ventana de entrada de datos del SIMPELEC Simulador, las tensiones de las fases y el consumo, así como las sucesivas modificaciones en los argumentos de la fase 2 en este caso, tal y como mostramos en la figura V.1. 


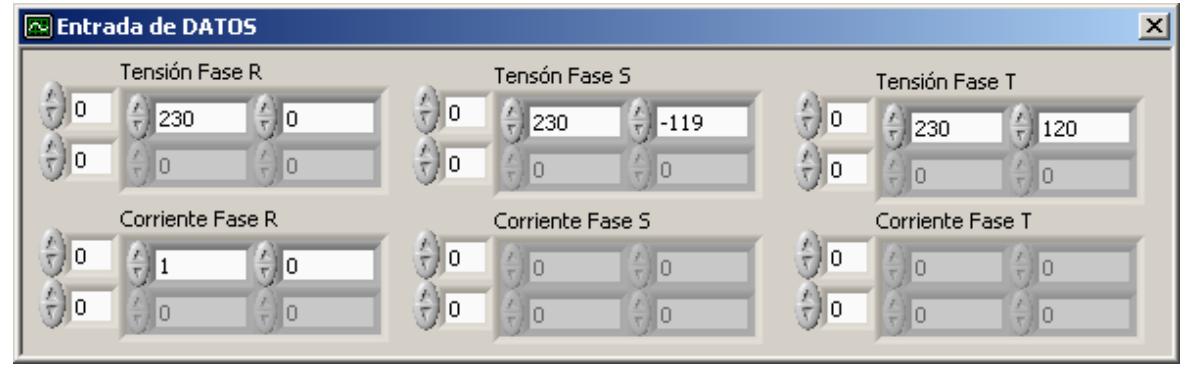

Figura V.1. Pantalla de entrada de datos.

Tenemos pues un sistema de tensiones en el que como ya hemos comentado de manera general dejamos fijos los módulos de las tensiones de las fases, y tenemos

una carga resistiva pura que consume $1 \angle 0^{\circ} \mathrm{A}$ conectada en la fase 1, y tan sólo variamos de manera gradual el argumento de la fase 2, logrando los pertinentes desequilibrios de tensión y produciendo la consiguiente potencia de desfase, tal y como podemos observar en la tabla V.1.

\begin{tabular}{||c|c|c||}
\hline $\begin{array}{c}\text { Argumento } \\
\text { Fase 2 }\end{array}$ & $\begin{array}{c}\text { Variación } \\
\text { Argumento } \mathbf{(}^{\mathbf{0}} \text { ) }\end{array}$ & $\begin{array}{c}\text { Potencia Desfase } \\
\text { Desequilibrio } \mathbf{Q}_{\text {dru }} \text { (VAr) }\end{array}$ \\
\hline$-119^{\circ}$ & 1 & 1,34 \\
\hline$-118^{\circ}$ & 2 & 2,68 \\
\hline$-115^{\circ}$ & 5 & 6,68 \\
\hline$-110^{\circ}$ & 10 & 13,31 \\
\hline$-105^{\circ}$ & 15 & 19,84 \\
\hline$-95^{\circ}$ & 25 & 32,40 \\
\hline$-85^{\circ}$ & 35 & 43,97 \\
\hline$-70^{\circ}$ & 50 & 58,73 \\
\hline$-50^{\circ}$ & 70 & 72,04 \\
\hline$-30^{\circ}$ & 90 & 76,67 \\
\hline$-20^{\circ}$ & 100 & 75,50 \\
\hline$-10^{\circ}$ & 110 & 72,04 \\
\hline $0^{\circ}$ & 120 & 66,40 \\
\hline
\end{tabular}

Tabla V.1. Potencia de Desfase \& VAriación Argumento.

Observando la tabla V.1, vemos que de manera general, al producirse el desequilibrio del sistema modificando gradualmente el argumento de la fase 2 yendo este desde $-120^{\circ}$ a $0^{\circ}$, la potencia de desfase que se pone de manifiesto en el sistema es inductiva, y es de resaltar que existe una total linealidad entre la variación de argumentos producida y la potencia de desfase, quedando patente 
de manera clara que la variación de un grado $\left(1^{\circ}\right)$, produce una potencia de desfase de 1,34 VAr.

Los datos anteriores para la primera variación en el argumento de la fase 2, generan información que podemos visualizar por medio de la figura V.2., esta pantalla nos muestra las potencias de desfase como se ve en la figura V.3, en la que podemos observar la potencia de desfase total, la potencia de desfase debida a las reactancias presentes en el sistema, y la potencia de desfase provocada por los desequilibrios, cuando la potencia de desfase es capacitiva, o alguna de sus componentes, se representa por medio del indicador "CAP", en la que corresponda.

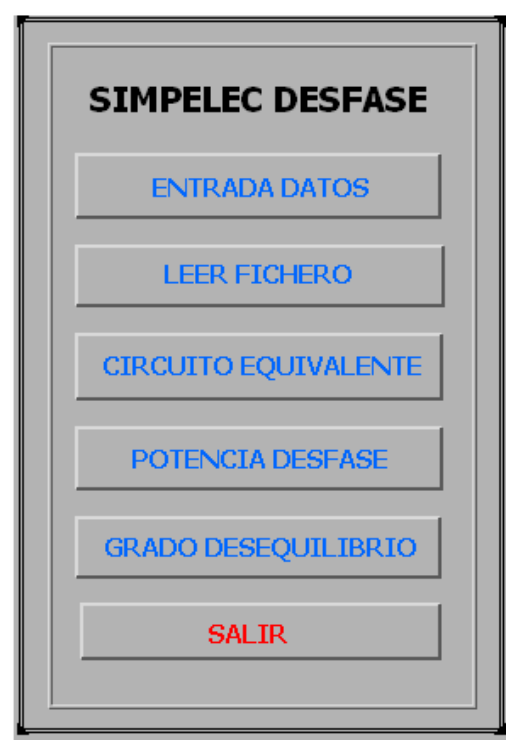

Figura V.2. Pantalla Principal. 


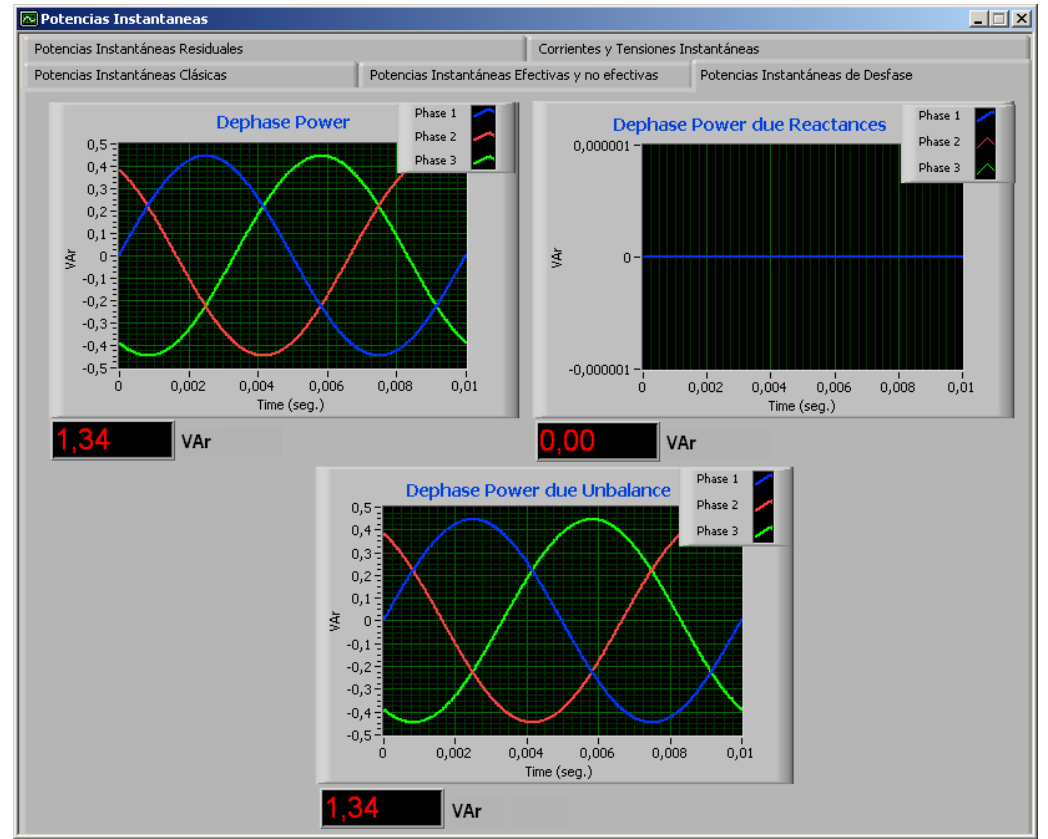

Figura V.3. Pantalla de Potencias de Desfase. 
Carga resistiva entre la fase 1 y el neutro, con un consumo de $1 \angle 0^{\circ} \mathrm{A}, \mathrm{y}$ producimos el desequilibrio sumando ángulos negativos al argumento de la fase 2.

Introducimos los datos en el SIMPELEC Simulador, como se muestra en la figura V.4.

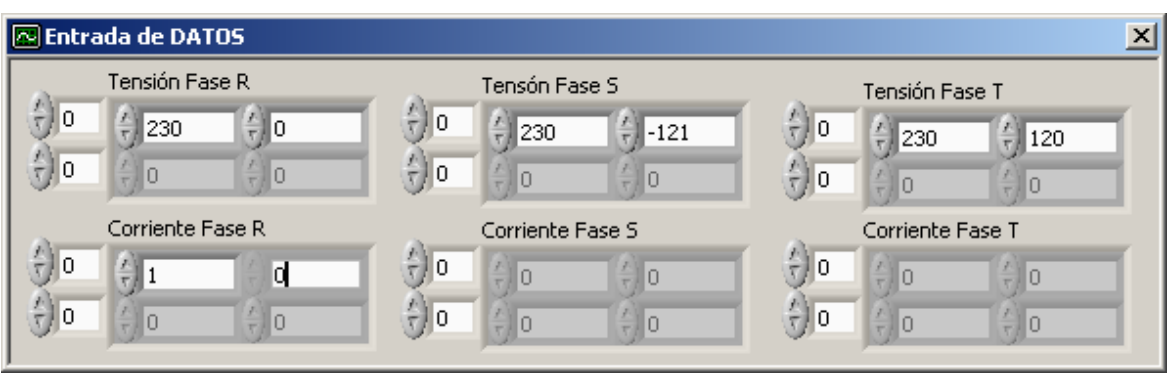

Figura V.4. Pantalla de entrada de datos.

Partimos de un sistema de tensiones en el que dejamos fijos los módulos de las fases, y tenemos una carga resistiva pura que consume $1 \angle 0^{\circ} \mathrm{A}$ conectada en la fase 1 , y tan sólo variamos de manera gradual el argumento de la fase 2, logrando los pertinentes desequilibrios de tensión y produciendo la consiguiente potencia de desfase, tal y como podemos observar en la tabla V.2, y en la figura V.5 para el caso concreto de la variación de un grado, siendo el argumento resultante de $-121^{\circ}$.

\begin{tabular}{||c|c|c||}
\hline $\begin{array}{c}\text { Argumento } \\
\text { Fase 2 }\end{array}$ & $\begin{array}{c}\text { Variación } \\
\text { Argumento }^{\left({ }^{\circ}\right)}\end{array}$ & $\begin{array}{c}\text { Potencia Desfase } \\
\text { Desequilibrio } \mathbf{Q d r u}_{\text {(VAr) }}\end{array}$ \\
\hline$-121^{\circ}$ & 1 & $-1,34$ \\
\hline$-122^{\circ}$ & 2 & $-2,68$ \\
\hline$-125^{\circ}$ & 5 & $-6,68$ \\
\hline$-130^{\circ}$ & 10 & $-13,31$ \\
\hline$-135^{\circ}$ & 15 & $-19,84$ \\
\hline$-145^{\circ}$ & 25 & $-32,40$ \\
\hline$-155^{\circ}$ & 35 & $-43,97$ \\
\hline$-170^{\circ}$ & 50 & $-58,73$ \\
\hline$-190^{\circ}$ & 70 & $-72,04$ \\
\hline$-210^{\circ}$ & 90 & $-76,67$ \\
\hline$-220^{\circ}$ & 100 & $-75,50$ \\
\hline$-230^{\circ}$ & 110 & $-72,04$ \\
\hline$-240^{\circ}$ & 120 & $-66,40$ \\
\hline
\end{tabular}

Tabla V.2. Potencia de Desfase \& Variación Argumento. 
De la observación de la tabla V.2, vemos que al modificar gradualmente el argumento de la fase 2, variando desde $-120^{\circ}$ a $-240^{\circ}$, sigue existiendo linealidad entre los incrementos de grados producidos y la potencia de desfase obtenida, se ha de resaltar en este caso que ahora la potencia de desfase pasa a tener carácter capacitivo, manteniéndose no obstante el ratio de que 1,34 VAr de potencia de desfase son obtenidos con la variación de un grado $\left(1^{\circ}\right)$.

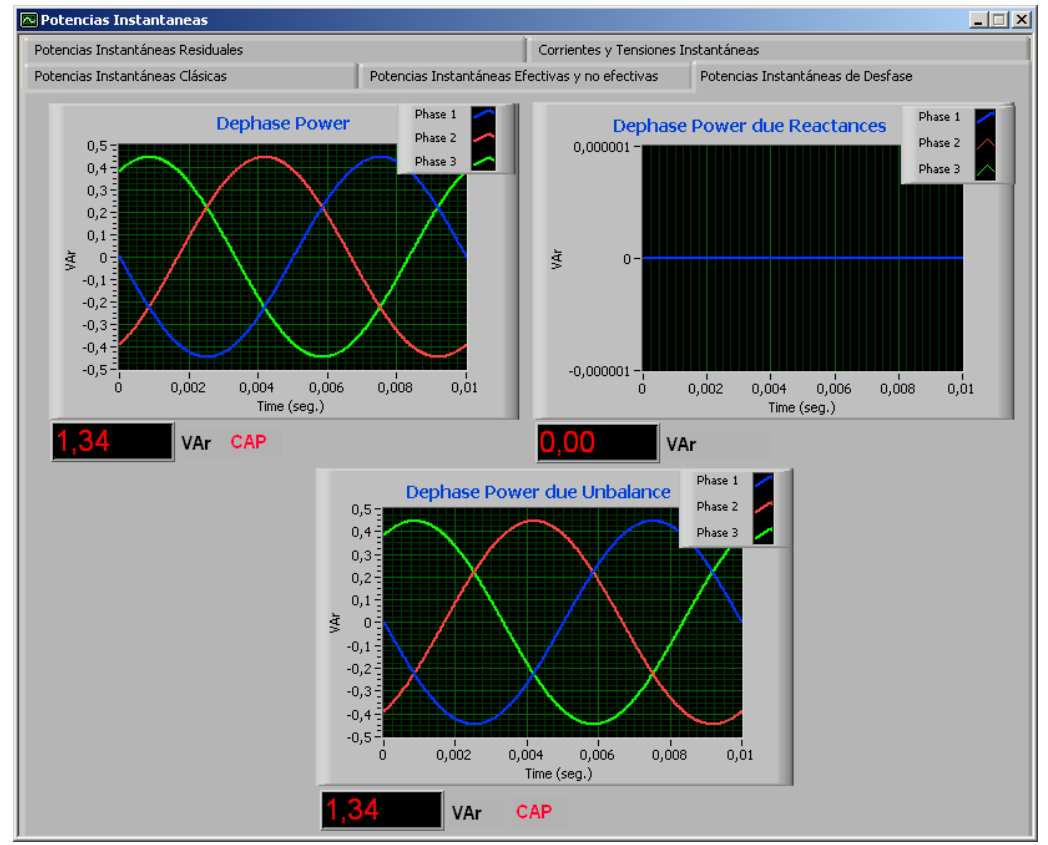

Figura V.5. Pantalla de Potencias de Desfase. 
Carga resistiva entre la fase 1 y el neutro, con un consumo de $1 \angle 0^{\circ} \mathrm{A}, \mathrm{y}$ producimos el desequilibrio sumando ángulos positivos al argumento de la fase 3.

Introducimos los datos en el SIMPELEC Simulador, como mostramos en la figura V.6,

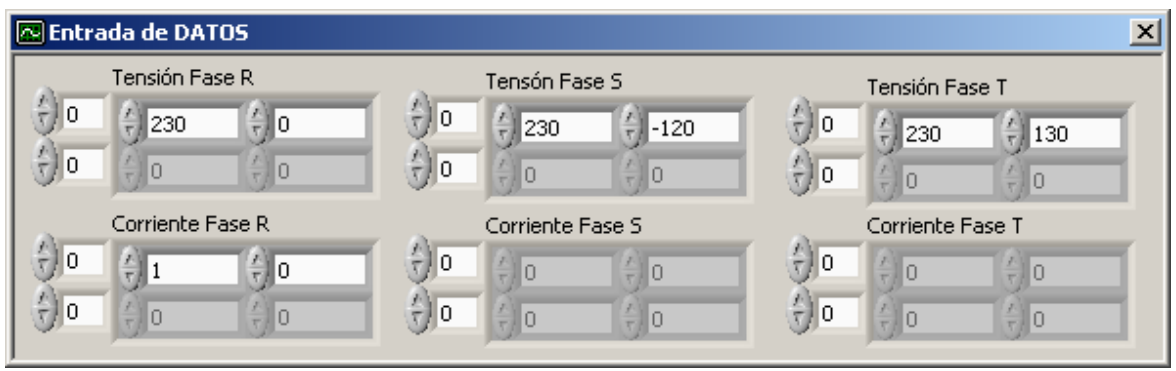

Figura V.6. Pantalla de entrada de datos.

Un sistema de tensiones en el que dejamos fijos los módulos de las fases, y tenemos una carga resistiva pura que consume $1 \angle 0^{\circ} \mathrm{A}$ conectada en la fase 1 , y tan sólo variamos de manera gradual el argumento de la fase 3, logrando los pertinentes desequilibrios de tensión y produciendo la consiguiente potencia de desfase, tal y como podemos observar en la tabla V.2, y en la figura V.7 para el caso concreto de que el argumento de la fase 3 tome el valor de $130^{\circ}$.

\begin{tabular}{||c|c|c||}
\hline $\begin{array}{c}\text { Argumento } \\
\text { Fase 3 }\end{array}$ & $\begin{array}{c}\text { Variación } \\
\text { Argumento } \mathbf{(}^{\circ} \text { ) }\end{array}$ & $\begin{array}{c}\text { Potencia Desfase } \\
\text { Desequilibrio } \mathbf{Q}_{\text {dru }} \text { (VAr) }\end{array}$ \\
\hline $121^{\circ}$ & 1 & 1,34 \\
\hline $122^{\circ}$ & 2 & 2,68 \\
\hline $125^{\circ}$ & 5 & 6,68 \\
\hline $130^{\circ}$ & 10 & 13,31 \\
\hline $135^{\circ}$ & 15 & 19,84 \\
\hline $145^{\circ}$ & 25 & 32,40 \\
\hline $155^{\circ}$ & 35 & 43,97 \\
\hline $170^{\circ}$ & 50 & 58,73 \\
\hline $190^{\circ}$ & 70 & 72,04 \\
\hline $210^{\circ}$ & 90 & 76,67 \\
\hline $220^{\circ}$ & 100 & 75,50 \\
\hline $230^{\circ}$ & 110 & 72,04 \\
\hline $240^{\circ}$ & 120 & 66,40 \\
\hline
\end{tabular}

Tabla V.3. Potencia de Desfase \& Variación Argumento. 
Observamos en la tabla V.3, que se obtienen idénticos resultados que los ya mencionados cuando producíamos el desequilibrio por medio de la variación del argumento de la fase 2 de $-120^{\circ}$ a $0^{\circ}$, siendo por lo tanto una potencia de desfase de carácter inductivo.

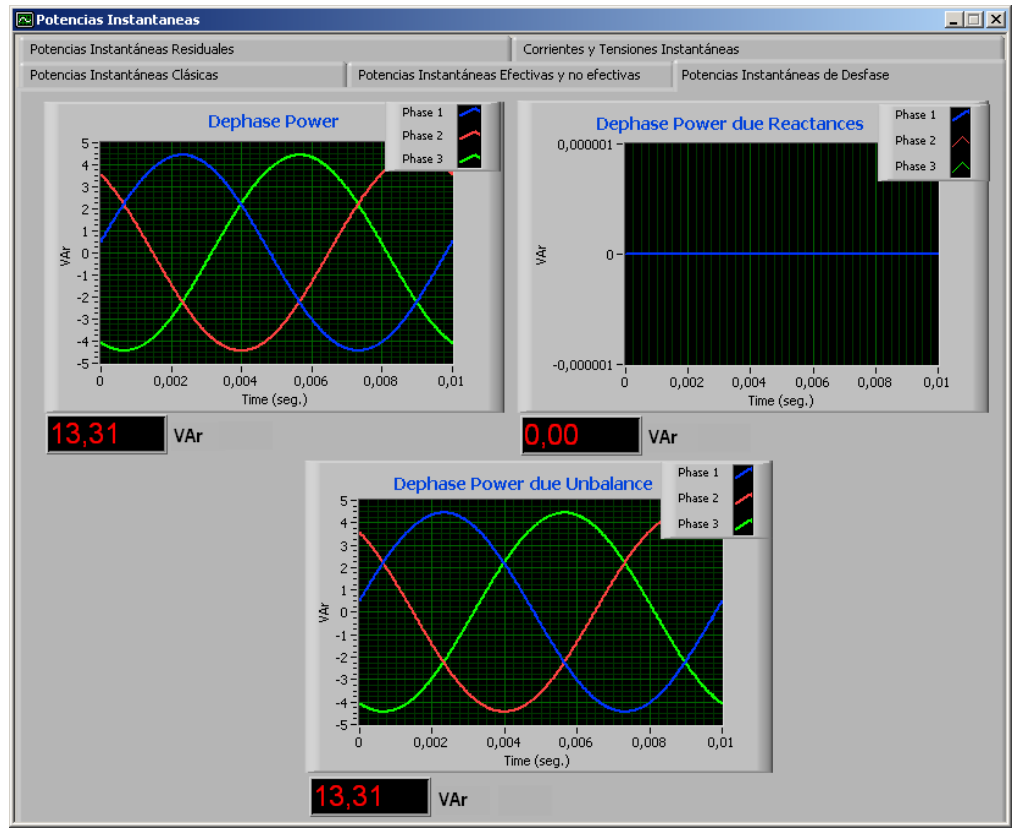

Figura V.7. Pantalla de Potencias de Desfase. 
Carga resistiva entre la fase 1 y el neutro, con un consumo de $1 \angle 0^{\circ} \mathrm{A}, \mathrm{y}$ producimos el desequilibrio sumando ángulos negativos al argumento de la fase 3.

Introducimos los datos en el SIMPELEC Simulador, como mostramos en la figura V.8,

\begin{tabular}{|c|c|c|c|c|c|c|c|c|}
\hline 圆 Entr & a de $\mathrm{D}$ & & & & & & & \\
\hline & ensión $\mathrm{F}$ & & & nnsón F & & & nsión I & \\
\hline (5) 0 & 230 & 0 & 5) 0 & 230 & -120 & (5) 0 & 230 & 70 \\
\hline 5) 0 & 0 & 0 & 5) 0 & 0 & 0 & 70 & 0 & 0 \\
\hline & orriente & & & orrients & & & orrient & \\
\hline (5) 0 & 1 & 0 & (3) 0 & 0 & 0 & 5) 0 & 0 & 0 \\
\hline (5) 0 & 0 & 0 & 50 & 0 & 0 & (3) 0 & 0 & 0 \\
\hline
\end{tabular}

Figura V.8. Pantalla de entrada de datos.

Un sistema de tensiones en el que dejamos fijos los módulos de las fases, y tenemos una carga resistiva pura que consume $1 \angle 0^{\circ} \mathrm{A}$ conectada en la fase 1 , y tan sólo variamos de manera gradual el argumento de la fase 3, logrando los pertinentes desequilibrios de tensión y produciendo la consiguiente potencia de desfase, tal y como podemos observar en la tabla V.4, y en la figura V.9 para el caso concreto de que el argumento de la fase 3 valga $70^{\circ}$.

\begin{tabular}{||c|c|c||}
\hline $\begin{array}{c}\text { Argumento } \\
\text { Fase 3 }\end{array}$ & $\begin{array}{c}\text { Variación } \\
\text { Argumento ( } \mathbf{(}^{\circ}\end{array}$ & $\begin{array}{c}\text { Potencia Desfase } \\
\text { Desequilibrio } \mathbf{Q}_{\text {dru }} \text { (VAr) }\end{array}$ \\
\hline $119^{\circ}$ & 1 & $-1,34$ \\
\hline $118^{\circ}$ & 2 & $-2,68$ \\
\hline $115^{\circ}$ & 5 & $-6,68$ \\
\hline $110^{\circ}$ & 10 & $-13,31$ \\
\hline $105^{\circ}$ & 15 & $-19,84$ \\
\hline $95^{\circ}$ & 25 & $-32,40$ \\
\hline $85^{\circ}$ & 35 & $-43,97$ \\
\hline $70^{\circ}$ & 50 & $-58,73$ \\
\hline $50^{\circ}$ & 70 & $-72,04$ \\
\hline $30^{\circ}$ & 90 & $-76,67$ \\
\hline $20^{\circ}$ & 100 & $-75,50$ \\
\hline $10^{\circ}$ & 110 & $-72,04$ \\
\hline $0^{\circ}$ & 120 & $-66,40$ \\
\hline
\end{tabular}

Tabla V.4. Potencia de Desfase \& Variación Argumento. 
De los resultados mostrados en la tabla V.4, observamos que son coincidentes con los obtenidos cuando el desequilibrio se realizaba modificando los argumentos de la fase 2 desde -120 a $-240^{\circ}$, y que la potencia de desfase es capacitiva.

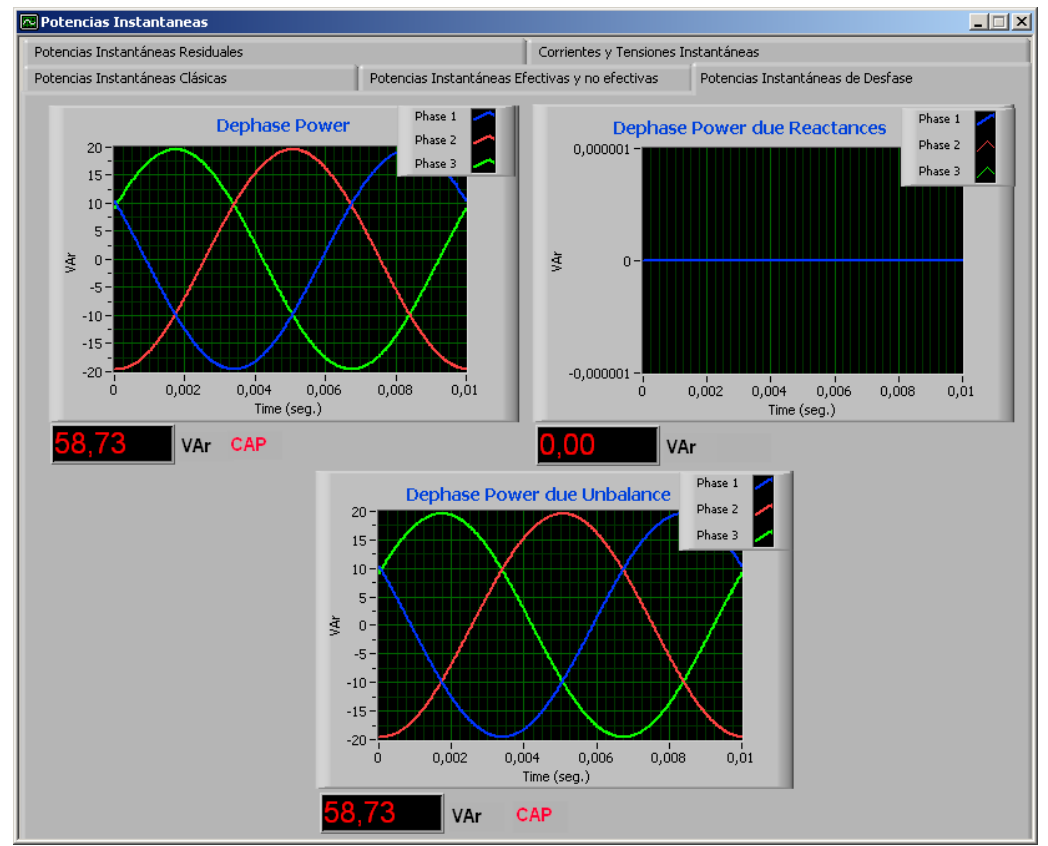

Figura V.9. Pantalla de Potencias de Desfase. 
Carga resistiva entre la fase 2 y el neutro, con un consumo de $1 \angle-120^{\circ} A$, y producimos el desequilibrio sumando ángulos positivos al argumento de la fase 1.

Introducimos los datos en el SIMPELEC Simulador, como observamos en la figura V.10,

\begin{tabular}{|c|c|c|c|c|c|c|c|c|}
\hline \multicolumn{9}{|c|}{ Entrada de DATOS } \\
\hline \multicolumn{3}{|c|}{ Tensión Fase R } & \multicolumn{3}{|c|}{ Tensón Fase S } & \multicolumn{3}{|c|}{ Tensión Fase T } \\
\hline 5) 0 & 230 & 5 & 7) 0 & 230 & -120 & (5) 0 & 230 & 120 \\
\hline (5) 0 & 0 & 0 & 5) 0 & 0 & 0 & (5) 0 & 0 & 0 \\
\hline \multicolumn{3}{|c|}{ Corriente Fase R } & \multicolumn{3}{|c|}{ Corriente Fase S } & \multicolumn{3}{|c|}{ Corriente Fase T } \\
\hline (5) 0 & 0 & 0 & (3) 0 & 1 & -120 & 7) 0 & 0 & 0 \\
\hline (5) 0 & 0 & 0 & 7) 0 & 0 & 0 & (3) 0 & 0 & 0 \\
\hline
\end{tabular}

Figura V.10. Pantalla de entrada de datos.

Tenemos pues un sistema de tensiones en el que como ya hemos comentado de manera general dejamos fijos los módulos de las tensiones de las fases, y tenemos una carga resistiva pura que consume $1 \angle-120^{\circ} A$ conectada en la fase 2, y tan sólo variamos de manera gradual el argumento de la fase 1, logrando los pertinentes desequilibrios de tensión y produciendo la consiguiente potencia de desfase, tal y como podemos observar en la tabla V.5, y en la figura V.11 para el caso concreto de que el argumento de la fase 1 tome el valor de $15^{\circ}$.

\begin{tabular}{|c|c|c||}
\hline $\begin{array}{c}\text { Argumento } \\
\text { Fase 1 }\end{array}$ & $\begin{array}{c}\text { Variación } \\
\text { Argumento }\left(^{(}\right)\end{array}$ & $\begin{array}{c}\text { Potencia Desfase } \\
\text { Desequilibrio } \mathbf{Q}_{\text {dru }} \text { (VAr) }\end{array}$ \\
\hline $1^{\circ}$ & 1 & 1,34 \\
\hline $2^{\circ}$ & 2 & 2,68 \\
\hline $5^{\circ}$ & 5 & 6,68 \\
\hline $10^{\circ}$ & 10 & 13,31 \\
\hline $15^{\circ}$ & 15 & 19,84 \\
\hline $25^{\circ}$ & 25 & 32,40 \\
\hline $35^{\circ}$ & 35 & 43,97 \\
\hline $50^{\circ}$ & 50 & 58,73 \\
\hline $70^{\circ}$ & 70 & 72,04 \\
\hline $90^{\circ}$ & 90 & 76,67 \\
\hline $100^{\circ}$ & 100 & 75,50 \\
\hline $110^{\circ}$ & 110 & 72,04 \\
\hline $120^{\circ}$ & 120 & 66,40 \\
\hline \hline
\end{tabular}

Tabla V.5. Potencia de Desfase \& Variación Argumento. 
Observamos que aunque ahora tenemos la carga resistiva conectada en la fase 2, los resultados de la tabla V.5, coinciden con los mostrados en las tablas V.1 y 3, y por lo tanto la potencia de desfase tiene carácter inductivo.

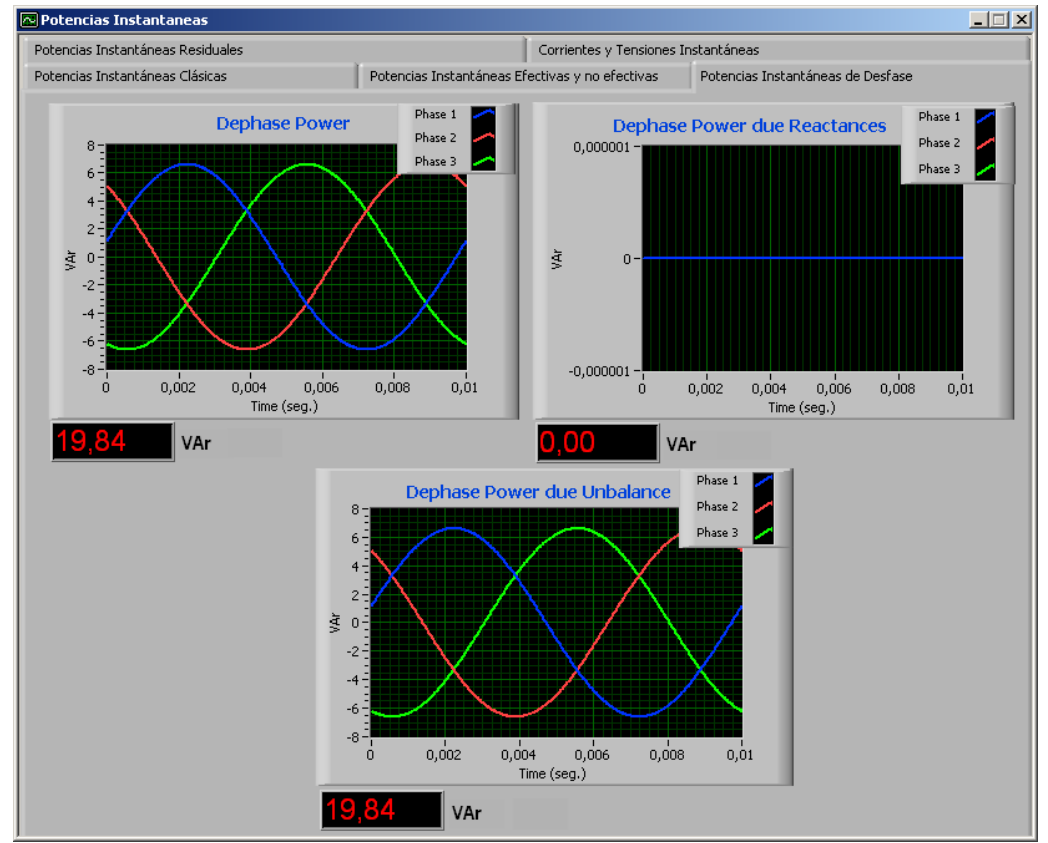

Figura V.11. Pantalla de Potencias de Desfase. 
Carga resistiva entre la fase 2 y el neutro, con un consumo de $1 \angle-120^{\circ} A$, y producimos el desequilibrio sumando ángulos negativos al argumento de la fase 1.

Introducimos los datos en el SIMPELEC Simulador, como se muestra en la figura V.12,

\begin{tabular}{|c|c|c|c|c|c|c|c|c|}
\hline \multicolumn{9}{|c|}{ Entrada de DATOS } \\
\hline \multicolumn{3}{|c|}{ Tensión Fase R } & \multicolumn{3}{|c|}{ Tensón Fase 5} & \multicolumn{3}{|c|}{ Tensión Fase T } \\
\hline 7) 0 & 1) 230 & -25 & 5) 0 & 230 & -120 & (5) 0 & 230 & 120 \\
\hline 5) 0 & 0 & 0 & (5) 0 & 0 & 0 & (3) 0 & 0 & 0 \\
\hline \multicolumn{3}{|c|}{ Corriente Fase R } & \multicolumn{3}{|c|}{ Corriente Fase S } & \multicolumn{3}{|c|}{ Corriente Fase $T$} \\
\hline (5) 0 & 0 & 0 & (3) 0 & 1 & -120 & (5) 0 & 0 & 0 \\
\hline 5) 0 & 0 & 0 & (3) 0 & 0 & 0 & (5) 0 & 0 & 0 \\
\hline
\end{tabular}

Figura V.12. Pantalla de entrada de datos.

Partimos de un sistema de tensiones en el que dejamos fijos los módulos de las fases, y tenemos una carga resistiva pura que consume $1 \angle-120^{\circ} A$ conectada en la fase 2, y tan sólo variamos de manera gradual el argumento de la fase 1 , logrando los pertinentes desequilibrios de tensión y produciendo la consiguiente potencia de desfase, tal y como podemos observar en la tabla V.6, y en la figura V.13 para el caso concreto de que el argumento de la fase 1 tome el valor de $25^{\circ}$.

\begin{tabular}{|c|c|c||}
\hline $\begin{array}{c}\text { Argumento } \\
\text { Fase 1 }\end{array}$ & $\begin{array}{c}\text { Variación } \\
\text { Argumento ( }\end{array}$ & $\begin{array}{c}\text { Potencia Desfase } \\
\text { Desequilibrio } \mathbf{Q}_{\text {dru }} \text { (VAr) }\end{array}$ \\
\hline$-1^{\circ}$ & 1 & $-1,34$ \\
\hline$-2^{\circ}$ & 2 & $-2,68$ \\
\hline$-5^{\circ}$ & 5 & $-6,68$ \\
\hline$-10^{\circ}$ & 10 & $-13,31$ \\
\hline$-15^{\circ}$ & 15 & $-19,84$ \\
\hline$-25^{\circ}$ & 25 & $-32,40$ \\
\hline$-35^{\circ}$ & 35 & $-43,97$ \\
\hline$-50^{\circ}$ & 50 & $-58,73$ \\
\hline$-70^{\circ}$ & 70 & $-72,04$ \\
\hline$-90^{\circ}$ & 90 & $-76,67$ \\
\hline$-100^{\circ}$ & 100 & $-75,50$ \\
\hline$-110^{\circ}$ & 110 & $-72,04$ \\
\hline$-120^{\circ}$ & 120 & $-66,40$ \\
\hline
\end{tabular}

Tabla V.6. Potencia de Desfase \& Variación Argumento. 
En la tabla V.6, se observa que los valores obtenidos para la potencia de desfase, tienen carácter capacitivo y coinciden con los mostrados en las tablas V.2 y 4.

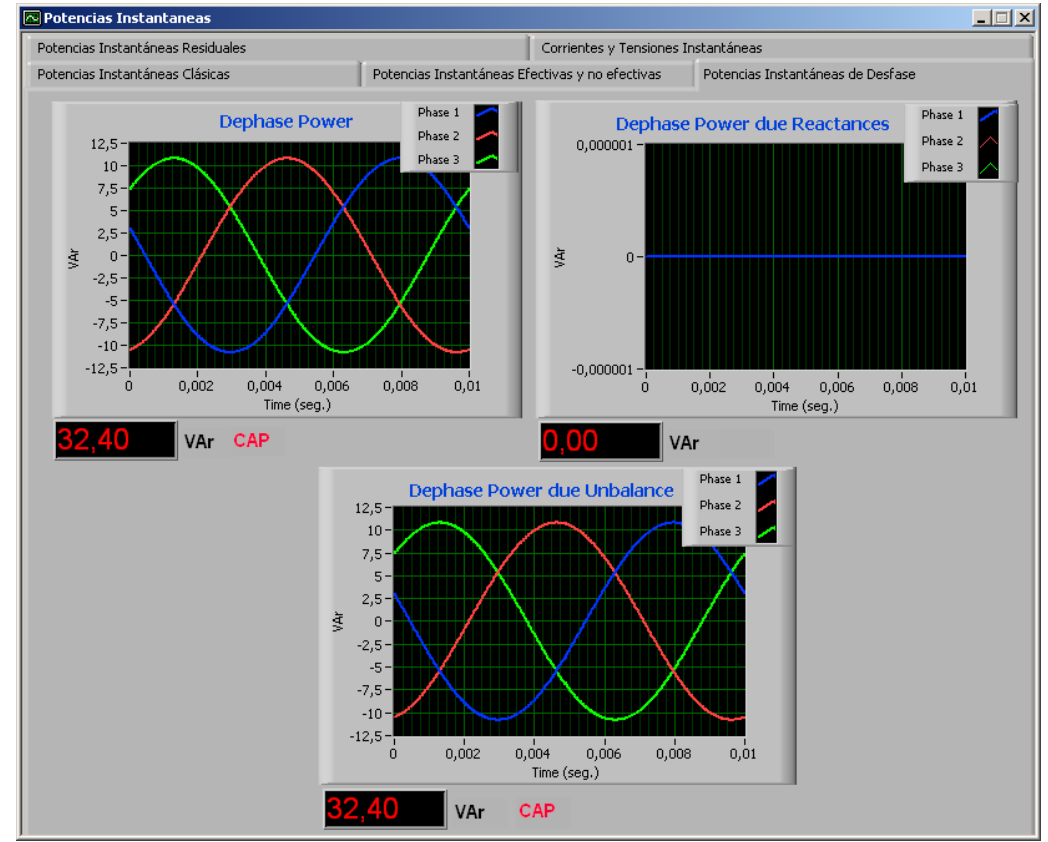

Figura V.13. Pantalla de Potencias de Desfase. 
Carga resistiva entre la fase 2 y el neutro, con un consumo de $1 \angle-120^{\circ} A$, y producimos el desequilibrio sumando ángulos positivos al argumento de la fase 3.

Introducimos los datos en el SIMPELEC Simulador, como mostramos en la figura V.14,

\begin{tabular}{|c|c|c|c|c|c|c|c|c|}
\hline \multicolumn{9}{|c|}{ 圆Entrada de DATOS } \\
\hline \multicolumn{3}{|c|}{ Tensión Fase R } & \multicolumn{3}{|c|}{ Tensón Fase S } & \multicolumn{3}{|c|}{ Tensión Fase T } \\
\hline 5) 0 & 230 & 0 & 5) 0 & 230 & -120 & 5) 0 & 230 & 145 \\
\hline 5) 0 & 0 & 0 & 5) 0 & 0 & 0 & 75) 0 & 0 & 0 \\
\hline \multicolumn{3}{|c|}{ Corriente Fase R } & \multicolumn{3}{|c|}{ Corriente Fase S } & \multicolumn{3}{|c|}{ Corriente Fase $\mathrm{T}$} \\
\hline (5) 0 & 0 & 0 & (5) 0 & 1 & -120 & 7) 0 & 0 & 0 \\
\hline 5) 0 & 0 & 0 & (3) 0 & 0 & 0 & (3) 0 & 0 & 0 \\
\hline
\end{tabular}

Figura V.14. Pantalla de entrada de datos.

Un sistema de tensiones en el que dejamos fijos los módulos de las fases, y tenemos una carga resistiva pura que consume $1 \angle-120^{\circ} A$ conectada en la fase 2, y tan sólo variamos de manera gradual el argumento de la fase 3, logrando los pertinentes desequilibrios de tensión y produciendo la consiguiente potencia de desfase, tal y como podemos observar en la tabla V.7, y en la figura V.15 para el caso concreto, de que el argumento de la fase 3 tome el valor de $145^{\circ}$.

\begin{tabular}{|c|c|c||}
\hline $\begin{array}{c}\text { Argumento } \\
\text { Fase 3 }\end{array}$ & $\begin{array}{c}\text { Variación } \\
\text { Argumento ( }\end{array}$ & $\begin{array}{c}\text { Potencia Desfase } \\
\text { Desequilibrio } \mathbf{Q}_{\text {dru }} \text { (VAr) }\end{array}$ \\
\hline $121^{\circ}$ & 1 & 1,34 \\
\hline $122^{\circ}$ & 2 & 2,68 \\
\hline $125^{\circ}$ & 5 & 6,68 \\
\hline $130^{\circ}$ & 10 & 13,31 \\
\hline $135^{\circ}$ & 15 & 19,84 \\
\hline $145^{\circ}$ & 25 & 32,40 \\
\hline $155^{\circ}$ & 35 & 43,97 \\
\hline $170^{\circ}$ & 50 & 58,73 \\
\hline $190^{\circ}$ & 70 & 72,04 \\
\hline $210^{\circ}$ & 90 & 76,67 \\
\hline $220^{\circ}$ & 100 & 75,50 \\
\hline $230^{\circ}$ & 110 & 72,04 \\
\hline $240^{\circ}$ & 120 & 66,40 \\
\hline
\end{tabular}

Tabla V.7. Potencia de Desfase \& Variación Argumento. 
Tras la observación de la tabla V.7, observamos que la potencia de desfase consumida posee carácter inductivo, al igual que sucedía en los resultados mostrados en las tablas V.1,3 y 5, y siendo sus valores idénticos.

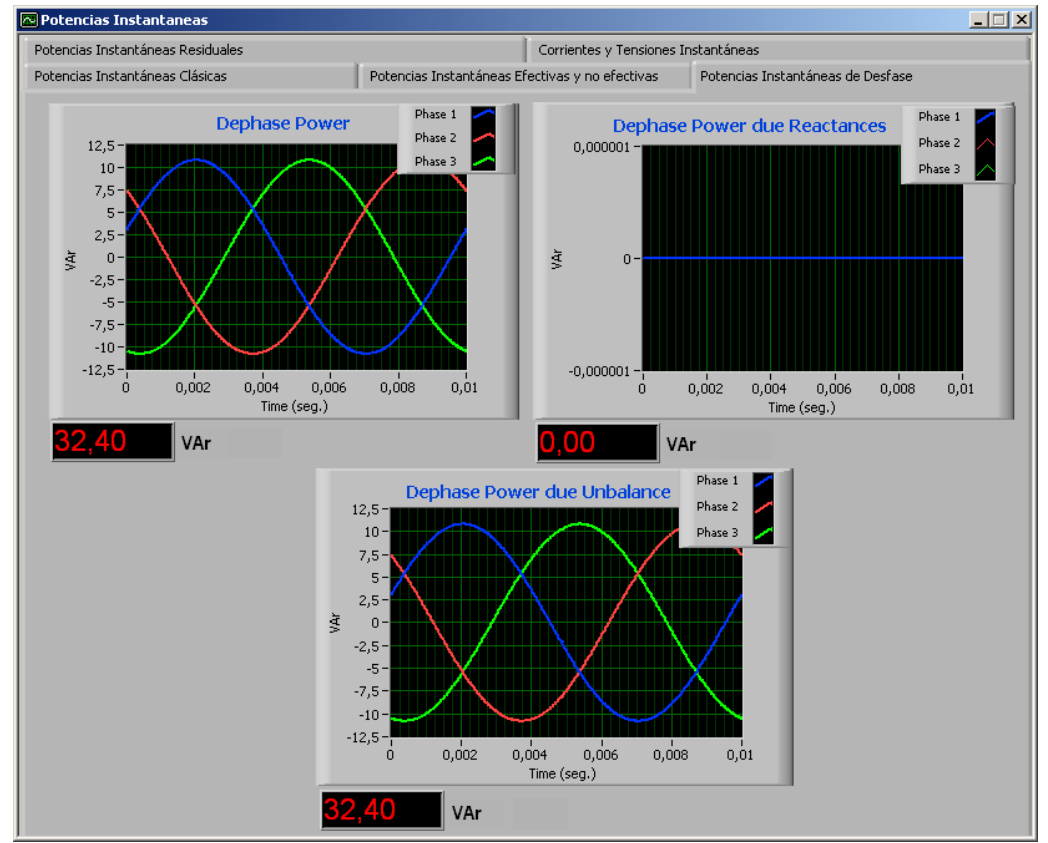

Figura V.15. Pantalla de Potencias de Desfase. 
Carga resistiva entre la fase 2 y el neutro, con un consumo de $1 \angle-120^{\circ} A$, y producimos el desequilibrio sumando ángulos negativos al argumento de la fase 3.

Introducimos los datos en el SIMPELEC Simulador, como mostramos en la figura V.16,

\begin{tabular}{|c|c|c|c|c|c|c|c|c|}
\hline \multicolumn{9}{|c|}{ Entrada de DATOS } \\
\hline \multicolumn{3}{|c|}{ Tensión Fase R } & \multicolumn{3}{|c|}{ Tensón Fase 5} & \multicolumn{3}{|c|}{ Tensión Fase T } \\
\hline 5) 0 & 230 & 0 & (5) 0 & 230 & -120 & (5) 0 & 230 & 85 \\
\hline (5) 0 & 0 & 0 & 5) 0 & 0 & 0 & (5) 0 & 0 & 0 \\
\hline \multicolumn{3}{|c|}{ Corriente Fase R } & \multicolumn{3}{|c|}{ Corriente Fase S } & \multicolumn{3}{|c|}{ Corriente Fase T } \\
\hline 5) 0 & 0 & 0 & (3) 0 & 1 & -120 & 7) 0 & 0 & 0 \\
\hline (5) 0 & 0 & 0 & (3) 0 & 0 & 0 & (3) 0 & 0 & 0 \\
\hline
\end{tabular}

Figura V.16. Pantalla de entrada de datos.

Un sistema de tensiones en el que dejamos fijos los módulos de las fases, y tenemos una carga resistiva pura que consume $1 \angle-120^{\circ} A$ conectada en la fase 2, y tan sólo variamos de manera gradual el argumento de la fase 3, logrando los pertinentes desequilibrios de tensión y produciendo la consiguiente potencia de desfase, tal y como podemos observar en la tabla V.8, y en la figura V.17 para el caso concreto de que el argumento de la fase 3 valga $85^{\circ}$.

\begin{tabular}{|c|c|c||}
\hline $\begin{array}{c}\text { Argumento } \\
\text { Fase 3 }\end{array}$ & $\begin{array}{c}\text { Variación } \\
\text { Argumento ( }\end{array}$ & $\begin{array}{c}\text { Potencia Desfase } \\
\text { Desequilibrio } \mathbf{Q}_{\text {dru }} \text { (VAr) }\end{array}$ \\
\hline $119^{\circ}$ & 1 & $-1,34$ \\
\hline $118^{\circ}$ & 2 & $-2,68$ \\
\hline $115^{\circ}$ & 5 & $-6,68$ \\
\hline $110^{\circ}$ & 10 & $-13,31$ \\
\hline $105^{\circ}$ & 15 & $-19,84$ \\
\hline $95^{\circ}$ & 25 & $-32,40$ \\
\hline $85^{\circ}$ & 35 & $-43,97$ \\
\hline $70^{\circ}$ & 50 & $-58,73$ \\
\hline $50^{\circ}$ & 70 & $-72,04$ \\
\hline $30^{\circ}$ & 90 & $-76,67$ \\
\hline $20^{\circ}$ & 100 & $-75,50$ \\
\hline $10^{\circ}$ & 110 & $-72,04$ \\
\hline $0^{\circ}$ & 120 & $-66,40$ \\
\hline
\end{tabular}

Tabla V.8. Potencia de Desfase \& Variación Argumento. 
Los resultados mostrados en la tabla V.8, nos muestran el comportamiento capacitivo de la potencia de desfase que resulta de este desequilibrio en esta carga resistiva, al igual que sucedía en lo mostrado en las tablas V.2, 4 y 6.

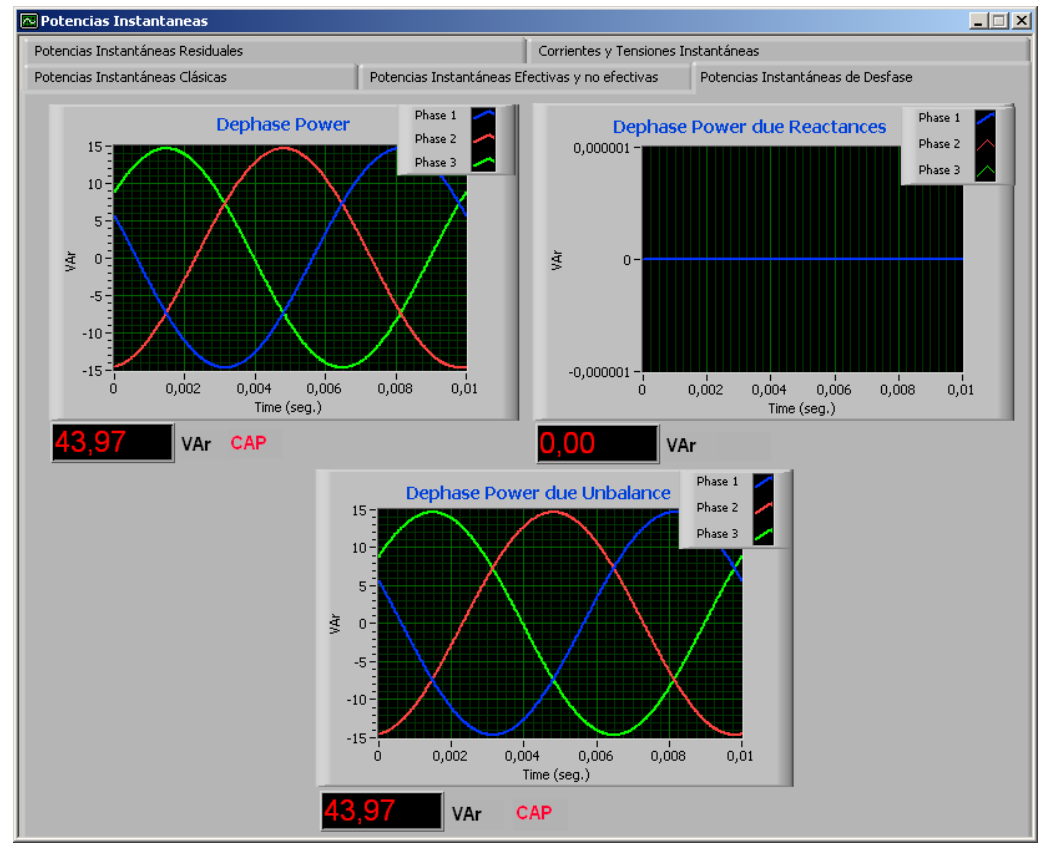

Figura V.17. Pantalla de Potencias de Desfase. 
Carga resistiva entre la fase 3 y el neutro, con un consumo de $1 \angle 120^{\circ} \mathrm{A}, \mathrm{y}$ producimos el desequilibrio sumando ángulos positivos al argumento de la fase 1.

Introducimos los datos en el SIMPELEC Simulador, como observamos en la figura V.18.

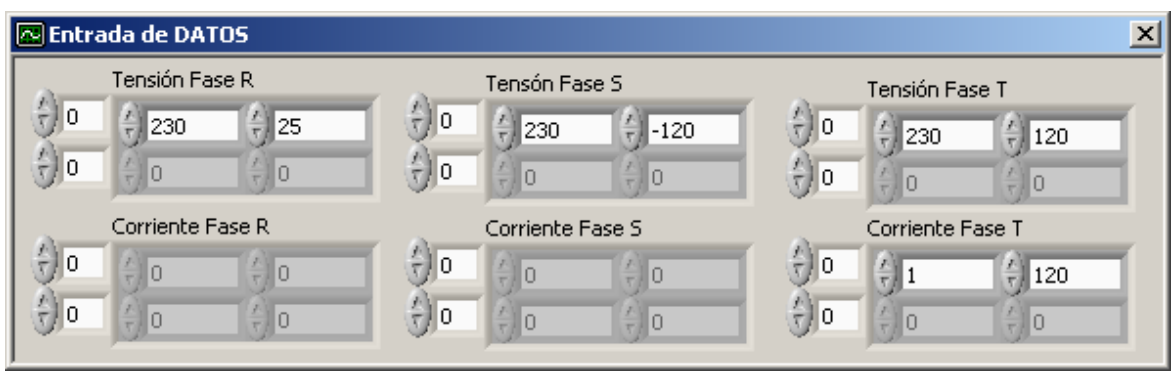

Figura V.18. Pantalla de entrada de datos.

Tenemos pues un sistema de tensiones en el que como ya hemos comentado de manera general dejamos fijos los módulos de las tensiones de las fases, y tenemos una carga resistiva pura que consume $1 \angle 120^{\circ} A$ conectada en la fase 3 , y tan sólo variamos de manera gradual el argumento de la fase 1, logrando los pertinentes desequilibrios de tensión y produciendo la consiguiente potencia de desfase, tal y como podemos observar en la tabla V.9, y en la figura V.19 para el caso concreto de que el argumento de la fase 1 tome el valor de $25^{\circ}$.

\begin{tabular}{||c|c|c||}
\hline $\begin{array}{c}\text { Argumento } \\
\text { Fase 1 }\end{array}$ & $\begin{array}{c}\text { Variación } \\
\text { Argumento ( } \mathbf{(}^{\circ}\end{array}$ & $\begin{array}{c}\text { Potencia Desfase } \\
\text { Desequilibrio } \mathbf{Q}_{\text {dru }} \text { (VAr) }\end{array}$ \\
\hline $1^{\circ}$ & 1 & 1,34 \\
\hline $2^{\circ}$ & 2 & 2,68 \\
\hline $5^{\circ}$ & 5 & 6,68 \\
\hline $10^{\circ}$ & 10 & 13,31 \\
\hline $15^{\circ}$ & 15 & 19,84 \\
\hline $25^{\circ}$ & 25 & 32,40 \\
\hline $35^{\circ}$ & 35 & 43,97 \\
\hline $50^{\circ}$ & 50 & 58,73 \\
\hline $70^{\circ}$ & 70 & 72,04 \\
\hline $90^{\circ}$ & 90 & 76,67 \\
\hline $100^{\circ}$ & 100 & 75,50 \\
\hline $110^{\circ}$ & 110 & 72,04 \\
\hline $120^{\circ}$ & 120 & 66,40 \\
\hline \hline
\end{tabular}

Tabla V.9. Potencia de Desfase \& Variación Argumento. 
De los resultados de la tabla V.9, apreciamos que la carga provoca que exista una potencia de desfase con carácter inductivo, al igual que sucedía con los resultados mostrados en las tablas $\mathrm{V} .1,3,5$ y 7.

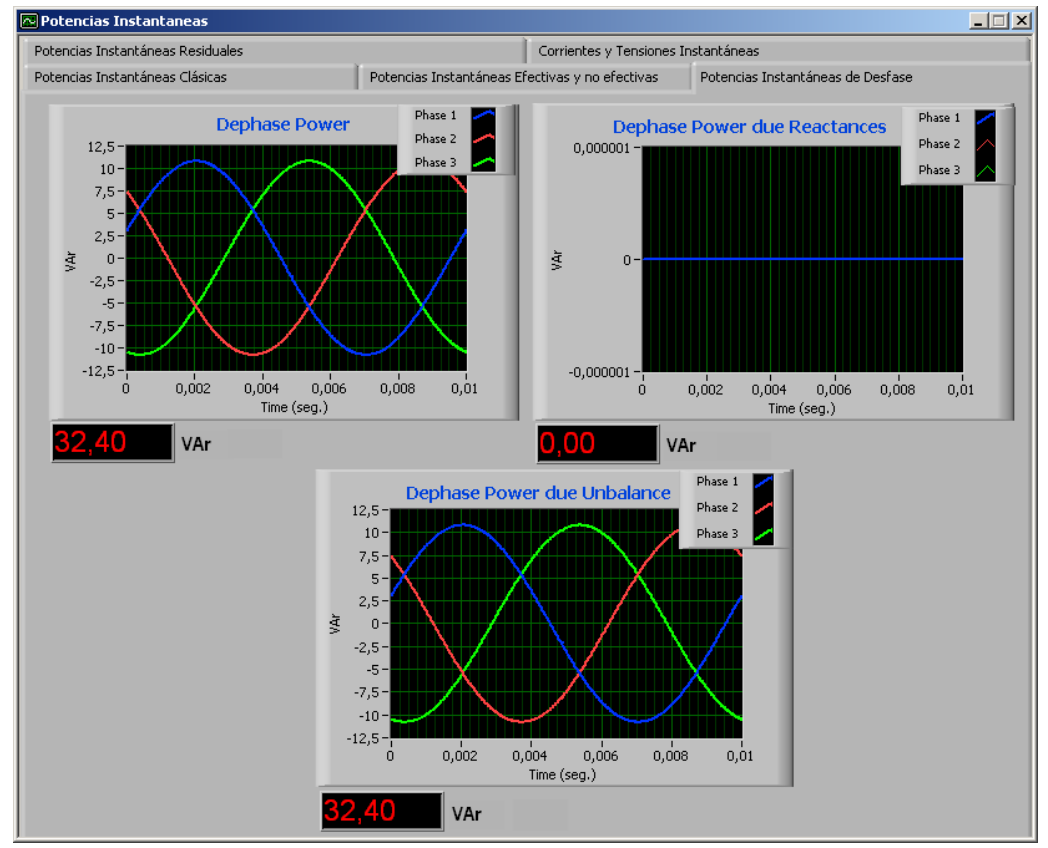

Figura V.19. Pantalla de Potencias de Desfase. 
Carga resistiva entre la fase 3 y el neutro, con un consumo de $1 \angle 120^{\circ} \mathrm{A}, \mathrm{y}$ producimos el desequilibrio sumando ángulos negativos al argumento de la fase 1.

Introducimos los datos en el SIMPELEC Simulador, como se muestra en la figura V.20.

\begin{tabular}{|c|c|c|c|c|c|c|c|c|}
\hline \multicolumn{9}{|c|}{ Entrada de DATOS } \\
\hline \multicolumn{3}{|c|}{ Tensión Fase $\mathrm{R}$} & \multicolumn{3}{|c|}{ Tensón Fase 5} & \multicolumn{3}{|c|}{ Tensión Fase T } \\
\hline 5) 0 & 230 & -5 & t) 0 & 230 & -120 & 5) 0 & 230 & 120 \\
\hline 5) 0 & 0 & 0 & (5) 0 & 0 & 0 & (5) 0 & 0 & 0 \\
\hline \multicolumn{3}{|c|}{ Corriente Fase R } & \multicolumn{3}{|c|}{ Corriente Fase S } & \multicolumn{3}{|c|}{ Corriente Fase T } \\
\hline (5) 0 & 0 & 0 & 5) 0 & 0 & 0 & (5) 0 & 1 & 120 \\
\hline 5) 0 & 0 & 0 & 5) 0 & 0 & 0 & (5) 0 & 0 & 0 \\
\hline
\end{tabular}

Figura V.20. Pantalla de entrada de datos.

Partimos de un sistema de tensiones en el que dejamos fijos los módulos de las fases, y tenemos una carga resistiva pura que consume $1 \angle 120^{\circ} A$ conectada en la fase 3 , y tan sólo variamos de manera gradual el argumento de la fase 1 , logrando los pertinentes desequilibrios de tensión y produciendo la consiguiente potencia de desfase, tal y como podemos observar en la tabla V.10, y en la figura V.21 para el caso concreto de que el argumento de la fase 1 tome el valor de -50 .

\begin{tabular}{|c|c|c||}
\hline $\begin{array}{c}\text { Argumento } \\
\text { Fase 1 }\end{array}$ & $\begin{array}{c}\text { Variación } \\
\text { Argumento ( }\end{array}$ & $\begin{array}{c}\text { Potencia Desfase } \\
\text { Desequilibrio } \mathbf{Q}_{\text {dru }} \text { (VAr) }\end{array}$ \\
\hline$-1^{\circ}$ & 1 & $-1,34$ \\
\hline$-2^{\circ}$ & 2 & $-2,68$ \\
\hline$-5^{\circ}$ & 5 & $-6,68$ \\
\hline$-10^{\circ}$ & 10 & $-13,31$ \\
\hline$-15^{\circ}$ & 15 & $-19,84$ \\
\hline$-25^{\circ}$ & 25 & $-32,40$ \\
\hline$-35^{\circ}$ & 35 & $-43,97$ \\
\hline$-50^{\circ}$ & 50 & $-58,73$ \\
\hline$-70^{\circ}$ & 70 & $-72,04$ \\
\hline$-90^{\circ}$ & 90 & $-76,67$ \\
\hline$-100^{\circ}$ & 100 & $-75,50$ \\
\hline$-110^{\circ}$ & 110 & $-72,04$ \\
\hline$-120^{\circ}$ & 120 & $-66,40$ \\
\hline
\end{tabular}

Tabla V.10. Potencia de Desfase \& Variación Argumento. 
Tras la observación de la tabla V.10, deducimos que existe un consumo de potencia de desfase capacitiva, con esta carga resistiva al igual que sucedía en los resultados de las tablas V.2, 4, 6 y 8 .

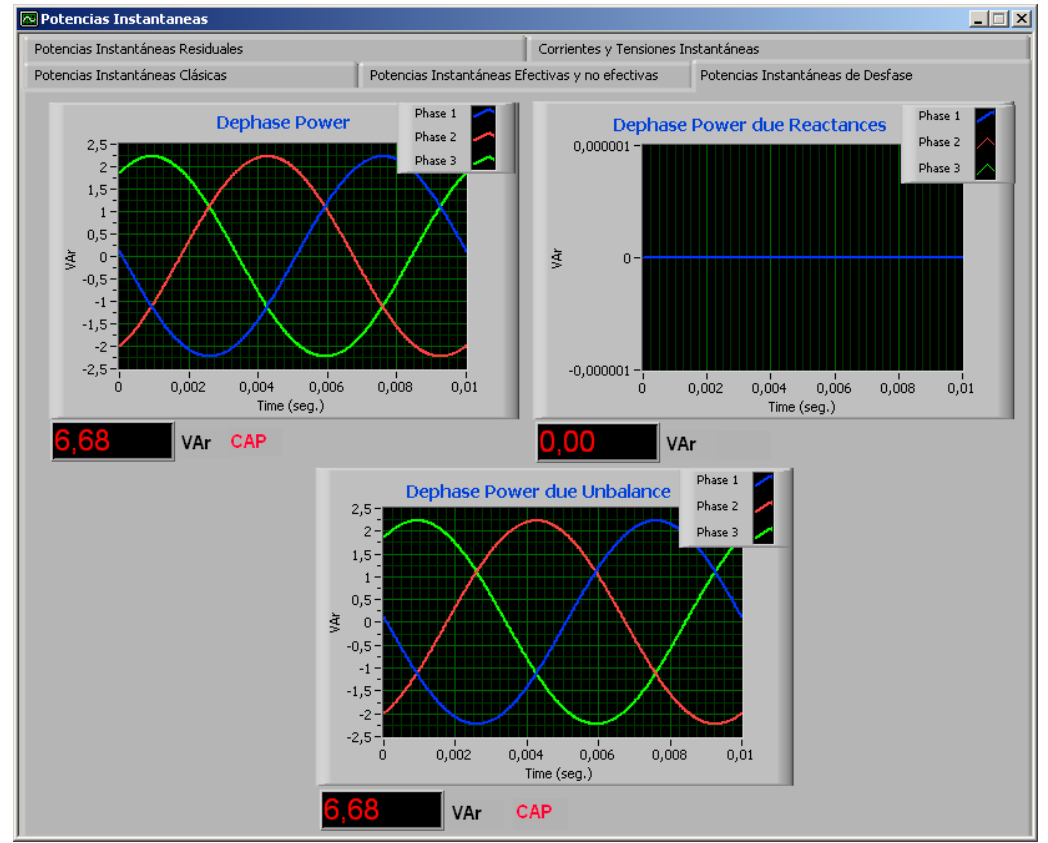

Figura V.21. Pantalla de Potencias de Desfase. 
Carga resistiva entre la fase 3 y el neutro, con un consumo de $1 \angle 120^{\circ} \mathrm{A}, \mathrm{y}$ producimos el desequilibrio sumando ángulos positivos al argumento de la fase 2.

Introducimos los datos en el SIMPELEC Simulador, como mostramos en la figura V.22.

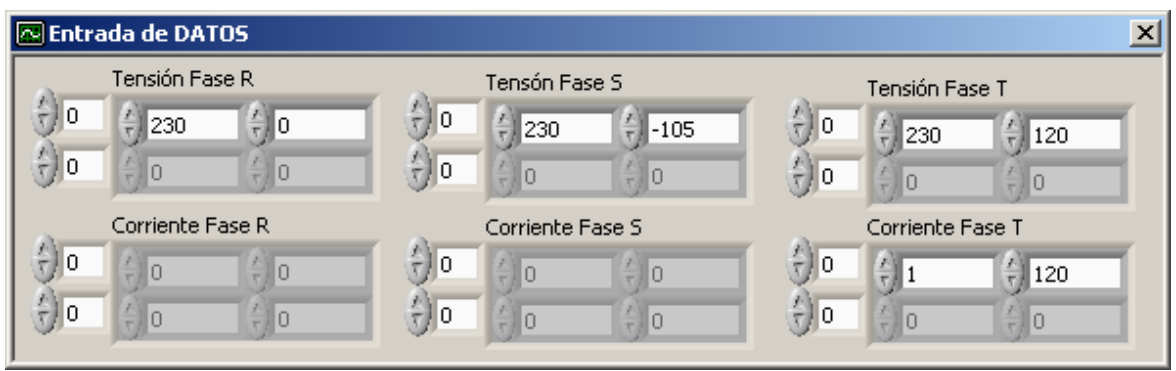

Figura V.22. Pantalla de entrada de datos.

Un sistema de tensiones en el que dejamos fijos los módulos de las fases, y tenemos una carga resistiva pura que consume $1 \angle 120^{\circ} A$ conectada en la fase 3, y tan sólo variamos de manera gradual el argumento de la fase 2, logrando los pertinentes desequilibrios de tensión y produciendo la consiguiente potencia de desfase, tal y como podemos observar en la tabla V.11, y en la figura V.23 para el caso concreto de que el argumento de la fase 3 tome el valor de $-105^{\circ}$.

\begin{tabular}{||c|c|c||}
\hline $\begin{array}{c}\text { Argumento } \\
\text { Fase 2 }\end{array}$ & $\begin{array}{c}\text { Variación } \\
\left.\text { Argumento } \mathbf{(}^{\circ}\right)\end{array}$ & $\begin{array}{c}\text { Potencia Desfase } \\
\text { Desequilibrio } \mathbf{Q}_{\text {dru }} \text { (VAr) }\end{array}$ \\
\hline$-119^{\circ}$ & 1 & 1,34 \\
\hline$-118^{\circ}$ & 2 & 2,68 \\
\hline$-115^{\circ}$ & 5 & 6,68 \\
\hline$-110^{\circ}$ & 10 & 13,31 \\
\hline$-105^{\circ}$ & 15 & 19,84 \\
\hline$-95^{\circ}$ & 25 & 32,40 \\
\hline$-85^{\circ}$ & 35 & 43,97 \\
\hline$-70^{\circ}$ & 50 & 58,73 \\
\hline$-50^{\circ}$ & 70 & 72,04 \\
\hline$-30^{\circ}$ & 90 & 76,67 \\
\hline$-20^{\circ}$ & 100 & 75,50 \\
\hline$-10^{\circ}$ & 110 & 72,04 \\
\hline $0^{\circ}$ & 120 & 66,40 \\
\hline
\end{tabular}

Tabla V.11. Potencia de Desfase \& Variación Argumento. 
Los resultados mostrados en la tabla V.11, ponen de manifiesto el carácter inductivo de la potencia de desfase, así como la coincidencia de valores con los mostrados en las tablas V.1, 3, 5, 7 y 9.

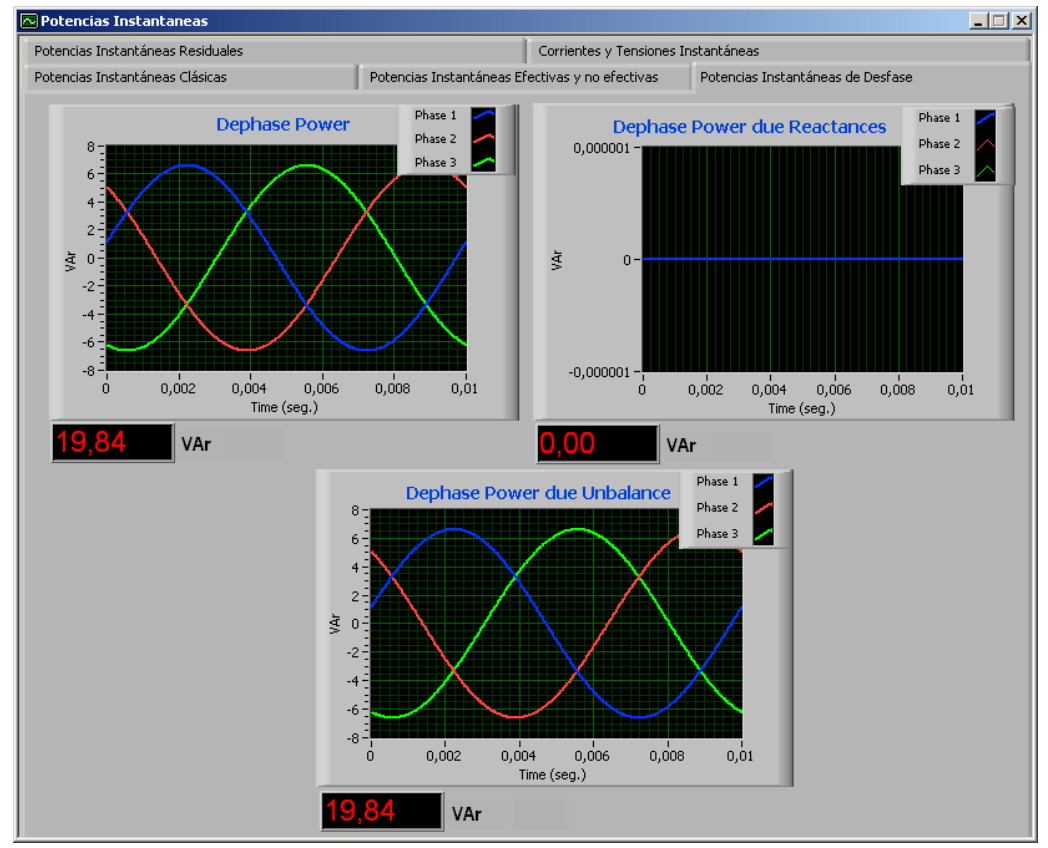

Figura V.23. Pantalla de Potencias de Desfase. 
Carga resistiva entre la fase 3 y el neutro, con un consumo de $1 \angle 120^{\circ} \mathrm{A}, \mathrm{y}$ producimos el desequilibrio sumando ángulos negativos al argumento de la fase 2.

Introducimos los datos en el SIMPELEC Simulador, como mostramos en la figura V.24.

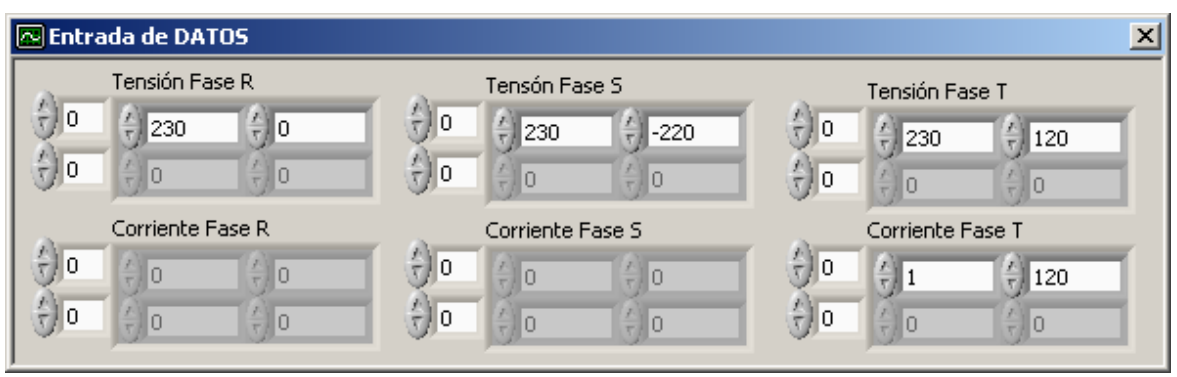

Figura V.24. Pantalla de entrada de datos.

Un sistema de tensiones en el que dejamos fijos los módulos de las fases, y tenemos una carga resistiva pura que consume $1 \angle 120^{\circ} A$ conectada en la fase 3, y tan sólo variamos de manera gradual el argumento de la fase 2, logrando los pertinentes desequilibrios de tensión y produciendo la consiguiente potencia de desfase, tal y como podemos observar en la tabla V.12, y en la figura V.25 para el caso concreto de que el argumento de la fase 2 tome el valor de $-220^{\circ}$.

\begin{tabular}{||c|c|c||}
\hline $\begin{array}{c}\text { Argumento } \\
\text { Fase 2 }\end{array}$ & $\begin{array}{c}\text { Variación } \\
\text { Argumento ( }\end{array}$ & $\begin{array}{c}\text { Potencia Desfase } \\
\text { Desequilibrio } \mathbf{Q}_{\text {dru }} \text { (VAr) }\end{array}$ \\
\hline$-121^{\circ}$ & 1 & $-1,34$ \\
\hline$-122^{\circ}$ & 2 & $-2,68$ \\
\hline$-125^{\circ}$ & 5 & $-6,68$ \\
\hline$-130^{\circ}$ & 10 & $-13,31$ \\
\hline$-135^{\circ}$ & 15 & $-19,84$ \\
\hline$-145^{\circ}$ & 25 & $-32,40$ \\
\hline$-155^{\circ}$ & 35 & $-43,97$ \\
\hline$-170^{\circ}$ & 50 & $-58,73$ \\
\hline$-190^{\circ}$ & 70 & $-72,04$ \\
\hline$-210^{\circ}$ & 90 & $-76,67$ \\
\hline$-220^{\circ}$ & 100 & $-75,50$ \\
\hline$-230^{\circ}$ & 110 & $-72,04$ \\
\hline$-240^{\circ}$ & 120 & $-66,40$ \\
\hline
\end{tabular}

Tabla V.12. Potencia de Desfase \& Variación Argumento. 
En la tabla V.12, nos encontramos con resultados que nos indican el consumo de una potencia de desfase capacitiva, como sucedía en las tablas V.2, 4, 6, 8 y 10.

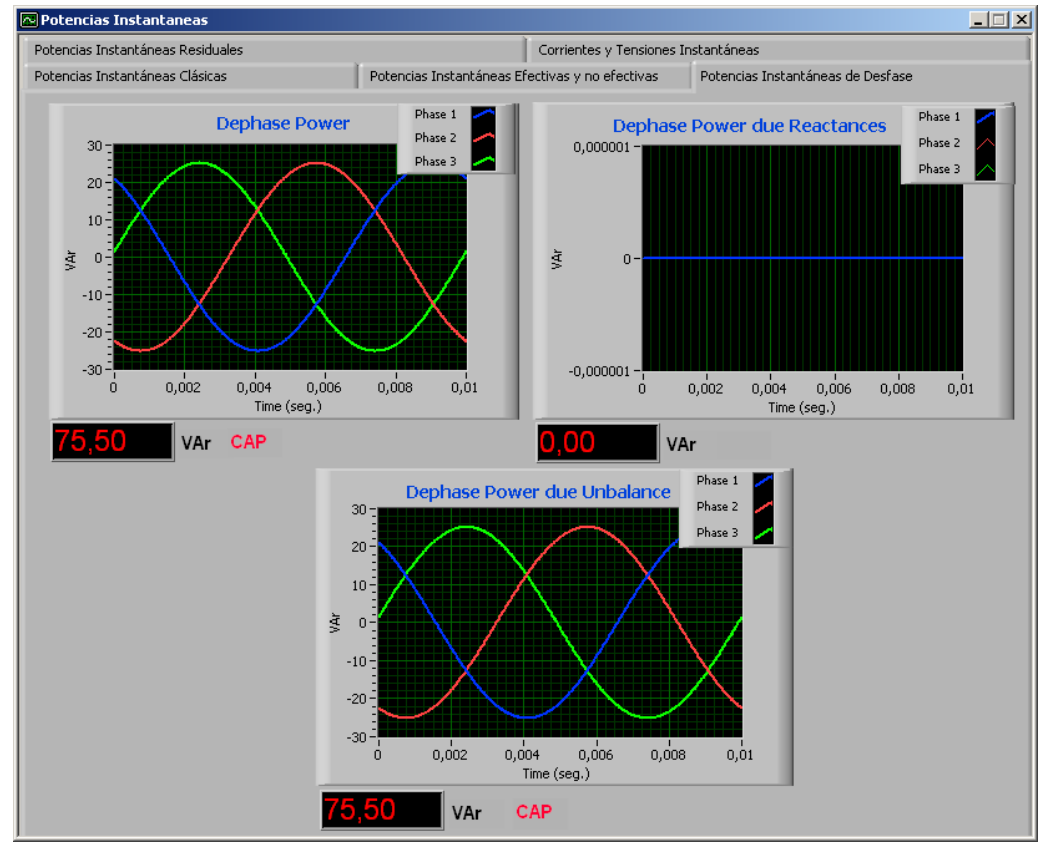

Figura V.25. Pantalla de Potencias de Desfase.

En la figura V.26, se pone de manifiesto lo comentado en las diversas gráficas y tablas vistas en este apartado, para el caso de tener conectada una carga resistiva en las diversas fases de un sistema trifásico a cuatro hilos y con diferentes grados de desequilibrio.

En ella se evidencia que con independencia de que en que fase se halle conectada la carga, se obtienen los mismos valores de potencia de desfase cuando provocamos los mismos grados de desequilibrio en base a variar los argumentos de la fase descargada. Y además, siempre que procedamos a sumar ángulos positivos a las fases descargadas, la potencia de desfase posee carácter inductivo y por el contrario si logramos el grado de desequilibrio del sistema en base a sumar ángulos negativos a las fases descargadas, la potencia de desfase obtenida tiene carácter capacitivo. 
En la figura V.26, observamos además diversas gráficas de la potencia de desfase para diversos casos en los que el consumo de la fase cargada sea de 1 , 2 y 4 Amperios, manteniéndose la linealidad de los valores obtenidos al trabajar con un sistema lineal, para cualquier otro valor de carga conectada y sometida al mismo grado de desequilibrio.

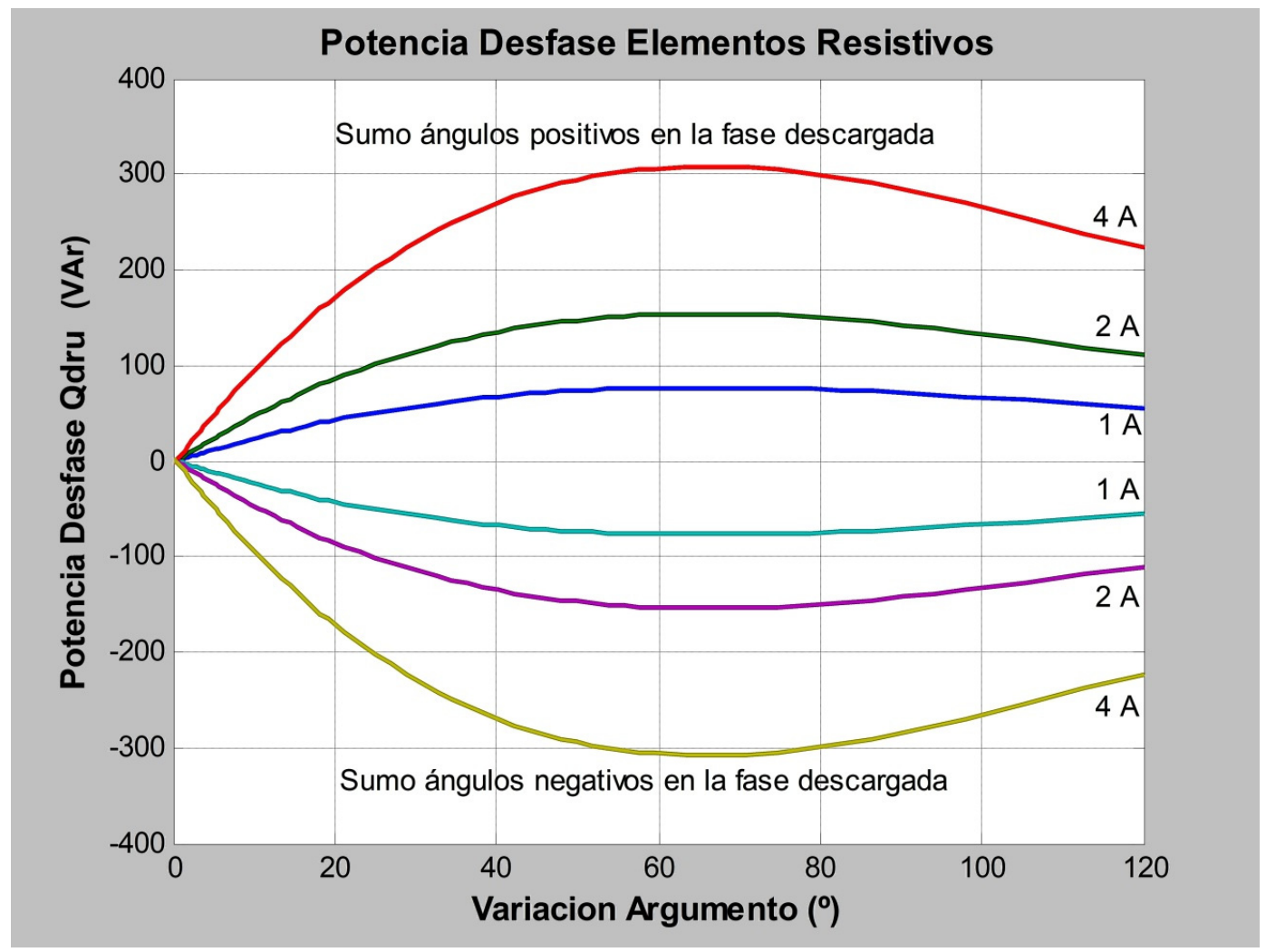

Figura V.26. Potencias de Desfase, para diversas variaciones de carga resistiva en una fase y grados de desequilibrio. 
b) Carga Inductiva: Se analiza el comportamiento de este elemento frente a diversos desequilibrios que se producen, estando esta carga de manera sucesiva en cada fase.

Una carga inductiva entre la fase 1 y el neutro, con un consumo de $1 \angle-45^{\circ} A$, y producimos el desequilibrio sumando ángulos positivos al argumento de la fase 2.

Introducimos los datos en el SIMPELEC Simulador, tal y como mostramos en la figura V.27.

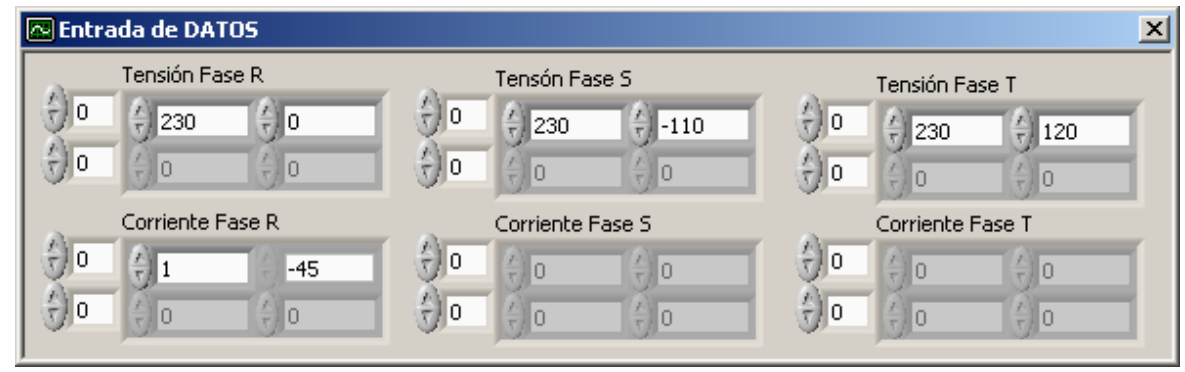

Figura V.27. Pantalla de entrada de datos.

Tenemos pues un sistema de tensiones en el que dejamos fijos los módulos de las tensiones de las fases, y tenemos una carga reactiva inductiva que consume $1 \angle-45^{\circ} A$ conectada en la fase $1, y$ tan sólo variamos de manera gradual el argumento de la fase 2, logrando los pertinentes desequilibrios de tensión y produciendo la consiguiente potencia de desfase, tal y como podemos observar en la tabla V.13, y en la figura V.28 para el caso concreto de que el argumento de la fase 2 tome el valor de $-110^{\circ}$. 


\begin{tabular}{|c|c|c||}
\hline $\begin{array}{c}\text { Argumento } \\
\text { Fase 2 }\end{array}$ & $\begin{array}{c}\text { Variación } \\
\text { Argumento } \mathbf{(}^{\mathbf{0}} \text { ) }\end{array}$ & $\begin{array}{c}\text { Potencia Desfase } \\
\text { Desequilibrio } \mathbf{Q}_{\text {dru }} \text { (VAr) }\end{array}$ \\
\hline$-119^{\circ}$ & 1 & 0,95 \\
\hline$-118^{\circ}$ & 2 & 1,90 \\
\hline$-115^{\circ}$ & 5 & 4,79 \\
\hline$-110^{\circ}$ & 10 & 9,69 \\
\hline$-105^{\circ}$ & 15 & 14,65 \\
\hline$-95^{\circ}$ & 25 & 24,60 \\
\hline$-85^{\circ}$ & 35 & 34,36 \\
\hline$-70^{\circ}$ & 50 & 47,98 \\
\hline$-50^{\circ}$ & 70 & 62,83 \\
\hline$-30^{\circ}$ & 90 & 72,28 \\
\hline$-20^{\circ}$ & 100 & 74,60 \\
\hline$-10^{\circ}$ & 110 & 75,19 \\
\hline $0^{\circ}$ & 120 & 74,05 \\
\hline
\end{tabular}

Tabla V.13. Potencia de Desfase \& Variación Argumento.

De los resultados de la tabla V.13, observamos que al producir modificaciones en el argumento de la fase 2, llevando esta desde $-120 \circ$ a $0^{\circ}$, se pone de manifiesto el consumo de potencia de desfase de carácter inductivo procedente del desequilibrio del sistema, que en este caso se suma a la consumida por la propia presencia de una carga inductiva, que también observamos en la figura V.28. El ratio de incremento de esta potencia de desfase debida al desequilibrio, se cuantifica en 0,95 VAr, con la variación de un grado (10) en el argumento, manteniéndose esta linealidad en todo el tramo de variación provocado.

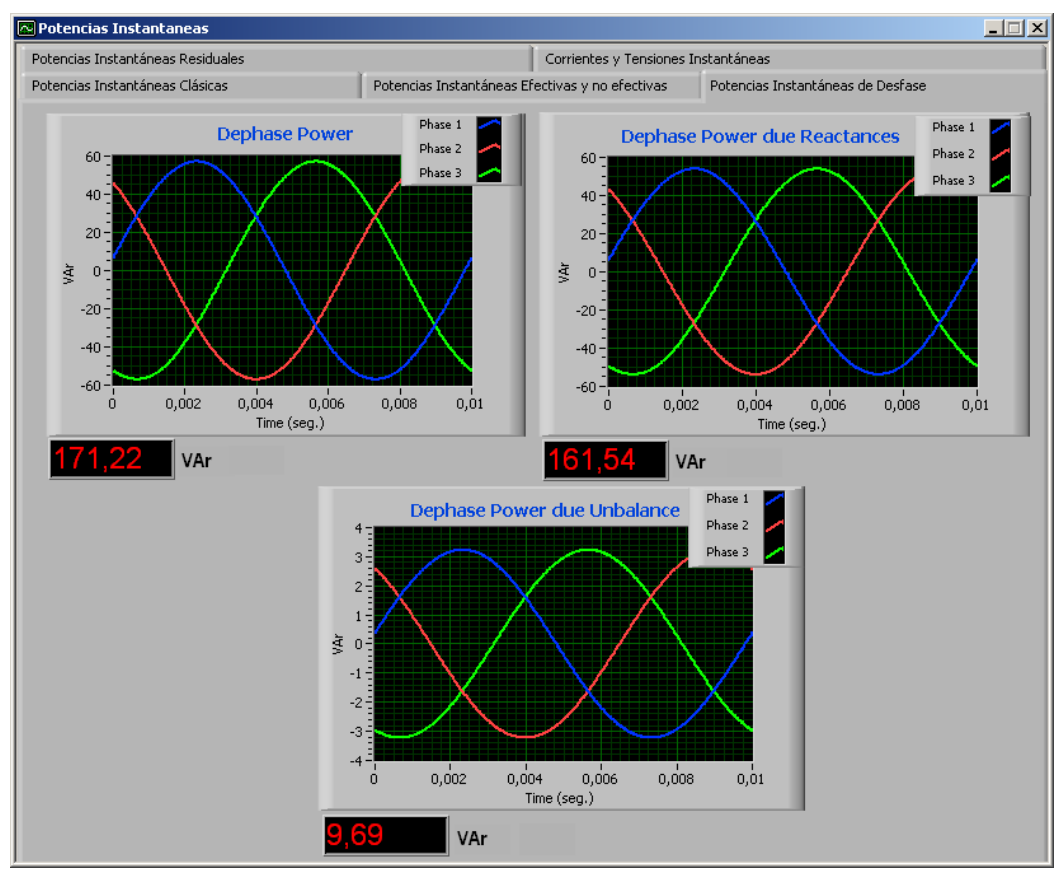

Figura V.28. Pantalla de Potencias de Desfase. 
Carga inductiva entre la fase 1 y el neutro, con un consumo de $1 \angle-45^{\circ} A, y$ producimos el desequilibrio sumando ángulos negativos al argumento de la fase 2.

Introducimos los datos en el SIMPELEC Simulador, tal y como mostramos en la figura V.29.

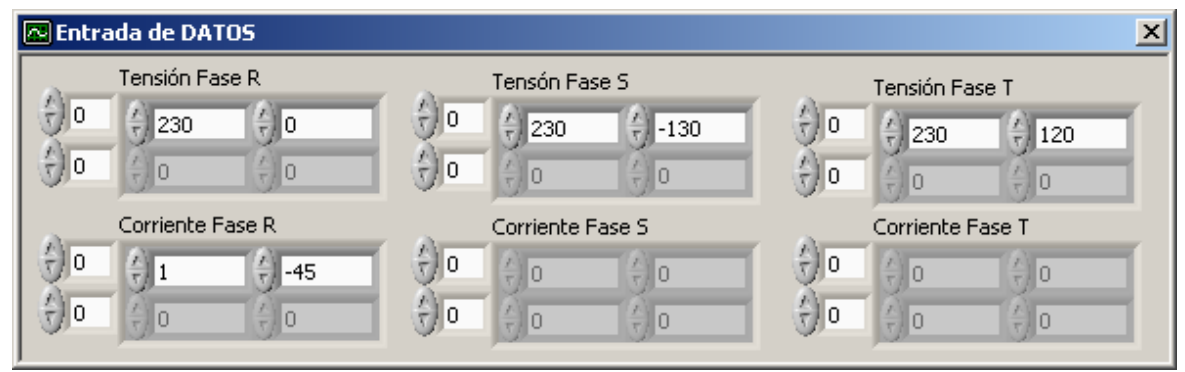

Figura V.29. Pantalla de entrada de datos.

Tenemos pues un sistema de tensiones en el que dejamos fijos los módulos de las tensiones de las fases, y tenemos una carga reactiva inductiva que consume $1 \angle-45^{\circ} A$ conectada en la fase $1, y$ tan sólo variamos de manera gradual el argumento de la fase 2, logrando los pertinentes desequilibrios de tensión y produciendo la consiguiente potencia de desfase, tal y como podemos observar en la tabla V.14, y en la figura V.30 para el caso de que el argumento de la fase 2 valga $-130^{\circ}$.

\begin{tabular}{||c|c|c||}
\hline $\begin{array}{c}\text { Argumento } \\
\text { Fase 2 }\end{array}$ & $\begin{array}{c}\text { Variación } \\
\left.\text { Argumento ( } \mathbf{(}^{\circ}\right)\end{array}$ & $\begin{array}{c}\text { Potencia Desfase } \\
\text { Desequilibrio } \mathbf{Q}_{\text {dru }} \text { (VAr) }\end{array}$ \\
\hline$-121^{\circ}$ & 1 & $-0,94$ \\
\hline$-122^{\circ}$ & 2 & $-1,88$ \\
\hline$-125^{\circ}$ & 5 & $-4,66$ \\
\hline$-130^{\circ}$ & 10 & $-9,14$ \\
\hline$-135^{\circ}$ & 15 & $-13,42$ \\
\hline$-145^{\circ}$ & 25 & $-21,22$ \\
\hline$-155^{\circ}$ & 35 & $-27,83$ \\
\hline$-170^{\circ}$ & 50 & $-35,07$ \\
\hline$-190^{\circ}$ & 70 & $-39,05$ \\
\hline$-210^{\circ}$ & 90 & $-36,14$ \\
\hline$-220^{\circ}$ & 100 & $-32,18$ \\
\hline$-230^{\circ}$ & 110 & $-26,69$ \\
\hline$-240^{\circ}$ & 120 & $-19,84$ \\
\hline \multicolumn{2}{|r|}{} \\
\hline
\end{tabular}

Tabla V.14. Potencia de Desfase \& Variación Argumento. 
De la observación de la tabla V.14, se pone de manifiesto por un lado la presencia de potencia de desfase debida al desequilibrio con carácter capacitivo, aún siendo la carga inductiva, y que el ratio que cuantifica la variación producida por un grado de variación en el argumento de la fase, es ahora de 0,94 VAr. La presencia de esta potencia de desfase debida al desequilibrio, observamos que produce que la carga en cuestión posea un comportamiento menos inductivo del esperado, lo que queda de manifiesto en la figura V.30.

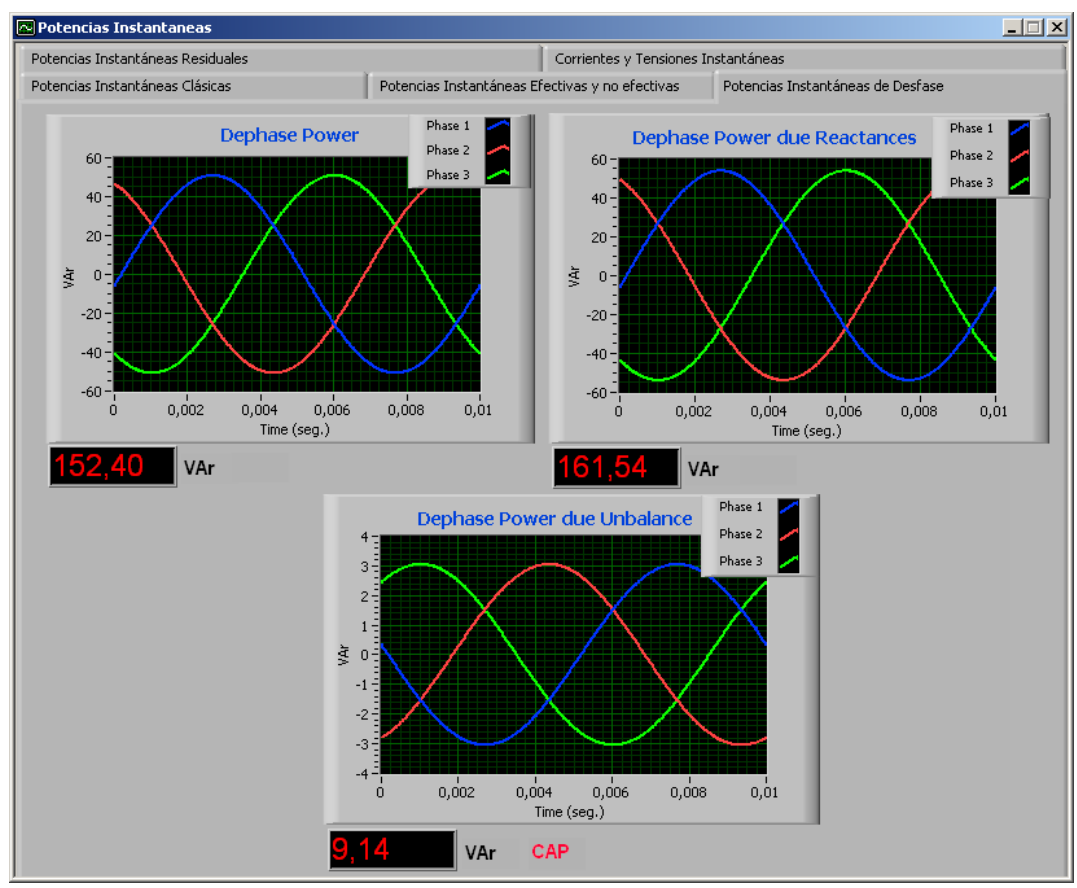

Figura V.30. Pantalla de Potencias de Desfase. 
Carga inductiva entre la fase 1 y el neutro, con un consumo de $1 \angle-45^{\circ} A, y$ producimos el desequilibrio sumando ángulos positivos al argumento de la fase 3.

Introducimos los datos en el SIMPELEC Simulador, tal y como mostramos en la figura V.31.

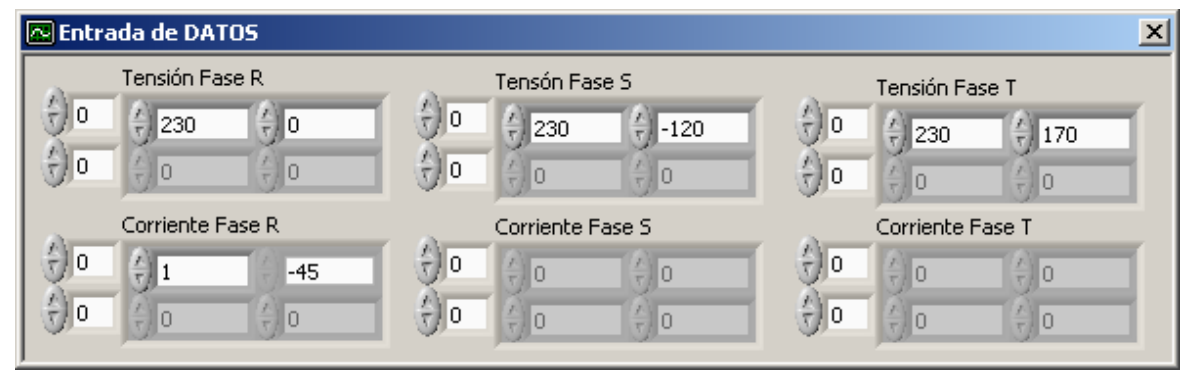

Figura V.31. Pantalla de entrada de datos.

Tenemos pues un sistema de tensiones en el que dejamos fijos los módulos de las tensiones de las fases, y tenemos una carga reactiva inductiva que consume $1 \angle-45^{\circ} A$ conectada en la fase $1, y$ tan sólo variamos de manera gradual el argumento de la fase 3, logrando los pertinentes desequilibrios de tensión y produciendo la consiguiente potencia de desfase, tal y como podemos observar en la tabla V.15, y en la figura V.32 para el caso concreto de que el argumento de la fase 3 tome el valor de $170^{\circ}$.

\begin{tabular}{||c|c|c||}
\hline $\begin{array}{c}\text { Argumento } \\
\text { Fase 3 }\end{array}$ & $\begin{array}{c}\text { Variación } \\
\text { Argumento ( } \mathbf{(}^{\circ}\end{array}$ & $\begin{array}{c}\text { Potencia Desfase } \\
\text { Desequilibrio } \mathbf{Q}_{\text {dru }} \text { (VAr) }\end{array}$ \\
\hline $121^{\circ}$ & 1 & 0,95 \\
\hline $122^{\circ}$ & 2 & 1,90 \\
\hline $125^{\circ}$ & 5 & 4,79 \\
\hline $130^{\circ}$ & 10 & 9,69 \\
\hline $135^{\circ}$ & 15 & 14,65 \\
\hline $145^{\circ}$ & 25 & 24,60 \\
\hline $155^{\circ}$ & 35 & 34,36 \\
\hline $170^{\circ}$ & 50 & 47,98 \\
\hline $190^{\circ}$ & 70 & 62,83 \\
\hline $210^{\circ}$ & 90 & 72,28 \\
\hline $220^{\circ}$ & 100 & 74,60 \\
\hline $230^{\circ}$ & 110 & 75,19 \\
\hline $240^{\circ}$ & 120 & 74,05 \\
\hline \multicolumn{2}{|r|}{} \\
\hline \multicolumn{2}{|r|}{} \\
\hline
\end{tabular}

Tabla V.15. Potencia de Desfase \& Variación Argumento. 
Los resultados mostrados en la tabla V.15, nos muestran que se produce un consumo de potencia de desfase con carácter inductivo, y el ratio que nos cuantifica esta variación de la potencia en función del desequilibrio del sistema coincide con lo obtenido en la tabla V.13.

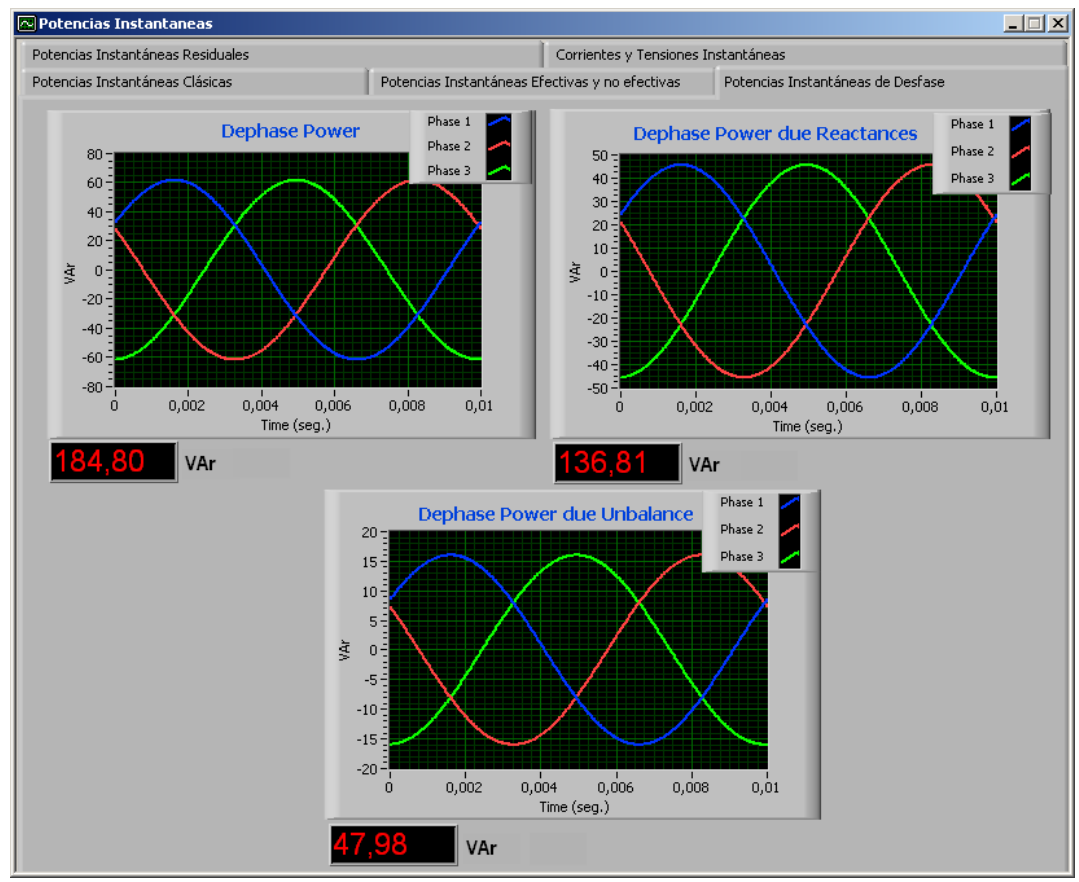

Figura V.32. Pantalla de Potencias de Desfase. 
Carga inductiva entre la fase 1 y el neutro, con un consumo de $1 \angle-45^{\circ} A, y$ producimos el desequilibrio sumando ángulos negativos al argumento de la fase 3.

Introducimos los datos en el SIMPELEC Simulador, tal y como mostramos en la figura V.33.

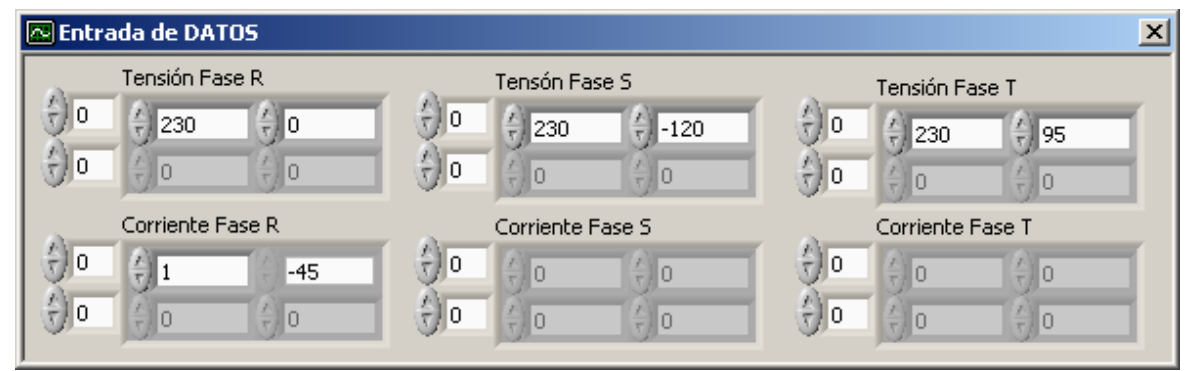

Figura V.33. Pantalla de entrada de datos.

Tenemos pues un sistema de tensiones en el que dejamos fijos los módulos de las tensiones de las fases, y tenemos una carga reactiva inductiva que consume $1 \angle-45^{\circ} A$ conectada en la fase $1, y$ tan sólo variamos de manera gradual el argumento de la fase 3, logrando los pertinentes desequilibrios de tensión y produciendo la consiguiente potencia de desfase, tal y como podemos observar en la tabla V.16, y en la figura V.34 cuando el argumento de la fase 3 adquiere el valor de $95^{\circ}$.

\begin{tabular}{||c|c|c||}
\hline $\begin{array}{c}\text { Argumento } \\
\text { Fase 3 }\end{array}$ & $\begin{array}{c}\text { Variación } \\
\left.\text { Argumento ( } \mathbf{(}^{\circ}\right)\end{array}$ & $\begin{array}{c}\text { Potencia Desfase } \\
\text { Desequilibrio } \mathbf{Q}_{\text {dru }} \text { (VAr) }\end{array}$ \\
\hline $119^{\circ}$ & 1 & $-0,94$ \\
\hline $118^{\circ}$ & 2 & $-1,88$ \\
\hline $115^{\circ}$ & 5 & $-4,66$ \\
\hline $110^{\circ}$ & 10 & $-9,14$ \\
\hline $105^{\circ}$ & 15 & $-13,42$ \\
\hline $95^{\circ}$ & 25 & $-21,22$ \\
\hline $85^{\circ}$ & 35 & $-27,83$ \\
\hline $70^{\circ}$ & 50 & $-35,07$ \\
\hline $50^{\circ}$ & 70 & $-39,05$ \\
\hline $30^{\circ}$ & 90 & $-36,14$ \\
\hline $20^{\circ}$ & 100 & $-32,18$ \\
\hline $10^{\circ}$ & 110 & $-26,69$ \\
\hline $0^{\circ}$ & 120 & $-19,84$ \\
\hline
\end{tabular}

Tabla V.16. Potencia de Desfase \& Variación Argumento. 
En la tabla V.16, se puede apreciar que con las variaciones provocadas en el argumento de la fase 3 , entre $120^{\circ}$ y $0^{\circ}$, provoca la presencia de una potencia de desfase de carácter capacitivo, y cuyos valores son idénticos a los obtenidos y mostrados en la tabla V.14, provocando que la potencia de desfase total del sistema sea menor que si no existieran desequilibrios en el sistema que provocan la presencia de la potencia de desfase debida al desequilibrio.

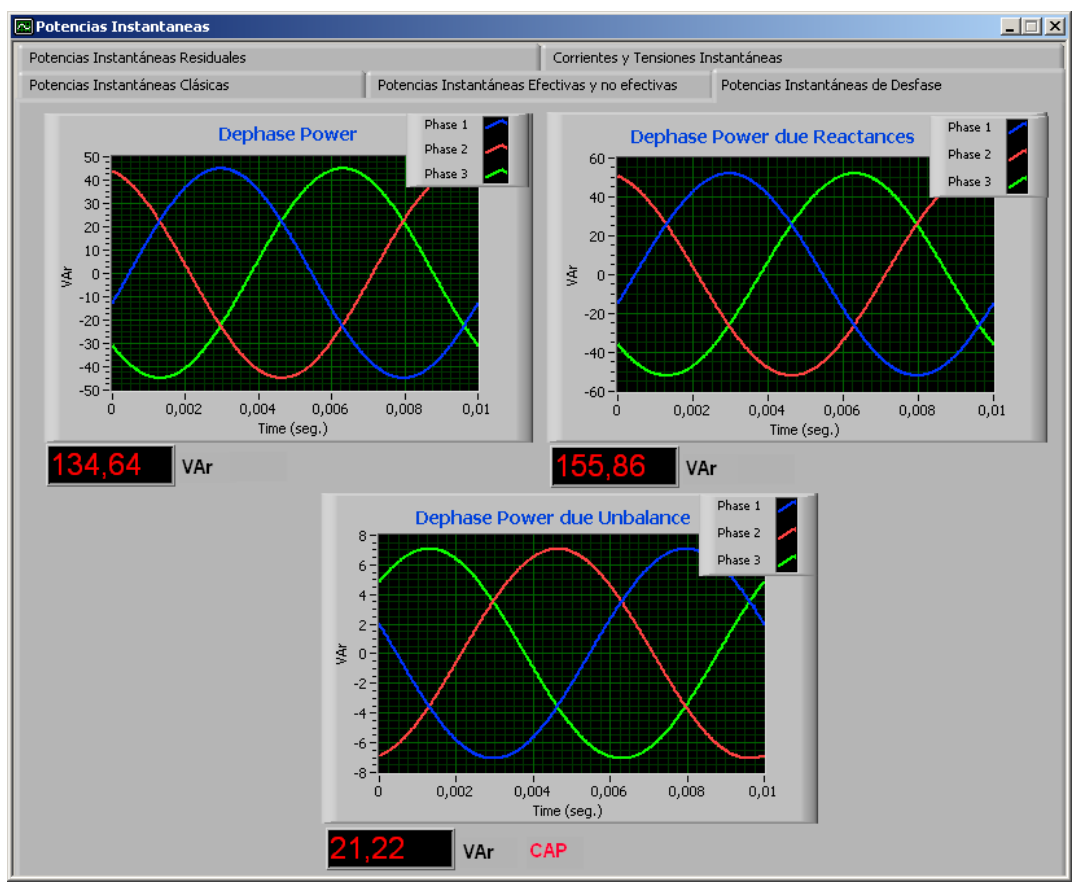

Figura V.34. Pantalla de Potencias de Desfase. 
Carga inductiva entre la fase 2 y el neutro, con un consumo de $1 \angle-165^{\circ} A, y$ producimos el desequilibrio sumando ángulos positivos al argumento de la fase 1.

Introducimos los datos en el SIMPELEC Simulador, tal y como mostramos en la figura V.35.

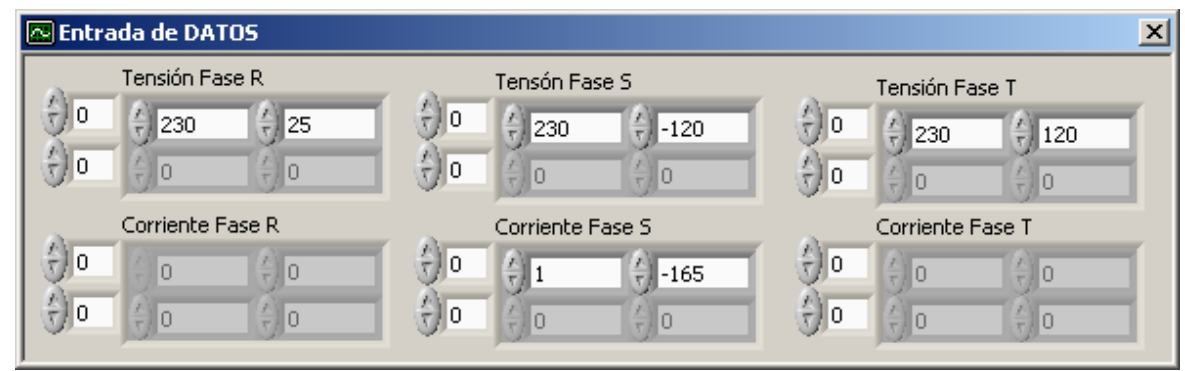

Figura V.35. Pantalla de entrada de datos.

Tenemos pues un sistema de tensiones en el que dejamos fijos los módulos de las tensiones de las fases, y tenemos una carga reactiva inductiva que consume $1 \angle-165^{\circ} A$ conectada en la fase 2 , y tan sólo variamos de manera gradual el argumento de la fase 1, logrando los pertinentes desequilibrios de tensión y produciendo la consiguiente potencia de desfase, tal y como podemos observar en la tabla V.17, y en la figura V.36 para el caso concreto de que el argumento de la fase 1 valga $25^{\circ}$.

\begin{tabular}{||c|c|c||}
\hline $\begin{array}{c}\text { Argumento } \\
\text { Fase 1 }\end{array}$ & $\begin{array}{c}\text { Variación } \\
\text { Argumento ( } \mathbf{(}^{\circ}\end{array}$ & $\begin{array}{c}\text { Potencia Desfase } \\
\text { Desequilibrio } \mathbf{Q}_{\text {dru }} \text { (VAr) }\end{array}$ \\
\hline $1^{\circ}$ & 1 & 0,95 \\
\hline $2^{\circ}$ & 2 & 1,90 \\
\hline $5^{\circ}$ & 5 & 4,79 \\
\hline $10^{\circ}$ & 10 & 9,69 \\
\hline $15^{\circ}$ & 15 & 14,65 \\
\hline $25^{\circ}$ & 25 & 24,60 \\
\hline $35^{\circ}$ & 35 & 34,36 \\
\hline $50^{\circ}$ & 50 & 47,98 \\
\hline $70^{\circ}$ & 70 & 62,83 \\
\hline $90^{\circ}$ & 90 & 72,28 \\
\hline $100^{\circ}$ & 100 & 74,60 \\
\hline $110^{\circ}$ & 110 & 75,19 \\
\hline $120^{\circ}$ & 120 & 74,05 \\
\hline \multicolumn{2}{|r|}{} \\
\hline
\end{tabular}

Tabla V.17. Potencia de Desfase \& Variación Argumento. 
De los resultados mostrados en la tabla V.17, observamos que la posición en la cual esta ubicada la carga, es independiente a la hora de producirse un consumo de potencia de desfase debida al desequilibrio, mientras provoquemos los mismos grados de desequilibrio, ya que los valores de potencia de desfase inductiva que obtenemos en esta ocasión son iguales a los obtenidos en las tablas V.13 y 15 .

En la figura V.36, podemos ver que esta potencia de desfase debida al desequilibrio del sistema, al poseer carácter inductivo, se suma a la debida al propio consumo de la carga inductiva.

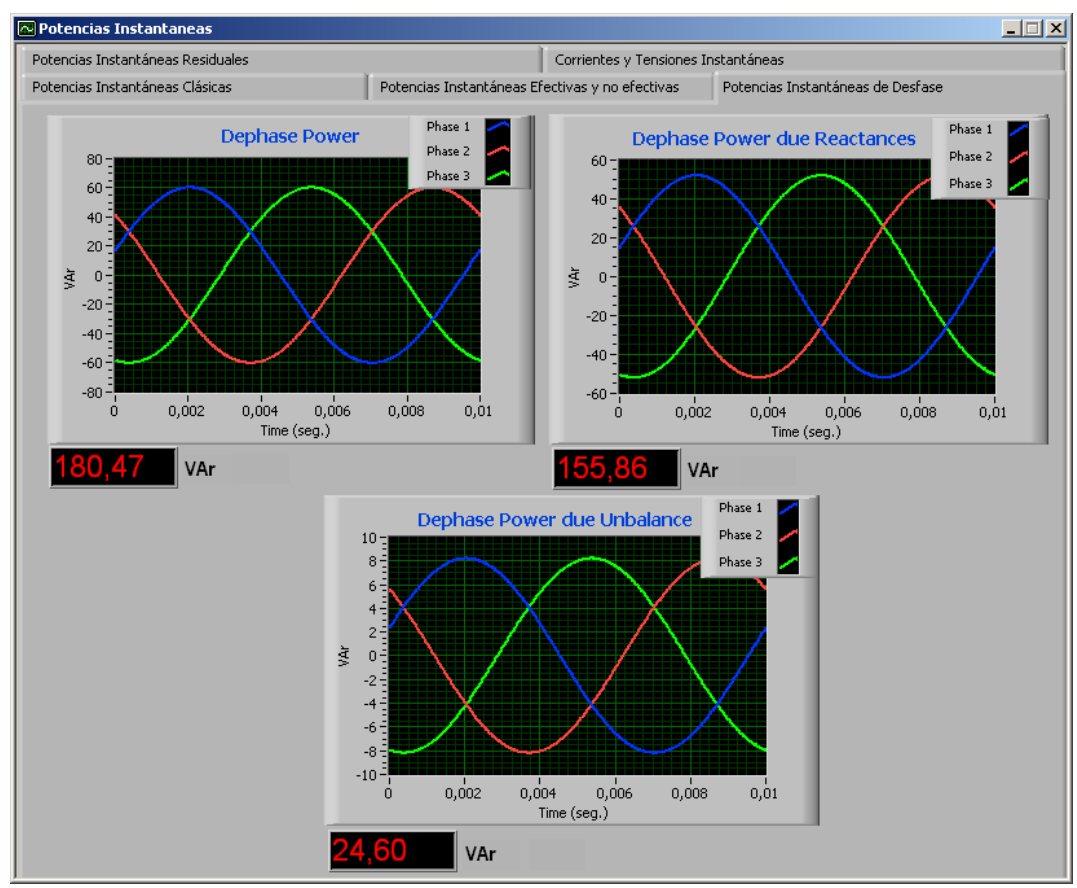

Figura V.36. Pantalla de Potencias de Desfase. 
Carga inductiva entre la fase 2 y el neutro, con un consumo de $1 \angle-165^{\circ} A, y$ producimos el desequilibrio sumando ángulos negativos al argumento de la fase 1.

Introducimos los datos en el SIMPELEC Simulador, tal y como mostramos en la figura V.37.

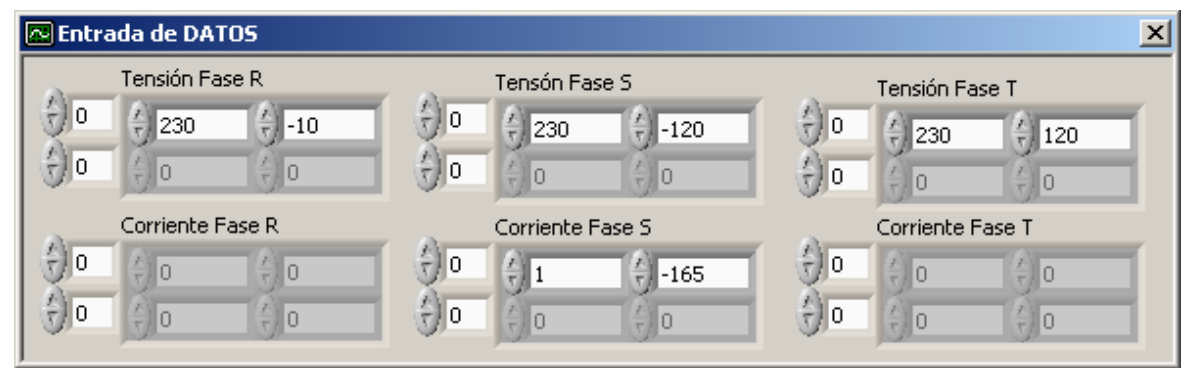

Figura V.37. Pantalla de entrada de datos.

Tenemos pues un sistema de tensiones en el que dejamos fijos los módulos de las tensiones de las fases, y tenemos una carga reactiva inductiva que consume $1 \angle-165^{\circ} A$ conectada en la fase 2 , y tan sólo variamos de manera gradual el argumento de la fase 1, logrando los pertinentes desequilibrios de tensión y produciendo la consiguiente potencia de desfase, tal y como podemos observar en la tabla V.18, y en la figura V.38 el caso particular de que el argumento de la fase 1 tome el valor de $-10^{\circ}$.

\begin{tabular}{||c|c|c||}
\hline $\begin{array}{c}\text { Argumento } \\
\text { Fase 1 }\end{array}$ & $\begin{array}{c}\text { Variación } \\
\left.\text { Argumento ( } \mathbf{(}^{\mathbf{}}\right)\end{array}$ & $\begin{array}{c}\text { Potencia Desfase } \\
\text { Desequilibrio } \mathbf{Q}_{\text {dru }} \text { (VAr) }\end{array}$ \\
\hline$-1^{\circ}$ & 1 & $-0,94$ \\
\hline$-2^{\circ}$ & 2 & $-1,88$ \\
\hline$-5^{\circ}$ & 5 & $-4,66$ \\
\hline$-10^{\circ}$ & 10 & $-9,14$ \\
\hline$-15^{\circ}$ & 15 & $-13,42$ \\
\hline$-25^{\circ}$ & 25 & $-21,22$ \\
\hline$-35^{\circ}$ & 35 & $-27,83$ \\
\hline$-50^{\circ}$ & 50 & $-35,07$ \\
\hline$-70^{\circ}$ & 70 & $-39,05$ \\
\hline$-90^{\circ}$ & 90 & $-36,14$ \\
\hline$-100^{\circ}$ & 100 & $-32,18$ \\
\hline$-110^{\circ}$ & 110 & $-26,69$ \\
\hline$-120^{\circ}$ & 120 & $-19,84$ \\
\hline
\end{tabular}

Tabla V.18. Potencia de Desfase \& Variación Argumento. 
En la tabla V.18, vemos los resultados de variar el argumento de la fase 1, que provoca una potencia de desfase debida al desequilibrio capacitiva, disminuyendo el carácter inductivo del receptor, como sucedía en las tablas V.14 y 16.

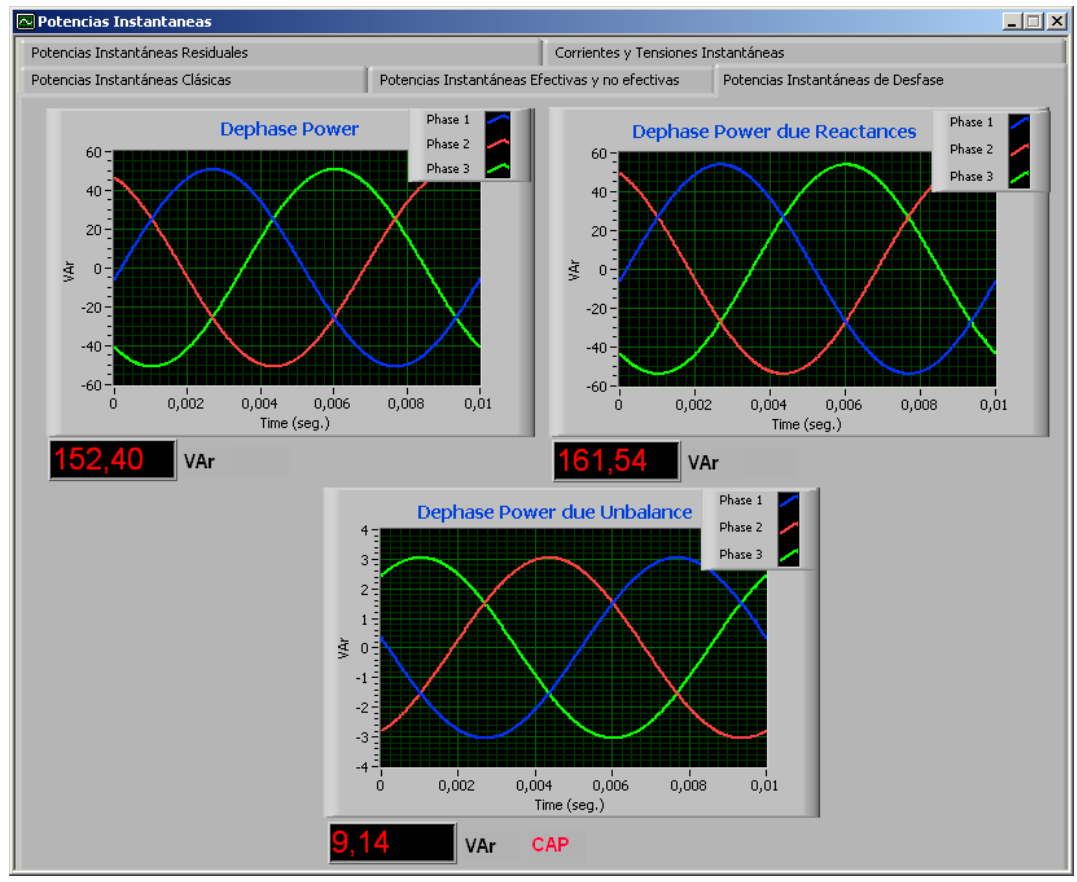

Figura V.38. Pantalla de Potencias de Desfase. 
Carga inductiva entre la fase 2 y el neutro, con un consumo de $1 \angle-165^{\circ} A, y$ producimos el desequilibrio sumando ángulos positivos al argumento de la fase 3.

Introducimos los datos en el SIMPELEC Simulador, tal y como mostramos en la figura V.39.

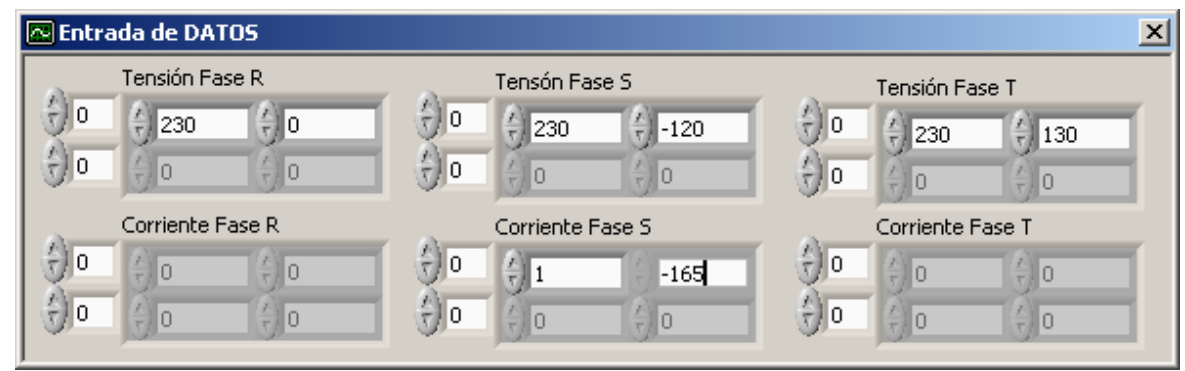

Figura V.39. Pantalla de entrada de datos.

Tenemos pues un sistema de tensiones en el que dejamos fijos los módulos de las tensiones de las fases, y tenemos una carga reactiva inductiva que consume $1 \angle-165^{\circ} A$ conectada en la fase 2 , y tan sólo variamos de manera gradual el argumento de la fase 3, logrando los pertinentes desequilibrios de tensión y produciendo la consiguiente potencia de desfase, tal y como podemos observar en la tabla V.19, y en la figura V.40 para el caso concreto de que el argumento de la fase 3 sea de $130^{\circ}$.

\begin{tabular}{||c|c|c||}
\hline $\begin{array}{c}\text { Argumento } \\
\text { Fase 3 }\end{array}$ & $\begin{array}{c}\text { Variación } \\
\text { Argumento ( } \mathbf{(}^{\circ}\end{array}$ & $\begin{array}{c}\text { Potencia Desfase } \\
\text { Desequilibrio } \mathbf{Q}_{\text {dru }} \text { (VAr) }\end{array}$ \\
\hline $121^{\circ}$ & 1 & 0,95 \\
\hline $122^{\circ}$ & 2 & 1,90 \\
\hline $125^{\circ}$ & 5 & 4,79 \\
\hline $130^{\circ}$ & 10 & 9,69 \\
\hline $135^{\circ}$ & 15 & 14,65 \\
\hline $145^{\circ}$ & 25 & 24,60 \\
\hline $155^{\circ}$ & 35 & 34,36 \\
\hline $170^{\circ}$ & 50 & 47,98 \\
\hline $190^{\circ}$ & 70 & 62,83 \\
\hline $210^{\circ}$ & 90 & 72,28 \\
\hline $220^{\circ}$ & 100 & 74,60 \\
\hline $230^{\circ}$ & 110 & 75,19 \\
\hline $240^{\circ}$ & 120 & 74,05 \\
\hline \multicolumn{2}{|r|}{} \\
\hline \multicolumn{2}{|r|}{} \\
\hline
\end{tabular}

Tabla V.19. Potencia de Desfase \& Variación Argumento. 
La tabla V.19, nos muestra los resultados de que la potencia de desfase debida al desequilibrio posee carácter inductivo, que se suma a la debida a la existencia de un elemento inductivo en el sistema, lo que apreciamos en la figura V.40 y como habíamos observado también en la tablas V.13, 15 y 17.

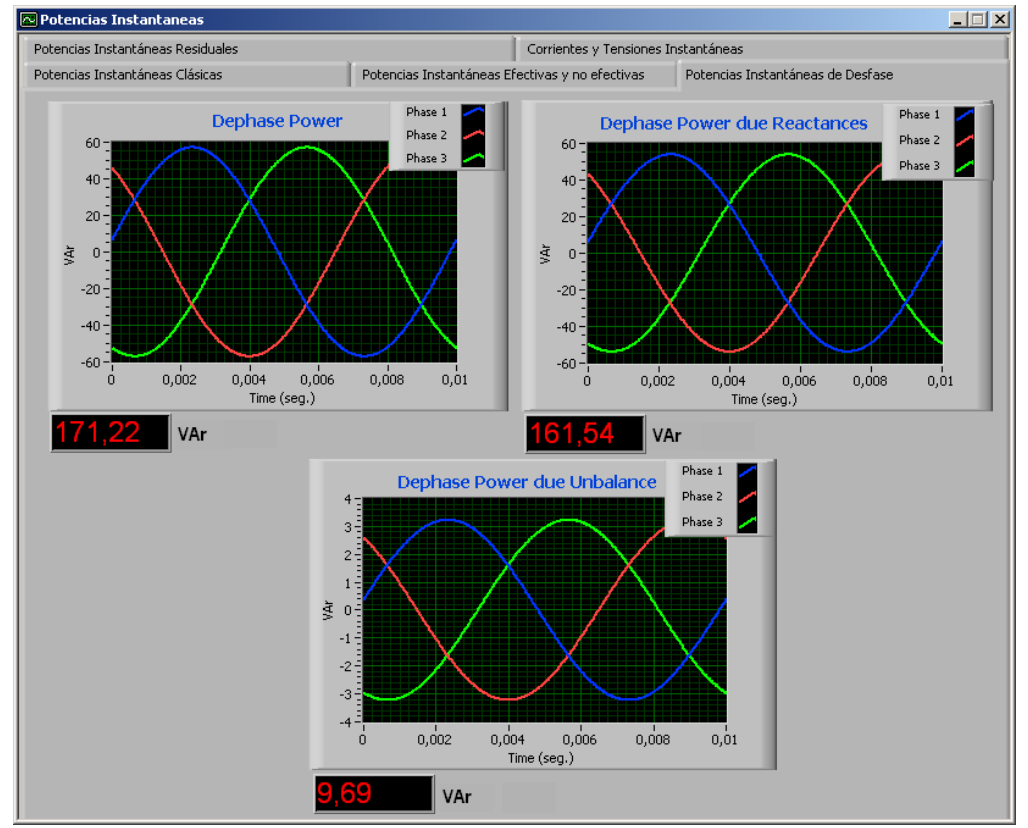

Figura V.40. Pantalla de Potencias de Desfase. 
Carga inductiva entre la fase 2 y el neutro, con un consumo de $1 \angle-165^{\circ} A, y$ producimos el desequilibrio sumando ángulos negativos al argumento de la fase 3.

Introducimos los datos en el SIMPELEC Simulador, tal y como mostramos en la figura V.41.

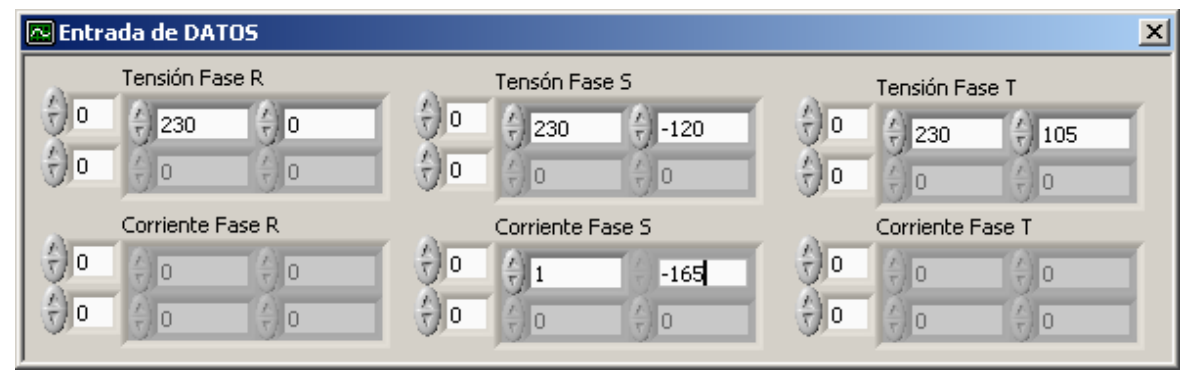

Figura V.41. Pantalla de entrada de datos.

Tenemos pues un sistema de tensiones en el que dejamos fijos los módulos de las tensiones de las fases, y tenemos una carga reactiva inductiva que consume $1 \angle-165^{\circ} A$ conectada en la fase 2 , y tan sólo variamos de manera gradual el argumento de la fase 3, logrando los pertinentes desequilibrios de tensión y produciendo la consiguiente potencia de desfase, tal y como podemos observar en la tabla V.20, y en la figura V.42 para el caso de que el argumento de la fase 3 tome el valor de $105^{\circ}$.

\begin{tabular}{||c|c|c||}
\hline $\begin{array}{c}\text { Argumento } \\
\text { Fase 3 }\end{array}$ & $\begin{array}{c}\text { Variación } \\
\text { Argumento ( } \mathbf{(}^{\circ}\end{array}$ & $\begin{array}{c}\text { Potencia Desfase } \\
\text { Desequilibrio } \mathbf{Q}_{\text {dru }} \text { (VAr) }\end{array}$ \\
\hline $119^{\circ}$ & 1 & $-0,94$ \\
\hline $118^{\circ}$ & 2 & $-1,88$ \\
\hline $115^{\circ}$ & 5 & $-4,66$ \\
\hline $110^{\circ}$ & 10 & $-9,14$ \\
\hline $105^{\circ}$ & 15 & $-13,42$ \\
\hline $95^{\circ}$ & 25 & $-21,22$ \\
\hline $85^{\circ}$ & 35 & $-27,83$ \\
\hline $70^{\circ}$ & 50 & $-35,07$ \\
\hline $50^{\circ}$ & 70 & $-39,05$ \\
\hline $30^{\circ}$ & 90 & $-36,14$ \\
\hline $20^{\circ}$ & 100 & $-32,18$ \\
\hline $10^{\circ}$ & 110 & $-26,69$ \\
\hline $0^{\circ}$ & 120 & $-19,84$ \\
\hline \multicolumn{2}{|c|}{} \\
\hline \multicolumn{2}{|l}{} \\
\hline
\end{tabular}

Tabla V.20. Potencia de Desfase \& Variación Argumento. 
En la tabla V.20, apreciamos el carácter capacitivo que tiene la potencia de desfase debida al desequilibrio, cuando procedemos a variar gradualmente el argumento de la fase 3, provocando esto como se observa en la figura V.42 que la potencia de desfase total del sistema disminuya, como también sucedía en los resultados mostrados en las tablas V.14, 16 y 18.

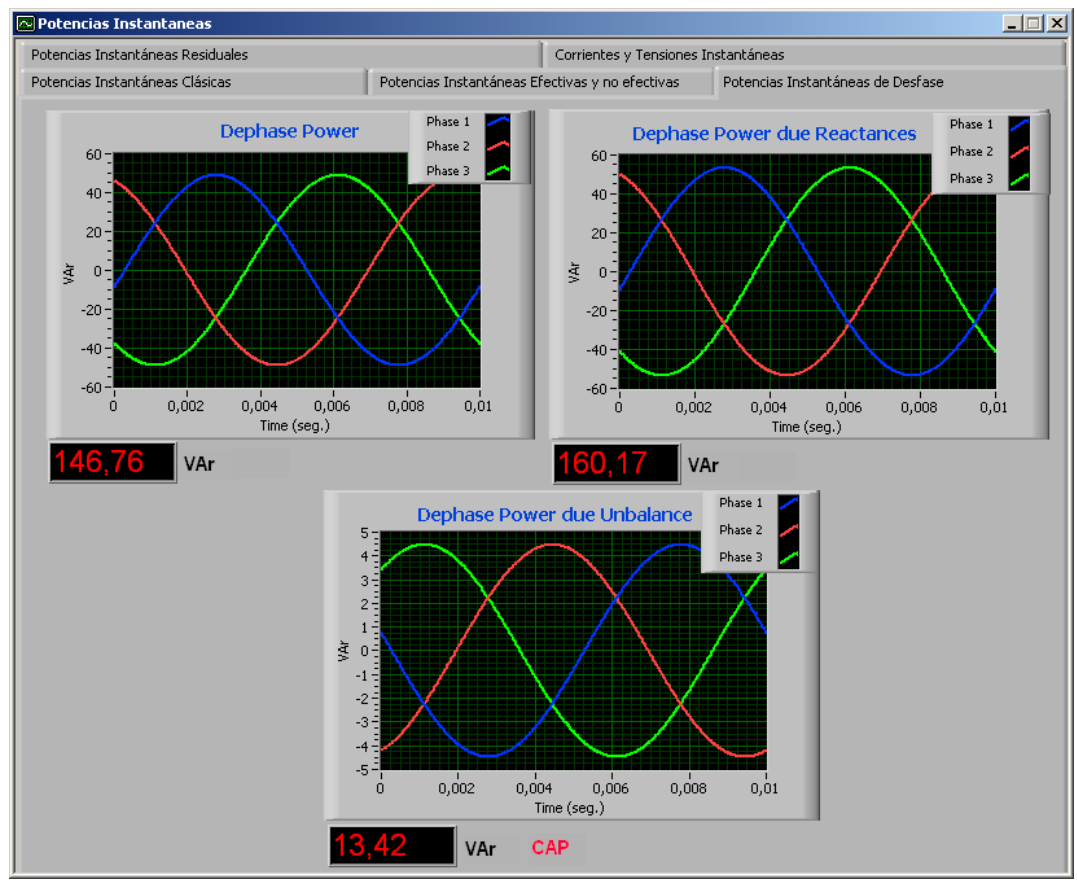

Figura V.42. Pantalla de Potencias de Desfase. 
Carga inductiva entre la fase 3 y el neutro, con un consumo de $1 \angle 75^{\circ} A, y$ producimos el desequilibrio sumando ángulos positivos al argumento de la fase 1.

Introducimos los datos en el SIMPELEC Simulador, tal y como mostramos en la figura V.43.

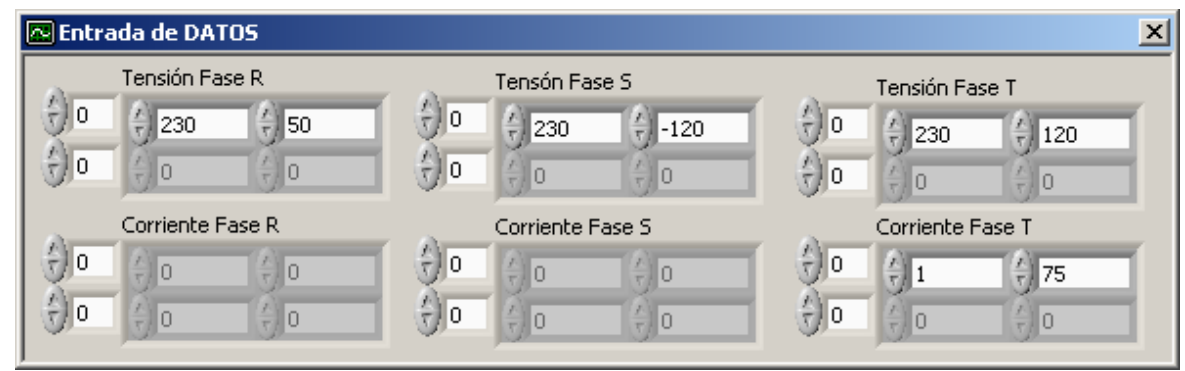

Figura V.43. Pantalla de entrada de datos.

Tenemos pues un sistema de tensiones en el que dejamos fijos los módulos de las tensiones de las fases, y tenemos una carga reactiva inductiva que consume $1 \angle 75^{\circ} A$ conectada en la fase 3 , y tan sólo variamos de manera gradual el argumento de la fase 1, logrando los pertinentes desequilibrios de tensión y produciendo la consiguiente potencia de desfase, tal y como podemos observar en la tabla V.21, y en la figura V.44 cuando el argumento de la fase 1 toma el valor de $50^{\circ}$.

\begin{tabular}{||c|c|c||}
\hline $\begin{array}{c}\text { Argumento } \\
\text { Fase 1 }\end{array}$ & $\begin{array}{c}\text { Variación } \\
\text { Argumento ( } \mathbf{(}^{\circ}\end{array}$ & $\begin{array}{c}\text { Potencia Desfase } \\
\text { Desequilibrio } \mathbf{Q}_{\text {dru }} \text { (VAr) }\end{array}$ \\
\hline $1^{\circ}$ & 1 & 0,95 \\
\hline $2^{\circ}$ & 2 & 1,90 \\
\hline $5^{\circ}$ & 5 & 4,79 \\
\hline $10^{\circ}$ & 10 & 9,69 \\
\hline $15^{\circ}$ & 15 & 14,65 \\
\hline $25^{\circ}$ & 25 & 24,60 \\
\hline $35^{\circ}$ & 35 & 34,36 \\
\hline $50^{\circ}$ & 50 & 47,98 \\
\hline $70^{\circ}$ & 70 & 62,83 \\
\hline $90^{\circ}$ & 90 & 72,28 \\
\hline $100^{\circ}$ & 100 & 74,60 \\
\hline $110^{\circ}$ & 110 & 75,19 \\
\hline $120^{\circ}$ & 120 & 74,05 \\
\hline \multicolumn{2}{|r|}{} \\
\hline
\end{tabular}

Tabla V.21. Potencia de Desfase \& Variación Argumento. 
En la tabla V.21, observamos el carácter inductivo de la potencia de desfase debida al desequilibrio, al igual que sucedía en las tablas V.13, 15, 17 y 19.

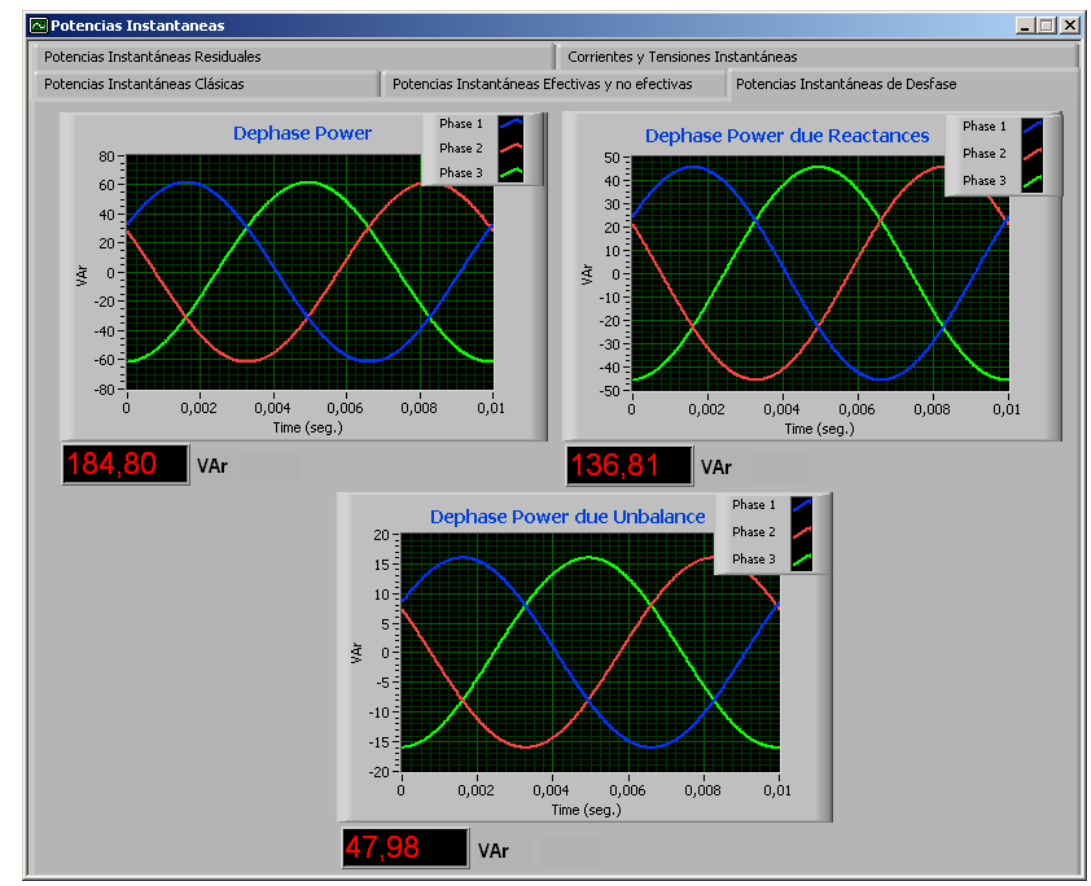

Figura V.44. Pantalla de Potencias de Desfase. 
Carga inductiva entre la fase 3 y el neutro, con un consumo de $1 \angle 75^{\circ} \mathrm{A}, \mathrm{y}$ producimos el desequilibrio sumando ángulos negativos al argumento de la fase 1.

Introducimos los datos en el SIMPELEC Simulador, tal y como mostramos en la figura V.45.

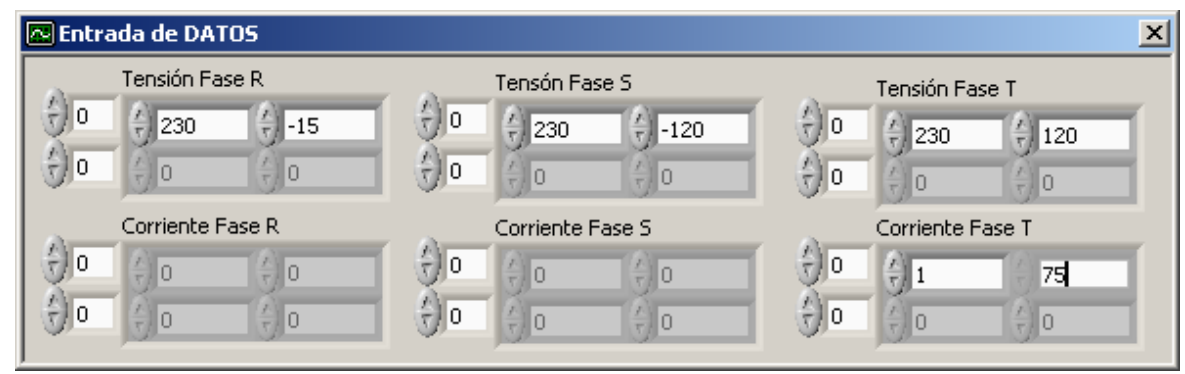

Figura V.45. Pantalla de entrada de datos.

Tenemos pues un sistema de tensiones en el que dejamos fijos los módulos de las tensiones de las fases, y tenemos una carga reactiva inductiva que consume $1 \angle 75^{\circ} A$ conectada en la fase 3 , y tan sólo variamos de manera gradual el argumento de la fase 1 , logrando los pertinentes desequilibrios de tensión y produciendo la consiguiente potencia de desfase, tal y como podemos observar en la tabla V.22, y en la figura V.46 para el caso concreto de que el argumento de la fase 1 adquiere el valor de $-15^{\circ}$.

\begin{tabular}{||c|c|c||}
\hline $\begin{array}{c}\text { Argumento } \\
\text { Fase 1 }\end{array}$ & $\begin{array}{c}\text { Variación } \\
\text { Argumento ( } \mathbf{(}^{\circ}\end{array}$ & $\begin{array}{c}\text { Potencia Desfase } \\
\text { Desequilibrio } \mathbf{Q}_{\text {dru }} \text { (VAr) }\end{array}$ \\
\hline$-1^{\circ}$ & 1 & $-0,94$ \\
\hline$-2^{\circ}$ & 2 & $-1,88$ \\
\hline$-5^{\circ}$ & 5 & $-4,66$ \\
\hline$-10^{\circ}$ & 10 & $-9,14$ \\
\hline$-15^{\circ}$ & 15 & $-13,42$ \\
\hline$-25^{\circ}$ & 25 & $-21,22$ \\
\hline$-35^{\circ}$ & 35 & $-27,83$ \\
\hline$-50^{\circ}$ & 50 & $-35,07$ \\
\hline$-70^{\circ}$ & 70 & $-39,05$ \\
\hline$-90^{\circ}$ & 90 & $-36,14$ \\
\hline$-100^{\circ}$ & 100 & $-32,18$ \\
\hline$-110^{\circ}$ & 110 & $-26,69$ \\
\hline$-120^{\circ}$ & 120 & $-19,84$ \\
\hline
\end{tabular}

Tabla V.22. Potencia de Desfase \& Variación Argumento. 
De la tabla V.22, se desprende el carácter capacitivo que toma la potencia de desfase debida al desequilibrio, como ya sucedía en las tablas V.14, 16, 18 y 20.

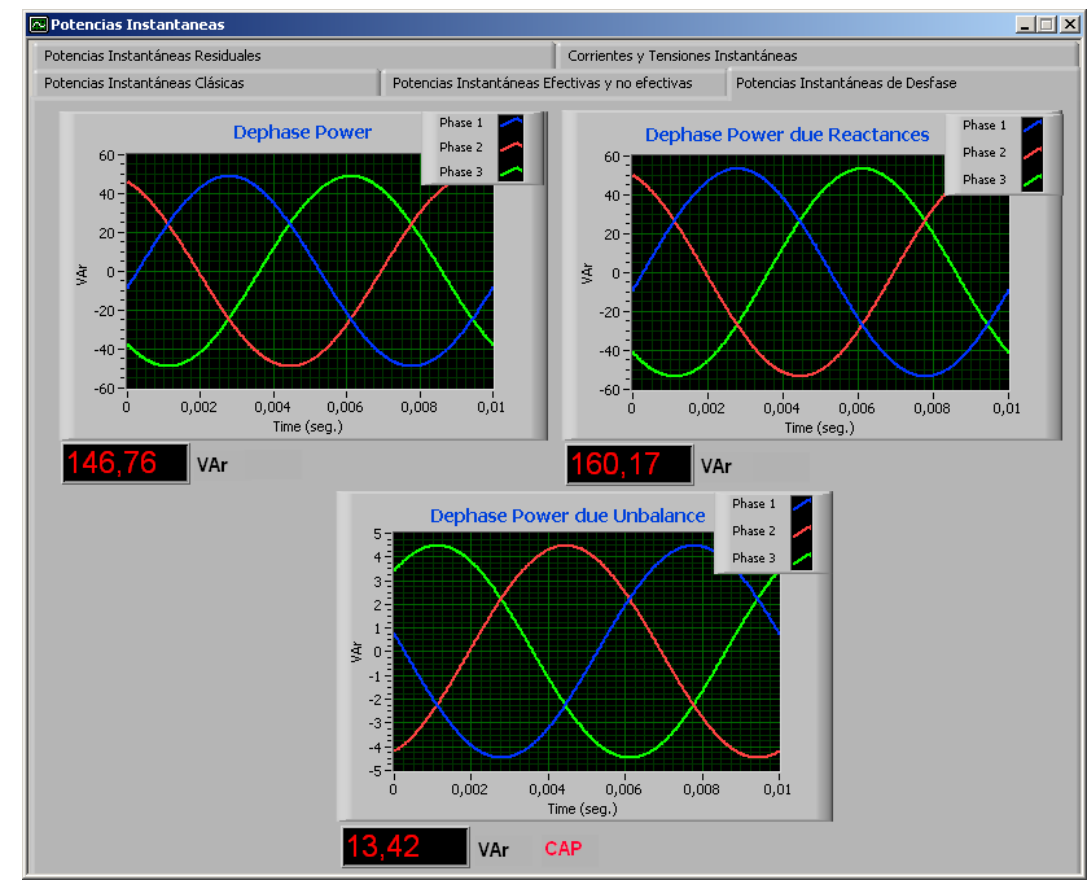

Figura V.46. Pantalla de Potencias de Desfase. 
Carga inductiva entre la fase 3 y el neutro, con un consumo de $1 \angle 75^{\circ} A, y$ producimos el desequilibrio sumando ángulos positivos al argumento de la fase 2.

Introducimos los datos en el SIMPELEC Simulador, tal y como mostramos en la figura V.47.

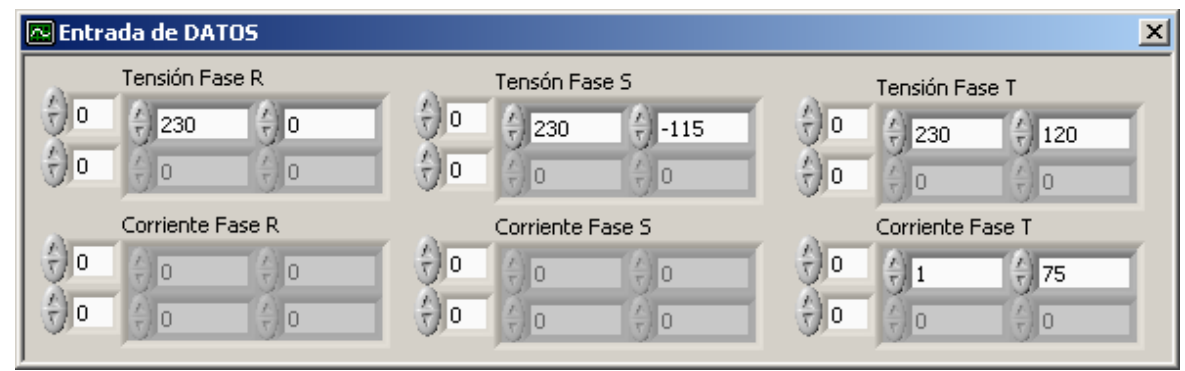

Figura V.47. Pantalla de entrada de datos.

Tenemos pues un sistema de tensiones en el que dejamos fijos los módulos de las tensiones de las fases, y tenemos una carga reactiva inductiva que consume $1 \angle 75^{\circ} A$ conectada en la fase $3, y$ tan sólo variamos de manera gradual el argumento de la fase 2, logrando los pertinentes desequilibrios de tensión y produciendo la consiguiente potencia de desfase, tal y como podemos observar en la tabla V.23, y en la figura V.48 para el caso de que el argumento de la fase 2 tome el valor de $-115^{\circ}$.

\begin{tabular}{||c|c|c||}
\hline $\begin{array}{c}\text { Argumento } \\
\text { Fase 2 }\end{array}$ & $\begin{array}{c}\text { Variación } \\
\left.\text { Argumento ( } \mathbf{(}^{\mathbf{}}\right)\end{array}$ & $\begin{array}{c}\text { Potencia Desfase } \\
\text { Desequilibrio } \mathbf{Q}_{\text {dru }} \text { (VAr) }\end{array}$ \\
\hline$-119^{\circ}$ & 1 & 0,95 \\
\hline$-118^{\circ}$ & 2 & 1,90 \\
\hline$-115^{\circ}$ & 5 & 4,79 \\
\hline$-110^{\circ}$ & 10 & 9,69 \\
\hline$-105^{\circ}$ & 15 & 14,65 \\
\hline$-95^{\circ}$ & 25 & 24,60 \\
\hline$-85^{\circ}$ & 35 & 34,36 \\
\hline$-70^{\circ}$ & 50 & 47,98 \\
\hline$-50^{\circ}$ & 70 & 62,83 \\
\hline$-30^{\circ}$ & 90 & 72,28 \\
\hline$-20^{\circ}$ & 100 & 74,60 \\
\hline$-10^{\circ}$ & 110 & 75,19 \\
\hline $0^{\circ}$ & 120 & 74,05 \\
\hline
\end{tabular}

Tabla V.23. Potencia de Desfase \& Variación Argumento. 
De la observación de la tabla V.23, vemos que la potencia de desfase debida al desequilibrio tiene un carácter inductivo, al igual que mostramos en los resultados de las tablas $\mathrm{V} .13,15,17,19$ y 21 , lo que provoca un incremento adicional de la potencia de desfase total del sistema, ya que se suma a la potencia de desfase debida a la presencia del elemento inductivo en el sistema, como se ve en la figura V.48.

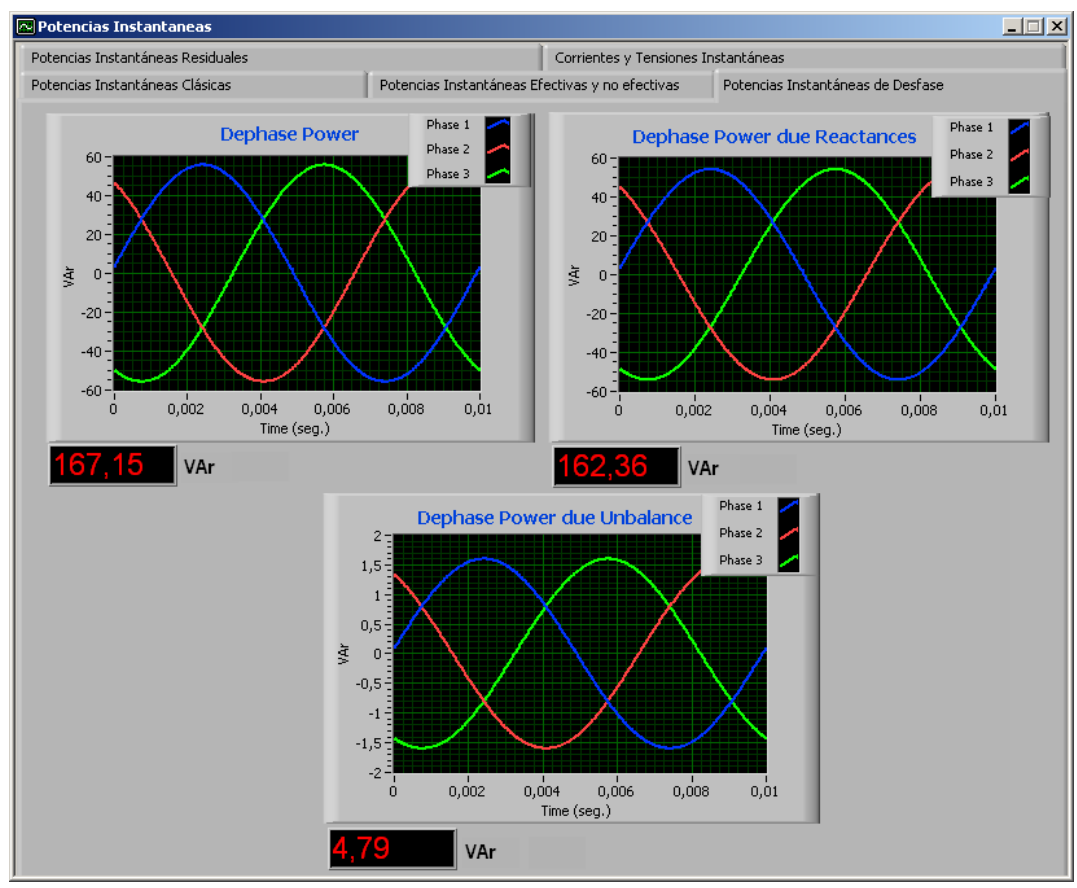

Figura V.48. Pantalla de Potencias de Desfase. 
Carga inductiva entre la fase 3 y el neutro, con un consumo de $1 \angle 75^{\circ} \mathrm{A}, \mathrm{y}$ producimos el desequilibrio sumando ángulos negativos al argumento de la fase 2.

Introducimos los datos en el SIMPELEC Simulador, tal y como mostramos en la figura V.49.

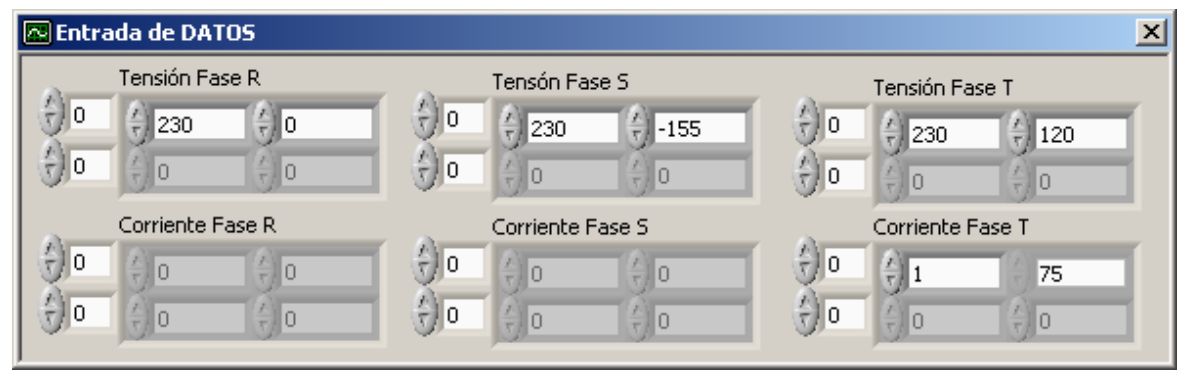

Figura V.49. Pantalla de entrada de datos.

Tenemos pues un sistema de tensiones en el que dejamos fijos los módulos de las tensiones de las fases, y tenemos una carga reactiva inductiva que consume $1 \angle 75^{\circ} A$ conectada en la fase 3 , y tan sólo variamos de manera gradual el argumento de la fase 2, logrando los pertinentes desequilibrios de tensión y produciendo la consiguiente potencia de desfase, tal y como podemos observar en la tabla V.24, y en la figura V.50 cuando el argumento de la fase 2 vale $-155^{\circ}$.

\begin{tabular}{||c|c|c||}
\hline $\begin{array}{c}\text { Argumento } \\
\text { Fase 2 }\end{array}$ & $\begin{array}{c}\text { Variación } \\
\text { Argumento } \mathbf{(}^{\circ} \text { ) }\end{array}$ & $\begin{array}{c}\text { Potencia Desfase } \\
\text { Desequilibrio } \mathbf{Q}_{\text {dru }} \text { (VAr) }\end{array}$ \\
\hline$-121^{\circ}$ & 1 & $-0,94$ \\
\hline$-122^{\circ}$ & 2 & $-1,88$ \\
\hline$-125^{\circ}$ & 5 & $-4,66$ \\
\hline$-130^{\circ}$ & 10 & $-9,14$ \\
\hline$-135^{\circ}$ & 15 & $-13,42$ \\
\hline$-145^{\circ}$ & 25 & $-21,22$ \\
\hline$-155^{\circ}$ & 35 & $-27,83$ \\
\hline$-170^{\circ}$ & 50 & $-35,07$ \\
\hline$-190^{\circ}$ & 70 & $-39,05$ \\
\hline$-210^{\circ}$ & 90 & $-36,14$ \\
\hline$-220^{\circ}$ & 100 & $-32,18$ \\
\hline$-230^{\circ}$ & 110 & $-26,69$ \\
\hline$-240^{\circ}$ & 120 & $-19,84$ \\
\hline
\end{tabular}

Tabla V.24. Potencia de Desfase \& Variación Argumento. 
La tabla V.24, nos muestra los resultados obtenidos por la variación del argumento de la fase 2 de $-120^{\circ}$ a $-240^{\circ}$, que produce una potencia de desfase debida al desequilibrio capacitiva, y cuyos valores coinciden con los de las tablas V.14, $16,18,20$ y 22.

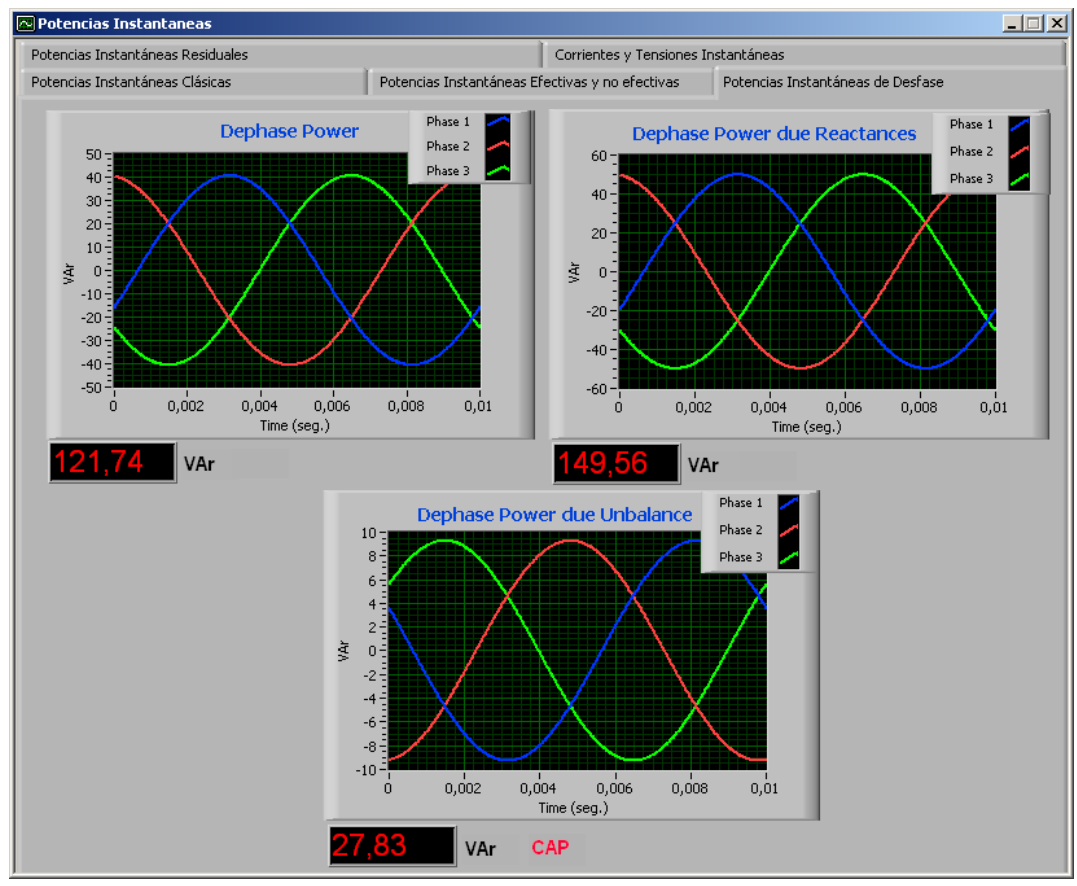

Figura V.50. Pantalla de Potencias de Desfase.

En la figura V.51, se resumen los resultados que fuimos obteniendo para el caso de tener conectada una carga inductiva. Se aprecia de manera clara que para los casos de desequilibrio del sistema logrados en base a sumar ángulos positivos en los argumentos de las fases descargadas, se refuerza el consumo de la potencia de desfase inductiva de la carga, así como cuando desequilibramos el sistema por medio de la suma de ángulos negativos en las fases descargadas, se llega a producir una inversión en el comportamiento de la carga pasando a observarse un consumo de la potencia de desfase de carácter capacitivo. 


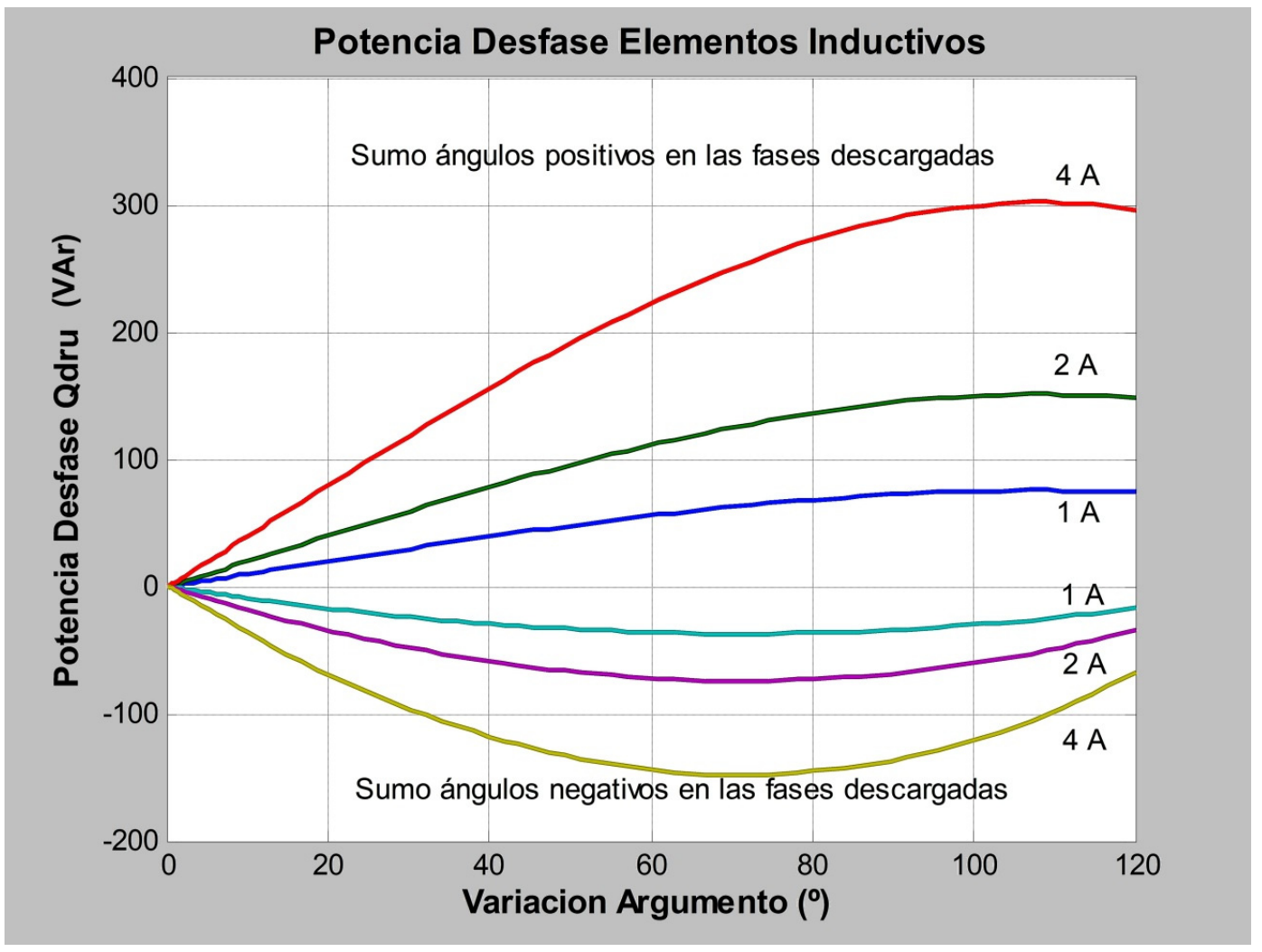

Figura V.51. Potencias de Desfase, para diversas variaciones de carga inductiva en una fase y grados de desequilibrio.

En las gráficas de la figura V.51, vemos que el comportamiento del sistema es independiente de en que fase se halle conectada la carga, y podemos apreciar las potencias de desfase para varios consumos de la carga de 1, 2 y 4 Amperios. 
b.1) Carga Inductiva Pura: Se analiza el comportamiento de este elemento frente a diversos desequilibrios que se producen, estando esta carga de manera sucesiva en cada fase.

El procedimiento realizado, es idéntico al usado para el caso que acabamos de analizar de carga inductiva con un consumo de $1 \angle-45^{\circ} A$, tan sólo que ahora la carga es inductiva pura con un consumo de $1 \angle-90^{\circ} A$, introducimos los datos en el simulador SIMPELEC, tal y como se muestra en la figura V.52, que se corresponde con el caso de estar la carga inductiva pura entre la fase 2 y el neutro, y siendo el desequilibrio de tensiones provocado por la suma de ángulos positivos al argumento de la fase 1 , que en este caso concreto es de una variación de $35^{\circ}$.

\begin{tabular}{|c|c|c|c|c|c|c|c|c|}
\hline 圆 Entr & a de D & & & & & & & \\
\hline & ensión & & & רร์́n F & & & nsión F & \\
\hline (5) 0 & 230 & 35 & 5) 0 & 230 & -120 & 5) 0 & 230 & 120 \\
\hline (5) 0 & 0 & 0 & (5) 0 & 0 & 0 & 3) 0 & 0 & 0 \\
\hline & orrient & & & rriente & & & irriente & \\
\hline 5) 0 & 0 & 0 & (5) 0 & 1 & -210 & 5) 0 & 0 & 0 \\
\hline (5) 0 & 0 & 0 & (5) 0 & 0 & 0 & 7) 0 & 0 & 0 \\
\hline
\end{tabular}

Figura V.52. Pantalla de entrada de datos.

Lo que nos da un consumo de potencia de desfase debida al desequilibrio con carácter inductivo, tal y como queda reflejado en la figura V.53, siendo el valor de esta de 4,62 VAr, que incrementan el debido a las reactancias del sistema. 


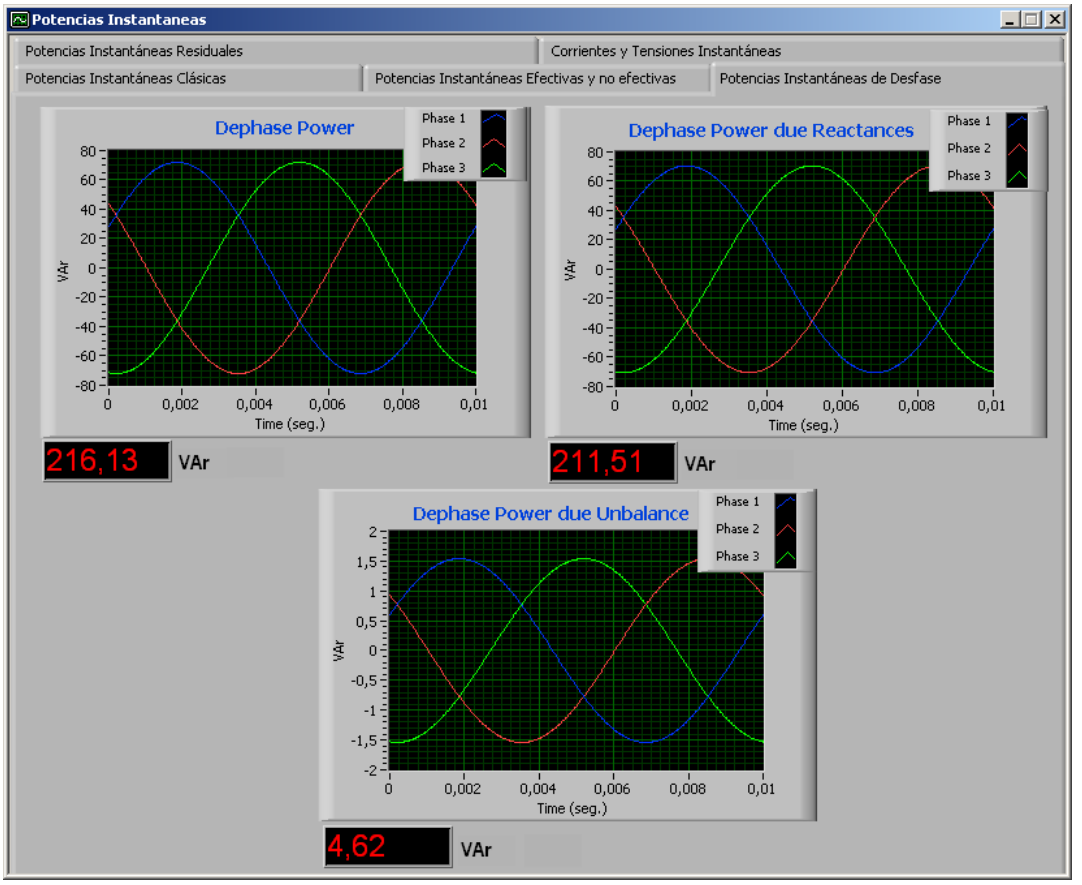

Figura V.53. Pantalla de Potencias de Desfase.

Con esta carga inductiva pura se han analizado los siguientes casos que pasamos a mencionar:

- Carga conectada entre la fase 1 y neutro, con variación de argumentos de la fase 2, variando estos de $-120^{\circ}$ a $0^{\circ}$. (sumo ángulos positivos)

- Carga conectada entre la fase 1 y neutro, con variación de argumentos de la fase 2 , variando estos de $-120^{\circ}$ a $-240^{\circ}$. (sumo ángulos negativos)

- Carga conectada entre la fase 1 y el neutro, con variación de argumentos de la fase 3 , variando estos de $120^{\circ}$ a $240^{\circ}$. (sumo ángulos positivos)

- Carga conectada entre la fase 1 y el neutro, con variación de argumentos de la fase 3 , variando estos de $120^{\circ}$ a $0^{\circ}$. (sumo ángulos negativos)

- Carga conectada entre la fase 2 y el neutro, con variación de argumentos de la fase 1 , variando estos de $0^{\circ}$ a $120^{\circ}$. (sumo ángulos positivos)

- Carga conectada entre la fase 2 y el neutro, con variación de argumentos de la fase 1 , variando estos de $0^{\circ} \mathrm{a}-120^{\circ}$. (sumo ángulos negativos)

- Carga conectada entre la fase 2 y el neutro, con variación de argumentos de la fase 3 , variando estos de $120^{\circ}$ a $240^{\circ}$. (sumo ángulo positivos) 
- Carga conectada entre la fase 2 y el neutro, con variación de argumentos de la fase 3 , variando estos de $120^{\circ}$ a $0^{\circ}$. (sumo ángulo negativos)

- Carga conectada entre la fase 3 y el neutro, con variación de argumentos de la fase 1 , variando estos de $0^{\circ}$ a $120^{\circ}$. (sumo ángulo positivos)

- Carga conectada entre la fase 3 y el neutro, con variación de argumentos de la fase 1 , variando estos de $0^{\circ}$ a $-120^{\circ}$. (sumo ángulos negativos)

- Carga conectada entre la fase 3 y el neutro, con variación de argumentos de la fase 2, variando estos de $-120^{\circ}$ a $0^{\circ}$. (sumo ángulos positivos)

- Carga conectada entre la fase 3 y el neutro, con variación de argumentos de la fase 2 , variando estos de $-120^{\circ}$ a $-240^{\circ}$. (sumo ángulos negativos)

En cada uno de los casos anteriores se fueron modificando de manera gradual, el incremento del argumento de la fase correspondiente, para provocar el desequilibrio, y a continuación se muestran los resultados obtenidos, en los cuales se evidencia que la potencia de desfase debida al desequilibrio, posee un carácter inductivo que refuerza a la del sistema.

En la tabla V.25 se muestran los incrementos de ángulos provocados, así como la potencia de desfase debida al desequilibrio que conlleva, que fueron iguales para todos los casos que acabamos de presentar.

\begin{tabular}{||c|c|}
\hline $\begin{array}{c}\text { Variación } \\
\left.\text { Argumento ( }{ }^{(}\right)\end{array}$ & $\begin{array}{c}\text { Potencia Desfase } \\
\text { Desequilibrio } \mathbf{Q}_{\text {dru }} \text { (VAr) }\end{array}$ \\
\hline 1 & 0 \\
\hline 2 & 0,02 \\
\hline 5 & 0,1 \\
\hline 10 & 0,39 \\
\hline 15 & 0,87 \\
\hline 25 & 2,21 \\
\hline 35 & 4,62 \\
\hline 50 & 9,13 \\
\hline 70 & 16,82 \\
\hline 90 & 25,26 \\
\hline 100 & 29,99 \\
\hline 110 & 34,30 \\
\hline 120 & 38,33 \\
\hline
\end{tabular}

Tabla V.25 Potencia de Desfase \& Variación Argumento 


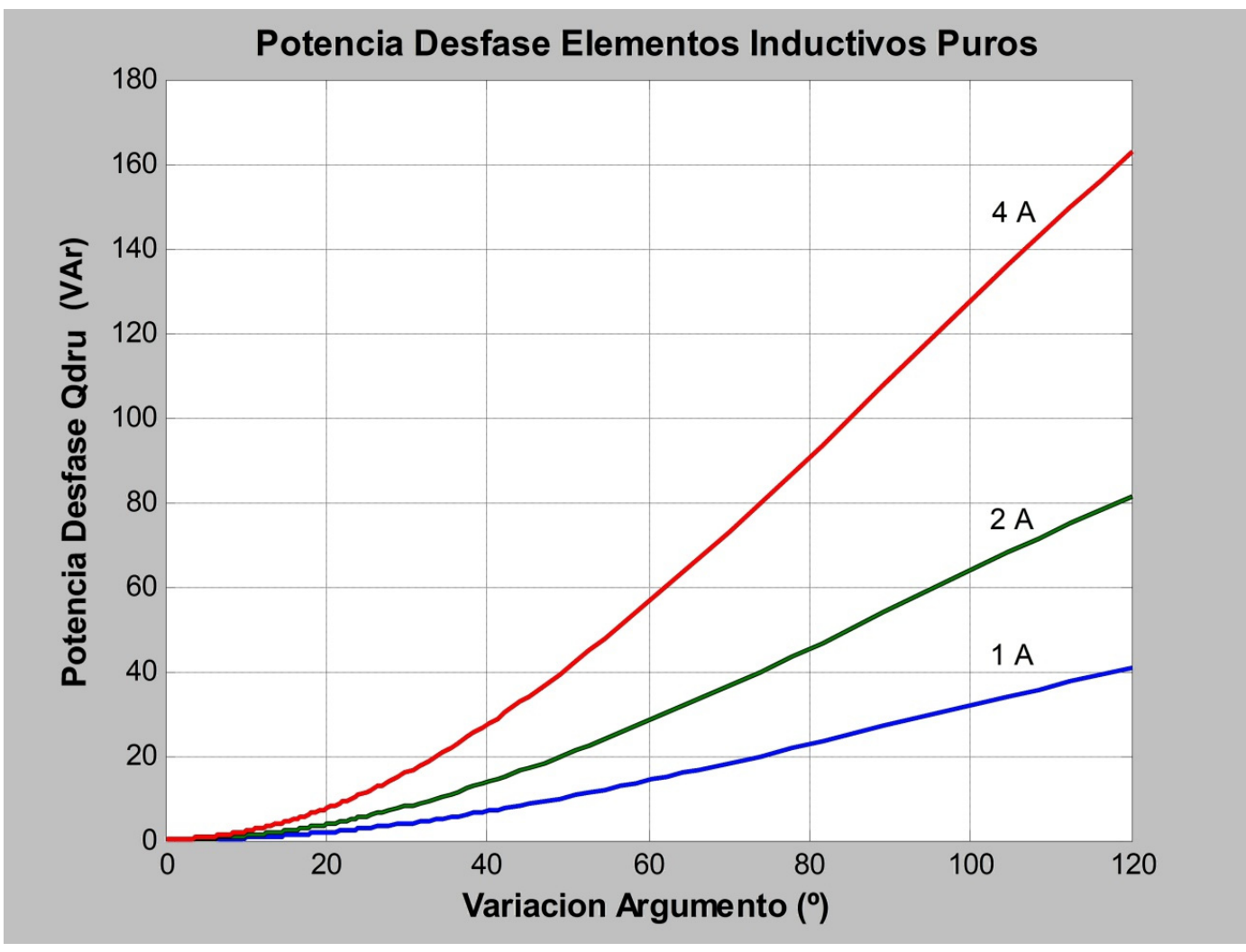

Figura V.54. Potencias de Desfase, para diversas variaciones de carga inductiva pura en una fase y variaciones de argumentos.

Podemos observar en la figura V.54, de manera gráfica los resultados obtenidos para el caso de que el sistema lo forme una carga inductiva pura, y que se pone de manifiesto que con independencia de que en que fase se halle la carga conectada, y de como obtengamos el desequilibrio del sistema, la potencia de desfase debida al desequilibrio siempre tiene carácter inductivo, y que por lo tanto se suma a la propia del sistema.

Se puede observar también en la figura V.54, las potencias de desfase debidas al desequilibrio, en los casos de cargas con consumos de 2 y 4 Amperios. 
c) Carga Capacitiva: Se analiza el comportamiento de este elemento frente a diversos desequilibrios que se producen, estando esta carga de manera sucesiva en cada fase.

Una carga capacitiva entre la fase 1 y el neutro, con un consumo de $1 \angle 45^{\circ} \mathrm{A}$, y producimos el desequilibrio sumando ángulos positivos al argumento de la fase 2.

Introducimos los datos en el SIMPELEC Simulador, tal y como mostramos en la figura V.55.

\begin{tabular}{|c|c|c|c|c|c|c|c|c|}
\hline \multicolumn{9}{|c|}{ 图Entrada de DATOS } \\
\hline \multicolumn{3}{|c|}{ Tensión Fase R } & \multicolumn{3}{|c|}{ Tensón Fase S } & \multicolumn{3}{|c|}{ Tensión Fase T } \\
\hline 5) 0 & $\frac{1}{2} 230$ & 0 & 5) 0 & 230 & -105 & 5) 0 & 230 & 120 \\
\hline 5) 0 & $\frac{7}{5} 0$ & 0 & (5) 0 & 0 & 0 & (5) 0 & 0 & 0 \\
\hline \multicolumn{3}{|c|}{ Corriente Fase R } & \multicolumn{3}{|c|}{ Corriente Fase 5} & \multicolumn{3}{|c|}{ Corriente Fase T } \\
\hline 5) 0 & $\frac{1}{1}$ & 45 & 5) 0 & 0 & 0 & 5) 0 & 0 & 0 \\
\hline (5) 0 & 0 & 0 & 50 & 0 & 0 & 5) 0 & 0 & 0 \\
\hline
\end{tabular}

Figura V.55. Pantalla de entrada de datos.

Tenemos pues un sistema de tensiones en el que dejamos fijos los módulos de las tensiones de las fases, y tenemos una carga reactiva capacitiva que consume $1 \angle 45^{\circ} A$ conectada en la fase 1, y tan sólo variamos de manera gradual el argumento de la fase 2, logrando los pertinentes desequilibrios de tensión y produciendo la consiguiente potencia de desfase, tal y como podemos observar en la tabla V.26, y en la figura V.56 para el caso concreto de que el argumento de la fase 2 tome el valor de $-105^{\circ}$. 


\begin{tabular}{||c|c|c||}
\hline $\begin{array}{c}\text { Argumento } \\
\text { Fase 2 }\end{array}$ & $\begin{array}{c}\text { Variación } \\
\left.\text { Argumento ( } \mathbf{(}^{\mathbf{}}\right)\end{array}$ & $\begin{array}{c}\text { Potencia Desfase } \\
\text { Desequilibrio } \mathbf{Q}_{\text {dru }} \text { (VAr) }\end{array}$ \\
\hline$-119^{\circ}$ & 1 & 0,94 \\
\hline$-118^{\circ}$ & 2 & 1,88 \\
\hline$-115^{\circ}$ & 5 & 4,66 \\
\hline$-110^{\circ}$ & 10 & 9,14 \\
\hline$-105^{\circ}$ & 15 & 13,42 \\
\hline$-95^{\circ}$ & 25 & 21,22 \\
\hline$-85^{\circ}$ & 35 & 27,83 \\
\hline$-70^{\circ}$ & 50 & 35,07 \\
\hline$-50^{\circ}$ & 70 & 39,05 \\
\hline$-30^{\circ}$ & 90 & 36,14 \\
\hline$-20^{\circ}$ & 100 & 32,18 \\
\hline$-10^{\circ}$ & 110 & 26,69 \\
\hline $0^{\circ}$ & 120 & 19,84 \\
\hline \hline
\end{tabular}

Tabla V.26. Potencia de Desfase \& Variación Argumento.

De los resultados de la tabla V.26 observamos que al producir modificaciones en el argumento de la fase 2, llevando esta desde $-120 \circ$ a $0^{\circ}$, se pone de manifiesto el consumo de potencia de desfase de carácter inductivo procedente del desequilibrio del sistema, que en este caso se resta a la consumida por la propia presencia de una carga capacitiva, que también observamos en la figura V.56. El ratio de incremento de esta potencia de desfase debida al desequilibrio, se cuantifica en 0,94 VAr, con la variación de un grado (10) en el argumento, manteniéndose esta linealidad en todo el tramo de variación provocado.

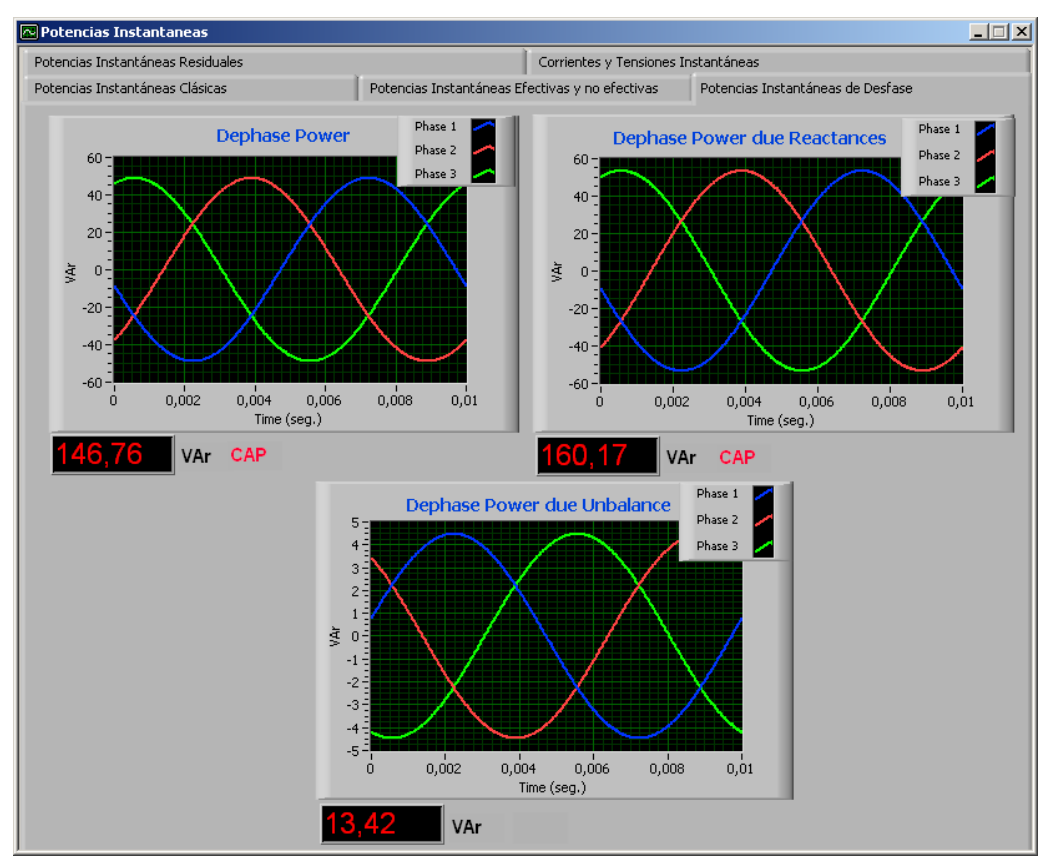

Figura V.56. Pantalla de Potencias de Desfase. 
Carga capacitiva entre la fase 1 y el neutro, con un consumo de $1 \angle 45^{\circ} \mathrm{A}, \mathrm{y}$ producimos el desequilibrio sumando ángulos negativos al argumento de la fase 2.

Introducimos los datos en el SIMPELEC Simulador, tal y como mostramos en la figura V.57.

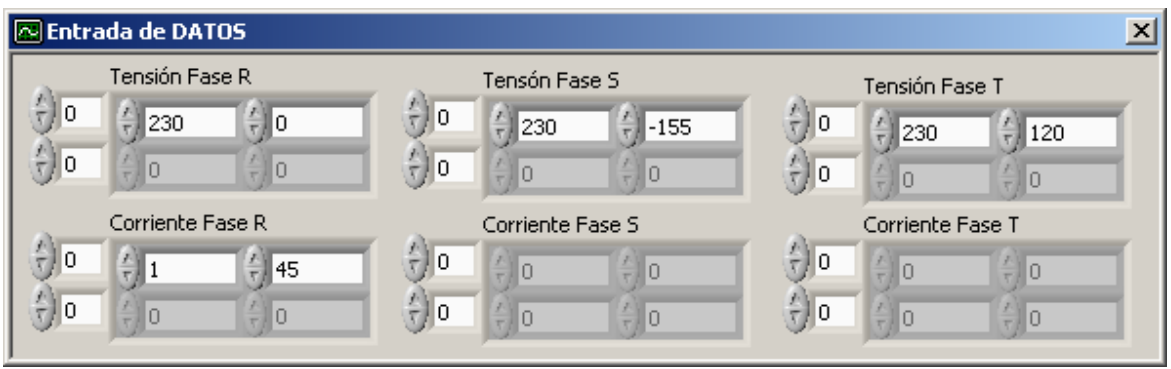

Figura V.57. Pantalla de entrada de datos.

Tenemos pues un sistema de tensiones en el que dejamos fijos los módulos de las tensiones de las fases, y tenemos una carga reactiva capacitiva que consume $1 \angle 45^{\circ} A$ conectada en la fase 1, y tan sólo variamos de manera gradual el argumento de la fase 2 , logrando los pertinentes desequilibrios de tensión y produciendo la consiguiente potencia de desfase, tal y como podemos observar en la tabla V.27, y en la figura V.58 para el caso de que el argumento de la fase 2 valga $-155^{\circ}$.

\begin{tabular}{|c|c|c||}
\hline $\begin{array}{c}\text { Argumento } \\
\text { Fase 2 }\end{array}$ & $\begin{array}{c}\text { Variación } \\
\text { Argumento ( }\end{array}$ & $\begin{array}{c}\text { Potencia Desfase } \\
\text { Desequilibrio } \mathbf{Q}_{\text {dru }} \text { (VAr) }\end{array}$ \\
\hline$-121^{\circ}$ & 1 & $-0,95$ \\
\hline$-122^{\circ}$ & 2 & $-1,90$ \\
\hline$-125^{\circ}$ & 5 & $-4,79$ \\
\hline$-130^{\circ}$ & 10 & $-9,69$ \\
\hline$-135^{\circ}$ & 15 & $-14,65$ \\
\hline$-145^{\circ}$ & 25 & $-24,60$ \\
\hline$-155^{\circ}$ & 35 & $-34,36$ \\
\hline$-170^{\circ}$ & 50 & $-47,98$ \\
\hline$-190^{\circ}$ & 70 & $-62,83$ \\
\hline$-210^{\circ}$ & 90 & $-72,28$ \\
\hline$-220^{\circ}$ & 100 & $-74,60$ \\
\hline$-230^{\circ}$ & 110 & $-75,19$ \\
\hline$-240^{\circ}$ & 120 & $-74,05$ \\
\hline
\end{tabular}

Tabla V.27. Potencia de Desfase \& Variación Argumento. 
De la observación de la tabla V.27, se pone de manifiesto por un lado la presencia de potencia de desfase debida al desequilibrio con carácter capacitivo, y que el ratio que cuantifica la variación producida por un grado de variación en el argumento de la fase, es ahora de 0,95 VAr. La presencia de esta potencia de desfase debida al desequilibrio, produce que la carga en cuestión posea un comportamiento más capacitivo que el esperado, lo que queda de manifiesto en la figura V.58.

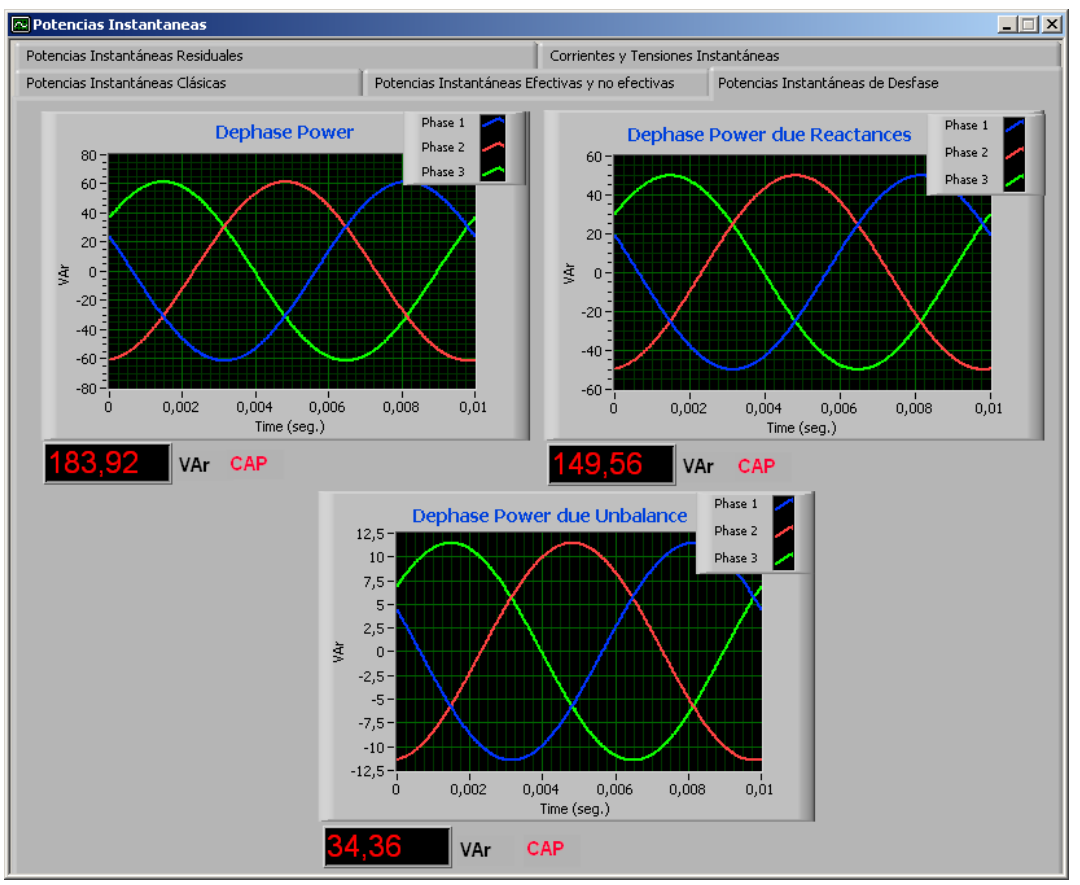

Figura V.58. Pantalla de Potencias de Desfase. 
Carga capacitiva entre la fase 1 y el neutro, con un consumo de $1 \angle 45^{\circ} \mathrm{A}, \mathrm{y}$ producimos el desequilibrio sumando ángulos positivos al argumento de la fase 3.

Introducimos los datos en el SIMPELEC Simulador, tal y como mostramos en la figura V.59.

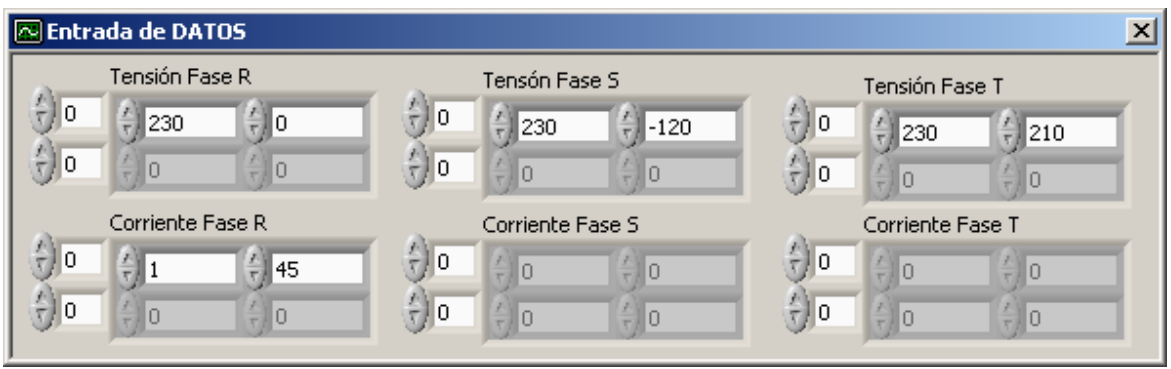

Figura V.59. Pantalla de entrada de datos.

Tenemos pues un sistema de tensiones en el que dejamos fijos los módulos de las tensiones de las fases, y tenemos una carga reactiva capacitiva que consume $1 \angle 45^{\circ} A$ conectada en la fase 1, y tan sólo variamos de manera gradual el argumento de la fase 3 , logrando los pertinentes desequilibrios de tensión y produciendo la consiguiente potencia de desfase, tal y como podemos observar en la tabla V.28, y en la figura V.60 para el caso concreto de que el argumento de la fase 3 tome el valor de $210^{\circ}$.

\begin{tabular}{||c|c|c||}
\hline $\begin{array}{c}\text { Argumento } \\
\text { Fase 3 }\end{array}$ & $\begin{array}{c}\text { Variación } \\
\text { Argumento } \mathbf{(}^{\circ} \text { ) }\end{array}$ & $\begin{array}{c}\text { Potencia Desfase } \\
\text { Desequilibrio } \mathbf{Q d r u}_{\text {(VAr) }}\end{array}$ \\
\hline $121^{\circ}$ & 1 & 0,94 \\
\hline $122^{\circ}$ & 2 & 1,88 \\
\hline $125^{\circ}$ & 5 & 4,66 \\
\hline $130^{\circ}$ & 10 & 9,14 \\
\hline $135^{\circ}$ & 15 & 13,42 \\
\hline $145^{\circ}$ & 25 & 21,22 \\
\hline $155^{\circ}$ & 35 & 27,83 \\
\hline $170^{\circ}$ & 50 & 35,07 \\
\hline $190^{\circ}$ & 70 & 39,05 \\
\hline $210^{\circ}$ & 90 & 36,14 \\
\hline $220^{\circ}$ & 100 & 32,18 \\
\hline $230^{\circ}$ & 110 & 26,69 \\
\hline $240^{\circ}$ & 120 & 19,84 \\
\hline
\end{tabular}

Tabla V.28. Potencia de Desfase \& Variación Argumento. 
Los resultados mostrados en la tabla V.28, nos muestran que se produce un consumo de potencia de desfase con carácter inductivo, viendo que el ratio que nos cuantifica esta variación de la potencia en función del desequilibrio del sistema coincide con lo obtenido en la tabla V.26.

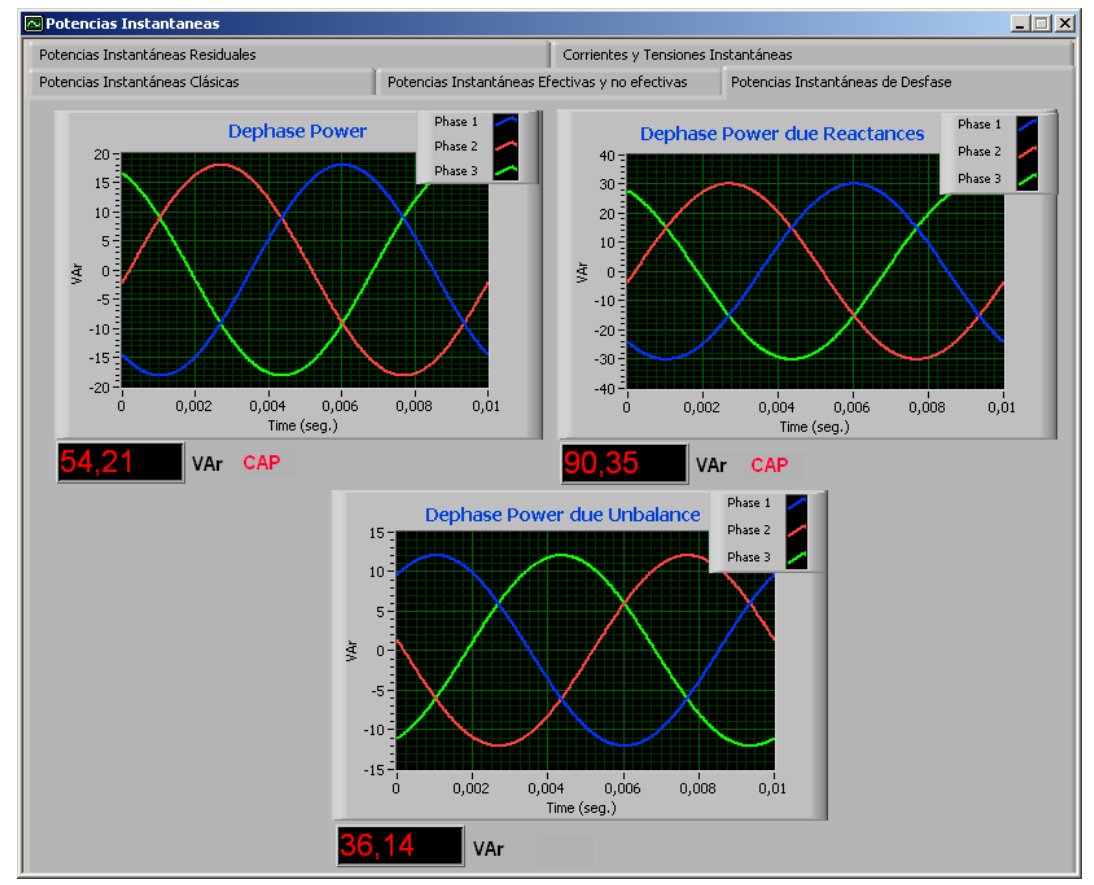

Figura V.60. Pantalla de Potencias de Desfase. 
Carga capacitiva entre la fase 1 y el neutro, con un consumo de $1 \angle 45^{\circ} \mathrm{A}, \mathrm{y}$ producimos el desequilibrio sumando ángulos negativos al argumento de la fase 3.

Introducimos los datos en el SIMPELEC Simulador, tal y como mostramos en la figura V.61.

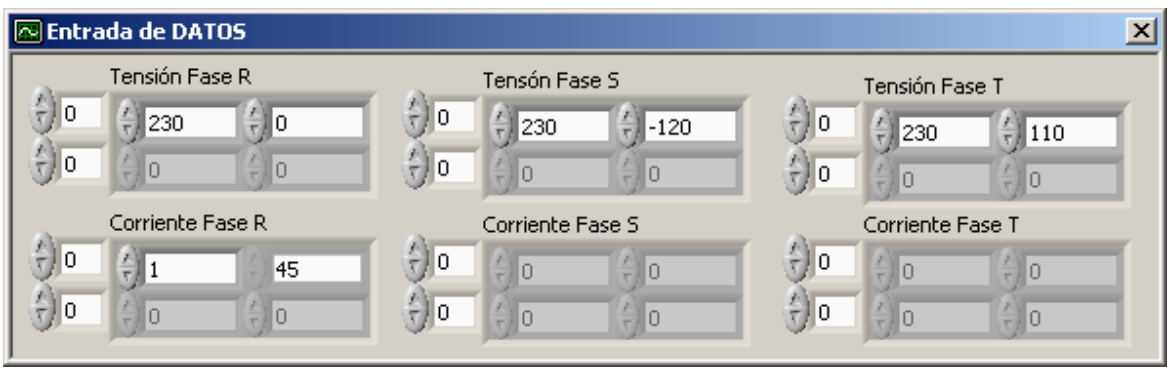

Figura V.61. Pantalla de entrada de datos.

Tenemos pues un sistema de tensiones en el que dejamos fijos los módulos de las tensiones de las fases, y tenemos una carga reactiva capacitiva que consume $1 \angle 45^{\circ} A$ conectada en la fase $1, y$ tan sólo variamos de manera gradual el argumento de la fase 3 , logrando los pertinentes desequilibrios de tensión y produciendo la consiguiente potencia de desfase, tal y como podemos observar en la tabla V.29, y en la figura V.62 cuando el argumento de la fase 3 adquiere el valor de $110^{\circ}$.

\begin{tabular}{|c|c|c||}
\hline $\begin{array}{c}\text { Argumento } \\
\text { Fase 3 }\end{array}$ & $\begin{array}{c}\text { Variación } \\
\text { Argumento ( }{ }^{\circ} \text { ) }\end{array}$ & $\begin{array}{c}\text { Potencia Desfase } \\
\text { Desequilibrio } \mathbf{Q}_{\text {dru }} \text { (VAr) }\end{array}$ \\
\hline $119^{\circ}$ & 1 & $-0,95$ \\
\hline $118^{\circ}$ & 2 & $-1,90$ \\
\hline $115^{\circ}$ & 5 & $-4,79$ \\
\hline $110^{\circ}$ & 10 & $-9,69$ \\
\hline $105^{\circ}$ & 15 & $-14,65$ \\
\hline $95^{\circ}$ & 25 & $-24,60$ \\
\hline $85^{\circ}$ & 35 & $-34,36$ \\
\hline $70^{\circ}$ & 50 & $-47,98$ \\
\hline $50^{\circ}$ & 70 & $-62,83$ \\
\hline $30^{\circ}$ & 90 & $-72,28$ \\
\hline $20^{\circ}$ & 100 & $-74,60$ \\
\hline $10^{\circ}$ & 110 & $-75,19$ \\
\hline $0^{\circ}$ & 120 & $-74,05$ \\
\hline
\end{tabular}

Tabla V.29. Potencia de Desfase \& Variación Argumento. 
En la tabla V.29, se puede apreciar que con las variaciones provocadas en el argumento de la fase 3 , entre $120^{\circ}$ y $0^{\circ}$, provocan la presencia de una potencia de desfase debida al desequilibrio de carácter capacitivo, y cuyos valores son idénticos a los obtenidos y mostrados en la tabla V.27, provocando que la potencia de desfase total del sistema sea mayor que si no existieran desequilibrios en el sistema que provocan la presencia de esta potencia de desfase debida al desequilibrio.

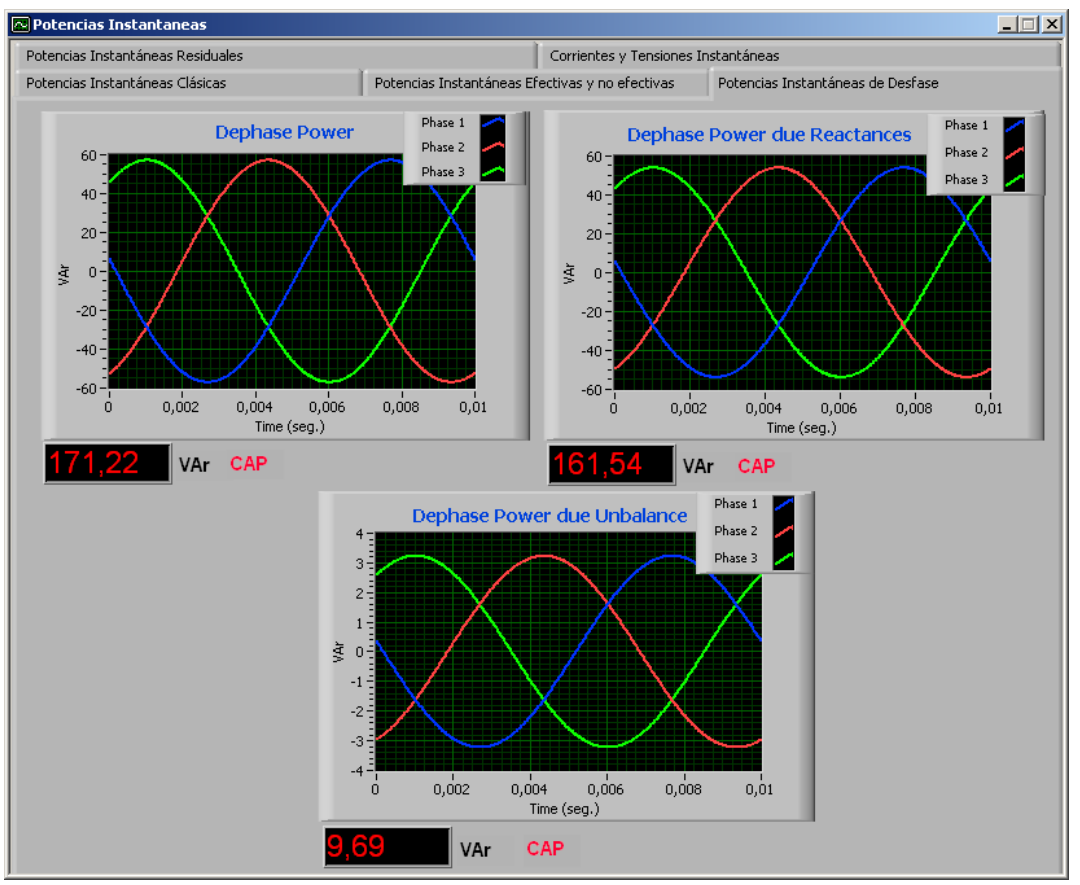

Figura V.62. Pantalla de Potencias de Desfase. 
Carga capacitiva entre la fase 2 y el neutro, con un consumo de $1 \angle-75^{\circ} A$, y producimos el desequilibrio sumando ángulos positivos al argumento de la fase 1.

Introducimos los datos en el SIMPELEC Simulador, tal y como mostramos en la figura V.63.

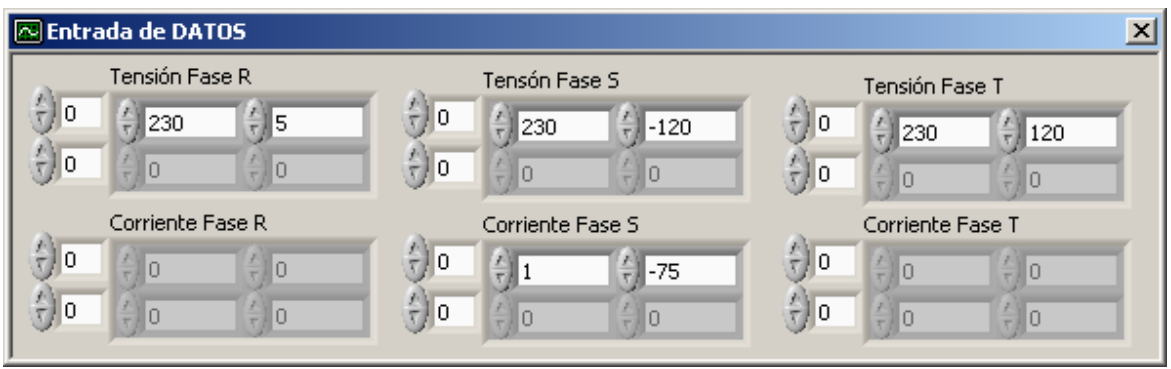

Figura V.63. Pantalla de entrada de datos.

Tenemos pues un sistema de tensiones en el que dejamos fijos los módulos de las tensiones de las fases, y tenemos una carga reactiva capacitiva que consume $1 \angle-75^{\circ} A$ conectada en la fase 2 , y tan sólo variamos de manera gradual el argumento de la fase 1 , logrando los pertinentes desequilibrios de tensión y produciendo la consiguiente potencia de desfase, tal y como podemos observar en la tabla V.30, y en la figura V.64 para el caso concreto de que el argumento de la fase 1 valga $5^{\circ}$.

\begin{tabular}{|c|c|c||}
\hline $\begin{array}{c}\text { Argumento } \\
\text { Fase 1 }\end{array}$ & $\begin{array}{c}\text { Variación } \\
\text { Argumento ( }\end{array}$ & $\begin{array}{c}\text { Potencia Desfase } \\
\text { Desequilibrio } \mathbf{Q}_{\text {dru }} \text { (VAr) }\end{array}$ \\
\hline $1^{\circ}$ & 1 & 0,94 \\
\hline $2^{\circ}$ & 2 & 1,88 \\
\hline $5^{\circ}$ & 5 & 4,66 \\
\hline $10^{\circ}$ & 10 & 9,14 \\
\hline $15^{\circ}$ & 15 & 13,42 \\
\hline $25^{\circ}$ & 25 & 21,22 \\
\hline $35^{\circ}$ & 35 & 27,83 \\
\hline $50^{\circ}$ & 50 & 35,07 \\
\hline $70^{\circ}$ & 70 & 39,05 \\
\hline $90^{\circ}$ & 90 & 36,14 \\
\hline $100^{\circ}$ & 100 & 32,18 \\
\hline $110^{\circ}$ & 110 & 26,69 \\
\hline $120^{\circ}$ & 120 & 19,84 \\
\hline
\end{tabular}

Tabla V.30. Potencia de Desfase \& Variación Argumento. 
De los resultados mostrados en la tabla V.30, vemos que la posición en la cual esta ubicada la carga, no influye a la hora de producirse un consumo de potencia de desfase debida al desequilibrio, mientras provoquemos los mismos grados de desequilibrio, ya que los valores de potencia de desfase inductiva que obtenemos en esta ocasión son iguales a los obtenidos en las tablas V.26 y 28.

En la figura V.64, podemos ver que esta potencia de desfase debida al desequilibrio del sistema, al poseer carácter inductivo, se resta a la debida al propio consumo de la carga capacitiva.

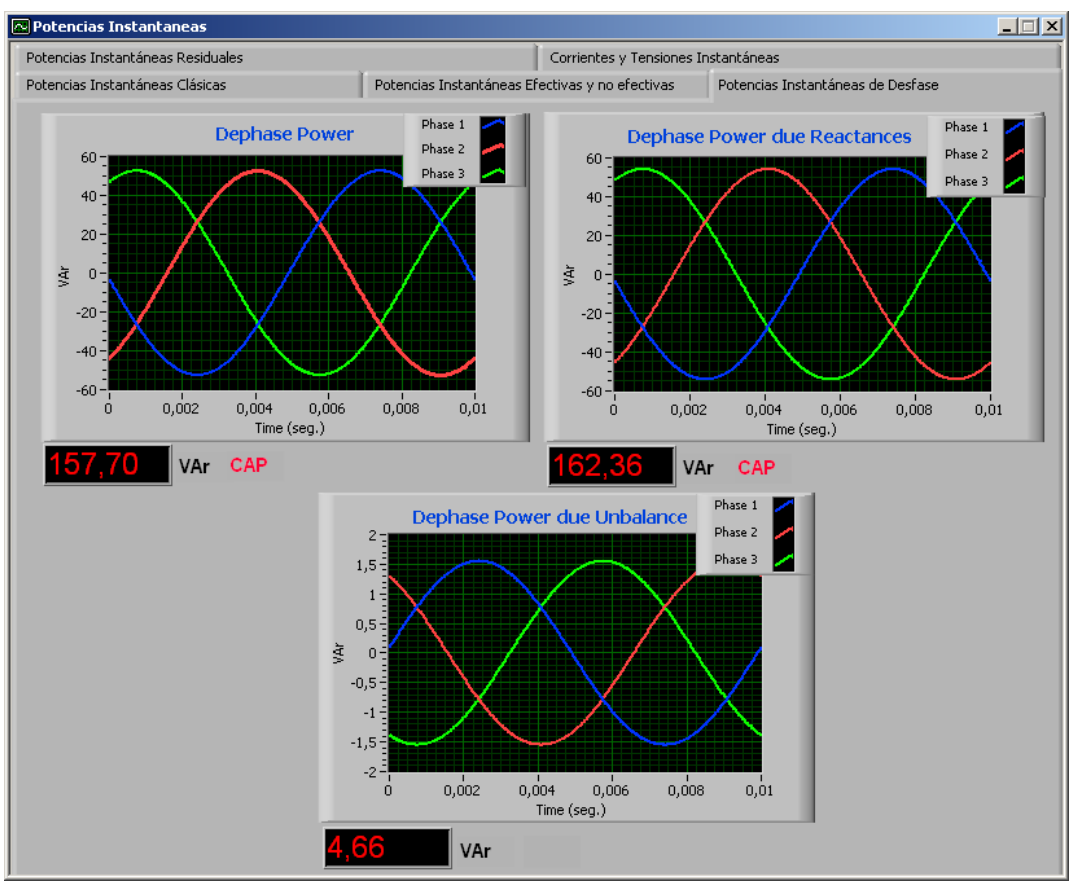

Figura V.64. Pantalla de Potencias de Desfase. 
Carga capacitiva entre la fase 2 y el neutro, con un consumo de $1 \angle-75^{\circ} A, y$ producimos el desequilibrio sumando ángulos negativos al argumento de la fase 1.

Introducimos los datos en el SIMPELEC Simulador, tal y como mostramos en la figura V.65.

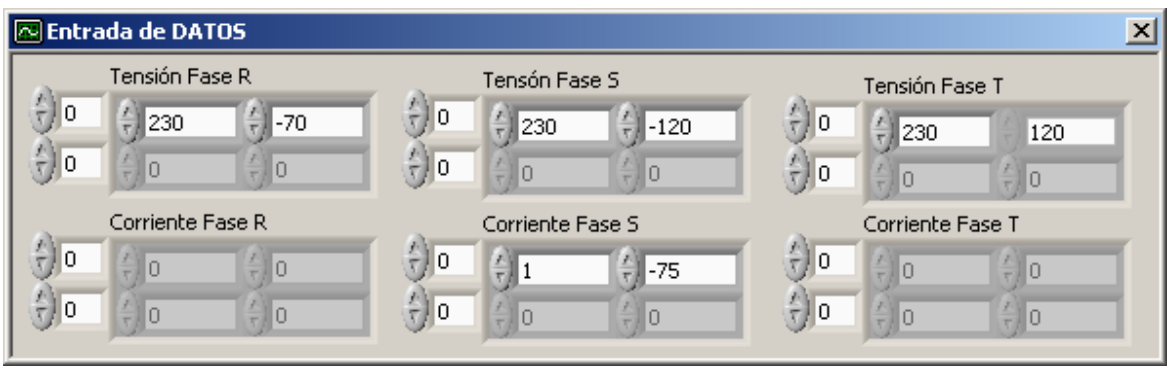

Figura V.65. Pantalla de entrada de datos.

Tenemos pues un sistema de tensiones en el que dejamos fijos los módulos de las tensiones de las fases, y tenemos una carga reactiva capacitiva que consume $1 \angle-75^{\circ} A$ conectada en la fase 2 , y tan sólo variamos de manera gradual el argumento de la fase 1 , logrando los pertinentes desequilibrios de tensión y produciendo la consiguiente potencia de desfase, tal y como podemos observar en la tabla V.31, y en la figura V.66 el caso particular de que el argumento de la fase 1 tome el valor de $-70^{\circ}$.

\begin{tabular}{||c|c|c||}
\hline $\begin{array}{c}\text { Argumento } \\
\text { Fase 1 }\end{array}$ & $\begin{array}{c}\text { Variación } \\
\text { Argumento ( }{ }^{\circ} \text { ) }\end{array}$ & $\begin{array}{c}\text { Potencia Desfase } \\
\text { Desequilibrio } \mathbf{Q}_{\text {dru }} \text { (VAr) }\end{array}$ \\
\hline$-1^{\circ}$ & 1 & $-0,95$ \\
\hline$-2^{\circ}$ & 2 & $-1,90$ \\
\hline$-5^{\circ}$ & 5 & $-4,79$ \\
\hline$-10^{\circ}$ & 10 & $-9,69$ \\
\hline$-15^{\circ}$ & 15 & $-14,65$ \\
\hline$-25^{\circ}$ & 25 & $-24,60$ \\
\hline$-35^{\circ}$ & 35 & $-34,36$ \\
\hline$-50^{\circ}$ & 50 & $-47,98$ \\
\hline$-70^{\circ}$ & 70 & $-62,83$ \\
\hline$-90^{\circ}$ & 90 & $-72,28$ \\
\hline$-100^{\circ}$ & 100 & $-74,60$ \\
\hline$-110^{\circ}$ & 110 & $-75,19$ \\
\hline$-120^{\circ}$ & 120 & $-74,05$ \\
\hline
\end{tabular}

Tabla V.30. Potencia de Desfase \& Variación Argumento. 
En la tabla V.31, vemos los resultados de variar el argumento de la fase 1, que provoca una potencia de desfase debida al desequilibrio capacitiva, aumentando el carácter capacitivo del receptor, como sucedía en las tablas V.27 y 29.

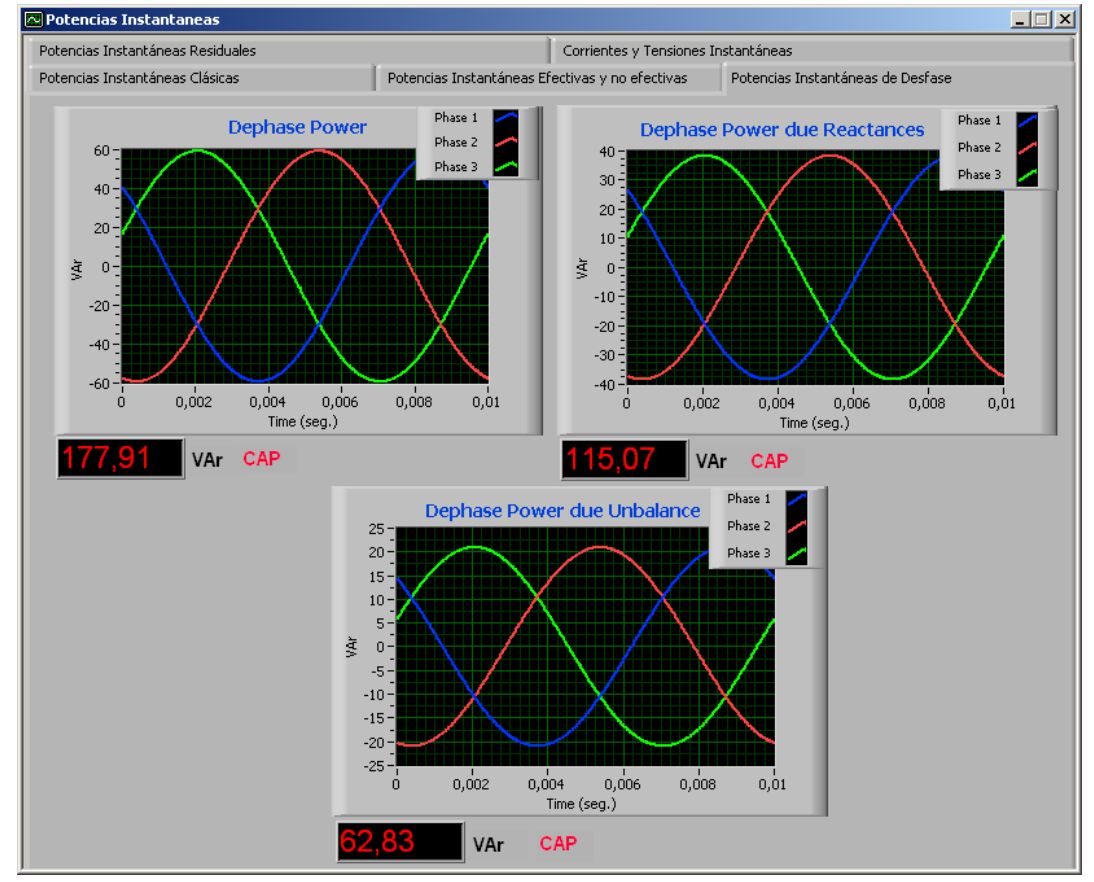

Figura V.66. Pantalla de Potencias de Desfase. 
Carga capacitiva entre la fase 2 y el neutro, con un consumo de $1 \angle-75^{\circ} \mathrm{A}, \mathrm{y}$ producimos el desequilibrio sumando ángulos positivos al argumento de la fase 3.

Introducimos los datos en el SIMPELEC Simulador, tal y como mostramos en la figura V.67.

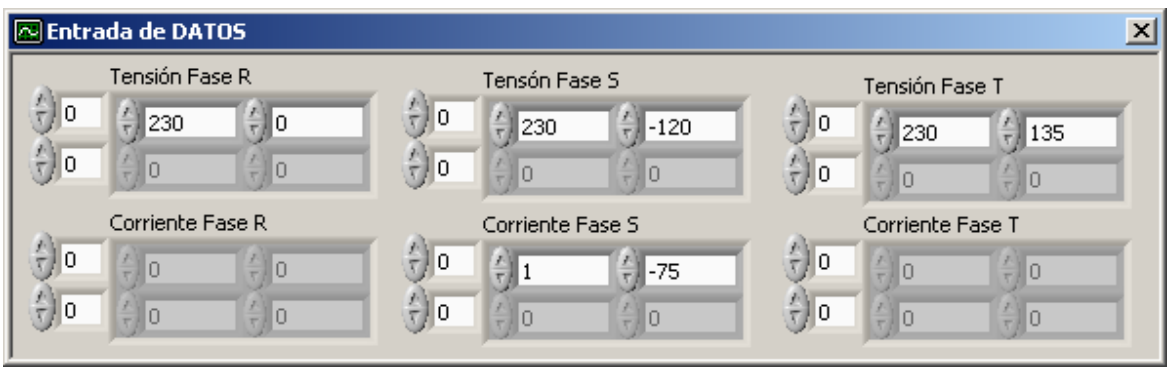

Figura V.67. Pantalla de entrada de datos.

Tenemos pues un sistema de tensiones en el que dejamos fijos los módulos de las tensiones de las fases, y tenemos una carga reactiva capacitiva que consume $1 \angle-75^{\circ} A$ conectada en la fase $2, y$ tan sólo variamos de manera gradual el argumento de la fase 3, logrando los pertinentes desequilibrios de tensión y produciendo la consiguiente potencia de desfase, tal y como podemos observar en la tabla V.32, y en la figura V.68 para el caso concreto de que el argumento de la fase 3 sea de $135^{\circ}$.

\begin{tabular}{|c|c|c||}
\hline $\begin{array}{c}\text { Argumento } \\
\text { Fase 3 }\end{array}$ & $\begin{array}{c}\text { Variación } \\
\text { Argumento ( }\end{array}$ & $\begin{array}{c}\text { Potencia Desfase } \\
\text { Desequilibrio } \mathbf{Q}_{\text {dru }} \text { (VAr) }\end{array}$ \\
\hline $121^{\circ}$ & 1 & 0,94 \\
\hline $122^{\circ}$ & 2 & 1,88 \\
\hline $125^{\circ}$ & 5 & 4,66 \\
\hline $130^{\circ}$ & 10 & 9,14 \\
\hline $135^{\circ}$ & 15 & 13,42 \\
\hline $145^{\circ}$ & 25 & 21,22 \\
\hline $155^{\circ}$ & 35 & 27,83 \\
\hline $170^{\circ}$ & 50 & 35,07 \\
\hline $190^{\circ}$ & 70 & 39,05 \\
\hline $210^{\circ}$ & 90 & 36,14 \\
\hline $220^{\circ}$ & 100 & 32,18 \\
\hline $230^{\circ}$ & 110 & 26,69 \\
\hline $240^{\circ}$ & 120 & 19,84 \\
\hline
\end{tabular}

Tabla V.32. Potencia de Desfase \& Variación Argumento. 
La tabla V.32, nos muestra los resultados de que la potencia de desfase debida al desequilibrio posee carácter inductivo, que resta a la debida a la existencia de un elemento capacitivo en el sistema, lo que apreciamos en la figura V.68 y como habíamos observado también en la tablas V.26, 28 y 30.

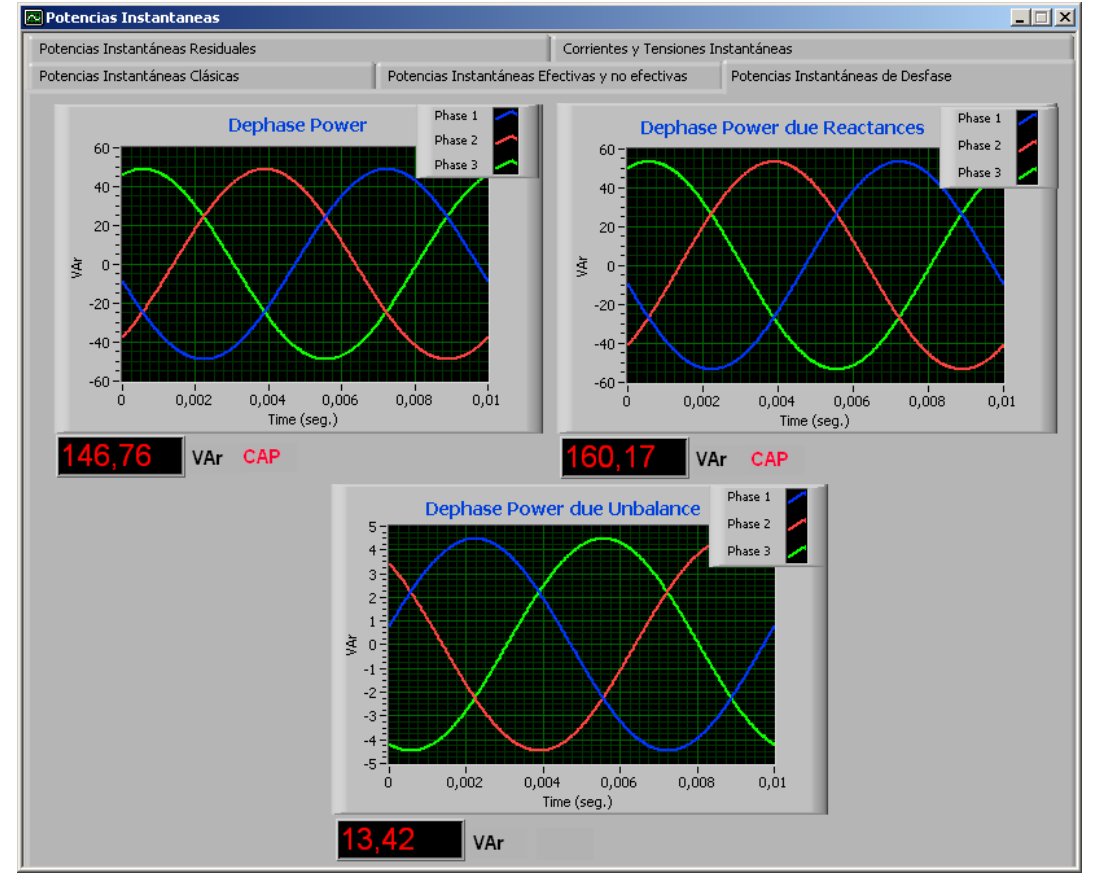

Figura V.68. Pantalla de Potencias de Desfase. 
Carga capacitiva entre la fase 2 y el neutro, con un consumo de $1 \angle-75^{\circ} A, y$ producimos el desequilibrio sumando ángulos negativos al argumento de la fase 3.

Introducimos los datos en el SIMPELEC Simulador, tal y como mostramos en la figura V.69.

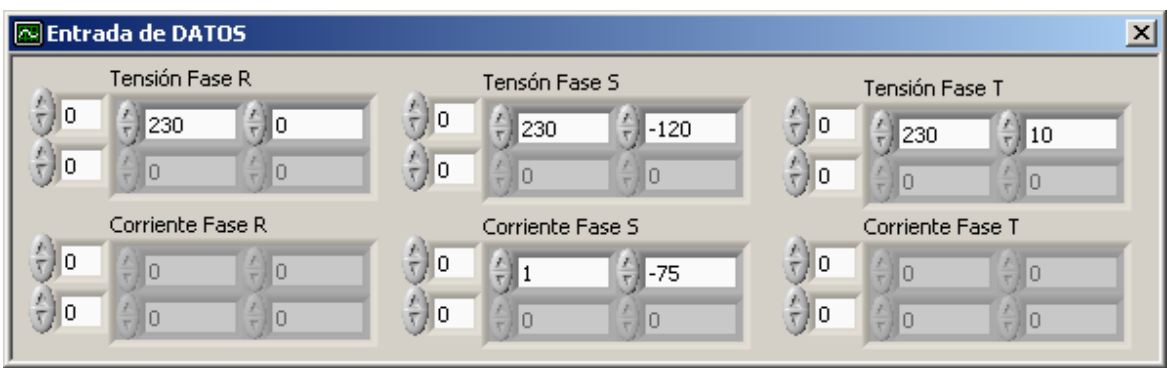

Figura V.69. Pantalla de entrada de datos.

Tenemos pues un sistema de tensiones en el que dejamos fijos los módulos de las tensiones de las fases, y tenemos una carga reactiva capacitiva que consume $1 \angle-75^{\circ} A$ conectada en la fase $2, y$ tan sólo variamos de manera gradual el argumento de la fase 3, logrando los pertinentes desequilibrios de tensión y produciendo la consiguiente potencia de desfase, tal y como podemos observar en la tabla V.33, y en la figura V.70 para el caso de que el argumento de la fase 3 tome el valor de $10^{\circ}$.

\begin{tabular}{|c|c|c||}
\hline $\begin{array}{c}\text { Argumento } \\
\text { Fase 3 }\end{array}$ & $\begin{array}{c}\text { Variación } \\
\text { Argumento ( }\end{array}$ & $\begin{array}{c}\text { Potencia Desfase } \\
\text { Desequilibrio } \mathbf{Q}_{\text {dru }} \text { (VAr) }\end{array}$ \\
\hline $119^{\circ}$ & 1 & $-0,95$ \\
\hline $118^{\circ}$ & 2 & $-1,90$ \\
\hline $115^{\circ}$ & 5 & $-4,79$ \\
\hline $110^{\circ}$ & 10 & $-9,69$ \\
\hline $105^{\circ}$ & 15 & $-14,65$ \\
\hline $95^{\circ}$ & 25 & $-24,60$ \\
\hline $85^{\circ}$ & 35 & $-34,36$ \\
\hline $70^{\circ}$ & 50 & $-47,98$ \\
\hline $50^{\circ}$ & 70 & $-62,83$ \\
\hline $30^{\circ}$ & 90 & $-72,28$ \\
\hline $20^{\circ}$ & 100 & $-74,60$ \\
\hline $10^{\circ}$ & 110 & $-75,19$ \\
\hline $0^{\circ}$ & 120 & $-74,05$ \\
\hline
\end{tabular}

Tabla V.33. Potencia de Desfase \& Variación Argumento. 
En la tabla V.33, apreciamos el carácter capacitivo que tiene la potencia de desfase debida al desequilibrio, cuando procedemos a variar gradualmente el argumento de la fase 3, provocando esto como se observa en la figura V.70 que la potencia de desfase total del sistema aumente, como también sucedía en los resultados mostrados en las tablas V.27, 29 y 31.

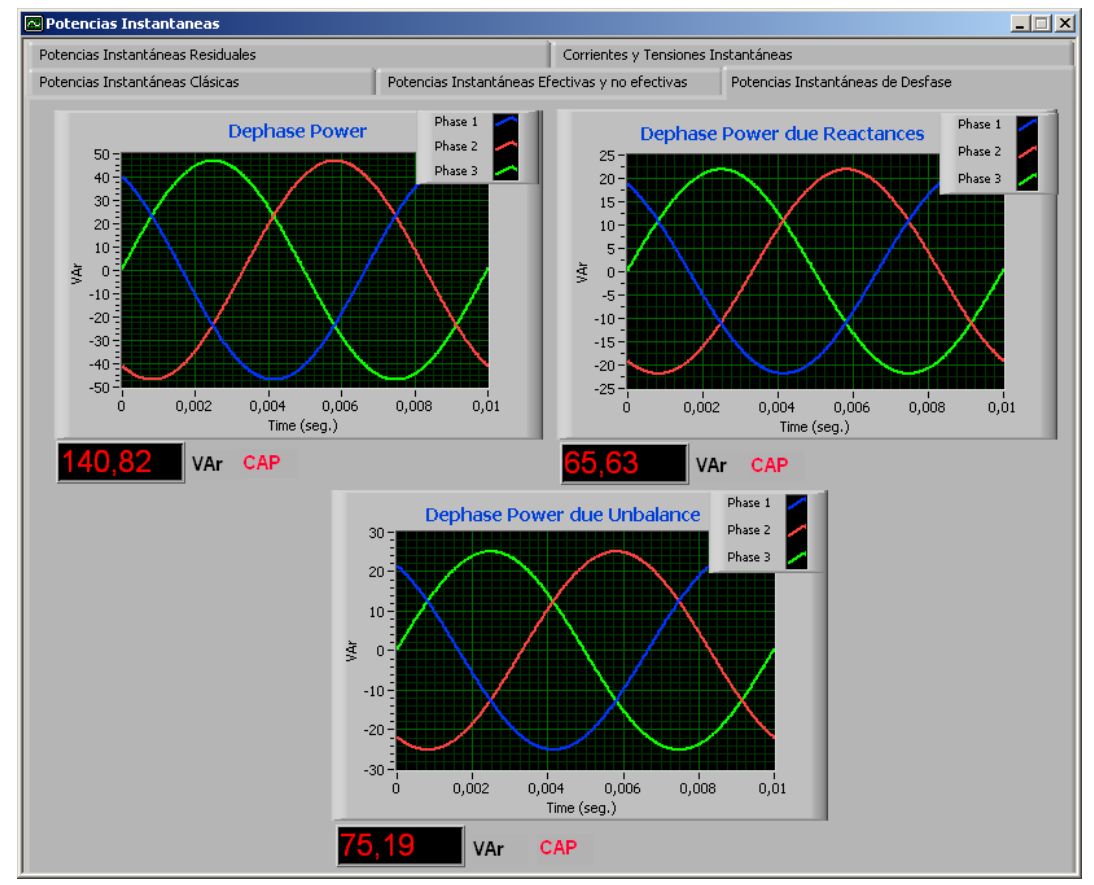

Figura V.70. Pantalla de Potencias de Desfase. 
Carga capacitiva entre la fase 3 y el neutro, con un consumo de $1 \angle 165^{\circ} \mathrm{A}, \mathrm{y}$ producimos el desequilibrio sumando ángulos positivos al argumento de la fase 1.

Introducimos los datos en el SIMPELEC Simulador, tal y como mostramos en la figura V.71.

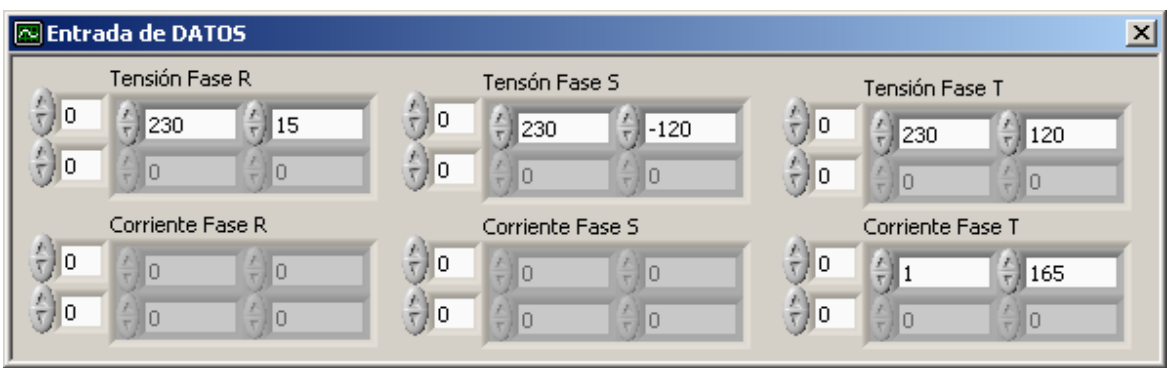

Figura V.71. Pantalla de entrada de datos.

Tenemos pues un sistema de tensiones en el que dejamos fijos los módulos de las tensiones de las fases, y tenemos una carga reactiva capacitiva que consume $1 \angle 165^{\circ} A$ conectada en la fase $3, y$ tan sólo variamos de manera gradual el argumento de la fase 1, logrando los pertinentes desequilibrios de tensión y produciendo la consiguiente potencia de desfase, tal y como podemos observar en la tabla V.34, y en la figura V.72 cuando el argumento de la fase 1 toma el valor de $15^{\circ}$.

\begin{tabular}{|c|c|c||}
\hline $\begin{array}{c}\text { Argumento } \\
\text { Fase 1 }\end{array}$ & $\begin{array}{c}\text { Variación } \\
\text { Argumento } \mathbf{(}^{\mathbf{0}} \text { ) }\end{array}$ & $\begin{array}{c}\text { Potencia Desfase } \\
\text { Desequilibrio } \mathbf{Q}_{\text {dru }} \text { (VAr) }\end{array}$ \\
\hline $1^{\circ}$ & 1 & 0,94 \\
\hline $2^{\circ}$ & 2 & 1,88 \\
\hline $5^{\circ}$ & 5 & 4,66 \\
\hline $10^{\circ}$ & 10 & 9,14 \\
\hline $15^{\circ}$ & 15 & 13,42 \\
\hline $25^{\circ}$ & 25 & 21,22 \\
\hline $35^{\circ}$ & 35 & 27,83 \\
\hline $50^{\circ}$ & 50 & 35,07 \\
\hline $70^{\circ}$ & 70 & 39,05 \\
\hline $90^{\circ}$ & 90 & 36,14 \\
\hline $100^{\circ}$ & 100 & 32,18 \\
\hline $110^{\circ}$ & 110 & 26,69 \\
\hline $120^{\circ}$ & 120 & 19,84 \\
\hline
\end{tabular}

Tabla V.34. Potencia de Desfase \& Variación Argumento. 
En la tabla V.34, observamos el carácter inductivo de la potencia de desfase debida al desequilibrio, al igual que sucedía en las tablas V.26, 28, 30 y 32.

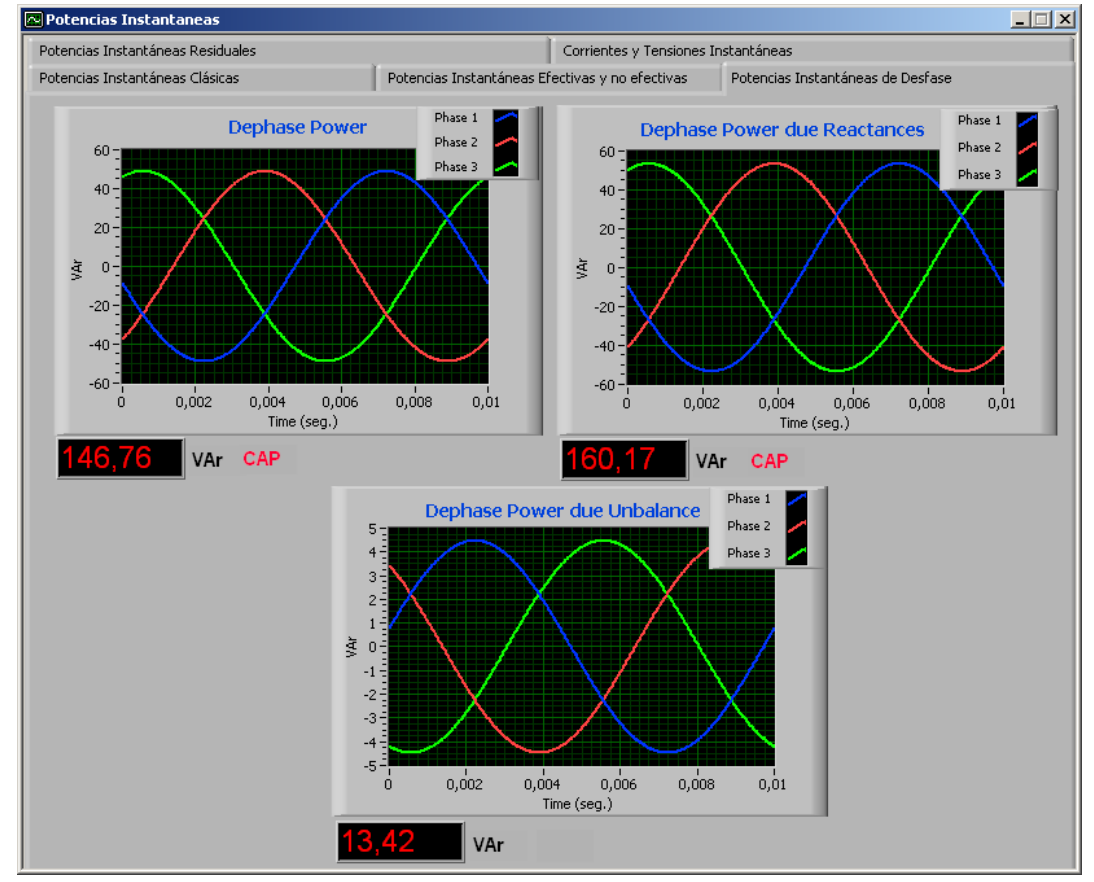

Figura V.72. Pantalla de Potencias de Desfase. 
Carga capacitiva entre la fase 3 y el neutro, con un consumo de $1 \angle 165^{\circ} \mathrm{A}, \mathrm{y}$ producimos el desequilibrio sumando ángulos negativos al argumento de la fase 1.

Introducimos los datos en el SIMPELEC Simulador, tal y como mostramos en la figura V.73.

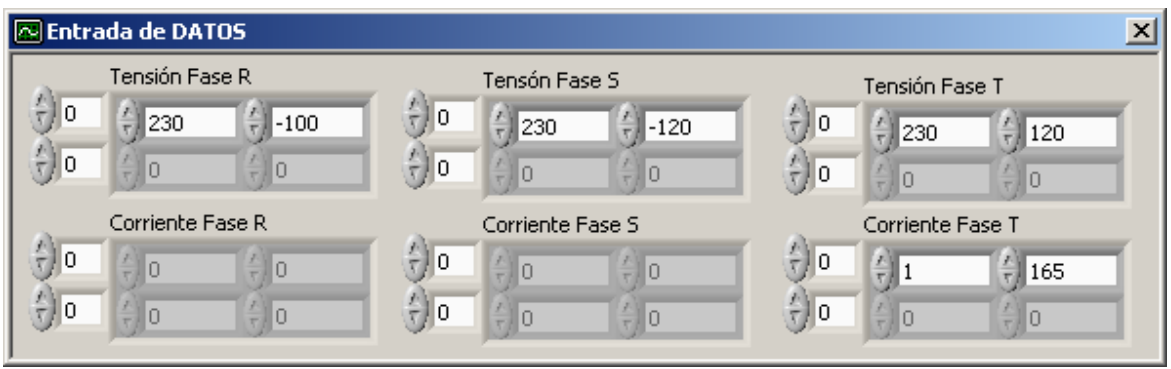

Figura V.73. Pantalla de entrada de datos.

Tenemos pues un sistema de tensiones en el que dejamos fijos los módulos de las tensiones de las fases, y tenemos una carga reactiva capacitiva que consume $1 \angle 165^{\circ} A$ conectada en la fase $3, y$ tan sólo variamos de manera gradual el argumento de la fase 1 , logrando los pertinentes desequilibrios de tensión y produciendo la consiguiente potencia de desfase, tal y como podemos observar en la tabla V.35, y en la figura V.74 para el caso concreto de que el argumento de la fase 1 toma el valor de $-100^{\circ}$.

\begin{tabular}{|c|c|c||}
\hline $\begin{array}{c}\text { Argumento } \\
\text { Fase 1 }\end{array}$ & $\begin{array}{c}\text { Variación } \\
\text { Argumento } \mathbf{(}^{\circ} \text { ) }\end{array}$ & $\begin{array}{c}\text { Potencia Desfase } \\
\text { Desequilibrio } \mathbf{Q}_{\text {dru }} \text { (VAr) }\end{array}$ \\
\hline$-1^{\circ}$ & 1 & $-0,95$ \\
\hline$-2^{\circ}$ & 2 & $-1,90$ \\
\hline$-5^{\circ}$ & 5 & $-4,79$ \\
\hline$-10^{\circ}$ & 10 & $-9,69$ \\
\hline$-15^{\circ}$ & 15 & $-14,65$ \\
\hline$-25^{\circ}$ & 25 & $-24,60$ \\
\hline$-35^{\circ}$ & 35 & $-34,36$ \\
\hline$-50^{\circ}$ & 50 & $-47,98$ \\
\hline$-70^{\circ}$ & 70 & $-62,83$ \\
\hline$-90^{\circ}$ & 90 & $-72,28$ \\
\hline$-100^{\circ}$ & 100 & $-74,60$ \\
\hline$-110^{\circ}$ & 110 & $-75,19$ \\
\hline$-120^{\circ}$ & 120 & $-74,05$ \\
\hline
\end{tabular}

Tabla V.35. Potencia de Desfase \& Variación Argumento. 
De la tabla V.35, se desprende el carácter capacitivo que toma la potencia de desfase debida al desequilibrio, y que se suma a la potencia de desfase debida a la presencia de un elemento capacitivo, al igual que en las tablas V.27, 29, 31 y 33.

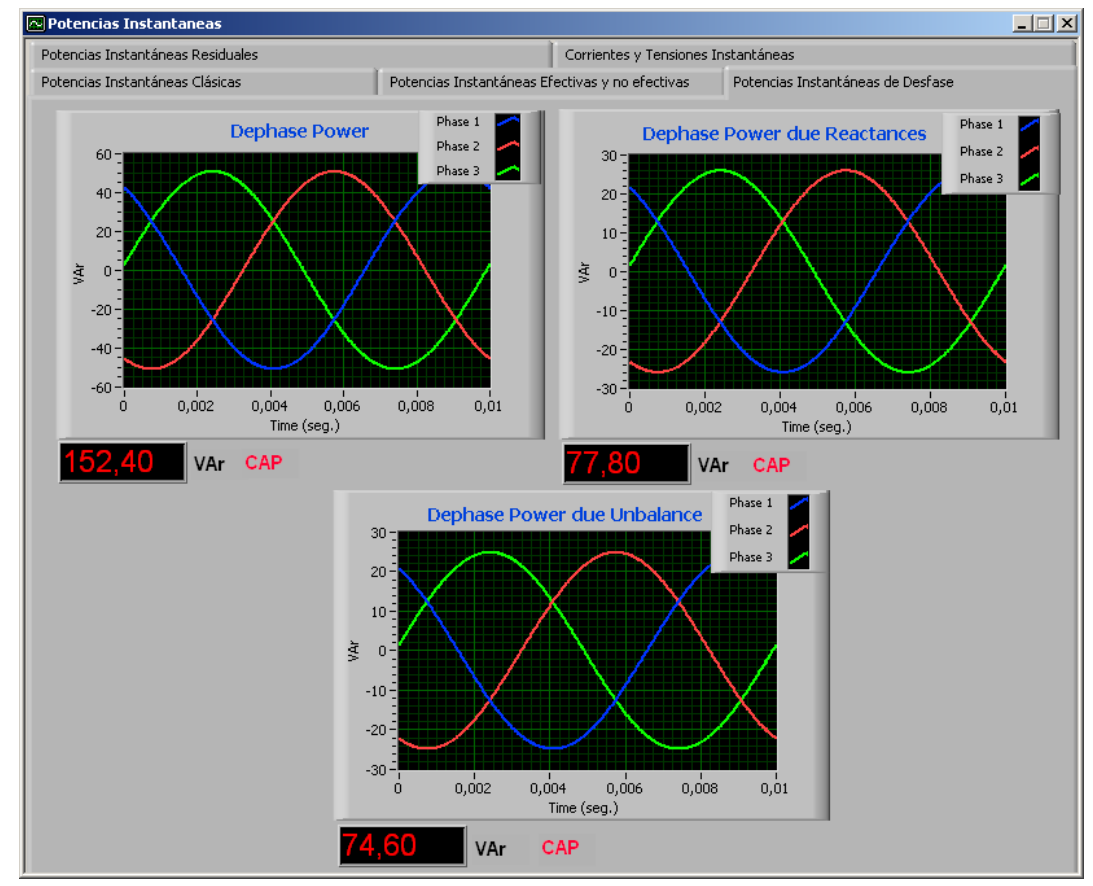

Figura V.74. Pantalla de Potencias de Desfase. 
Carga capacitiva entre la fase 3 y el neutro, con un consumo de $1 \angle 165^{\circ} \mathrm{A}, \mathrm{y}$ producimos el desequilibrio sumando ángulos positivos al argumento de la fase 2.

Introducimos los datos en el SIMPELEC Simulador, tal y como mostramos en la figura V.75.

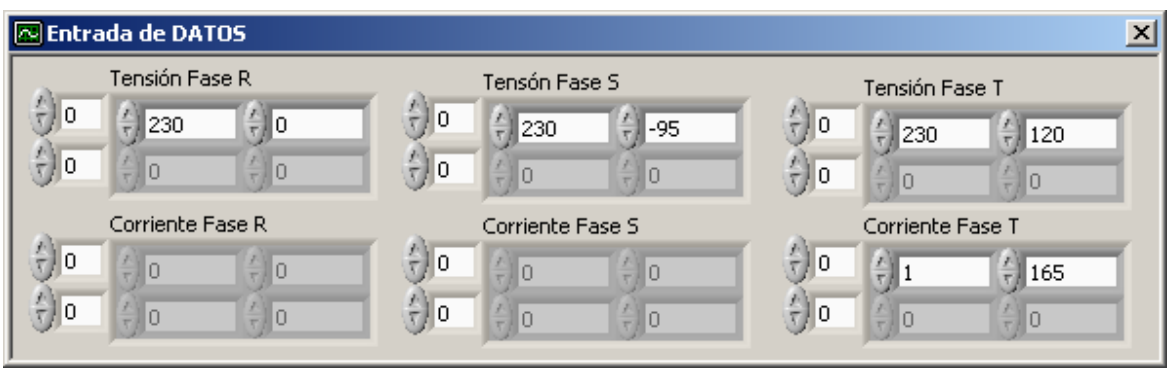

Figura V.75. Pantalla de entrada de datos.

Tenemos pues un sistema de tensiones en el que dejamos fijos los módulos de las tensiones de las fases, y tenemos una carga reactiva capacitiva que consume $1 \angle 75^{\circ} A$ conectada en la fase 3 , y tan sólo variamos de manera gradual el argumento de la fase 2 , logrando los pertinentes desequilibrios de tensión y produciendo la consiguiente potencia de desfase, tal y como podemos observar en la tabla V.36, y en la figura V.76 para el caso de que el argumento de la fase 2 tome el valor de $-95^{\circ}$.

\begin{tabular}{|c|c|c||}
\hline $\begin{array}{c}\text { Argumento } \\
\text { Fase 2 }\end{array}$ & $\begin{array}{c}\text { Variación } \\
\text { Argumento } \mathbf{(}^{\circ} \text { ) }\end{array}$ & $\begin{array}{c}\text { Potencia Desfase } \\
\text { Desequilibrio } \mathbf{Q}_{\text {dru }} \text { (VAr) }\end{array}$ \\
\hline$-119^{\circ}$ & 1 & 0,94 \\
\hline$-118^{\circ}$ & 2 & 1,88 \\
\hline$-115^{\circ}$ & 5 & 4,66 \\
\hline$-110^{\circ}$ & 10 & 9,14 \\
\hline$-105^{\circ}$ & 15 & 13,42 \\
\hline$-95^{\circ}$ & 25 & 21,22 \\
\hline$-85^{\circ}$ & 35 & 27,83 \\
\hline$-70^{\circ}$ & 50 & 35,07 \\
\hline$-50^{\circ}$ & 70 & 39,05 \\
\hline$-30^{\circ}$ & 90 & 36,14 \\
\hline$-20^{\circ}$ & 100 & 32,18 \\
\hline$-10^{\circ}$ & 110 & 26,69 \\
\hline $0^{\circ}$ & 120 & 19,84 \\
\hline \multicolumn{2}{|c|}{} \\
\hline
\end{tabular}

Tabla V.36. Potencia de Desfase \& Variación Argumento. 
De la observación de la tabla V.36, vemos que la potencia de desfase debida al desequilibrio tiene un carácter inductivo, al igual que sucedía en los resultados de las tablas V.26, 28, 30, 32 y 34, lo que provoca un decremento de la potencia de desfase total del sistema, ya que se resta a la potencia de desfase debida a la presencia del elemento capacitivo en el sistema, como se ve en la figura V.76.

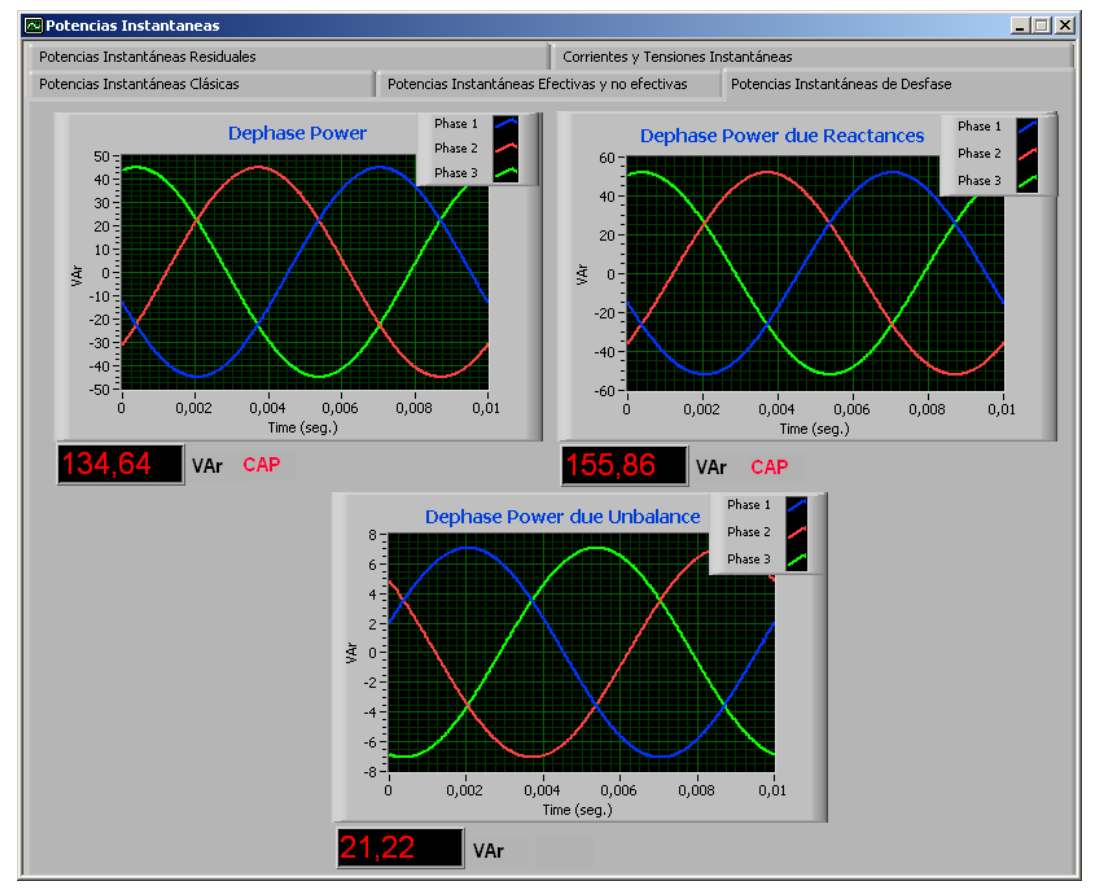

Figura V.76. Pantalla de Potencias de Desfase. 
Carga capacitiva entre la fase 3 y el neutro, con un consumo de $1 \angle 165^{\circ} \mathrm{A}, \mathrm{y}$ producimos el desequilibrio sumando ángulos negativos al argumento de la fase 2.

Introducimos los datos en el SIMPELEC Simulador, tal y como mostramos en la figura V.77.

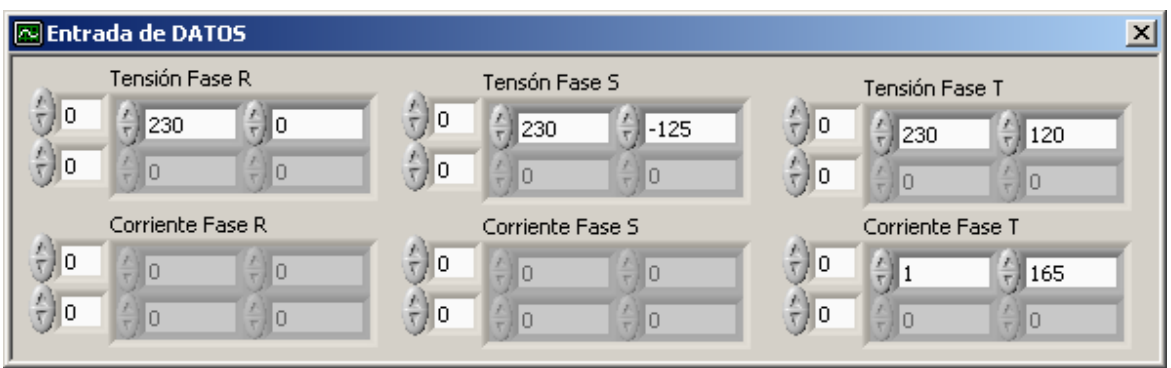

Figura V.77. Pantalla de entrada de datos.

Tenemos pues un sistema de tensiones en el que dejamos fijos los módulos de las tensiones de las fases, y tenemos una carga reactiva capacitiva que consume $1 \angle 165^{\circ} A$ conectada en la fase 3 , y tan sólo variamos de manera gradual el argumento de la fase 2, logrando los pertinentes desequilibrios de tensión y produciendo la consiguiente potencia de desfase, tal y como podemos observar en la tabla V.37, y en la figura V.78 cuando el argumento de la fase 2 vale $-125^{\circ}$.

\begin{tabular}{|c|c|c||}
\hline $\begin{array}{c}\text { Argumento } \\
\text { Fase 2 }\end{array}$ & $\begin{array}{c}\text { Variación } \\
\text { Argumento } \mathbf{(}^{\circ} \text { ) }\end{array}$ & $\begin{array}{c}\text { Potencia Desfase } \\
\text { Desequilibrio } \mathbf{Q}_{\text {dru }} \text { (VAr) }\end{array}$ \\
\hline$-121^{\circ}$ & 1 & $-0,95$ \\
\hline$-122^{\circ}$ & 2 & $-1,90$ \\
\hline$-125^{\circ}$ & 5 & $-4,79$ \\
\hline$-130^{\circ}$ & 10 & $-9,69$ \\
\hline$-135^{\circ}$ & 15 & $-14,65$ \\
\hline$-145^{\circ}$ & 25 & $-24,60$ \\
\hline$-155^{\circ}$ & 35 & $-34,36$ \\
\hline$-170^{\circ}$ & 50 & $-47,98$ \\
\hline$-190^{\circ}$ & 70 & $-62,83$ \\
\hline$-210^{\circ}$ & 90 & $-72,28$ \\
\hline$-220^{\circ}$ & 100 & $-74,60$ \\
\hline$-230^{\circ}$ & 110 & $-75,19$ \\
\hline$-240^{\circ}$ & 120 & $-74,05$ \\
\hline
\end{tabular}

Tabla V.37. Potencia de Desfase \& Variación Argumento. 
La tabla V.37, nos muestra los resultados obtenidos por la variación del argumento de la fase 2 de $-120^{\circ}$ a $-240^{\circ}$, que produce una potencia de desfase debida al desequilibrio capacitiva, y cuyos valores coinciden con los de las tablas V.27, 29, 31, 33 y 35.

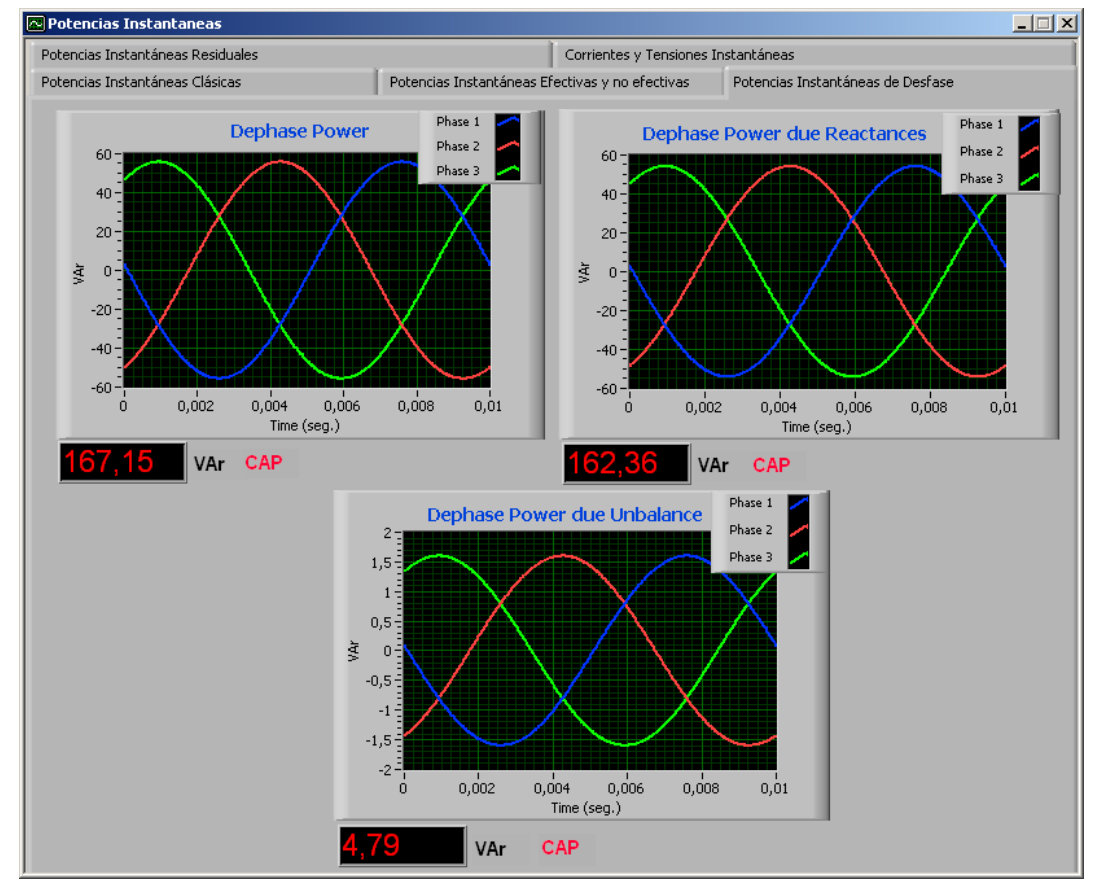

Figura V.78. Pantalla de Potencias de Desfase.

Podemos observar en la figura V.79, las gráficas que resultan de los resultados obtenidos cuando el sistema tiene cargas capacitivas de manera sucesiva en cada fase, y lo sometemos a diversos grados de desequilibrio. Se ve claramente que el sistema posee comportamientos que le llevan a consumir potencia de desfase capacitiva (cuando sumamos ángulos negativos a los argumentos de las fases descargadas), que hace que se incremente el valor teórico que debería de producirse en el sistema si no existieran desequilibrios en el sistema, y un comportamiento que lleva a consumir potencia de desfase de carácter inductivo (si se suman ángulos positivos a los argumentos de las fases descargadas), lo que provoca que el sistema tenga un comportamiento inductivo cuando teóricamente debiera de ser capacitivo, sino existieran estos desequilibrios. 


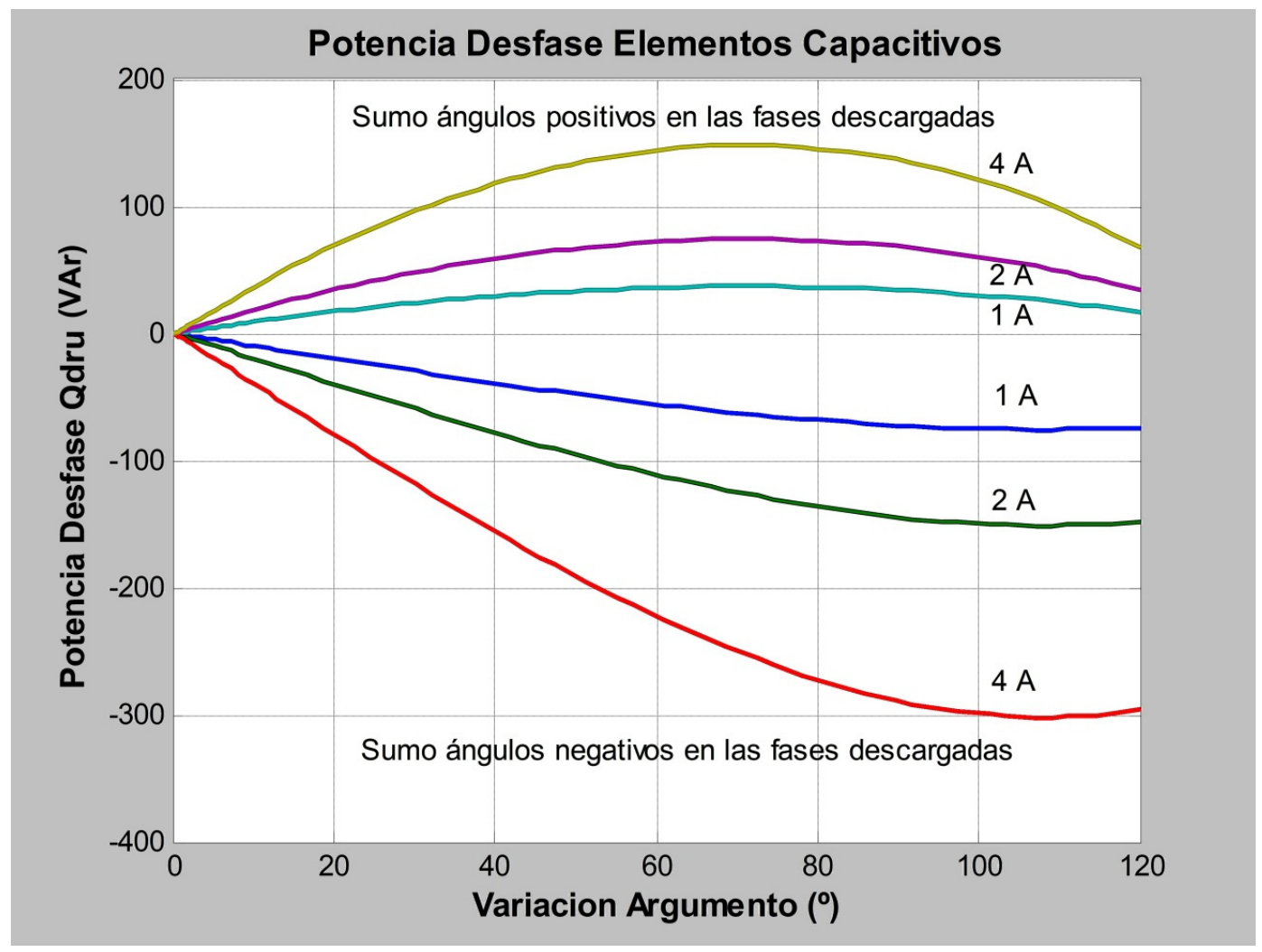

Figura V.79. Potencias de Desfase, para diversas variaciones de carga capacitiva en una fase y grados de desequilibrio.

En la figura V.79, también se muestran las gráficas obtenidas cuando la carga posee consumos de 1,2 y 4 Amperios. 
c.1) Carga Capacitiva Pura: Se analiza el comportamiento de este elemento frente a diversos desequilibrios que se producen, estando esta carga de manera sucesiva en cada fase.

El procedimiento realizado, es idéntico al usado para el caso que acabamos de analizar de carga capacitiva con un consumo de $1 \angle 45^{\circ} \mathrm{A}$, tan sólo que ahora la carga es capacitiva pura con un consumo de $1 \angle 90^{\circ} A$, introducimos los datos en el simulador SIMPELEC, tal y como se muestra en la figura V.80, que se corresponde con el caso de estar la carga capacitiva pura entre la fase 1 y el neutro, y siendo el desequilibrio de tensiones provocado por la suma de ángulos positivos al argumento de la fase 3, que en este caso concreto es de una variación de $70^{\circ}$.

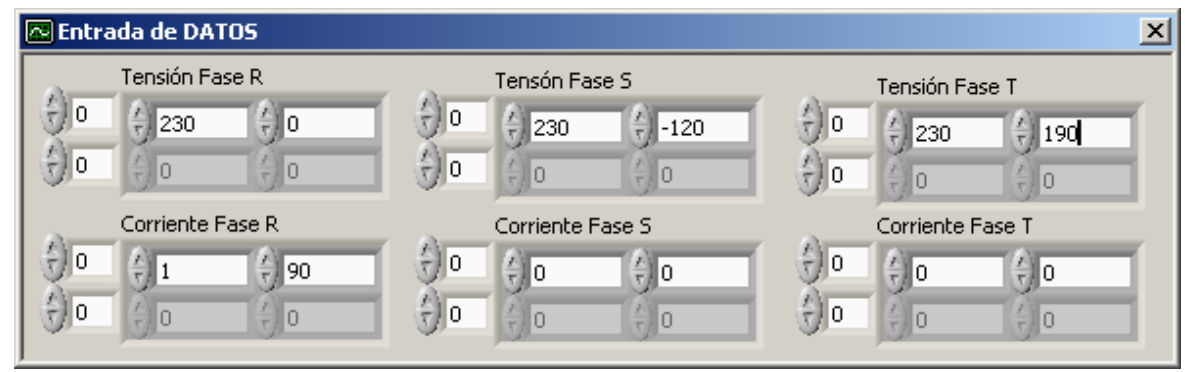

Figura V.80. Pantalla de entrada de datos.

Lo que nos da un consumo de potencia de desfase debida al desequilibrio con carácter capacitivo, tal y como queda reflejado en la figura V.81, siendo el valor de esta de 16,82 VAr, que incrementan el que ya posee el sistema. 


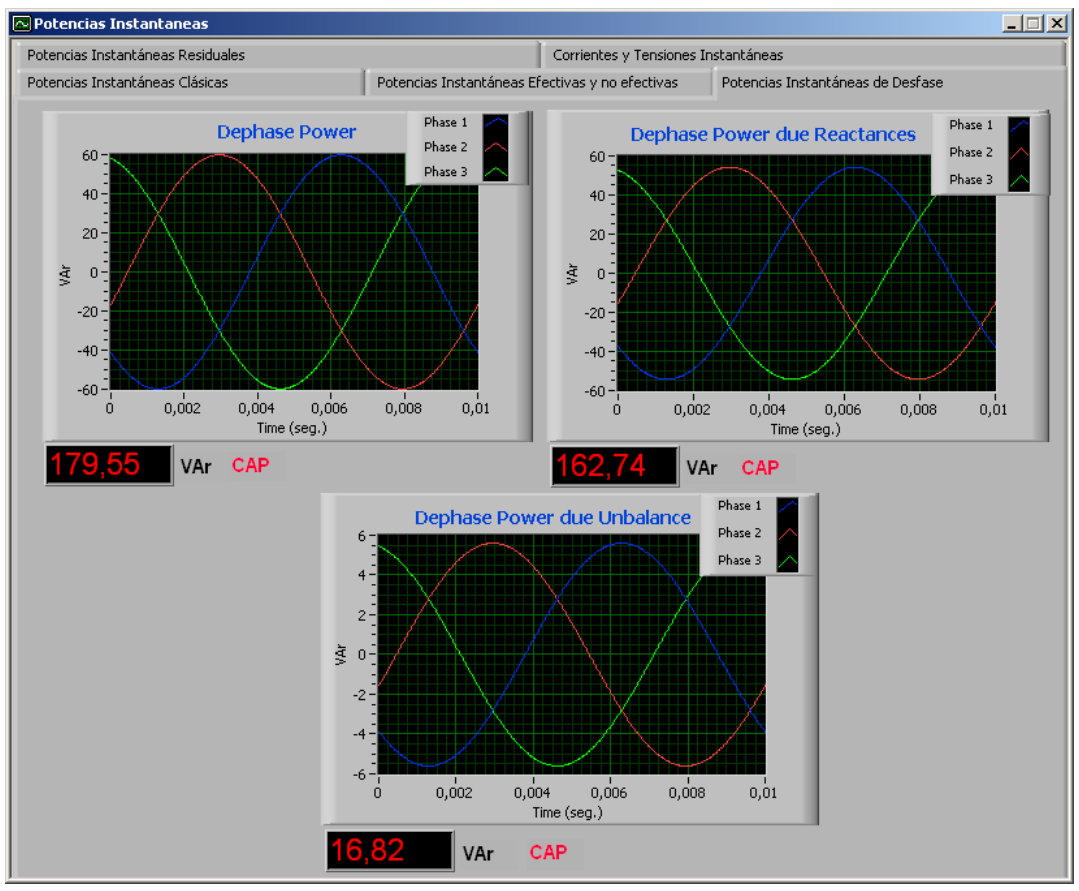

Figura V.81. Pantalla de Potencias de Desfase.

Con esta carga capacitiva pura se han analizado los siguientes casos que pasamos a mencionar:

- Carga conectada entre la fase 1 y neutro, con variación de argumentos de la fase 2, variando estos de $-120^{\circ}$ a $0^{\circ}$. (sumo ángulos positivos)

- Carga conectada entre la fase 1 y neutro, con variación de argumentos de la fase 2 , variando estos de $-120^{\circ}$ a $-240^{\circ}$. (sumo ángulos negativos)

- Carga conectada entre la fase 1 y el neutro, con variación de argumentos de la fase 3 , variando estos de $120^{\circ}$ a $240^{\circ}$. (sumo ángulos positivos)

- Carga conectada entre la fase 1 y el neutro, con variación de argumentos de la fase 3 , variando estos de $120^{\circ}$ a $0^{\circ}$. (sumo ángulos negativos)

- Carga conectada entre la fase 2 y el neutro, con variación de argumentos de la fase 1 , variando estos de $0^{\circ}$ a $120^{\circ}$. (sumo ángulos positivos)

- Carga conectada entre la fase 2 y el neutro, con variación de argumentos de la fase 1 , variando estos de $0^{\circ} \mathrm{a}-120^{\circ}$. (sumo ángulos negativos)

- Carga conectada entre la fase 2 y el neutro, con variación de argumentos de la fase 3, variando estos de $120^{\circ}$ a $240^{\circ}$. (sumo ángulo positivos) 
- Carga conectada entre la fase 2 y el neutro, con variación de argumentos de la fase 3 , variando estos de $120^{\circ}$ a $0^{\circ}$. (sumo ángulo negativos)

- Carga conectada entre la fase 3 y el neutro, con variación de argumentos de la fase 1 , variando estos de $0^{\circ}$ a $120^{\circ}$. (sumo ángulo positivos)

- Carga conectada entre la fase 3 y el neutro, con variación de argumentos de la fase 1 , variando estos de $0^{\circ}$ a $-120^{\circ}$. (sumo ángulos negativos)

- Carga conectada entre la fase 3 y el neutro, con variación de argumentos de la fase 2, variando estos de $-120^{\circ}$ a $0^{\circ}$. (sumo ángulos positivos)

- Carga conectada entre la fase 3 y el neutro, con variación de argumentos de la fase 2 , variando estos de $-120^{\circ}$ a $-240^{\circ}$. (sumo ángulos negativos)

En cada uno de los casos anteriores se fueron modificando de manera gradual, el incremento del argumento de la fase correspondiente, para provocar el desequilibrio, y a continuación se muestran los resultados obtenidos, en los cuales se evidencia que la potencia de desfase debida al desequilibrio, posee un carácter capacitivo que refuerza a la del sistema.

En la tabla V.38 se muestran los incrementos de ángulos provocados, así como la potencia de desfase debida al desequilibrio que conlleva, que fueron iguales para todos los casos que acabamos de presentar.

\begin{tabular}{|c|c|}
\hline $\begin{array}{c}\text { Variación } \\
\text { Argumento ( }{ }^{\circ} \text { ) }\end{array}$ & $\begin{array}{c}\text { Potencia Desfase } \\
\text { Desequilibrio } \mathbf{Q}_{\text {dru }} \text { (VAr) }\end{array}$ \\
\hline 1 & 0 \\
\hline 2 & $-0,02$ \\
\hline 5 & $-0,10$ \\
\hline 10 & $-0,39$ \\
\hline 15 & $-0,87$ \\
\hline 25 & $-2,21$ \\
\hline 35 & $-4,62$ \\
\hline 50 & $-9,13$ \\
\hline 70 & $-16,82$ \\
\hline 90 & $-25,26$ \\
\hline 100 & $-29,99$ \\
\hline 110 & $-34,30$ \\
\hline 120 & $-38,33$ \\
\hline
\end{tabular}

Tabla V.38 Potencia de Desfase \& Variación Argumento 


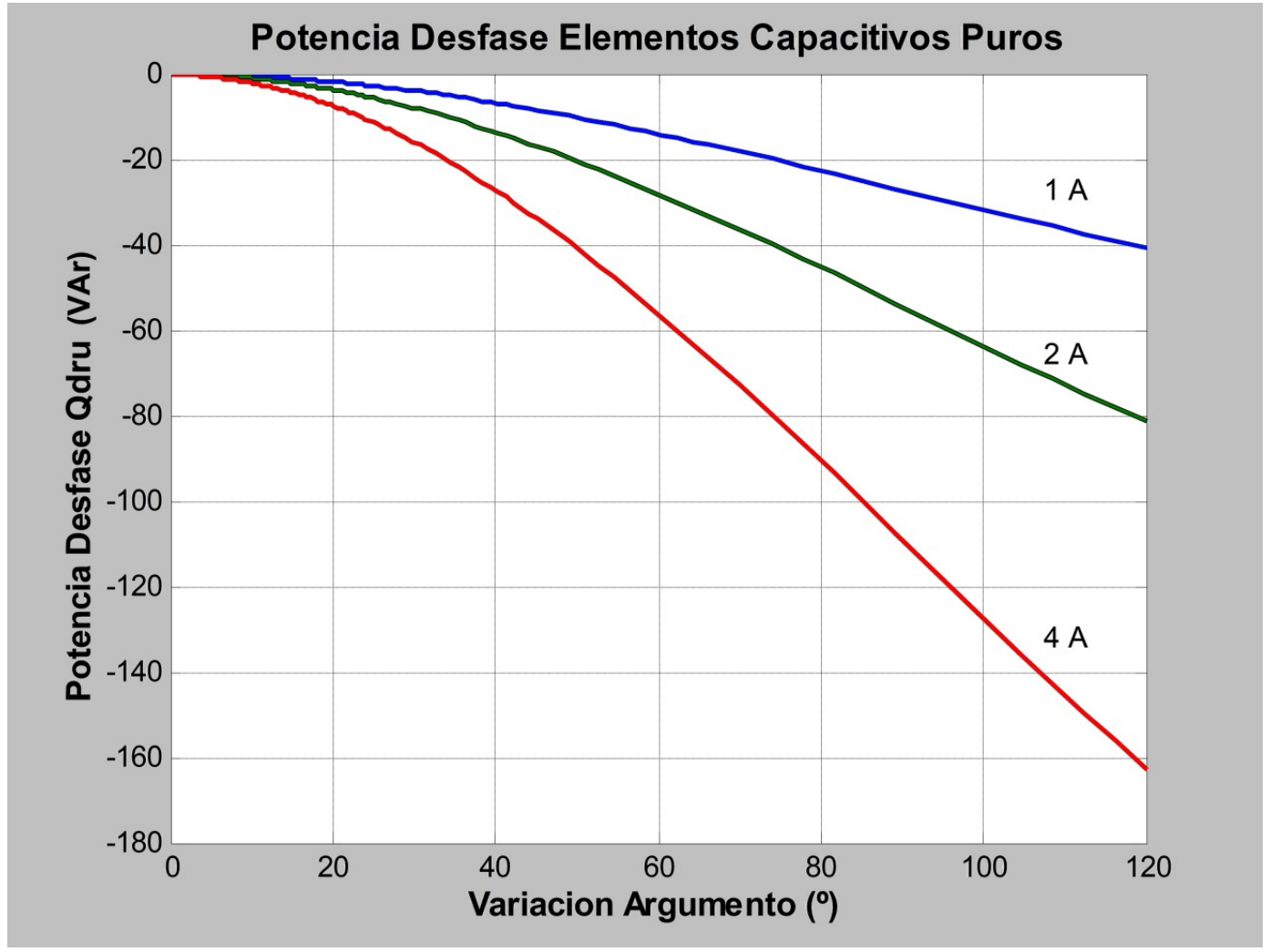

Figura V.82. Potencias de Desfase, para diversas variaciones de carga capacitiva pura en una fase y variaciones de argumento.

En la figura V.82, se muestran los resultados obtenidos para el caso de que ahora el sistema lo forme una carga capacitiva pura, se observa que con independencia de como hayamos conseguido el desequilibrio del sistema o en que fase se halle conectada la carga, el consumo de potencia de desfase debida al desequilibrio es siempre capacitiva. Lo que provoca que la potencia de desfase total, sea mayor que la debida a la que correspondería a la presencia de cargas capacitivas, ya que en este caso se suma también la potencia de desfase debida al desequilibrio. En la gráfica se puede ver también las potencias de desfase debidas al desequilibrio, cuando los consumos de las cargas son de 2 y 4 Amperios. 


\section{V.3.2.- Cargas conectadas entre dos fases y el neutro.}

El proceso usado para esta comprobación, es como el efectuado en los apartados anteriores, quedando por lo tanto los módulos de las tensiones constantes, y en este caso se provocan desequilibrios en el sistema variando el argumento de la fase descargada, por medio de la suma de ángulos crecientes y decrecientes en la misma.

a) Cargas Resistivas: Se analiza el comportamiento de este sistema compuesto por dos cargas resistivas frente a diversos desequilibrios provocados en el sistema de alimentación.

Cargas resistivas entre las fase 1,2 y el neutro, con un consumo de $1 \angle 0^{\circ} \mathrm{A}$ y de $1 \angle-120^{\circ} A$ y producimos el desequilibrio sumando ángulos positivos al argumento de la fase 3.

Introducimos gracias a la ventana de entrada de datos del SIMPELEC Simulador, las tensiones de las fases y el consumo, así como las sucesivas modificaciones en los argumentos de la fase 3 en este caso, tal y como mostramos en la figura V.83.

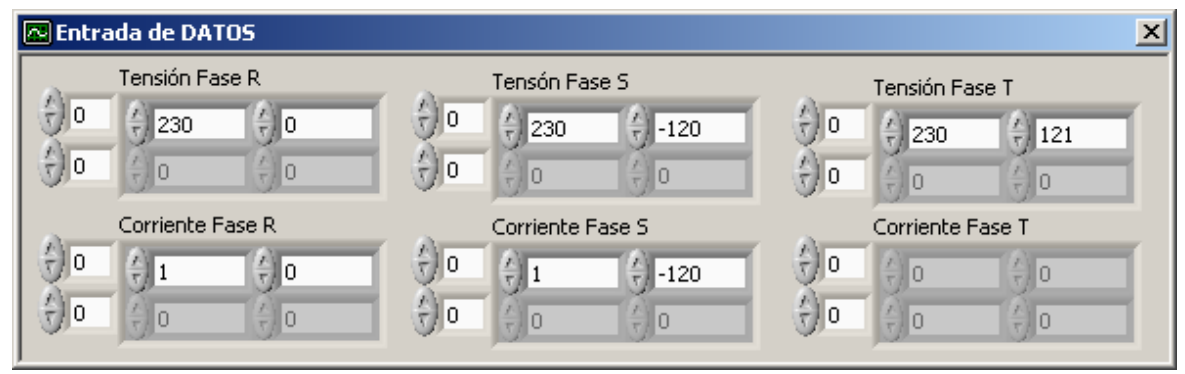

Figura V.83. Pantalla de entrada de datos.

Tenemos pues un sistema de tensiones en el que como ya hemos comentado de manera general dejamos fijos los módulos de las tensiones de las fases, y tenemos dos cargas resistivas puras que consumen $1 \angle 0^{\circ} \mathrm{A}$ y $1 \angle-120^{\circ} \mathrm{A}$ conectadas en las fases 1 y 2 , variando de manera gradual el argumento de la 
fase 3, logrando los pertinentes desequilibrios de tensión y produciendo la consiguiente potencia de desfase, tal y como podemos observar en la tabla V.39.

\begin{tabular}{|c|c|c||}
\hline $\begin{array}{c}\text { Argumento } \\
\text { Fase 3 }\end{array}$ & $\begin{array}{c}\text { Variación } \\
\left.\text { Argumento ( } \mathbf{(}^{\mathbf{}}\right)\end{array}$ & $\begin{array}{c}\text { Potencia Desfase } \\
\text { Desequilibrio } \mathbf{Q}_{\text {dru }} \text { (VAr) }\end{array}$ \\
\hline $121^{\circ}$ & 1 & 2,68 \\
\hline $122^{\circ}$ & 2 & 5,35 \\
\hline $125^{\circ}$ & 5 & 13,36 \\
\hline $130^{\circ}$ & 10 & 26,63 \\
\hline $135^{\circ}$ & 15 & 39,69 \\
\hline $145^{\circ}$ & 25 & 64,80 \\
\hline $155^{\circ}$ & 35 & 87,95 \\
\hline $170^{\circ}$ & 50 & 117,46 \\
\hline $190^{\circ}$ & 70 & 144,09 \\
\hline $210^{\circ}$ & 90 & 153,33 \\
\hline $220^{\circ}$ & 100 & 151,00 \\
\hline $230^{\circ}$ & 110 & 144,09 \\
\hline $240^{\circ}$ & 120 & 132,79 \\
\hline
\end{tabular}

Tabla V.39. Potencia de Desfase \& Variación Argumento.

Observando la tabla V.39, vemos que de manera general, al producirse el desequilibrio del sistema modificando gradualmente el argumento de la fase 3 yendo este desde $120^{\circ}$ a $240^{\circ}$, la potencia de desfase que se pone de manifiesto en el sistema es inductiva, y es de resaltar que existe una total linealidad entre la variación de argumentos producida y la potencia de desfase, quedando patente de manera clara que la variación de un grado $\left(1^{\circ}\right)$, produce una potencia de desfase de 2,68 VAr.

Produciendo una variación en el argumento de la fase 3, de modo que valga $121^{\circ}$ tenemos un consumo de potencia de desfase inductiva provocada por el desequilibrio tal como se muestra en la figura V.84, y en la que observamos que es el doble que la que provocaba este mismo desequilibrio con una sola carga resistiva en el sistema. 


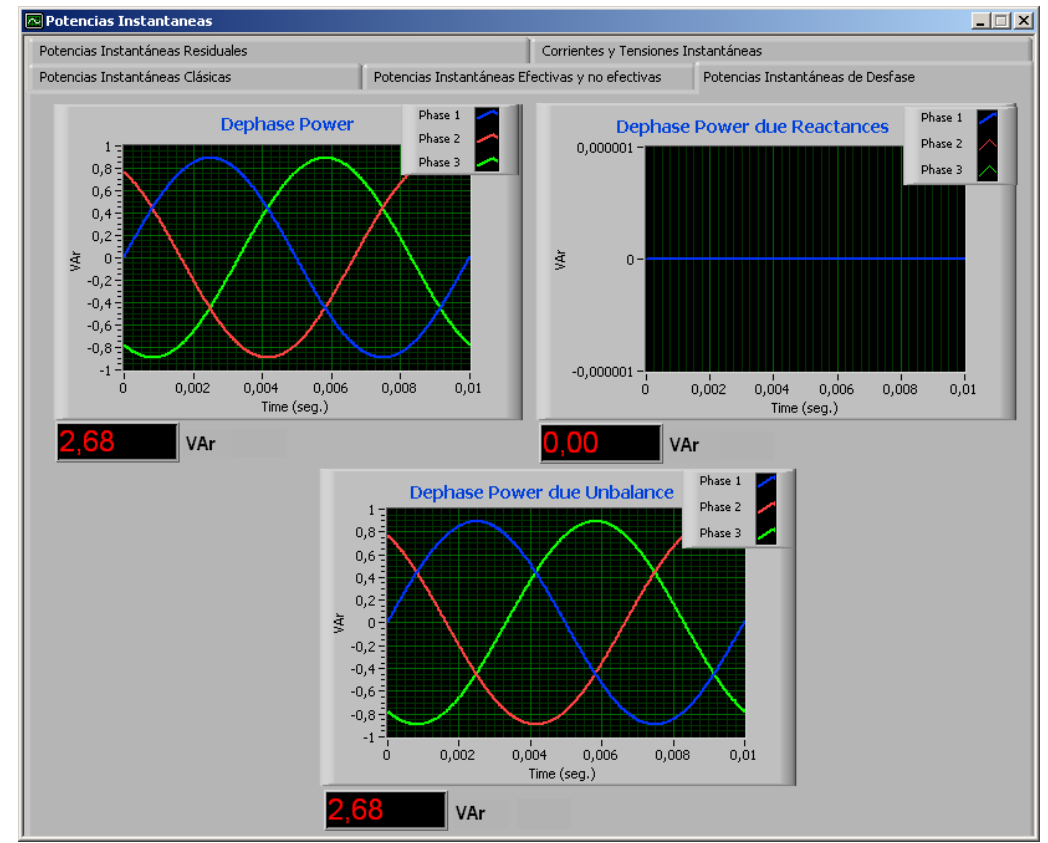

Figura V.84. Pantalla de Potencias de Desfase.

Cargas resistivas entre las fase 1,2 y el neutro, con un consumo de $1 \angle 0^{\circ} \mathrm{A}$ y de $1 \angle-120^{\circ} \mathrm{A}$ y producimos el desequilibrio sumando ángulos negativos al argumento de la fase 3.

Introducimos los datos en el SIMPELEC Simulador, como se muestra en la figura V.85.

\begin{tabular}{|c|c|c|c|c|c|c|c|c|c|}
\hline \multicolumn{6}{|c|}{ 圆Entrada de DATOS } & \multicolumn{4}{|c|}{$x$} \\
\hline \multicolumn{3}{|c|}{ Tensión Fase R } & \multicolumn{3}{|c|}{ Tensón Fase 5} & \multicolumn{3}{|c|}{ Tensión Fase T } & \\
\hline (5) 0 & $\sqrt[5]{230}$ & 0 & 7) 0 & 230 & -120 & 5) 0 & 230 & 119 & \\
\hline (5) 0 & 50 & 0 & 7) 0 & 0 & 0 & (5) 0 & 0 & 0 & \\
\hline \multicolumn{3}{|c|}{ Corriente Fase R } & \multicolumn{3}{|c|}{ Corriente Fase 5} & \multicolumn{3}{|c|}{ Corriente Fase T } & \\
\hline (5) 0 & $\frac{1}{1}$ & 0 & 5) 0 & 1 & $-12 d$ & 5) 0 & 0 & 0 & \\
\hline (3) 0 & 0 & 0 & 7) 0 & 0 & 0 & (3) 0 & 0 & 0 & \\
\hline
\end{tabular}

Figura V.85. Pantalla de entrada de datos.

Partimos de un sistema de tensiones en el que dejamos fijos los módulos de las fases, y tenemos una carga resistiva en la fase 1 que consume $1 \angle 0^{\circ} \mathrm{A}$ y otra carga resistiva en la fase 2 que consume $1 \angle-120^{\circ} A$, y se provocan variaciones 
de manera gradual en el argumento de la fase 3, logrando los pertinentes desequilibrios de tensión y produciendo la consiguiente potencia de desfase, tal y como podemos observar en la tabla V.40, y en la figura V.86 para el caso concreto de la variación de un grado, siendo el argumento resultante de $119^{\circ}$.

\begin{tabular}{|c|c|c||}
\hline $\begin{array}{c}\text { Argumento } \\
\text { Fase 3 }\end{array}$ & $\begin{array}{c}\text { Variación } \\
\left.\text { Argumento ( } \mathbf{(}^{\mathbf{}}\right)\end{array}$ & $\begin{array}{c}\text { Potencia Desfase } \\
\text { Desequilibrio } \mathbf{Q}_{\text {dru }} \text { (VAr) }\end{array}$ \\
\hline $119^{\circ}$ & 1 & $-2,68$ \\
\hline $118^{\circ}$ & 2 & $-5,35$ \\
\hline $115^{\circ}$ & 5 & $-13,36$ \\
\hline $110^{\circ}$ & 10 & $-26,63$ \\
\hline $105^{\circ}$ & 15 & $-39,69$ \\
\hline $95^{\circ}$ & 25 & $-64,80$ \\
\hline $85^{\circ}$ & 35 & $-87,95$ \\
\hline $70^{\circ}$ & 50 & $-117,46$ \\
\hline $50^{\circ}$ & 70 & $-144,09$ \\
\hline $30^{\circ}$ & 90 & $-153,33$ \\
\hline $20^{\circ}$ & 100 & $-151,00$ \\
\hline $10^{\circ}$ & 110 & $-144,09$ \\
\hline $0^{\circ}$ & 120 & $-132,79$ \\
\hline \multicolumn{2}{|c|}{} \\
\hline \multicolumn{2}{|l}{} \\
\hline
\end{tabular}

Tabla V.40. Potencia de Desfase \& Variación Argumento.

De la observación de la tabla V.40, vemos que al modificar gradualmente el argumento de la fase 3, variando desde $120^{\circ}$ a $0^{\circ}$, sigue existiendo linealidad entre los incrementos de grados producidos y la potencia de desfase obtenida, se ha de resaltar en este caso que ahora la potencia de desfase pasa a tener carácter capacitivo, pero manteniéndose no obstante el ratio de que $-2,68 \mathrm{VAr}$ de potencia de desfase son obtenidos con la variación de un grado $\left(1^{\circ}\right)$. 


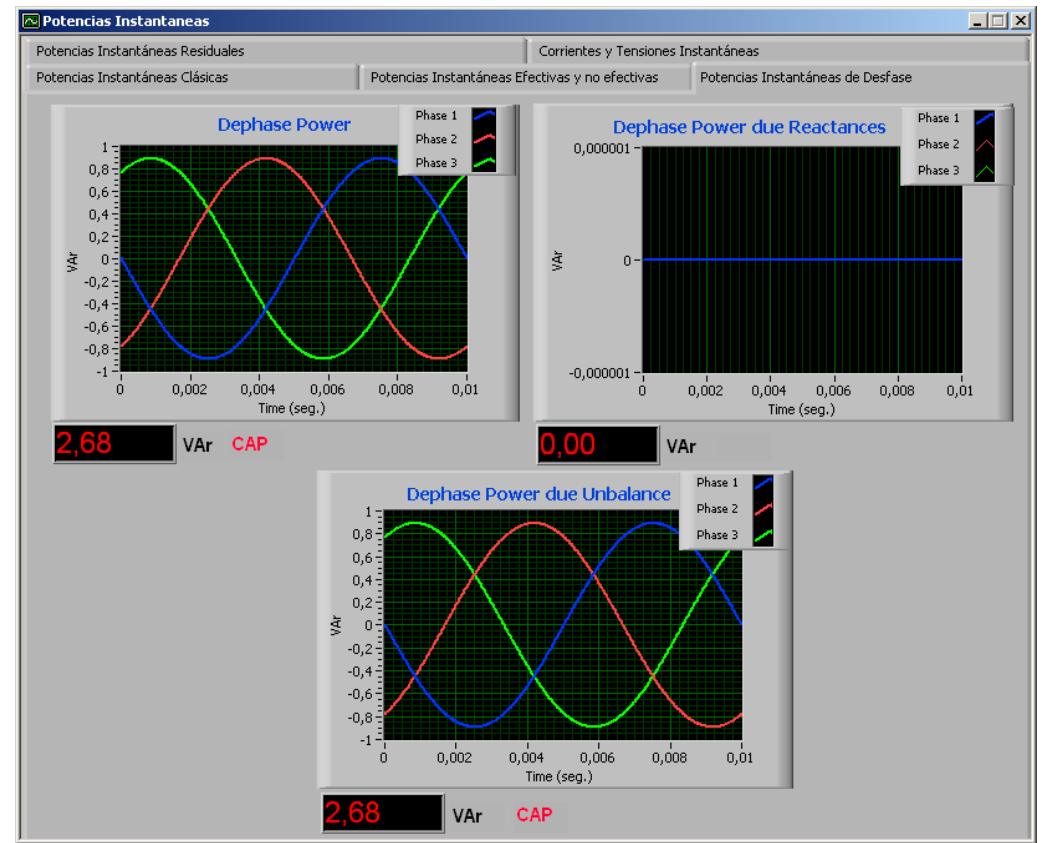

Figura V.86. Pantalla de Potencias de Desfase.

Cargas resistivas entre las fase $1,3 \mathrm{y}$ el neutro, con un consumo de $1 \angle 0^{\circ} \mathrm{A}$ y de $1 \angle 120^{\circ} \mathrm{A}$ y producimos el desequilibrio sumando ángulos positivos al argumento de la fase 2.

Introducimos los datos en el SIMPELEC Simulador, como mostramos en la figura V.87.

\begin{tabular}{|c|c|c|c|c|c|c|c|c|}
\hline \multicolumn{6}{|c|}{ Entrada de DATOS } & \multirow{2}{*}{\multicolumn{3}{|c|}{ Tensión Fase T }} \\
\hline \multicolumn{3}{|c|}{ Tensión Fase R } & \multicolumn{3}{|c|}{ Tensón Fase 5} & & & \\
\hline 5) 0 & $\frac{1}{230}$ & 0 & 5) 0 & 230 & -110 & \multicolumn{2}{|c|}{$\begin{array}{l}\text { Tensión Fase T } \\
230\end{array}$} & 120 \\
\hline (5) 0 & 15 & 0 & 5) 0 & 0 & 0 & (5) 0 & 0 & 0 \\
\hline \multicolumn{3}{|c|}{ Corriente Fase R } & \multicolumn{3}{|c|}{ Corriente Fase 5} & \multicolumn{3}{|c|}{ Corriente Fase T } \\
\hline (5) 0 & $\frac{1}{5} 1$ & 0 & 5) 0 & 0 & 0 & (5) 0 & 1 & 120 \\
\hline (5) 0 & 0 & 0 & 5) 0 & 0 & 0 & (5) 0 & 0 & 0 \\
\hline
\end{tabular}

Figura V.87. Pantalla de entrada de datos.

Un sistema de tensiones en el que dejamos fijos los módulos de las fases, y tenemos una carga resistiva que consume $1 \angle 0^{\circ} A$ conectada en la fase 1 , y otra carga resistiva que consume $1 \angle 120^{\circ} \mathrm{A}$ y tan sólo variamos de manera gradual el argumento de la fase 2, logrando los pertinentes desequilibrios de tensión y 
produciendo la consiguiente potencia de desfase, tal y como podemos observar en la tabla V.41, y en la figura V.88 para el caso concreto de que el argumento de la fase 2 tome el valor de $-110^{\circ}$.

\begin{tabular}{|c|c|c||}
\hline $\begin{array}{c}\text { Argumento } \\
\text { Fase 2 }\end{array}$ & $\begin{array}{c}\text { Variación } \\
\text { Argumento } \mathbf{(}^{\mathbf{0}} \text { ) }\end{array}$ & $\begin{array}{c}\text { Potencia Desfase } \\
\text { Desequilibrio } \mathbf{Q}_{\text {dru }} \text { (VAr) }\end{array}$ \\
\hline$-119^{\circ}$ & 1 & 2,68 \\
\hline$-118^{\circ}$ & 2 & 5,35 \\
\hline$-115^{\circ}$ & 5 & 13,36 \\
\hline$-110^{\circ}$ & 10 & 26,63 \\
\hline$-105^{\circ}$ & 15 & 39,69 \\
\hline$-95^{\circ}$ & 25 & 64,80 \\
\hline$-85^{\circ}$ & 35 & 87,95 \\
\hline$-70^{\circ}$ & 50 & 117,46 \\
\hline$-50^{\circ}$ & 70 & 144,09 \\
\hline$-30^{\circ}$ & 90 & 153,33 \\
\hline$-20^{\circ}$ & 100 & 151,00 \\
\hline$-10^{\circ}$ & 110 & 144,09 \\
\hline $0^{\circ}$ & 120 & 132,79 \\
\hline \multicolumn{2}{|c|}{} \\
\hline \multicolumn{2}{|c|}{} \\
\hline
\end{tabular}

Tabla V.41. Potencia de Desfase \& Variación Argumento.

Observamos en la tabla V.41, que obtenemos una potencia de desfase de carácter inductivo, y cuyos valores son idénticos a los mostrados en la tabla V.39.

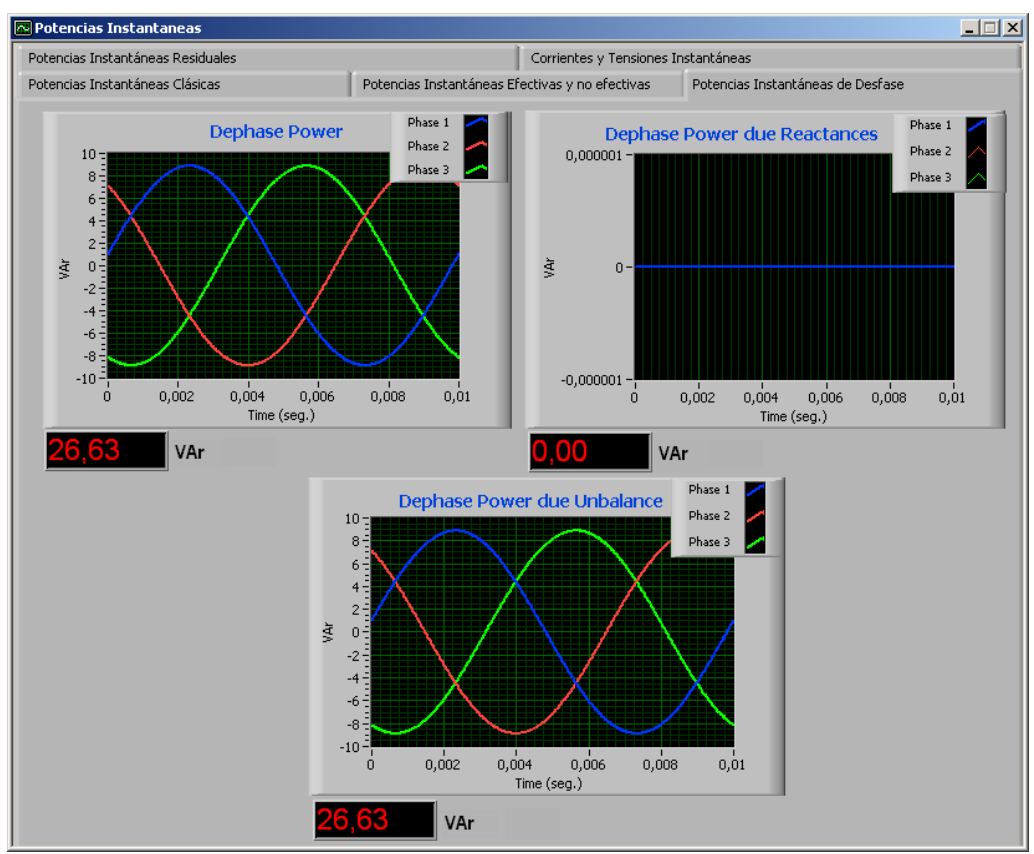

Figura V.88. Pantalla de Potencias de Desfase. 
Cargas resistivas entre las fase 1,3 y el neutro, con un consumo de $1 \angle 0^{\circ} \mathrm{A}$ y de $1 \angle 120^{\circ} \mathrm{A}$ y producimos el desequilibrio sumando ángulos negativos al argumento de la fase 2.

Introducimos los datos en el SIMPELEC Simulador, como mostramos en la figura V.89.

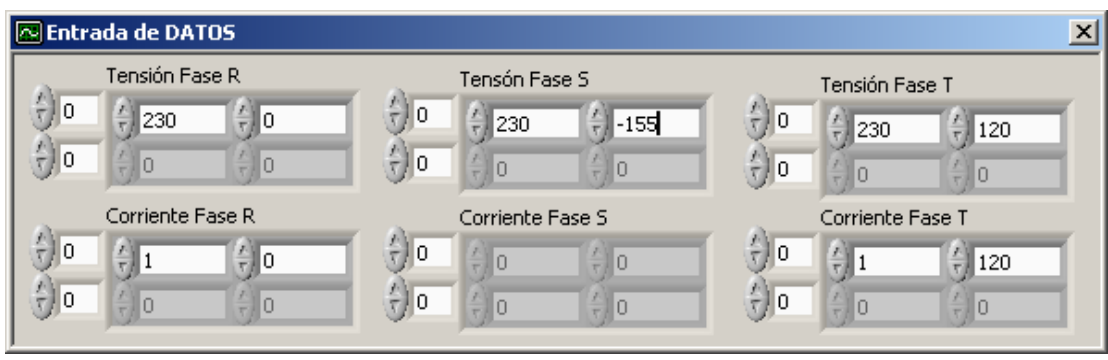

Figura V.89. Pantalla de entrada de datos.

Un sistema de tensiones en el que dejamos fijos los módulos de las fases, y tenemos una carga resistiva que consume $1 \angle 0^{\circ} A$ conectada en la fase $1, y$ otra carga resistiva conectada en la fase 3 que consume $1 \angle 120^{\circ} A$ y provocamos variaciones de manera gradual en el argumento de la fase 2, logrando los pertinentes desequilibrios de tensión y produciendo la consiguiente potencia de desfase, tal y como podemos observar en la tabla V.42, y en la figura V.90 para el caso concreto de que el argumento de la fase 2 valga $-155^{\circ}$.

\begin{tabular}{||c|c|c||}
\hline $\begin{array}{c}\text { Argumento } \\
\text { Fase 2 }\end{array}$ & $\begin{array}{c}\text { Variación } \\
\text { Argumento } \mathbf{(}^{\circ} \text { ) }\end{array}$ & $\begin{array}{c}\text { Potencia Desfase } \\
\text { Desequilibrio } \mathbf{Q}_{\text {dru }} \text { (VAr) }\end{array}$ \\
\hline$-121^{\circ}$ & 1 & $-2,68$ \\
\hline$-122^{\circ}$ & 2 & $-5,35$ \\
\hline$-125^{\circ}$ & 5 & $-13,36$ \\
\hline$-130^{\circ}$ & 10 & $-26,63$ \\
\hline$-135^{\circ}$ & 15 & $-39,69$ \\
\hline$-145^{\circ}$ & 25 & $-64,80$ \\
\hline$-155^{\circ}$ & 35 & $-87,95$ \\
\hline$-170^{\circ}$ & 50 & $-117,46$ \\
\hline$-190^{\circ}$ & 70 & $-144,09$ \\
\hline$-210^{\circ}$ & 90 & $-153,33$ \\
\hline$-220^{\circ}$ & 100 & $-151,00$ \\
\hline$-230^{\circ}$ & 110 & $-144,09$ \\
\hline$-240^{\circ}$ & 120 & $-132,79$ \\
\hline
\end{tabular}

Tabla V.42. Potencia de Desfase \& Variación Argumento. 
De los resultados mostrados en la tabla V.42, observamos que la potencia de desfase es capacitiva, y sus valores coinciden con los obtenidos y presentados en la tabla V.40.

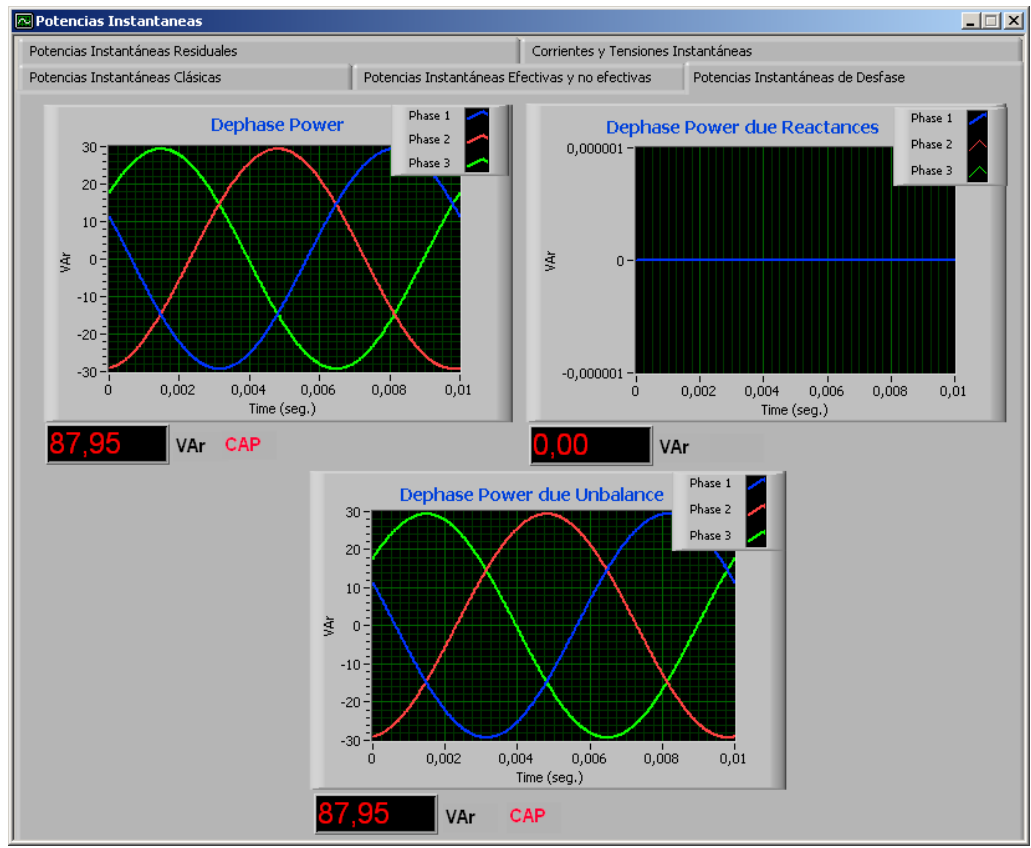

Figura V.90. Pantalla de Potencias de Desfase.

Cargas resistivas entre la fase 2,3 y el neutro, con un consumo de $1 \angle-120^{\circ} \mathrm{A}$ y de $1 \angle 120^{\circ} \mathrm{A}$ y producimos el desequilibrio sumando ángulos positivos al argumento de la fase 1.

Introducimos los datos en el SIMPELEC Simulador, como observamos en la figura V.91.

\begin{tabular}{|c|c|c|c|c|c|c|c|c|}
\hline \multicolumn{9}{|c|}{ Entrada de DATOS } \\
\hline \multicolumn{3}{|c|}{ Tensión Fase R } & \multicolumn{3}{|c|}{ Tensón Fase 5} & \multicolumn{3}{|c|}{ Tensión Fase T } \\
\hline (5) 0 & $\sqrt[7]{230}$ & 5 & (5) 0 & 230 & -120 & (7) 0 & 230 & 120 \\
\hline 5) 0 & 0 & 0 & (5) 0 & 0 & 0 & (5) 0 & 0 & 0 \\
\hline \multicolumn{3}{|c|}{ Corriente Fase R } & \multicolumn{3}{|c|}{ Corriente Fase 5} & \multicolumn{3}{|c|}{ Corriente Fase T } \\
\hline 5) 0 & 0 & 0 & 3) 0 & 1 & -120 & 3) 0 & 1 & $12 \mathrm{~d}$ \\
\hline 7) 0 & 0 & 0 & 5) 0 & 0 & 0 & 5) 0 & 0 & 0 \\
\hline
\end{tabular}

Figura V.91. Pantalla de entrada de datos. 
Tenemos pues un sistema de tensiones en el que como ya hemos comentado de manera general dejamos fijos los módulos de las tensiones de las fases, y tenemos una carga resistiva que consume $1 \angle-120^{\circ} A$ conectada en la fase $2, y$ otra carga resistiva en la fase 3 que consume $1 \angle 120^{\circ} A$, y tan sólo variamos de manera gradual el argumento de la fase 1, logrando los pertinentes desequilibrios de tensión y produciendo la consiguiente potencia de desfase, tal y como podemos observar en la tabla V.43, y en la figura V.92 para el caso concreto de que el argumento de la fase 1 tome el valor de $5^{\circ}$.

\begin{tabular}{||c|c|c||}
\hline $\begin{array}{c}\text { Argumento } \\
\text { Fase 1 }\end{array}$ & $\begin{array}{c}\text { Variación } \\
\text { Argumento } \mathbf{(}^{\circ} \text { ) }\end{array}$ & $\begin{array}{c}\text { Potencia Desfase } \\
\text { Desequilibrio } \mathbf{Q}_{\text {dru }} \text { (VAr) }\end{array}$ \\
\hline $1^{\circ}$ & 1 & 2,68 \\
\hline $2^{\circ}$ & 2 & 5,35 \\
\hline $5^{\circ}$ & 5 & 13,36 \\
\hline $10^{\circ}$ & 10 & 26,63 \\
\hline $15^{\circ}$ & 15 & 39,69 \\
\hline $25^{\circ}$ & 25 & 64,80 \\
\hline $35^{\circ}$ & 35 & 87,95 \\
\hline $50^{\circ}$ & 50 & 117,46 \\
\hline $70^{\circ}$ & 70 & 144,09 \\
\hline $90^{\circ}$ & 90 & 153,33 \\
\hline $100^{\circ}$ & 100 & 151,00 \\
\hline $110^{\circ}$ & 110 & 144,09 \\
\hline $120^{\circ}$ & 120 & 132,79 \\
\hline
\end{tabular}

Tabla V.43. Potencia de Desfase \& Variación Argumento.

Observamos de los resultados mostrados en la tabla V.43 que la potencia de desfase tiene carácter inductivo, y sus valores coinciden con los de las tablas V.39 y 41. 


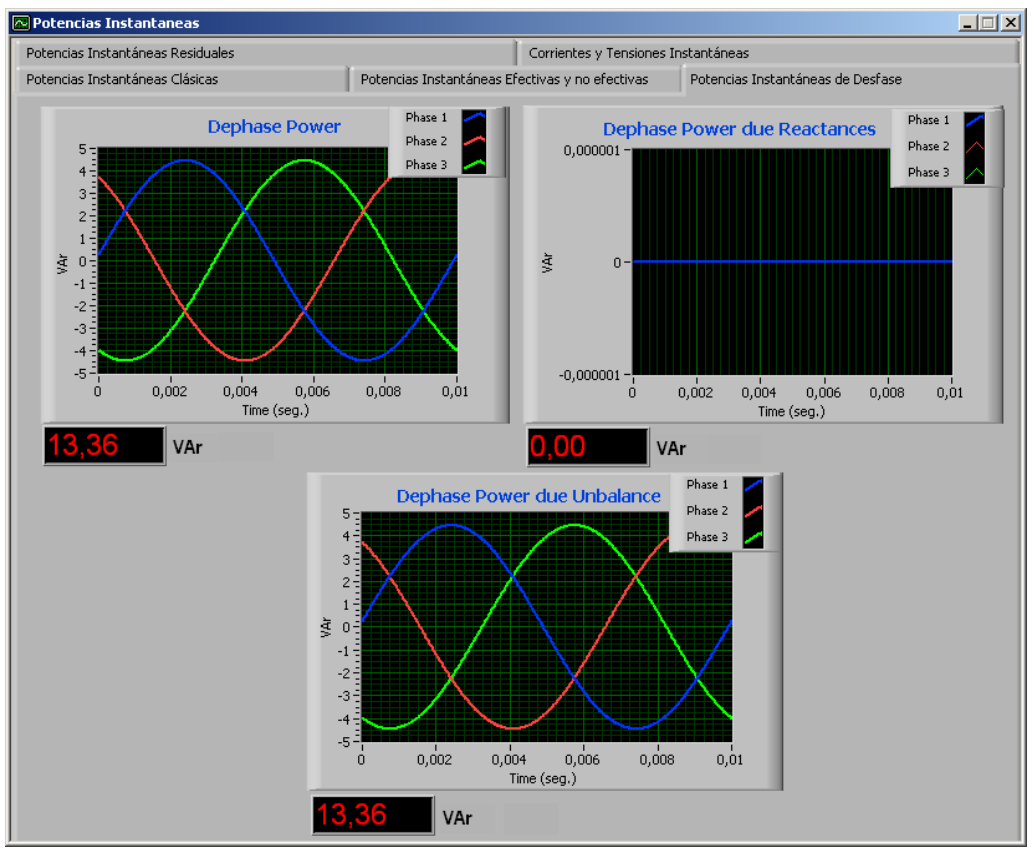

Figura V.92. Pantalla de Potencias de Desfase.

Cargas resistivas entre la fase 2,3 y el neutro, con un consumo de $1 \angle-120^{\circ} \mathrm{A}$ y de $1 \angle 120^{\circ} \mathrm{A}$ y producimos el desequilibrio sumando ángulos negativos al argumento de la fase 1.

Introducimos los datos en el SIMPELEC Simulador, como se muestra en la figura V.93.

\begin{tabular}{|c|c|c|c|c|c|c|c|c|}
\hline \multicolumn{9}{|c|}{ 司Entrada de DATOS } \\
\hline \multicolumn{3}{|c|}{ Tensión Fase $\mathrm{R}$} & \multicolumn{3}{|c|}{ Tensón Fase 5} & \multicolumn{3}{|c|}{ Tensión Fase T } \\
\hline (5) 0 & $\sqrt[1]{230}$ & -25 & 5) 0 & 230 & -120 & (5) 0 & t) 230 & 120 \\
\hline (5) 0 & $\frac{1}{5} 0$ & 0 & (5) 0 & 0 & 0 & (5) 0 & 0 & 0 \\
\hline \multicolumn{3}{|c|}{ Corriente Fase R } & \multicolumn{3}{|c|}{ Corriente Fase 5} & \multicolumn{3}{|c|}{ Corriente Fase T } \\
\hline 7) 0 & 0 & 0 & 5) 0 & 1 & -120 & (5) 0 & 1 & 120 \\
\hline (5) 0 & 0 & 0 & 5) 0 & 0 & 0 & 7) 0 & 0 & 0 \\
\hline
\end{tabular}

Figura V.93. Pantalla de entrada de datos.

Partimos de un sistema de tensiones en el que dejamos fijos los módulos de las fases, y tenemos una carga resistiva pura que consume $1 \angle-120^{\circ} A$ conectada en la fase 2 , y otra en la fase 3 que consume $1 \angle 120^{\circ} A$, y tan sólo variamos de manera gradual el argumento de la fase 1, logrando los pertinentes 
desequilibrios de tensión y produciendo la consiguiente potencia de desfase, tal y como podemos observar en la tabla V.44, y en la figura V.94 para el caso concreto de que el argumento de la fase 1 tome el valor de $-25^{\circ}$.

\begin{tabular}{||c|c|c||}
\hline $\begin{array}{c}\text { Argumento } \\
\text { Fase 1 }\end{array}$ & $\begin{array}{c}\text { Variación } \\
\text { Argumento ( }\end{array}$ & $\begin{array}{c}\text { Potencia Desfase } \\
\text { Desequilibrio } \mathbf{Q}_{\text {dru }} \text { (VAr) }\end{array}$ \\
\hline$-1^{\circ}$ & 1 & $-2,68$ \\
\hline$-2^{\circ}$ & 2 & $-5,35$ \\
\hline$-5^{\circ}$ & 5 & $-13,36$ \\
\hline$-10^{\circ}$ & 10 & $-26,63$ \\
\hline$-15^{\circ}$ & 15 & $-39,69$ \\
\hline$-25^{\circ}$ & 25 & $-64,80$ \\
\hline$-35^{\circ}$ & 35 & $-87,95$ \\
\hline$-50^{\circ}$ & 50 & $-117,46$ \\
\hline$-70^{\circ}$ & 70 & $-144,09$ \\
\hline$-90^{\circ}$ & 90 & $-153,33$ \\
\hline$-100^{\circ}$ & 100 & $-151,00$ \\
\hline$-110^{\circ}$ & 110 & $-144,09$ \\
\hline$-120^{\circ}$ & 120 & $-132,79$ \\
\hline
\end{tabular}

Tabla V.44. Potencia de Desfase \& Variación Argumento.

En la tabla V.44 se observa que los valores obtenidos para la potencia de desfase, tienen carácter capacitivo y coinciden con los mostrados en las tablas V. 40 y 42.

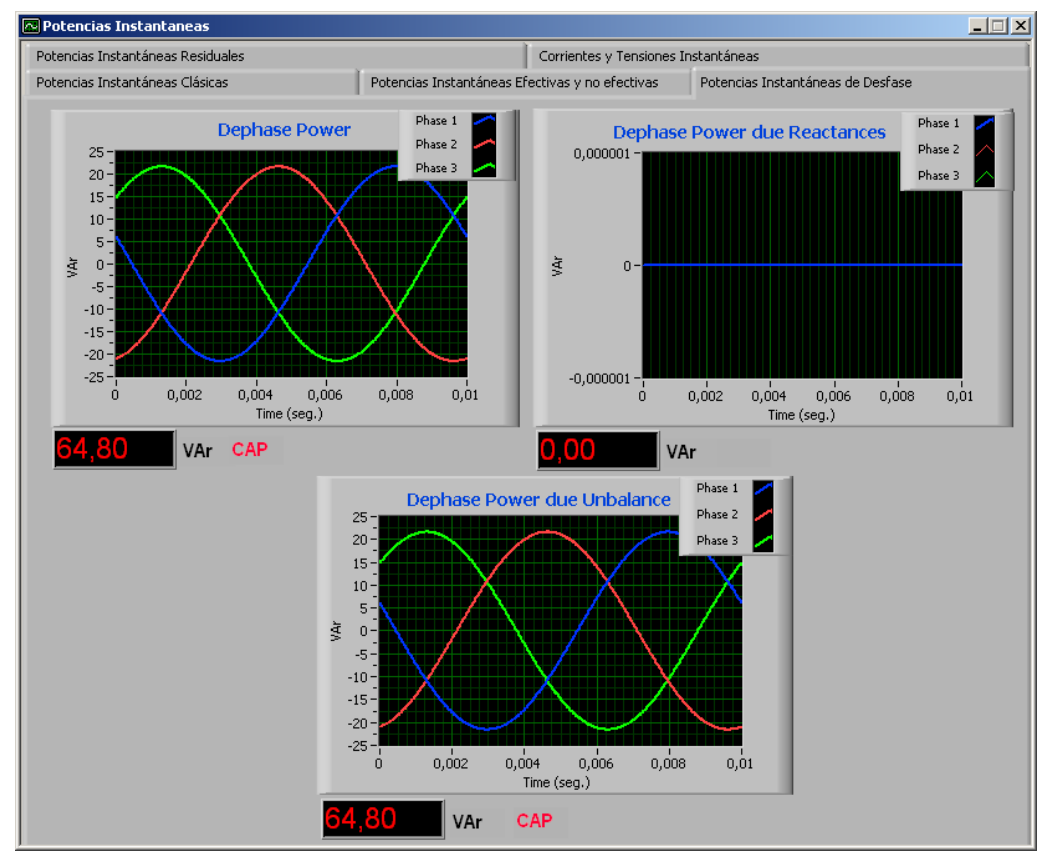

Figura V.94. Pantalla de Potencias de Desfase. 
En la figura V.95, se muestran las gráficas basadas en los datos obtenidos para el caso de someter al sistema cargado por dos elementos resistivos a diversos grados de desequilibrio, y vemos la similitud existente con el caso de que en el sistema hubiera tan sólo una carga resistiva, pero en este caso al existir dos cargas resistivas, las potencias de desfase que se muestran en las gráficas se duplican en valor con relación al caso de una sola carga resistiva, aunque se mantiene el carácter inductivo o capacitivo de esta potencia de desfase que consumen estas cargas, según los desequilibrios provocados.

Asimismo, se reflejan en esta figura las gráficas cuando los consumos de las cargas son de 1,2 y 4 Amperios.

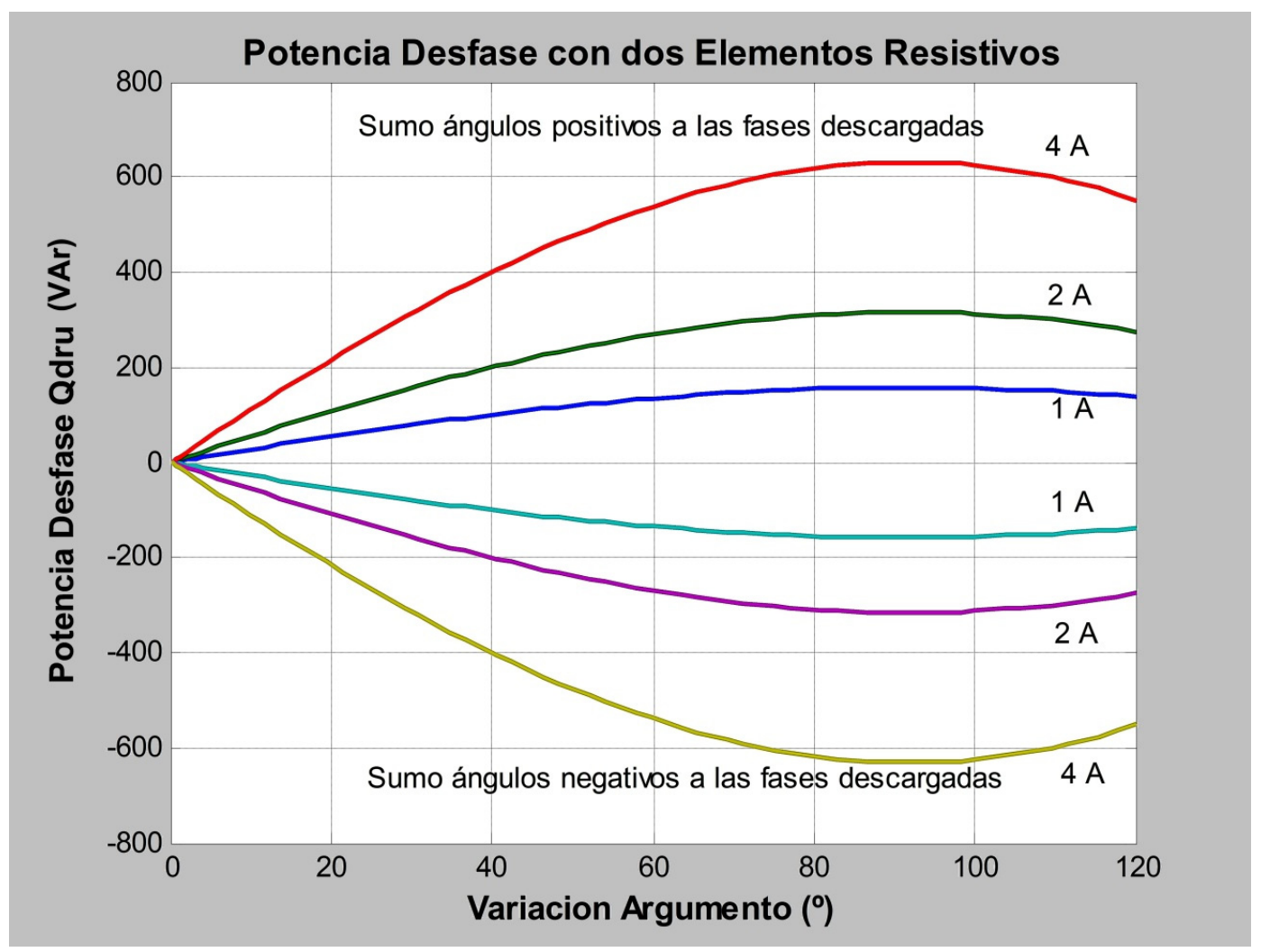

Figura V.95. Potencias de Desfase, para variaciones de dos cargas resistivas, una en cada fase y diversos grados de desequilibrio. 
b) Carga Resistiva e Inductiva: Se analiza el comportamiento de este sistema compuesto por dos cargas: una resistiva y la otra inductiva frente a diversos desequilibrios provocados en el sistema de alimentación.

Carga resistiva entre la fase 1 y el neutro, con un consumo de $1 \angle 0^{\circ} \mathrm{A}$ y otra carga inductiva entre la fase 2 y el neutro, con un consumo de $1 \angle-165^{\circ} \mathrm{A}$ y producimos el desequilibrio sumando ángulos positivos al argumento de la fase 3.

Introducimos gracias a la ventana de entrada de datos del SIMPELEC Simulador, las tensiones de las fases y el consumo, así como las sucesivas modificaciones en los argumentos de la fase 3 en este caso, tal y como mostramos en la figura V.96.

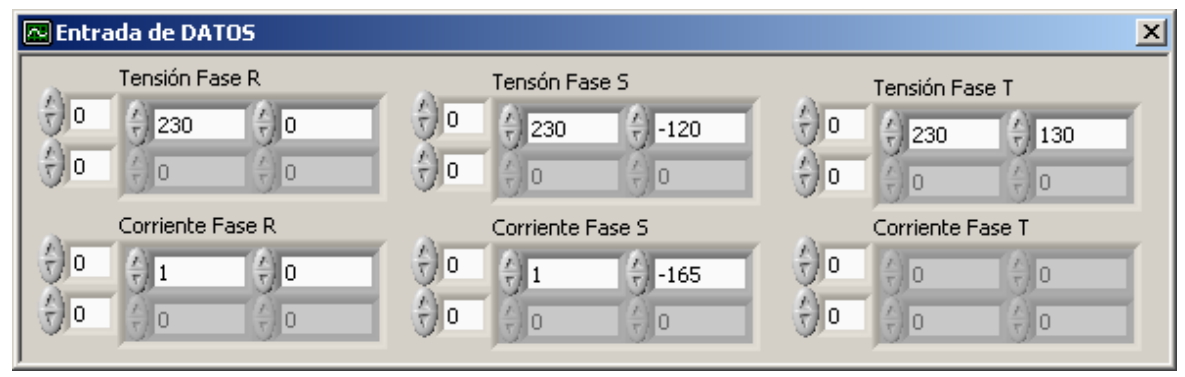

Figura V.96. Pantalla de entrada de datos.

Tenemos pues un sistema de tensiones en el que como ya hemos comentado de manera general dejamos fijos los módulos de las tensiones de las fases, y tenemos una carga resistiva que consume $1 \angle 0^{\circ} \mathrm{A}$ y otra carga inductiva que consume $1 \angle-165^{\circ} A$ conectadas en las fases 1 y 2 , variando de manera gradual el argumento de la fase 3, logrando los pertinentes desequilibrios de tensión y produciendo la consiguiente potencia de desfase, tal y como podemos observar en la tabla V.45. 


\begin{tabular}{|c|c|c||}
\hline $\begin{array}{c}\text { Argumento } \\
\text { Fase 3 }\end{array}$ & $\begin{array}{c}\text { Variación } \\
\text { Argumento ( }{ }^{\circ} \text { ) }\end{array}$ & $\begin{array}{c}\text { Potencia Desfase } \\
\text { Desequilibrio } \mathbf{Q}_{\text {dru }} \text { (VAr) }\end{array}$ \\
\hline $121^{\circ}$ & 1 & 2,29 \\
\hline $122^{\circ}$ & 2 & 4,58 \\
\hline $125^{\circ}$ & 5 & 11,48 \\
\hline $130^{\circ}$ & 10 & 23,00 \\
\hline $135^{\circ}$ & 15 & 34,49 \\
\hline $145^{\circ}$ & 25 & 57,00 \\
\hline $155^{\circ}$ & 35 & 78,34 \\
\hline $170^{\circ}$ & 50 & 106,71 \\
\hline $190^{\circ}$ & 70 & 134,88 \\
\hline $210^{\circ}$ & 90 & 148,95 \\
\hline $220^{\circ}$ & 100 & 150,10 \\
\hline $230^{\circ}$ & 110 & 147,24 \\
\hline $240^{\circ}$ & 120 & 140,45 \\
\hline
\end{tabular}

Tabla V.45. Potencia de Desfase \& Variación Argumento.

Observando la tabla V.45, vemos que de manera general, al producirse el desequilibrio del sistema modificando gradualmente el argumento de la fase 3 yendo este desde $120^{\circ}$ a $240^{\circ}$, la potencia de desfase debida al desequilibrio que se pone de manifiesto en el sistema es inductiva.

Para cualquiera de los valores que obtenemos con cada grado de desequilibrio, podemos comprobar que debido a que estamos trabajando con sistemas lineales, la potencia de desfase demandada por el sistema, se puede ver como el consumo de manera independiente de cada una de las dos cargas que lo constituyen.

Así pues usando los valores obtenidos en anteriores simulaciones de los consumos de la carga resistiva e inductiva, sometidas a el mismo desequilibrio de tensiones y conectadas de manera independiente en las mismas fases, obtendríamos los valores mostrados en la tabla V.43, concretamente para el grado de desequilibrio de un 0,58\% y las misma cargas conectadas en las fases, teníamos que la potencia de desfase de la carga resistiva era de 1,34 VAr, y la de la carga inductiva era de 0,95 VAr, siendo la potencia de desfase total del sistema formada por la suma de estas dos, y por lo tanto de 1,29 $\mathrm{VAr}$, valor que coincide con el obtenido cuando conectamos las dos cargas de manera 
simultánea en el sistema y lo sometemos al mismo desequilibrio, tal y como mostramos en la mencionada tabla V.45.

Los datos anteriores, para el caso de que el argumento de la fase 3 valga 130 , generan la pantalla principal que podemos observar en la figura V.97.

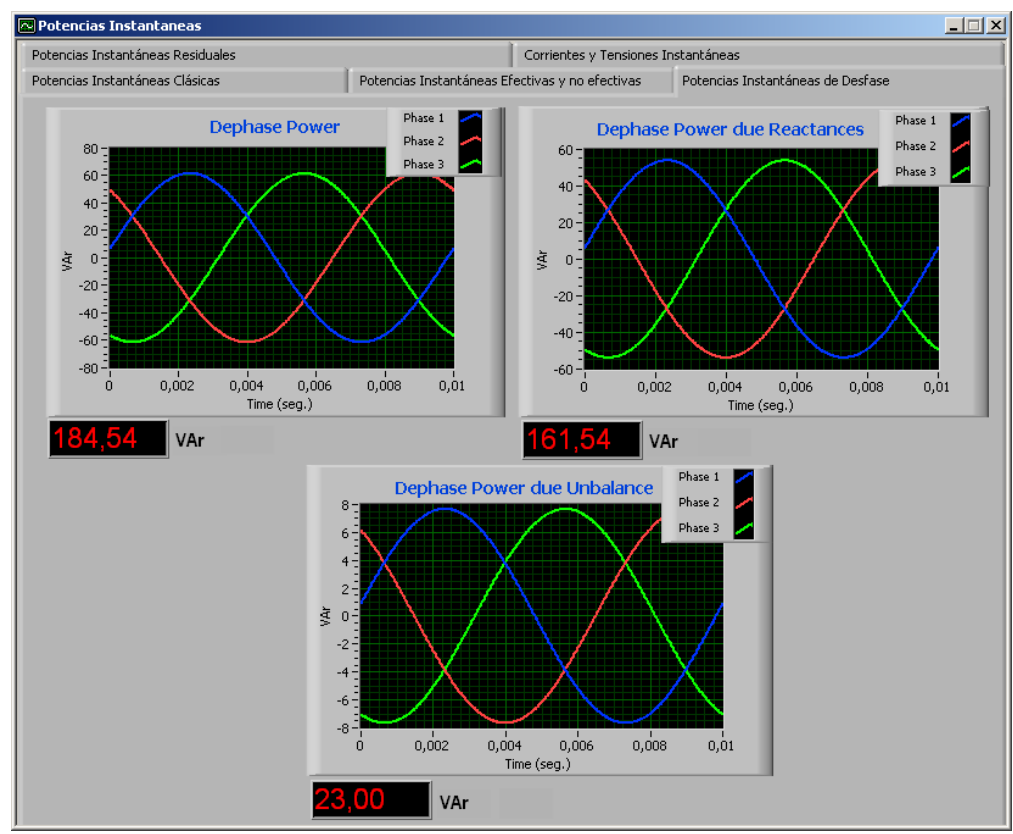

Figura V.97. Pantalla de Potencias de Desfase.

Carga resistiva entre la fase 1 y el neutro, con un consumo de $1 \angle 0^{\circ} \mathrm{A}$ y otra carga inductiva entre la fase 2 y el neutro, con un consumo de $1 \angle-165^{\circ} \mathrm{A}$ y producimos el desequilibrio sumando ángulos negativos al argumento de la fase 3.

Introducimos gracias a la ventana de entrada de datos del SIMPELEC Simulador, las tensiones de las fases y el consumo, así como las sucesivas modificaciones en los argumentos de la fase 3 en este caso, tal y como mostramos en la figura V.98. 


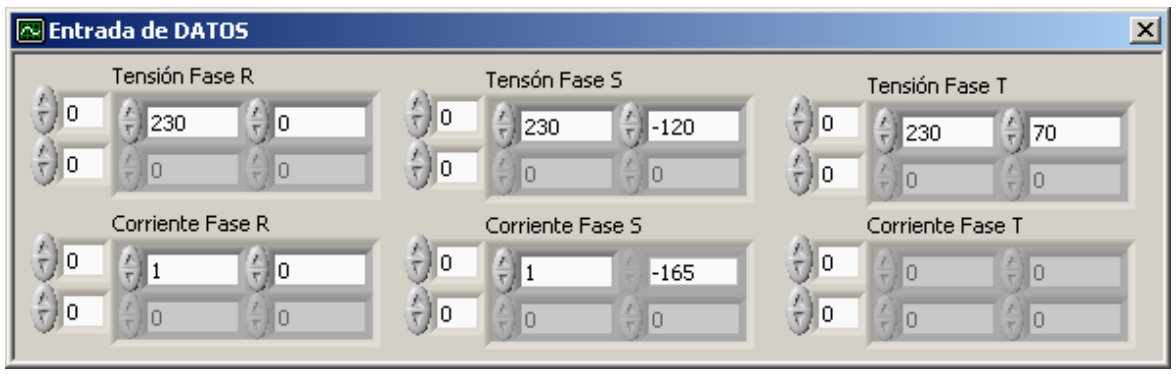

Figura V.98. Pantalla de entrada de datos.

Tenemos pues un sistema de tensiones en el que como ya hemos comentado de manera general dejamos fijos los módulos de las tensiones de las fases, y tenemos una carga resistiva que consume $1 \angle 0^{\circ} A$ y otra inductiva con un consumo de $1 \angle-165^{\circ} A$ conectadas en las fases 1 y 2 , variando de manera gradual el argumento de la fase 3, logrando los pertinentes desequilibrios de tensión y produciendo la consiguiente potencia de desfase, tal y como podemos observar en la tabla V.46.

\begin{tabular}{|c|c|c||}
\hline $\begin{array}{c}\text { Argumento } \\
\text { Fase 3 }\end{array}$ & $\begin{array}{c}\text { Variación } \\
\text { Argumento }\left(^{(}\right)\end{array}$ & $\begin{array}{c}\text { Potencia Desfase } \\
\text { Desequilibrio } \mathbf{Q}_{\text {dru }} \text { (VAr) }\end{array}$ \\
\hline $119^{\circ}$ & 1 & $-2,28$ \\
\hline $118^{\circ}$ & 2 & $-4,56$ \\
\hline $115^{\circ}$ & 5 & $-11,34$ \\
\hline $110^{\circ}$ & 10 & $-22,45$ \\
\hline $105^{\circ}$ & 15 & $-33,26$ \\
\hline $95^{\circ}$ & 25 & $-53,62$ \\
\hline $85^{\circ}$ & 35 & $-71,80$ \\
\hline $70^{\circ}$ & 50 & $-93,80$ \\
\hline $50^{\circ}$ & 70 & $-111,10$ \\
\hline $30^{\circ}$ & 90 & $-107,68$ \\
\hline $20^{\circ}$ & 100 & $-98,73$ \\
\hline $10^{\circ}$ & 110 & $-98,73$ \\
\hline $0^{\circ}$ & 120 & $-86,24$ \\
\hline
\end{tabular}

Tabla V.46. Potencia de Desfase \& Variación Argumento.

De la tabla V.46, vemos que de manera general, al producirse el desequilibrio del sistema modificando gradualmente el argumento de la fase 3 desde $120^{\circ}$ a $0^{\circ}$, la potencia de desfase debida al desequilibrio en el sistema es capacitiva. 
Que como se ha comentado en el caso anterior, se pueden obtener los resultados de la tabla V.46, como suma de los consumos individuales de las cargas sometidas a esos desequilibrios de manera independiente, valiéndonos de que estamos trabajando con un sistema lineal.

Para el caso de que el argumento de la fase 3 tome el valor de $70^{\circ}$, se genera la pantalla principal que podemos observar en la figura V.99, en la que también queda patente, que el hecho de este comportamiento capacitivo del sistema debido al desequilibrio, provoca una disminución de la potencia total de desfase del sistema.

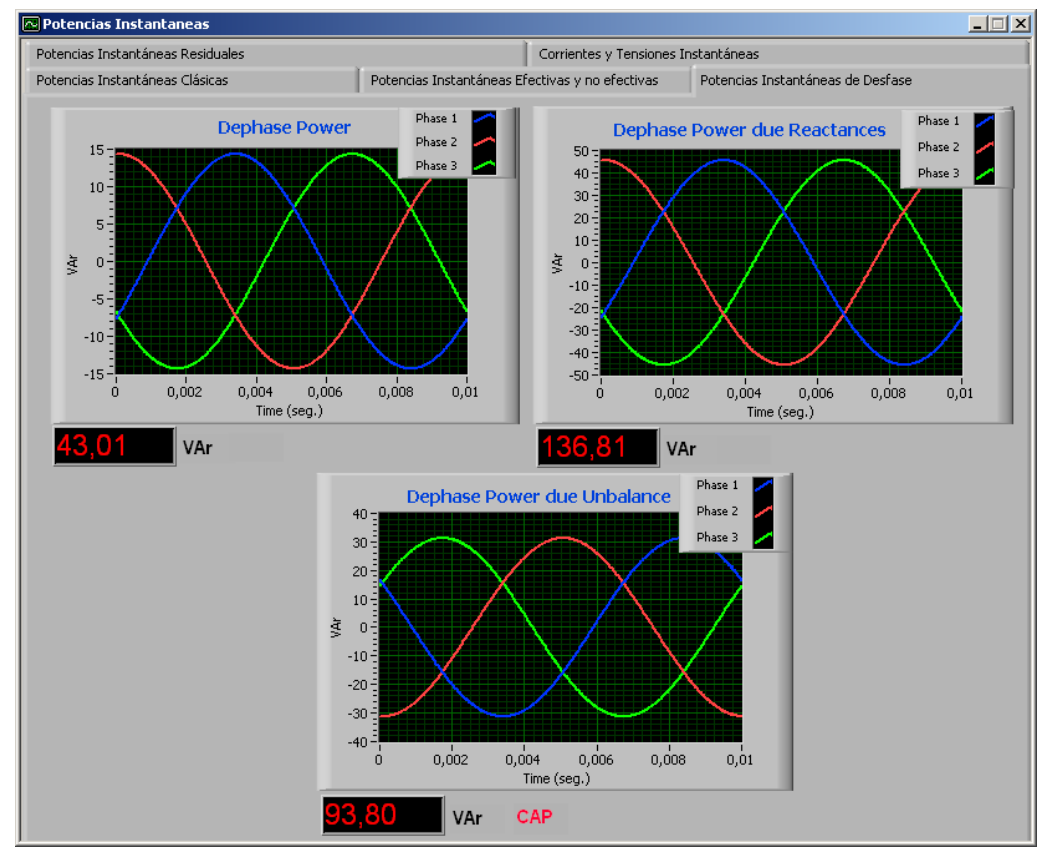

Figura V.99. Pantalla de Potencias de Desfase. 


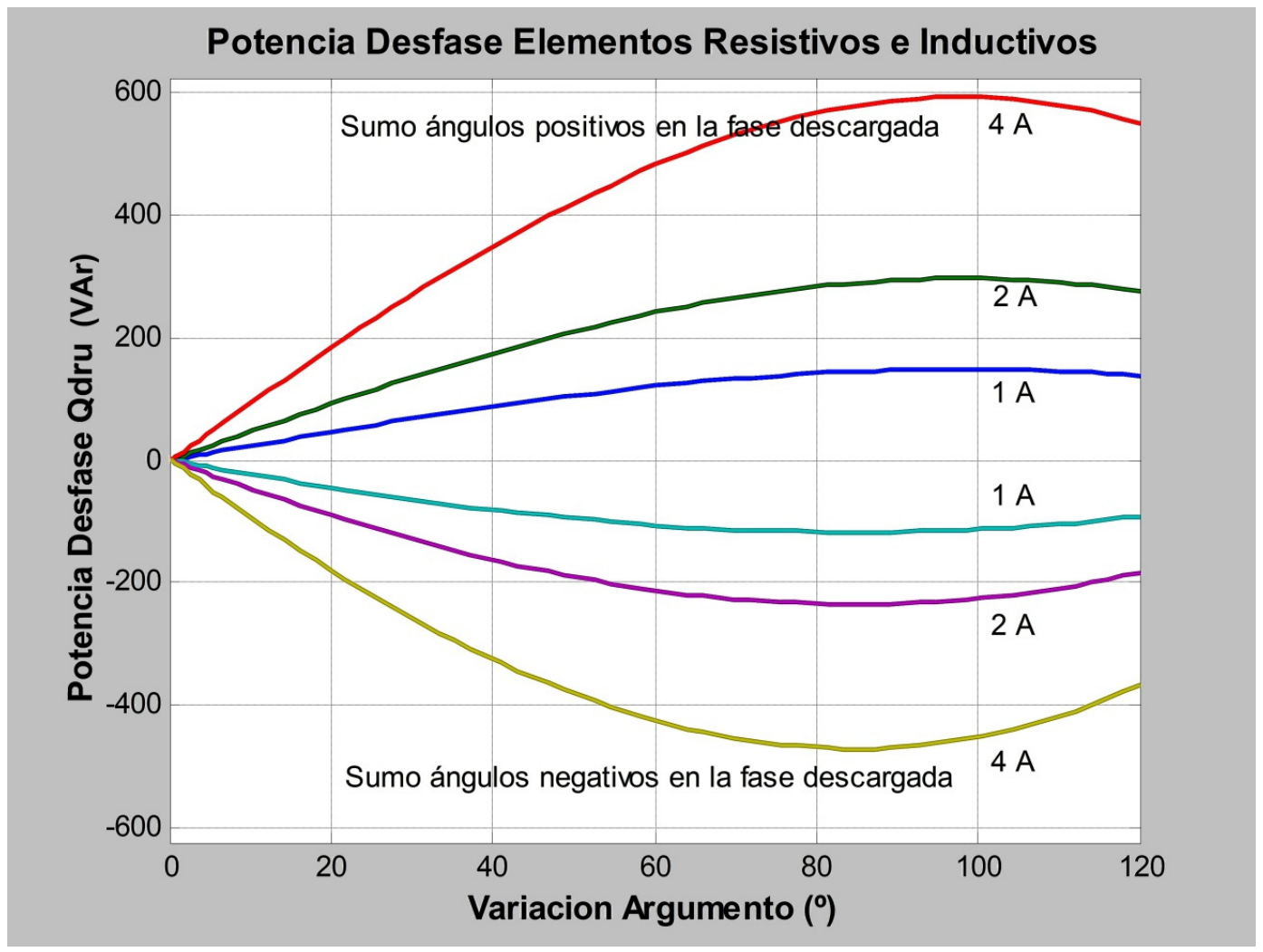

Figura V.100. Potencia de Desfase, para variaciones de una carga resistiva, y otra inductiva, en diversas fases y grados de desequilibrio.

Aprovechando esta propiedad de linealidad del sistema, junto con los datos obtenidos previamente para las cargas individuales resistivas e inductivas, conectadas de forma sucesiva en diversas fases y sometidas a diferentes grados de desequilibrio, hemos obtenido los valores de la potencia de desfase que nos permiten mostrar la gráfica que se ve en la figura V.100, así como las gráficas de las potencias de desfase para los consumos de 1, 2 y Amperios. 
c) Carga Resistiva y Capacitiva: Se analiza el comportamiento de este sistema compuesto por dos cargas: una resistiva y la otra capacitiva frente a diversos desequilibrios provocados en el sistema de alimentación.

Carga resistiva entre la fase 1 y el neutro, con un consumo de $1 \angle 0^{\circ} \mathrm{A}$ y otra carga capacitiva entre la fase 2 y el neutro, con un consumo de $1 \angle-75^{\circ} \mathrm{A}$ y producimos el desequilibrio sumando ángulos positivos al argumento de la fase 3.

Introducimos gracias a la ventana de entrada de datos del SIMPELEC Simulador, las tensiones de las fases y el consumo, así como las sucesivas modificaciones en los argumentos de la fase 3 en este caso, tal y como mostramos en la figura V.101.

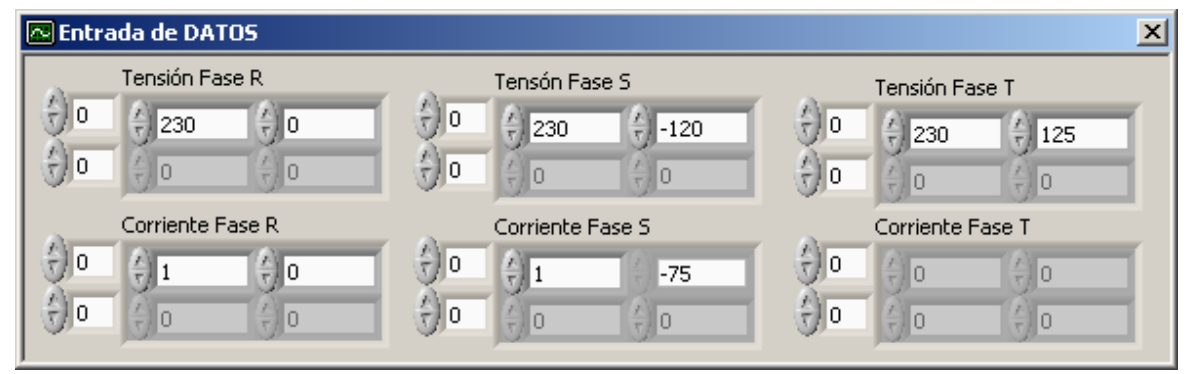

Figura V.101. Pantalla de entrada de datos.

Tenemos pues un sistema de tensiones en el que como ya hemos comentado de manera general dejamos fijos los módulos de las tensiones de las fases, y tenemos una carga resistiva que consume $1 \angle 0^{\circ} A$ y otra carga capacitiva que consume $1 \angle-75^{\circ} A$ conectadas en las fases 1 y 2 , variando de manera gradual el argumento de la fase 3, logrando los pertinentes desequilibrios de tensión y produciendo la consiguiente potencia de desfase, tal y como podemos observar en la tabla V.47. 


\begin{tabular}{|c|c|c||}
\hline $\begin{array}{c}\text { Argumento } \\
\text { Fase 3 }\end{array}$ & $\begin{array}{c}\text { Variación } \\
\text { Argumento ( }{ }^{\circ} \text { ) }\end{array}$ & $\begin{array}{c}\text { Potencia Desfase } \\
\text { Desequilibrio } \mathbf{Q}_{\text {dru }} \text { (VAr) }\end{array}$ \\
\hline $121^{\circ}$ & 1 & 2,28 \\
\hline $122^{\circ}$ & 2 & 4,56 \\
\hline $125^{\circ}$ & 5 & 11,34 \\
\hline $130^{\circ}$ & 10 & 22,45 \\
\hline $135^{\circ}$ & 15 & 33,26 \\
\hline $145^{\circ}$ & 25 & 53,62 \\
\hline $155^{\circ}$ & 35 & 71,80 \\
\hline $170^{\circ}$ & 50 & 93,80 \\
\hline $190^{\circ}$ & 70 & 111,09 \\
\hline $210^{\circ}$ & 90 & 112,81 \\
\hline $220^{\circ}$ & 100 & 107,68 \\
\hline $230^{\circ}$ & 110 & 98,73 \\
\hline $240^{\circ}$ & 120 & 86,24 \\
\hline \multicolumn{2}{|r|}{} \\
\hline \multicolumn{2}{|l|}{} \\
\hline
\end{tabular}

Tabla V.47. Potencia de Desfase \& Variación Argumento.

Observando la tabla V.47, vemos que de manera general, al producirse el desequilibrio del sistema modificando gradualmente el argumento de la fase 3 yendo este desde $120^{\circ}$ a $240^{\circ}$, la potencia de desfase debida al desequilibrio posee carácter inductivo.

Para cualquiera de los valores que obtenemos con cada grado de desequilibrio, podemos comprobar que debido a que estamos trabajando con sistemas lineales, la potencia de desfase debida al desequilibrio demandada por el sistema, se puede ver como el consumo de manera independiente de cada una de las dos cargas que lo constituyen.

En la pantalla de la figura V.102, podemos observar el caso concreto de que el argumento de la fase 3 tome el valor de $125^{\circ}$, lo que nos da un consumo de potencia de desfase debida al desequilibrio de 11,34 VAr, con carácter como ya se ha mencionado inductivo, esto provoca que los valores de consumo de potencia de desfase totales del sistema sean menores que los esperados. Al igual que mencionamos en el caso analizado anteriormente, este valor lo podríamos haber conseguido como la aportación de manera individual de las dos cargas sometidas a las mismas condiciones de desequilibrio. 


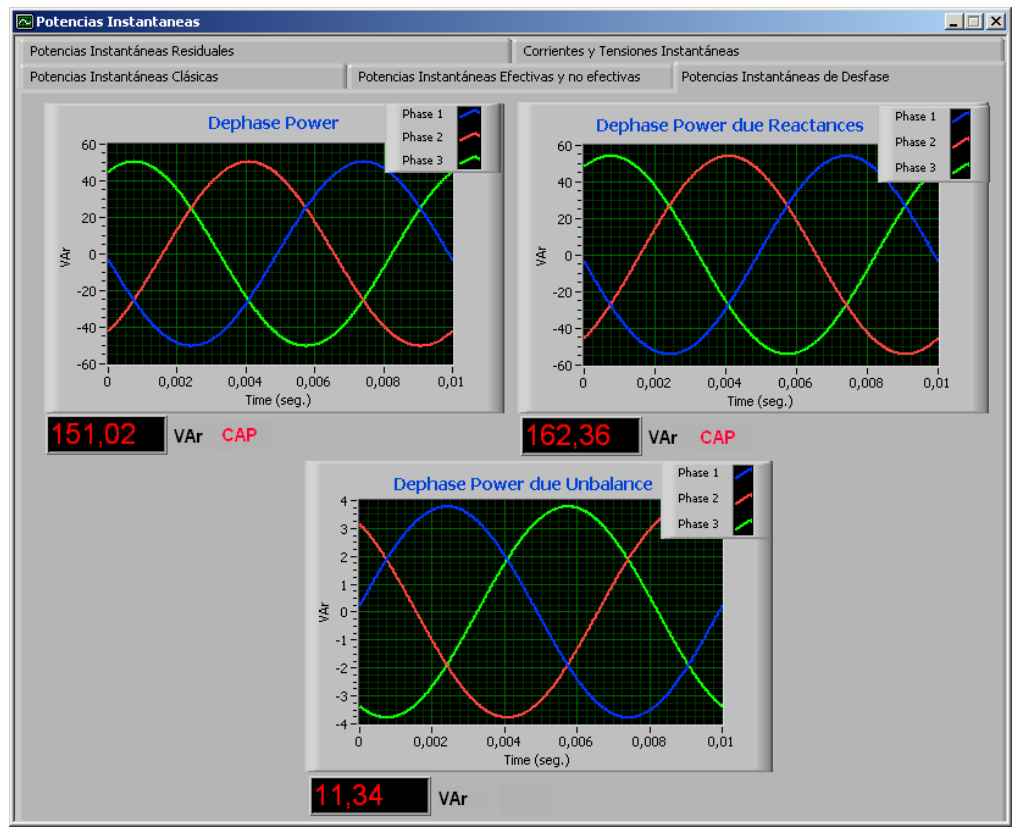

Figura V.102. Pantalla de Potencias de Desfase.

Carga resistiva entre la fase 1 y el neutro, con un consumo de $1 \angle 0^{\circ} \mathrm{A}$ y otra carga capacitiva entre la fase 2 y el neutro, con un consumo de $1 \angle-75^{\circ} A$ y producimos el desequilibrio sumando ángulos negativos al argumento de la fase 3.

Introducimos gracias a la ventana de entrada de datos del SIMPELEC Simulador, las tensiones de las fases y el consumo, así como las sucesivas modificaciones en los argumentos de la fase 3 en este caso, tal y como mostramos en la figura V.103.

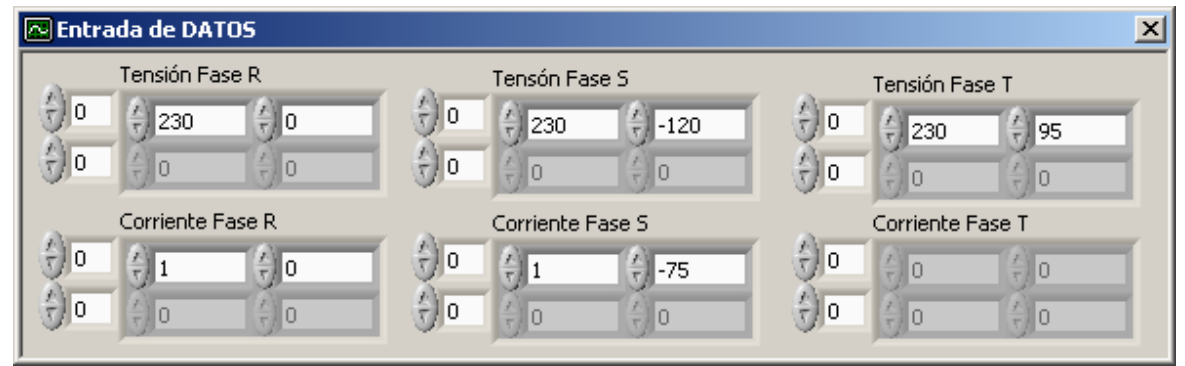

Figura V.103. Pantalla de entrada de datos. 
Tenemos pues un sistema de tensiones en el que como ya hemos comentado de manera general dejamos fijos los módulos de las tensiones de las fases, y tenemos una carga resistiva que consume $1 \angle 0^{\circ} \mathrm{A}$ y otra capacitiva con un consumo de $1 \angle-75^{\circ} A$ conectadas en las fases 1 y 2 , variando de manera gradual el argumento de la fase 3, logrando los pertinentes desequilibrios de tensión y produciendo la consiguiente potencia de desfase, tal y como podemos observar en la tabla V.48.

\begin{tabular}{|c|c|c||}
\hline $\begin{array}{c}\text { Argumento } \\
\text { Fase 3 }\end{array}$ & $\begin{array}{c}\text { Variación } \\
\text { Argumento ( }{ }^{\circ} \text { ) }\end{array}$ & $\begin{array}{c}\text { Potencia Desfase } \\
\text { Desequilibrio } \mathbf{Q}_{\text {dru }} \text { (VAr) }\end{array}$ \\
\hline $119^{\circ}$ & 1 & $-2,29$ \\
\hline $118^{\circ}$ & 2 & $-4,58$ \\
\hline $115^{\circ}$ & 5 & $-11,48$ \\
\hline $110^{\circ}$ & 10 & $-23,00$ \\
\hline $105^{\circ}$ & 15 & $-34,49$ \\
\hline $95^{\circ}$ & 25 & $-57,00$ \\
\hline $85^{\circ}$ & 35 & $-78,34$ \\
\hline $70^{\circ}$ & 50 & $-106,71$ \\
\hline $50^{\circ}$ & 70 & $-134,88$ \\
\hline $30^{\circ}$ & 90 & $-148,95$ \\
\hline $20^{\circ}$ & 100 & $-150,10$ \\
\hline $10^{\circ}$ & 110 & $-147,24$ \\
\hline $0^{\circ}$ & 120 & $-140,45$ \\
\hline \multicolumn{2}{|l}{} \\
\hline
\end{tabular}

Tabla V.48. Potencia de Desfase \& Variación Argumento.

De la tabla V.48, vemos que de manera general, al producirse el desequilibrio del sistema modificando gradualmente el argumento de la fase 3 desde $120^{\circ}$ a $0^{\circ}$, la potencia de desfase debida al desequilibrio en el sistema es capacitiva.

Que como se ha comentado en el caso anterior, se pueden obtener los resultados de la tabla V.48, como suma de los consumos individuales de las cargas sometidas a esos desequilibrios de manera independiente, valiéndonos de que estamos trabajando con un sistema lineal.

La variación en el argumento de la fase 3 , de manera que valga $95^{\circ}$, genera la pantalla principal que podemos observar en la figura V.104, en la que en este caso se puede observar que se refuerza el carácter capacitivo del sistema, al 
sumarse la potencia de desfase debida a la reactancia capacitiva con la provocada por el desequilibrio del sistema.

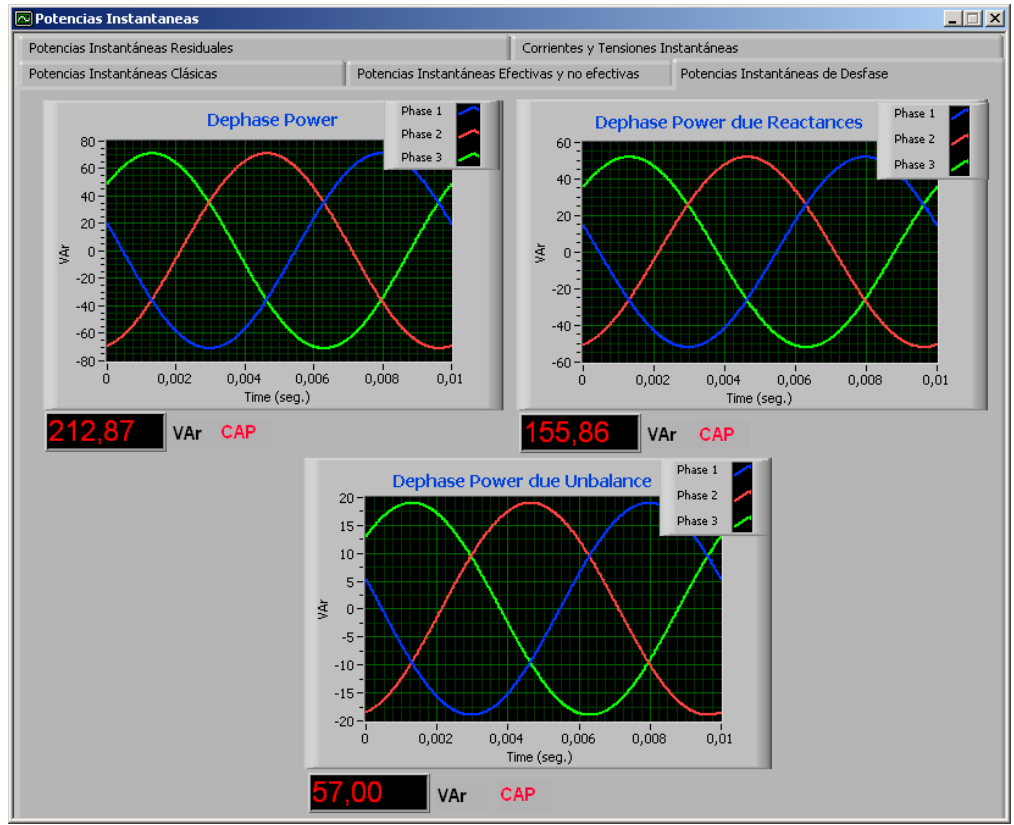

Figura V.104. Pantalla de Potencias de Desfase.

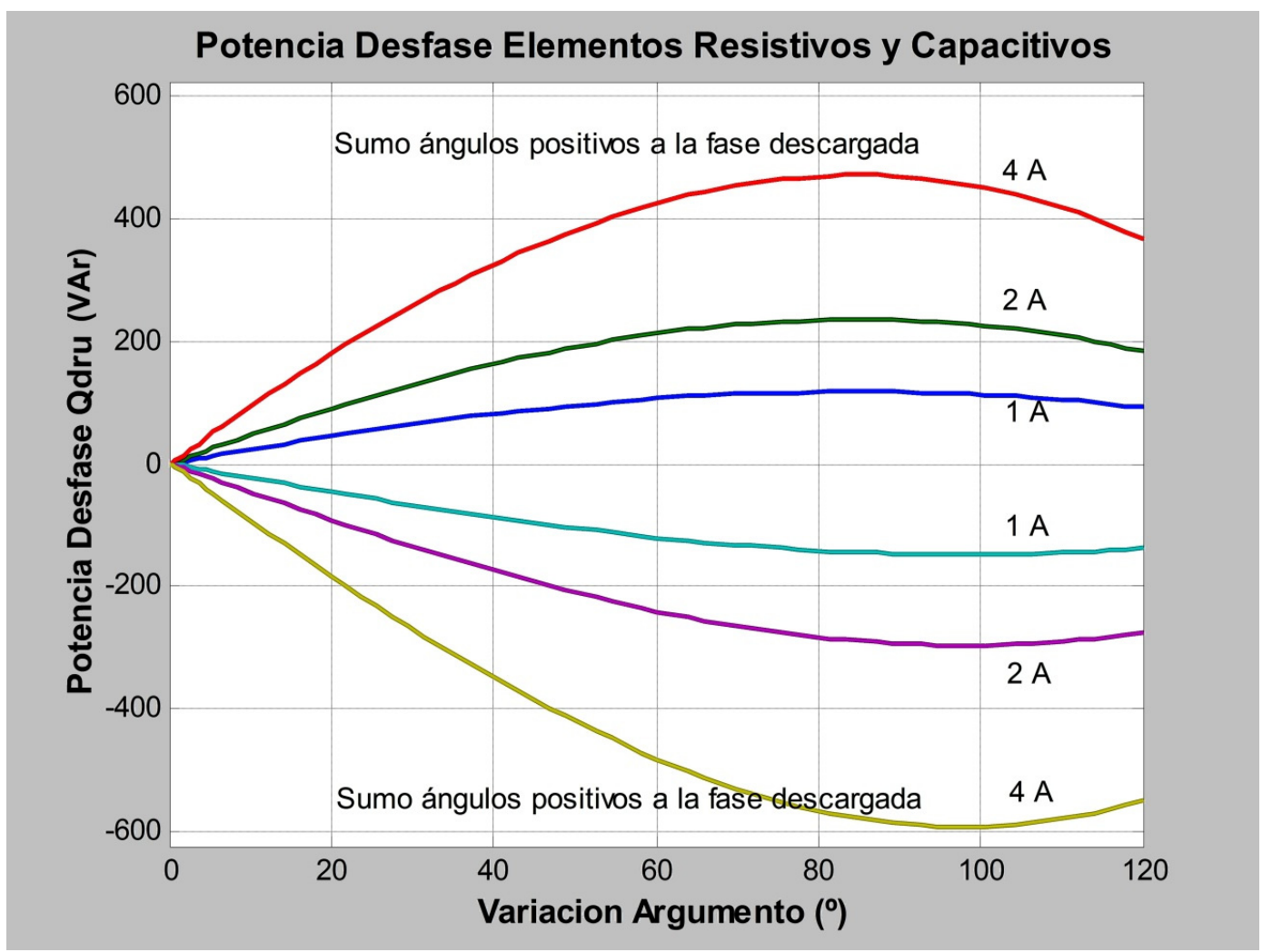

Figura V.105. Potencia de Desfase, para variaciones de una carga resistiva, y otra capacitiva, en diversas fases y grados de desequilibrio. 
Aprovechando la linealidad del sistema, y los datos obtenidos previamente para las cargas individuales resistivas y capacitivas, conectadas de forma sucesiva en diversas fases y sometidas a diferentes grados de desequilibrio, se han obtenido los valores de la potencia de desfase que nos permiten mostrar las gráficas que se ve en la figura V.105, en la que también se muestran las potencias de desfase debidas al desequilibrio para consumos de 1, 2 y 4 Amperios. 


\section{V.3.3.- Cargas monofásicas entre fase y neutro. Vario módulos de tensión.}

El proceso seguido en esta comprobación, difiere de los anteriormente usados, ya que ahora se va proceder a ver la evolución de la potencia de desfase debida al desequilibrio, cuando este es provocado por la modificación de los módulos de las tensiones del sistema, sin ser variados los argumentos de las mismas. Se va por lo tanto a aumentar y disminuir los módulos de las tensiones de las fases descargas de forma sucesiva, así como luego pasar a realizar esta variación en la fase que se halle cargada. Los resultados obtenidos para cada caso, usando el simulador SIMPELEC, se muestran en forma tabular y gráfica para los diversos casos analizados.

a) Carga Resistiva: Se ha estudiado el comportamiento del sistema cuando se introduce una carga resistiva, con un consumo de $1 \angle 0^{\circ} \mathrm{A}$, de forma sucesiva en cada una de las fases del sistema y se le somete a desequilibrios provocados por tan sólo la variación de los módulos de las tensiones en las fases descargadas y posteriormente en la fase que se halla cargada. Para todos los casos analizados, se ha observado que estas modificaciones en los módulos de las tensiones del sistema, no conllevan asociado en este caso la presencia de potencia de desfase.

b) Carga Inductiva: Se ha analizado el comportamiento del sistema cuando es introducido en el mismo una carga inductiva pura de consumo $1 \angle-90^{\circ} \mathrm{A}$, en cada una de las fases y provocamos desequilibrios en el sistema por medio de aumentar y disminuir las tensiones de las fases descargadas y luego de la fase cargada de manera sucesiva.

Usando el SIMPELEC Simulador, se ha constatado que con independencia de que en que fase se halle conectada la carga, y mientras que sea sometida a el mismo desequilibrio obtenemos el mismo valor de la potencia de desfase debida al desequilibrio, a modo de ejemplo se muestra en la figura V.106, los datos de entrada de uno de los múltiples valores usados, en el que se aumenta la tensión 
en una de las fases descargadas, así como en la figura V.107, se observa la pantalla de potencias de desfase.

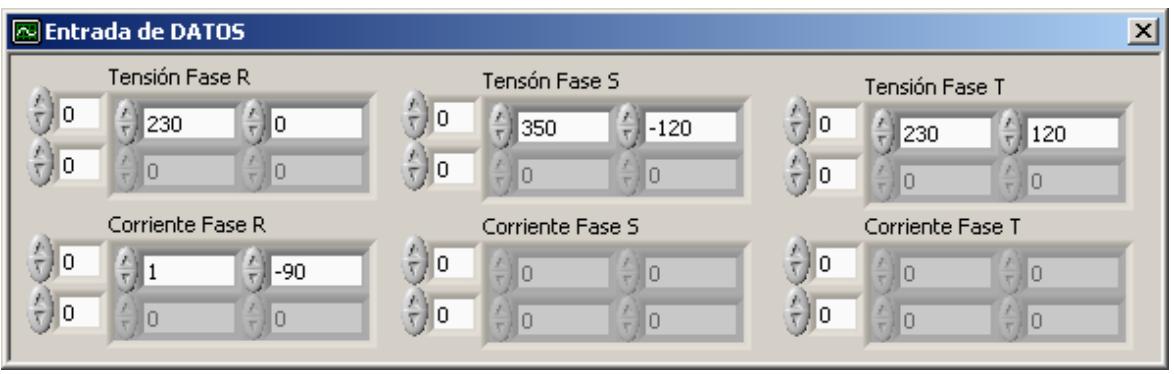

Figura V.106. Pantalla de entrada de datos.

Se observa en la figura V.107, que la potencia de desfase debida al desequilibrio en este caso, aún siendo la carga inductiva, posee carácter capacitivo, lo que provoca que el efecto inductivo de la carga se reduzca.

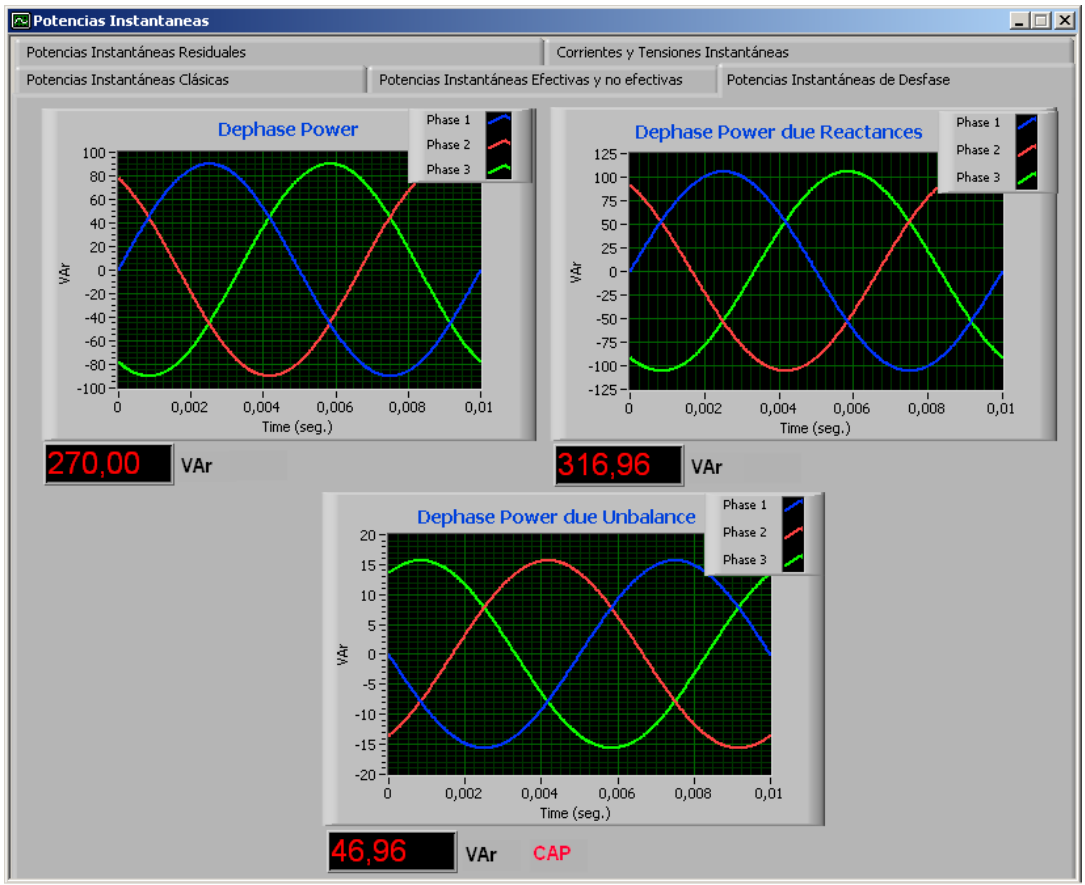

Figura V.107. Pantalla de Potencias de Desfase.

Los datos obtenidos se muestran la tabla V.49, que se han conseguido en base a ir aumentando y disminuyendo la tensión en las fases descargadas, y variando de manera sucesiva la fase cargada. 


\begin{tabular}{||c|c|c|c||}
\hline $\begin{array}{c}\text { Variación } \\
\text { De Tensión (V) }\end{array}$ & $\begin{array}{c}\text { Potencia Desfase } \\
\text { Deseq. } \mathbf{Q}_{\text {dru }} \text { (VAr) } \\
\text { Aumento Tensión }\end{array}$ & $\begin{array}{c}\text { Variación } \\
\text { De Tensión (V) }\end{array}$ & $\begin{array}{c}\text { Potencia Desfase } \\
\text { Deseq. } \mathbf{Q}_{\text {dru }} \text { (VAr) } \\
\text { Disminuyo Tensión }\end{array}$ \\
\hline 4 & $-1,34$ & 4 & 1,33 \\
\hline 8 & $-2,70$ & 8 & 2,64 \\
\hline 21 & $-7,21$ & 20 & 6,47 \\
\hline 43 & $-15,23$ & 38 & 11,97 \\
\hline 66 & $-24,10$ & 55 & 16,87 \\
\hline 120 & $-46,96$ & 88 & 25,59 \\
\hline 182 & $-76,67$ & 118 & 32,61 \\
\hline 300 & $-143,48$ & 160 & 40,97 \\
\hline
\end{tabular}

Tabla V.49. Potencia de Desfase \& Variación de Tensión

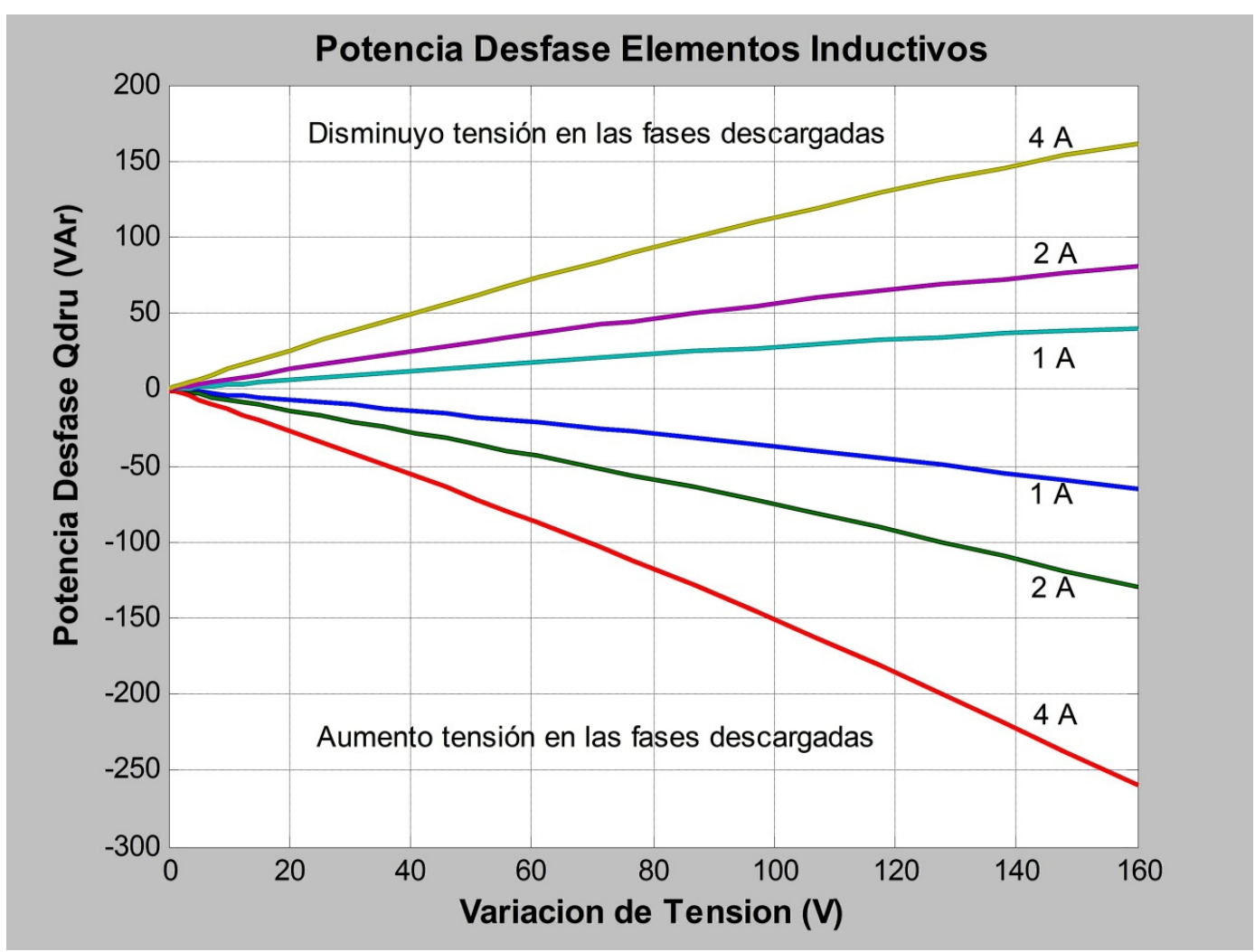

Figura V.108. Potencia de Desfase, con una carga inductiva, en diversas fases y grados de desequilibrio, en base a variaciones de tensión en fases descargadas.

En la figura V. 108, podemos ver las gráficas que se obtienen de la tabla V.49, añadiendo los consumos de 2 y $4 \mathrm{~A}$, usando que el sistema es lineal, en ella podemos observar que de manera general hay potencia de desfase debida al desequilibrio de carácter inductivo cuando se disminuye tensión en las fases 
descargadas y en cambio esta potencia de desfase debida al desequilibrio pasa a tener carácter capacitivo se produce un aumento de tensión en estas fases descargadas.

Pasamos ahora a analizar el caso de que las tensiones de las fases descargadas permanezcan constantes y lo que hagamos sea variar la tensión de la fase en la que se halle conectada la carga, cuyos resultados se presentan después de la simulación pertinente, en la tabla V.50.

\begin{tabular}{||c|c|c|c||}
\hline $\begin{array}{c}\text { Variación } \\
\text { De Tensión (V) }\end{array}$ & $\begin{array}{c}\text { Potencia Desfase } \\
\text { Deseq. Qdru (VAr) } \\
\text { Aumento Tensión }\end{array}$ & $\begin{array}{c}\text { Variación } \\
\text { De Tensión (V) }\end{array}$ & $\begin{array}{c}\text { Potencia Desfase } \\
\text { Deseq. Qdru (VAr) } \\
\text { Disminuyo Tensión }\end{array}$ \\
\hline 4 & 2,64 & 4 & $-2,70$ \\
\hline 8 & 5,21 & 8 & $-5,46$ \\
\hline 21 & 13,22 & 20 & $-14,18$ \\
\hline 43 & 25,66 & 38 & $-28,68$ \\
\hline 66 & 37,46 & 55 & $-44,35$ \\
\hline 120 & 61,71 & 88 & $-82,90$ \\
\hline 182 & 85,60 & 118 & $-133,92$ \\
\hline 300 & 124,53 & 160 & $-269,21$ \\
\hline
\end{tabular}

Tabla V.50. Potencia de Desfase \& Variación de Tensión

En la figura V.109, podemos observar las gráficas obtenidas en base a lo datos de la tabla V.50, en ella se aprecia claramente, que cuando dejamos fijas las tensiones de las fases descargadas y producimos aumentos y disminuciones de la tensión en la fase en la que se halla conectada la carga, existe potencia de desfase debida al desequilibrio, pero esta potencia varia su carácter en función de como se provoque el desequilibrio, así tenemos, que al aumentar la tensión en la fase cargada, esta posee un carácter inductivo, que refuerza el ya propio de la carga, mientras que si procedemos a disminuir la tensión en la fase en la que se halla la carga, el carácter es capacitivo, que contrarresta un poco el carácter inductivo de la carga. Esto se puso de manifiesto en las simulaciones llevadas a cabo, con independencia de en que fase se hubiera conectado la carga. 


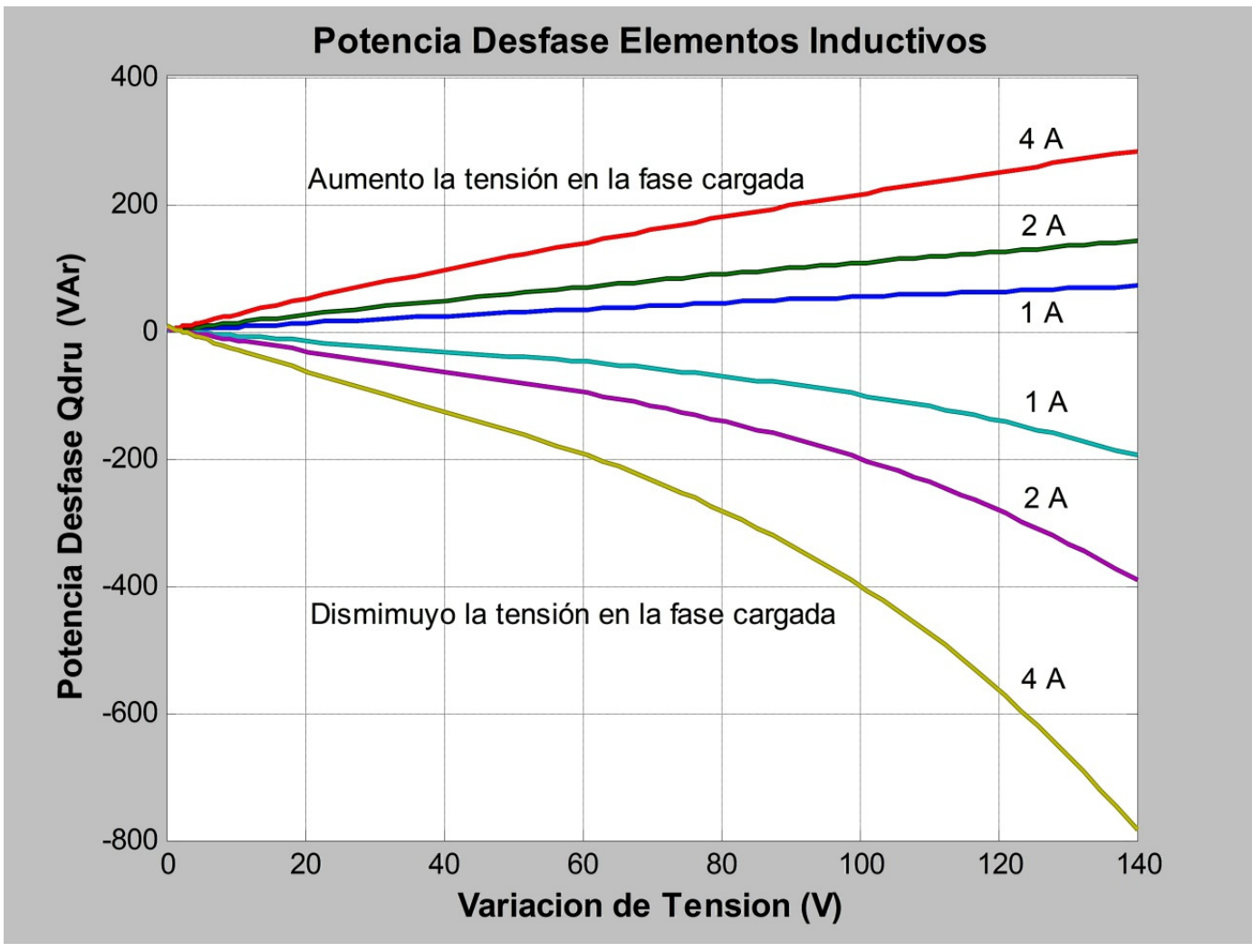

Figura V.109. Potencia de Desfase, con una carga inductiva, en diversas fases y grados de desequilibrio, en base a variaciones de tensión en la fase cargada.

c) Carga Capacitiva: Se ha analizado el comportamiento del sistema cuando es introducido en el mismo una carga capacitiva de consumo $1 \angle 90^{\circ} \mathrm{A}$, en cada una de las fases y provocamos desequilibrios en el sistema por medio de aumentar y disminuir las tensiones de las fases descargadas y luego de la fase cargada de manera sucesiva.

Al igual que sucedía con la carga inductiva, haciendo uso del SIMPELEC Simulador, se ha observado que los resultados que se obtenían de la potencia de desfase debida al desequilibrio, eran iguales con independencia de en que fase se hallara la carga conectada, mientras estuviera sometida a los mismos desequilibrios, desequilibrios estos que los lográbamos en base a variar los módulos de las fases descargadas, para luego provocar la variación de tensión en la fase cargada. 
Los resultados obtenidos, se muestran en la tabla V.51, en la que podemos apreciar que la potencia de desfase debida al desequilibrio, toma carácter diferente en función de como produzcamos el desequilibrio.

\begin{tabular}{||c|c|c|c||}
\hline $\begin{array}{c}\text { Variación } \\
\text { De Tensión (V) }\end{array}$ & $\begin{array}{c}\text { Potencia Desfase } \\
\text { Deseq. Qdru (VAr) } \\
\text { Aumento Tensión }\end{array}$ & $\begin{array}{c}\text { Variación } \\
\text { De Tensión (V) }\end{array}$ & $\begin{array}{c}\text { Potencia Desfase } \\
\text { Deseq. Qdru (VAr) } \\
\text { Disminuyo Tensión }\end{array}$ \\
\hline 4 & 1,34 & 4 & $-1,33$ \\
\hline 8 & 2,70 & 8 & $-2,64$ \\
\hline 21 & 7,21 & 20 & $-6,47$ \\
\hline 43 & 15,23 & 38 & $-11,97$ \\
\hline 66 & 24,10 & 55 & $-16,87$ \\
\hline 120 & 46,96 & 88 & $-25,59$ \\
\hline 182 & 76,67 & 118 & $-32,61$ \\
\hline 300 & 143,48 & 160 & $-40,97$ \\
\hline
\end{tabular}

Tabla V.51. Potencia de Desfase \& Variación de Tensión

Podemos apreciar, que se obtienen justamente los valores contrarios a los obtenidos cuando en el sistema introducíamos una carga inductiva.

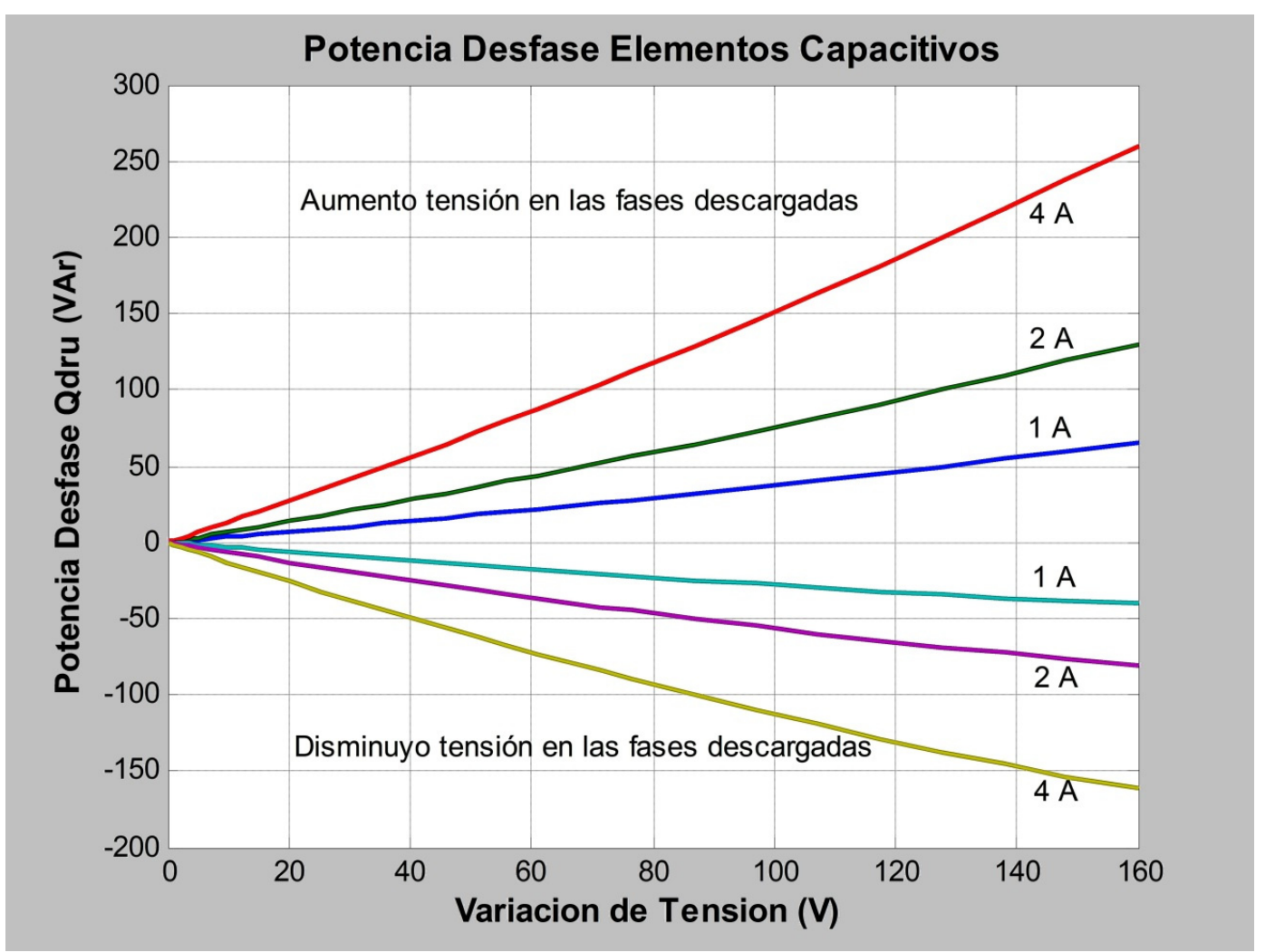

Figura V.110. Potencia de Desfase, con una carga capacitiva, en diversas fases y grados de desequilibrio, en base a variaciones de tensión en fases descargadas. 
De la figura V.110, observamos que ahora al producir un desequilibrio en base a variar aumentando el módulo de las fases descargadas, provoca la aparición de potencia de desfase debida al desequilibrio con carácter inductivo, aún siendo la carga capacitiva, mientras que si procedemos a disminuir la tensión en las fases descargadas la potencia de desfase debida al desequilibrio toma carácter capacitivo.

Veamos ahora el caso de producir variaciones en la tensión de la fase cargada, mientras que en las fases libres permanecen constantes, los resultados obtenidos se muestran en la tabla V.52.

\begin{tabular}{|c|c|c|c||}
\hline $\begin{array}{c}\text { Variación } \\
\text { De Tensión (V) }\end{array}$ & $\begin{array}{c}\text { Potencia Desfase } \\
\text { Deseq. Qdru (VAr) } \\
\text { Aumento Tensión }\end{array}$ & $\begin{array}{c}\text { Variación } \\
\text { De Tensión (V) }\end{array}$ & $\begin{array}{c}\text { Potencia Desfase } \\
\text { Deseq. Qdru (VAr) } \\
\text { Disminuyo Tensión }\end{array}$ \\
\hline 4 & $-2,64$ & 4 & 2,70 \\
\hline 8 & $-5,21$ & 8 & 5,46 \\
\hline 21 & $-13,22$ & 20 & 14,18 \\
\hline 43 & $-25,66$ & 38 & 28,68 \\
\hline 66 & $-37,46$ & 55 & 44,35 \\
\hline 120 & $-61,71$ & 88 & 82,90 \\
\hline 182 & $-85,60$ & 118 & 133,92 \\
\hline 300 & $-124,53$ & 160 & 269,21 \\
\hline
\end{tabular}

Tabla V.52. Potencia de Desfase \& Variación Argumento

En la figura V.111, se muestran las gráficas que nos indican como evoluciona la potencia de desfase debida al desequilibrio, para el caso de que produzcamos variaciones de tensión en la fase cargada, así vemos que se produce el efecto contrario al reflejado en la figura V.109, ya que un aumento de tensión en la fase cargada provoca que la potencia de desfase debida al desequilibrio tenga carácter capacitivo, añadiéndose a la de la propia carga, por el contrario, si disminuyo tensión en la fase cargada la potencia de desfase debida al desequilibrio posee carácter inductivo. 


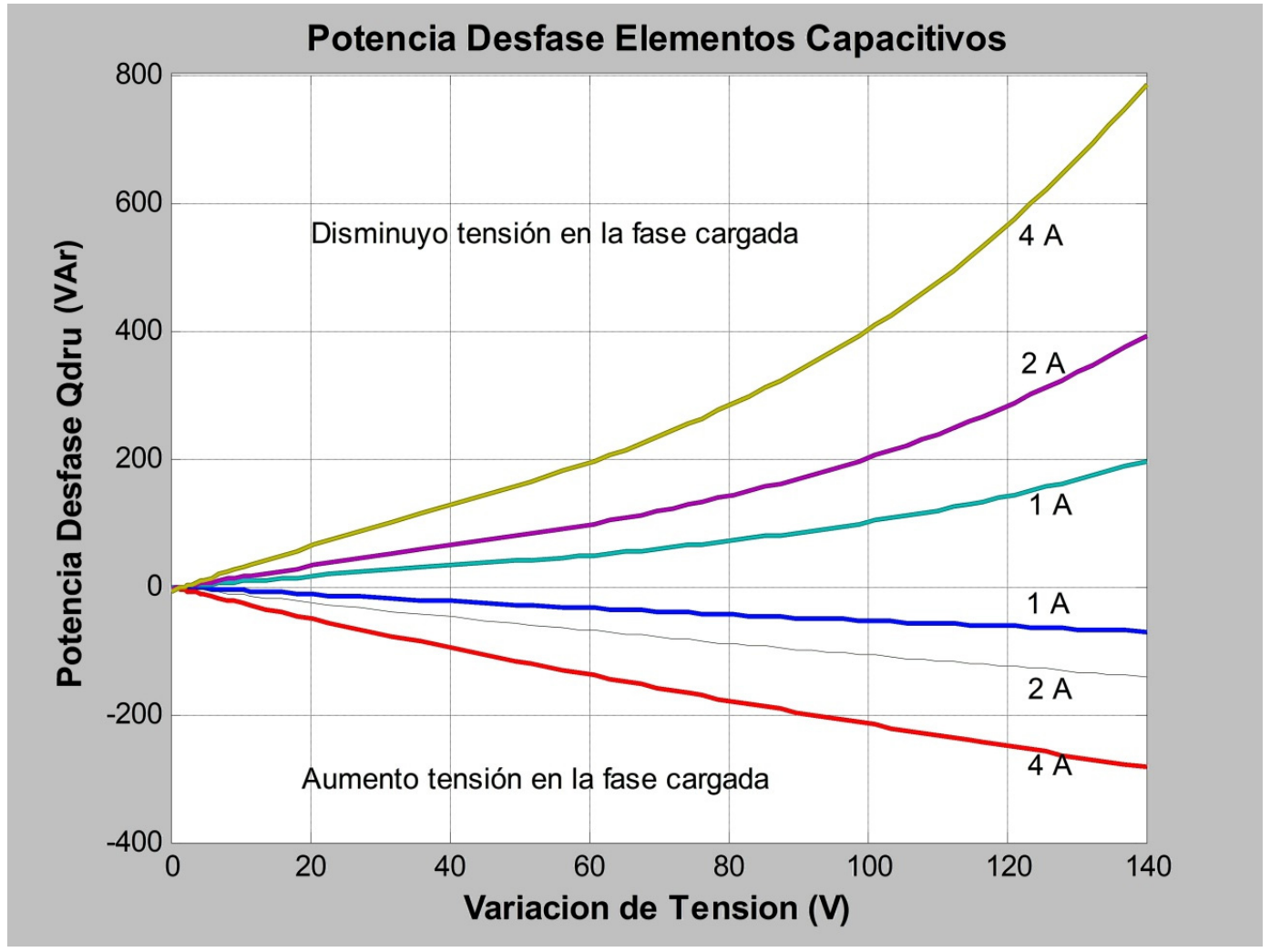

Figura V.111. Potencia de Desfase, con una carga capacitiva, en diversas fases y grados de desequilibrio, en base a variaciones de tensión en la fase cargada. 


\section{V.4.- Medidas Experimentales Obtenidas en Centros de Transformación.}

Hasta ahora las medidas mostradas estaban realizadas en base a la simulación, por medio del SIMPELEC Simulador, en este apartado se van a mostrar medidas efectuadas en redes de baja tensión de industrias, y de zonas residenciales alimentadas desde sus correspondientes centros de transformación, en las cuales se han llevado a cabo medidas realizadas con el dispositivo de medida SIMPELEC Desfase, usando para ello la configuración de medida indirecta y por medio del uso de sondas de corriente de uso comercial.

En estas redes alimentadas por los centros de transformación de zonas industriales y de zonas residenciales, se han registrado más de 30 mil medidas con el dispositivo de medida SIMPELEC, y a continuación se muestran algunos casos, en los que se pone de manifiesto el fenómeno del desfase, provocado por los desequilibrios presentes en los sistemas eléctricos reales.

En las medidas que hemos llevado a cabo, se ha comprobado que en mayor o menor medida los sistemas reales se encuentran desequilibrados, lo que lleva a que exista presencia de potencia de desfase debida al desequilibrio, que como se vera en algunos casos de desequilibrios importantes, esta potencia adquiere unos valores significativos, en relación con el total de la potencia de desfase del sistema. 


\section{V.4.1.- CT Industrial no 1.}

En esta medida, podemos observar según la figura V.112, que el sistema presenta unos valores bastante equilibrados, tanto en corrientes, como en tensiones, siendo el desequilibrio en tensiones del 0,56\%, lo que provoca una pequeña presencia de potencia de desfase debida al desequilibrio, como se muestra en la figura V.113, con un valor de 4,64 VAr, de carácter capacitivo, contrario al producido por las reactancias del sistema, pero cuyo valor en este caso en el que las tensiones están prácticamente equilibradas es muy pequeño, lo que no llega a influir en el carácter total de la potencia de desfase.

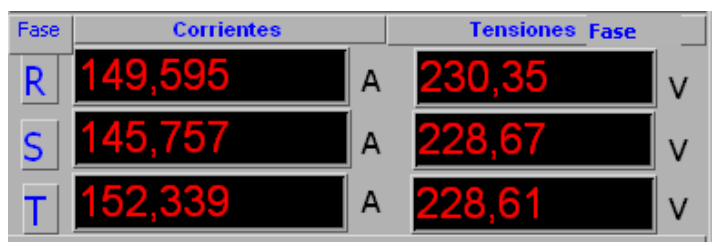

Figura V.112. Pantalla Tensiones y Corrientes, CT Industrial $n^{0} 1$.

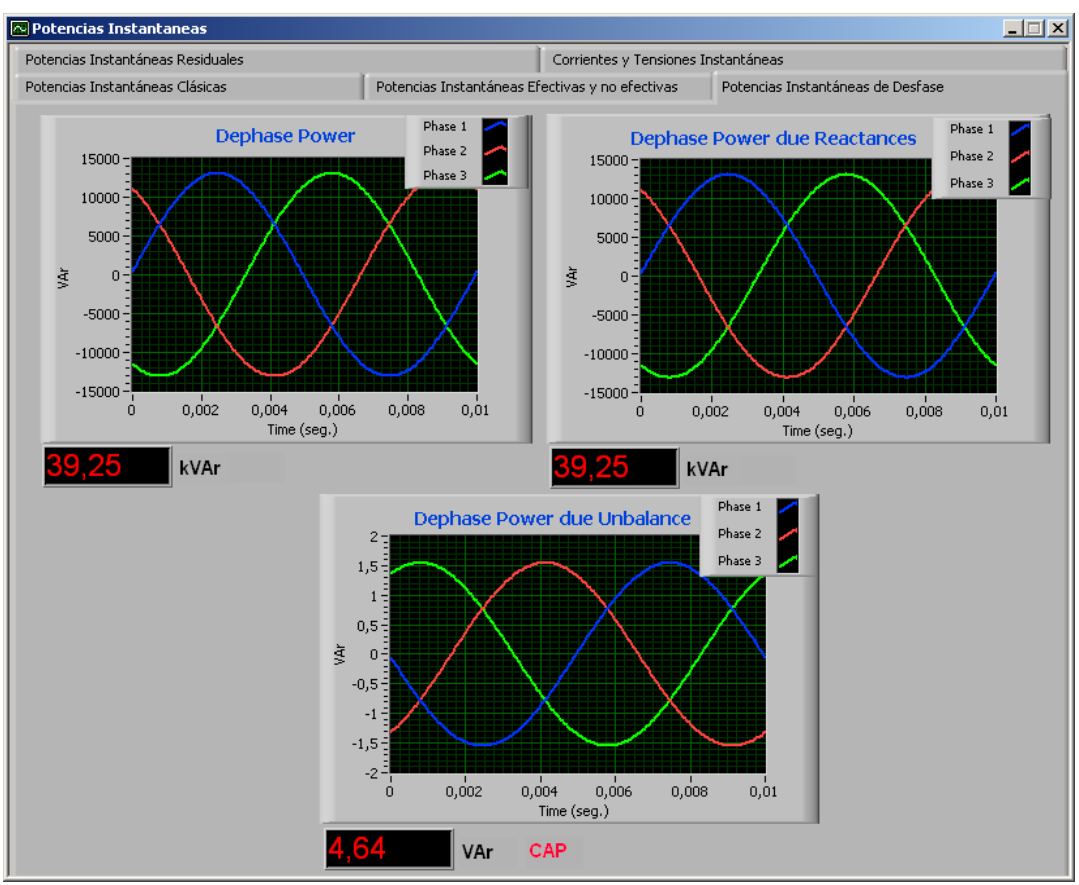

Figura V.113. Pantalla Potencias Desfase, CT Industrial $n^{\circ} 1$. 


\section{V.4.2.- CT Industrial $n \circ 2$.}

En la figura V.114, vemos que el sistema se halla bastante desequilibrado, tanto en corrientes, como en tensiones, siendo el desequilibrio en tensiones del 6,41\%, lo que provoca potencia de desfase debida al desequilibrio, como se muestra en la figura V.115, que podemos apreciar que tiene un valor apreciable de 6,88 kVAr, de carácter capacitivo, lo que supone un 3,06 \% de la potencia de desfase de las reactancias y que además la contrarresta, provocando que la potencia de desfase total del sistema sea menor.

\begin{tabular}{|c|c|c|c|c|}
\hline Fase & Corrientes & & Tensiones Fase & \\
\hline $\mathrm{R}$ & 576,148 & A & 204,48 & v \\
\hline S & 413,559 & $A$ & 229,02 & V \\
\hline $\mathrm{T}$ & 508,600 & A & 205,88 & V \\
\hline
\end{tabular}

Figura V.114. Pantalla Tensiones y Corrientes, CT Industrial no 2.

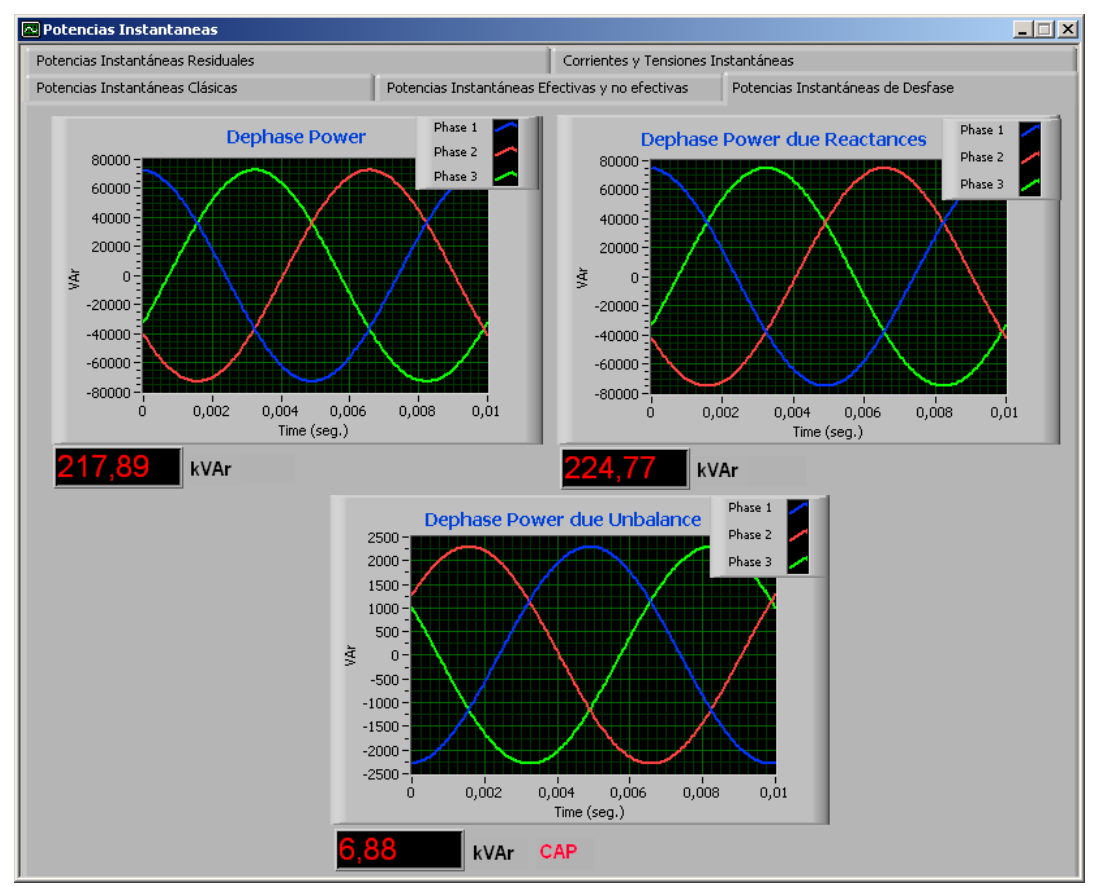

Figura V.115. Pantalla Potencias Desfase, CT Industrial no 2. 


\section{V.4.3.- CT Industrial no 3.}

Se muestran en la figura V.116, los valores capturados en esta instalación, en la que se observan unos pequeños desequilibrios tanto en corrientes como en tensiones, siendo este último de tan sólo el $0,6 \%$, lo que provoca potencia de desfase debida al desequilibrio de carácter inductivo de valor 51,20 $\mathrm{VAr}$, como vemos en la figura V.117, que se suma a la potencia de desfase debida a las reactancias del sistema, lo que provoca que la potencia de desfase total del sistema sea mayor.

\begin{tabular}{|c|c|c|}
\hline Fase & Corrientes & Tensiones Fase \\
\hline $\mathrm{R}$ & 594,034 & 228,96 \\
\hline S & 583,970 & 226,71 \\
\hline $\mathrm{T}$ & 552,333 & A 228,26 \\
\hline
\end{tabular}

Figura V.116. Pantalla Tensiones y Corrientes, CT Industrial no 3.

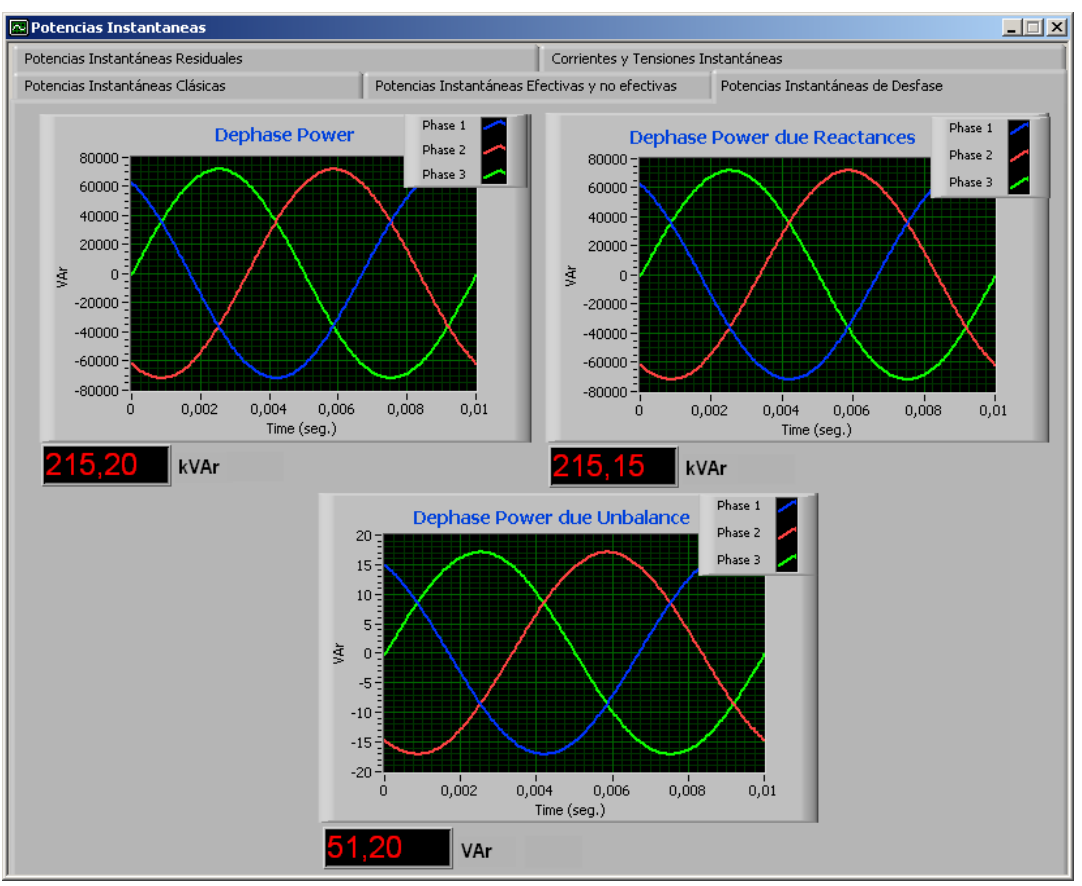

Figura V.117. Pantalla Potencias Desfase, CT Industrial no 3. 


\section{V.4.4.- CT Industrial no 4.}

Podemos apreciar en esta instalación que existían importantes desequilibrios en corrientes y en tensiones $6,26 \%$, como vemos en la figura V.118, que provoca la presencia de potencia de desfase debida al desequilibrio de carácter capacitivo y de un valor importante 6,12 kVAr tal y como se muestra en la figura V.119, lo que representa un $3,15 \%$ de la potencia de desfase debida a las reactancias, lo que provoca una potencia de desfase total del sistema menor que la esperada, al ser contraria la potencia de desfase debida al desequilibrio, y la debida a las reactancias del sistema.

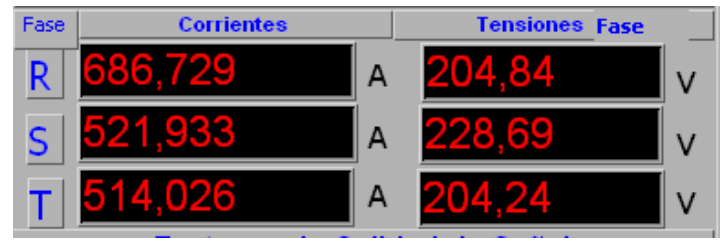

Figura V.118. Pantalla Tensiones y Corrientes, CT Industrial no 4.

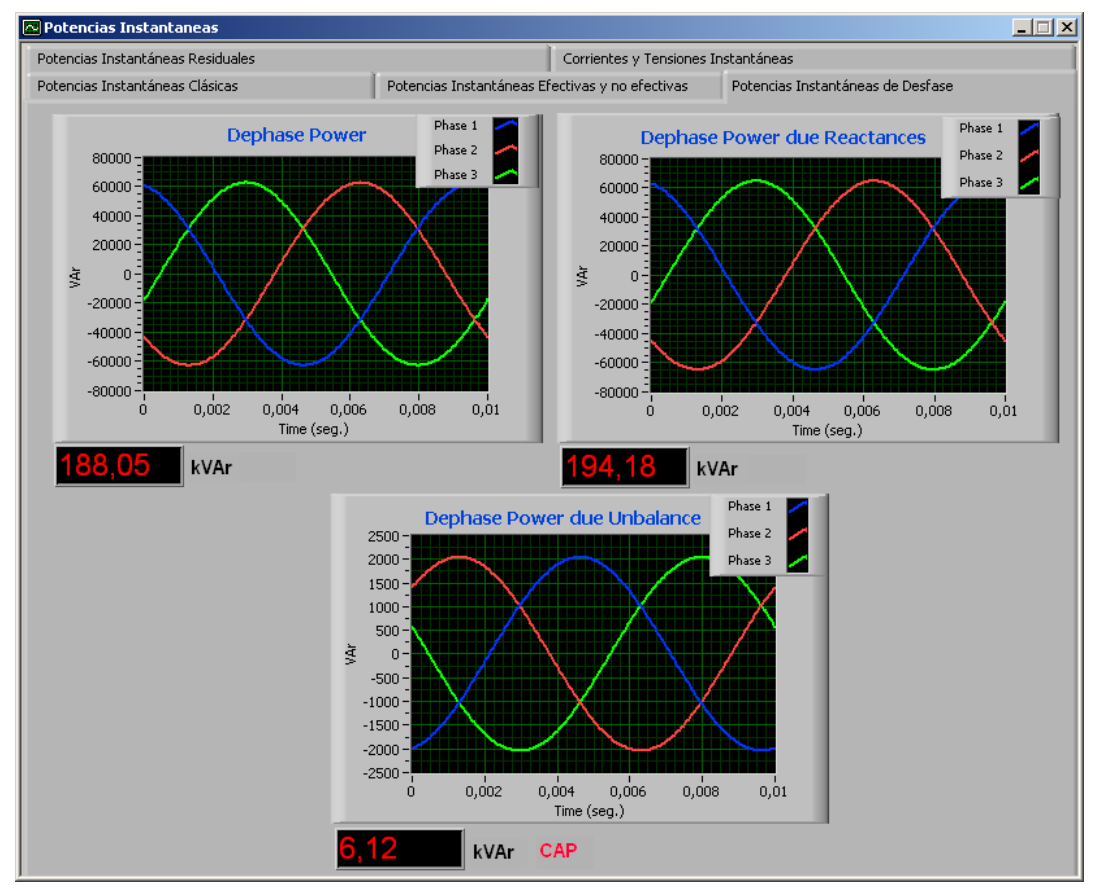

Figura V.119. Pantalla Potencias Desfase, CT Industrial no 4. 


\section{V.4.5.- CT Higinio Noja.}

Observamos en la figura V.120, que el sistema se halla sometido a un desequilibrio bastante grande tanto en tensiones como en corrientes, siendo el de tensiones de un $4,91 \%$, lo que pone de manifiesto una potencia de desfase debida al desequilibrio de un valor elevado de unos $5 \mathrm{kVAr}$, y con carácter capacitivo, que supone un $164,47 \%$ la potencia de desfase del sistema, y que en este caso se suma a la potencia de desfase también capacitiva del sistema, debida seguramente a la conexión de condensadores de compensación, lo que podemos observar en la figura V.121.

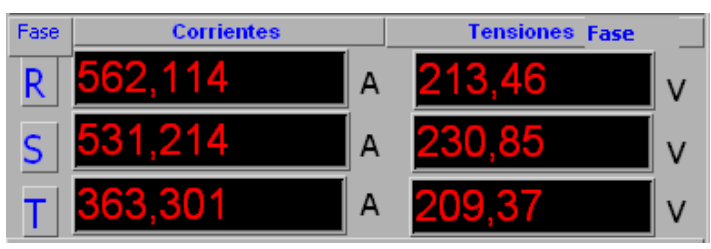

Figura V.120. Pantalla Tensiones y Corrientes, CT Higinio Noja.

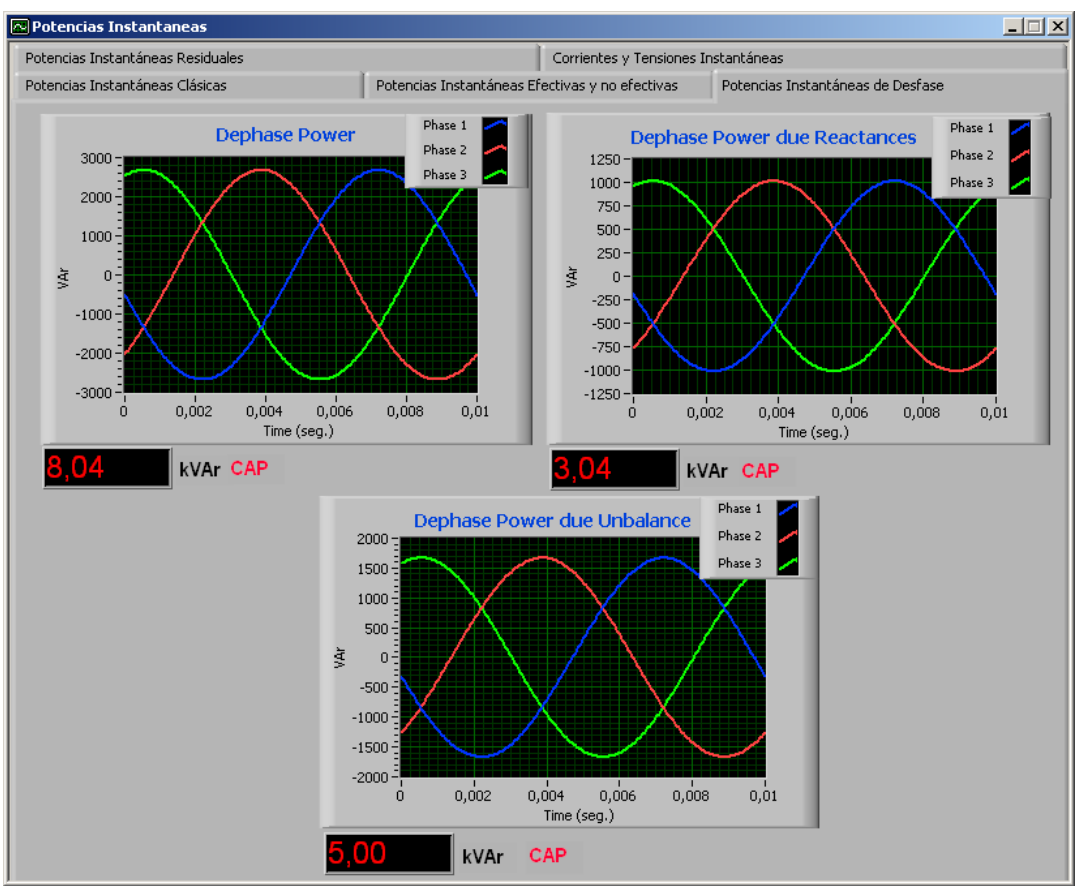

Figura V.121. Pantalla Potencias Desfase, CT Higinio Noja. 


\section{V.4.6.- CT Polígono de Viviendas.}

De la medida que hemos realizado en este polígono de viviendas, observamos en la figura V.122, que los desequilibrios son importantes, siendo el de tensiones de un $6,79 \%$, que conlleva la presencia de una potencia de desfase debida al desequilibrio de $696,46 \operatorname{VAr}$ y de carácter capacitivo, que representa un $29,14 \%$ la potencia de desfase debida a las reactancias, y que provoca que la potencia total del sistema disminuya de manera significativa tal y como se muestra en la figura V.123.

\begin{tabular}{|c|c|c|c|c|}
\hline Fase & Corrientes & & Tensiones Fase & \\
\hline $\mathrm{R}$ & 32,249 & A & 200,45 & V \\
\hline S & 29,605 & A & 225,46 & V \\
\hline $\mathrm{T}$ & 28,335 & A & 201,92 & V \\
\hline
\end{tabular}

Figura V.122. Pantalla Tensiones y Corrientes, CT Polígono de Viviendas.

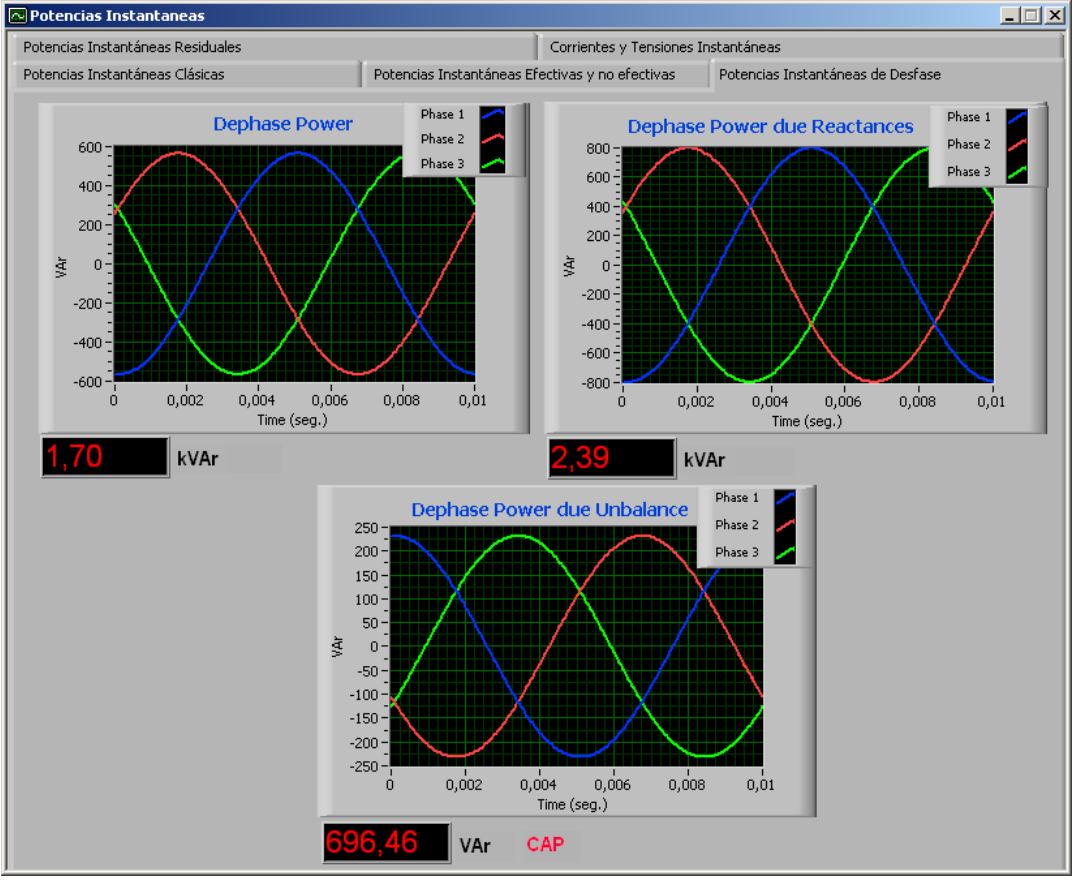

Figura V.123. Pantalla Potencias Desfase, CT Polígono de Viviendas. 


\section{V.4.7.- CT Línea Residencial no 56 de la Calle Paz}

La medida efectuada en esta línea residencial, ha mostrado que existía un fuerte desequilibrio en corrientes y en tensiones, siendo este último de un 8,69\%, tal y como se puede observar en la figura V.124, lo que provoca una potencia de desfase de carácter capacitivo de 736,53 VAr, valor que es un $12,48 \%$ de la potencia de desfase debida a las reactancias del sistema, y que hace que la potencia de desfase total del sistema sea menor que la esperada si sólo tuviéramos en cuenta la debida a la presencia de elementos reactivos en el sistema, como se evidencia en la figura V.125.

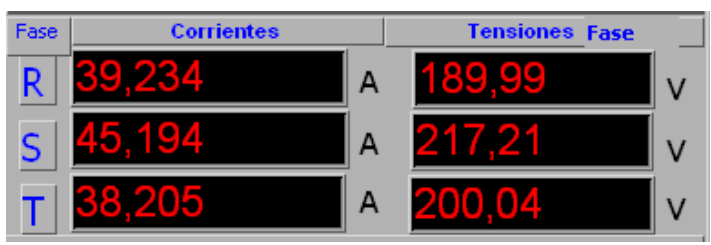

Figura V.124. Pantalla Tensiones y Corrientes, CT Línea 56 C/ Paz.

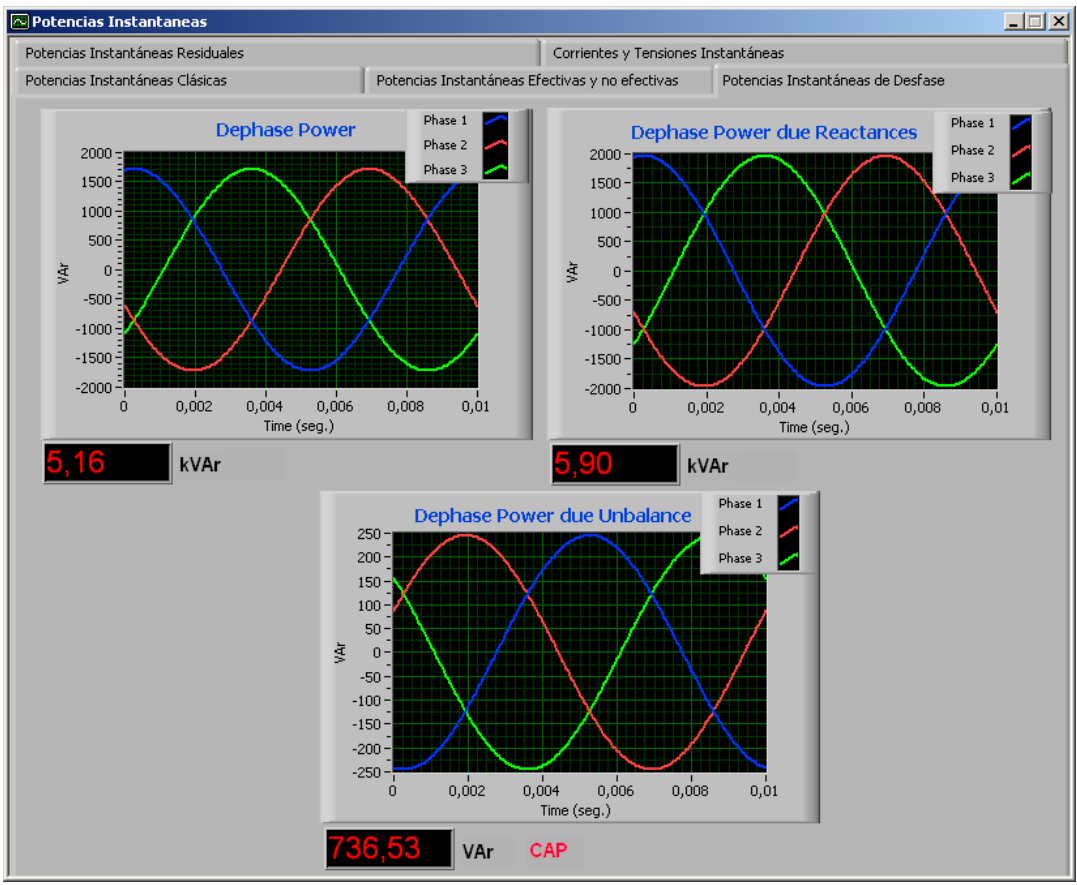

Figura V.125. Pantalla Potencias Desfase, CT Línea 56 C/ Paz. 


\section{V.4.8.- CT Línea Residencial no 57 de la Calle Paz.}

Al igual que en la otra línea residencial, se pone de manifiesto el alto grado de desequilibrio de la misma, ya que podemos observar en la figura V.125, que el desequilibrio en tensiones es de un 8,06\%, y por lo tanto tenemos presencia de potencia de desfase debida al desequilibrio con un valor de 728,98 $\operatorname{VAr}$ de carácter capacitivo, lo que nos da un $15,57 \%$ de la potencia de desfase debida a las reactancias del sistema, y tal como observamos en la figura V.126, reduce el efecto inductivo que tendría el sistema si este estuviera equilibrado.

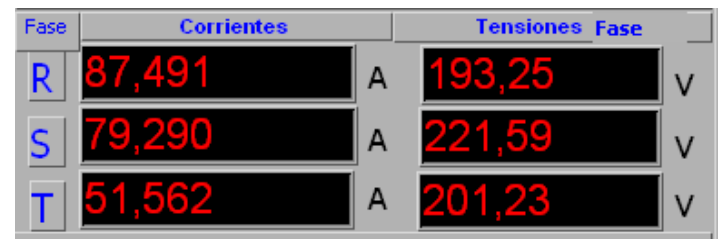

Figura V.125. Pantalla Tensiones y Corrientes, CT Línea 57 C/ Paz.

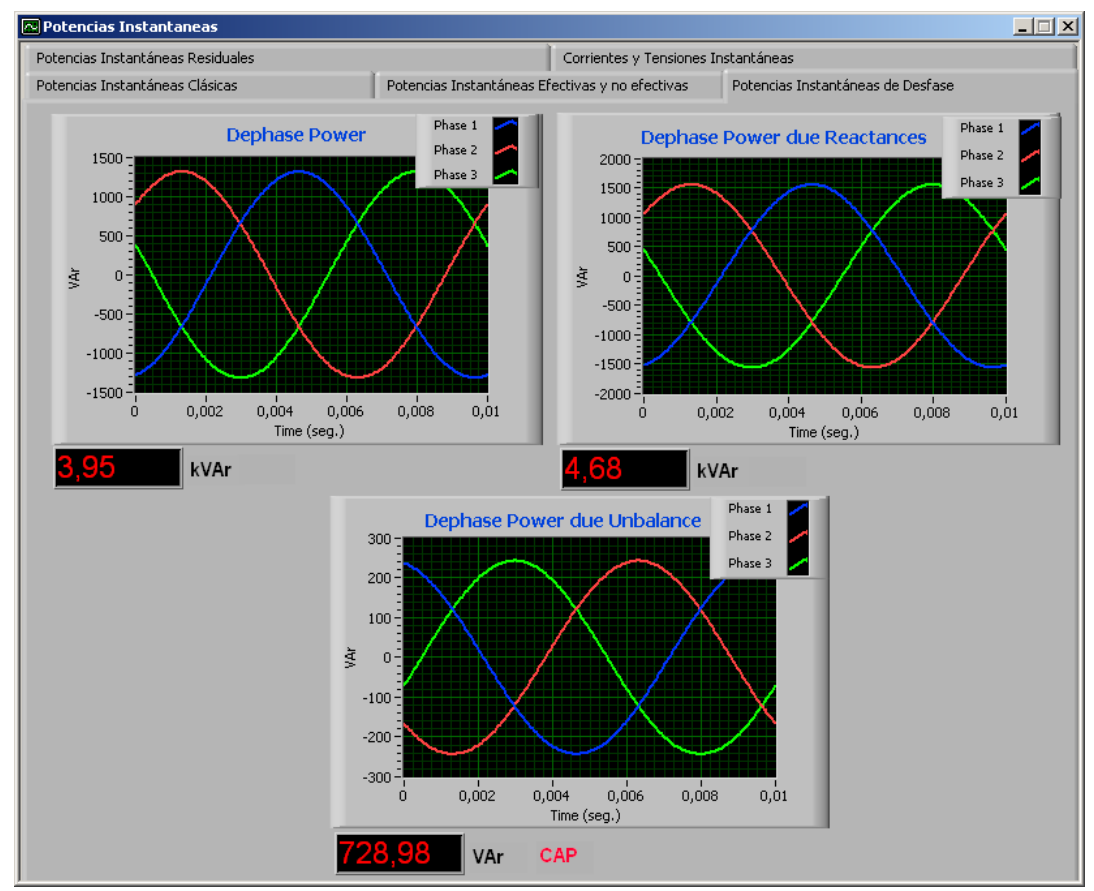

Figura V.126. Pantalla Potencias Desfase, CT Línea 57 C/ Paz. 


\section{V.5.- Fuente Trifásica Programable.}

El GINTEC, tal y como se menciono en la introducción de este capítulo, cuenta con una fuente trifásica conmutada programable de la casa Chroma, de la que pasamos a indicar algunas de sus principales características técnicas:

Modelo 61704

- Potencia de salida en AC:

- Potencia máxima: 6000 VA

- Por fase: 2000 VA.

- Tensión:

- Rango: 150 V / 300 V.

- Precisión: 0,2\% + 0,2\% F.S.

○ Resolución: 0,1 V.

○ Distorsión: 0,3\% @ 50 / $60 \mathrm{~Hz}$.

- Corriente máxima por fase:

- R.M.S. : 16 A / 8 A.

- Pico: 96 A / 48 A.

- Frecuencia:

- Rango: CC, $15 \div 1,2 \mathrm{kHz}$.

○ Precisión: $0,15 \%$.

- Ángulo de Fase:

- Rango: $0 \div 360^{\circ}$.

- Resolución: 0,30 .

○ Precisión: <0,80 @ 50 / $60 \mathrm{~Hz}$.

- Potencia de salida en CC:

- Potencia: $1000 \mathrm{~W}$.

○ Tensión: 212 / 424 V.

- Corriente: 8 A / 4 A.

De estas características, podemos observar que se pueden programar la tensión y la frecuencia, así como el usuario puede regular el ángulo de fase entre los $0^{\circ}$ 
y los $360^{\circ}$, lo que nos permite poder simular de una manera muy práctica el estudio de los sistemas trifásicos desequilibrados. También nos permite añadir una componente de continua a la señal de alterna, con lo que podemos simular condiciones reales de trabajo que con frecuencia nos podemos encontrar.

Esta fuente programable la 61704 de la casa Chroma, nos ha permitido realizar las comprobaciones experimentales con el aparato de medida SIMPELEC Desfase, de las medidas experimentales efectuadas con el SIMPELEC Simulador, obteniendo la concordancia de resultados esperada, ya que gracias a esta fuente se han podido ensayar todo tipo de condiciones de desequilibrios en el sistema.

A continuación se muestran una serie de fotografías en las que podemos observar la fuente de alimentación, y el equipo de medida SIMPELEC Desfase con un sistema en estudio.

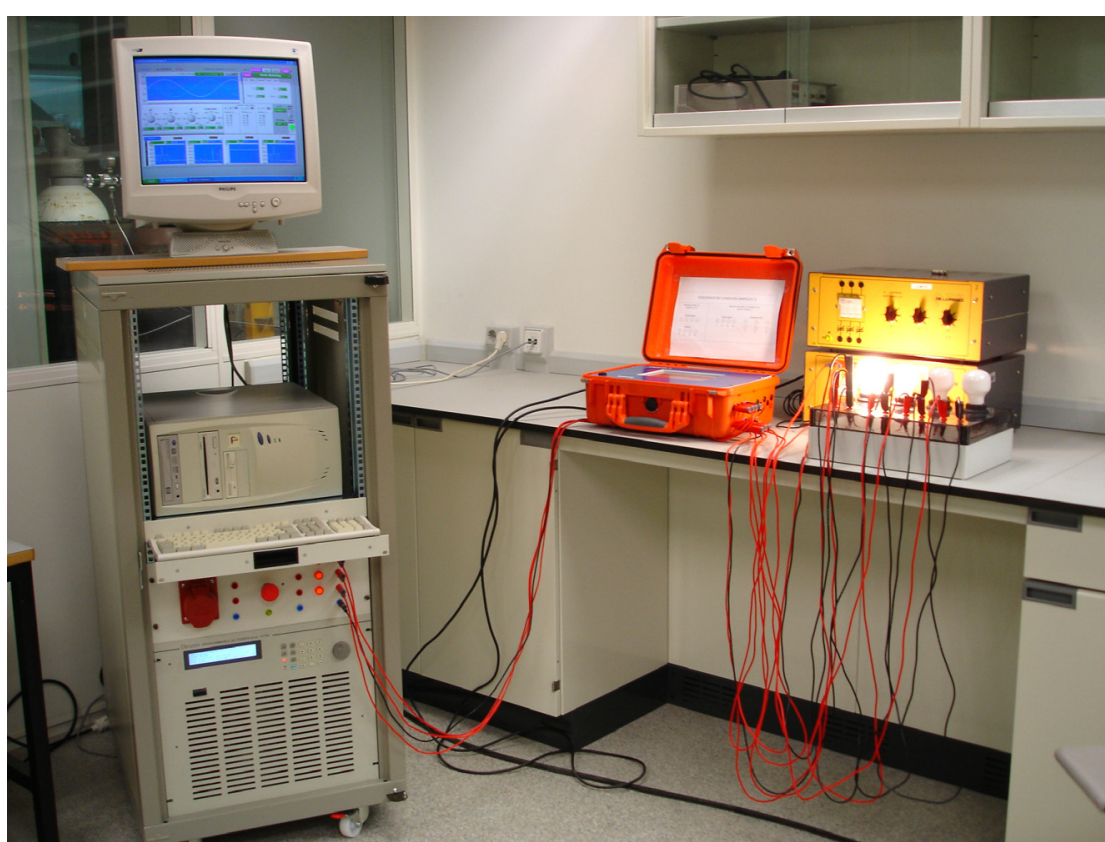

Figura V.127. Vista general de uno de los montajes. 


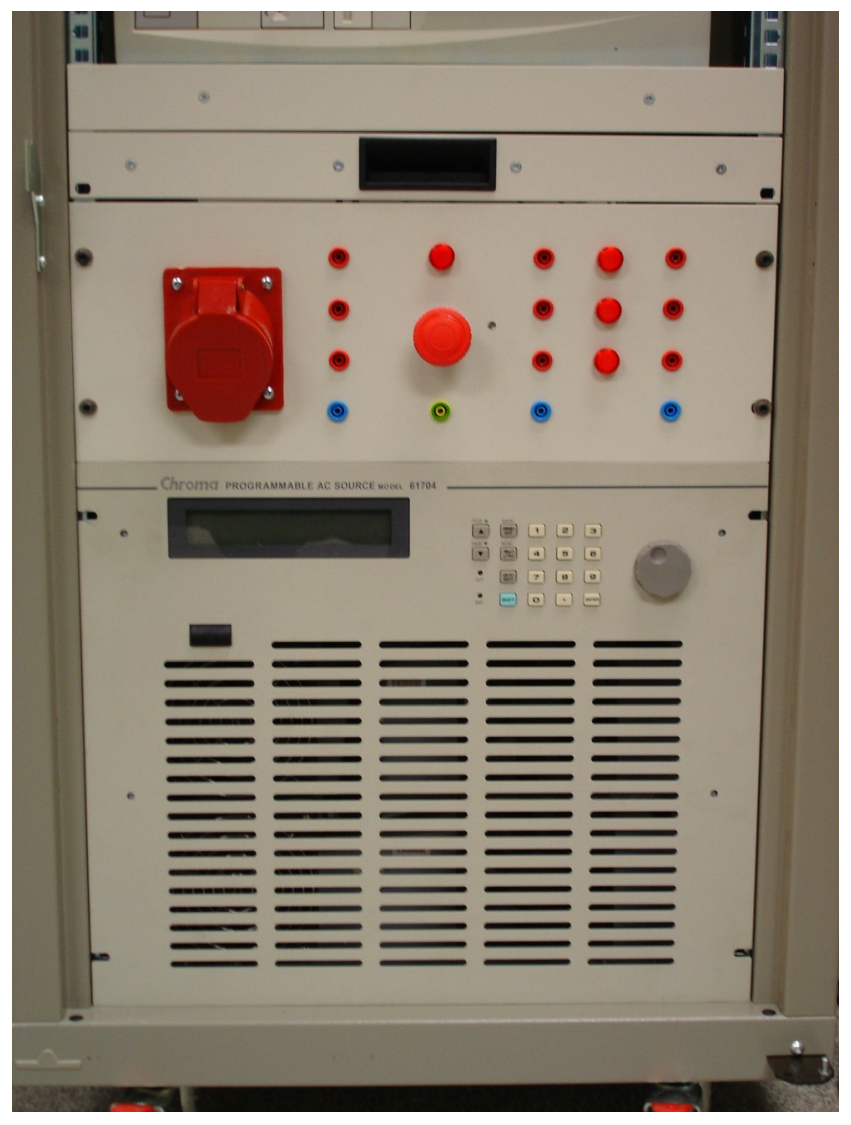

Figura V.128. Detalle de la fuente trifásica programable. 


\section{V.6.- Medidas experimentales obtenidas en el laboratorio.}

Se presentan a continuación una serie de medidas experimentales muy ilustrativas, llevadas a cabo en el laboratorio, para lo cual se ha usado el dispositivo de medida SIMPELEC, conjuntamente con la fuente trifásica conmutada programable descrita en al apartado anterior que nos ha permitido reproducir casos difíciles de ejecutar en instalaciones reales y que corroboran los resultados obtenidos y presentados por medio de las medidas experimentales obtenidas por la simulación mediante el SIMPELEC simulador.

1. Montaje con carga resistiva: conectamos una carga monofásica resistiva, (formada por dos estufas conectadas en paralelo), entre la fase $\mathrm{R}$ y el neutro, tal y como se puede apreciar en la figura V.129.

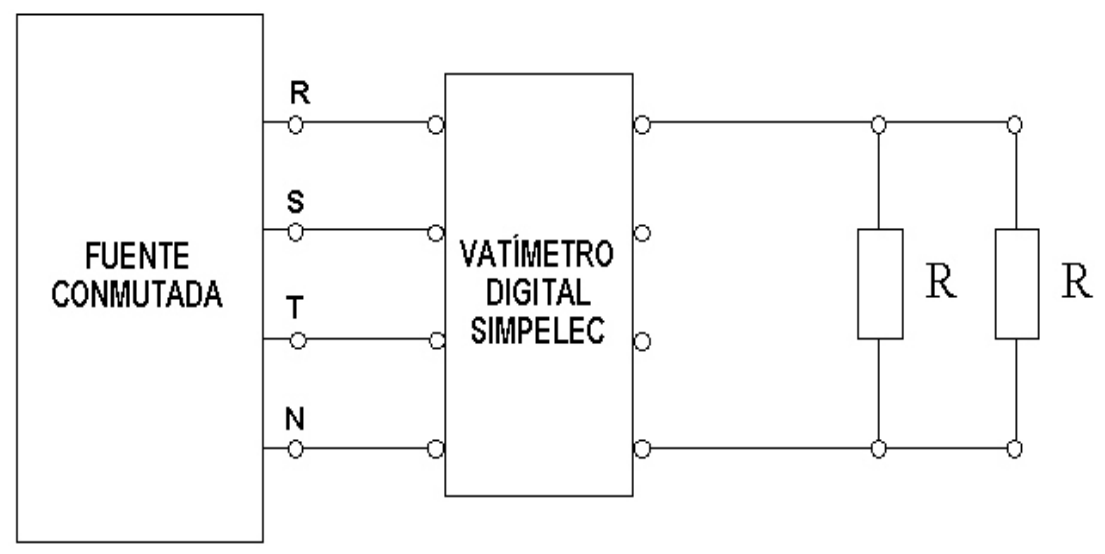

Figura V.129. Montaje con carga resistiva.

A continuación partimos de un sistema equilibrado en tensiones y analizamos el consumo de potencia de desfase (debida a los desequilibrios y la total), en función de los desequilibrios del sistema de alimentación, tal y como se muestra en la tabla V.53. 


\begin{tabular}{|c|c|c|c|c|c|}
\hline Casos & 0 & 1 & 2 & 3 & 4 \\
\hline $\mathrm{V}_{1}(\mathrm{~V})$ & $229,50_{\angle 0^{\circ}}$ & $229,45_{\angle 0^{\circ}}$ & $229,45_{\angle 0^{\circ}}$ & $229,45_{\angle 0^{\circ}}$ & $229,32_{\angle 0^{\circ}}$ \\
\hline$\overline{V_{2}(V)}$ & $229,89_{\angle-120^{\circ}}$ & $229,92_{\angle-120,04^{\circ}}$ & $229,92_{\angle-120,06^{\circ}}$ & $229,92_{\angle-120,08^{\circ}}$ & $229,91_{\angle-120,08^{\circ}}$ \\
\hline $\mathrm{V}_{3}(\mathrm{~V})$ & $230,16_{\angle 120^{\circ}}$ & $230,12_{\angle 120^{\circ}}$ & $230,12_{\angle 120^{\circ}}$ & $230,04_{\angle 120^{\circ}}$ & $230,04_{\angle 120^{\circ}}$ \\
\hline $\mathrm{I}_{1}(\mathrm{~A})$ & $3,066_{\angle 0^{\circ}}$ & $3,066_{\angle 0^{\circ}}$ & $3,066_{\angle 0^{\circ}}$ & $3,066_{\angle 0^{\circ}}$ & $3,066_{\angle 0^{\circ}}$ \\
\hline $\begin{array}{l}\mathrm{I}_{2}(\mathrm{~A}) \\
\mathrm{I}_{3}(\mathrm{~A}) \\
\end{array}$ & 0 & 0 & 0 & 0 & 0 \\
\hline $\begin{array}{c}\text { Potencia } \\
\text { Desfase } \\
\text { Reactancias } \\
\boldsymbol{Q}_{\boldsymbol{d r r}}(\boldsymbol{V A r}) \\
\end{array}$ & 1,96 & 1,96 & 1,95 & 1,93 & 1,91 \\
\hline $\begin{array}{c}\text { Potencia } \\
\text { Desfase } \\
\text { Desequilibrio } \\
\boldsymbol{Q}_{\text {dru }}(\boldsymbol{V A r})\end{array}$ & 1,74 & 0,11 & $-0,82$ & $-1,84$ & $-4,17$ \\
\hline $\begin{array}{l}\text { Potencia } \\
\text { Desfase } \\
\text { Total } \\
\boldsymbol{Q}_{\boldsymbol{d}}(\boldsymbol{V A r}) \\
\end{array}$ & 3,69 & 2,07 & 1,13 & 0,09 & $-2,26$ \\
\hline
\end{tabular}

Tabla V.53. Evolución potencias desfase, carga resistiva.

En la tabla V.53, observamos de una manera clara y evidente, como podemos modificar la potencia de desfase debida a los desequilibrios $\left(\mathrm{Q}_{\mathrm{dru}}\right)$, por medio de ligeras variaciones en los argumentos de las tensiones de alimentación. Se parte de un sistema equilibrado en tensiones (caso 0), y vemos como evoluciona la potencia de desfase debida a los desequilibrios y como esta modifica la potencia de desfase total del sistema, pudiendo anularse su valor o cambiar su carácter tal y como se ve llegando a ser capacitiva.

En las figuras V.130, V.131, V.132, V.133 y V.134, que se muestran a continuación se observan las pantallas de las potencias de desfase, obtenidas para cada uno de los casos anteriormente mostrados. 


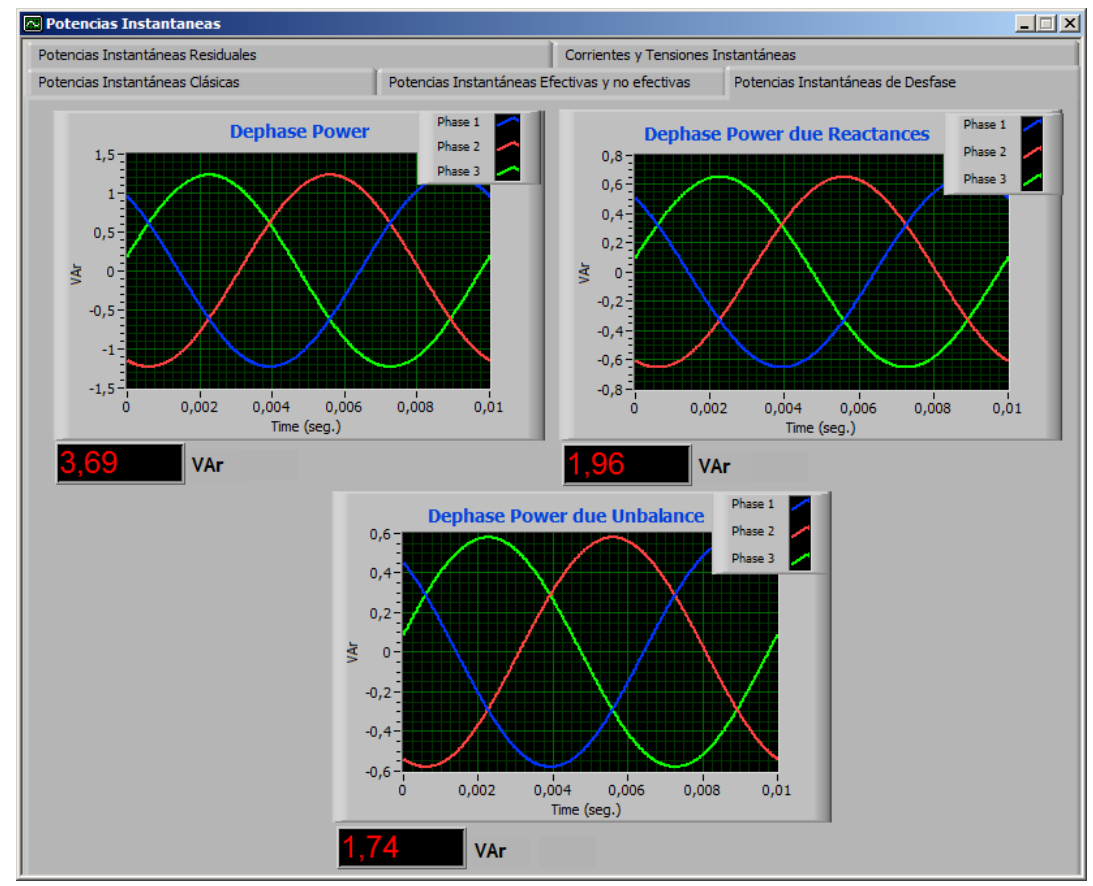

Figura V.130. Pantalla Potencias Desfase. Caso 0.

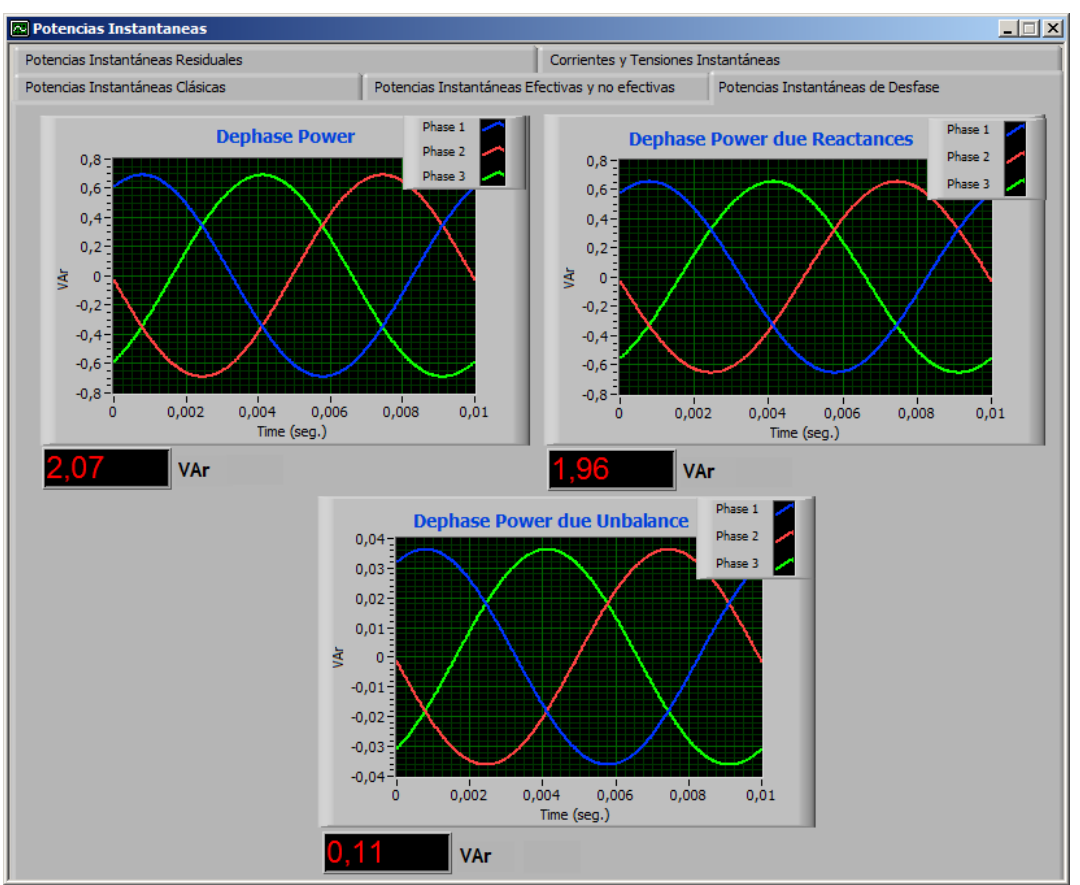

Figura V.131. Pantalla Potencias Desfase. Caso 1. 


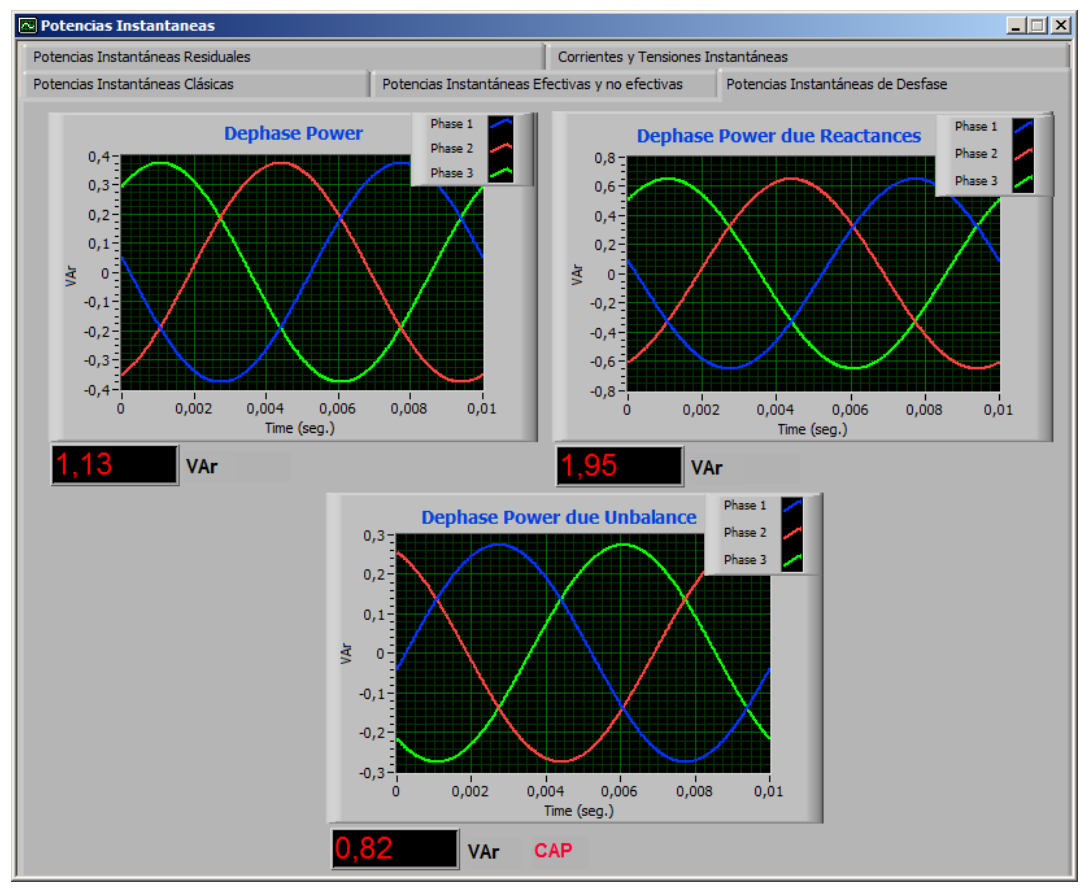

Figura V.132. Pantalla Potencias Desfase. Caso 2.

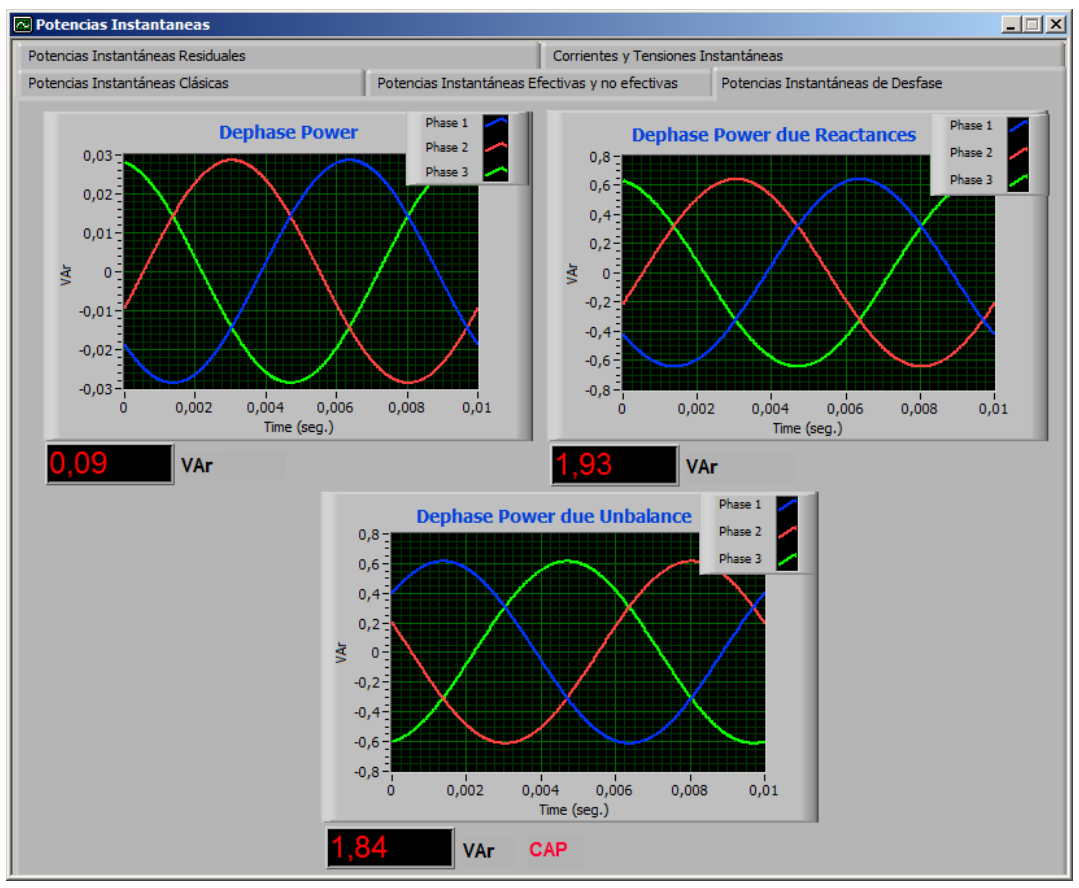

Figura V.133. Pantalla Potencias Desfase. Caso 3. 


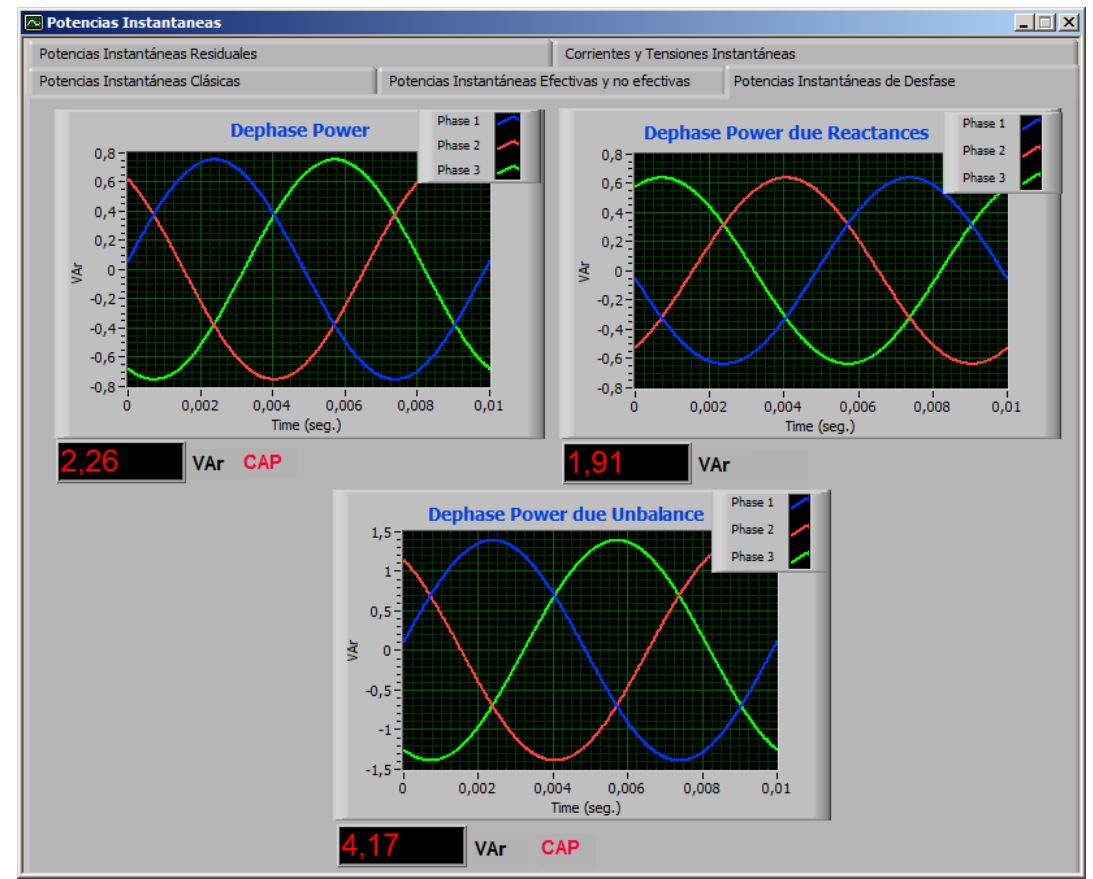

Figura V.134. Pantalla Potencias Desfase. Caso 4.

2. Montaje con carga capacitiva: ahora conectamos un condensador de $2 \mu \mathrm{F}$ al montaje anterior, estando este también entre la fase $\mathrm{R}$ y el neutro, tal y como se puede apreciar en la figura V.135.

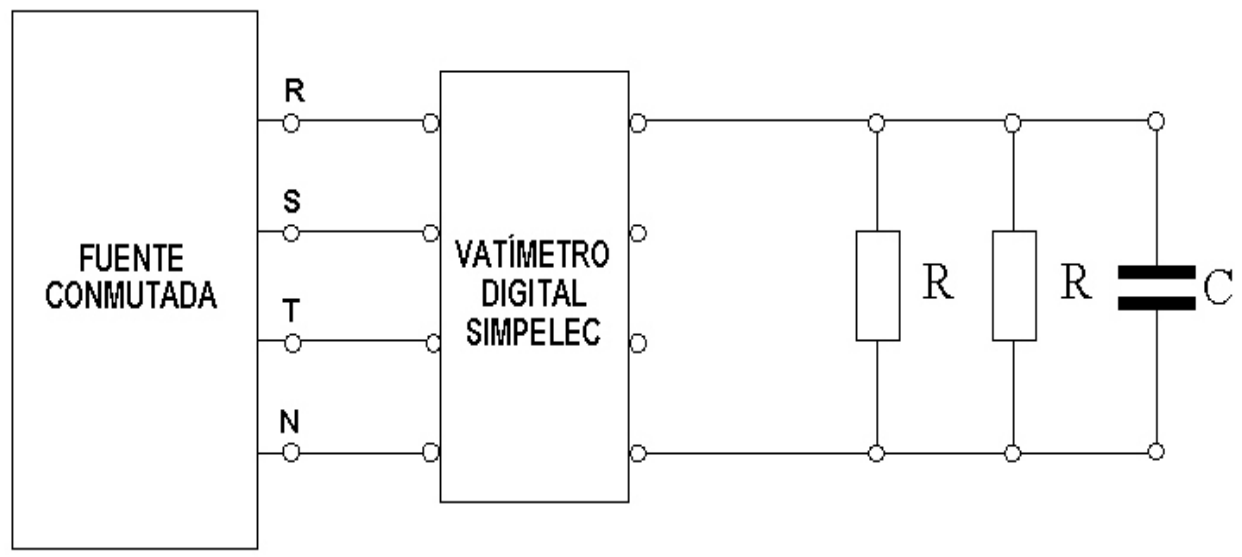

Figura V.135. Montaje con carga capacitiva. 
Como en el anterior montaje analizado, se muestra a continuación como evoluciona la potencia de desfase debida al desequilibrio $\left(Q_{d r u}\right)$, según vayamos desequilibrando el sistema de alimentación y cuyos resultados se muestran en la tabla V.54 que seguidamente mostramos.

\begin{tabular}{|c|c|c|c|c|}
\hline Casos & 0 & 1 & 2 & 3 \\
\hline$V_{1}(V)$ & $229,54_{\angle 0^{\circ}}$ & $229,48_{\angle 0^{\circ}}$ & $229,66_{\angle 0^{\circ}}$ & $229,52_{\angle 0^{\circ}}$ \\
\hline $\mathrm{V}_{2}(\mathrm{~V})$ & $229,81_{\angle-120^{\circ}}$ & $229,89_{\angle-118,1^{\circ}}$ & $230,0_{\angle-116^{\circ}}$ & $229,80_{\angle-115,2^{\circ}}$ \\
\hline$V_{3}(V)$ & $230,04_{\angle 120^{\circ}}$ & $229,99_{\angle 120^{\circ}}$ & $230,13_{\angle 117^{\circ}}$ & $229,99_{\angle 115,6^{\circ}}$ \\
\hline$I_{1}(A)$ & $3,071_{\angle 2,48^{\circ}}$ & $3,070_{\angle 2,48^{\circ}}$ & $3,068_{\angle 2,48^{\circ}}$ & $3,070_{\angle 2,48^{\circ}}$ \\
\hline $\begin{array}{l}\mathrm{I}_{2}(\mathrm{~A}) \\
\mathrm{I}_{3}(\mathrm{~A}) \\
\end{array}$ & 0 & 0 & 0 & 0 \\
\hline $\begin{array}{c}\text { Potencia } \\
\text { Desfase } \\
\text { Reactancias } \\
\boldsymbol{Q}_{\text {drr }}(\boldsymbol{V A r})\end{array}$ & $-30,58$ & $-30,60$ & $-30,44$ & - 30,54 \\
\hline $\begin{array}{c}\text { Potencia } \\
\text { Desfase } \\
\text { Desequilibrio } \\
\boldsymbol{Q}_{d r u}(\boldsymbol{V A r}) \\
\end{array}$ & 1,71 & 9,27 & 30,22 & 39,32 \\
\hline $\begin{array}{l}\text { Potencia } \\
\text { Desfase } \\
\text { Total } \\
\boldsymbol{Q}_{\boldsymbol{d}}(\boldsymbol{V A r})\end{array}$ & - 28,87 & $-21,33$ & $-0,22$ & 8,78 \\
\hline
\end{tabular}

Tabla V.54. Evolución potencias desfase, carga capacitiva.

Es de remarcar, a raíz de la observación de la tabla V.54 que partiendo de un sistema equilibrado (caso 0 ) como por medio del desequilibrio inducido en el sistema de alimentación se consigue una potencia de desfase de carácter inductivo, que en función del mismo anula el carácter capacitivo de la carga y se llega a que la potencia de desfase total del sistema sea inductiva.

En las figuras V.136, V.137, V.138 y V.139, que se muestran a continuación se observan las pantallas de las potencias de desfase, obtenidas para cada uno de los casos anteriormente mostrados. 


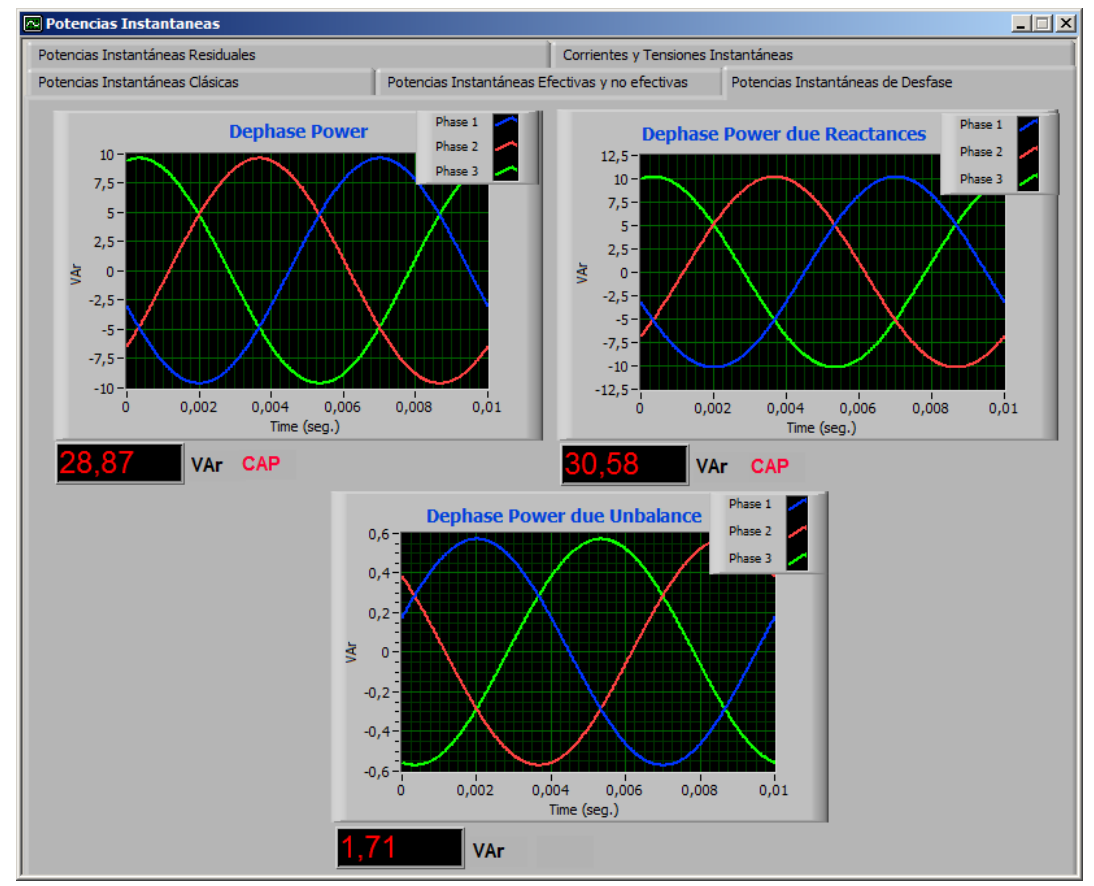

Figura V.136. Pantalla Potencias Desfase. Caso 0.

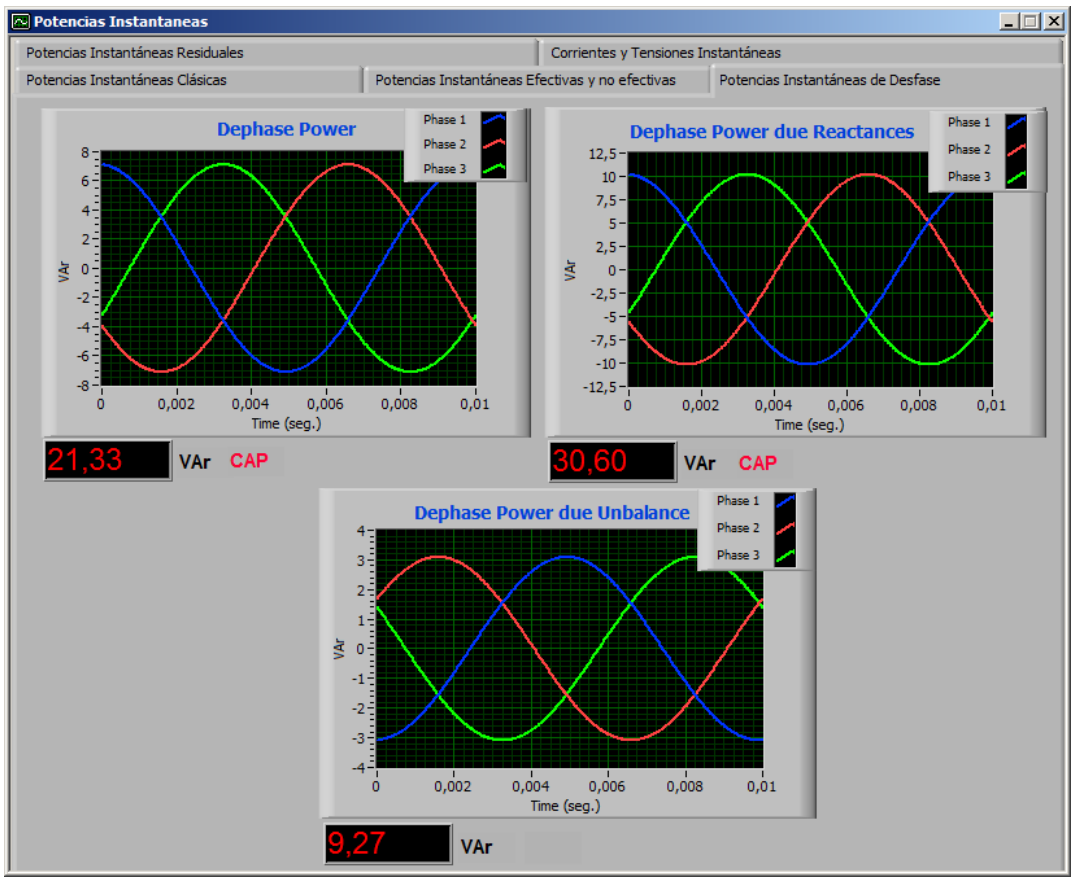

Figura V.137. Pantalla Potencias Desfase. Caso 1. 


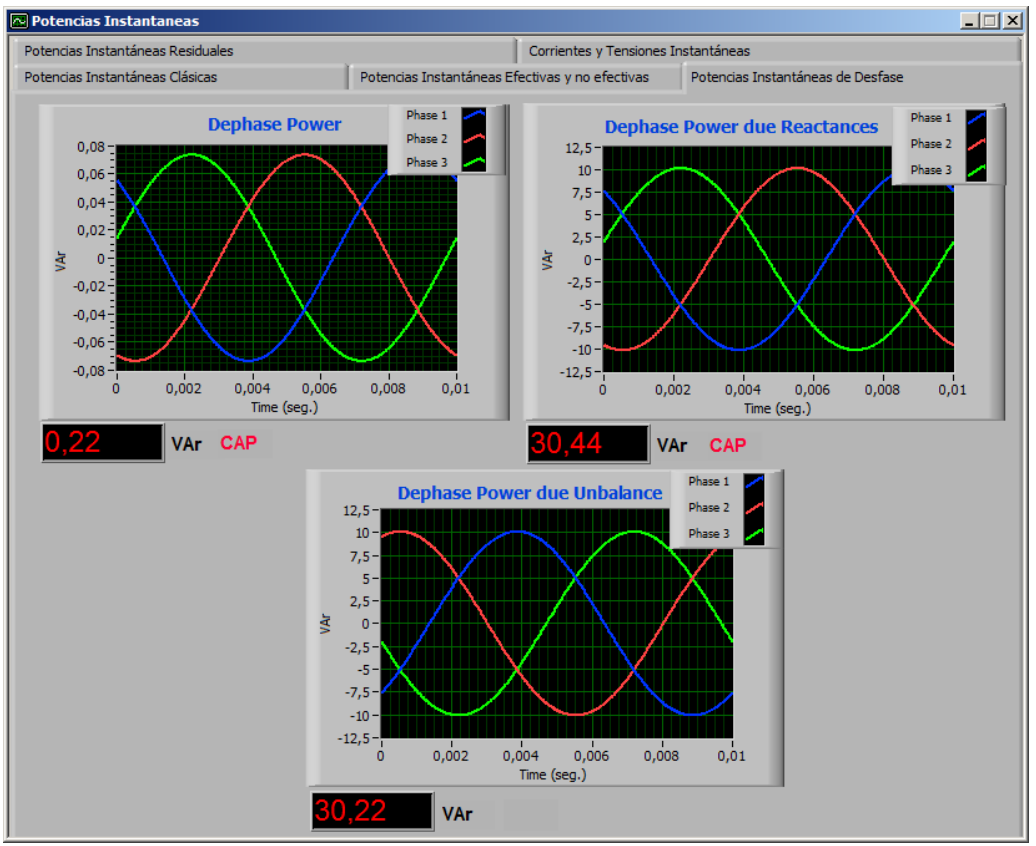

Figura V.138. Pantalla Potencias Desfase. Caso 2.

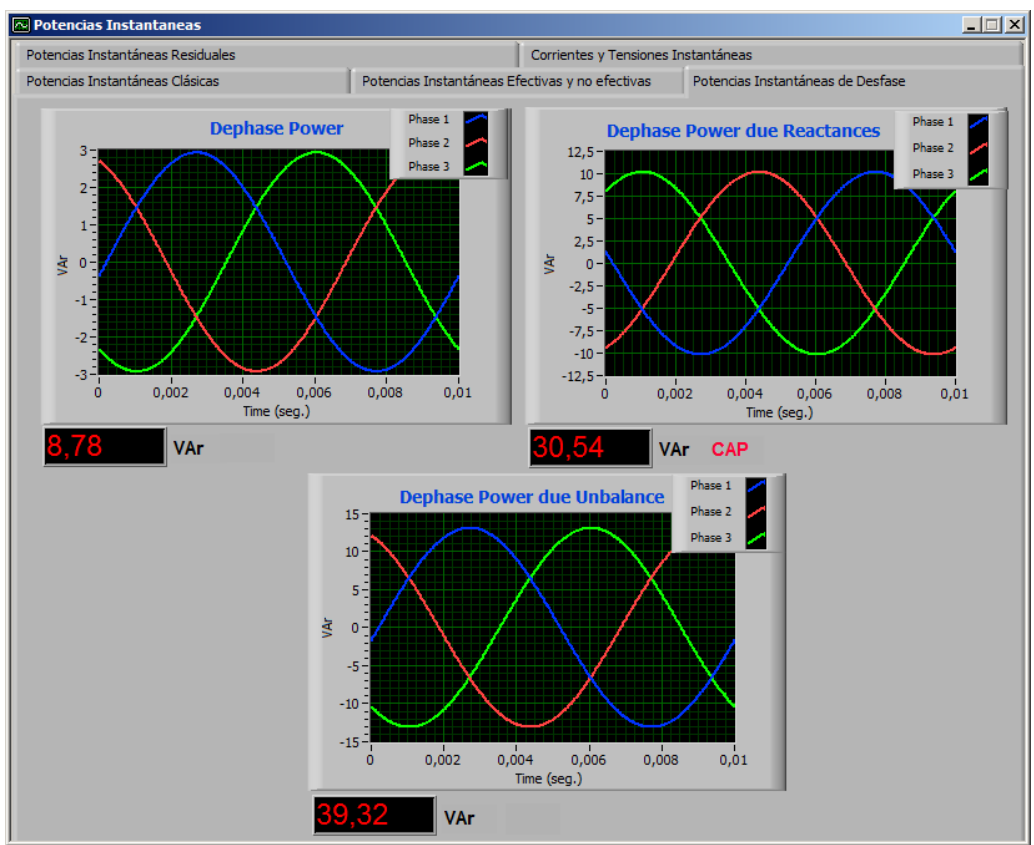

Figura V.139. Pantalla Potencias Desfase. Caso 3. 


\section{V.7.- Conclusiones.}

En este capítulo, se ha mostrado la presencia del fenómeno energético de la potencia de desfase debida al desequilibrio, en sistemas desequilibrados que alimentan cargas resistivas, inductivas y capacitivas.

La comprobación experimental se ha llevado a cabo mediante dos caminos, en uno de ellos se han efectuado medidas virtuales mediante el SIMPELEC Simulador, y en el otro se han hecho uso de medidas reales registradas mediante el instrumento de medida SIMPELEC Desfase en diversas instalaciones industriales y residenciales así como en el laboratorio.

Mediante el uso del SIMPELEC Simulador, hemos provocado desequilibrios muy grandes en los sistemas que se han estudiado, y que son difícilmente reproducibles en instalaciones reales (pero si han podido ser efectuados en el laboratorio del GINTEC, mediante el uso de la fuente trifásica programable). Estos desequilibrios fueron conseguidos en base a modificaciones de los argumentos de las tensiones y a los módulos de las mismas, observándose los siguientes patrones:

- Desequilibrios provocados mediante la suma de ángulos positivos en los argumentos de las fases descargadas, con independencia del sistema estudiado (resistivo, inductivo o capacitivo), dan lugar a la presencia de potencia de desfase debida al desequilibrio de carácter inductivo, mientras que si el desequilibrio se lleva a cabo por medio de la suma de ángulos negativos, la potencia de desfase debida al desequilibrio posee un carácter capacitivo.

- Desequilibrios en sistemas inductivos puros, por medio de la modificación de los argumentos de las fases con independencia de como se produzca el mismo, conllevan siempre la presencia de potencia de desfase debida al desequilibrio de carácter inductivo, si el desequilibrio se lleva a cabo en un sistema capacitivo puro, la potencia de desfase debida al desequilibrio tiene carácter capacitivo. 
- Desequilibrios en sistemas inductivos puros, provocados por aumentos de tensión en las fases descargadas, producen potencia de desfase debida al desequilibrio de carácter capacitivo, mientras que si lo que se hace es disminuir la tensión de las fases descargadas la potencia de desfase debida al desequilibrio posee un carácter inductivo.

- Desequilibrios en sistemas inductivos puros, provocados por aumentos de tensión en la fase cargada provoca la presencia de potencia de desfase debida al desequilibrio de carácter inductivo, mientras que si lo que se realiza es disminuir la tensión de la fase cargada, la potencia de desfase debida al desequilibrio pasa a ser capacitiva.

- Desequilibrios en sistemas capacitivos puros, provocados por aumentos de tensión en la fase cargada provoca la presencia de potencia de desfase debida al desequilibrio de carácter capacitivo, mientras que si lo que se realiza es disminuir la tensión de la fase cargada, la potencia de desfase debida al desequilibrio pasa a ser inductiva.

De las medidas reales registradas, en los centros de transformación industriales y comerciales, también se ha puesto de manifiesto la presencia de potencia de desfase debida al desequilibrio.

- En las medidas efectuadas en instalaciones industriales, se han encontrado casos de potencia de desfase debida al desequilibrio con carácter inductivo y capacitivo, siendo los valores de la potencia de desfase debida al desequilibrio del orden del $3 \%$ de la potencia de desfase debida a las reactancias del sistema, lo que provoca en algunos casos una disminución y en otros un aumento de la potencia de desfase total del sistema.

- En las medidas efectuadas en instalaciones residenciales, y en los casos que se evidenciaron unos desequilibrios mayores del 6\%, observamos unos porcentajes superiores al $12 \%$ de potencia de desfase debida al desequilibrio en relación con la potencia de desfase debida a las reactancias del sistema, lo que influye claramente en la potencia de desfase total del sistema. 



\section{CAPÍTULO VI}

CONCLUSIONES Y FUTURAS

REALIZACIONES. 



\section{VI.- Conclusiones y futuras realizaciones.}

\section{VI.1.- Conclusiones.}

A lo largo de los diversos capítulos de esta Tesis, se ha desarrollado el concepto de la potencia de desfase, separando que parte del fenómeno corresponde a las reactancias y el que corresponde al desequilibrio. Esto último constituye la principal aportación de esta Tesis dado que no era conocido, para ello se tomo como base la Teoría Unificadora de la Potencia Eléctrica, que luego se ha usado para realizar medidas experimentales, de todo ello podemos extraer las siguientes conclusiones:

- La descripción, identificación y cuantificación del fenómeno físico del desfase asociado con los desequilibrios, hasta ahora desconocido por la comunidad científica.

- La definición de la potencia de desfase basándose en la Teoría Unificadora, que coincide para esta magnitud con la Teoría de Emanuel contemplada en la IEEE Std. 1459/2000.

- Existencia de dos flujos de potencia de desfase, ambos provocados por los desfases entre la tensión y la corriente, que son debidos a:

- Presencia de reactancias en el sistema.

- Presencia de desequilibrios en el sistema.

- La Potencia de desfase, se muestra por medio de tres flujos sinusoidales y equilibrados, que son provocados por las corrientes $y$ tensiones de secuencia directa y de frecuencia fundamental, independientemente de que el sistema sea lineal o no, y que el sistema de alimentación se halle equilibrado. 
- Se ha puesto de manifiesto la presencia de potencia de desfase debida al desequilibrio, con carácter capacitivo e inductivo, incluso en elementos resistivos, siempre que exista desfase entre las corrientes y las tensiones de secuencia directa y frecuencia fundamental.

- Se han comprobado comportamientos de refuerzo o de compensación de los caracteres capacitivo e inductivo de los elementos reactivos, provocados por los desequilibrios del sistema.

- Incrementos iguales de potencia de desfase debida al desequilibrio, pero de carácter contrario (inductivo o capacitivo) según como se provoque el desequilibrio en el sistema en estudio, y de forma que el grado de desequilibrio sea el mismo.

- Importancia del fenómeno del desfase debido al desequilibrio en las redes eléctricas reales, ya que se ha comprobado en instalaciones industriales que con desequilibrios en el sistema de generación cercanos al $6 \%$, esto conlleva que la potencia de desfase producida por el desequilibrio sea del orden del $3 \%$, del provocado por la potencia de desfase debida a las reactancias presentes en el sistema, y en instalaciones residenciales y con desequilibrios superiores al $6 \%$, se han encontrado potencias de desfase debidas al desequilibrio superiores al $12 \%$ de la debida a las reactancias del sistema. 


\section{VI.2.- Futuras realizaciones.}

En esta Tesis, se ha tratado de dar una visión general de la potencia de desfase, incluyendo el fenómeno del desfase debido a los desequilibrios, y la importancia que este puede tener, y se han llevado a cabo las comprobaciones experimentales por medio del SIMPELEC Simulador, en un futuro inmediato, caben abordar las siguientes realizaciones:

- Seguir el desarrollo del SIMPELEC Simulador, de manera que su tamaño se reduzca considerablemente, y sobre todo el poder trabajar en régimen transitorio para poder proceder al análisis de huecos de tensión, que se dan fundamentalmente en las centrales eólicas, y ver como influyen en la potencia reactiva de las mismas.

- La posible implantación de las expresiones usadas ahora en el SIMPELEC Desfase, basadas en la Teoría Unificadora, en un DSP, a la hora de poder desarrollar un dispositivo muy portátil.

- Aplicar el conocimiento de los incrementos de reactiva, para obtener dispositivos de generación de reactiva para diversas aplicaciones. 



\section{CAPÍTULO VII}

BIBLIOGRAFÍA. 

Bibliografía.

[1] AENOR.

UNE-EN 62053-23. Equipos de medida de la energía eléctrica (c.a.). Requisitos particulares. Parte 23: Contadores estáticos de energía reactiva (clases 2 y 3 ).

AENOR. Noviembre 2003.

[2] Akagi, H.; Ogasawara, S.; Kim, H.

The theory of instantaneous power in three-phase four-wire systems and its applications.

Electrical Engineering in Japan, Vol. 135, nº 3, 2001, pp. 74-86.

[3] Akagi, H.; Fujita H.

The unified power quality conditioner: the integration of series and shunt active filters.

IEEE Trans. on Power Electronics, Vol. 13, n 2, March 1998, pp. 315-322.

[4] Akagi, H.; Fujita L.

A new power line conditioner for harmonic compensation in power systems.

IEEE Trans. on Power Delivery, Vol. 10, no 3, July 1995, pp. 1570-1575.

[5] Akagi, H.; Nabae, A.

The $p-q$ theory three-phase systems under non-sinusoidal conditions.

ETEP, n01, 1993, pp. 27-31.

[6] Akagi, H.; Kazanawa, Y.; Nabae, A.

Instantaneous reactive compensators comprising switching devices without energy storage components.

IEEE Trans. On Indust. Applications, Vol. 20, no 3, May/June 1984, pp. 625-630

[7] Angrisani, L.; D’Apuzzo, M.; D’Arco, M.

A new method for power measurement in digital wireless communication systems.

IEEE Trans. on Instrumentation and Measurement, Vol. 52, no 4, August 2003, pp. 1097-1106. 
[8] Angrisani, L.; Ferrigno, L.

Reducing the uncertainty in real-time impedance measurement. Measurement, Vol. 31, 2001, pp. 307-315.

[9] Angrisani, L.; Daponte, P.; D'Apuzzo, M.; Pietrosanto, A.

A VXI power quality analyser implementing a wavelet transformbased measurement procedure.

Measurement, Vol. 26, 1999, pp. 91-102.

[10] Angrisani, L.; Baccigalupi, A.; Pietrosanto, A.

A DSP based VXI instrument for impedance measurement.

IEEE Instrumentation and Measurement Technology Conference, Belgium 1996, pp. 38-43.

[11] Angrisani, L.; Baccigalupi, A.; Pietrosanto, A.

A digital signal-processing instrument for impedance measurement.

IEEE Trans. on Instrumentation and Measurement, Vol. 45, no 6, December 1996, pp. 930-934

[12] Arroyo J.; Gomollón, J.; Mayo, J.; Nievas, J.; Pastor, A.

Equipos para el análisis del estado de las redes de energía eléctrica.

Automática e Instrumentación, Marzo 1987, pp. 109-120.

[13] Arseneau R.; Filipski, P.S.; Zelle, J.

An improved three-phase digital recorder system for calibrating power instrumentation.

IEEE Transactions on Instrumentation and Measurement, Vol. 46, n⿳ 2, April 1997, pp.399-402.

[14] Arseneau, R.

The performance of demand meters under varying load conditions.

IEEE Transactions on Power Delivery, Vol. 8, no 4, October 1993, pp.1708-1711. 
[15] Arseneau R.; Filipski, P.S.; Zelle, J.

A VA-meter error analysis.

IEEE Transactions on Power Delivery, Vol. 6, no 4, October 1991, pp.1769-1773.

[16] Arseneau R.; Filipski, P.S.

Application of a three phase nonsinusoidal calibration system for testing energy and demand meters under simulated field conditions.

IEEE Transactions on Power Delivery, Vol. 3, n 3, July 1988, pp.874-879.

[17] Aumala, O.

Fundamentals and trends of digital measurement.

Measurement, Vol. 26, 1999, pp. 45-54.

[18] Bishop, R. H.

LabView Student Edition 6i.

Prentice Hall, New Jersey 2001.

[19] Bollen, M.H.; Goossens, P.; Robert, A.

Assessment of voltage dips in HV-networks: deduction of complex voltages from the measured RMS voltages.

IEEE Transactions on Power Delivery, Vol. 19, no 2, April 2004, pp.783790.

[20] Bollen, M.H.

What is the power quality?.

Electric Power System Research, Vol. 66, 2003, pp. 5-14.

[21] Bollen, M.H.

Algorithms for characterizing measured three phase unbalanced voltage dips.

IEEE Transactions on Power Delivery, Vol. 18, no 3, Julyl 2003, pp. 937944.

[22] Bollen, M.H.

Definitions of voltage unbalance.

IEEE Power Engineering Review, November 2002, pp.49-50. 
[23] Budeanu, C. I.

Puissances reactives et fictives.

Institut Romain de L'Energie, Bucharest 1927.

[24] Chugani, M. L.; Samant, A. R.; Cerna, M.

\section{LabView. Signal Processing.}

Prentice Hall, New Jersey 1998.

[25] Cohen, J.; León, F.; Hernández, L.

Physical time domain representation of powers in linear and nonlinear electrical circuits.

IEEE Transactions on Power Delivery, Vol. 14, no 4, October 1999, pp.1240-1249.

[26] Cox, M.D.; Williams, T.B.

Induction varhour and solid-state varhour meters performances on nonlinear loads.

IEEE Transactions on Power Delivery, Vol. 5, no 4, November 1990, pp.1678-1686.

[27] Cristaldi, L.; Ferrero, A.; Ottoboni, R.

A distributed system for electric power quality measurement.

IEEE Transactions on Instrumentation and Measurement, Vol. 51, no 4, August 2002, pp.776-781.

[28] Cristaldi, L.; Lazzaroni, M.; Salicone, S.

A calibration procedure for a digital instrument for electric power quality measurement.

IEEE Transactions on Instrumentation and Measurement, Vol. 51, no 4, August 2002, pp.716-722.

[29] Cristaldi, L.; Ferrero, A.

A method and related digital instrument for the measurement of the electric power quality.

IEEE Transactions on Power Delivery, Vol. 10, no 3, July 1995, pp.11831189. 
[30] Cristaldi, L.; Ferrero, A.

Current decomposition in asymmetrical, unbalanced three-phase systems under nonsinusoidal conditions.

IEEE Transactions on Instrumentation and Measurement, Vol. 43, no 1, February 1994, pp.63-68.

[31] Cristaldi, L.; Ferrero, A.; Ottoboni, R.

Measuring equipment for the electric quantities at the terminals of an inverter-fed induction motor.

IEEE Transactions on Instrumentation and Measurement, Vol. 45, no 2, April 1996, pp.449-452

[32] Czarnecki, L.S.

Physical Interpretation of the Reactive Power in Terms of the CPC Power Theory.

Electrical Power Quality and Utilisation, Journal, Vol. XIII, no 1, 2007, pp.89-95.

[33] Czarnecki, L.S.

On some misinterpretations of the instantaneous reactive power p-q theory.

IEEE Transactions on Power Delivery, Vol. 19, n 3, May. 2004, pp.828836.

[34] Czarnecki, L.S.

Comparison of instantaneous reactive power $p-q$ theory with theory of the current's physical components.

Electrical Engineering, Vol. 85, 2002, pp. 21-28.

[35] Czarnecki, L.S.

Energy flow and power phenomena in electrical circuits illusions and reality.

Electrical Engineering, Vol. 82, 2000, pp. 119-126.

[36] Czarnecki, L.S.

Phantom load: a simulator of non periodic currents.

IEEE Power Engineering Society Summer Meeting, Vol. 2, 2000, pp. 988993. 
[37] Czarnecki, L.S.

Budeanu and Fryze: two frameworks for interpreting power properties of circuits with nonsinusoidal voltages and currents.

Electrical Engineering, Vol. 80, 1997, pp. 359-367.

[38] Czarnecki, L.S.

Comments on active power flow and energy accounts in electrical systems with nonsinusoidal waveforms and asymmetry.

IEEE Transactions on Power Delivery, Vol. 11, n 3, July 1996, pp.12441250.

[39] Czarnecki, L.S.; Staroszcyk, Z.

On line measurement of equivalent parameters for harmonic frequencies of a power distribution system and load.

IEEE Transactions on Instrumentation and Measurement, Vol. 45, no 2, April 1996, pp.467-472.

[40] Czarnecki, L.S.

Equivalent circuits of unbalanced loads supplied with symmetrical and asymmetrical voltage and their identification.

Electrical Engineering, Vol. 78, 1995, pp. 165-168.

[41] Czarnecki, L.S.

Power related phenomena in three-phase unbalanced systems.

IEEE Transactions on Power Delivery, Vol. 10, nº 3, July 1995, pp.11681176.

[42] Czarnecki, L.S.; Min, S.; Chen, G.

Adaptative balancing compensator.

IEEE Transactions on Power Delivery, Vol. 10, no 3, July 1995, pp.16631669.

[43] Czarnecki, L.S.

Misinterpretations of some power properties of electric circuits.

IEEE Trans. on Power Delivery, Vol. 9, no 4, October 1994, pp. 17601769. 
[44] Czarnecki, L.S.

Physical reasons of currents RMS value increase in power systems with nonsinusoidal voltage.

IEEE Transactions on Power Delivery, Vol. 8, no 1, January 1993, pp. 437447.

[45] Czarnecki, L.S.

Minimisation of unbalanced and reactive currents in three-phase asymmetrical circuits with nonsinusoidal voltage.

IEEE Proceedings B, Vol. 139, no 4, July 1992, pp. 347-354.

[46] Czarnecki, L.S.

Distortion power in systems with nonsinusoidal voltage.

IEEE Proceedings B, Vol. 139, nº 3, May 1992, pp. 276-280.

[47] Czarnecki, L.S.

Scatterred and reactive current, voltage, and power in circuits with nonsinusoidal waveforms and their compensation.

IEEE Transactions on Instrumentation and Measurement, Vol. 40, n० 3, June 1991, pp. 563-567.

[48] Czarnecki, L.S.

A time-domain approach to reactive current minimization in nonsinusoidal situations.

IEEE Transactions on Instrumentation and Measurement, Vol. 39, no 5, October 1990, pp. 698-703.

[49] Czarnecki, L.S.; Swietlicki, T.

Powers in nonsinusoidal networks: their interpretation, analysis, and measurement.

IEEE Transactions on Instrumentation and Measurement, Vol. 39, no 2, April 1990, pp. 340-345.

[50] Czarnecki, L.S.

Reactive and unbalanced currents compensation in three-phase asymmetrical circuits under nonsinusoidal conditions.

IEEE Transactions on Instrumentation and Measurement, Vol. 38, no 3, June 1989, pp. 754-759. 
[51] Czarnecki, L.S.

Active, reactive, and scattered current in circuits with nonperiodic voltage of a finite energy.

IEEE Transactions on Instrumentation and Measurement, Vol. 37, no 3, September 1988, pp. 398-402.

[52] Czarnecki, L.S.

Ortogonal Decomposition of the Currents in a 3-Phase Nonlinear Asymmetrical Circuit with a Nonsinusoidal Voltage Source.

IEEE Transactions on Instrumentation and Measurement, Vol. 37, $\mathrm{n}^{0} 1$, March 1988, pp. 30-34.

[53] Czarnecki, L.S.

What is wrong with the Budeanu concept of Reactive and Distortion Power and why it should be abandoned.

IEEE Transactions on Instrumentation and Measurement, Vol. 36, no 3, September 1987, pp. 834-837.

[54] Dai, X.; Liu, G.; Gretsch, R.

Generalized theory of instantaneous reactive quantity for multiphase power system.

IEEE Transactions on Power Delivery, Vol. 19, no 3, July 2004, pp. 965972.

[55] De Capua, C.; Landi, C.

Quality assessment of electrical drives with strongly deformed supply waveform.

Measurement, Vol. 30, 2001, pp. 269-278.

[56] Dell'Aquilla, M.; Vito Giuseppe, M.; Zanchetta, P.

New power-quality assessment criteria for supply systems under unbalanced and nonsinusoidal conditions.

IEEE Transactions on Power Delivery, Vol. 19, no 3, July 2004, pp.12841290. 
[57] Depenbrock, M., Staudt, V. ; Wrede, H.

A theoretical investigation of original and modified instantaneous power theory applied to four-wire systems.

IEEE Trans. on Industry Applications, Vol. 39, no 4, July 2003, pp.11601167.

[58] Depenbrock, M. ; M., Staudt, V. ; Wrede, H.

A concise assessment of original and modified instantaneous power theory applied to four-wire systems.

PCC-Osaka 2002, pp.60-67.

[59] Depenbrock, M. ; M., Staudt, V.

The FBD-Method as tool for compensating total non active currents.

$8^{\circ}$ ICHQP 1998, pp. 320-324.

[60] Depenbrock, M.

The FBD-Method, a generally applicable tool for analyzing power relations.

IEEE Transactions on Power Delivery, Vol. 8, no 2, May 1993, pp. 381387.

[61] Djokic, B.; Bosnjakovic, P.

A high accuracy reactive power and reactive energy meter.

Electrical Engineering, Vol. 81, 1998, pp. 43-44.

[62] Djokic, B.; Bosnjakovic, P.

Two methods for improved measurements of reactive power and reactive energy insensitive to frequency variations.

IEEE Transactions on Instrumentation and Measurement, Vol. 47, no 1 , February 1998, pp. 215-218.

[63] Djokic, B.; Bosnjakovic, P.

New method for reactive power and energy measurement.

IEEE Transactions on Instrumentation and Measurement, Vol. 41, no 2, April 1992, pp. 280-285. 
[64] Domijan, A.; Embriz-Santander, E.; Lamer, G.; Stiles, C.; Williams, C.

Watthour meter accuracy under controlled unbalanced harmonic voltage and current conditions.

IEEE Transactions on Power Delivery, Vol. 11 no 1, January 1996, pp. 6472.

[65] Driesen, J.; Van Craenenbroeck, T.

The registration of harmonic power by analog and digital power meters.

IEEE Transactions on Instrumentation and Measurement, Vol. 47, $\mathrm{n}^{\circ} 1$, February 1998, pp. 195-198.

[66] Emanuel, A.

Addendum to the Apparent Power Concept and the IEEE Standard 1549-2000.

IEEE Transactions on Power Delivery, Vol. 20, n 2, April 2005, pp. 885886.

[67] Emanuel, A.

Clarke's Alpha, Beta and Zero Components: A Possible Approach for the Conceptual Design of Instrumentation Compatible with IEEE Standard 1459-2000.

IMTC 2004- Instrumentation and Measurement Technology Conference, Como, Italy, 18-20 May 2004, pp. 1614-1619.

[68] Emanuel, A.

Summary of IEEE Standard 1459: definitions for the measurement of electric power quantities under sinusoidal, nonsinusoidal, balanced or unbalanced conditions.

IEEE Transactions on Industry Applications, Vol. 40, no 3, May/June 2004, pp. 869-876.

[69] Emanuel, A.

IEEE Standard 1459: Questions and Future Direction- - Quo Vadis?.

Proc. IEEE Power Engineering Society General Meeting, 13-17 July 2003, Vol. 1, pp. 82-86. 
[70] Emanuel, A.

Introduction to IEEE Trial - Use Standard 1459.

Proc. IEEE Power Engineering Society Summer Meeting, 2002, Vol. 3, pp. 1674-1676.

[71] Emanuel, A.

Nonactive power due to randommess.

IEEE Power Engineering Review, August 2001, pp. 43-45.

[72] Emanuel, A.; Cakareski, Z.

On the physical meaning of nonactive powers in three phase systems.

IEEE Power Engineering Review, July 1999, pp. 46-47.

[73] Emanuel, A.

Apparent power definitions for three-phase systems.

IEEE Transactions on Power Delivery, Vol. 14 no 3, July 1999, pp. 767772.

[74] Emanuel, A.

The Buchholz-Goodhue apparent power definition: the practical approach for nonsinusoidal and unbalanced systems.

IEEE Transactions on Power Delivery, Vol. 13 no 2, April 1998, pp. 344350.

[75] Emanuel, A.

Actual measurements of apparent power and its components at low and medium voltage buses.

ETEP, Vol.4, no 5, September/October 1994, pp. 371-380.

[76] Emanuel, A.

On the definition of power factor and apparent power in unbalanced polyphase circuits with sinusoidal voltage and currents.

IEEE Transactions on Power Delivery, Vol. 8 n 3, July 1993, pp. 841-852.

[77] Emanuel, A.

Apparent and reactive powers in three-phase systems: In search of a physical meaning and a better resolution.

ETEP, Vol.3, no 1, January/February 1993, pp. 7-14. 
[78] Emanuel, A.

Powers in nonsinusoidal situations. A review of definitions and physical meaning.

IEEE Transactions on Power Delivery, Vol. 5 no 3, July 1990, pp. 13771389.

[79] Enslin, J. H. R.; Van Wyk, J. D.

Measurement and compensation of fictitious power under nonsinusoidal voltage and current conditions.

IEEE Transactions on Instrumentation and Measurement, Vol. 37, no 3, September 1988, pp. 403-408.

[80] Essick, J.

Advanced LabView Labs.

Prentice Hall, New Jersey 1999.

[81] Fairney, W.; Eng, F.

Reactive power, real or imaginary ?.

Power Engineering Journal, April, 1994, pp. 69-75.

[82] Ferrero, A.; Lazzaroni, M.; Salicone, S.

A calibration procedure for a digital instrument for electric power quality measurement.

IEEE Transactions on Instrumentation and Measurement, Vol. 51, no 4, August 2002, pp. 716-722.

[83] Filipski, P.S.; Baghzouz, Y.; Cox, M.D.

Discussion of power definitions contained in the IEEE dictionary.

IEEE Transactions on Power Delivery, Vol. 9, n 3, July 1992, pp. 12371244.

[84] Filipski, P.S.; Labaj, P.W.

Evaluation of reactive power meters in the presence of high harmonic distortion.

IEEE Transactions on Power Delivery, Vol. 7, no 4, July 1994, pp. 17931799. 
[85] Filipski, P.S.

Polyphase apparent power and power factor under distorted waveform conditions.

IEEE Transactions on Power Delivery, Vol. 6, no 3, July 1991, pp. 11611165.

[86] Filipski, P.S.

Comments on "Measurement and compensation of ficticious power under nonsinusoidal voltage and current conditions.

IEEE Transactions on Instrumentation and Measurement, Vol. 38, $n^{\circ} 3$, June 1989, pp. 837-841.

[87] Finkelstein, F.; Morawski, R.

Fundamental concepts of measurement.

Measurement, Vol. 34, 2003, pp. 1-2.

[88] Furuhashi, T.; Okuma, S.; Uchikawa, Y.

A study on the theory of instantaneous reactive power.

IEEE Transactions on Industrial Electronics, Vol. 37, no 1, February 1990, pp. 86-90.

[89] García, R.; Regueiro, A.; Laiz, H.

A sampling wattmeter with a capacitive shunt for the measurement of power under highly distorted waveforms.

IEEE MOP 2000, pp. 36-37.

[90] Ghassemi, F.

New concept in AC power theory.

IEEE Proc. Gener. Transm. Distrib., Vol. 147, n6, November 2000, pp. 417-424.

[91] Ghassemi, F.

Verification of the new concept in ac power theory using energy conversion medium.

90 Conference on Harmonics and Quality of Power, Vol. 1, 2000, pp. 155161. 
[92] Ghassemi, F.

What is wrong with electric power theory and how it should be modified.

Metering and Tariffs for Energy Supply, no 462, May 1999, pp. 109-114.

[93] Gherasim, C.; Van den Keybus, J.; Driesen, J.; Belmans, R.

DSP implementation of power measurements according to the IEEE Trial-Use Standard 1459.

IEEE Trans. on Instr. and Meas., Vol. 53, no 4, August 2004, pp. 10861092.

[94] Gherasim, C.; Van den Keybus, J.; Driesen, J.; Belmans, R.

Power measurements based on the IEEE Trial-Use Standard 1459.

IMTC 2003-Instrumentation and Measurement Technology Conference, May 2003, pp. 1042-1047.

[95] Ghosh, A.; Joshi, A.; Mishra, M.

A new compensation algorithm for balanced and unbalanced distribution systems using generalized instantaneous reactive power theory.

Electric Power Systems Research, Vol. 60, 2001, pp. 29-37.

[96] Ghosh, A.; Joshi, A.

The use of instantaneous symmetrical components for balancing a delta connected load and power factor correction.

Electric Power Systems Research, Vol. 54, 2000, pp. 67-74.

[97] Ghosh, A.; Joshi, A.

A new approach to balancing and power factor correction in power distribution system.

IEEE Transactions on Power Delivery, Vol. 15, no 1, January 2000, pp. 417-422. 
[98] Gómez, A.; González, F.; Izquierdo, C.

Microprocessor based control o fan SVC for optimal load compensation.

IEEE Transactions on Power Delivery, Vol. 7, n 2, April 1992, pp. 706712.

[99] Henderson, I.A.; McGhee, J.; Szaniawski, W.; Domaradzki, P.

Incorporating high reliability into the design of microprocessor based instrumentation.

IEE Proceedings A, Vol. 138, no 2, March 1991, pp. 105-112.

[100] Ibáñez Talens, Enrique.

Contribución al conocimiento del comportamiento energético de receptores lineales funcionando en sistemas eléctricos con tensiones sinusoidales y desequilibradas.

Tesis Doctoral, Servicio de Publicaciones de la UPV, 2003.

[101] IEEE Published by Standards Network IEEE Press.

IEEE 100 The Autoritative Dictionary of IEEE Standards Terms.

7 a Edition, 2000.

[102] IEEE Published by Standards Network IEEE Press.

IEEE Std. 1459-2000, IEEE Trial Use Standard Definitions for the Measurement of Electric Power Quantities Under Sinusoidal, Nonsinusoidal, Balanced, or Unbalanced Conditions.

January 2000.

[103] IEEE Working group on nonsinusoidal situations: effects on meter performance and definitions of power.

Practical definitions for powers in systems with nonsinusoidal waveforms and unbalanced loads: a discussion.

IEEE Transactions on Power Delivery, Vol. 11, no 1, January 1996, pp. 79101.

[104] Jeon, Seong-Jeub.

Considerations on a Reactive Power Concept in a Multiline System.

IEEE Trans. on Power Delivery, Vol. 21, n 2, April 2006, pp. 551-559. 
[105] Jouanne, A.; Banerjee, B.

Assessment of voltage unbalanced.

IEEE Trans. on Power Delivery, Vol. 16, no 4, October 2001, pp. 782-790.

[106] Karimi-Ghartemani, M.; Mokhtari, H.; Reza Iravini, M.; Sedighy, M.

A signal processing system for extraction of harmonics and reactive current of single-phase systems.

IEEE Transactions on Power Delivery, Vol. 19, no 3, July 2004, pp. 979986.

[107] Karimi-Ghartemani, M.; Reza Iravini, M.

A signal processing module for power systems applications.

IEEE Transactions on Power Delivery, Vol. 18, no 4, October 2003, pp. 1118-1126.

[108] Knowlton, A. E.

Reactive power concepts in need of clarification.

Transactions A.I.E.E., September 1933, pp. 744-747.

[109] Kolanko, J.K.

Accurate measurement of power, energy, and true RMS voltage using synchronous counting.

IEEE Trans. on Instr. and Measurement, Vol. 42, no 3, June 1993, pp. 752-754.

[110] Lario García, J.;Pallás Areny, R.

Analysis of a three component impedance using two sine waves.

IMTC 2003 Instrumentation and Measurement, May 2003, pp. 1282-1284.

[111] Layer, E.

Non-standard input signals for the calibration and optimisation of the measuring systems.

Measurement, Vol. 34, 2003, pp. 179-186.

[112] Lázaro, A. M.

LabView. Programación gráfica para el control de instrumentación.

Editorial Paraninfo ITP, Madrid 1997. 
[113] León, V.; Montañana, J.; Giner, J.; Cazorla, A.; Roger, J.; Graña, M.

Power Quality Effects on the Measurement of Reactive Power in Three-Phase Power Systems in the Ligth of the IEEE Standard 1459-2000.

9th International Conference. Electrical Power Quality and Utilisation. Barcelona, 9-11. October, 2007. Libro de actas.

[114] León, V.; Graña, M.; Chouza, J.; Cazorla, A.; Montañana, J.; Giner, J. Modelización de fenómenos energéticos de desfase en sistemas trifásicos desequilibrados, lineales a tres hilos.

Conferencia Internacional de Energía y Mediambiente. TIEMA 2005. Camagüey, Cuba, Junio 2005. Universidad de Camagüey. Libro de actas.

[115] León, V.; Giner, J.; Montañana, J.; Cazorla, A.

Cortes en el suministro de energía eléctrica. Una solución a las sobrecargas de los transformadores de CT.

Mundo Electrónico, Vol. 1, no 327, 2002, pp. 34-38.

[116] León, V.; Giner, J.; Montañana, J.; Cazorla, A.

Ineficiencias de los sistemas eléctricos. Efectos, cuantificación y dispositivos de mejora de la eficiencia.

Editorial Universidad Politécnica de Valencia. 2001.

[117] León, V.; Giner, J.; Montañana, J.; Cazorla, A.

Eficiencia en las instalaciones eléctricas. Nuevas definiciones de potencia.

Mundo Electrónico, Vol. 1, no 322, 2001, pp. 28-32.

[118] León, V.; Giner, J.; Montañana, J.; Cazorla, A.

Compensación de reactiva en instalaciones desequilibradas. Optimización de prestaciones de los equipos de compensación. Mundo Electrónico, Vol. 1, no 317, 2001, pp. 54-57.

[119] León, V.; Giner, J.; Montañana, J.; Cazorla, A.

Medida de energías asociadas a desequilibrios de tensiones.

Conferencia Internacional "La eficiencia energética y el medio ambiente", 2001, Camagüey. Libro de actas. 
[120] León, V.; Giner, J.; Montañana, J.; Cazorla, A., Ibañez, E.

Efectos de los desequilibrios de tensiones sobre receptores lineales, en redes eléctricas trifásicas a tres hilos.

Eléctrica 2000 - Conferencia Científica Internacional FIE 2000, Santiago de Cuba. Libro de actas.

[121] León, V.; Giner, J.; Montañana, J.; Cazorla, A.

Revisión de los conocimientos de la potencia eléctrica en la docencia universitaria.

9a Reunión Nacional de Grupos de Investigación en Ingeniería Eléctrica, 1999, Terrassa, pp. 33-38.

[122] León, V.; Giner, J.; Montañana, J.; Cazorla, A.

Mejora de la eficiencia en sistemas lineales en B.T. con tensiones desequilibradas.

8a Reunión Nacional de Grupos de Investigación en Ingeniería Eléctrica, 1998, Cádiz, pp. 361-369.

[123] León Martínez, Vicente.

Método integral para la mejora de la eficiencia y la calidad del suministro de la energía eléctrica en Baja Tensión.

Tesis Doctoral, Servicio de Publicaciones de la UPV, 1997.

[124] Lu, S.; Lin, C.; Huang, C.

Suggested power definition and measurement due to harmonic load.

Electric Power Systems Research, Vol. 53, 2000, pp. 73-78.

[125] Lyon, W.V.

Reactive power and unbalanced circuits.

Electrical World, Vol. 75, no 25, June 1920.

[126] Mañana, M.

Instrumentación de muestreo. Aplicación a la medida de potencia.

XVII Cursos de Verano de la Universidad de Cantabria. Julio 2001, Libro de actas. 
[127] Marei, M.I.; El-Saadany, E.F.; Salama, M.

A processing unit for symmetrical components and harmonics estimation based on a new adaptative linear combiner structure. IEEE Transactions on Power Delivery, Vol. 19, no 3, July 2004, pp. 12451252.

[128] Martos, J.; Castelo, J.

Vatihorímetro electrónico con conexión en red.

Mundo Electrónico, Vol. 329, Marzo 2002, pp. 42-46.

[129] Martos, J.; Castelo, J.

Consideraciones sobre la medida digital de energía eléctrica.

Mundo Electrónico, Vol. 327, Enero 2002, pp. 50-53.

[130] Masud, S.

Error analysis of digital phase measurement of distorted waves.

IEEE Transactions on Instrumentation and Measurement, Vol. 38, no 1 , February 1989, pp. 6-9.

[131] Milanez, D.;

New concepts of the power received by ideal energy storage elements: the instantaneous complex power approach.

IEEE Proceedings A, 1997, pp. 1038-1041.

[132] Milanez, D.; Emanuel, A.E.

The instantaneous space phasor a powerful diagnosis tool.

IEEE Transactions on Instrumentation and Measurement, Vol. 52, $\mathrm{n}^{0} 1$, February 2003, pp. 143-148.

[133] Mirri, D.; Iuculano, G.; Menchetti, A.; Filicori, F.; Catelani, M.

Recursive random sampling strategy for a digital wattmeter.

IEEE Trans. on Instr. and Meas., Vol. 41, no 6, December 1992, pp. 979984.

[134] Montañana, J.; León, V.; Giner, J.; Cazorla, A.

Tratamiento de señales eléctricas mediante FFT y Wavelets.

4a Jornadas Investigación y Fomento de la Multidisciplinariedad, Valencia 2002, Libro de actas. 
[135] Montañana, J.; León, V.; Giner, J.; Cazorla, A.

Measurement of energies associated of power quality with unbalanced and nonsinusoidal voltages in electric power systems.

$14^{\text {th }}$ Power Systems Computation Conference PSCC 02, July 2002, pp. 1-5.

[136] Montañana, J.; León, V.; Giner, J.; Cazorla, A.

Medida de las potencias, eficiencia y calidad de suministro en los sistemas eléctricos de potencia con tensiones desequilibradas.

SAAEI 01, Septiembre 2001, Matanzas (Cuba), pp. 1-4.

[137] Montañana, J.; León, V.; Giner, J.; Cazorla, A.

Estudio energético de lámparas de bajo consumo comerciales mediante el instrumento de medida SIMPELEC.

Eléctrica 2000 - Conferencia Científica Internacional FIE 2000, Santiago de Cuba. Libro de actas.

[138] Montañana, J.; León, V.; Giner, J.; Cazorla, A.

Modelos equivalentes de Pspice de lámparas de bajo consumo comerciales.

Eléctrica 2000 - Conferencia Científica Internacional FIE 2000, Santiago de Cuba. Libro de actas.

[139] Montañana, J.; León, V.; Giner, J.; Cazorla, A.

Simulación de lámparas de bajo consumo con Pspice.

Revista Española de Electrónica, no 550, 2000, pp. 106-109.

[140] Montañana, J.; León, V.; Giner, J.; Cazorla, A.

Instrumento de medida de la eficiencia en el suministro de la energía eléctrica en baja tensión.

INTERCON 99, Lima (Perú), 1999, Libro de actas.

[141] Montañana, J.; León, V.; Giner, J.; Cazorla, A.

Estudio comparativo de lámparas de bajo consumo.

9a Reunión Nacional de Grupos de Investigación en Ingeniería Eléctrica, 1999, Terrassa, pp. 22-27. 
[142] Montañana, J.; León, V.; Giner, J.; Cazorla, A.

Medida de la potencia eléctrica en sistemas trifásicos asimétricos no lineales con tensiones desequilibradas no senoidales.

6a Jornadas Luso-Espanholas de engenharia electrotecnica, 1999, Lisboa, pp. 157-164.

[143] Montañana Romeu, Joaquín.

Medida Integral de la eficiencia en el suministro de energía eléctrica en baja tensión.

Tesis Doctoral, Servicio de Publicaciones de la UPV, 1999.

[144] Montaño, J.C.; Salmerón, P.; Vázquez, J.; Prieto, J.; Pérez, A.

Compensation in nonsinusoidal, unbalanced three phase four wire systems with active power line conditioner.

IEEE Transactions on Power Delivery, Vol. 19, no 4, October 2004, pp. 1968-1974.

[145] Montaño, J.C.; Salmerón, P.

Strategies of instantaneous compensation for three phase four wire circuits.

IEEE Transactions on Power Delivery, Vol. 17, no 4, October 2002, pp. 1079-1084.

[146] Montaño, J.C.; Gutiérrez, J.; Castilla, M.; López, A.

Power quality improvement in reactive power control using FCTCR circuits.

IECON 02, Vol. 2, 2002, pp. 880-885.

[147] Montaño, J.C.; Salmerón, P.

Identification of instantaneous current components in three phase systems.

IEEE Proc. Sci. Meas. Technol., Vol. 146, nº 5, September 1999, pp. 227233.

[148] Montaño, J.C.; Gutiérrez, J.; Castilla, M.; López, A.

DSP based algorithm for electric power measurement.

IEEE Proceedings A, Vol. 140, nº 6, November 1993, pp. 485-490. 
[149] Montaño, J.C.; Ibáñez, M.C.; Ojeda, A.; Benítez, J.

A TMS320 based reactive power meter.

IEEE Proceedings, 1990, pp. 268-272.

[150] Montaño, J.C.; Ibáñez, M.C.; Ojeda, A.; Benítez, J.

Measurement of apparent power components in the frequency domain.

IEEE Trans. on Instrumentation and Measurement, Vol. 39, no 4, August 1990, pp. 583-587.

[151] Moreno-Eguilaz, J.; Peracaula, ].

Understanding AC power using generalizad instantaneous reactive power theory: considerations for instrumentation of three phase electronic converters.

ISIE 99, Slovenia, 1999, pp. 1273-1277.

[152] Nabae, A.; Tanaka, T.; Funabiki, S.

A new approach to an individual phase reactive power compensator for nonsinusoidal and unbalanced three phase systems.

Electrical Engineeering in Japan, Vol. 139, no 3, 2002, pp. 73-81.

[153] Nabae, A.; Tanaka, T.

A Quasi instantaneous reactive power compensator for unbalanced and nonsinusoidal three phase systems.

ICHPQ 98, Greece, 1998, pp. 823-828.

[154] Nabae, A.; Tanaka, T.; Yoshikawa, A.; Cao, L.

A new definition of instantaneous active reactive current and power in three phase circuits, and its application.

Electrical Engineeering in Japan, Vol. 121, no 2, 1997, pp. 83-90.

[155] Nabae, A.; Tanaka, T.

A new definition of instantaneous active reactive current and power based on the instantaneous space vectors on polar coordinates in three phase circuits.

IEEE Transactions on Power Delivery, Vol. 11, July 1996, pp. 1218-1243. 
[156] Petrovic, P.

New digital multimeter for accurate measurement of synchronously sampled AC signals.

IEEE Transactions on Instrumentation and Measurement, Vol. 53, no 3, June 2004, pp. 716-725.

[157] Picinbono, B.

On instantaneous amplitude and phase of signals.

IEEE Trans. on Signal Processing, Vol. 45, no 3, March 1997, pp. 552-560.

[158] Pindado, R.; Rodríguez, P.; Pou, J.

Incidencia del desequilibrio de la carga en convertidores trifásicos SPWM DC-AC con tres y cuatro hilos.

XIII Reunión Nacional de Grupos de Investigación en Ingeniería Eléctrica, 2003, Vigo, Libro de actas.

[159] Piotrowski, T.

Instantaneous components of power and actual physical phenomena in sinusoidal circuits.

ICECS 2000, Vol. 2, 2000, pp. 805-808.

[160] Podestá, L.; Sangiovanni, S.; Zappitelli, E.

A new system for distorted three phase sinusiodal quantities measurement.

Measurement, Vol. 32, 2002, pp. 1-6.

[161] Pogliano, U.

Use of integrative analog to digital converters for high precision measurement of electrical power.

IEEE Transactions on Instrumentation and Measurement, Vol. 50, $n^{\circ} 5$, October 2001, pp. 1315-1318.

[162] Ramm, G.; Moser, H.; Braun, A.

A new scheme for generating and measuring active, reactive, and apparent power at power frequencies with uncertainties of $2,5 \times 10 e^{-6}$.

IEEE Transactions on Instrumentation and Measurement, Vol. 48, no 2, April 1999, pp. 422-426. 
[163] Redondo, F.

Energía Reactiva y disminución de las pérdidas en distribución de energía eléctrica.

Energía, Julio-Agosto 1998, pp. 91-94.

[164] Ribeiro, M.; Romano, J.; Duque, C.

An improved method for signal procesing and comprensión in power quality evaluation.

IEEE Transactions on Power Delivery, Vol. 19, no 2, April 2004, pp. 464471.

[165] Saranovac, L.

Digital realization of frequency insensitive phase shifter for reactive Var-hour meters.

IEEE Transactions on Instrumentation and Measurement, Vol. 49, no 4, August 2000, pp. 802-808.

[166] Sharon, D.

Power factor definitions and power transfer quality in nonsinusoidal situations.

IEEE Transactions on Instrumentation and Measurement, Vol. 45, $n^{\circ} 3$, June 1996, pp. 728-733.

[167] Sidhu, T.

Accurate measurement of power system frequency using a digital signal processing technique.

IEEE Transactions on Instrumentation and Measurement, Vol. 48, $n^{0} 1$, February 1999, pp. 75-81.

[168] Slomovitz, D.; Carbia G.

Medición de energía y otros parámetros eléctricos basada en sistemas electrónicos.

IEEE Proceedings of $5^{\circ}$ Encuentro de Potencia, Instrumentación y Medidas, 1999, Uruguay. 
[169] Slonim, M.A.; Van Wyk, J.D.

Power components in a system with sinusoidal and nonsinusoidal voltages and or currents.

IEEE Proceedings B, Vol. 135, no 2, March 1988, pp. 76-84.

[170] Sun, S. Q.; Jiang, C. G.

Decomposition of Czarnecki's reactive current and reactive power.

IEE Proceedings B, Vol. 138, no 3, May 1991, pp. 125-128.

[171] Sun, S. Q.; Kiyokawa, H.

Decomposition of voltage, current and power.

IEE Proceedings B, Vol. 138, no 1, Juanary 1991, pp. 35-39.

[172] Sydenham, P.H.

Relationship between measurement, knowledge and advancement.

Measurement, Vol. 34, 2003, pp. 3-16.

[173] Tarafdar, M.; Ise, T.

Implementation of single phase pq theory.

PCC Osaka, 2002, pp. 761-765.

[174] Terzija, V.V.; Stanojevic, V.

Power quality indices estimation using robust estimation approach.

Electrical Engineering, April 2004.

[175] Tarafdar, M.; Ise, T.

Reactive power and energy measurement in the frequency domain using random pulse arithmetic.

IEEE Proc. Sci. Meas. Technol., Vol. 148, no 2, March 2001, pp. 63-67.

[176] Torrents, J.M.; Pallás, R.

Uncertainty analysis in two terminal impedeance measurements with residual correction.

IEEE Instrumentation and Measurement Technology Conference, Hungry 2001, pp. 1450-1453. 
[177] Watanabe, E. H.; Stephan, R.; Aredes, M.

New concepts of instantaneous active and reactive powers in electrical systems with generic loads.

IEEE Transactions on Power Delivery, Vol. 8, no 2, April 1993, pp. 697703.

[178] Wilczynski, E.

Total apparent power of the electrical system for periodic, deformed waveforms.

IEE Proc. Electr. Power Appl., Vol. 147, no 4, July 2000, pp. 281-285.

[179] Willems, J. L.

Reflections on apparent power and power factor in nonsinusoidal and polyphase situations.

IEEE Transactions on Power Delivery, Vol. 19, no 2, April 2004, pp. 835840.

[180] Willems, J. L.; Aeyeles, D.

New decomposition for three phase currents in power systems.

IEEE Proceedings C, Vol. 140, no 4, July 1993, pp. 307-310.

[181] Willems, J. L.

A new interpretation of the Akagi-Nabae power components for nonsinusoidal three phase situations.

IEEE Transactions on Instrumentation and Measurement, Vol. 41, no 4, August 1992, pp. 523-527.

[182] Yang, J. Z.; Liu, C. W.

A precise calculation of power system frequency and phasor.

IEEE Transactions on Power Delivery, Vol. 15, n 2, April 2000, pp. 494499.

[183] Yoon, W. K.; Devaney, M. J.

Reactive power measurement using the wavelet transform.

IEEE Transactions on Instrumentation and Measurement, Vol. 49, n⿳ 2, April 2000, pp. 246-252. 
[184] Zheng, T.; Makram, E. B.; Girgis, A. A.

Evaluating power system unbalance in the presence of harmonic distortion.

IEEE Transactions on Power Delivery, Vol. 18, n 2, April 2003, pp. 393397.

[185] Zheng, F.; Ott, G. W.; Adams, D. J.

Harmonic and reactive power compensation based on the generalized instantaneous reactive power theory for three phase four wire systems.

IEEE Transactions on Power Electronics, Vol. 13, no 6, November 1998, pp. 1174-1181.

[186] Zheng, F.; Lai, J. S.

Generalized instantaneous reactive power theory for three phase power system.

IEEE Transactions on Instrumentation and Measurement, Vol. 45, $\mathrm{n}^{0} 1$, February 1996, pp. 293-297. 


